\title{
IMPLEMENTATION OF PHYSIOLOGIC PRESSURE CONDITIONS IN A BLOOD VESSEL MIMIC BIOREACTOR SYSTEM
}

\author{
A Thesis \\ presented to \\ the Faculty of California Polytechnic State University, \\ San Luis Obispo
}

\author{
In Partial Fulfillment \\ of the Requirements for the Degree \\ Master of Science in Biomedical Engineering \\ By Kevin Mark Okarski
}

April 2010 
(C) 2010 Kevin M. Okarski

ALL RIGHTS RESERVED 
THESIS COMMITTEE MEMBERSHIP

Title: IMPLEMENTATION OF PHYSIOLOGIC PRESSURE CONDITIONS IN A BLOOD VESSEL MIMIC BIOREACTOR SYSTEM FOR THE EVALUATION OF INTRAVASCULAR DEVICES

Author: Kevin M. Okarski

Submitted: April, 2010

Committee Chair: Dr. Kristen O’Halloran Cardinal

Committee Member: Dr. Trevor Cardinal

Committee Member: Dr. Lily Laiho 


\author{
ABSTRACT \\ Implementation of Physiologic Pressure Conditions in \\ a Blood Vessel Mimic Bioreactor System \\ Kevin Mark Okarski
}

Tissue engineering has traditionally been pursued as a therapeutic science intended for restoring or replacing diseased or damaged biologic tissues or organs. Cal Poly’s Blood Vessel Mimic Laboratory is developing a novel application of tissue engineering as a tool for the preclinical evaluation of intravascular devices. The blood vessel mimic (BVM) system has been previously used to assess the tissue response to deployed stents, but under non-physiologic conditions. Since then, efforts have been made to improve the vessel and bioreactor's ability to emulate in vivo conditions. The ability to tissue engineer constructs similar to their native tissue counterparts is heavily reliant upon controlling the environment and mechanical stimuli the construct is exposed to. Mimicking physiologic conditions influences cellular growth, proliferation, and differentiation. Two important mechanical stimuli are cyclic strain and wall shear stress. Previous work sought to improve these factors within the BVM bioreactor and resulted in the implementation of pulsatile perfusion and increased fluid viscosity. These previous bioreactor design modifications generated pulsatile pressures of approximately $80 \mathrm{mmHg}$ and a wall shear stress of 6.4 dynes $/ \mathrm{cm}^{2}$. However, physiologic pressure waveforms were not achieved.

Studies in this thesis were carried out to implement an effective means of establishing a more physiologic pressure wave within the bioreactor that is accurate, 
consistent, and easily adjustable. As a result of conducting the present studies, modifications to the bioreactor system were made that uphold the overall goals of efficacy and efficiency. The desired pressure wave was created by setting the degree of pump tubing occlusion on the 3-roller peristaltic pump head and using a water column to backpressure the bioreactor chamber. Maintaining a desired backpressure within the system necessitated the development of a new bioreactor chamber with increased extraluminal leak pressure resistance. The opportunity was also used to further improve upon the bioreactor chamber design to allow for $360^{\circ}$ rotation to reduce cell sedimentation. Modifications to the bioreactor system required quantitative evaluation to assess their impact upon local flow dynamics to the tissue construct. A system model was created and evaluated using computational modeling.

Through the work performed in this thesis, pulsatile pressure waves of approximately $120 / 80 \mathrm{mmHg}$ were successfully established within the bioreactor. The ability to accurately model physiologic pressures will ultimately help yield tissue constructs more similar to native tissues - both healthy and pathological. The newly designed bioreactor chamber and computational model for the system will be helpful tools for implementing or evaluating future bioreactor developments or improvements. While the main objective of the thesis has been completed by creating a system capable of emulating physiologic pressure fluctuations, there still remains room for further improvements in back-pressuring and scaling the system, refining the rotational bioreactor chamber design, and building upon the complexity and accuracy of the computational model. 


\section{ACKNOWLEDGEMENTS}

First, I would like to thank Dr. Kristen O'Halloran Cardinal for the time, support, and guidance she has given me over the duration of my project. I also greatly appreciate the opportunity to work in the BVM Lab and contribute to the research. I am excited to see what the future may hold for the laboratory.

Additionally, I would like to thank Dr. Lily Laiho and Dr. Trevor Cardinal for their time and willingness to undertake the task of serving as committee members, evaluating the thesis, and providing input.

I would also like to thank Dr. David Clague for teaching me the intricacies of computational modeling, providing guidance, and where to find relevant resources. 


\section{TABLE OF CONTENTS}

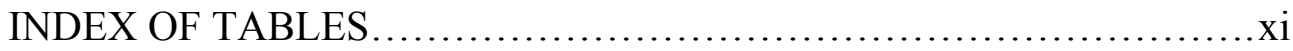

INDEX OF FIGURES............................................

\section{BACKGROUND}

\section{CHAPTER 1: INTRODUCTION}

Cardiovascular Physiology: Blood Vessels........................1

Tissue Engineering: Blood Vessels...............................

Contemporary Work Engineering Blood Vessels...................9

Establishing Physiologic Pressure Waves....................10

Effects of Pulsatile Conditions...............................14

Summary and Goals of the Thesis................................19

Original BVM System...................................21

Goals...................................................... 26

\section{A NEW BIOREACTOR CHAMBER}

\section{CHAPTER 2: TESTING ORIGINAL BIOREACTOR CHAMBER}

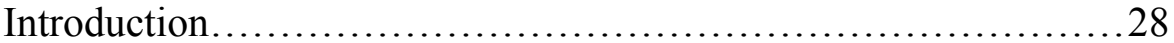

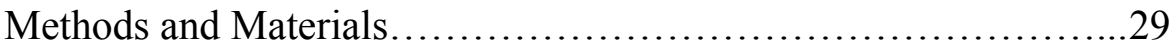

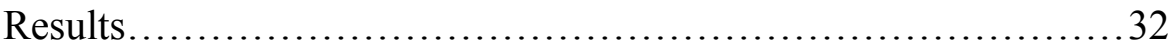

Discussion and Conclusion.......................................... 33

CHAPTER 3: DESIGNING THE NEW BIOREACTOR CHAMBER

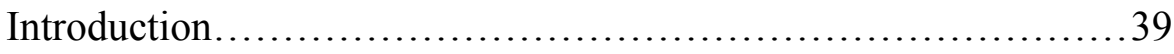

Methods and Materials.......................................... 40

Material Selection...................................... 40

The Design............................................. 44

Fabrication........................................... 46

Assembly ......................................................... 
End Plates...........................................54

Dumbbell............................................54

Adding Acrylic Wall................................55

End Caps.............................................56

CHAPTER 4: CHARACTERIZING NEW BIOREACTOR CHAMBER Introduction............................................... 58

Methods and Materials ...................................... 60

Results...................................................... 64

Discussion and Conclusion.................................... 66

\section{BACK-PRESSURING THE SYSTEM}

CHAPTER 5: QUANTITATIVE LEAK DIAGNOSTICS

Introduction................................................... 68

Methods and Materials.................................... 70

Results...................................................... 75

Pilot study ......................................... 75

Main study........................................ 77

Discussion and Conclusion...................................... 81

CHAPTER 6: SELF REGULATING INJECTION

Introduction.............................................. 86

Methods and Materials........................................... 88

Results................................................... 90

Discussion and Conclusion................................... 92

CHAPTER 7: WATER COLUMN

Introduction............................................ 101

Methods and Materials..................................... 102

Results.................................................. 105

Discussion and Conclusion .................................. 105

CHAPTER 8: WATER COLUMN II

Introduction............................................... 108 
Methods and Materials...................................... 109

Results................................................... 110

Discussion and Conclusion......................................... 111

CHAPTER 9: WATER COLUMN III

Introduction.............................................. 112

Methods and Materials......................................... 114

Results............................................... 118

Discussion and Conclusion..................................... 120

CHAPTER 10: WATER COLUMN IV

Introduction........................................... 126

Methods and Materials........................................ 127

Results.................................................... 128

Discussion and Conclusion....................................... 130

CHAPTER 11: WATER COLUMN V

Introduction............................................... 132

Methods and Materials..................................... 132

Results.................................................. 134

Discussion and Conclusion...................................... 136

\section{BACKPRESSURE TECHNIQUE EVALUATION}

CHAPTER 12: 72 HOUR TEST

Introduction............................................. 140

Methods and Materials.................................... 141

Results............................................... 141

Discussion and Conclusion....................................... 142

CHAPTER 13: 72 HOUR TEST II

Introduction................................................. 145

Methods and Materials...................................... 145

Results............................................... 146

Discussion and Conclusion...................................... 152 
CHAPTER 14: BIOREACTOR FLUID DYNAMICS EVALUATION

Introduction.............................................. 157

Methods and Materials................................... 159

Results .............................................. 162

Discussion and Conclusion................................. 164

\section{CONCLUDING REMARKS}

CHAPTER 15: DISCUSSION AND CONCLUSION

Future Work........................................ 172

Conclusion............................................ 175

LIST OF REFERENCES......................................... 177

APPENDIX A: RAW DATA.................................. 182

APPENDIX B: QUALITATIVE LEAK ASSESSMENT ............... 248

APPENDIX C: STATISTIC DESIGN OF EXPERIMENT............. 256

APPENDIX D: DESIGN CONSIDERATIONS ..................... 260 


\section{INDEX OF TABLES}

Table 1.1 Mechanical properties of tissue engineered vessels................... 18

Table 2.1 Lock N Lock Leak pressure test results.......................... 33

Table 4.1 Leak pressure test of new bioreactor chamber.................... 65

Table 5.1 Pilot study pressure loss measurements.......................... 76

Table 5.2 Least square means for factors and treatment combinations........... 80

Table $7.150 \mathrm{ml}$ reservoir water column trial................................. 105

Table 8.1 T225 reservoir water column trial.............................. 111

Table 9.1 Systolic and diastolic pressure measurements...................... 119

Table 10.1 Systolic and diastolic pressures reported hourly for a 31 hour test.... 129

Table 11.1 Systolic and diastolic pressures reported hourly for an 18 hour test.... 135

Table 12.1 Systolic and diastolic pressures test terminated after 13 hours........ 141

Table 13.1 Systolic and diastolic pressures reported hourly for 72 hours........ 147

Table A.1: 25 hour test............................................... 183

Table A.2: 31 hour test............................................ 194

Table A.3: 18 hour test................................................ 208

Table A.4: 72 hour test............................................ 216

Table A.5 Weighted syringe.......................................... 247

Table A.6 General linear model ANOVA table............................ 256

Table A.7 Least squares means for pressure drop........................ 256

Table A.8 Tukey pairwise comparison of treatment factors.................. 257

Table A.9 Data measurements for pressure leakage....................... 258 


\section{INDEX OF FIGURES}

Figure 1.1 Visual illustration of the multiple layers that make up a blood vessel... 1

Figure 1.2 An example of a blood vessel tissue engineering bioreactor .......... 4

Figure 1.3 Bioreactor setup with scalable design............................ 5

Figure 1.4 Initial concept driving bioreactor design......................... 10

Figure 1.5 The actual bioreactor system using ventilator mechanism............ 11

Figure 1.6 Pressure wave generated by bioreactor versus an actual heart......... 12

Figure 1.7 Bioreactor setup for novel pulse wave generator system............. 13

Figure 1.8 Presssure wave/flow rate measurements from wave generator......... 14

Figure 1.9 Mechanical stimulus and signal transduction..................... 17

Figure 1.10 Rabbits are used for assessing intravascular devices............... 20

Figure 1.11 Acrylic bioreactor chamber from original bioreactor system........ 22

Figure 1.12 Sara Leifer's bioreactor chamber design......................... 24

Figure 2.1 Data acquisition hardware ................................... 29

Figure 2.2 Setup for pressurized leakage test.............................. 31

Figure 2.3 Locations of fluid loss......................................... 33

Figure 2.4 Zip-tie placement to reduce fluid loss from bioreactor chamber....... 36

Figure 2.5 Silicone sealant placement.................................... 37

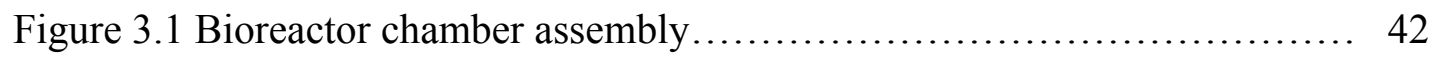

Figure 3.2 The progression of machining the aluminum end caps............... 53

Figure 3.3 Dumbbell assembly of bioreactor chamber........................ 55

Figure 3.4 Integration of dumbbell assembly with acrylic wall.................. 56

Figure 3.5 Final assembly of bioreactor chamber.......................... 57 
Figure 4.1 Bioreactor chamber system for pressure testing................. 61

Figure 4.2 The leak pressure test setup............................... 63

Figure 5.1 Experimental setup for pressure leak studies................... 73

Figure 5.2 Power curve generated from pilot study $\ldots \ldots \ldots \ldots \ldots \ldots \ldots \ldots \ldots \ldots . \ldots \ldots$

Figure 5.3 Normality and variance plots............................. 78

Figure 5.4 Interaction plot....................................... 79

Figure 6.1 Weighted syringe assembly used to backpressure system.......... 89

Figure 6.2 Measured backpressure over time........................... 92

Figure 6.3 Modeled weighted syringe backpressure setup.................. 93

Figure 6.4 Observational evaluation of trapped bubble..................... 96

Figure 6.5 Hypothetical weighted syringe setup.......................... 98

Figure 7.1 The horizontal assembly of the water column system.............. 103

Figure 7.2 Water column backpressure setup with $50 \mathrm{ml}$ reservoir............. 104

Figure 7.3 Actual water column setup used to backpressure the system......... 104

Figure 7.4 Negligible height difference within a cross-section of tubing........ 106

Figure 8.1 Water column backpressure setup with $250 \mathrm{ml}$ reservoir........... 109

Figure 8.2 Water column backpressure setup with $250 \mathrm{ml}$ reservoir........... 110

Figure 9.1 Independently attached water column........................ 113

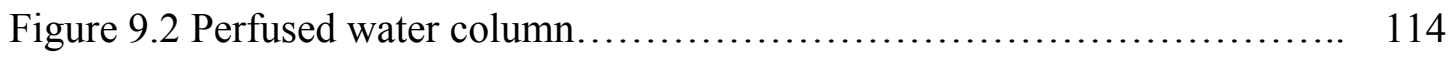

Figure 9.3 Bioreactor setup used to generate physiologic pressure waveforms... 116

Figure 9.4 Sampled pressure waveform after 2 hours.................... 120

Figure 9.5 An example of the system's ability to self-regulate............... 122

Figure 10.1 System modification to allow venting........................ 128 
Figure 10.2 An example of the generated pressure $\ldots \ldots \ldots \ldots \ldots \ldots \ldots \ldots \ldots \ldots . \ldots \ldots$

Figure 11.1 Reservoir cap with 3 ports............................... 133

Figure 11.2 Recorded pressure wave during 18 hour test................... 135

Figure 13.1 Recorded pressure waveform for 72 hour study................. 148

Figure 13.2 Illustration of the pressure transducer removal process............ 148

Figure 13.3 Example of drifting pressure transducer calibration............... 151

Figure 14.1 Bioreactor with reservoir, pump, and bioreactor chamber.......... 158

Figure 14.2 A simplified illustration of fitting/scaffold interface.............. 159

Figure 14.3 Fluid velocity profile at time 0.5 seconds..................... 163

Figure 14.4 Radial and longitudinal cross sections of wall shear stress......... 164

Figure 14.5 An illustration of how velocity profile relates to wall shear stress.... 166

Figure 14.6 Longitudinal cross section of velocity profile with inlet bias....... 167

Figure 15.1 Characteristic coronary artery pressure waveform.............. 174

Figure A.1 Three luer lock valves used in study........................ 255

Figure A.2 Statistical charts used for assessing quality of data.............. 259

Figure A.3 Back-pressure regulator schematic......................... 266

Figure A.4 SRC fitting from Colder Products Company..................... 268

Figure A.5 Bioreactor chamber rotary rack.......................... 272

Figure A.6 Three stages of elevating the bioreactor chamber............... 273 


\section{Chapter 1: INTRODUCTION}

\section{Cardiovascular Physiology: Blood Vessels}

It is impossible to engineer a tissue without an understanding of its native physiology. The cardiovascular system essentially includes the heart and blood vessels. For the purpose of this thesis, the focus is primarily on arteries. Larger blood vessels (Figure 1.1), such as arteries, are comprised of three separate layers: the tunica intima, tunica media, and tunica adventitia $(33,34)$. The tunica intima consists of a monolayer of endothelial cells supported by connective tissue. This monolayer lines the inner lumen of the vessel and is thus the blood contacting surface. The individual endothelial cells are sealed together by tight junctions. Tight junctions play a role in mass transport as they restrict diffusion of large molecules across the endothelium (15). Endothelial cells control vascular permeability, vasoconstriction, angiogenesis, and regulation of coagulation (15).

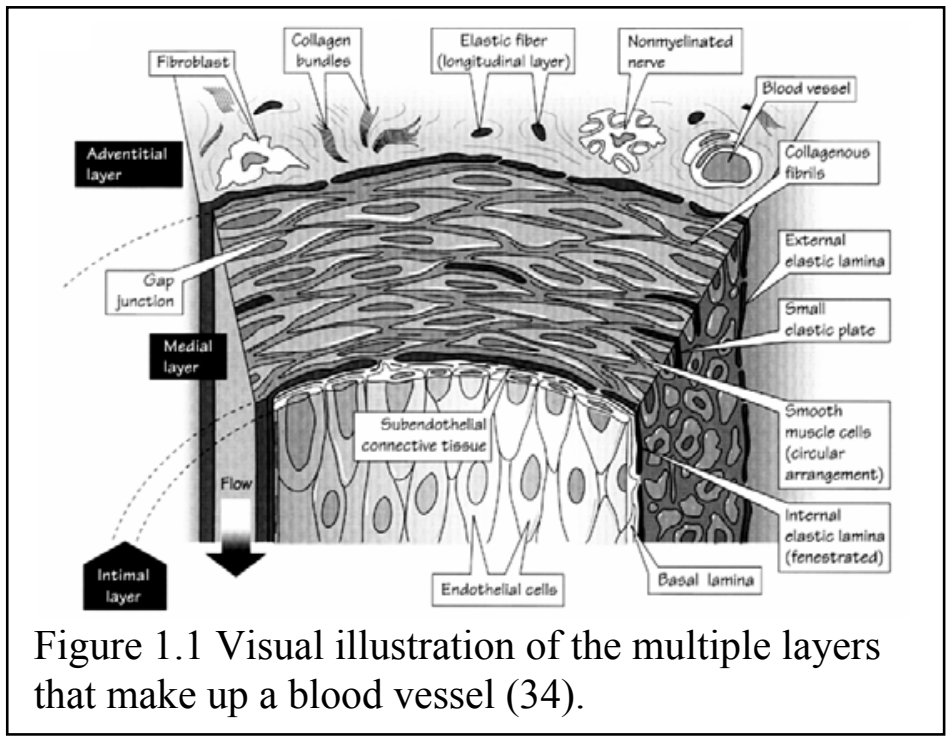


The tunica intima's thickness is proportional to vessel size. Larger vessels have thicker layers, but the layer still is only a monolayer of endothelial cells. The larger vessels even contain some smooth muscle cells (SMCs) within this innermost layer (34, 38). The basal lamina of the tunica intima is primarily composed of type IV collagen. The tunica media is the middle layer of the vessel. It is separated from the tunica intima by the internal elastic lamina, which is a fenestrated sheath composed primarily of elastin (15). The tunica media is made up of SMCs supported by extracellular matrix (ECM) made of mostly type III collagen, elastin, and proteoglycans $(4,20,38,39)$. The SMCs are shaped liked irregular cylinders with tapered ends and are typically between 15-100 $\mu \mathrm{m}$ in length. The SMCs are oriented in a low-pitch spiral which allows the vessels to constrict when the cells contract. While endothelial cells have tight junctions, adjacent SMCs form gap junctions. Gap junctions are characterized as areas of close cellular contact where connexons span the adjacent cell membranes (34). These channels allow ions to flow between cells and play an important role in intercellular communication (34). The cells thus form a syncitium where depolarization can spread from one cell throughout the entire network of SMCs. The third and outermost layer, the tunica adventitia, is separated from the tunica media by an external elastic lamina. The tunica adventitia contains type I and VIII collagen which supports fibroblasts and nerves $(4,10,20,38,39)$. The outer layer in larger arteries also contains the vasa vasorum, which supplies the vessel with oxygen and nutrients.

The function of the cardiovascular system is more complex than just an interconnected network of tubular conduits transporting fluids. The fluid transport is not steady; blood pulses with the synchronous beats of the heart and pressure waves are 
generated. While pulsatility and fluctuating pressure can cause blood vessel movement, vasomotion (dilation or constriction) can occur independently of the heart rhythm or internal pressure (36). When attempting to recreate blood vessels, the complexity of the system is modeled as accurately as possible using a bioreactor. The bioreactor typically contains a scaffold upon which the tubular vessel is cultivated. With some tissue engineered blood vessels, the role of the tunica media and adventitia are replaced by that scaffold $(17,22,41,42,51)$. The scaffold provides the necessary physical support and topographical environment that endothelial and/or smooth muscle cells require to attach, grow, and to proliferate. However, just like the native physiology, tissue engineering blood vessels is more complex than just a scaffold, and successful tissue cultivation heavily relies upon environmental conditions and the ability to control them within a bioreactor system.

\section{Tissue Engineering: Blood Vessels}

Tissue engineering is a relatively new and growing field of research. Currently, promising progress is being made in cultivating blood vessels, cartilage, skin and many other types of tissue $(2,24,44)$. The technology of particular interest to this thesis involves the systems that are used to generate blood vessels. Most research facilities that are tissue engineering blood vessels use perfusion bioreactors. A perfusion bioreactor is generally made up of a pump, a closed circuit loop of silicone tubing, a bioreactor chamber, and a reservoir. The bioreactor chamber and reservoir are attached to the system in-line with the silicone tubing. The bioreactor chamber contains a scaffold seeded with cells for cultivation. The reservoir contains excess media which allows the 
bioreactor to continuously run for extended periods of time. The pump acts as the driving force of the perfusion system by circulating the media through the closed loop.

There are many different types of perfusion bioreactors (Figure 1.2) used for tissue engineering blood vessels, with variations possible by altering the pumps, and/or the chambers. For the pump, many different styles can be incorporated into the bioreactor depending on the desired fluid dynamics within the system.

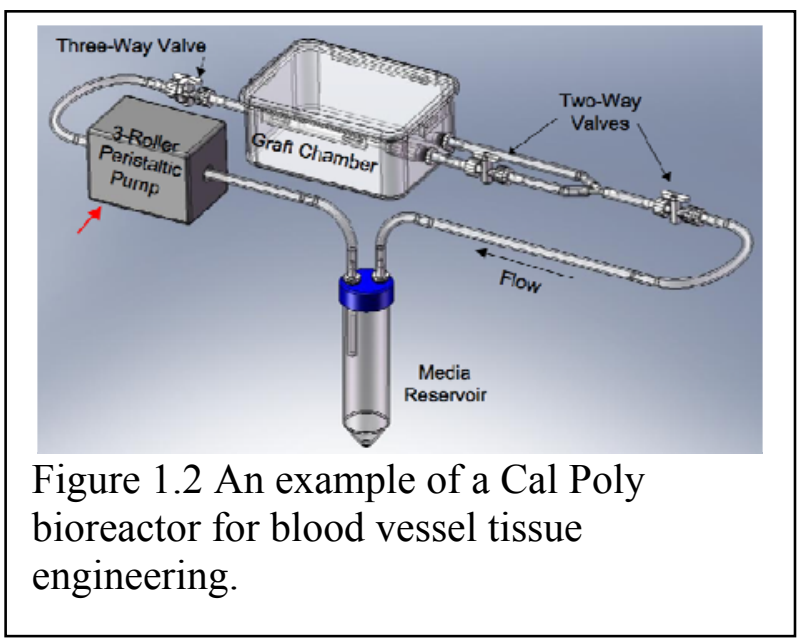

Researchers use many different styles of bioreactor chambers as well. For example, bioreactor chambers can be designed to accommodate different vessel sizes or they can be designed with the intention of scalability. Scalable chambers (Figure 1.3) can contain multiple tissue engineering scaffolds at one time or simply conserve space $(8,22)$. Even the reservoirs have multiple designs that can be potentially used within the bioreactor. Most reservoirs are just simple containers of various geometries, while others contain different styles of agitators that help ensure a homogenous mixture of media $(17,40)$. The material properties (stiff or flexible) of the reservoir can play an important role in 
dictating system conditions. A flexible wall reservoir has been used to dampen or eliminate pressure fluctuations within a perfusion bioreactor (18).

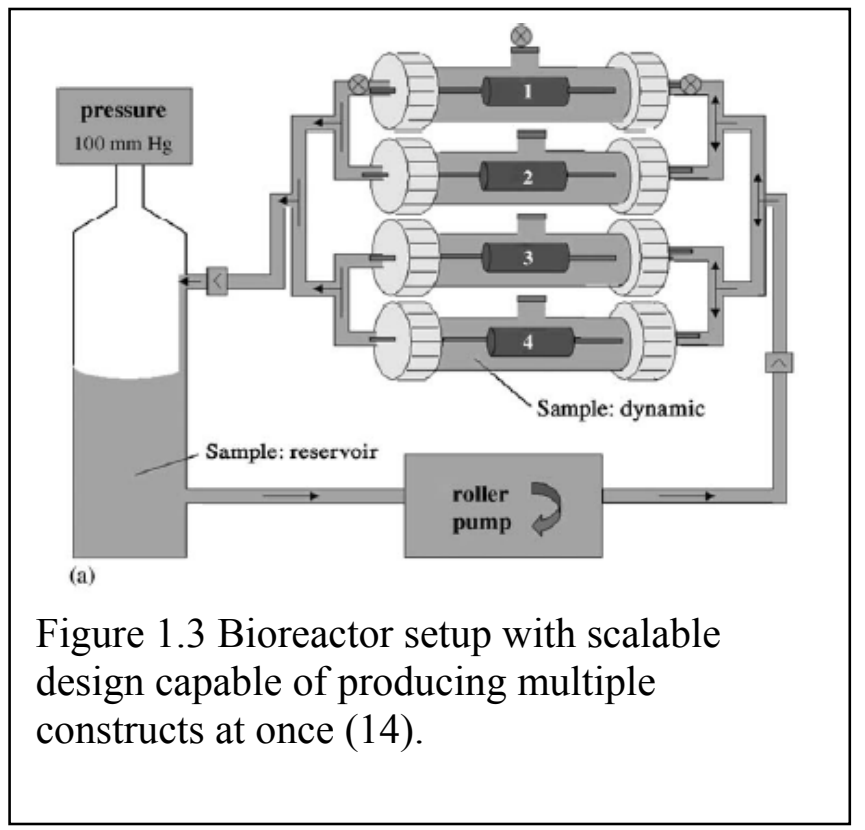

In order for cells to adhere or attach to the scaffolding, the scaffolding must have certain key properties, such as a similar porosity to that of native tissue $(3,27)$. Once cells are able to adhere to the surface of the scaffolding, the environment they are in affects their ability to proliferate and survive. Some of these conditions are easily controlled. For example, the bioreactors are maintained in incubators that regulate temperature and $\mathrm{C} 02$ content, which helps regulate $\mathrm{pH}$. Flow rates are also easily controlled using pumps. If physiologic conditions are not emulated in the bioreactor the cells may not develop the proper phenotypic expression, alignment, or grow into a vessel with mechanical properties comparable to native vessels - if the cells survive at all $(3,5$, 29). Physiologic pressures are much more difficult to recreate in vitro. The cyclic 
changes in pressure create varying hoop stresses on the walls of native vessels generating cyclic strains. These strains are believed to induce cell differentiation during vessel development and later dictate mechanical properties $(43,46,49)$.

Tissue engineering, in general, consists of a basic process which includes determining a cell source, obtaining and isolating the cells, seeding or sodding cells upon a scaffold, and then culturing the cells into tissue constructs within a bioreactor. There are a wide variety of cell sources with varying potencies (ability to differentiate into other cells). In general, stem and progenitor cells constitute the cell sources for many tissue engineering applications $(3,41,48,54)$. The list of stem cells includes hemopoietic, epithelial, mesenchymal, neuronal, and embryonic. These cells can be used in their original form for tissue engineering, but they require intrinsic and extrinsic factors in order to differentiate (3). Differentiated versions of these cells are known as progenitor cells, which are also widely used in tissue engineering. Progenitor cells have already differentiated down a particular cell lineage and can divide many times.

Once a cell source and type have been selected, the cells must be accessed and isolated. Isolation typically involves enzymatic digestion or mincing and centrifugation of sample tissue taken from a specific region of the body (3). The process of cell isolation rarely yields enough cells to be able to immediately place them upon a scaffold and generate a tissue. The cells are therefore cultured and passed until enough population doublings have occurred to provide the necessary amount of cells necessary to cultivate the desired tissue. The cells are then coupled with a scaffold in one of two-ways. Cell sodding is a technique where a high number of cells are placed upon a scaffold in a blanketing fashion. Cell seeding involves fewer cells which are inserted into or onto the 
matrix of the scaffold (3). After cell deposition by either technique, the scaffold is then placed within a bioreactor that mimics the native conditions of the desired tissue and the tissue is cultivated for an extended period of time until, ideally, the tissue resembles the mechanical and structural properties of the native tissue.

When applying the tissue engineering process to blood vessels specifically, the correct cell type and source to use must first be determined. For example, an embryonic stem cell could serve as a cell source for the specific goal of blood vessel cultivation. An embryo is stratified into three separate layers. The layers include the ectoderm, mesoderm, and endoderm. Endothelial cells differentiate within the splachnic mesoderm that faces the endoderm (34). The process is known as vasculogenesis. The endothelial precursors assemble and form a plexus. The plexus later gives rise to embryonic and extra-embryonic vessels on the yolk sac and the placenta. The endothelial plexus expands into the somatic mesoderm through angiogenesis. The plexus will eventually grow and develop into organ-specific vasculatures under the right conditions (intrinsic and extrinsic factors). Fetal liver kinase 1 (Flk-1) endothelial progenitor cells can be derived from embryonic stem cells and differentiated into endothelial cells in vitro on a type IV collagen-coated dish. Vascular endothelial growth factor and VE-cadherin can also added to the system to aid differentiation (21).

The endothelial cells isolated from this particular cell source must be sodded on a scaffold. It is important to achieve a confluent and uniform cell deposition within the scaffold because it ultimately translates into proportional and uniform tissue formation. A number of research facilities utilize rotary deposition models to achieve the desired uniformity $(14,17,22,35,58,60)$. Failure to achieve a uniform distribution of cells may 
have varied results depending on the cells. Endothelial cells grow to develop a monolayer. The ECs would have to migrate and proliferate in order to redistribute uniformly across the scaffold. Smooth muscle cells, on the other hand, do not require a monolayer arrangement to proliferate and survive. As a result, presumably, the SMCs will not redistribute on their own accord as readily and this may initially result in uneven tissue growth and require longer time periods for cultivation.

With the understanding that the plexus will develop into vasculatures under the proper conditions, the next logical step would be to simulate those conditions using a bioreactor - as discussed above. This is a seemingly simple task that is much more complex than some may realize. There are a variety of environmental factors that play a significant role in cell proliferation, differentiation, and phenotypic expression. These factors can include temperature, $\mathrm{CO} 2$ levels, $\mathrm{O} 2$ levels, $\mathrm{pH}$, pressure, growth factors, cell signaling, and mechanical stimulation including shear stress or cyclic strain $(3,5,29)$. Having identified some of the important conditions, the next step is controlling them. This is due to the desirability of replicating physiological conditions as closely as possible in order to promote development of a tissue construct with the appropriate properties. Of the factors listed, cyclic strain and wall shear stress are particularly crucial to mechanically stimulating and engineering a vessel construct with desired physical and mechanical properties $(6,11,18,37,43,46,49)$. The cyclic strain affects the SMCs and helps condition them into developing physical and mechanical properties similar to those found in native vessels. The wall shear stress affects the endothelial cells and their ability to organize, align, and mature $(43,46,49)$. 


\section{Contemporary Work Engineering Blood Vessels}

As the field of tissue engineering progresses, conditions necessary for cultivating replacement blood vessels are becoming better understood. Shear stress and cyclic strain have been found to be among the most important factors contributing to enhanced EC and SMC differentiation, phenotypic expression, and mechanical properties $(6,11,18,37,43$, $46,49,59)$. The wall shear stress is a mechanical stimulus that specifically applies to conditioning endothelial cells because they are in constant contact with the circulating flow of blood within the body. The continual exposure this stimulus implies wall shear stress is crucial factor guiding proper endothelial cell development $(11,37,54)$. Shear stress has been found to promote EC differentiation with greater cell adhesion and a more confluent layer on the scaffold surface - it even promotes the proper organization and alignment of cells $(37,54,59)$.

Preconditioning by cyclic strain has been found to reduce in vivo graft thrombosis and neointimal hyperplasia in decellularized arteries sodded with endothelial cells (59). Thrombosis formation can be a deadly side effect of various intravascular treatments or replacements since the clots may lead to heart attacks or strokes. Neointimal hyperplasia refers to the occlusion of the vessel. The reduced blood flow resulting from neointimal hyperplasia also has its fair share of consequences. For example, the increased resistance to blood flow results in elevated blood pressures. The cyclic strain stimulates the SMCs to differentiate and produce greater cell density, increased extracellular matrix, maturation, and organization $(46,49)$. It also increases the ability of endothelial cells to resist blood flow induced shear stress and fluid particulate wall adhesion (6). Since the focus of the thesis is on the implementation of physiologic pressure waves, it is 
imperative that previously utilized system designs and modifications are understood as well as their potential or expected benefits. Accordingly, methodologies used in the field of tissue engineering for establishing physiologic pressure waves and their effects will be explored.

\section{Establishing Physiologic Pressure Waves}

Over the past 10 years, a number of research groups have been introducing physiologic pressures in their bioreactors in order to help produce tissue constructs with more physiologic properties. In 2002, Thompson et al. incorporated a mechanical ventilator into their bioreactor. The goal of the study was to establish physiologic pressure and flow conditions. The ventilator generated both pulsatile flow and physiologic pressure waves (Figures 1.4 and 1.5) that more closely resembled the waves generated by the heart rather than a peristaltic pump (52). In essence, having achieved their goal, the researchers intended to further study the effects of these more realistic pressure waves and fluid pulsatility upon cell signaling and tissue cultivation.

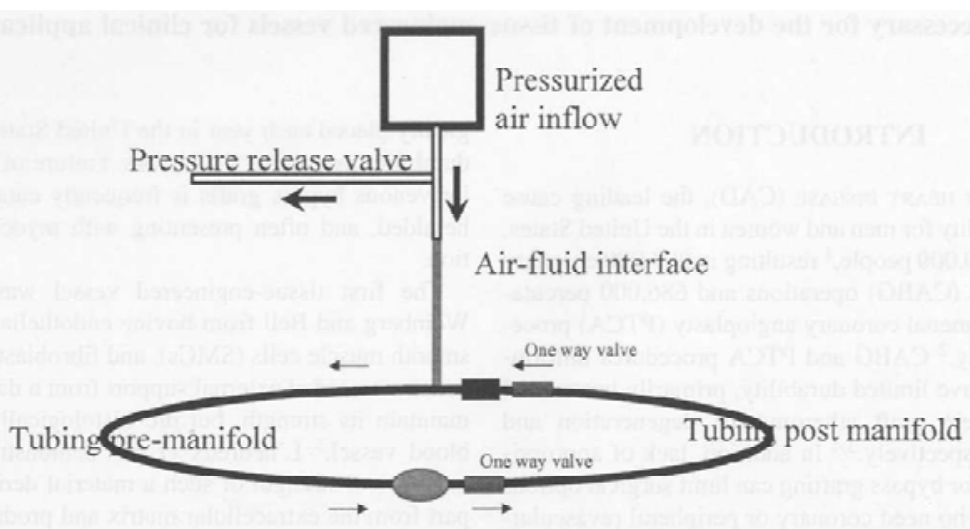

Figure 1.4 Initial concept by Thompson et al for their bioreactor design (52). 


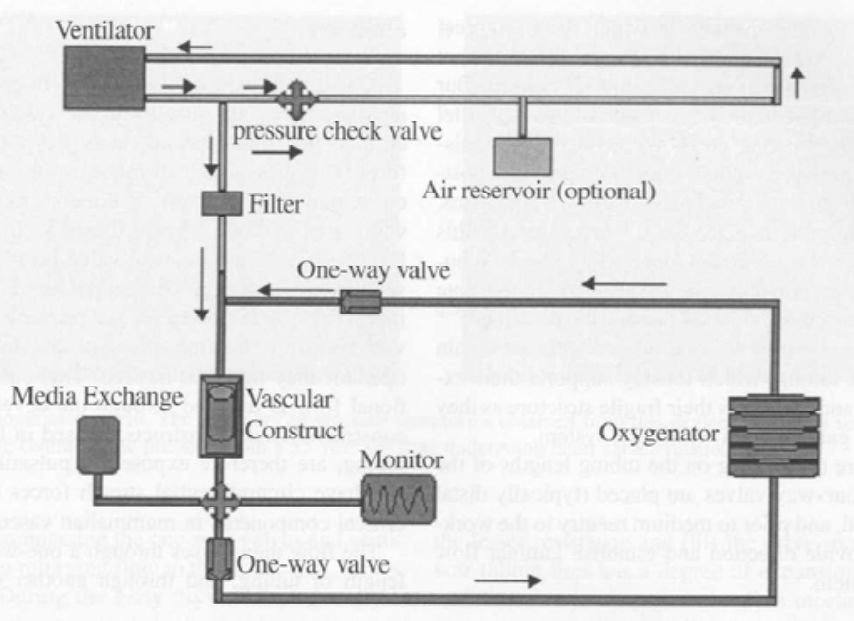

Figure 1.5 The actual bioreactor system used in Thompson et al study. The ventilator generates pulsatile flow and pressure within the system as the gasses impart their force upon the fluid column (52).

The research team ultimately found that their system modeled intravascular pressure conditions more accurately (Figure 1.6) than a roller-pump. They proposed that this system may be able to provide superior signaling mechanisms for the creation of blood vessels and heart valve. They intend on investigating the effects of compliance, pressure, pressure acceleration, flow, preseeding versus dynamic seeding, and duration of bioreactor cultivation on vascular tissue formation. 

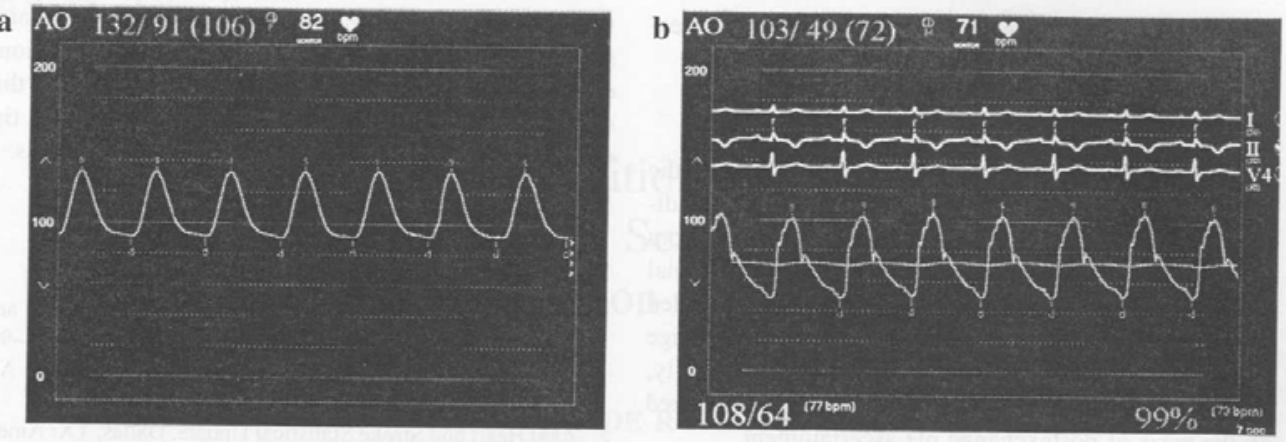

Figure 1.6 Pressure wave generated by Thompson bioreactor (a) versus an actual heart (b) (52).

Slightly more recently, Hahn et al. began focusing specifically on using physiologic pressures to help cultivate blood vessels. For their system, a Cellmax pump was placed in line with a peristaltic pump, compliant reservoir, bioreactor chamber, multiple valves, and silicone tubing. The pressure waves generated by the peristaltic pump were eliminated by incorporating a flexible reservoir. The Cellmax pumps would then impart pressure and flow pulsatility within the system. A valve was employed to restrict flow and backpressure the system. The system was able to accurately generate 120/80 mmHg pressure waveforms (18). Cellmax is commonly used instead of a peristaltic pump primarily because of the wear debris that is generated from extended use of the peristaltic pump breaking down the silicone tubing wall.

In 2005, Warnock et al. designed a sterile organ culture system for studying aortic heart valves. While heart valves are quite different from blood vessels they are both still part of the cardiovascular system as a whole. As a result, the ex vivo heart valves must experience similar environmental conditions of pulsatility and pressure that blood vessels are exposed to. This research team used a programmable waveform generator from Hewlett Packard to create physiologic waveforms that interface with a piston pump 
(Superpump, Vivitro Systems Inc.) which imparts the wave upon the bioreactor (55). The results of this study as they relate to the heart valve are not necessarily of interest, but the physiologic wave generation is. This model is of particular interest because the pressure wave is modeled to emulate a physiologic pressure wave (Figure 1.7). The wave is not just an effect of a functioning pump that is scaled to fit the desired physiologic boundaries - the peaks and troughs span the correct range without regard to the specific waveform.

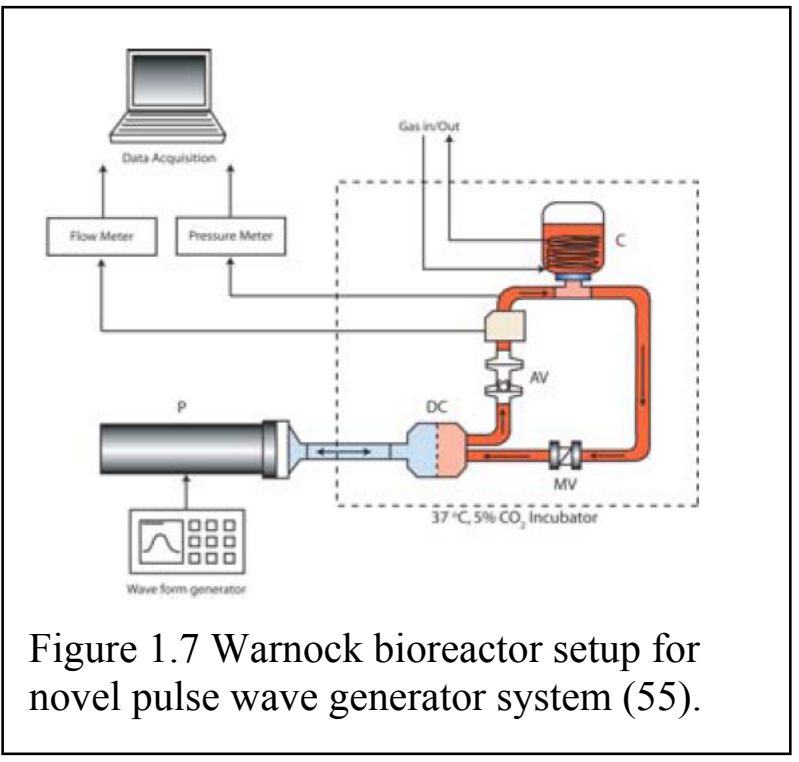

They found that the mechanical performance of the system allowed for independent adjustment of pressure, flow rate, and frequency. The mean pressure within the system could be adjusted to account for physiological and pathophysiological ranges (Figure 1.8 below), but the pressure amplitude is increased when pump stroke volume is increased. This issue could be avoided by using more compliant tubing for great pump stroke volumes. 

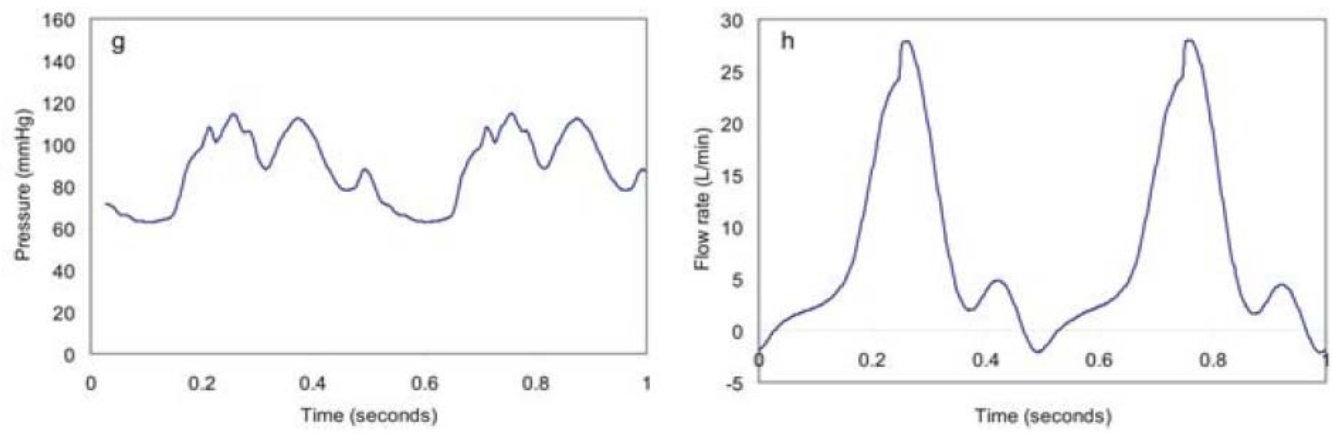

Figure 1.8 Presssure wave/flow rate measurements recorded from wave generator in study by Warnock et al (55).

Finally, in 2005, Engbers-Buijtenhuijs et al. cultured SMCs under pulsatile conditions and found that they improved mass transport of nutrients and waste to and from the cells of the vessel construct. In addition, like the other tests, tissue formation was stimulated which resulted in greater numbers of SMCs and a more uniform distribution across the scaffold. More importantly, the research team was able to establish physiologic pressures by pressurizing their reservoir to $100 \mathrm{mmHg}$ and then their peristaltic pump generated a wave of $124 / 82 \mathrm{mmHg}(14)$. The pressure was established through the application of compressed air and regulated using and electronically controlled Venturi valve. Their bioreactor is not much different than the one used in the Cal Poly BVM lab since they both utilize peristaltic roller-pumps (see Figure 1.3), and more importantly, they were able to produce physiologic pressure waves with the pump.

\section{Effects of Pulsatile Conditions}

The pressure within a system is related to pulsatility, and therefore pulsatile conditions will be discussed. Pulsatile conditions can be broken down into two 
complimentary factors - velocity and pressure. Pulsatile conditions result in fluctuating velocities. Velocity and the coefficient of friction of the endothelial cells are the primary factors dictating wall shear stress (see Equation 14.1 in subsequent section). "Q" is equal to volumetric flow rate and is determined by the product of a fluid's average velocity and the cross-sectional area of the conduit through which it passes. Since volumetric flow is in the numerator of the equation, greater volumetric flow results in greater wall shear stress. The method by which pulsatile flow is generated within a system has an impact upon system pressure. The pressures produced within the system typically result from a pressure-specific volume relationship. For a closed system, as the volume within the system increases the pressure also increases, and as the volume decreases the pressure decreases. Decreasing the volume and increasing the pressure within the system strains the system's walls causing them to stretch. This gives rise to the cyclic strain that blood vessels are exposed to. The amount of strain caused by the system pressure can be estimated using the equation for hoop stress (Equation 1.1).

$$
\mathrm{T}=\operatorname{Pr} / \mathrm{h}
$$

In the above equation, tension ( $\mathrm{T}$ ) is determined by dividing the product of the system pressure (P) and the radius of the vessel (r) by the thickness of vessel wall (h) (29).

The importance of these hemodynamic forces for vascular cell differentiation was recognized first in the early 1990's. In fact, between 1990 and 1997, vascular tissue engineering studies found that cyclic strain (stretch) produced an increase in markers of differentiated smooth muscle cell phenotypes and caused changes in cellular orientation 
and alignment within the construct $(46,49)$. Further investigation into the effects of pulsatile conditions found that shear stress promoted endothelial cell adherence and increased markers of endothelial differentiation representing mature endothelial phenotypes $(11,37,54,59)$.

As tissue engineering progressed, and the understanding of the roles hemodynamic factors played in guiding tissue growth increased, studies began integrating cyclic strain, growth factors, and preconditioning to modulate SMC and EC phenotypic and cellular differentiation promotion $(6,11,18,37,43,46,49,59)$. With the variety of cell types and sources available to tissue engineer blood vessels, studies sought to understand how different flow conditions influence specific types of cells. Shear stresses were found to result in the differentiation of progenitor cells along endothelial cell lineages $(41,48,54)$ and cyclic strain was found to initiate progenitor cells to differentiate along SMC lineages $(4,38)$. While cyclic strain and shear stress have been largely associated specifically with regard to SMCs or ECs, it is important to know that these are not exclusive relationships. There have been some effects seen in endothelial cell differentiation based on exposure to cyclic strain. Endothelial cells in cyclic strain environments have been shown to express phenotypic markers of smooth muscle cells (18).

Research has gone beyond only exploring the cause and effect relationship between mechanical stimuli and vessel tissue response and the cell signaling transduction pathways governing these effects have been characterized. The process begins with membrane signal transduction. Mechanotransduction begins with a force or stimulus imparted upon the cell. The cytoskeleton, focal adhesion sites, integrins, cellular 
junctions, and the extracellular matrix are then able to transmit and modulate tension within the cell. The cytoskeleton of the cell is composed of microtubules, microfilaments, and intermediate filaments that interconnect nearly all of the cellular structures. As result, structural modifications due to mechanical stimuli are capable of initiating complex signal transduction cascades that can lead to functional changes within the cell shown in Figure 1.9 (29). The stimulus activates the MAPK pathway that ends with protein synthesis. The synthesized proteins are specific to the cell type - endothelial or smooth muscle cells.

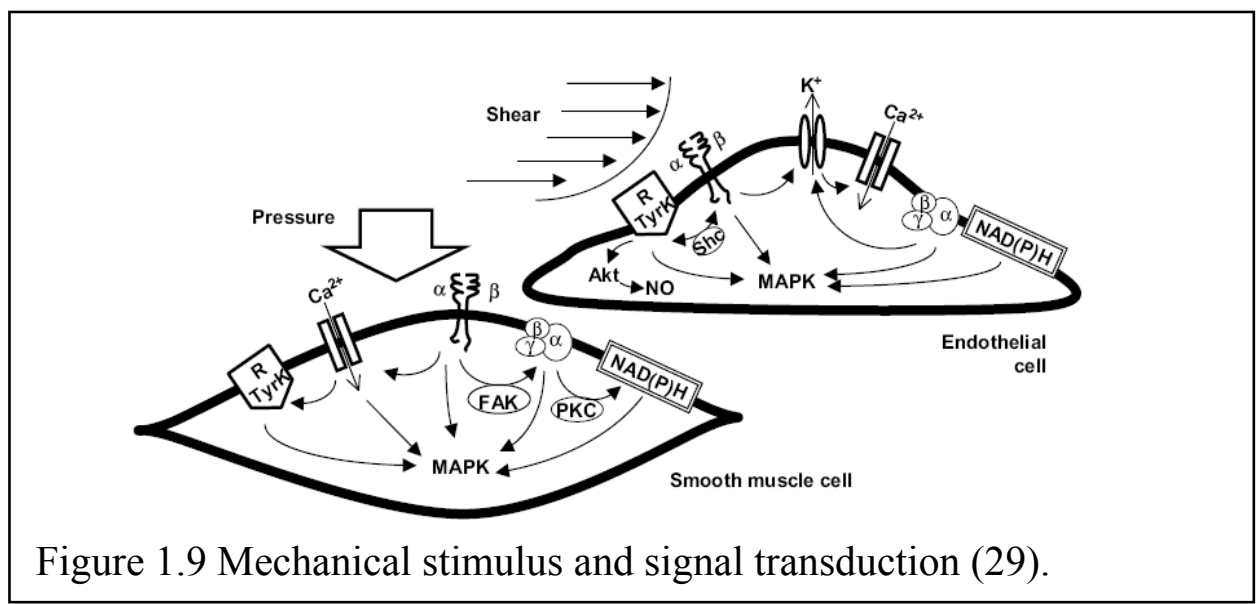

In 2001, S.P. Hoerstrup et al. cultivated blood vessels under pulsatile conditions. The bioreactor included a dual-phase air pump and a "novel pulse duplicator" which they did not elaborate on, but that resulted in pressure pulses ranging from $30 \mathrm{mmHg}$ to 50 $\mathrm{mmHg}$ in magnitude and flow rates from $125-750 \mathrm{ml} / \mathrm{min}$ - both incrementally increased over time. Endothelial cells were obtained from ovine carotid arteries and sodded upon a bioabsorbable PGA mesh scaffold. Constructs exposed to pulsatile conditions exhibited greater tissue growth than constructs cultured under static conditions. Further vessel 
assessment found that the pulsatile blood vessels had higher burst strength and suture retention strength (22) - ultimately greater mechanical properties (see Table 1.1 below). While the influence of pulsatility alone upon the mechanical properties of the vascular grafts cannot be discerned from this particular experiment, it is evident that flow greatly improved the mechanical properties of the vessels.

Table 1.1 Mechanical properties of tissue engineered vessels (dynamic/static) determined by Hoerstrup et al illustrating enhanced properties due to pulsatile flow.

\begin{tabular}{|c|c|c|c|c|c|c|}
\hline \multirow[t]{2}{*}{ Time in vitro (days) } & \multicolumn{2}{|c|}{ Burst strength (mmHg) } & \multicolumn{2}{|c|}{ Suture retention (g) } & \multicolumn{2}{|c|}{ Wall thickness (mm) } \\
\hline & Pulsatile flow & Control (static) & Pulsatile flow & Control (static) & Pulsatile flow & Control (static) \\
\hline 7 & $177.5( \pm 10)$ & $178.8( \pm 4)$ & $74.5( \pm 4)$ & $67.3( \pm 5)$ & $0.85( \pm 0.08)$ & $0.98( \pm 0.08)$ \\
\hline 14 & $240.0( \pm 28)$ & $110.0( \pm 18)$ & $56.8( \pm 5)$ & $42.0( \pm 5)$ & $0.75( \pm 0.05)$ & $0.63( \pm 0.13)$ \\
\hline 21 & $262.5( \pm 26)$ & $90.0( \pm 8)$ & $64.8( \pm 3)$ & $25.0( \pm 8)$ & $0.63( \pm 0.04)$ & $0.50( \pm 0.05)$ \\
\hline 28 & $326.3( \pm 24)$ & $50.0( \pm 5)$ & $64.3( \pm 5)$ & $12.0( \pm 3)$ & $0.73( \pm 0.04)$ & $0.50( \pm 0.1)$ \\
\hline
\end{tabular}

In 2009, Zhang et al. also cultivated vascular grafts using pulsatile conditions (60). The research team implemented a peristaltic pump to generate pulsatile perfusion. Physiologic pulsatile flow conditions resulted in enhanced tissue formation and extracellular matrix production. In addition, vessels displayed endothelial cell alignment and retained differentiated cell phenotypes. As with Hoerstrup, Zahng's experiment compared tissue engineered vascular constructs cultivated under pulsatile flow conditions to static conditions. Once again dynamic flow proves important for vessel cultivation, but the effect of pulsatility alone is not characterized. It is well established that the dynamic conditions have beneficial effects upon the tissue engineered blood vessels (60). 


\section{Summary and Goals of the Thesis}

In order to further provide the motivation for this thesis, it is important to understand the future goals of the BVM lab. Laboratories and research facilities around the world are working on developing efficient bioreactor systems to grow human blood vessels in vitro $(51,53,56)$. The primary goal of the majority of such groups, excluding Cal Poly, is to engineer tissue engineered vascular grafts for human implantation as bypass conduits $(28,35)$. However, the novel application of the BVM Lab's bioreactor system places this technology in a separate category of its own. It is not lab's intention to develop blood vessels for human implantation. The Cal Poly Blood Vessel Mimic Laboratory is using tissue engineered blood vessels to develop an in vitro assay for evaluating intravascular devices and drugs, with the goal to eventually provide results that are predictive of those obtained in vivo. The existing BVM bioreactor that is currently used to cultivate tissues within the lab is simplistic compared to more intricately designed systems used to make vessels for grafts $(14,22,24)$. Although the Cal Poly bioreactor may not be as complex as other systems currently in operation, the system is intentionally simple to allow for scale-up, and is progressive in its intended use as a preclinical testing device.

The use of a bioreactor system to emulate in vivo conditions and produce predictive results is a new application of blood vessel tissue engineering. Creating such a testing model would reduce the cost and time associated with preclinical trials that rely on animal models. The BVM system could be a beneficial tool for the medical community and intravascular device companies. As this application is refined by the Cal Poly BVM lab, it can be used to gather preclinical testing data for safety and efficacy. Currently, 
preclinical trials include bench top testing (in vitro) and animal testing (in vivo). These trials are performed to ensure or establish both safety and efficacy of a device before clinical trials, as required by the FDA. Animal testing is the gold standard for preclinical device assessment and must be satisfactorily completed in order to move on to clinical trials. Such studies generally take 2-3 years and can cost anywhere from $\$ 10-20$ million (26). Devices must undergo escalating phases of testing regulated by the FDA before they can be approved for the market. Rabbits (Figure 1.10) and pigs are typically used for animal testing involving stents $(13,32)$.

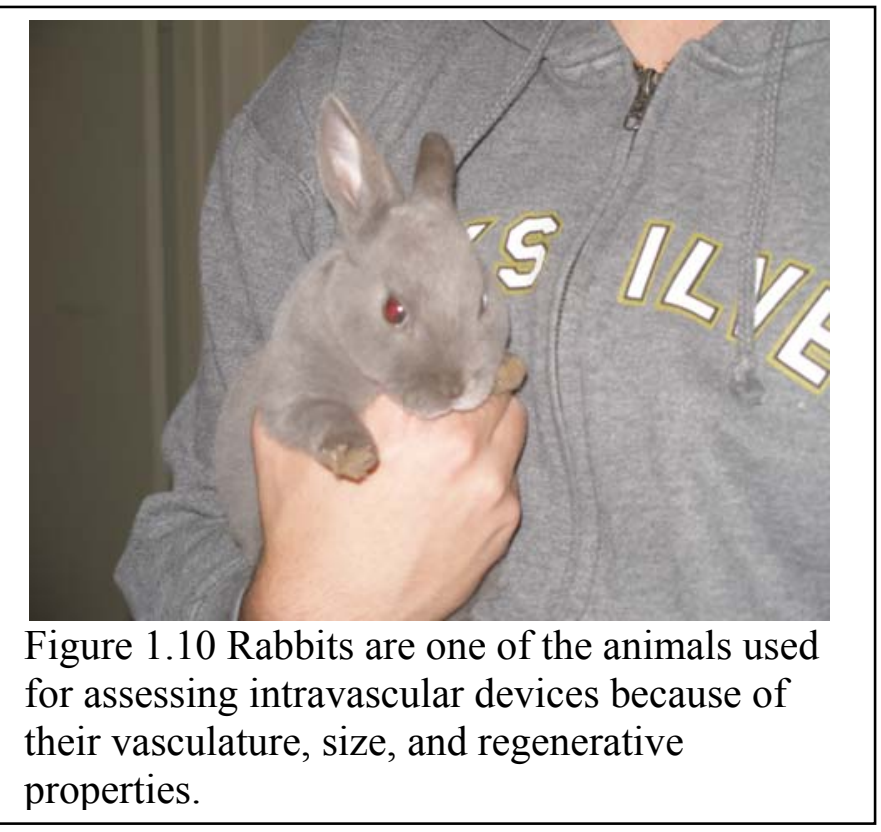

During these trials, stents are deployed in the various animals and excised at different points in time over the course of the trial. The excised stents are then assessed for biocompatibility and performance. There are two main problems associated with the animal model for device testing. The first problem is that animals are not humans and 
they do not provide an entirely accurate example of what will occur when the device is implanted into a human patient $(1,13,32)$. The other problem is the high cost associated with animal testing. The cost of purchasing animals for testing varies, but according to Charles River Laboratories, the least expensive available rabbit for purchase is $\$ 92.35$. When size and sex are taken into consideration and are specifically required for a trial, the rabbits can be as much as $\$ 200.35$ each (Charles River: Products and Service Guide). These prices are simply for the animals and do not include costs associated with resources, surgery, facilities, and care which are mandated by Insitutional Animal Care and Use Committees (IACUC).

The BVM system could potentially provide additional useful information more characteristic of the human response since human cells would be used to culture the blood vessels. The bioreactor system would also help reduce any unnecessary animal testing - animals are expensive and there are some ethical issues at play. This is not an indictment of animal testing, but a concern for efficiency as it relates to time and expenses and upholding the humane treatment of animals for testing.

\section{Original BVM System}

To provide the context for which this thesis is based and the general technological progression of system design, it is necessary to understand the history of the Cal Poly BVM bioreactor. The system is intended for preclinical testing and had its beginnings at the University of Arizona. Dr. Kristen Cardinal began working with the original bioreactor system as a part of her graduate studies and dissertation. Since that time, a number of years have been dedicated to the development of the technology. When Dr. 
Cardinal began teaching at Cal Poly, she was given permission to bring the tissue engineered blood vessel mimic project and research with her. In 2007, the BVM Lab consisted primarily of Dr. Cardinal and four students and has grown to include 12 students today.

The original bioreactor system design was made up of a peristaltic pump with an 8 roller pump head, silicone tubing, luer lock fittings, an ePTFE scaffold, an acrylic bioreactor chamber (Figure 1.11), and a $50 \mathrm{ml}$ flask style reservoir. This system ran using a continuous flow style pump and operated at the pressure induced by the pump. Pressure waves generated by the pump tubing occlusion were so small compared to physiologic pressures that they were largely disregarded and flow was considered "steady". The acrylic bioreactor chamber used in this setup was successful for much of the previous research, but had several features that could be improved upon. The chambers were difficult to manufacture, not scalable, and challenging to assemble using sterile techniques.

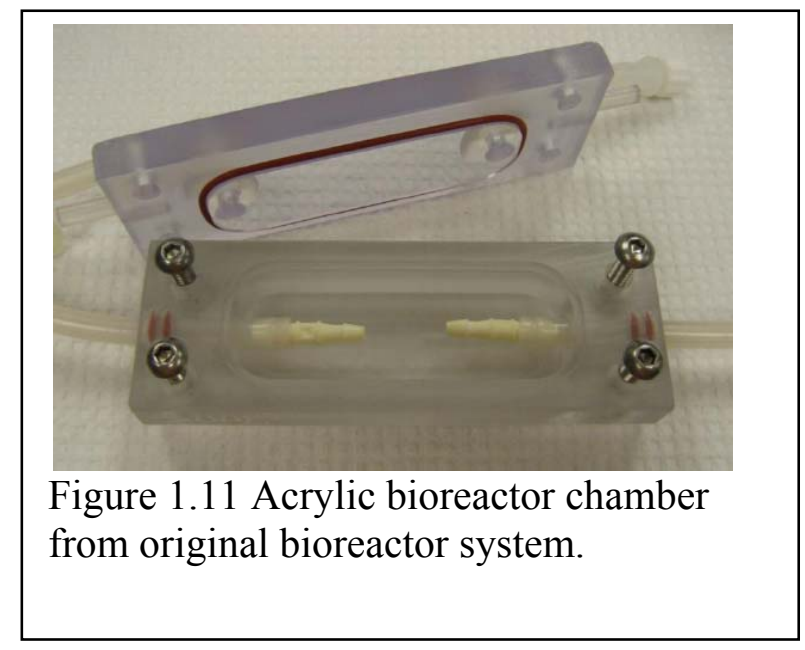


Students working in the BVM Laboratory have performed a substantial amount of research and design in order to develop a bioreactor that is both easy to use and effective for engineering blood vessel mimics. While a number of students are conducting research, much of this work depends on the quality of the bioreactor providing them data and results to analyze. Sara Leifer and Marc Dawson dedicated their time to improving the bioreactor.

Sara Leifer's goal was to design a more practical bioreactor based on the system previously used by Dr. Kristen Cardinal. The older system was tried and true through the research conducted by the tissue engineering lab at the University of Arizona. A drawback to the previous system was the inability to stack the bioreactor chambers. Other drawbacks included having to fabricate the individual chambers and the small extraluminal space. The small extra-luminal space made securing the luer lock fittings and scaffolding difficult. As a result, running multiple systems would take up an excessive amount of space. Sara wanted to create a system that could accommodate a wider range of stent lengths and diameters. It was also important to make it easy to insert scaffolds and seal the system. Sara also wanted to build a strong foundation for future research and experimentation with the bioreactor by being able to test various vessel geometries such as bends and bifurcations.

Sara evaluated various designs using the criteria as follows: user friendly design, easy scaffold insertion and removal, easy to seal/leak-proof bioreactor chamber, adjustable scaffold length and diameter, ability to be sterilized with ethylene oxide gas, optically clear bioreactor chamber, easy to scale-up and space effective, low cost, easy to manufacture, use of readily available materials, and use of biocompatible materials. 
Using these criteria, Sara narrowed down her design selection to the Lock N Lock bioreactor chamber connected in series with a reservoir and peristaltic pump. This BVM system is now used to conduct testing and is the basis for new research testing different scaffold geometries, assessing electrospun scaffolds, and simulating the effects of different pathologies. The Leifer system is illustrated below in Figure 1.12. The Lock N Lock bioreactor chamber is a rectangular plastic container for perishable foods. Sara also researched new panel mount luer lock fittings to allow scaffold suspension and chamber tubing connections to be easily made. The entire top of the container is easily removed and gives the user enough space to attach the scaffolding without much difficulty. The design was also beneficial in that it allowed bioreactor chambers to be stacked upon one another. The conservation of space and inexpensive materials made this design scalable and feasible.

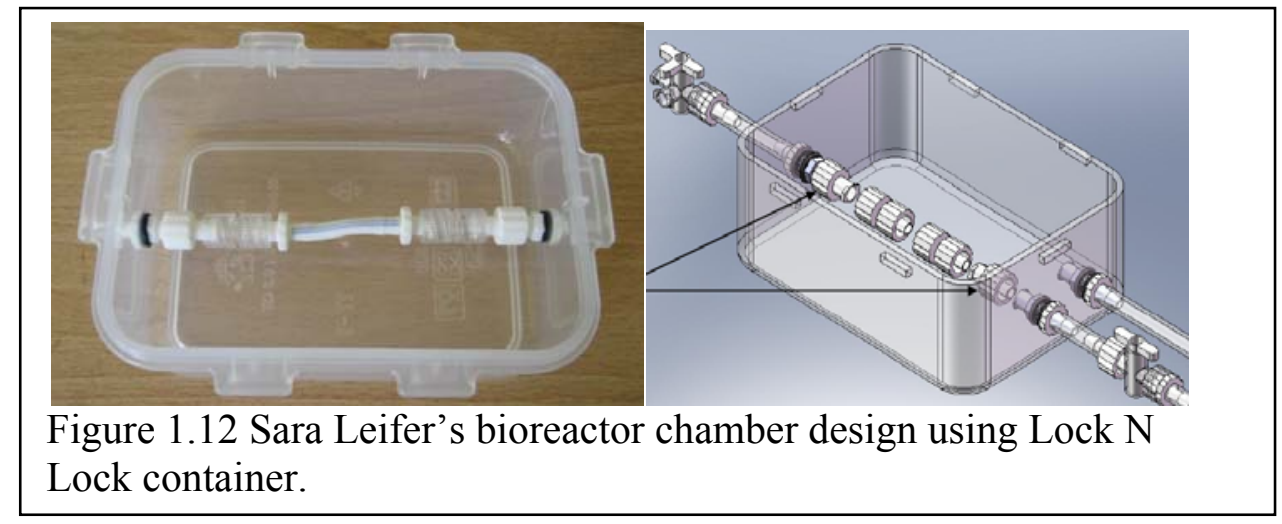

Following Sara's design and implementation of the new chamber, Marc Dawson made further modifications to the system as a part of his master's thesis. Now that the system had been made scalable and user friendly, the next avenue to pursue was emulating physiologic flow conditions. Marc worked on a variety of parameters including 
viscosity and pulsatility. Marc changed the peristaltic pump head from an 8 roller configuration to a 3 roller configuration. Having only three rollers made the fluid flow within the system pulse rather than continuously flow. By increasing media viscosity, Marc was able to also develop physiologic wall shear stresses within the scaffolding of the bioreactor chamber. Some additional effort was dedicated to establishing a pressure wave within the system that emulated human systolic and diastolic pressures. Progress was made in establishing a basal backpressure, but it was insufficient in physiologic terms - lacking both consistency and sustainability.

Following both Sara's and Marc's bioreactor developments, further modifications to the bioreactor system were desirable. Testing and previous experience found that the Lock N Lock bioreactor chambers suffered from leaks. Some of the leaks could be easily repaired, but the fundamental design of the chamber was not conducive to or durable enough to support physiologic pressure conditions. Consequently, it was necessary to redesign the bioreactor chamber so that it was more durable, could easily withstand a wide range of pressures, accommodate various vessel geometries, and would be compatible with future modifications and improvements upon the system. In addition to this, it was desirable to develop a method of back-pressuring the system that could be easily adjusted, accurate, and consistent for extended periods of time.

Overall, the bioreactor evolution started with Dr. Cardinal's arrival at Cal Poly and has continued ever since; with the current thesis work being the most recent addition. During this time, other members of the BVM lab have also performed numerous cell sodding, attachment, and proliferation tests to assess tissue cultivation. A well rounded 
understanding of the previously performed work and the future goals of the BVM lab are important to keep in mind as further developments are designed into the system.

\section{$\underline{\text { Goals }}$}

As discussed throughout this introduction, the bioreactor plays an integral role in cultivating blood vessel constructs under appropriate flow conditions. Theoretically, a bioreactor that closely simulates physiological conditions will produce blood vessels with mechanical properties more similar to native vessels. For research in the Cal Poly Blood Vessel Mimic lab, there are two areas of opportunity that should be resolved in order to provide a sound foundation for further progress: current environmental pressure conditions, and cell sedimentation. These areas led to the development of 4 specific aims for this thesis:

1) Design a new bioreactor chamber that can withstand backpressures

2) Establish a realistic base-line pressure to mimic systolic/diastolic conditions.

3) Characterize flow conditions through computational modeling

4) Propose a methodology to prevent cell sedimentation

As discussed in the introduction, published research suggests that pressure conditions are essential to developing vessels with the necessary properties. Previous studies conducted by Marc Dawson reaffirm this assertion. The current system can accurately achieve peak systolic pressures but then drops to zero. The other issue that should be resolved is cell sedimentation. Cultured vessels could potentially exhibit 
uneven cell distribution with a greater cell density toward the bottom of the scaffold. An even distribution along the entire circumferential surface area is desirable.

Overall, the specified aims were addressed using qualitative analysis, quantitative experimentation, equipment provided by Cal Poly's machine shop, and by conceptual design. The original bioreactor system was characterized based on applied projected environmental conditions. Results from the initial testing were then used to illustrate potential bioreactor weaknesses and were taken into consideration when designing a new bioreactor chamber. The bioreactor chamber was produced using Cal Poly's machine shop. The new chamber was incorporated into the bioreactor and back-pressuring techniques were then evaluated qualitatively for efficacy. Potential solutions were narrowed down to the most promising option and quantitative testing was performed. Computational modeling was then used to estimate the fluid dynamics of the custom bioreactor. Lastly, a conceptual design to eliminate cell sedimentation during the seeding process was developed to provide uniform cell distributions. This work will be described in the following chapters. 


\section{Chapter 2: TESTING THE LOCK N LOCK BIOREACTOR CHAMBER \\ Introduction}

In order to meet the goal of establishing physiologic pressure, the capabilities and characteristics of the current Lock N Lock bioreactor had to be better understood. Marc Dawson evaluated base-line pressure within the chamber as a function of flow restriction. Flow restriction on the discharge side of the positive displacement peristaltic pump increases pressure as fluid is continuously circulated through the reservoir. At a given flow rate, this flow restriction will cause the fluid to stress the walls of the system between the pump and the point of restriction. Creating the flow restriction by occluding the tubing results in increased pressure, but it is difficult to regulate since the tools used to clamp down on the tubing do not offer fine adjustment (12). The internal pressure of the system can shoot from a negligible base-line to $500 \mathrm{mmHg}$ with a fraction of a turn of the adjustment on the clamping device.

Two concerns are raised by this degree of sensitivity. The first is how the system structurally responds to elevated pressure and the second is the capacity to regulate pressure. The pressure must adhere to a desired range at acceptable levels of accuracy and repeatability. Clearly, the system is constrained by reliance on variable flow restriction to generate target pressures - the concept is sound, but available hardware is too imprecise. The questions surrounding the concern of the system's response to high pressures can be readily answered by performing a leak pressure test. Therefore, the initial goals of this project - and the work that will be presented in this chapter - involved testing the existing Lock N Lock bioreactor chamber's ability to withstand physiologic pressures. 


\section{Materials and Methods}

A Lock N Lock bioreactor chamber was used to conduct the chamber leak pressure test. The chamber itself is compromised of essentially two parts - the lid and the semi-rigid container. The container is made of translucent, polypropylene plastic and has locking nodes for the lid. The lid has a built-in gasket and four flexible locking arms that interlock with the nodes of the container. When the pieces are locked together, the edge of the container is pressed into the lid's gasket - sealing the two parts together. For these tests, the bioreactor chamber was assembled with the necessary luer lock fittings and the ePTFE scaffolding normally used during vessel cultivation and data was recorded using LabChart 7 data acquisition software. The data acquisition equipment included both hardware and software. The hardware included the pressure transducer, data acquisition unit, and the bridge pod amplifier (Figure 2.1). The hardware was assembled and attached to the software (LabChart 7) that was run using a Dell laptop.

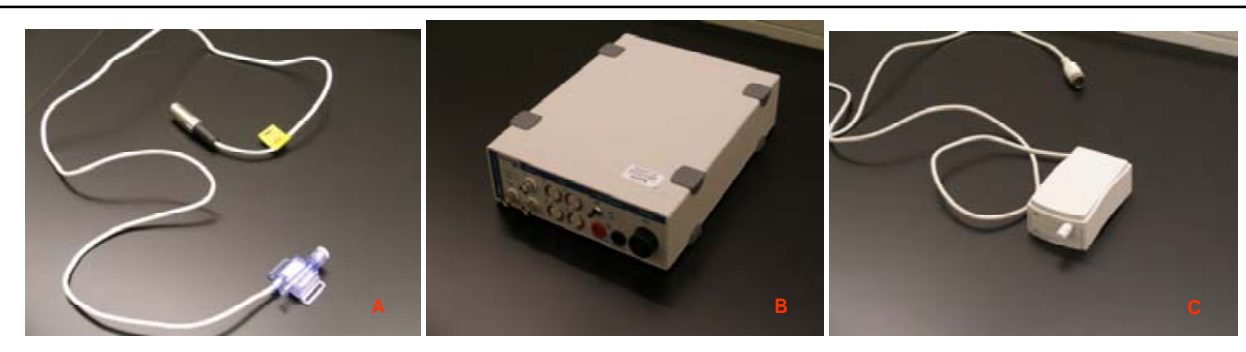

Figure 2.1 Data acquisition hardware: pressure transducer (A), data acquisition unit (B), and bridge pod amplifier (C).

The next step was calibrating the pressure transducer to measure gauge pressure in mmHg. A two point calibration was performed to accomplish the task. The ADI pressure transducer for the system was calibrated with a mercury monometer. The pressure 
transducer was connected in line with the monometer and pressurized from 0 to 200 mmHg while the LabChart data acquisition software recorded the data. The two point calibration technique required selecting the minimum $(0 \mathrm{mmHg})$ and maximum $(200$ $\mathrm{mmHg}$ ) points of the recorded data to establish accurate measurements with the proper units. The system settings were saved and later confirmed using a pressure cuff. The pressure transducer was accordingly ready to be implanted within the system to record data.

The set up is illustrated in Figure 2.2 below. Three sections of silicone tubing (1/8 inch inner diameter, $1 / 4$ inch outer diameter) were connected respectively to the one inlet and two outlets of the chamber. The two outlet tubes then converged to a single tube using a "Y" style barbed connector with an additional length of tubing after the intersection. A stopcock was placed at the exit end of the "Y" connection, as well as at the entrance of the inlet chamber tube. The stopcocks allowed the luminal and transluminal spaces to be flushed and filled. The ADI pressure transducer was placed in line attached to the outlet stop valve. A luer lock syringe full of water was then attached to the inlet tube stop valve and water was passed through the abbreviated system. Once water exited the pressure transducer on the luminal outlet tube, a luer plug was attached to the transducer, and the stop valve with the syringe attached was closed. The extra-luminal space of the bioreactor chamber was then filled with water. This was accomplished by removing the snap-on lid of the chamber and pouring water into the extra-luminal space surrounding the scaffold. 

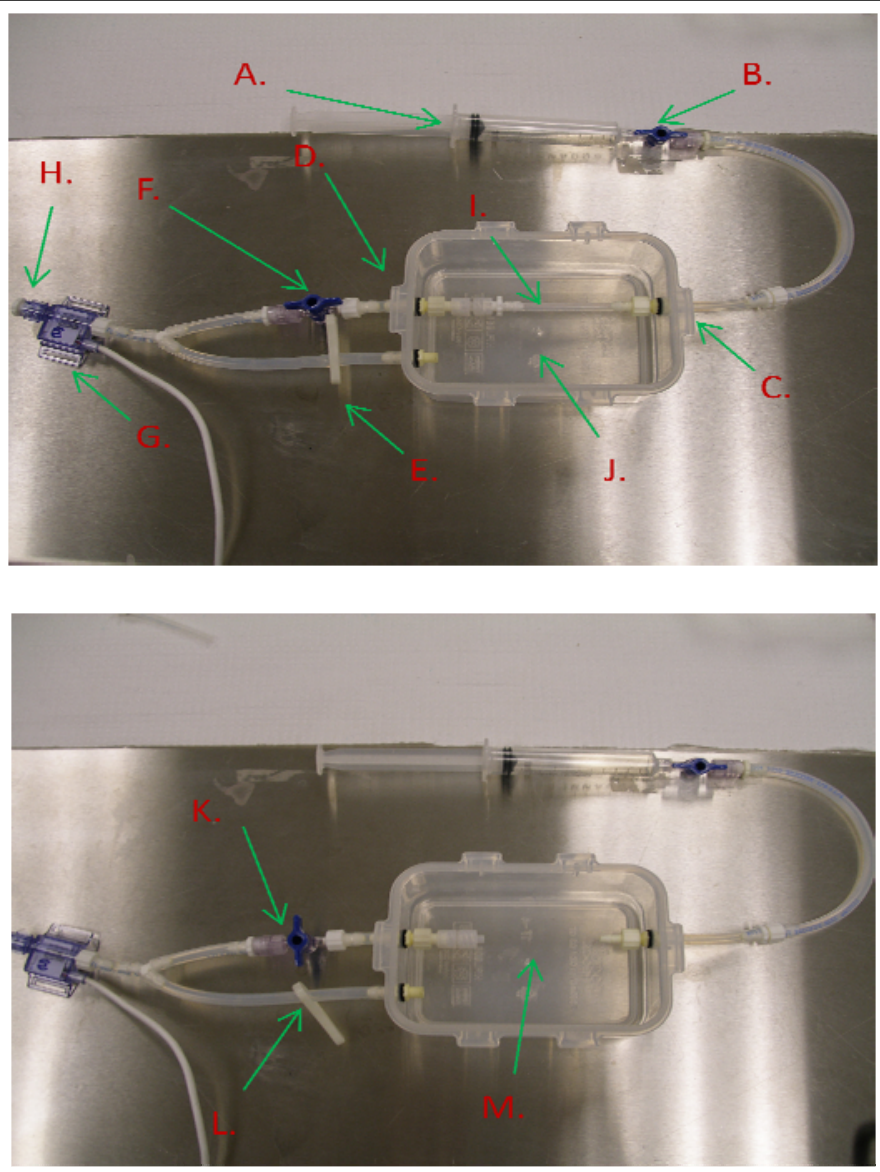

Figure 2.2 Setup for pressurized leakage test. Luer lock syringe (A) injects fluid into the system which can be sealed by the two-way valve (B) on the side of the inlet port (C). To test luminal leak pressures, the scaffold (I) is suspended within the chamber. The extra-luminal space $(\mathrm{J})$ is sealed off with a tubing clamp (E). On the dual port outlet (D) side of the chamber, the two-way valve $(F)$ is open leading to the pressure transducer $(\mathrm{G})$. The pressure transducer is capped with a male luer lock cap $(\mathrm{H})$. Testing the extra-luminal space $(\mathrm{J})$ involves removing the scaffold $(\mathrm{M})$, closing the two-way valve $(\mathrm{K})$, and releasing the tubing clamp (L) on the outlet side of the chamber.

The extra-luminal outlet was closed using a hemostat ahead of the "Y" connector in order to assess luminal leak pressure. The scaffolding and clamp were removed from the bioreactor chamber to test extra-luminal leak pressures. The pressure transducer, located on the bioreactor chamber outlet, was attached to data acquisition hardware and 
displayed by LabChart 7 software. While LabChart recorded live data, the valve on the inlet tube was opened and the syringe, described above, was used to force fluid into the system until failure was achieved. Failure would be evidenced and defined by the onset of fluid leakage at any of the tubing connections, luer lock fittings, chamber seals, or any combination thereof.

To check the extra-luminal leak pressure, the hemostat was removed from the extra-luminal outlet and the scaffold was removed from the bioreactor chamber. Since the ePTFE scaffolding is porous, if it were included in the trans-luminal test, the generated luminal pressure would diffuse across the barrier slowly into the extra-luminal cavity of the bioreactor chamber and eventually reach equilibrium. As a result, the test would have to be performed slow enough to allow equilibrium to be reached between luminal and extra-luminal sections in order to exert the desired forces upon the rigid walls of the container. Removing both the scaffold and the hemostat allows the pressure to rapidly spread and distribute homogeneously throughout the system and stress the walls of the chamber during the test. As with the luminal test, the syringe was again used to inject fluid until the point of system failure was reached. In the extra-luminal evaluation, the definition of failure remained the same and was indicated by fluid leakage. Six leak pressure tests were conducted to characterize luminal and extra-luminal pressure limits, three tests per parameter.

\section{$\underline{\text { Results }}$}

Three tests were performed to assess luminal leak pressure, and three tests were performed to assess extra-luminal leak pressure - six tests in total. The results of the tests 
are listed below in terms of pressure readings at the point of failure. Catastrophic failure for the luminal leak pressure tests was characterized by silicone tubing releasing from the luer lock barb connection. Extra-luminal leak pressure failure exhibited leaks around the panel-mount chamber wall penetrations and the seal on the bioreactor chamber lid. The common sites of failure are indicated below in Figure 2.3.

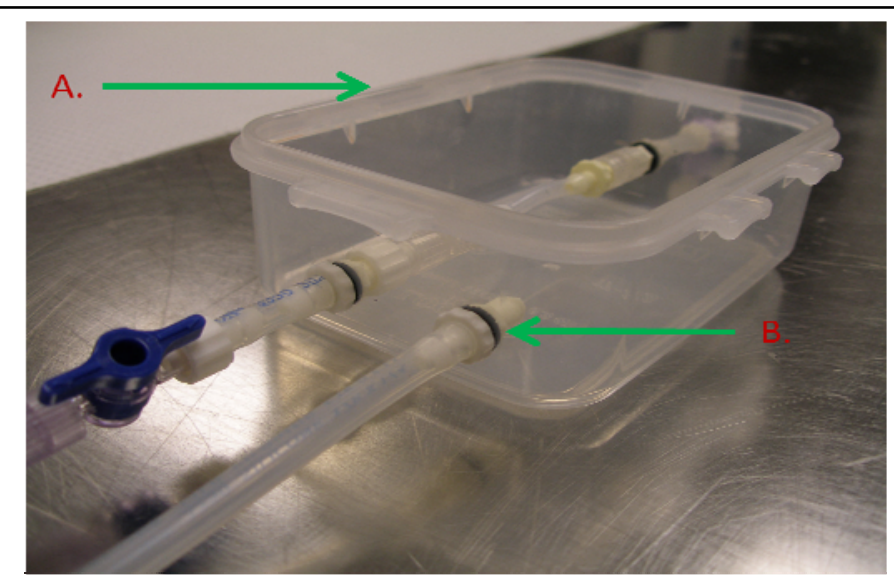

Figure 2.3 Locations of fluid loss. During the extraluminal pressurization fluid would drip from under the lid (A) and from around the panel mounts (B).

Table 2.1 Lock N Lock leak pressure test results.

\begin{tabular}{|c|c|c|}
\hline Trial & Luminal $(\mathrm{mmHg})$ & Extra-luminal $(\mathrm{mmHg})$ \\
\hline 1 & 1257 & 100 \\
\hline 2 & 1204 & 102 \\
\hline 3 & 1282 & 101 \\
\hline
\end{tabular}

\section{Discussion and Conclusion}

These results are quite significant in the context of human physiologic pressure conditions. A normal human systolic and diastolic pressure ratio would be 120/80 $\mathrm{mmHg}$ 
and can range (not necessarily with good health) from $230 / 140 \mathrm{mmHg}$ to a mean arterial pressure of $45 \mathrm{mmHg}$ based on severe hypertensive and hypotensive pathologies $(19,50)$. The data presented in this chapter shows that the luminal leak pressure is significantly greater than this prospective range and therefore noncritical. However, the extra-luminal pressure failure threshold of approximately $101 \mathrm{mmHg}$ is below the systolic $120 \mathrm{mmHg}$ of an average healthy adult. This pressure limitation would hinder the ability to test pathological conditions involving high systolic pressures, or even normotensive pressures of $120 \mathrm{mmHg}$, and could be a constraint in the pursuit of "optimized" pressure and flow parameters that would require exceeding $100 \mathrm{mmHg}$.

For the luminal test, failure occurred where the tubing connected to the barb of a luer lock fitting. In this case, the failure occurred at the same place each time on the extra-luminal outlet side of the "Y" connector. While the failure occurred in the same place each time, it is not necessarily indicative of a structural weakness that is located solely in that area. Any tubing and barb coupling would be at similar risk of failure. As the fluid injected into the system stretches the walls of the tubing, the barbs lose contact with the inner wall of the tube. Whichever coupling is the weakest (e.g. tubing not pressed as far onto the barb) will fail. Once failure has occurred, the resulting burst of water lubricates the subsequent couplings at that spot adding to the likelihood of repeated failures in the near future.

During the extra-luminal pressure test, leaks were seen around the inlet and outlet panel mount Buna-N O-ring gaskets. The Buna-N O-rings used to seal the wall penetrations were cracked and degraded. While any leak is bad for maintaining pressure, the leaks at each of the panel mounts were more of a nuisance in nature compared to the 
other source of leakage, as these leaks did not lead to significant fluid loss. This, however, could be a major issue for contamination concerns. Ultimate failure occurred at the seal of the lid to the Lock N Lock bioreactor chamber. Fluid would seemingly dribble out at the same rate it was injected in. The panel mount leaks would only become wet and produce an occasional tear.

While the luminal leak pressure is acceptable, the extra-luminal leak pressure is problematic. The tissue constructs are cultivated with the extra-luminal outlet closed and the luminal outlet open on the bioreactor chamber during normal operation. The initial sodding and preparatory stages are performed with the outlets switched (luminal closed, extra-luminal open) to force transmural flow. The pressures occurring during transmural flow are minimal and would not approach $100 \mathrm{mmHg}$ and should not compromise the extra-luminal seal. During normal operation, the outlet configuration would tend to buffer the extra-luminal cavity from rapid pressure changes resulting from elevated pressures in the luminal portion of the system. However, ePTFE is porous. Eventually the bioreactor chamber will reach equilibrium with the luminal portion of the system when under constant luminal pressure. When this happens, the lid and panel mounts cannot withstand the pressures and will leak. With pulsatile flow and pressure, the trans-equilibrium pressure will be somewhere between the high and low luminal values. Given a pressure wave of $120 / 80 \mathrm{mmHg}$, this average would be at least $100 \mathrm{mmHg}$ and very close to the failure point. Accordingly, a new bioreactor chamber should be designed to address the pressure limitation and propensity to leak of the current model. In the mean time, some temporary fixes could be used to mitigate the described failure modes, by including zip ties and silicone sealant. For example, zip ties could be applied to the bioreactor chamber 
to better secure the lid to the chamber with additional force. The zip ties can be applied along both the length and width of the chamber (Figure 2.4). Silicone sealant could be applied to the extra-luminal panel mount penetrations on the exterior of the chambercovering the base of the luer lock fitting (Figure 2.5).

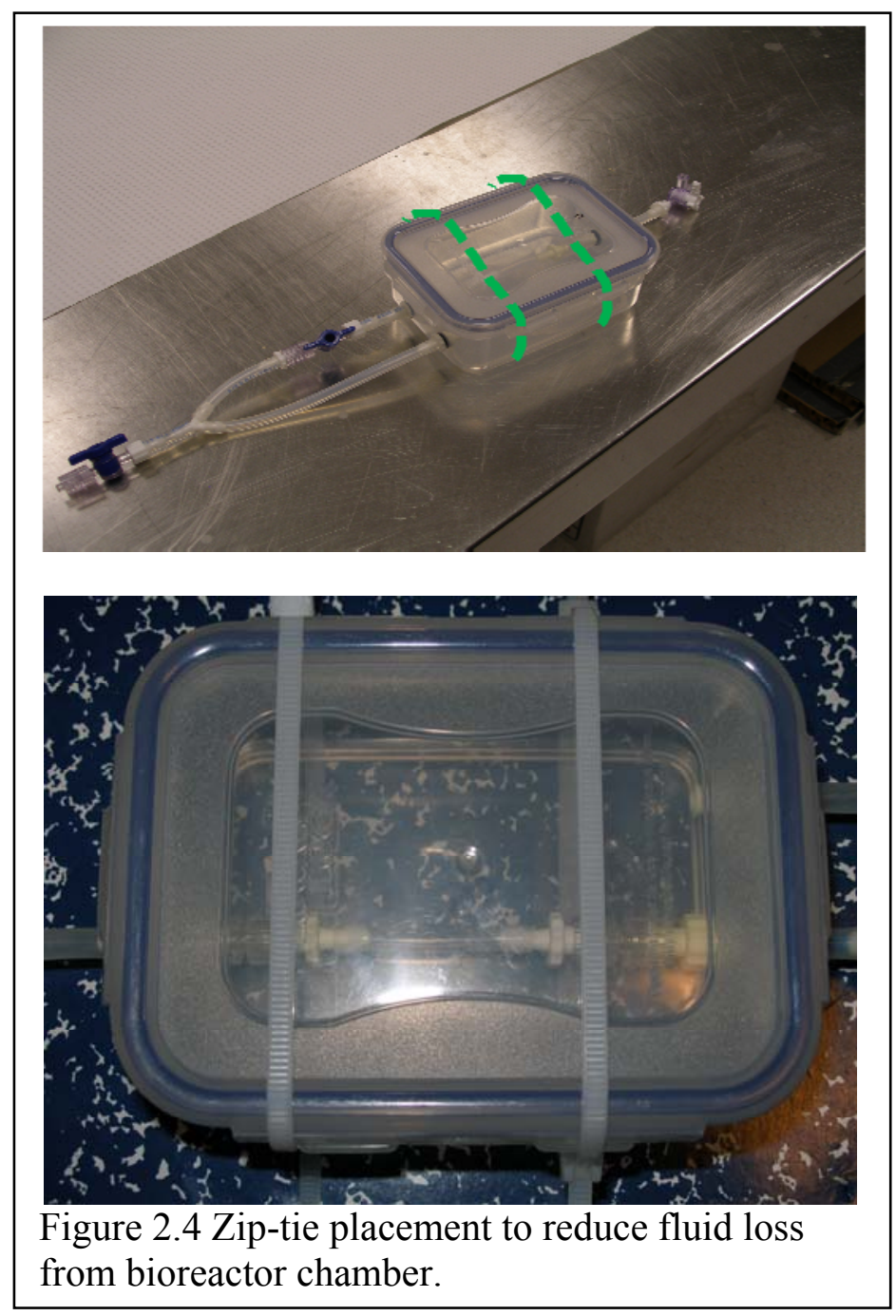



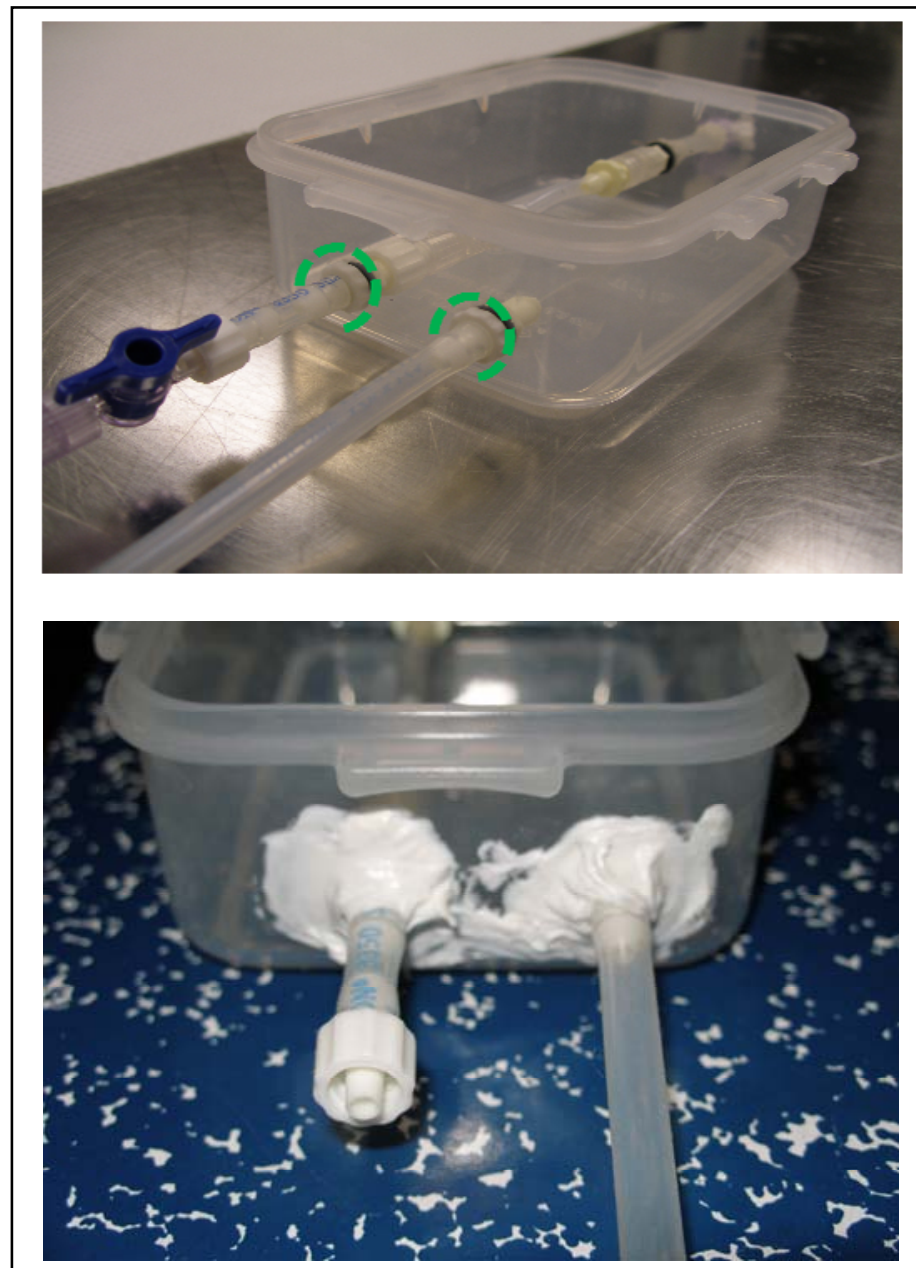

Figure 2.5 Silicone sealant placement to reduce fluid loss from bioreactor chamber.

In addition, due to the poor condition of the Buna-N O-rings, further investigation was performed. It was found that they are incompatible with ethylene oxide (EtO) gas the method currently used to sterilize the materials (www.efunda.com). The ethylene leaches the oils from the rubber gasket. This causes the material to become brittle and crack. Every gasket inspected showed signs of degradation. The panel mounts are permanently attached to the bioreactor chamber walls with adhesive after sandwiching a rubber gasket on each side of the chamber wall. The number of times these chambers 
have been used is unknown. It is safe to assume that they have been sterilized more than once. Either the gasket material needs to be changed, or the panel mounts must be removable so gaskets can be periodically exchanged.

In conclusion, the work in this chapter revealed a critical problem in the Lock $\mathrm{N}$ Lock bioreactor chamber's inability to withstand extra-luminal pressures at or above 100 mmHg. As a result, a new bioreactor chamber must be designed. 


\section{Chapter 3: DESIGNING THE NEW BIOREACTOR CHAMBER \\ Introduction}

The initial assessment of the Lock N Lock bioreactor chamber revealed pressure limitations that are problematic when replicating physiologic pressure wave conditions in vitro. While zip ties and silicone sealant may offer short term compensation for the problem, a new bioreactor chamber must be designed in order to reliably replicate physiologic pressures while maintaining a sterile closed system. Sara Leifer developed her bioreactor chamber to be easy to assemble and use, and to accommodate a variety of vessel geometries. In order to provide a bioreactor chamber with necessary attributes to withstand physiologic pressure conditions, some sacrifices need to be made in ease of creation and operation.

Sarah's previous work outlined a number of criteria that the bioreactor chamber must fulfill in order to be considered successful (30):

1) An overall user friendly design so that assembly is simple and straightforward

2) Easy scaffold insertion and removal without the need for bending or twisting

3) Easy to seal leak-proof chamber for quick and sterile assembly

4) Adjustable scaffold length and diameter for greater testing variability

5) Sterilizable by ethylene oxide gas

6) Optically clear chamber for easy visibility of scaffold, media and stent placement

7) Easy scale-up so that multiple chambers can be configured in the incubator

8) Low cost

9) Easy to manufacture without the need for adhesive or special tooling 
10) Use of materials and components that are readily available and easy to obtain

11) Use of biocompatible materials so that cells are able to grow and function

Each and every one of these criterion is important to the design of an efficient and practical bioreactor chamber. However, criteria 1, 8 , and 9 are not as important in this phase of the design process, because it is better to have a design that achieves current and potentially future goals rather than to fall short on account of simplicity and cost. In order to obtain a chamber that can withstand the necessary environmental pressure conditions, the cost of the system will undoubtedly increase. Designing a system to withstand these pressures will also likely involve a slightly more complicated manufacturing and assembly process. Within reason, these can be acceptable consequences for an improved design. While it is still immensely important that the system remain simple and easy to use, this requirement is balanced by performance expectations. The design should account for ease of use, but also achieve the important functional goal of supporting physiologic pressures.

\section{$\underline{\text { Methods and Materials }}$}

\section{Material Selection}

As mentioned previously, there is a list of design criteria that the bioreactor chamber must fulfill. The chamber must be capable of being sterilized. There cannot be any leaks and seals must be able to withstand the entire range of physiologic pressure. It has been noted that cell sedimentation occurs during vessel cultivation. In accordance, the bioreactor chamber should be capable of being rotated 360 degrees. 
Overall, the design that was created and implemented consisted of the following components: cylindrical housing, solid caps, and flexible end plates. A basic diagram of the new design is provided in Fig 3.1 below. The specific materials selected for these components will be described and justified below, followed by a detailed description of the design, manufacturing process, and assembly of the bioreactor chamber. 


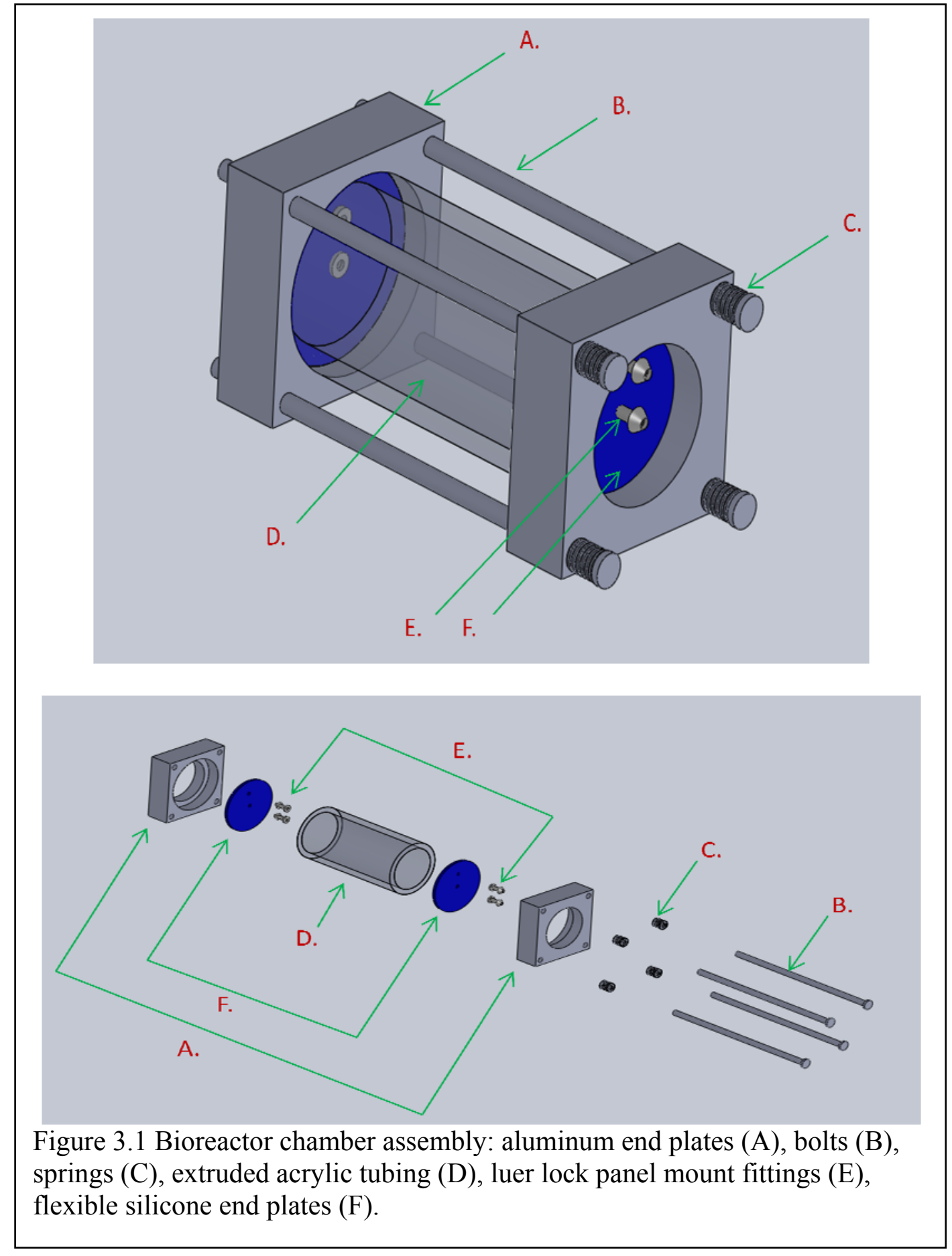

An extruded acrylic tube was chosen for the bioreactor chamber wall (McMasterCarr, $\mathrm{P} / \mathrm{N}$ 8486K547). The tube has a 2 inch outer diameter and a $13 / 4$ inch inner diameter. Having a 1/4 inch wall thickness is important for not only ensuring that the wall 
can withstand the necessary pressures, but it provides structural support to allow the tightening of end caps without cracking. Acrylic is optimal because of its optical clarity, but the material is brittle. Acrylic can only withstand up to roughly 200 degrees Fahrenheit (www.mcmaster.com). Accordingly, the acrylic will have to be sterilized by EtO gas rather than the autoclave. Acrylic is a widely used material in the research community and has been shown to be biocompatible with cells (47).

6061 aluminum is an all purpose aluminum (McMaster-Carr, P/N 8975K313) that was selected for end cap fabrication of the bioreactor chamber. It is easily machined and known for its strong structural properties and relatively light weight (25). The weight of the bioreactor chamber is important for ease of use and for potential future scaling of the system. Being able to be easily machined, the metal lends itself to reproducibility. The 6061 aluminum can be sterilized using an autoclave or EtO gas. There are a wide variety of aluminum alloys that are considered biocompatible. The aluminum, in this design, will not come into contact with cells or media and therefore its biocompatibility is of less significance. The aluminum will be used to make the end caps illustrated in Figure 3.1 above.

A pharmaceutical grade polysiloxane (silicone) sheet was selected to provide stock to cut out circular end pieces for the bioreactor chamber (McMaster-Carr, P/N 5812T145). The silicone is rated to withstand a temperature range from negative 50 up to 400 degrees Fahrenheit (www.mcmaster.com). This range will accommodate the autoclave process for sterilization. The silicone end pieces were selected for their biocompatibility based on the fact that silicone tubing is used in the bioreactor. The rubber pieces also act as a gasket between the acrylic and aluminum as well as the panel 
mounts. This helps reduce the number of parts and potential for leaks. If future bioreactor chambers must be built, the silicone can be purchased in bulk as stamped out blanks with the desired outer diameter.

Four 7 inch bolts (Ace Hardware, 7" x 1/4") were used to tighten down the end caps. The bolt material is not necessarily important from a biocompatibility point of view since it will not come into contact with the fluid of the system. The bolts were $1 / 4$ inch in diameter and four nuts (Ace Hardware, 1/4”) were used to tighten down on the threads. In addition, 4 stainless steel springs (Ace Hardware, 1/2"x 1/4") that are 1/2 inch with a 1/4 inch inner diameter were slid onto the bolts to prevent over tightening and stripping of threads. The springs had an added bonus of allowing users to evenly tighten the bolts down without inadvertently loosening nearby bolts or creating a bind.

\section{$\underline{\text { The Design }}$}

The bioreactor chamber was primarily designed to withstand the entire range of physiologic pressure without leaking. The shortcomings of the Lock N Lock are largely do the inability to apply sufficient compressive force to seal the lid of the chamber, the use of luer lock panel mounts to bridge two different environments (which is not their intended purpose), and the incompatibility of o-rings with the sterilization process. These specific factors inhibit the chambers' ability to function under the intended conditions. A secondary issue is the inability to rotate the Lock N Lock chamber. This could potentially result in cell sedimentation, and would be ideally improved by having a design that could be rotated. 
The new bioreactor design addresses each of these specific issues in order to provide the bioreactor system with the necessary equipment to operate under the desired conditions, and to do so efficiently. The acrylic cylinder serves as the bioreactor chamber wall, the silicone end plates act as gaskets, and the bolt/spring/cap assembly allows sufficient compressive forces to be generated to seal the interior of the bioreactor chamber from the outside environment. This aspect of the design specifically addresses the issue of the lid seal integrity - which proved to be a critical flaw in the previous design. The issue of leaks at the inlets and outlets of the bioreactor chamber wall is addressed with the flexible silicone end plate. Even though the panel mounts are not intended to bridge different environments (and maintain their separation), selecting a silicone material with ideal mechanical properties effectively compensates for the problem. The silicone is autoclavable so there is no issue with EtO gas sterilization degrading the polymer. The silicone is also very flexible, is rated 50A on the durometer scale, and has a tensile strength of $700 \mathrm{psi}$. The silicone also acts as a gasket for the panel mounts. The flexibility and compressibility of the material allow it to stretch and accommodate the luer lock fitting while maintaining a very tight seal across its surface.

The design of the bioreactor chamber also allows for future improvements, modifications, and customizations. The flexible end plates allow multiple constructs to be assembled in a single bioreactor chamber, custom geometries and orientations of the scaffolds, and accommodation of bulkhead fittings (intended to bridge separate environments without cross contamination), which could be used to replace the panel mounts. The flexible end plates also allow for longitudinal tensile forces to be applied to the scaffold/tissue construct if ever needed or desired. The cylindrical design of the 
bioreactor chamber allows easy rotation of the chamber to prevent cell sedimentation. The square end caps allow manual 90 degree incremental rotations of the chamber during the sodding process. All of the benefits derived from the design are realized through the fabrication process.

\section{$\underline{\text { Fabrication }}$}

Cutting the acrylic tube to the appropriate lengths could have been achieved a number of ways, but the method chosen was to use the horizontal band saw. The horizontal band saw uses a vice to secure the material intended for alteration in place. This is a nice feature because it allows for a straight cut. One must be careful, however, that the vice is angled appropriately. The vice can be angled to give a wide range of diagonal cuts. It is important to make sure the vice is perpendicular ( 90 degrees) to the saw blade after tightening the acrylic tube into place. The vice may turn slightly while securing the material in place. Although the acrylic tube is round, the vice will still hold it tightly in place. Currently, the Cal Poly Hanger has two horizontal band saws. One has a saw blade with small teeth; the other has a blade with medium sized teeth. The smaller saw teeth are good for finer cuts and more brittle materials. Accordingly, the acrylic tube was cut using the finer saw. The rate at which the saw blade cuts through the material is based upon the amount of weight placed at the free end of the band saw. There are usually two to three weights that may be positioned along the band saw to determine the rate at which the saw blade drops and cuts through the material. The weights were moved toward the saw's axis of rotation to slow down the rate at which the material would be cut. 
The acrylic tube was measured using a tape ruler, and a mark was placed at 4.5 inches. The tube was measured multiple times to ensure accuracy, but dimensional tolerancing to the nearest $1 / 16$ inch was adequate for tube length. A fine tip sharpie worked for marking the acrylic. The tube was placed into the horizontal band saw vice and secured in place. The orientation of the tube was double checked to ensure it was perpendicular to the saw. The saw blade was lowered to make sure it would cut at the marked 4.5 inches. The saw was then turned on and the tube was slowly cut through. In this case, there was a small burr that was easily filed off. The bioreactor chamber wall was complete.

Cutting the aluminum down to size for the end caps followed a similar process. The aluminum block was placed in the vice. It was beneficial to have it lying across its 1 inch height dimension because the vice had more surface contact with the block this way. The aluminum was marked off in 3 inch increments along its length using a tape measure. The measurements were taken twice to ensure accuracy. It was important not to mark off all of the three inch increments immediately, as the saw removed about an $1 / 8$ " of stock where it cuts. The aluminum block had to be removed and marked again after each cut. With the aluminum block aligned and tightened into place, the band saw was turned on. The metal was not brittle, so the speed that the saw cut the block was substantially increased. In this case the teeth on the saw were not critical to cutting the material. Larger teeth take larger bites out of the material. Since the material was 3 inches wide, only two 3 inch cuts were necessary to make two 3" x 3" x 1" blocks. The smaller blade was used to cut the material so that the cuts were clean. If many cuts were required, the band saw with larger teeth would have been used. 
With the two aluminum blocks cut down to size, they were machined out using the mill. The mill provided tooling accuracy within thousandths of an inch. Unlike the dimensional tolerance of the chamber tube length, end cap bore was within ten thousandths of an inch from nominal. The aluminum blocks had to be machined to fit over each end of the acrylic tube. There were two steps to the process. First, there was a hole cut all the way through the center of each block. This hole was 2 inches in diameter. It had to match the inner diameter of the acrylic tube in order to provide a flush seal compressing the silicone end plates between the end cap and acrylic wall. Next, a 2.53 inch diameter hole was cut concentrically over the first hole. This was not a "through hole" as it only cut $1 / 2$ inch through the block. This, in effect, created a $1 / 4$ inch shelf for the acrylic tube to rest upon in order to provide greater surface area to seal against the silicone end plate. The outer diameter of the larger hole cut into the aluminum was slightly larger than the outer diameter of the acrylic tube to ensure the tube will slide into the end cap.

After obtaining a mill, a rotary vice was necessary to hold the aluminum in place. The rotary vice was secured to the mill's work top using the supplied rail bolts and nuts. This type of vice was necessary to cut out large diameter holes in the aluminum. A handcrank was used to move the vice 360 degrees. Two bisecting lines were drawn across the aluminum block at 1 1/2 inches from each edge - forming four equal quadrants. The lines should cross each other at the center of the block. Measuring out from the center, a mark was placed on each line exactly 1 inch out. These measurements are important for centering the mill later. The aluminum block was then placed in the center of the vice on top of sacrificial material. Using the $\mathrm{x}$ and $\mathrm{y}$ directional controls, the table was moved so 
the center of the block aligned with the center of the end mill. The end mill of choice for this application was a 1/2 inch center-cutting, 2 flute end mill.

The block was secured in place using t-nuts, bolts, nuts, end hold downs, and step blocks. The t-nut was threaded onto the bolt and then slid down the t-slot of the working surface of the vice. The end holder was placed across and edge or corner of the aluminum block to hold it in place. The step block was used to support the other end of the end holder so it could be tightened down to hold the aluminum block in place. The step block and end holder both have stepped edges that resemble teeth and were interlocked together. The same setup was used to pin down the other side of the aluminum block as well. Having centered the aluminum block on the vice and with the end mill, the work surface was moved in the x-direction until the outer edge of the end mill aligned with one of the 1inch marks. Once the block was lined up properly, the vice was rotated 180 degrees so that it lined up with the other 1 inch mark along the same line but on the other side. If the mill did not line up properly, slight adjustments were made to the position of the table in the $\mathrm{x}$-direction until it did. The vice was then rotated another 180 degrees back to the original mark to check the alignment. The process was repeated until the two marks line up properly with the end mill. Next, the vice was rotated 90 degrees to align the other mark in the y-direction. The alignment was checked in reference to the end mill and adjustments were made in the y-direction until both of the marks in this plane were aligned appropriately. The end goal was to be able to spin the vice in 360 degrees and having the outer edge of the end mill pass through the center of every 1 inch mark. Once this was achieved, the end mill was aligned with one of the four marks and the digital read out was zeroed. This provided a point of reference if alignment was lost. 
The milling process was straight forward, but somewhat time consuming. There was a depth gauge on the drilling portion of the mill that measures the depth of a cut. The end mill was lowered down until it almost touched the surface of the aluminum block. A small piece of paper was slid under the end mill and wiggled back and forth freely. The end mill was lowered using one hand while the other hand was sliding the paper. The end mill was lowered until the end mill barely snagged the paper and could no longer slide freely. The depth gauge was then zeroed. This was done in this fashion because the mill exerts enough force to embed the end mill into the material and give a false reading of the surface depth to be zeroed. The depth of each cut was then measured using the gauge. The RPM of the machine was set to 1700 . The speed affects the finish on the cut surface slightly, but more importantly the RPM contributes to heating up the end mill. A spray bottle of coolant was necessary to keep the bit from overheating and a brush was used to clean shavings from the block and end mill. The end mill could only penetrate 50 thousandths of an inch of the aluminum block safely at a time. Larger cuts would run the risk of breaking the mill, ruining the end mill, or fouling the material. Once the set depth was reached, the end mill was locked in place and sprayed with coolant. The rotary vice was spun 360 degrees and sprayed with coolant by hand. Roughly every quarter turn the end mill had to be sprayed with the coolant to prevent over-heating. Whether the vice was spun in the left or right directions, it did not really matter. One direction was "up milling" and the other direction was "down milling". They dictate the direction the shavings are flung and the quality of the finish. The only finish that was of particular importance was the shelf where the silicon end plate would be seated. That is dictated by the tip of the end mill and is not directionally influenced. Once a full circle had been cut, the end mill 
was raised and brushed off. The process was repeated going an additional 50 thousandths deeper into the material each time. This continued until the block was cut through entirely and the small aluminum slug was removed from the center.

The next step was to create the shelf in the aluminum block. From the zeroed position, the table was moved over 0.25 inches. The end result was that the end mill's edge was exactly 1.25 inches away from the center of the block. This would provide the correct 2.5 inch outer diameter required to fit the acrylic cylinder. The rotary vice was spun 360 degrees to ensure the center of the end mill aligned with the entire perimeter of the 1 inch circular edge. The same process was used to remove 50 thousands of the block with each pass. The only difference with this phase was that the machining ended when a half inch of the aluminum block was removed. This process was performed to fabricate both end caps.

Once this had been done to both end caps, it was time to drill a hole through all four corners of the two blocks. Using a straight edge, diagonal lines were penciled on the block from each corner. Then using digital calipers, the distance from the outer diameter of the hole to the nearest corner's point was measured. Each corner was measured accordingly, and a mark was placed at half the distance between the outer diameter's perimeter edge and corner point - measured from the perimeter edge. This ensured that the bolt-hole pattern would be centered relative to the acrylic cylinder and would not be thrown off by variation in side lengths of the aluminum block. These marks were used to drill the holes for the securing bolts. The measurements and marks were only performed on one of the two blocks because both blocks were to be drilled through at the same time. This helped reduce the time spent machining and ensured alignment later. 
To do the drilling, the mill was fitted with the drill bit adaptor. This allowed the mill to use the various bits necessary to drill the holes in the block. Once again, sacrificial material was placed upon the vice to prevent damaging it. The two end caps were placed together - sandwiching a short acrylic tube (1 inch tall) cut from the excess acrylic material. The tube helped maintain critical alignment during the drilling process. It prevented any slippage and ensured that the bolts would align properly when the larger acrylic tube is placed between them. The end caps were not machined exactly the same dimensionally and this compensated for variations in the location of the machined holes relative to the block center-line. The blocks were secured to the rotary vice the same way as before using end hold downs and step blocks. The rotary vice was used here as well because it allowed the blocks to be moved without having to unclamp them. Unclamping would cause unwanted shifts and variation in alignment. Next, a small bit was placed in the mill drill chuck. A 1/8 inch bit was selected because it was small enough to accurately align with measured marks and sturdy enough to not break off into the material. With the smaller bit, it was important not to apply too much pressure to the bit. The bit may bend and break. The same RPM (1700) used for milling was also used for drilling the pilot holes. After the pilot holes were drilled, the small bit was swapped out for the $1 / 4$ inch bit. The $1 / 4$ inch bit was aligned with each pilot hole and, using the same RPM as before (1700), each hole was drilled using the larger bit. The holes were drilled using a technique called "peck-drilling" where the drill cuts through the material in quarter inch increments. The bit was brushed and sprayed with coolant in between cuts (Figure 3.2). 


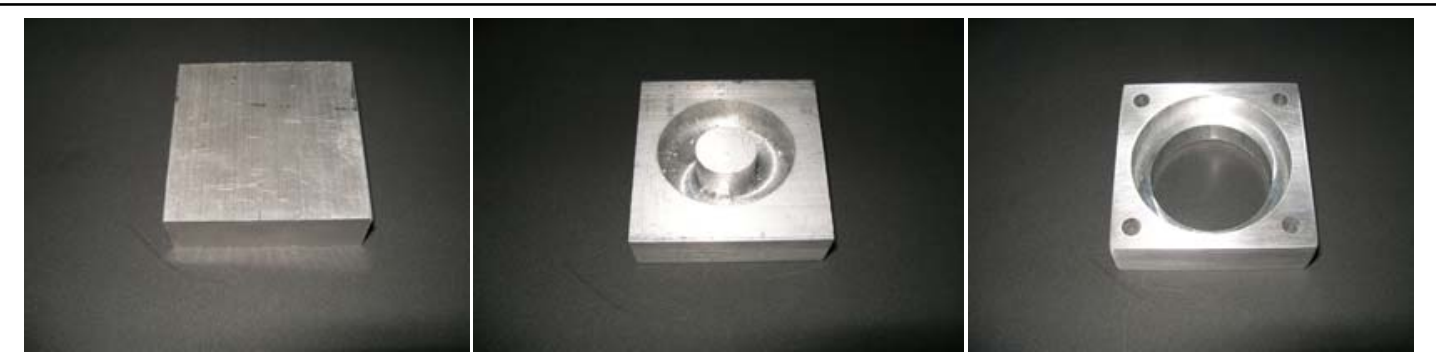

Figure 3.2 The progression of machining the aluminum end caps.

The hardware store was out of stock for the $1 / 2$ inch steel springs that were needed. As a result, 1 inch long springs were purchased and cut down to size using a pneumatic grinder. A pair of needle nose pliers was necessary to hold the spring for safety reasons. The spring heated up considerably and the grinder itself was not gentle to flesh. Care was taken to recognize the direction in which the grinder spun because it is the same direction that the sparks are thrown. Safety glasses were worn to protect the eyes from flying metal debris.

To prepare the silicone end plates, the acrylic tube was used as a stencil to produce an outline for cutting out the disks. The silicone was cut using sharp scissors. If the design is scaled up, the silicone disks can be purchased with the desired diameters and thicknesses. A leather hole-punch was used to cut holes in the silicone end plates to accommodate the luer lock style fittings. The second to smallest punch was used to provide a snug fit for the panel mounts.

\section{$\underline{\text { Assembly }}$}

The assembly is simple and straight forward and has been broken down into a logical series of steps. Figures 3.3, 3.4, and 3.5 illustrate completion of these steps. 


\section{End Plates}

1. Take a silicone end plate and bend it in half

2. Insert the panel mount into the silicone, barb first, until the silicone reaches the threads

3. While pushing on the panel mount, screw it into place using a twisting motion

4. The stop on the fitting should be in contact with the silicone, be careful not to over-tighten and warp the plate

5. Once in place, bend the plate in half again exposing the barb end

6. Thread the nut onto the panel mount until snug

7. Repeat this process for both end plates and all the necessary holes

\section{Dumbbell}

8. Depending on the length of scaffold, add the appropriate luer lock fittings to suspend the scaffold

9. After fitting the scaffold into place, use suture to tie the scaffold down onto each barb 


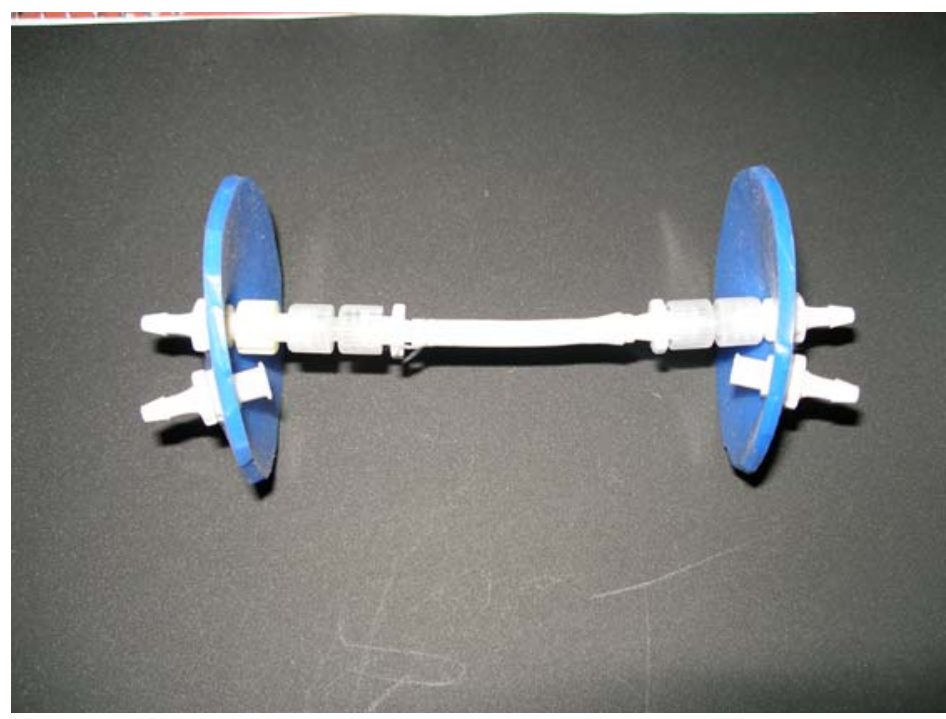

Figure 3.3 Dumbbell assembly of silicone end plates, luer lock fittings, and scaffolding.

\section{Adding Acrylic Wall}

10. Taking one end of the dumbbell assembly, bend the end plate in half toward the scaffold

11. The folded end plate is then threaded through the acrylic tube at a diagonal angle

12. The folded end plate can be grasped from the opposite end of the acrylic tube and pulled through far enough to expose the lip of the plate

13. Once the lip of the plate has cleared the end of the tube, the rest of the folded disk can be pulled out until it is flush with the tube

14. Now both end plates should align perfectly with the outer diameter of the acrylic tube 


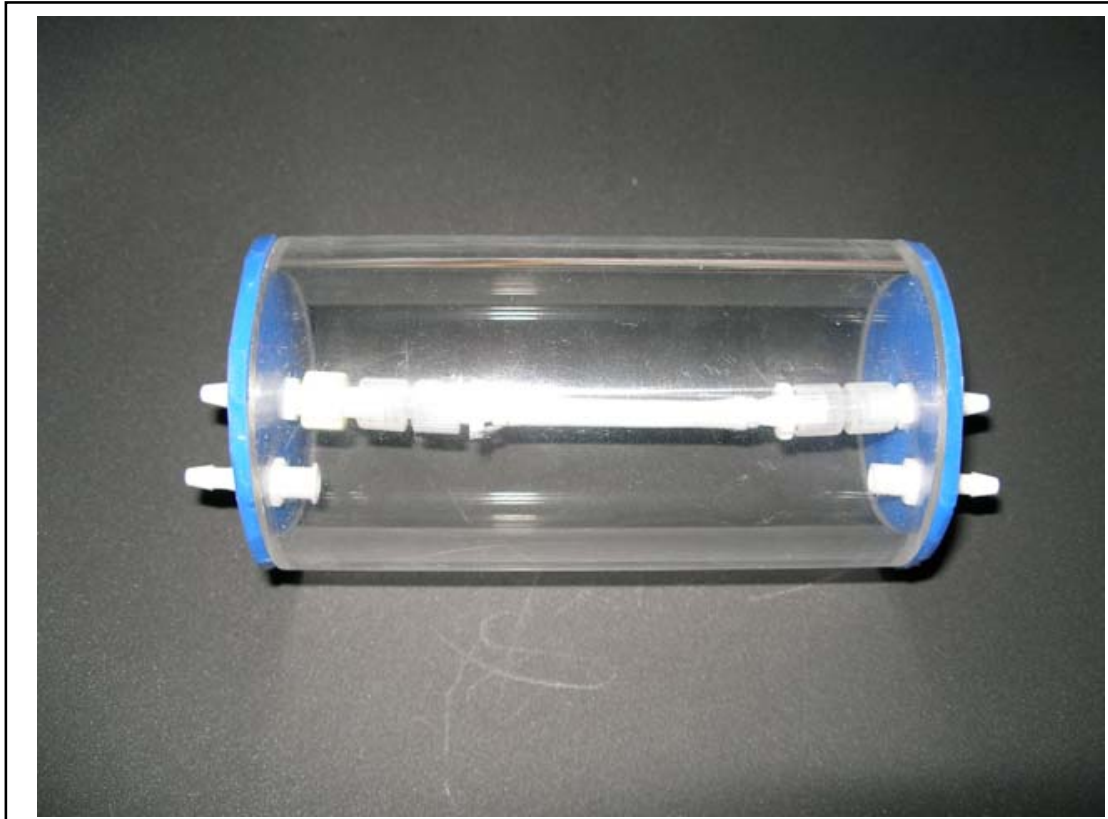

Figure 3.4 Integration of dumbbell assembly with acrylic wall.

\section{End Caps}

15. The aluminum end caps can now be positioned over the end plates and acrylic tube on both ends

16. The $1 / 2$ " steel springs should now be slid onto the 7 " bolts

17. The bolts are then threaded through the 4 corner holes in the end caps

18. Once in place, nuts are threaded onto the bolts and tightened down 


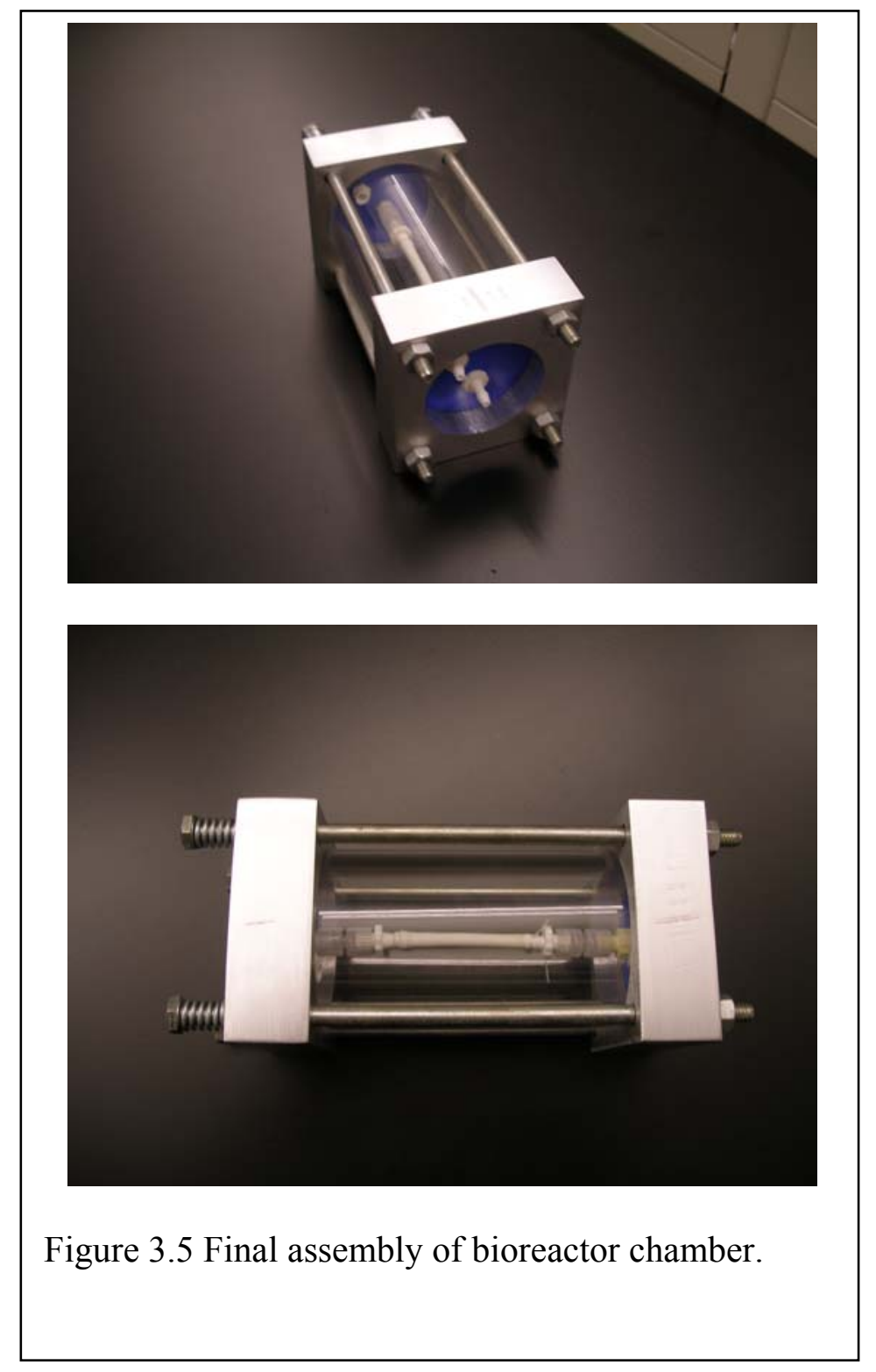




\section{Chapter 4: CHARACTERIZING THE PROPERTIES OF THE NEW BIOREACTOR CHAMBER}

\section{Introduction}

With the newly designed bioreactor chamber manufactured and assembled, tests were conducted to analyze the bioreactor chamber's capacity to accommodate a wide range of pressures. Initially, the bioreactor chamber was just evaluated at elevated pressures well above expected operating conditions. Once the chamber proved durable in these tests, a leak test was planned and performed. A leak pressure test was not conducted first with the bioreactor chamber for two reasons. The first reason was that there was only one bioreactor chamber fabricated. As a result, the bioreactor chamber was not expendable. A pressure leak test may result in a catastrophic mode of failure that would ultimately damage the chamber. It was unlikely that this would happen, but not worth the risk. The second reason was a safety concern. Once again, even though the bioreactor chamber was designed to withstand elevated pressures, there existed the extremely remote possibility of the pressurized chamber exploding. Such is the risk of having any container pressurized and the reason why warning labels are placed on products with contents under pressure. There was no reason to believe the chamber would fail catastrophically, but a conservative protocol was adopted in light of the many anticipated tests using the newly designed chamber. The silicone plates were regarded as the most likely sites of failure at high pressures. The flexibility of the silicone might compromise the seal between the acrylic tube and end cap shelves at elevated pressures near the point of failure. The panel mounts are not designed to be leak proof. They are not intended to bridge two separate environments (e.g. media and air). They also pose a threat and may 
leak, but the silicone itself is intended to act as a sealing gasket on the fitting. At high pressures, silicone distortion may compromise the seal between the silicone and panel mount.

Initially, the goal of this project was to use "manual pressure injection" to backpressure the system, and thus it was the focus of work in this chapter. Manual pressure injection involves straining the system by increasing the internal system fluid volume within a closed system. The increased volume would strain the walls of the closed system and generate an elevated pressure. It is similar to inflating a bicycle tire. The peristaltic pump would effectively decrease the size of the constraining system while, ideally, the internal volume remained constant. The fluctuating compressions would result in the formation of a pressure wave. Based on a finite system and volume, determining the necessary amount of fluid required to be injected into the system to achieve the desired backpressure could be easily done. The success of the technique is contingent upon the system's ability to maintain pressure and is entirely ineffective in the presence of a leak. Therefore, assessing the bioreactor chamber's ability to withstand elevated pressures would help draw conclusions regarding whether or not manual pressure injection would be a reasonable backpressure generating methodology. This setup did not take into consideration the bioreactor chamber's ability to withstand pressures with more complex vessel geometries or multiple vessels. The additional panel mounts placed in the silicone end plates that would be necessary to accommodate multiple scaffolds or different vessel geometries may decrease the integrity of the bioreactor chamber seal. As a result, a separate series of tests would be needed to assess these factors and their potential influence on the bioreactor chamber's ability to withstand 
pressure. Results and conclusions drawn from this study should not be extended to include the more complex configurations just mentioned.

\section{Materials and Methods}

The bioreactor chamber was assembled as described previously. One end plate contains a single panel mount (entry side) while the other has two (exit side). This simulated the setup likely to be used during tissue cultivation. 12 inches of silicone tubing was attached to the single barb of the panel mount luer lock fitting located on the entry side of the bioreactor chamber. The two barbs on the other end of the chamber were fitted with two 3 inch lengths of silicone tubing. One length of tubing was capped with a barbed female luer lock fitting coupled with a male luer lock plug. The other length of tubing was fitted with a barbed female luer lock fitting to accommodate the pressure transducer which was capped with a male luer lock plug fitting as well. The 12 inch length of silicone tubing was fitted with a two-way stop valve. A diagram of this set up is illustrated in Figure 4.1. The settings from previous calibrations could have been reused, but it was worthwhile to ensure accuracy and simply calibrate the transducer before each use. Before the transducer was hooked in line with the open system, the pressure transducer was calibrated and zeroed using a two point calibration. 


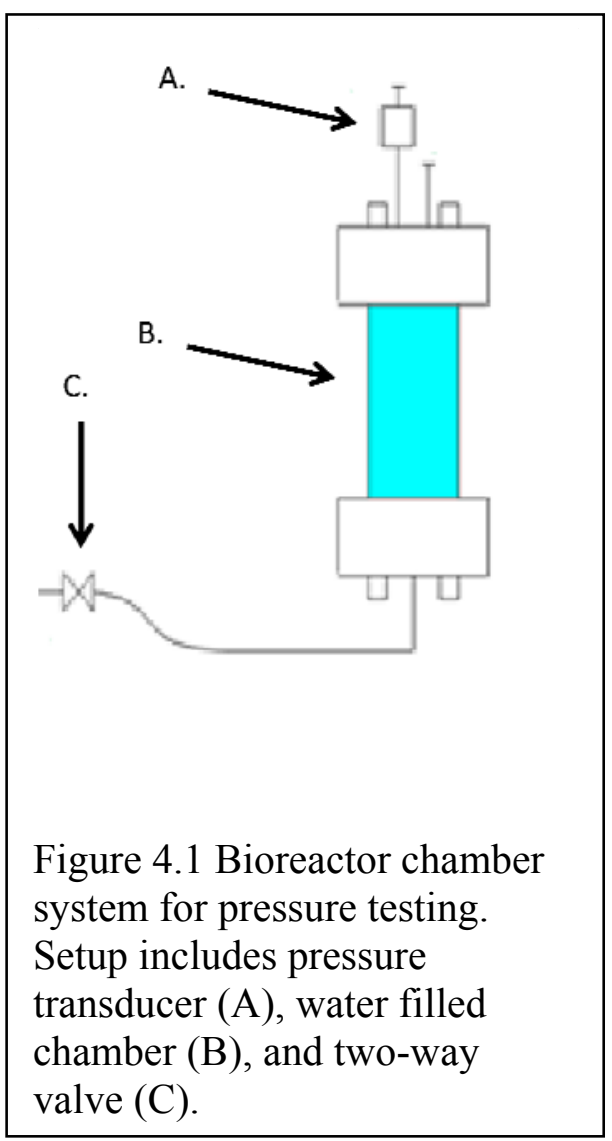

The luer lock plug was removed from the pressure transducer and the stop valve was opened. Using a luer lock syringe, the bioreactor chamber was filled with water. To accomplish this task, the syringe was filled with water, attached to the stop valve, and emptied into the system. The valve was closed and the syringe was removed, refilled, reattached and then emptied into the system again. The process was repeated until water shot out from the opposing end of the bioreactor chamber when the chamber was only half full. This occurred because the chamber was resting on its side. It was necessary to stand the bioreactor chamber on end to fill it completely. Once the fluid leaked out from the end of the pressure transducer (i.e. the system was completely full), it was capped off. 
Before removing the syringe, the stop valve was also closed to prevent fluid leakage. The system was then ready to proceed with testing and evaluation.

The pressure range capability of the bioreactor chamber was tested first. Another syringe full of fluid was attached to the stop valve. The valve was opened and fluid was slowly injected into the system. The internal pressure of the system was monitored on the computer using LabChart 7 data acquisition software. The pressure was cycled from 0 $\mathrm{mmHg}$ to $1000 \mathrm{mmHg}$ by pushing down the plunger on the syringe and releasing it repeatedly. The bioreactor chamber was then inspected for any signs of leakage. Next, the pressure was set at $1000 \mathrm{mmHg}$ and the two-way valve was closed to maintain the pressure. The bioreactor chamber was left pressurized for 5 minutes and inspected for signs of leaks. The bioreactor chamber was then left pressurized for an hour and checked every 5 minutes for any signs of fluid loss.

The final stage of assessment involved the leak pressure evaluation of the bioreactor chamber. Testing proceeded with the leak pressure test because the bioreactor chamber had performed well as it endured the elevated pressures from the previous tests. It was determined that the leak pressure test would not jeopardize the bioreactor chamber, surrounding lab equipment, or laboratory personnel. It was also inferred that the critical leak pressure test evaluated the extra-luminal seals of the bioreactor chamber. The luminal pressure tests distributed the forces across the luminal portion of the system stressing only the tubing and fitting connections. As a result, no scaffolding was included in the leak pressure test performed. The chamber was still assembled in the same configuration as previously mentioned. The exit side of the bioreactor chamber had two lengths of tubing - one capped and the other attached to the pressure transducer. The 
other end of the chamber was fitted with a length of silicone tubing with a two-way valve, a length of platinum cured silicone pump tubing attached, and another length of regular silicone tubing attached to its end. The platinum cured silicone pump tubing was clamped into the peristaltic pump. The free end of the tubing exiting the peristaltic pump was then placed into a beaker full of water. The two-way valve was opened and the pump was primed. This removed all of the air from the lines. The setup is illustrated below in Figure 4.2.

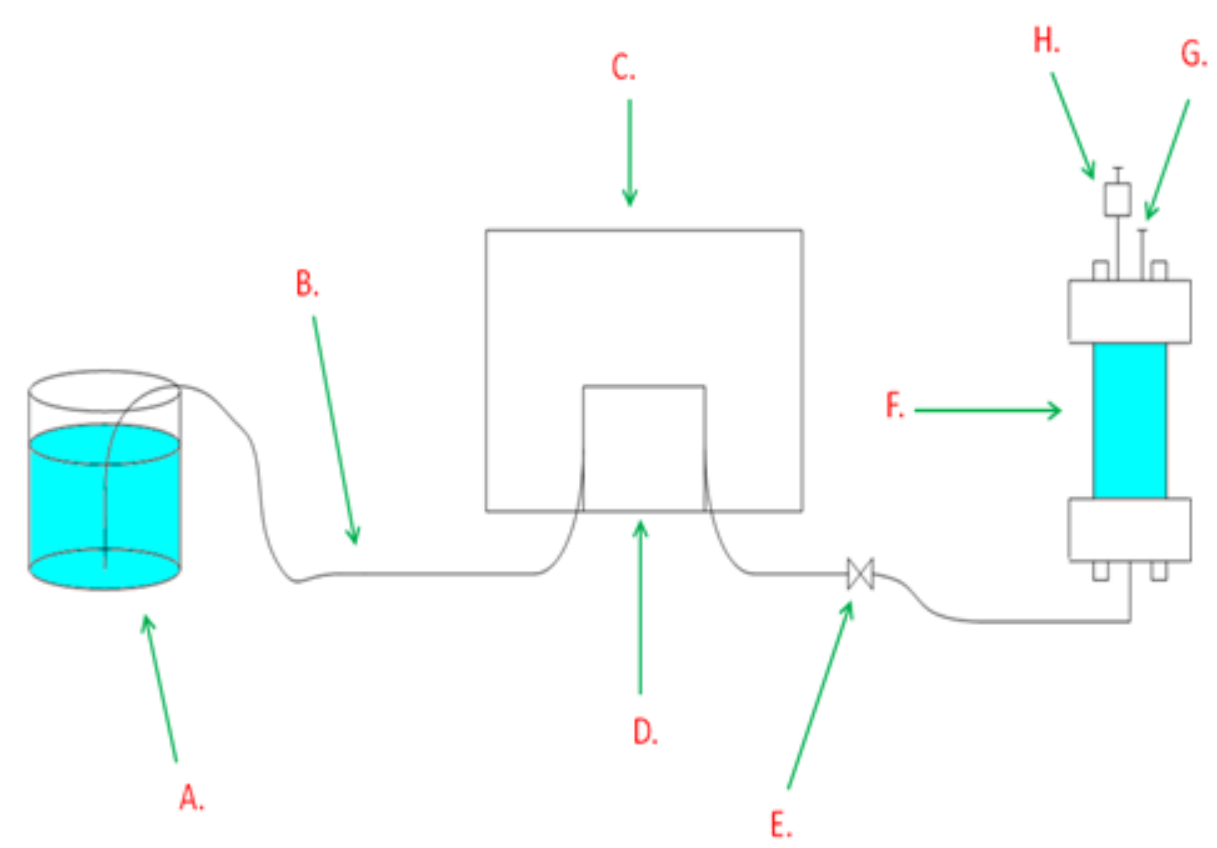

Figure 4.2 The leak pressure test setup used to fill the system with fluid and evacuate air from the lines. A beaker (A) was filled with water and the free end of the silicone tubing (B) was placed into the beaker. The silicone tubing ran to the peristaltic pump (C) and was connected to the platinum cured pump tubing which was clamped into the peristaltic pump head (D). The tubing then transitioned back into general purpose silicone tubing and was connected to a two-way valve (E). tubing connected the valve to the bioreactor chamber $(\mathrm{F})$ which was stood on end and filled with water. The extra-luminal port $(\mathrm{G})$ on the exit side of the bioreactor chamber was capped, and the luminal port with the pressure transducer $(\mathrm{H})$ was capped after the system was entirely filled with fluid. 
Once this step was completed, the RPM of the pump was increased and fluid was forced into the chamber until catastrophic failure occurred. Catastrophic failure was defined as the formation of any leak resulting in the loss of fluid from the system at any of the tubing connections, luer lock fittings, chamber seals, or any such combination. The initial plan was to use the peristaltic pump to rapidly pressurize the bioreactor chamber. It was not taken into consideration, however, that the cyclic compression of the closed system and the high RPM of the pump would generate a highly erratic pressure wave that quickly increased in magnitude until system failure. The erratic pressure measurements generated by the cyclic pump made analysis exceedingly difficult and drawing accurate conclusions practically impossible. Based on the issues that resulted from the process, the leak pressure test had to be performed by hand, just like the other trials. The manual test was performed three times in order to more accurately characterize the actual leak pressure of the bioreactor chamber.

\section{$\underline{\text { Results }}$}

The bioreactor chamber withstood the pressure range of 0-1000 $\mathrm{mmHg}$. There were no signs of leaks during cycling, but the pressure inside the chamber dropped roughly $1 \mathrm{mmHg}$ in 5 minutes. After the hour long test, the system lost roughly $12 \mathrm{mmHg}$ and still showed no signs of leakage. Catastrophic failure did not occur until the leak pressure tests were performed. Even then, there were no leaks from the chamber. Failure only occurred at the interface between the luer lock barb and the silicone tubing connecting to it. Table 4.1 (below) contains the recorded pressure measurements obtained during the pressure leak tests by the data acquisition equipment. The maximum pressures 
for each of the three tests were $1213.61 \mathrm{mmHg}, 1215.75 \mathrm{mmHg}$, and $1196.39 \mathrm{mmHg}$ - all reached within at least 11 seconds.

Table 4.1 Leak pressure test of new
bioreactor chamber.
\begin{tabular}{|c|c|c|c|}
\hline Time(s) & $\begin{array}{c}\text { Test 1 } \\
(\mathrm{mmHg})\end{array}$ & $\begin{array}{c}\text { Test } 2 \\
(\mathrm{mmHg})\end{array}$ & $\begin{array}{c}\text { Test 3 } \\
(\mathrm{mmHg})\end{array}$ \\
\hline 0 & 14.88 & 21.11 & 25.76 \\
\hline 0.5 & 20.19 & 34.39 & 33.68 \\
\hline 1 & 24.38 & 95.810 & 49.31 \\
\hline 1.5 & 49.41 & 234.06 & 185.52 \\
\hline 2 & 106.18 & 376.05 & 272.33 \\
\hline 2.5 & 210.00 & 475.63 & 340.49 \\
\hline 3 & 247.19 & 591.45 & 441.24 \\
\hline 3.5 & 315.50 & 695.94 & 524.93 \\
\hline 4 & 428.01 & 732.82 & 622.36 \\
\hline 4.5 & 506.13 & 765.22 & 682.86 \\
\hline 5 & 584.91 & 775.69 & 792.04 \\
\hline 5.5 & 660.53 & 816.97 & 860.35 \\
\hline 6 & 745.50 & 858.56 & 923.70 \\
\hline 6.5 & 795.57 & 875.37 & 965.86 \\
\hline 7 & 852.84 & 952.11 & 990.89 \\
\hline 7.5 & 918.80 & 1018.23 & 1083.12 \\
\hline 8 & 950.12 & 1066.36 & 1082.35 \\
\hline 8.5 & 1041.07 & 1126.19 & 1139.37 \\
\hline 9 & 1106.82 & 1144.94 & 1196.39 \\
\hline 9.5 & 1156.18 & 1164.40 & 25.86 \\
\hline 10 & 1157.15 & 1193.68 & -1.00 \\
\hline 10.5 & 1168.85 & 1173.40 & - \\
\hline 11 & 1213.61 & 1215.75 & - \\
\hline 11.5 & -2.33 & 48.49 & - \\
\hline & & & \\
\hline
\end{tabular}




\section{Discussion and Conclusion}

Based on the results generated in this chapter, was demonstrated that the bioreactor chamber is able to operate under and withstand physiologic pressures over at least an hour. The bioreactor chamber did not show any signs of fluid loss within the range of $0-1000 \mathrm{mmHg}$ during the leak pressure test or the hour-long pressurization. Thus, the newly designed bioreactor chamber is considered a success, based on the initial goal of creating a chamber that could accommodate physiologic pressure fluctuations. However, it is problematic that the pressure within the system dropped. If this system cannot hold a constant pressure, the "manual pressure injection" method will have to be changed. The dropping pressure indicated that there was a leak somewhere in the system. Wherever the leak was, it was very small, but suspicion focused on the tubing connection that failed during the leak tests. Water is incompressible. Accordingly, when small amounts of fluid are forced into closed systems, the internal pressure is greatly affected. In addition, a very small loss of fluid from a similar system would result in a large drop in pressure. There are many potential sources of leaks within the experimental setup used in this study that will have to be further explored.

In summary, the bioreactor chamber was able to withstand physiologic pressures without failing. Testing at pressures in excess of ten times human physiologic conditions were successful as well. However, the leak within the system was an issue. Therefore, the source needed to be identified and eliminated in order for pressurization of the system through manual pressure injection to be reasonable. The large number of variables within the experimental setup used in this study made determining the location or cause of the leak difficult and impractical. Although the bioreactor chamber displayed no signs of 
leakage, it could not be ruled out as potential source with any reasonable degree of certainty. To better understand the source of fluid loss, further testing on simplified systems with reduced numbers of variables would be necessary. This leak "diagnostic" testing will be described in the next chapter. 


\section{Chapter 5: QUANTITATIVE LEAK DIAGNOSTICS}

\section{$\underline{\text { Introduction }}$}

As stated in Chapter 4, manual pressure injection was the preferred technique to be used for back-pressuring the system. Ideally, a specified volume of fluid would be injected into the closed bioreactor system to elevate the base-line pressure to $100 \mathrm{mmHg}$ - or any other desired pressure. If all bioreactor systems were built uniformly in accordance with specifications and filled with a predetermined volume of fluid, then it would be feasible to determine a specified volume to be injected to reach desired pressures accurately in all systems. The potential benefit of such a system would be that it would require minimal changes to the original Lock N Lock bioreactor setup while maintaining simplicity and scalability. The major fault with the manual pressure injection technique is that it is extremely sensitive to the presence of any leaks within the system. The study performed in the previous chapter identified the existence of a leak within a simplified experimental setup that included the newly designed bioreactor chamber, silicone tubing, and luer lock fittings. No physical evidence of fluid loss was found relating to the new bioreactor chamber within the range of $0-1000 \mathrm{mmHg}$, but the source of the leak had to be determined in order to proceed with effectively back-pressuring the system. If the bioreactor was the source of leakage, the problem could be addressed with further design modifications or improvements, and manual pressure injection would still be a reasonable method for pressurizing the system. If the leak was related to a more fundamental portion of the system, such as the tubing or fittings, manual pressure injection would require a complete overhaul of the entire system and would no longer be reasonable. 
After taking a thorough, qualitative look at various simplified bioreactor models, a general understanding of the technical difficulties concerning manual pressure injection was established (See Appendix B for summaries of Qualitative assessments of various bioreactor components). The next step was to apply this understanding and perform a study to assess practical potential solutions. By determining the factors that influence pressure drop, their significance could be assessed. Then the factor, or combination of factors, that provides the greatest improvement in reducing the rate of pressure loss within the system may be determined. The intent behind this study was based on the idea that once a problem is well defined, steps can be taken to directly address it or maneuver around it.

The factors of interest for the newly designed bioreactor system included tubing permeability, and the interface between tubing and luer lock barb fittings. The silicone tubing used within the system is gas permeable to allow $\mathrm{CO} 2$ gas exchange within the incubator, which is maintained at $5 \% \mathrm{CO} 2$ content. This exchange allows the media within the system to maintain a desirable level of $\mathrm{pH}$. The permeability may influence system pressure by allowing trapped air bubbles to diffuse out of the system while maintaining the fluid within the system. This would cause a decrease in internal system fluid volume (gas is a fluid) stressing the constraining walls of the bioreactor. As volume inside decreases, pressure would decrease as well.

The other potential problem arises from the coupling of the silicone tubing and fittings. The silicone tubing is compliant and readily capable of deformation. The barbed tip of the fitting is slanted and focuses internal pressure in a ring around the tip - right 
around the circumference of the barb. Once the connection between the tubing and the barb are compromised, the tubing and fitting may separate.

In order to determine the impact these two factors have on leaks, studies in this chapter focused on applying proposed treatments on a simplified bioreactor subsystem that would impair the factors' contribution to pressure loss when compared to an uninhibited system. The simplified bioreactor subsystems are intended to reduce the number of potential variables influencing the drop in pressure. The simplified system essentially consisted of only luer lock fittings and a single length of tubing suspended between them. Based on information gained from Value Plastics technical support, the luer lock fittings are designed to be leak-proof up to 40 psi. As a result, the simplified system should only have two potential sources of leakage - the permeability of the tubing and the interface between the fitting barbs and tubing. The reservoir, peristaltic pump, and new bioreactor chamber were not included in the testing as they would introduce more variables and potentially confound results. If the tubing and connections maintained pressure, the testing would move on to include the bioreactor and more complex system designs.

\section{Methods and Materials}

In order to conduct this test, an experiment was designed with a treatment structure of two factors - each with multiple levels. Factors refer to the experimental treatments applied to the experimental unit. The experimental unit is the most basic element of an experiment to which the experimental treatments are applied. In this study, the experimental unit was the simplified bioreactor subsystem. The first factor was 
bioreactor subsystem modification. Factors can have multiple levels. Levels are equivalent to variations of the applied treatment. The subsystem modification factor in this study had three levels: a control, tubing spring clamps (Ace Hardware, 1/4"), and non-permeable micro tubing (HomeDepot, 1/4" x 25'). The control was necessary for comparative purposes when assessing the capacity of other bioreactor subsystem modifications to reduce pressure loss. The tubing spring clamps were used as a treatment because they showed promise as a potential solution for leaks associated with silicone tubing and luer lock barb interfaces during the primary stages of qualitative testing (Appendix B). Non-permeable tubing was used as another bioreactor subsystem modification because the silicone tubing used in the original bioreactor is permeable in order to facilitate $\mathrm{CO} 2$ exchange with the environment within the incubator. It was thought that this permeability might have played a role in pressure escaping from the system.

The second factor was system size and had two levels: 12 inch (small), and 24 inch (long) lengths of tubing. Since the simplified models of the bioreactor systems were much smaller than the original bioreactors that are being used in the laboratory, it was necessary to take this difference into consideration during testing. The experimental unit was the simplified bioreactor model, and the response variable was the pressure drop over a one minute time frame.

The testing structure was a multi-factorial complete randomized block (CRB) design. Transducers were blocked on because they were potentially controllable nuisance factors that could have introduced significant variation into the test. In human studies, for example, it is generally recognized that person-to-person variation is significant and has 
the potential to reduce the sensitivity of a statistical test and result in a type II error. Type II errors are characterized by a failure to detect statistically significant differences between or among treatments when one, in fact, truly exists. Not knowing whether or not significant variation existed transducer-to-transducer, it was reasonable to take precautions just in case the pressure transducers did not provide uniform measurements relative to one another. As a result, transducers were blocked on in the study. Essentially, this means that each transducer used in the trial would receive all treatment combinations. This is a beneficial technique, when applied correctly, because the statistical analysis of the data can then remove the random variation attributed to each pressure transducer and compare treatment combinations without the added variability. In the end, it makes the test more sensitive to detecting significant differences between treatment combinations and their response variable measurements. All experimental data was analyzed using Minitab (statistical software) at a standard alpha of 0.05 . Uncontrolled nuisance factors did exist. Water temperature and trapped gas would be examples - even though all water used came from the tap and bubbles were removed from the lines during system assembly. The potential variation the nuisance factors may have added to the test was reduced through randomization of treatment combination and transducer run orders. There were six treatment combinations because there were two factors, one with three levels and the other with two. The primary interest of the experiment was to identify which treatment or treatment combination would drop the pressure the least.

Before the main study could be conducted, a pilot study was performed in order to determine the number of samples necessary to produce results with an acceptable power. The power of a test refers to the probability that the test will reject the null hypothesis 
when the alternative hypothesis is true. Rejecting the null hypothesis in this case would indicate that one or more of the factors are significant at one or more of their levels, or that there is a significant interaction between factors. To conduct the pilot study, all six treatment combinations were randomly assigned to two transducers using a random number generator. The combinations were labeled one through six. The number generator would produce integers from one to six (inclusive) for one transducer until all six treatment combinations were represented one time. The run order between transducers was also randomized. The transducers were labeled one and two. The number generator produced a series of ones and twos that dictated which transducer would be used and in what order. Numbers were generated until all treatment combinations were tested for by each transducer.

While conducting the testing, the various treatment combinations were connected to $\mathrm{AD}$ Instruments pressure transducers, which were in turn connected to the LabChart data acquisition module and software (Figure 5.1). Each pressure transducer was independently calibrated using the two point calibration technique, and the settings were saved to separate files in order to prevent repeatedly recalibrating the transducers. 


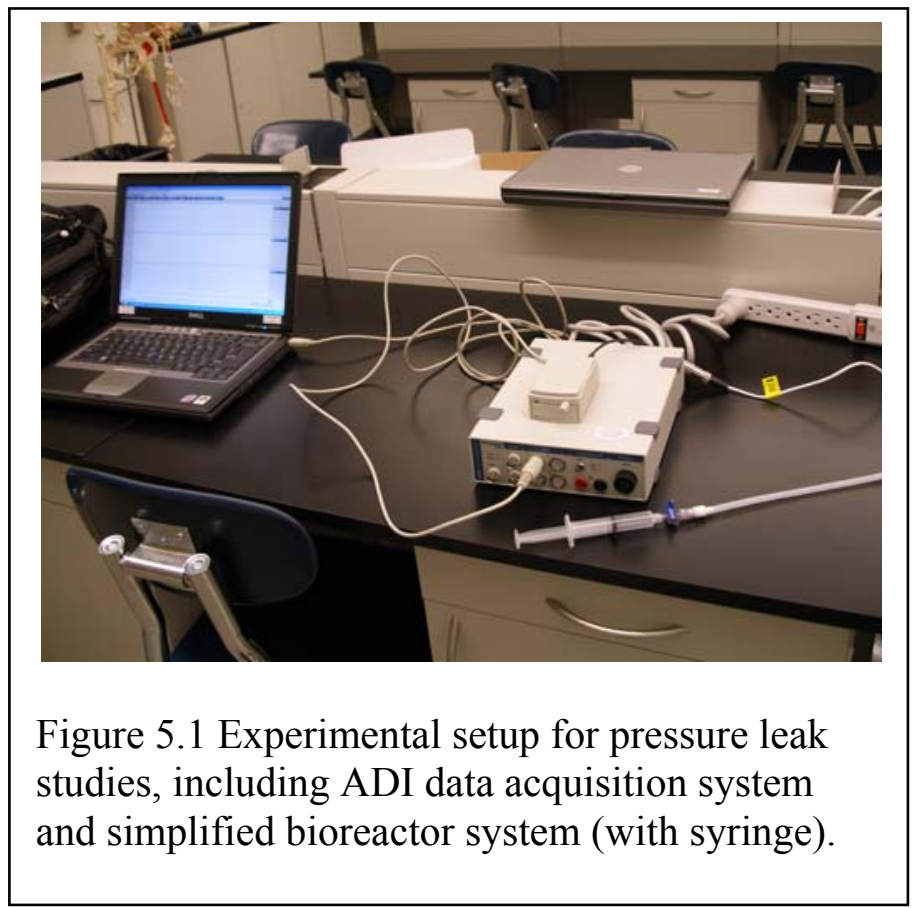

The signal measured by the pressure transducer was amplified using a bridge pod amplifier. The amplifier was necessary because the experiment had to accurately record measurements involving low pressures. Once the simplified bioreactor subsystem was connected to the system, it was flooded with fluid and then sealed using valves. Using a syringe full of water, additional fluid was then forced into the system to increase the pressure. Pressure readings were monitored on the LabChart software. Pressure was initially set to $110 \mathrm{mmHg}$. The system was allowed to settle until it reached $100 \mathrm{mmHg}$. The pressure drop over the following minute was then recorded. Performing the pilot study yielded 12 sample measurements - one measurement for each combination and on each transducer.

The generated data was averaged together for each treatment combination to produce mean estimates. The 12 measurements were accordingly reduced to 6 means. These means were then used to conduct a power analysis using Minitab. Based on the 
results, adequate sample sizes were then selected for performing the main study. The analysis was performed with the intention of establishing a power of 0.90 .

Performing the main study was similar to conducting the pilot study. The only difference was in the number of treatment combinations tested and the number of transducers used. Randomization was still established using random number generated sequences. Every transducer was independently calibrated using the same method as before, and each the settings were saved to separate files for later use. Recalibrating each transducer before use would have been time consuming. The data points were collected and recorded. They were later transferred into Minitab to be analyzed. The statistical analysis of the data was performed using the General Liner Model (GLM) for a two-way ANOVA. The two-way analysis of variance (ANOVA) was useful because this experiment was assessing the affects of multiple factors and treatment levels with potential interactions. The factors and treatment combinations were then compared and conclusions were drawn.

\section{$\underline{\text { Results }}$}

\section{Pilot study}

From the pilot study, 12 data points were gathered for the power analysis. The corresponding measurements are listed in the Table 5.1 below. The points were averaged together for each of the treatment combinations. In addition, the mean range and data standard deviation were also calculated. This information was then used to perform the power analysis in Minitab. The resulting graph is listed in Figure 5.2. Based on the experimental design, the tabulated sample size indicated the number of blocking groups 
required in order to obtain the desired power level of 0.90 . The power analysis indicated that a sample size of six was needed. As a result, it was necessary to block on six different transducers. "Blocking" on six different transducers meant that six separate transducers would each be used to test all six treatment combinations.

Table 5.1 Pilot study pressure loss measurements for
treatment combinations for each transducer.
\begin{tabular}{|l|r|r|}
\hline $\begin{array}{l}\text { Treatment } \\
\text { combination }\end{array}$ & $\begin{array}{l}\text { Transducer 1 } \\
\text { (mmHg/min) }\end{array}$ & $\begin{array}{l}\text { Transducer } 2 \\
\text { (mmHg/min) }\end{array}$ \\
\hline Control-12" & 0.85 & 2.50 \\
\hline Non-permeable-12" & 1.30 & 1.10 \\
\hline Control-24" & 1.00 & 1.80 \\
\hline Non-permeable-24" & 5.90 & 3.50 \\
\hline Spring clamp-12" & 0.30 & 0.70 \\
\hline Spring clamp-24" & 0.10 & 0.20 \\
\hline
\end{tabular}




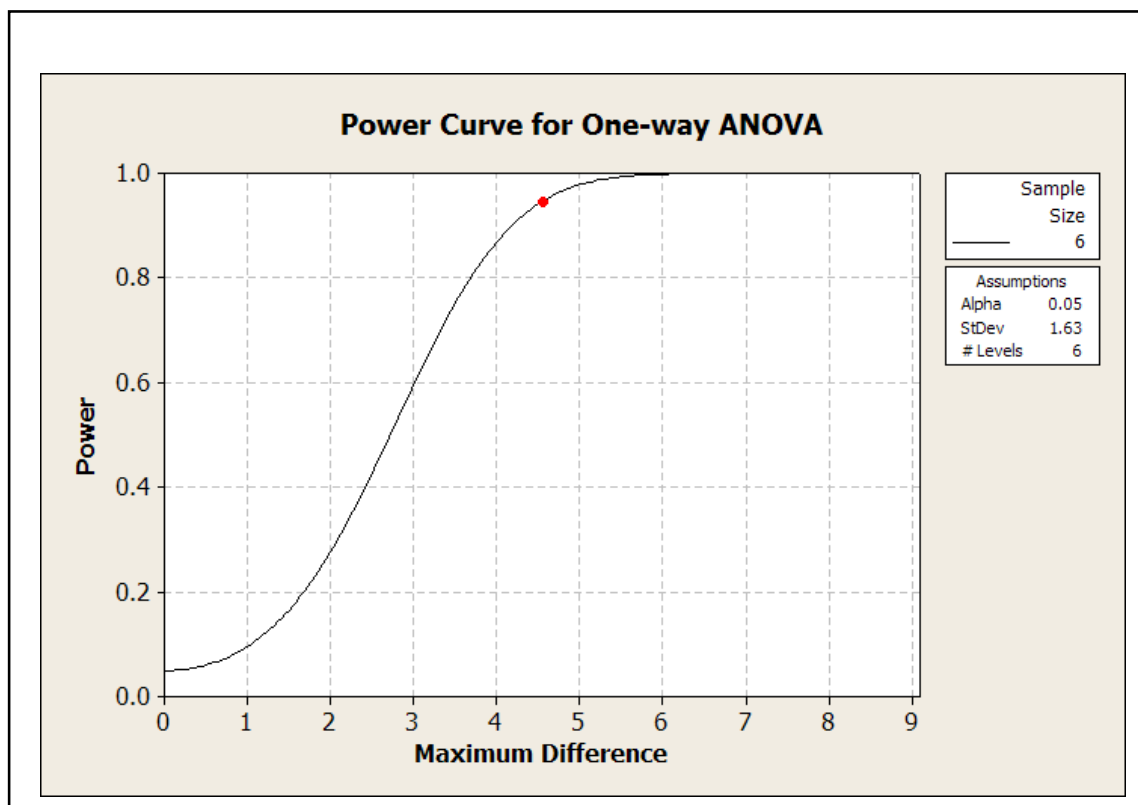

Figure 5.2 Power curve generated from pilot study, indicating that 6 transducers must be included in the main study testing each of the treatment combinations.

\section{Main Study}

For the main study, six transducers were used to record pressure drops for the treatment combinations. Since there was potential for human error, two measurements were taken for each treatment combination with each transducer. The entire data set can be found in Appendix C. Before proceeding with any analysis, the assumptions necessary for the model had to be checked. In Figure 5.3 below, normality and residual plots characterizing the data supported the necessary assumptions made to conduct the statistical analysis of the gathered data points. The linear nature of the normality plot indicated that the data was normally distributed. The ranges for the residuals on the "Versus Fits" plot were all relatively the same, and the residuals for the "Versus Order" plot were random and void of any megaphone or arcing patterns. As a result, the 
assumption of equal variance was satisfied. The randomization process used in performing the testing satisfies the requirement for sample independence. Accordingly, the assumptions were met and analysis could be performed.

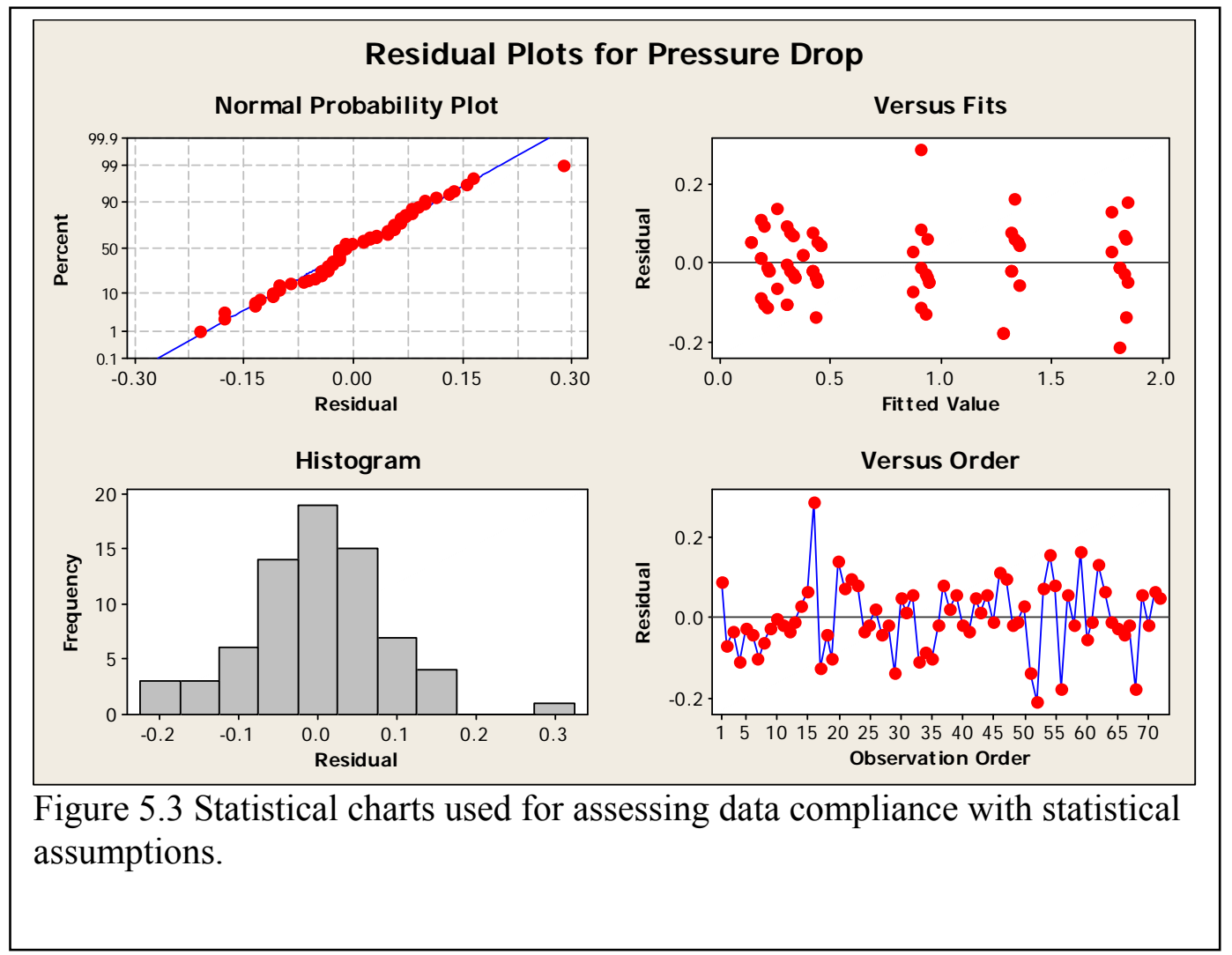

The output from the two-way ANOVA showed that blocking on the transducer was not necessary. Since the blocking variable received a p-value greater than alpha at 0.439 , the average measurements taken by each transducer were not significantly different from one another. The ANOVA also indicated that there was a significant interaction between the two factors since the combination "Size*Treatment" received a pvalue smaller than 0.001. The corresponding interaction plot is illustrated in Figure 5.4. 


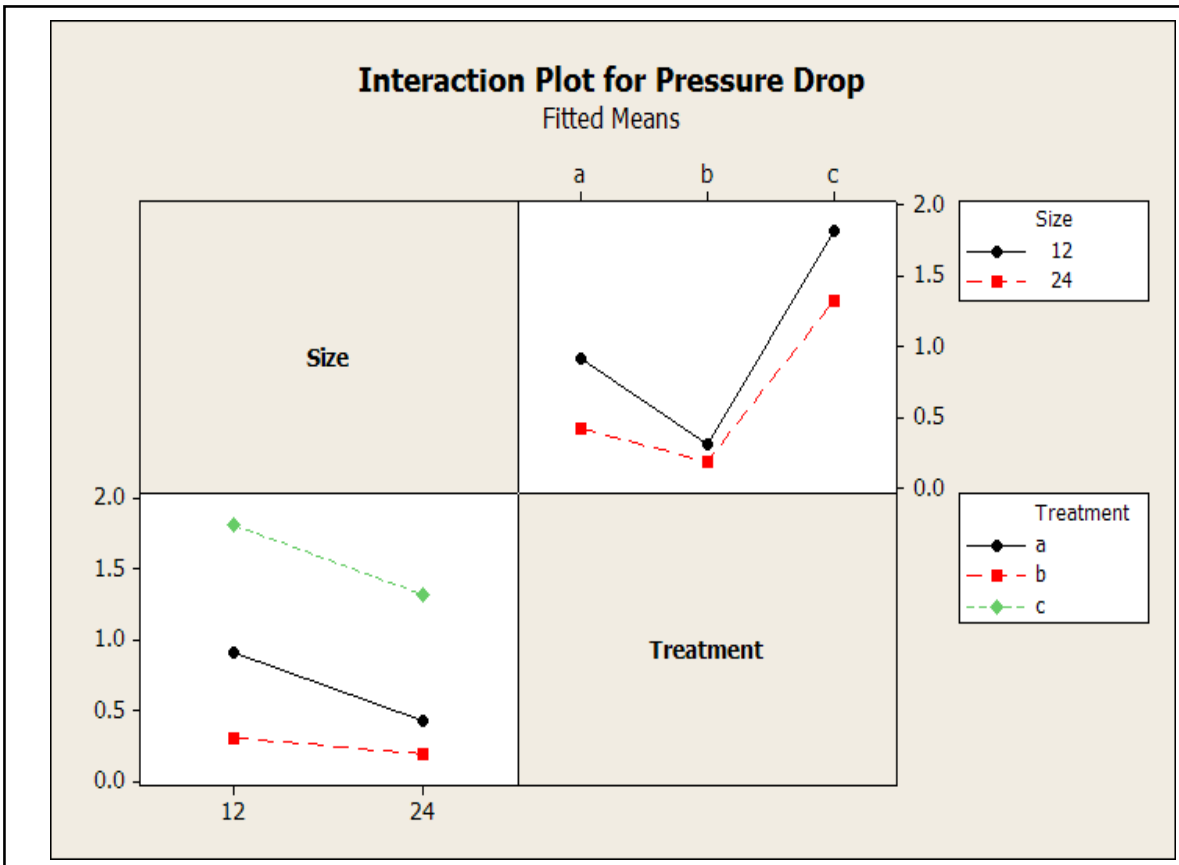

Figure 5.4 Interaction plot illustrating how system size and applied treatment affect pressure loss. Sizes 12 and 24 correlate to the 12 and 24 inch lengths of tubing used within the bioreactor subsystems. Treatments $\mathrm{a}, \mathrm{b}$, and c correspond to the control, tubing spring clamps, and non-permeable tubing respectively.

While each of the individual factors also had small p-values that were less than alpha, statistically significant interactions will always dominate the effects on the response variable. Accordingly, they did not need to be considered in any further analysis. Accordingly, while size and system modification had effects on the rate of pressure drop, they would not have an effect greater than their combination. Results indicated that the larger bioreactor subsystems lost less pressure over time compared to the smaller systems, tubing spring clamps resulted in decreased pressure loss while nonpermeable tubing increased pressure loss over time compared to the control subsystem, and most importantly that the combination of large system size and the use of tubing spring clamps resulted in the lowest pressure drop of all (Figure 5.4). The treatment 
combinations were compared using a Tukey pairwise comparison to assess their similarities. The pairwise comparison also illustrates the results shown in the interaction plot, but in a numerical form. This data can be found in its entirety in Appendix C. All of the comparisons resulted in p-values less than alpha. This indicated that no two treatment combinations were alike. Accordingly, the lowest least square mean listed below Table 5.2 corresponds to the best treatment combination for reducing pressure loss.

\begin{tabular}{|c|c|}
\hline & $\begin{array}{l}\text { Mean Drop in } \\
\mathrm{mmHg} / \mathrm{Min}\end{array}$ \\
\hline \multicolumn{2}{|c|}{ System Modification } \\
\hline $\mathrm{a}$ & 0.67 \\
\hline $\mathrm{b}$ & 0.25 \\
\hline $\mathrm{c}$ & 1.57 \\
\hline \multicolumn{2}{|l|}{ Size } \\
\hline 12 & 1.01 \\
\hline 24 & 0.64 \\
\hline \multicolumn{2}{|c|}{ Size*Treatment } \\
\hline $12 \mathrm{a}$ & 0.91 \\
\hline $12 \mathrm{~b}$ & 0.30 \\
\hline $12 \mathrm{c}$ & 1.81 \\
\hline $24 \mathrm{a}$ & 0.42 \\
\hline $24 \mathrm{~b}$ & 0.19 \\
\hline $24 \mathrm{c}$ & 1.32 \\
\hline
\end{tabular}




\section{Discussion and Conclusion}

Since there was an interaction between treatment factors, the interactions plot was the most appropriate graphical representation of the data. The two graphs illustrated the same relationship, but the size plot made the comparison easier to understand. In Figure 5.4, the black line represented the smaller sized bioreactor models, while the red was the larger. Since the black line is elevated above the red at all points, it can be seen that the smaller systems tended to leak faster. Letters "a", "b", and "c" refer to the control, tubing spring clamp, and non-permeable tubing subsystem modifications, respectively. For both sizes, the tubing spring clamp treatment resulted in less pressure loss than the control. It can also be seen that for both of the system sizes, the non-permeable tubing had a much greater loss in pressure than the control. The two points for the tubing spring clamp system modification were very close together. This is where the Tukey pairwise comparison became important. The comparison assessed whether or not those two points, among others, were significantly different from each other. Based on the comparison, it is clear that they are (their comparison yielded a p-value less than alpha). The difference between those two points was better represented in the interaction plot comparing treatments (Figure 5.4). The very small slope connecting the two points for the tubing spring clamp treatments showed their similarity.

The high rates of pressure loss seen for the non-permeable tube treatment were surprising. The silicone tubing typically used for the bioreactors is gas permeable. If there is any gas within the system (bubbles), the permeable tubing may allow the gas to escape and result in lost pressure. Pressure lost from this variable may be small or take an extended period of time to occur, but it was still worth assessing. Non-permeable tubing 
should have prevented the leakage from occurring, if it existed, and either resulted in no significant difference compared to the control or a slight drop in the rate of loss. Counter intuitively, the rate increased. This was possibly due to the material properties of the tubing and the effects on the interface between tubing and barb. When the barbs were inserted into the tubing, there was adequate tube deformation and stretch to indicate an acceptable fit. The outer diameter of the tubing was $1 / 4$ inch, but no specifications were listed for the inner diameter. Logically, luer lock barbs are intentionally sized larger than the inner diameter of the tubing they are meant to connect to in order to ensure a snug fit. Not having a specification on the inner diameter, it was unknown whether the two pieces actually fit together properly. Once the fittings were inserted into the non-permeable tubing, however, the fittings could not be removed. On the other hand, the silicone of the original tubing interfaced well with the barb and practically stuck or adhered to the surface of the plastic. The non-permeable tubing used was vinyl and had a very smooth surface that may have compromised the seal and supported capillary action.

When comparing the square means for each of the treatment combinations, it is easy to see that the 24 inch system size and tubing spring clamp treatment combination bioreactor model reduced the rate of pressure loss the greatest. This has a number of implications. The first is that the pressure was lost in the bioreactor system at tubing and fitting connections. While clamping the tubing to the barb provided a reduction in pressure loss, it did not altogether prevent it. Either pressure was still leaking at the tubing and fitting connection, or there were other sources of pressure drop present. Since the bioreactor had been reduced to a simplified model, the other potential sources were easy to deduce. Because of the material selection and results for the non-permeable 
tubing, tubing permeability is still potentially a source for pressure drop. The luer lock fitting connections themselves are also potential sources of leakage. This was also noted when performing the qualitative testing described in Appendix B.

The results obtained make sense. Larger systems contain greater volumes of water. The water lost in a smaller volume system comprises a greater percentage of water compared to the larger system assuming the leak volumes in each system are equivalent. This results in a greater rate of pressure loss in the smaller systems. Also, the reduction in pressure loss achieved through tying the tubing down on the barb is reasonable when considering how the tubing reacts under pressure. As the silicone tubing is pressurized, there is a ballooning effect because of the material compliance. This ballooning can compromise the seal between the barb and tubing. The non-permeable tubing is much less compliant than the silicone. While ballooning should not be an issue for the vinyl at these pressures, the non compliant nature of the material may result in greater force concentrated at the tubing and barb interfaces. Especially when compared to a more compliant material that can expand redistribute the forces. In this case, it should not play much of a role since both systems are pressurized at relatively low levels.

Ultimately, it would not be reasonable to tie off all of the connections for the normal bioreactor. In addition, and more importantly, there are still other sources of leakage that would have to be addressed. The manual pressure injection method for backpressuring the systems is not feasible using the either the older Lock N Lock or recently designed bioreactor systems due to their dependence on silicone tubing and luer lock fittings. As mentioned previously, the manual pressure injection technique is highly sensitive to any fluid loss within the bioreactor system. Small leaks result in large drops 
in pressure due to the incompressibility of liquids. Even though larger bioreactor systems and tubing spring clamps have shown to be pivotal in reducing pressure loss from the system, addressing these two issues did not entirely prevent pressure loss. Further work dedicated to improving these system fortifications would not likely yield a leak-proof system. As a result, a new method of pressurizing the systems will have to be developed to replace the previously intended method of manual pressure injection, but this is only considering the simplified systems.

The issues become even greater for identifying the many potential sources of micro leaks within the full bioreactor system. Locating and effectively resolving each of these leaks would be extremely time-consuming. Furthermore, this commitment of effort would be necessary every time the system was assembled or after any partial disassembly - too inefficient to be practical. In an ideal system, absent any leaks, forcing additional fluid into the system would effectively pressurize the system and establish a base-line pressure that would be maintained indefinitely. However, the leaks are substantial enough that fluid would have to be injected every few minutes to maintain the necessary levels in the laboratory setting. Obviously, that is too intensive to be addressed manually. Other options must be identified.

Following the same line of reasoning (injecting additional fluid to maintain desired pressure), something must take the place of the unfortunate laboratory assistant equipped with a syringe. While injecting additional fluid into the bioreactor system at short intervals of time for potentially multiple days in a row is not reasonable for a laboratory assistant, an automated system would likely be much more suitable. If the rate of pressure drop is consistent and accurately determinable, a syringe pump may prove 
effective. Syringe pumps can be fitted with a wide array of syringes - including those with the luer lock design. A barbed male luer lock fitting could be attached to the syringe. Next, a length of silicone tubing could be attached to the barb. The free end of the tubing would then be placed on the center barb of a three-way barbed fitting. The bioreactor's tubing would then be cut at a specified point and the two free barbs of the fitting would be inserted to span the gap. As the system would run, it would leak at a specific rate and the syringe pump would replace the leaking fluid at the same rate. Thus, the leak would be nullified for all practical purposes and the base-line pressure would be maintained.

There would be many difficulties with using this syringe pump setup. First, an extensive battery of tests would have to be conducted to accurately characterize the cumulative leak behavior of the system. This would be both time consuming and complex. The difficulty arises from the potential variability from one system to another and the repeatability in generating the same leak rates. Even with all of the same parts, each assembly would be slightly different. Any discrepancy in the rate of fluid entering the system relative to the rate of fluid exiting the system would result in a drift in pressure over time. Also, over extended periods of time, the leaks may not be constant. The rate of leakage could change over time and the syringe pump would not be able to adjust to the varying rates. Another issue is the scalability of the syringe pump. Tying multiple systems into a single pump may deplete resources too quickly and cause problems. Plus, syringe pumps are expensive and take up a lot of space. Finally, in order to ensure agreement between injection and loss rates, auto regulation utilizing pressure feedback could be incorporated in the design but would be extremely expensive. 
Therefore, the syringe pump and similar fluid injection techniques would not solve the problem, but they are an important conceptual step in the correct direction.

Overall, upon completion of the pressure loss experiments described in this chapter, it was concluded that fluid injection would not be a viable option for backpressuring the bioreactor system. The system had a continuous leak so it would require a source of continuous supply in order to sustain consistent pressure. A number of potential alternate solutions will be explored in the next chapter. 


\section{Chapter 6: SELF-REGULATING INJECTION PROCESS}

\section{$\underline{\text { Introduction }}$}

The concept of continually replacing lost fluid from the bioreactor system by injecting a complementary volume of fluid back into the system, as discussed at the end of Chapter 5, is explored further in this chapter. The syringe pump serves as an example of a "blind injection process." For the purposes of the studies in this chapter, blind injection processes are defined as techniques or methodologies of injecting fluid into a system at a constant or preset rate without any negative feedback or self-regulating properties. Blind injection processes are not suitable for the highly variable demands of the new or old bioreactor systems that would be necessary for maintaining desired baseline pressures. For example, if a system variably leaks at rate within a range of 1 to 2 $\mathrm{ml} / \mathrm{hr}$ and an attached syringe pump attempts to compensate for the loss at a constant rate of $2 \mathrm{ml} / \mathrm{hr}$, the syringe pump will outpace the leak. This would ultimately result in an increasing system pressure which would have its own associated risks and problems.

Therefore, it is the goal of this chapter to introduce the idea of self-regulating injection processes as potential solutions to the back-pressuring objective and to develop an initial design to assess the feasibility of implementing such methods. Self-regulating injection processes, within the context of this study, are the opposite of their blind counterparts. They will be loosely defined as any technique or methodology of injecting fluid into a system at a rate adjusted by negative feedback or some other regulatory means. The major benefit to such systems or processes is that they "know" when to stop. Creating a self-regulating injection process for testing was a complicated task. The basic idea and guiding principles used in this study to develop the technique will 
now be described. A three-way valve would be placed in line with the bioreactor. Its location with respect to the bioreactor chamber would not be important as it would pressurize the entire system homogenously. Attached to the three-way valve would be a luer lock syringe full of fluid. A custom stand would be necessary to hold the syringe perpendicular to the bioreactor tubing within the incubator, but a ring stand is sufficient for bench top testing. A weight could then be attached to the plunger of the syringe to apply a constant force to the system. This would, in theory, continually replace the lost fluid and prevent pressure loss as a constant force is applied. This method could be applied in conjunction with manual pressure injection to sustain the backpressure or as an independent means of obtaining the desired pressure levels. The implementation and evaluation of this concept and theory are described in the subsequent sections of this chapter.

\section{Methods and Materials}

In order to test this method, a simplified bioreactor system was used once again. The system utilized one 6 inch length of silicone tubing, one two-way valve, one threeway valve, a pressure transducer, two luer lock syringes, a ring stand, a plastic CD case, and a partially filled $32 \mathrm{oz}$ plastic bottle of water. One end of the 6 inch length of tubing was connected to the pressure transducer using luer lock fittings. The free end of tubing was then connected to one end of the straight-through flow path of a three-way valve with a barbed female luer lock fitting. The free end of the pressure transducer was fitted with a two-way valve. Then, a luer lock syringe was filled with water and attached to the vertically oriented " $\mathrm{T}$ " branch connection of the three-way valve - this vertical branch 
remained closed initially. A second luer lock syringe filled with water was connected to the two-way valve on the pressure transducer and fluid was injected into the system and allowed to vent through the open end of the three-way valve until the system was completely filled with fluid and no air bubbles could be seen. The luer lock syringe attached to the two-way valve on the transducer was then removed, and both the two-way and three-way valves were closed to prevent fluid loss from the system. The three-way valve was closed by shutting off the outlet port of the valve - simultaneously opening the path connecting the tubing to the luer lock syringe. A ring stand was used to secure the syringe attached to the three-way valve into a vertical position. The plastic CD case was balanced atop of the syringe plunger, and the partially fill plastic bottle was balanced upon the CD case. This set up is illustrated in Figure 6.1.

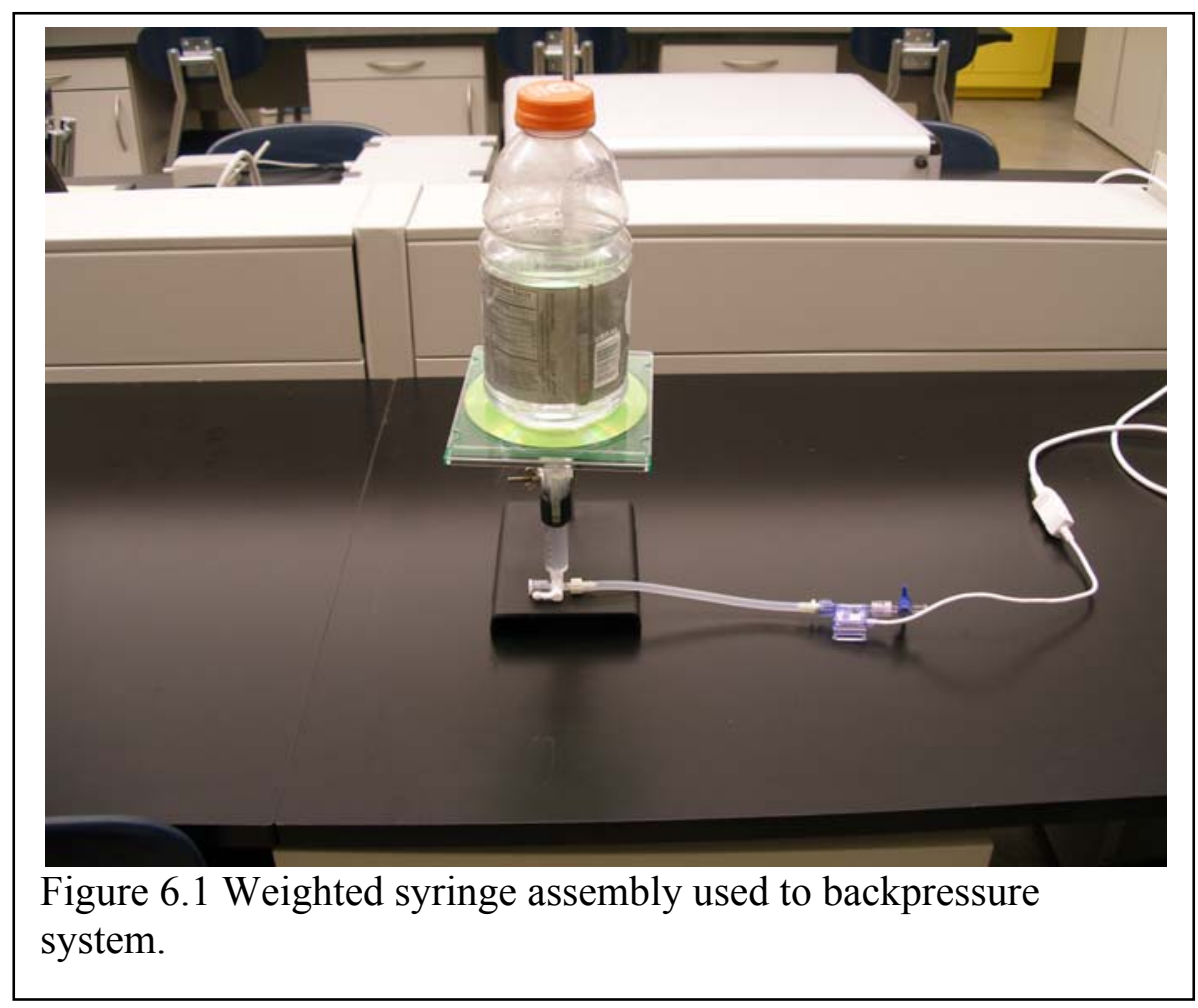


Initially, a force was first applied by hand to the syringe plunger to see how well the system responded to force without the use of the partially filled plastic bottle and CD case. Next, the $32 \mathrm{oz}$ bottle was used as a weight to impart constant force upon the plunger in the setup described and illustrated above. The sturdy CD case was balanced on top of the plunger to augment the surface upon which the water bottle rested. $33.8 \mathrm{oz}$ of fluid is equal to 1 liter. 1 liter of water weighs approximately $2.2 \mathrm{lbs}$. Accordingly, the 32 oz plastic bottle could provide the range and magnitude of force necessary to produce the ideal backpressures based on Equation 6.1 below.

$$
\mathrm{P}=\mathrm{F} / \mathrm{A}
$$

The outcome of this test set up was assessed by pressure recordings taken by the data acquisition equipment.

\section{$\underline{\text { Results }}$}

By applying more or less force by hand, desired pressures were easily reached. In addition, by monitoring the system pressure recorded by the data acquisition software, a relatively constant pressure was maintained. However, applying force to the system using the $32 \mathrm{oz}$ plastic bottle was far less effective. The pressure readings (illustrated in Figure 6.2) show a trend of decreasing rates of pressure loss over time. Nonetheless, the system failed to generate a constant pressure. A complete set of data is provided in Appendix A. The average rate of pressure loss was $0.318 \mathrm{mmHg} / \mathrm{min}$ based solely on initial and final pressure measurements. Interestingly, the rate of pressure loss was far from constant. At 
the beginning of the experiment, measurements recorded by the data acquisition equipment indicated an approximate $4 \mathrm{mmHg} / \mathrm{min}$ rate of pressure loss. Measurements recorded at the end of the test showed rates of pressure loss as low as $0.11 \mathrm{mmHg} / \mathrm{min}$. While the trend of the data points did indicate a decreasing rate of pressure over time, it did not occur constantly. Rates continually fluctuated in magnitude every couple of minutes - increasing and decreasing at times. The presence of a small air bubble was also noticed after 30 minutes of the experiment. No quantitative measurements were taken to characterize the size or volume of the trapped gas, but it was periodically checked over the course of the study and found to decrease over time. The bubble began as approximately half of the size of an eraser on the end of a pencil. At the end of the experiment, only $1 / 6$ of its original size appeared to remain. 


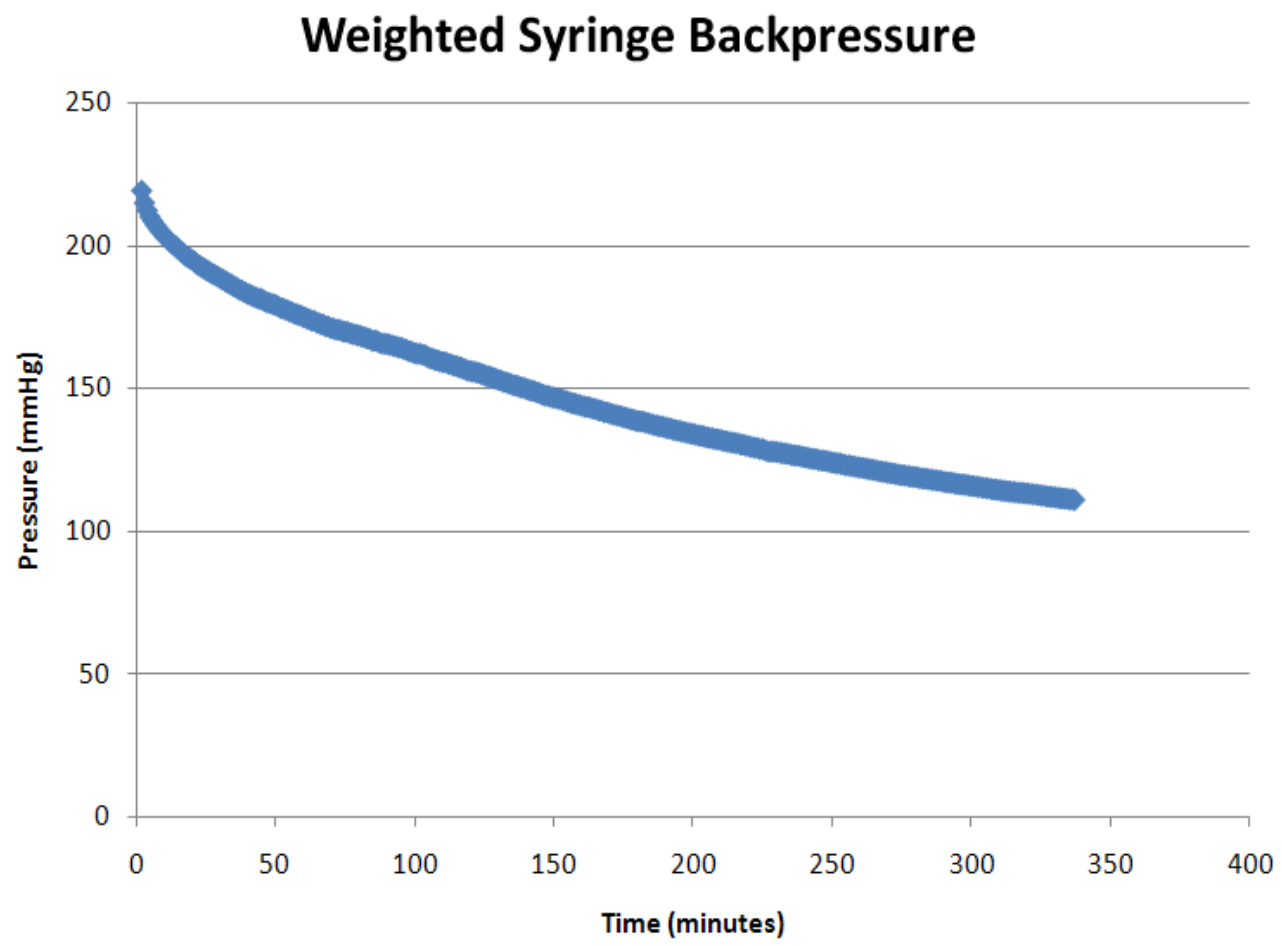

Figure 6.2 Measured backpressure over time - induced by weighted syringe setup.

\section{Discussion and Conclusion}

The constant backpressure measured and maintained in the manual force trial was not reproduced with the weighted syringe. By pushing down the plunger manually, it was possible to minimize the resistance stemming from physical and frictional forces generated by the seal of the syringe plunger and wall. Monitoring the pressure measurements recorded by the data acquisition equipment, a constant pressure of 100 $\mathrm{mmHg}$ was maintained with relative ease. Even so, it was difficult to estimate the amount of force that was applied by hand while the manual test was conducted. It was also difficult to determine whether or not a constant force was actually applied. As a result, 
the manual test was only beneficial in crudely testing the theory and providing results that supported further inquiry and evaluation with the weighted syringe. Accordingly, the next step involved conducting the trial with an object of mass rather than by hand, as illustrated in Figures 6.1 and 6.3.

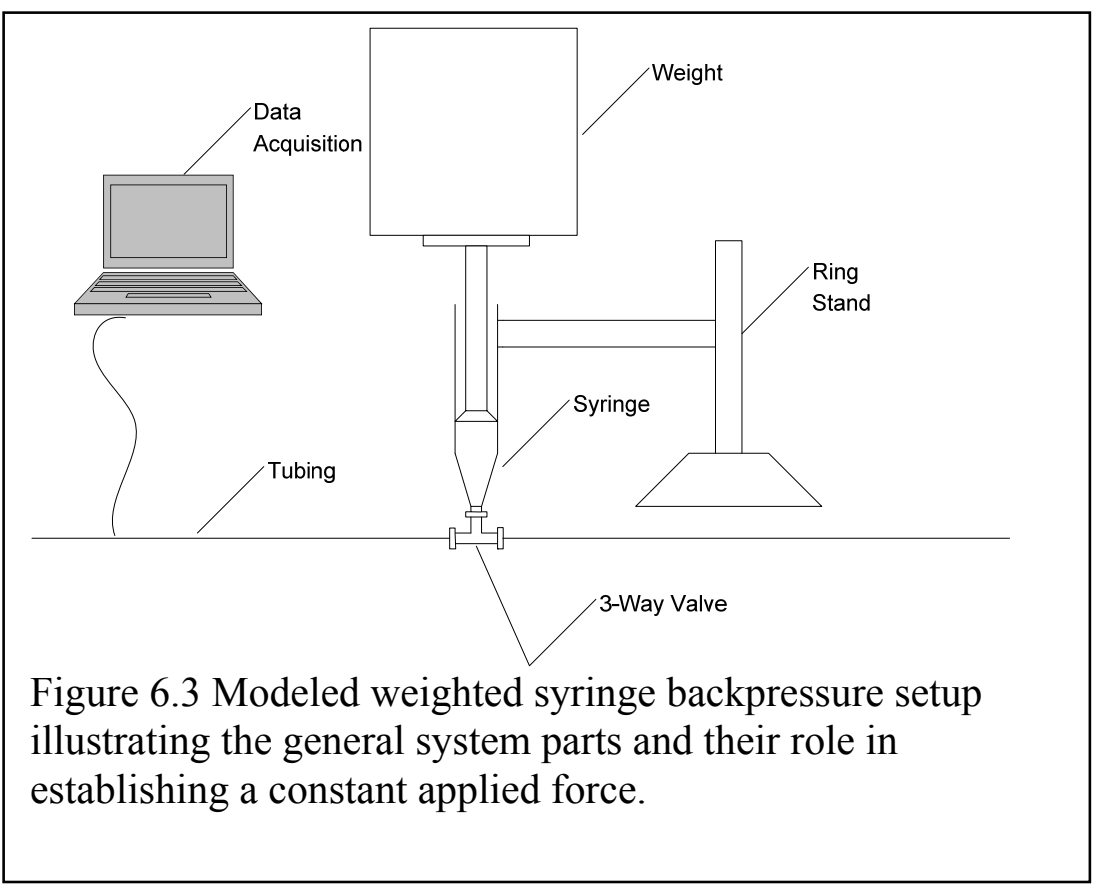

The failure to generate a constant backpressure appeared to be partially due to applying an insufficient weight to entirely overcome the plunger's frictional forces. This conclusion was based on the varying rates of pressure loss observed during the trial. As illustrated by the graph in Figure 6.2, the rate of pressure loss started out high. The rate appeared to decrease as the study reached the 100 minute mark, and then the rate slowly increased and decreased once more. As a result, it was hypothesized that the water bottle may have been too light to prevent the plunger from sticking to the syringe walls. 
Fluctuation between static and kinetic coefficients of friction could be a reasonable explanation for the varying rates of pressure loss.

Equation 6.1 was used to help resolve the question of whether or not frictional forces played a role in the pressure measurements. The necessary force required to generate a desired pressure within the system can be estimated using Equation 6.1.Based on the equation, pressure $(\mathrm{P})$ is equal to the force $(\mathrm{F})$ applied to a specified area $(\mathrm{A})$. It would require $0.49 \mathrm{lbs}$ to generate a pressure of $100 \mathrm{mmHg}$ using a $10 \mathrm{ml}$ syringe with a plunger surface area of $0.256 \mathrm{in}^{2}$ (Syringe Selection Guide, www.harvardapparatus.com). Approximately half of the $32 \mathrm{oz}$ bottle was filled with water before it was placed on the syringe. Based on the previously mentioned conversions, the bottle likely weighed a little over a $1 \mathrm{lb}$. Equation 6.1 would estimate the pressure generated to be slightly over 200 $\mathrm{mmHg}$. This is close to the actual initial pressure measurements recorded from the trial by the data acquisition equipment. Since the calculation does not take into consideration the frictional forces counteracting the force supplied by the water bottle, the pressure recorded by the pressure transducer would be expected to yield a lower pressure than the estimate. The pressure recorded by the data acquisition equipment was nearly equal if not slightly larger than the estimate. As a result, the frictional forces were likely negligible and contributed very little to the pressure variations. Accordingly, other potential explanations were considered.

Another potential source of pressure loss was the diminishing height of the water column within the syringe used in the study. Even so, this factor should have had only a minimal impact upon the system pressure. Considering the setup in Figure 6.3, the pressure at the base of the three-way valve depends upon two contributing factors. The 
first factor is the water column height within the syringe. The $10 \mathrm{ml}$ syringe can only hold 3-4 inches worth of fluid. 1 inch of water roughly correlates to $1.85 \mathrm{mmHg}$. Therefore, the 4 inch column of water accounted for about $7.4 \mathrm{mmHg}$ of the total backpressure. The second factor is the $1 \mathrm{lb}$. weight resting on the syringe plunger that accounts for nearly $200 \mathrm{mmHg}$. The weight of the water within the syringe is only a fraction (3.7\%) of the applied force attributed to the weight. Accordingly, as the fluid is lost from the system and the water column weight diminishes, variation in pressure should be minimal. As a result, this implicates another factor as being the source of the majority of the lost pressure during the experiment.

Previously, in Chapter 5, various factors influencing pressure drop were explored. One such factor was permeability of tubing. The silicone tubing is permeable in order to facilitate $\mathrm{CO} 2$ exchange with the environment within the incubator. Accordingly, it was hypothesized that trapped air bubbles could pose an issue for back-pressuring the bioreactor system as the gasses may be forced out of the tubing - lowering system volume and decreasing pressure. The way this system was designed, however, it would compensate for those volumetric losses. As fluid is lost from the system, a complimentary volume of fluid is passed from the syringe back into the system. The pressure lost due to the decreasing height of the water column is negligible compared to the pressure provided by the weight. During this study, a small air bubble was noted at the end of the luer lock fitting connected to the three-way valve (illustrated in Figure 6.4). The size of the trapped pocket of gas significantly decreased over time. Interestingly, the rate of pressure loss also significantly decreased over the course of the study. It would be easy to jump to the conclusion that the two factors were interrelated. However, it was 
only a coincidence. As just mentioned, fluid volume lost from the system is readily compensated for by system design. More importantly, the magnitude of the pressure lost from the system (almost $100 \mathrm{mmHg}$ ) is equivalent to a 54 inch tall water column. The weighted syringe only operates with a 4 inch water column. Accordingly, for this much pressure to be lost, there would have to have been no fluid left in the syringe. Oddly enough, by the end of the study, the syringe experienced no measurable loss of fluid. This left one additional solution.

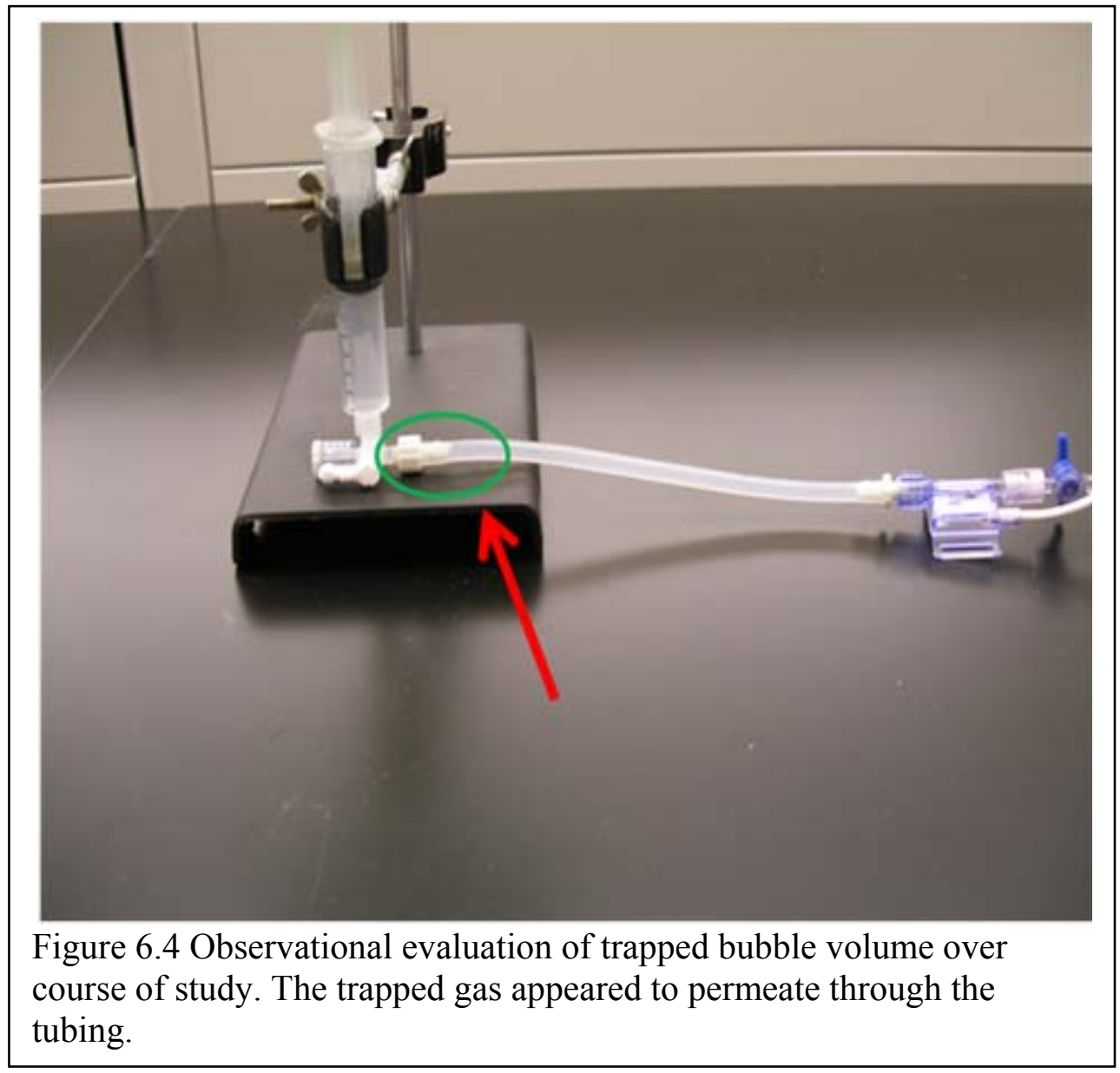


The force applied to the syringe was inconsistent. The reason why there was such a large variation in pressure but no change in system volume was because over time less and less force was applied to the syringe plunger. One may wonder how this was possible, but it became clear when evaluating the methods used to assemble the system. The weighted syringe system is one large balancing act. The fluid-filled syringe was placed on end and held upright by the ring stand. A platform (the CD case) was balanced upon the plunger, and a weight (the bottle of water) was balanced upon the platform. Any slight misalignment would cause the precariously unstable plunger stem to lean or flex. The more fluid contained within the syringe, the less stable the extruding plunger would become. As the system leans, the full force of the weight no longer directly acts upon the fluid within the syringe. This ultimately resulted in reduced pressure levels. If the system were allowed to have continued to operate indefinitely, it would have likely fallen over or found a resting spot.

Based on the previous analysis of applied forces and potential factors influencing pressure loss, more care must be taken in future work when considering how forces are applied to the system. The weighted syringe method was a step in the right direction, but it had a flaw - the means by which force was applied. Other sources of force could be considered in future trials. For example, a shallow tray of water with a large surface area could be fixed above the weighted plunger. The tray would be fitted with a cylindrical port extending down toward the weight atop the plunger. The weight itself would have a second plunger attached to its top. The plunger would fit into the tube above connected to the tray so that it would be flush with the flat surface (Figure 6.5). The necessary steps to properly secure and align the weight would also be accomplished using this setup. 
Assuming the two plungers were centered on the weight, the cylinders that the plungers fit into act as guide rails - preventing any changes in applied forces.

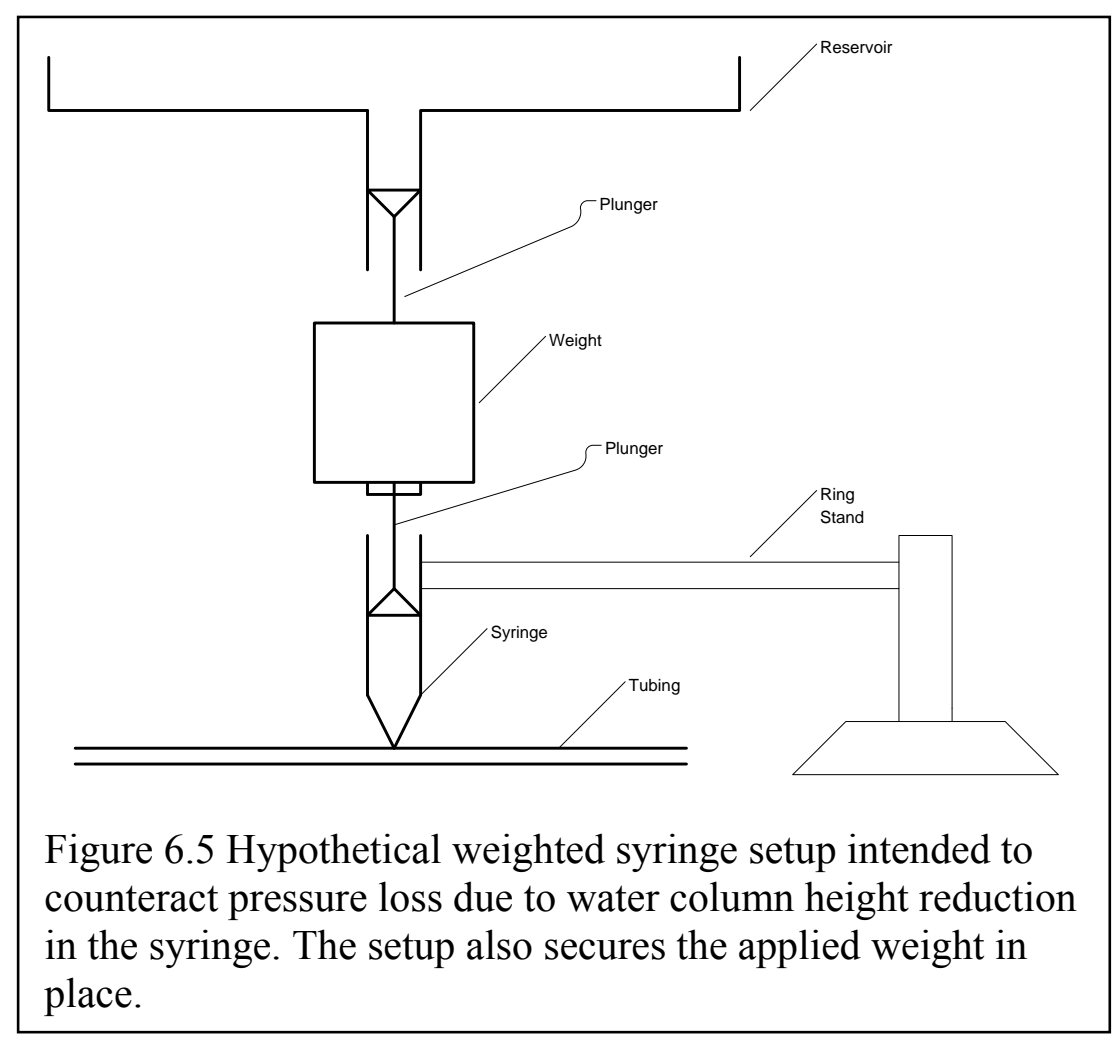

As the plunger falls and fluid is pressed out of the syringe below, water from the tray refills the cylinder above. The cylinder would be proportional to the syringe. As the syringe loses fluid, the cylinder gains the same amount and keeps the force constant. This would maintain a constant pressure based on the weight of the object attached to the plungers. Even though the water column only accounts for a fraction of the overall force, a highly accurate system is desired. The problem is that the pressure would be constant only as long as there is water left in the syringe. Once the water is gone, the fluid bleeding out of the system would no longer be replenished. The other problem is the 
amount of surface area required by the tray. The larger the tray is, the shallower the water will be above the plunger. This is important because that level will drop to compensate for the lowering plunger. The smaller that height is, the less weight variation that will occur. Another option would be to use a syringe pump, but that requires knowing the rate at which the system leaks. As discussed previously, the systems do not leak at the same rates all the time. Even if the average rate of leakage for one system could be accurately determined, no two systems will be the same. It would be difficult to scale the method to accommodate multiple bioreactors. Refilling the weighted syringe system would also be difficult to scale to accommodate other bioreactors. This method is not practical for the application. In addition, the floating weight would probably act as a dampener and oscillate with pressure waves. A base-line pressure would be maintained, but there would be no wave to speak of.

Another problem would be scaling the setup. A stand would have to be created to support all of the weights, and weights would consume a lot of space. In addition, weights can be hard to manage - especially lifting them above head, which also poses safety concerns. A single two pound weight would not cause any laboratory assistants to worry. 32 two pound weights loaded into a fully loaded incubator would not be forgiving if they were to fall upon someone. Finally, the incubator might not be able to support all of the weight necessary to back pressure all of the bioreactors.

In summary, the work in this chapter has demonstrated that self regulating injection processes are worth pursuing for back-pressuring the bioreactor system. The specific weighted syringe technique did not work as well as intended, but provided promising data to help guide future efforts. Since the weighted syringe technique is 
potentially highly variable and did not perform well during the study, the specific design will no longer be pursued. Therefore, the next step was to test and develop other potential techniques for back-pressuring the bioreactor system. The following chapter will discuss one such method that was derived from one of the more fundamental principles involved in the weighted syringe technique - the water column. 


\section{Chapter 7: WATER COLUMN}

\section{$\underline{\text { Introduction }}$}

As described in the previous chapter, the next step involved developing and exploring a new methodology for back-pressuring the bioreactor system since the weighted syringe setup did not perform adequately. Therefore, the goal of this chapter was to take the information gained from previous studies to design and evaluate a new technique that could be used to accurately and consistently backpressure the system.

While working on the weighted syringe method for pressurizing the system, it was noted that the weight of the water column provided some of the pressure in the system. As stated by a law explored in fluid dynamics, pressure is equal to the density of the fluid multiplied by gravity and the height of the column of fluid plus the atmospheric pressure (Equation 7.1).

$$
\mathrm{P}=\rho g h+\mathrm{P}_{\mathrm{ATM}}
$$

Gauge pressure, or the pressure within the system independent of the atmosphere, is the only pressure measurement relevant to the bioreactor system and experimental setups used in this research. Accordingly, the atmospheric term can be dropped from the equation. This was physically accomplished during the two point calibration technique used while setting up the data acquisition equipment. With the conversion of roughly 760 mmHg equal to $101.32 \mathrm{kPa}$ and the density of water at STP being $998.23 \mathrm{~kg} / \mathrm{m}^{\wedge} 3$, it is possible to calculate the height needed to generate any desired pressure. For example, in order to generate $100 \mathrm{mmHg}, 54$ inches is required. $100 \mathrm{mmHg}$ is a desirable 
backpressure because it will exploit the symmetry of the pressure wave generated by the peristaltic pump, and it has been shown to work in practice (11). The study described in this chapter involved a simple trial to assess the feasibility of back-pressuring the system by utilizing a water column.

\section{Methods and Materials}

The materials necessary to test the theory were few. All that was required was a $50 \mathrm{ml}$ reservoir, a 54 inch length of silicone tubing, a pressure transducer (calibrated before use), a short 1 inch length of tubing, a barbed luer lock fitting, a luer lock cap, and the data acquisition software. To conduct the experiment, the reservoir was first filled with water. The cap was then screwed on. This cap had two panel mounts attached to it. On one panel mount the 1 inch length of silicone tubing was attached along with the barbed luer lock fitting. The luer lock fitting was then capped off. This essentially plugged one of the panel mounts.

The following steps were performed laying the materials horizontally flat against the laboratory countertop to prevent water from spilling, as illustrated in Figure 7.1. Using a luer style syringe, enough fluid was injected into the 54 inch length of tubing until it was full of water and contained no air bubbles. The 54 inch length of tubing was then attached to the remaining panel mount. At the base of the long length of tubing, the pressure transducer was connected. This required additional luer lock fittings in order to make the connection. 


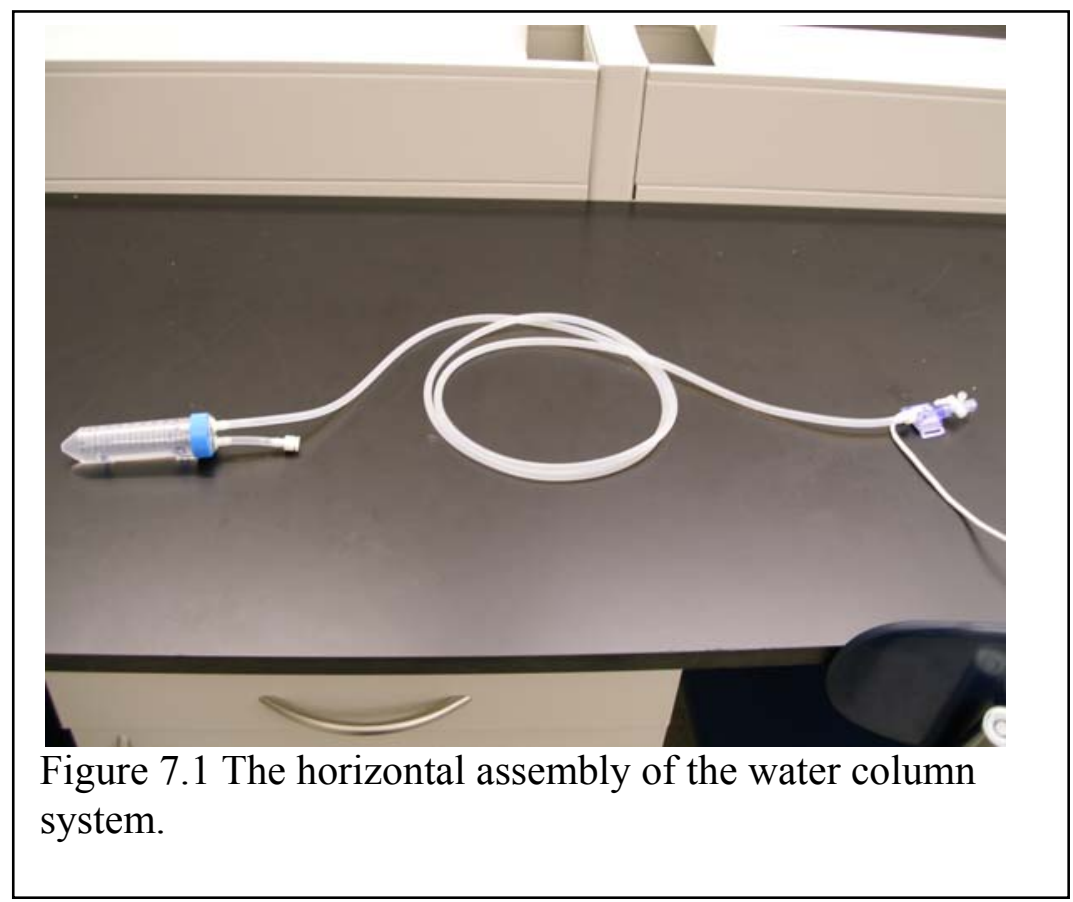

The whole system was then inverted in order to generate an elevated pressure at the location of the pressure transducer, as illustrated in Figures 7.2 and 7.3. The reservoir was held using a ring stand placed on top of the laboratory countertop. The data acquisition software was then turned on to record live data. 

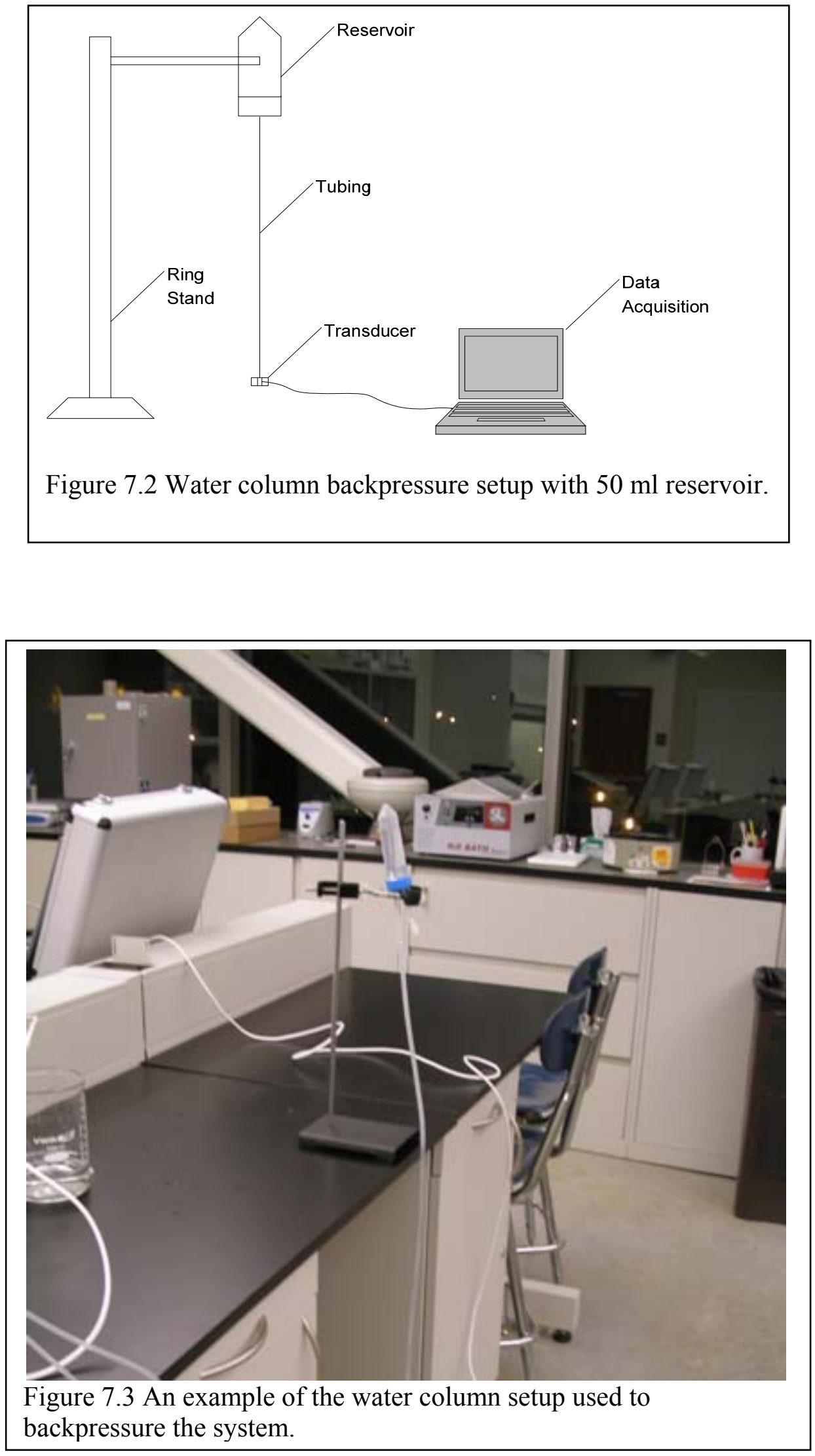


\section{$\underline{\text { Results }}$}

The pressure measured using the equipment was greater than $100 \mathrm{mmHg}$, but this was because of the additional fluid within the reservoir that effectively increased the water column height. The pressure remained constant for nearly an hour before $2 \mathrm{mmHg}$ was lost. The system was left in place for 6 hours. When checked after 6 hours, the pressure had decreased an additional $12 \mathrm{mmHg}$. Over the duration of the study, the fluid level within the reservoir was also monitored. While there were no significant changes in fluid height, the level in the reservoir had dropped slightly. Table 7.1 contains pressure measurements and characteristic parameters obtained during the first part of the trial.

\begin{tabular}{|c|c|}
\hline Start & $100.98 \mathrm{mmHg}$ \\
\hline End & $96.53 \mathrm{mmHg}$ \\
\hline $\begin{array}{l}\text { Total } \\
\text { Drop }\end{array}$ & $4.45 \mathrm{mmHg}$ \\
\hline Average & $0.029 \mathrm{mmHg} / \mathrm{min}$ \\
\hline $\begin{array}{l}\text { Hourly } \\
\text { Rate }\end{array}$ & $1.78 \mathrm{mmHg} / \mathrm{hr}$ \\
\hline
\end{tabular}

\section{Discussion and Conclusion}

The $2 \mathrm{mmHg}$ pressure drop described above was attributed to factors identified in the earlier statistical experiments dedicated to quantitative diagnostics of fluid leaks. Specifically, the tubing/barb interface (Chapter 5) and permeability of the silicone tubing (Chapter 6) contribute to losses in pressure. In this vertically oriented configuration, 
however, only the connections at the transducer were exposed to pressures approaching $100 \mathrm{mmHg}$ and the overall system volume was greater than that of the "large" statistical trial by roughly a factor of three. In the statistical experiment, the entire length of tubing experienced a uniform pressure that was measured by the pressure transducer. The uniform pressure is based on the level (horizontal) orientation of the experimental setups. Technically, there will be a small variation in pressure based on the fluid at the top of the cross section of tubing versus the bottom. However, the small diameter of the tubing limits the contribution of fluid level height to pressure variation in the vertical plane (Figure 7.4) to the point where it can be considered negligible. As a result of the uniform force distribution of the entirely horizontal system setup, all fittings and connections were exposed to these same forces. This is in contrast to the inverted, vertical system that would have one fitting at $100 \mathrm{mmHg}$ while another is at $0 \mathrm{mmHg}$.

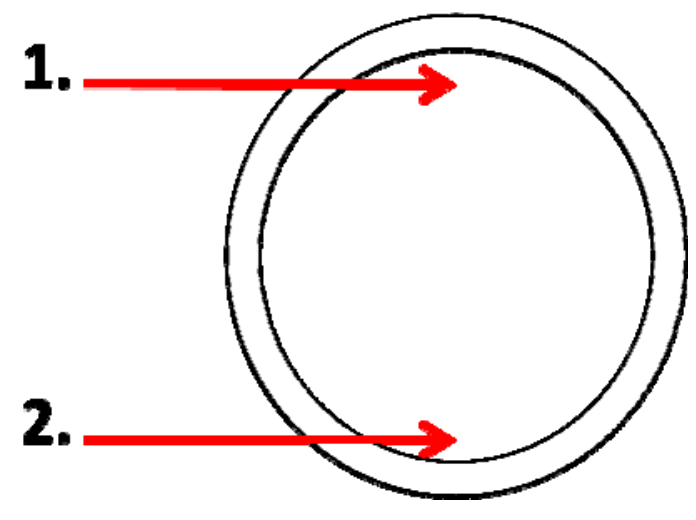

Figure 7.4 An illustration of the negligible height difference within a cross-section of tubing. Arrows indicate the height differential between the fluid at the top and bottom of the tubing cross-section that would lead to a pressure difference between points 1 and 2 based on $\mathrm{P}=\rho \mathrm{gh}$. 
It was interesting to note that the pressure dropped roughly $12 \mathrm{mmHg}$ over 6 hours, but the fluid level remained relatively constant. This was an odd result. A 12 $\mathrm{mmHg}$ decrease in pressure should correspond to roughly 6.48 inches of fluid loss within the setup. There is a discrepancy between the recorded measurement and the physical condition of the system at the end of the trial. While it remains unclear why this problem occurred, a possible explanation may be found in an old party trick. The trick involves a straw and a glass of water. Water is sucked up into the straw and the same end is quickly plugged by the person's thumb. The straw can then be removed from the glass of water without the water falling out of the straw. Perhaps, this setup is analogous to a large plugged straw. The inverted reservoir is sealed and as any fluid is lost at the base of the water column attached to the transducer a vacuum is formed within the reservoir. The vacuum generated imparts a force upon the water that counteracts the force of gravity that also acts upon the water. Accordingly, the fluid level may not have changed as much as the pressure did.

In spite of the lack of fluid loss per pressure drop, the results from this study were a major step in the right direction. The rate of pressure decline decreased to $1 \mathrm{mmHg}$ per 30 minutes. This is much closer to the goal of constant pressure, but still excessive over a 24 hour period--too much to be considered successful. Water or media would have to be added to the systems periodically throughout the day to maintain desired levels. Maintaining the fluid level would ensure consistent system base-line pressure. Therefore, the next step was to modify the water column design in order to significantly reduce the rate at which the water level would decrease. 


\section{Chapter 8: WATER COLUMN II}

\section{Introduction}

The study performed in Chapter 7 found the water column technique to significantly reduce the rate of pressure loss that occurred in the back pressured system. While it represented a significant improvement in system design, it still fell short of accomplishing a constant or even relatively consistent back pressure that could be used in the laboratory setting. A loss of $1 \mathrm{mmHg}$ every 30 minutes would still require aroundthe-clock supervision and maintenance by laboratory assistants. As a result, this chapter focuses on improving upon the original water column design. The experiment in Chapter 7 utilized a $50 \mathrm{ml}$ conical tube as a reservoir. The reservoir has a particularly small crosssectional area. Accordingly, a relatively small loss in system volume correlates to a significant drop in fluid level height. An example would be filling a $1 \mathrm{ml}$ micropipette from the same $50 \mathrm{ml}$ reservoir. The fluid taken from the $50 \mathrm{ml}$ reservoir will fill a 10 inch long $1 \mathrm{ml}$ pipette while barely dropping the fluid level height of the reservoir. Accordingly, modifying the reservoir to reduce the drop in fluid level height was the focus of this chapter.

One potential solution was to increase the reservoir volume and specifically the cross-sectional area, so that the rate of pressure loss would be decreased. Since the columnar height dictates the base-line pressure, reducing the rate at which the water level drops would make the pressure drop at a slower rate. This chapter will further explore this concept. 


\section{Methods and Materials}

The same setup was used as in water column experiment I, except a new reservoir replaced the original. A T225 culture flask was used as the new reservoir in hopes of significantly delaying the loss of pressure, and the culture flask was specifically chosen to be able to simply exchange caps between the two reservoirs. The threads for the T225 culture flask and $50 \mathrm{ml}$ conical tube were different and such a trade was not possible. However, the T225 culture flask cap has a cloth filter concentrically aligned with the end of the cap, and a barbed luer lock fitting was pressed through the filter without significantly compromising the seal. While this may have leaked slightly, the effect of the larger reservoir should still be noticeable. The reservoir was filled with fluid, capped, and connected to the 54 inch length of tubing still connected to the data acquisition software. Once again the system is inverted and positioned using the ring stand clamp (Figure 8.1 and 8.2).

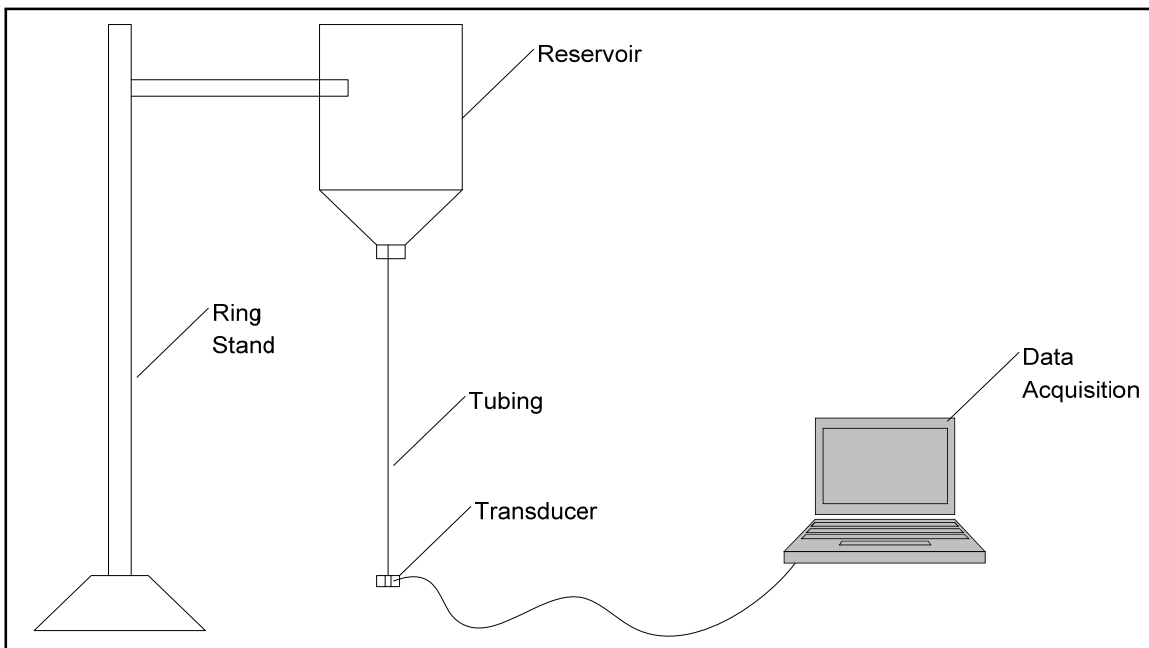

Figure 8.1 Water column backpressure setup with T225 culture flask reservoir. 


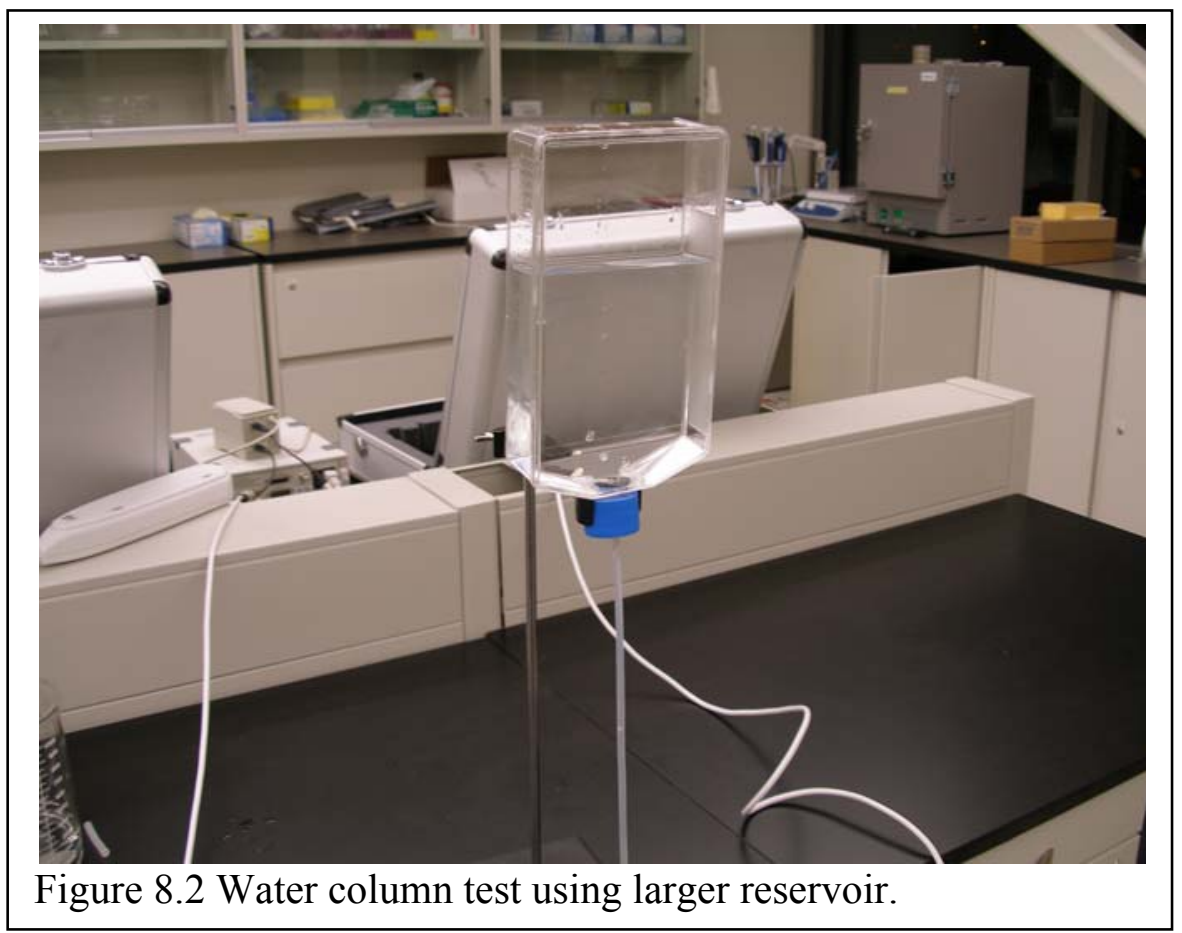

\section{$\underline{\text { Results }}$}

Surprisingly, no leaks were seen at the point where the barbed luer lock fitting was pressed through the filter. The $0.22 \mu \mathrm{m}$ filter is intended to prevent bacteria from entering the culture flask, but over the course of the trial it seemed to be fine enough to prevent water from seeping through as well. At the end of the test it was a little damp, but no leaks were noted. The first pressure drop was noticed at 2 hours when $1 \mathrm{mmHg}$ was lost. The system was allowed to hang for an additional 6 hours and 3 additional $\mathrm{mmHg}$ were lost. Table 8.1 contains characteristic pressure measurements recorded during part of the trial. 


Table $8.1250 \mathrm{ml}$ reservoir water column
trial conducted over 2 hours and 30
minutes.
\begin{tabular}{|c|c|}
\hline Start & $103.90 \mathrm{mmHg}$ \\
\hline End & $102.47 \mathrm{mmHg}$ \\
\hline Total Drop & $1.43 \mathrm{mmHg}$ \\
\hline Average & $0.0095 \mathrm{mmHg} / \mathrm{min}$ \\
\hline Hourly Rate & $0.57 \mathrm{mmHg} / \mathrm{hr}$ \\
\hline
\end{tabular}

\section{$\underline{\text { Discussion and Conclusion }}$}

The results from this study were quite interesting. Just by a visual comparison, the flask had roughly four times the surface area as the original reservoir. Based on the data, it is apparent that the rate of pressure drop turned out to be four times greater with the smaller flask than with the larger. While these tests have been more qualitative in nature, the empirical evidence shows promise. Although the larger reservoir provided a slower rate of pressure drop, losing $1 \mathrm{mmHg}$ every couple hours is still not desirable.

While improving the consistency of the pressure, large reservoirs consume a lot of space within the incubator and require more media. In addition, there is the added challenge of developing mounting hardware in order to suspend these containers within the incubator. The real issue, however, is that the leak rate is still too great. Losing 1 $\mathrm{mmHg}$ every 2 hours will result in a $12 \mathrm{mmHg}$ drop in 1 day. This would require that the system receive daily attention, which is both expensive and time consuming. Also, a 10 $\mathrm{mmHg}$ drop can be the difference between modeling slight hypertension at 130/90 $\mathrm{mmHg}$ and normal conditions at 120/80 $\mathrm{mmHg}$. A more accurate and consistent model can and must be developed. 


\section{Chapter 9: WATER COLUMN III}

\section{Introduction}

Changes over time in the height of the fluid level within the column have been a consistent problem in each of the water column based backpressure systems. The water levels in the reservoirs have been decreasing as a result of leaks in the trial evaluations of simplified bioreactors. It was also noted during regular laboratory trials that the bioreactors used to cultivate BVMs in the incubator tend to run low on media over time. Whether this was due to leaks, some evaporative process, tissue metabolizing the nutrients, or fluid redistribution within the system, it was not known. These losses posed a concern when utilizing an independently attached water column to provide a backpressure for the system. "Independently attached" is intended to describe an addition to the bioreactor perfusion loop that does not have fluid directly flowing through it (Figure 9.1). Fluid losses or redistribution would cause decreasing fluid level heights within the attached reservoir and result in an inconsistent backpressure. The water column trials up to this point had all been based on static conditions for the system. Static conditions were useful for reducing variables influencing pressure drop and as well as accurately monitoring pressure levels. Dynamic conditions, applied correctly, may resolve the issue and this chapter will explore the effects. 


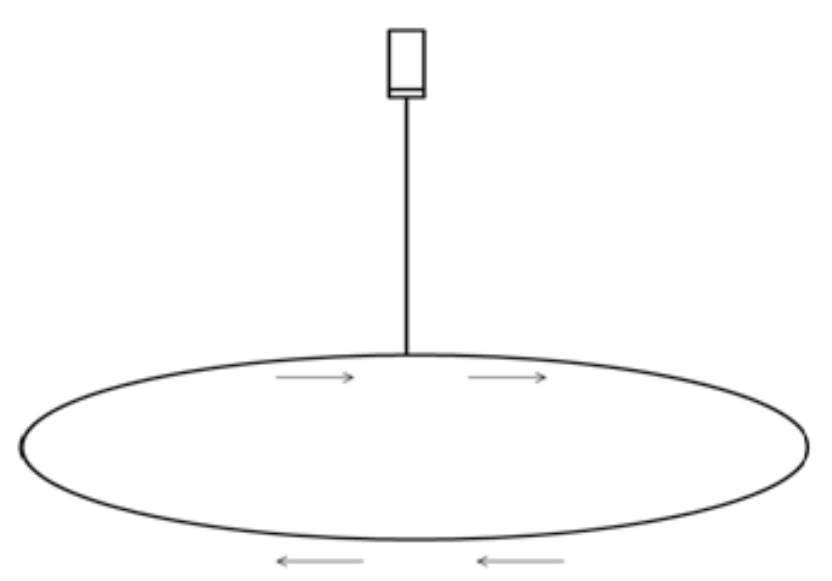

Figure 9.1 Independently attached water column. Arrows indicate the directional flow of fluid within the bioreactor system. Flow within the loop does not enter the water column.

Theoretically, the problem of decreasing fluid level heights within the water column could be solved by simply establishing a flow-through reservoir with the requisite height differential relative to the bioreactor chamber - thus creating a perfused water column (Figure 9.2). A dynamic system will continually perfuse fluid through the tubing of the bioreactor system. Consequently, there would always be fluid in the tubing connecting the bioreactor chamber to the reservoir inlet as long as the fluid level in the reservoir remained above the outlet to the supply line (the tubing section that connects the reservoir to the pump inlet). In this type of system, the backpressure will still be provided by the water column height of the silicone tubing connecting to the reservoir inlet. Any fluid that is lost from the system during use would result in a decrease in the fluid level height in the reservoir and not the water column leading to it. The amount of fluid in the reservoir would not have any impact upon the column of fluid feeding into the reservoir 
through the inlet side tube. This essentially creates a water column between the separated bioreactor chamber and reservoir that never loses its height. With this in mind, the water column backpressure technique explored in the previous studies will be applied to a running bioreactor system in its entirety.

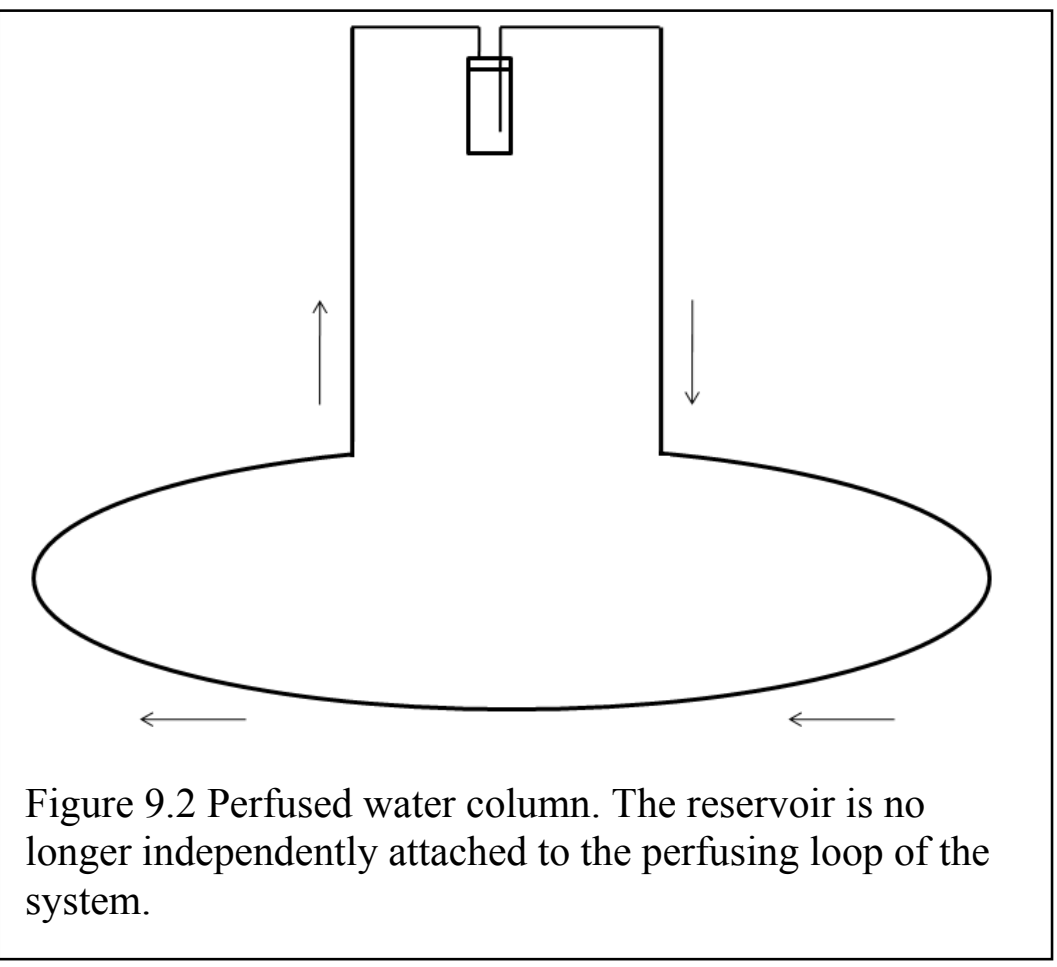

\section{Methods and Materials}

In order to conduct this experiment, numerous materials were required. To begin, the setup included a $50 \mathrm{ml}$ reservoir, Masterflex peristaltic pump with a 3 roller pump head, and a Lock N Lock bioreactor chamber. The reservoir was suspended using a ring stand affixed with a test-tube clamp. The cap of the reservoir had two panel mount luer lock fittings inserted in it. One was the inlet (system return) and the other was the outlet 
(system supply). The outlet fitting had a length of silicone tubing attached that extended down deep into the reservoir and was well below the surface level of the liquid within it. This configuration was intended to compensate for fluid losses and subsequent drops in fluid levels within the reservoir. If the fluid level would drop below the tubing extension, fluid would cease to be returned to the pump and the system would run dry. A length of silicone tubing was used to connect the outlet side of the reservoir to the platinum cured pump tubing secured to the pump. Male and female barbed luer lock fittings were used to connect the silicone tubing to the pump tubing. The free end of the pump tubing was fitted with an additional male and female barbed luer lock fitting combination and attached to another length of silicone tubing. This silicone tubing was then connected to the inlet side of the bioreactor chamber. The inlet side of the bioreactor chamber had a single panel mount barb connection for attaching the tubing. The outlet side of the bioreactor chamber had two panel mounts with short lengths of tubing joining at a "Y" fitting connection. The center-line luminal fitting had a two-way valve placed between the "Y" fitting and the bioreactor chamber wall. A second valve was located just after the combined branch of the "Y" fitting. A long length of silicone tubing was connected to the second valve using another luer lock fitting. The free end of the tubing was then connected back to the inlet fitting in the reservoir cap. The reservoir height was set at 54 inches, measured from the top of the cap to the chamber center-line. Next, a length of ePTFE was suspended within the bioreactor chamber. Once the loop had been completed, the ring stand and pump were set upon the countertop while the bioreactor chamber rested upon the floor (Figure 9.3). 


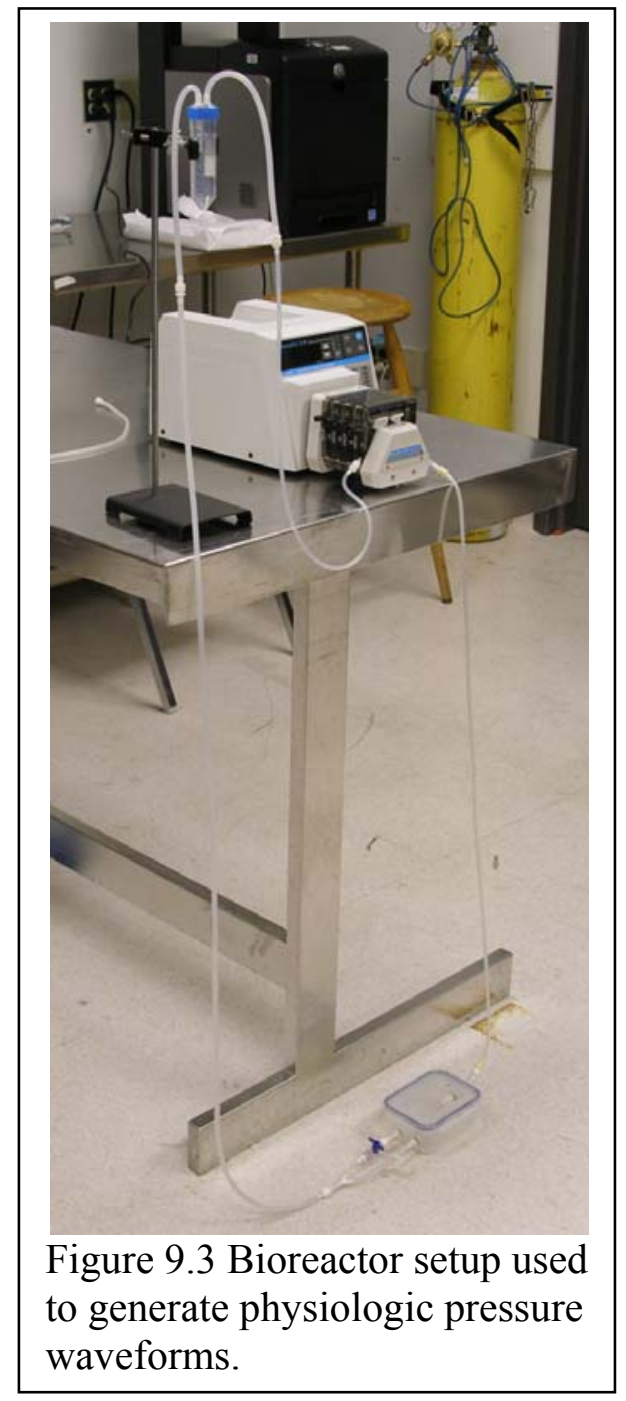

Introducing fluid into the system required a couple of steps. Fluid was first added to the extra-luminal space of the bioreactor chamber. Water was poured into this space around the scaffold until it was up to the brim of the Lock N Lock container. The snap on lid was then secured. There were a number of ways to fill the tubing with fluid, but the easiest utilized the pump. A selected cartridge head was removed from the pump. The pump tubing was then laid across the pump head and the cartridge was reinstalled compressing the tubing. Even with the tubing gates dropped down into place on the pump 
cartridge, the tubing "walked" out the side of the cartridge when the pump was turned on. It was necessary to turn the pump on at a low RPM in the clockwise direction to perfuse fluid toward inlet side of the bioreactor chamber. The pump tubing was then pulled snug in the cartridge in the direction of fluid flow so that there was no slack. This was done so that the luer lock fitting connecting the pump tubing to the silicone tubing was flush with the tubing drop gate on the cartridge. The fitting acted as a stop to prevent the tubing from being further pulled through the cartridge. The occlusion adjustment knob on the cartridge was set so that there was a 1/4 inch gap between the occlusion wedges. This had two important consequences. It set the pressure wave amplitude to $20 \mathrm{mmHg}$ - which corresponded to a $40 \mathrm{mmHg}$ fluctuation between the pressure wave highs and lows. It also ensured that fluid was adequately pushed through the system.

To prime the system, the cap to the reservoir was unscrewed and placed in a beaker full of water. The end of the supply tubing on the outlet of the reservoir was fully immersed. The pump was then turned on and the speed set at 45 RPM. The speed was not critical - it was dictated by the patience of the operator. A slower rate produced fewer bubbles in the line, but required more time. Once the system was full of water, it was necessary to throttle the pump on prime a few times to pass any trapped gas through the lines and out of the system. The cap was then removed from the beaker and affixed to the reservoir.

To measure the internal pressure of the system, the data acquisition equipment was tied into the system. The pressure transducer was positioned downstream of the bioreactor chamber and in horizontal alignment with the chamber. To install the transducer, the chamber outlet two-way valve was closed, and a hemostat was used to 
clamp off the tubing connecting to the valve. The transducer was injected with fluid to prevent the introduction of air bubbles into the system. Once the tubing and valve were sealed, they were disconnected and attached to the transducer - which bridged the gap. The reservoir, initially on a horizontal plane with the chamber, was elevated with a 54 inch offset to generate the desired $100 \mathrm{mmHg}$ base-line pressure (Figure 9.3). Data was then collected over a 25 hour period running the system at 20 RPM.

The data acquisition equipment included both hardware and software. It was assembled and calibrated using the same procedure as before. A confirmation of measurement accuracy was obtained from comparing the measured pressure generated by the 54 inch water column to the estimated pressure the transducer should read based on the water column (100 $\mathrm{mmHg})$. Data points were entered into the table after the experiment was concluded by scrolling through the first 72 hours of data and manually recording the pressure measurements every hour.

\section{$\underline{\text { Results }}$}

With the initial setup completed and under static conditions, the pressure of the low point of the system (the bioreactor chamber) measured between 101-102 $\mathrm{mmHg}$. As soon as the pump was started, the systolic and diastolic pressure measurements were 125/85 mmHg. Over the following 25 hours, measurements were taken continuously with the data acquisition equipment and recorded every hour. Table 9.1 displays the recorded data beginning after the first hour of operation. Figure 9.4 depicts a sample of the recorded waveform after two hours of system operation. 
Table 9.1 Systolic and diastolic pressure measurements reported hourly for a 25 hour test.

\begin{tabular}{|c|c|c|}
\hline hour & $\begin{array}{l}\text { systolic pressure } \\
(\mathrm{mmHg})\end{array}$ & $\begin{array}{l}\text { diastolic pressure } \\
(\mathrm{mmHg})\end{array}$ \\
\hline 1 & 120.44 & 80.56 \\
\hline 2 & 117.94 & 78.19 \\
\hline 3 & 116.46 & 76.86 \\
\hline 4 & 115.79 & 75.89 \\
\hline 5 & 114.67 & 75.28 \\
\hline 6 & 113.39 & 73.90 \\
\hline 7 & 112.17 & 72.57 \\
\hline 8 & 117.12 & 77.27 \\
\hline 9 & 115.95 & 76.50 \\
\hline 10 & 115.03 & 75.33 \\
\hline 11 & 114.52 & 75.00 \\
\hline 12 & 114.06 & 75.18 \\
\hline 13 & 113.09 & 75.63 \\
\hline 14 & 113.75 & 74.10 \\
\hline 15 & 113.95 & 74.72 \\
\hline 16 & 113.80 & 74.31 \\
\hline 17 & 113.39 & 75.02 \\
\hline 18 & 113.29 & 74.26 \\
\hline 19 & 112.93 & 74.36 \\
\hline 20 & 117.12 & 78.19 \\
\hline 21 & 116.05 & 77.07 \\
\hline 22 & 114.72 & 75.99 \\
\hline 23 & 113.24 & 76.09 \\
\hline 24 & 117.73 & 78.44 \\
\hline 25 & 116.61 & 77.32 \\
\hline
\end{tabular}




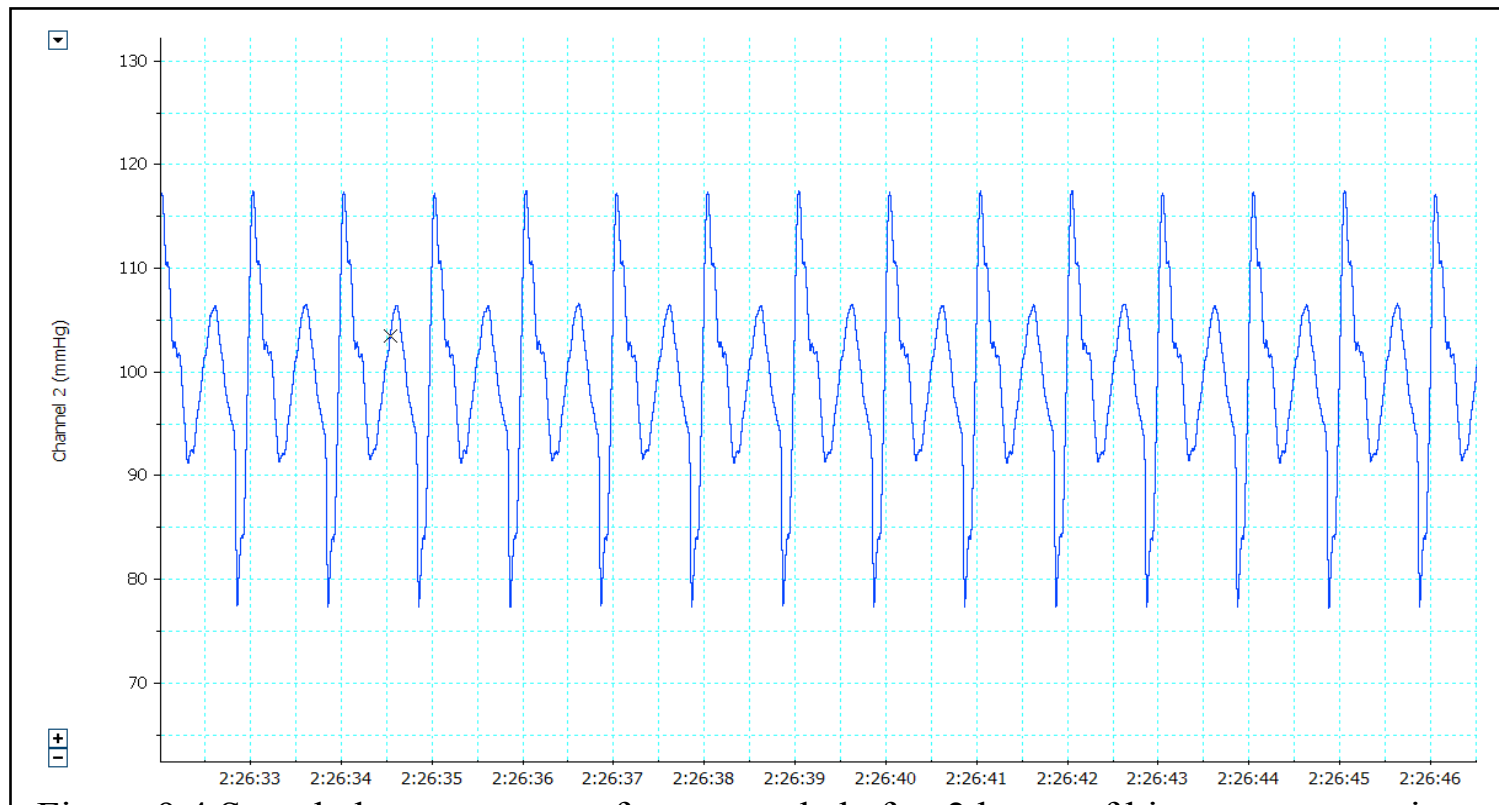

Figure 9.4 Sampled pressure waveform recorded after 2 hours of bioreactor operation.

\section{$\underline{\text { Discussion and Conclusion }}$}

Once the 25 hour test was completed, the pump head was disassembled. Upon disassembly the pump tubing was examined. Significant deformation of the tubing had occurred. The side in contact with the cartridge head had an accumulation of black wear debris and the tube no longer retained a cylindrical shape. While this is not ideal, it is normal and should not have any effect upon the results of the measurements. No cuts or breaks were found in the tubing. In addition, the tubing is "crushed" as a part of normal peristaltic function. Accordingly, the shape (deformed or otherwise) of the tubing crushed within the pump should not significantly affect pressure or flow provided the flow volume is not substantially altered under these circumstances. However, the tubing does undergo a break-in period and this phenomenon may affect initial measurements versus data recorded at a later time. 
Initial readings were high compared to the rest of the measured systolic and diastolic pressures. The pressures began at approximately $125 / 85 \mathrm{mmHg}$ and then settled at a peak of roughly $117 / 78 \mathrm{mmHg}$. The drop is most likely due to the tubing breaking in over the first hours of use. The other source of variation was due to capping the reservoir. When the cap was screwed on, additional gasses were trapped within the chamber and compressed. This led to an artificial spike in pressure that could not be maintained by the system. As a result, while running the bioreactor over a period of time, any leaks present within the system decayed the magnitude of the initial pressure that was in excess of the pressure the pump and water column were able to generate. For example, the water column is capable of generating and maintaining $100 \mathrm{mmHg}$ while the pump can produce a pressure wave of $\pm 20 \mathrm{mmHg}$. Accordingly, the system should be able to sustain 120/80 mmHg. However, the system began at $125 / 85 \mathrm{mmHg}$ after the cap was attached to the reservoir. The additional $5 \mathrm{mmHg}$ was not generated by normal system operation and, therefore, could not be maintained with the presence of leaks. Even with leaks, the system should have been able to maintain the $120 / 80 \mathrm{mmHg}$ pressure wave.

If one looks closely at the data, there are pressure shifts that occur between hours 7 and 8,19 and 20, as well as 23 and 24 . At 7 hours and roughly 41 minutes, systolic pressure had dropped all the way to $111.76 \mathrm{mmHg}$. Over the period of 11 seconds, the systolic pressure climbed up to $116 \mathrm{mmHg}$ (Figure 9.5). At 19 hours and roughly 15 minutes, systolic pressure had decreased to $112.47 \mathrm{mmHg}$. Over a period of 8 seconds, the systolic pressure climbed up to $116.30 \mathrm{mmHg}$. At 23 hours and roughly 32 minutes, the systolic pressure once again dropped and reached $112.83 \mathrm{mmHg}$. Over a period of 8 seconds, the systolic pressure climbed up to $116.36 \mathrm{mmHg}$. The amount of time leading 
up to the minimum pressures varied and occurred over extended periods of time. The self-regulated restoration of pressure, however, was abrupt and relatively consistent. In addition, the systolic pressures consistently oscillated between $112 \mathrm{mmHg}$ and 117 mmHg. As one can see, the pressure would continue to climb after the initial drop until the systolic pressure would reach $117 \mathrm{mmHg}$. The gaps between the drops in pressure were not steady, but the pressure would fluctuate within a relatively consistent range.

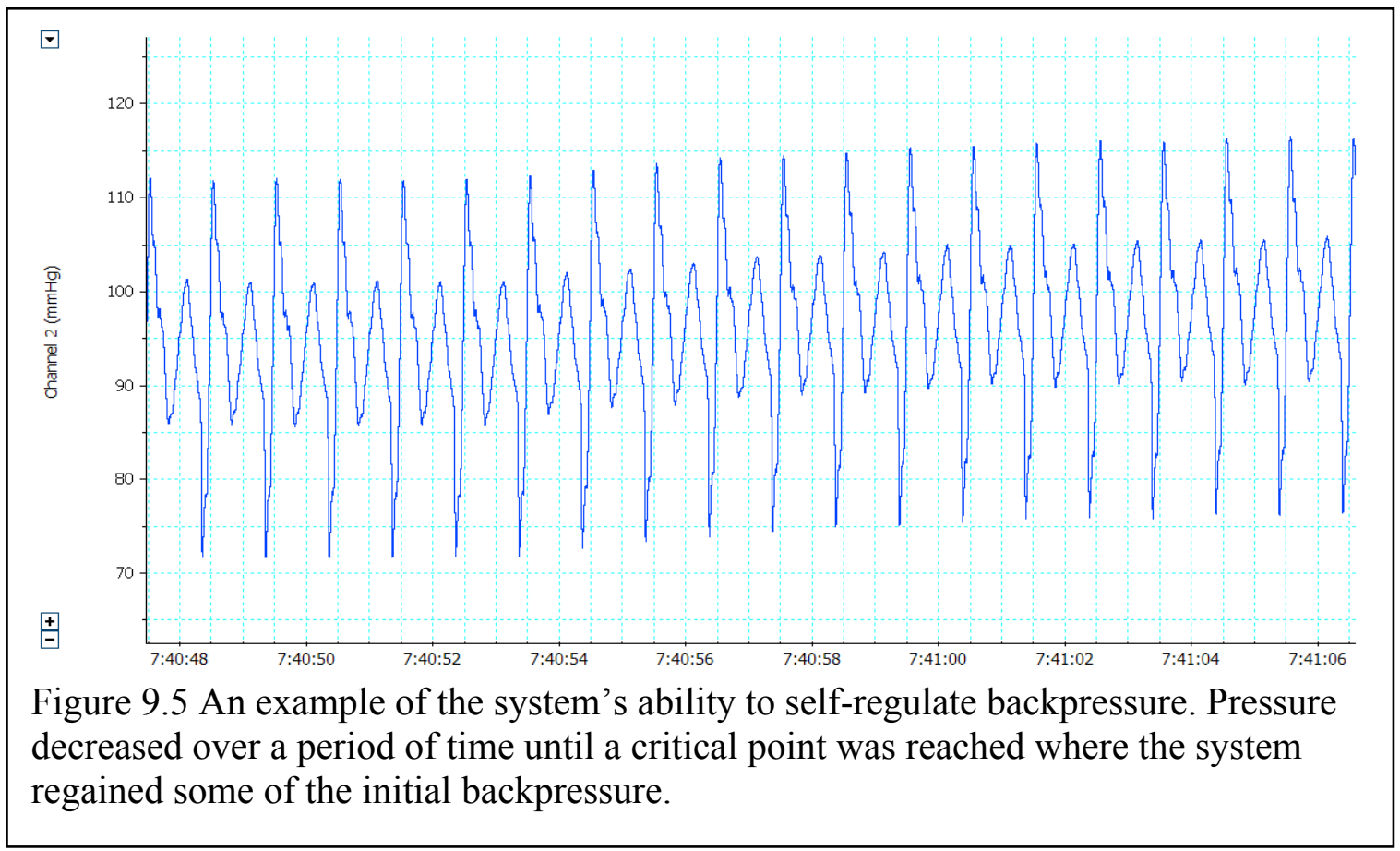

Based on the results, it appears that the closed system was generating a small vacuum in the reservoir as fluid was lost from the system. The reservoir was the highest point in the system and, as a result, the location of the lowest pressure. The system has been shown to leak in the previously conducted studies - especially the Lock N Lock bioreactor chambers at pressures at $100 \mathrm{mmHg}$ and above. Upon inspection of the 
bioreactor chamber, a water ring could be seen where it had been sitting. This was an obvious indication that fluid was lost from the system and supports the conclusion that a vacuum was generated in the reservoir - the high point of the system. Interestingly, the data also suggests that there was potentially a limit to the magnitude of the vacuum that the reservoir could withstand without compromising any of the seals. Once this point was exceeded, approximately $-8 \mathrm{mmHg}$ (from $120 \mathrm{mmHg}$ ), the panel mount seals on the cap were compromised and air was drawn back into the reservoir over an 8-10 second period until nearly the original pressure was restored. The reason the pressure does not return all the way back to $125 \mathrm{mmHg}$ is because this pressure was an artificial peak caused by the capping of the reservoir, and the compromised seal became functional at a pressure slightly less than $120 \mathrm{mmHg}$. During disassembly of the reservoir, a sucking sound could be heard once the cap seal was broken.

This study confirmed earlier suspicions of the Lock N Lock bioreactor chamber's inability to withstand elevated pressures. $120 \mathrm{mmHg}$ is not much greater than the reported leak pressure for the extra-luminal space of the bioreactor chamber, and it is only momentary. Since the pressure is not maintained at a constant elevated pressure, the chamber does not leak as drastically as it did during the leak pressure test. The study also confirmed that the pressure is able to diffuse beyond the scaffold barrier into the surrounding extra-luminal space. While the leak was not catastrophic, it did exist. The Lock N Lock bioreactor chambers could be successfully used with this experimental setup in future laboratory trials, but they generate vacuums within the system and a small mess. This issue qualifies the use the newly designed bioreactor chamber in the future 
studies performed for this project. Even so, an additional study will be performed with the Lock N Lock bioreactor chamber in order to confirm observations made in this study.

The leaks in the system would cause a vacuum because the system is run by a positive displacement pump. The pump will push the same amount of fluid that it pulls. If there is a disparity between what the pump draws and pushes, the system will either pressurize or depressurize. When the operations are balanced, the pressure is constant. During the experiment the pump was pulling a certain amount of fluid from the reservoir, but that same amount was not being returned to the reservoir because of the slight leaks downstream of the pump. Since the bioreactor used in the experiment was a closed loop system, the loss of fluid created a vacuum.

Overall, this experiment had some flaws, but they can be corrected. The significant positive result of this study was that it functioned within a consistent pressure range and was relatively insensitive to the minor leaks that have proven critical flaws in the other configurations. The system was insensitive to the leaks in the sense that pressure was lost over a period of hours rather than minutes and fell out of the systolic range of $117 \mathrm{mmHg}$ to $112 \mathrm{mmHg}$. Based on this, it is worthwhile to continue using this basic backpressure technique but to proceed with a few modifications. Since the sealed reservoir was where the vacuum was generated, venting the reservoir would prevent the phenomena from occurring. More testing is required to validate the results obtained in this study. While leaks were found and a drop in pressure was detected, this was only a single study. Another similar test will be performed to confirm the results. The next experiment performed in the following chapter will include an improved bioreactor 
design that will specifically eliminate the capping pressurization and reevaluate the potential vacuum formation. 


\section{Chapter 10: WATER COLUMN IV}

\section{$\underline{\text { Introduction }}$}

Based on the observation of pressure fluctuations in the previous study and the suspicions of vacuum formation, the experiment needed to be repeated to verify the accuracy of data obtained. The goal of this study was to recreate the fluctuating pressures observed in Chapter 9 and to obtain additional information to validate and better characterize the hypothesis of vacuum formation. Effort can then be dedicated to assessing the frequency and severity of the problem. If observations made during the last test were anomalies, it would not make sense to modify the system to compensate for them. Accurately defining system behavior now will lead to more efficient solutions later. Therefore, the focus of this study is to confirm the existence of vacuum formation within the bioreactor system and to better characterize its occurrence.

To accomplish this, the system was only slightly modified. It was important that the system from the last study was not drastically changed as it may have had an impact on the results that were obtained and phenomena that were observed. However, the last study began with artificially elevated pressures due to capping the reservoir. The initial spike in pressure may have caused instability within the system that may have impacted the rate at which the vacuum formation occurred. With this in mind, only a small modification was implemented in this study to the reservoir. This modification allowed the reservoir to be manually vented in order to prevent elevated pressure generation during the reservoir capping process. 


\section{Materials and Methods}

The materials and protocol were the same as described in the previous round of testing. The only differences were that this test was conducted for a slightly longer period of time (31 hours instead of 25), and that a three-way valve was installed at the inlet to the reservoir in order to vent any pressure generated while capping the reservoir (Figure 10.1). As was just mentioned, the bioreactor system was assembled using the same method as mentioned as in Chapter 9, with one small change. Before the cap was attached to the reservoir, the 3 way valve attached to the reservoir's inlet was opened to the atmosphere by closing the inlet port of the valve. This configuration allowed excess gasses to escape the reservoir and vent to the open air during capping. Even though the Lock N Lock bioreactor chamber had shown a propensity for leaking at elevated pressures, it was used again to assess its operation a second time and to gather more data to evaluate its ability to function under operating conditions. In addition, during the previous study the leaking bioreactor was implicated in vacuum formation. Since this study was focusing on better characterizing and understanding the potential vacuum, it was important to keep the system the same and not remove the potential cause. The pressure transducer used to collect data from the system was calibrated and zeroed before use. Data points were entered into the table after the experiment was concluded by scrolling through the 31 hours of data and manually recording the pressure measurements every hour. 


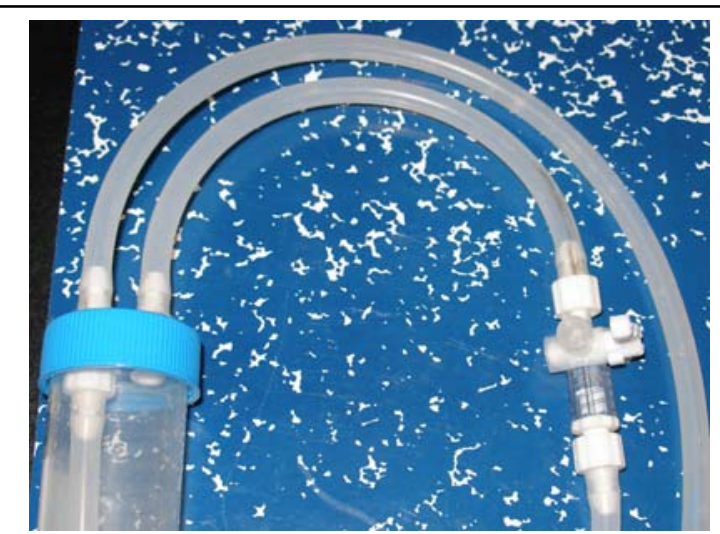

Figure 10.1 System modification to allow venting of initial pressurization from capping process.

\section{$\underline{\text { Results }}$}

The initial pressure without the pump running was $102 \mathrm{mmHg}$. Once the pump was started, pressure was measured at 121.46/82.99 $\mathrm{mmHg}$. The pressure recorded over the following 31 hours is listed below in Table 10.1. A sample of the generated pressure waveform measured at 31 hours is illustrated in Figure 10.2.

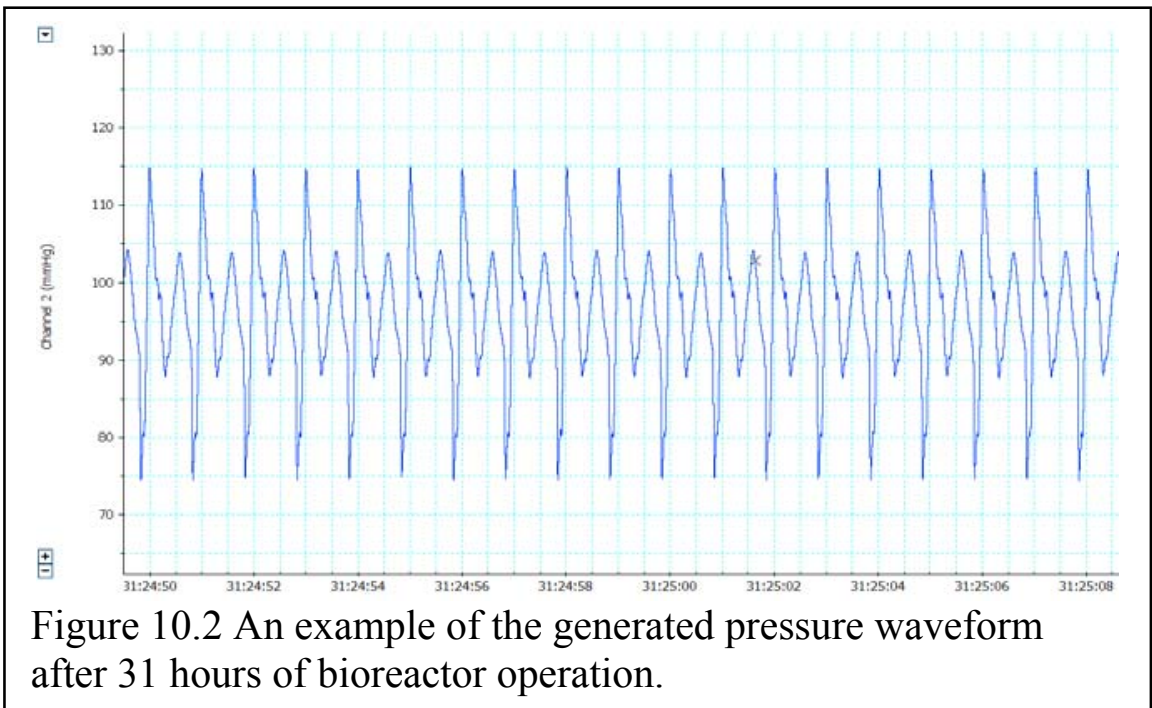


From Table 10.1 it can be seen that the pressure remained much more consistent over time. The pressure varied very little, only a fraction of $1 \mathrm{mmHg}$, in the first 14 hours of system operation. However, after this point, pressure appeared to decrease and then fluctuate between highs and lows (Figure10.2).

\begin{tabular}{|c|c|c|}
\hline \multicolumn{3}{|c|}{$\begin{array}{l}\text { Table } 10.1 \text { Systolic and diastolic pressures } \\
\text { reported hourly for a } 31 \text { hour test. }\end{array}$} \\
\hline Hour & $\begin{array}{l}\text { Systolic Pressure } \\
(\mathrm{mmHg})\end{array}$ & $\begin{array}{l}\text { Diastolic Pressure } \\
(\mathrm{mmHg})\end{array}$ \\
\hline 1 & 122.18 & 83.25 \\
\hline 2 & 121.98 & 83.15 \\
\hline 3 & 122.38 & 82.89 \\
\hline 4 & 122.49 & 83.20 \\
\hline 5 & 122.54 & 83.50 \\
\hline 6 & 122.08 & 83.96 \\
\hline 7 & 122.69 & 84.07 \\
\hline 8 & 122.74 & 83.09 \\
\hline 9 & 123.25 & 83.20 \\
\hline 10 & 122.38 & 83.71 \\
\hline 11 & 122.90 & 84.58 \\
\hline 12 & 122.64 & 84.52 \\
\hline 13 & 122.38 & 85.34 \\
\hline 14 & 122.49 & 85.90 \\
\hline 15 & 121.52 & 86.98 \\
\hline 16 & 121.21 & 87.49 \\
\hline 17 & 121.26 & 85.00 \\
\hline 18 & 118.30 & 78.19 \\
\hline 19 & 114.87 & 75.12 \\
\hline 20 & 117.99 & 78.50 \\
\hline 21 & 114.11 & 77.42 \\
\hline 22 & 119.73 & 83.66 \\
\hline 23 & 120.34 & 84.52 \\
\hline 24 & 120.19 & 84.93 \\
\hline 25 & 120.19 & 85.04 \\
\hline 26 & 120.90 & 85.80 \\
\hline 27 & 124.12 & 85.14 \\
\hline 28 & 124.73 & 85.19 \\
\hline 29 & 121.00 & 82.79 \\
\hline 30 & 118.30 & 78.96 \\
\hline 31 & 115.69 & 76.25 \\
\hline
\end{tabular}




\section{Discussion and Conclusion}

By removing the initial peak pressure generated during the capping process, the system modification and change in protocol seem to have had an effect on the stability of the pressure within the system. The pressure remained relatively constant for the first 14 hours of testing. After 14 hours, a vacuum began forming until pressure was once again reestablished. After the first fluctuation, two more fluctuations occurred. One occurred almost immediately after the pressure was restored. The next began to occur at the end of the test.

It appears that the frequency of fluctuations was a result of the system selfcorrecting. The previous test began with a pressure that was elevated slightly above the vented state. Accordingly, pressure quickly dropped in the first hour and a new set point was reached. Since the system adjusts and corrects as a function of fluid loss and the integrity of the seals on the reservoir, there is some variation in the period over which it may occur. This test started with the air space in the reservoir vented to atmosphere and the initial system pressure was subsequently lower than in the previous test. The cap was put in place with the valve open so that pressure would not build as a result of the capping process. Once in place, however, the valve was closed to the atmosphere so that the fluid within the system would circulate. As a result, the pressure built up over the following 9 hours after testing began. There are three potential explanations for the buildup of pressure within the system. The first potential cause is the presence of a few trapped air bubbles within the water column portion of the bioreactor system. These bubbles would detract from the pressure generated within the bioreactor chamber because they reduce the weight of the water column since air is less dense than water. After a few 
hours of operation, these bubbles may have been cleared from the line and the homogenous water column exerted a greater pressure within the bioreactor. Another potential explanation is that numerous air bubbles trapped throughout the bioreactor loop were dislodged over time and compressed within the bioreactor reservoir. The other explanation is that the pump pulled air into the system through a compromised seal or cracked tubing somewhere between the pump and the reservoir. As the trial progressed, the pressure fluctuations were likely due to system self-correction. There are system limitations to maximum and minimum pressures. Once these limits were surpassed, the system automatically restored resting conditions to some degree. The amount of correction that the system has to go through might result in over correction and affect the rate at which the fluctuation occurs as well as the magnitude. It is more likely, based on the data, that once the system limits are surpassed a seal somewhere in the system (probably the reservoir) is compromised. The variability of what seal is compromised, and to what degree, affects the pressure fluctuations. The same seal might not always respond the same way to the dropping pressures.

Based on this experiment, simply venting the system a single time before running the pump is not sufficient to maintain a constant pressure throughout system operation. In addition, vacuum formation was not an anomaly. Results in this experiment were similar to those observed in the previous experiment. Accordingly, the problem associated with leaks and fluctuating pressures still existed and must be dealt with. The next study will address the problem using a permanently vented reservoir. 


\section{Chapter 11: WATER COLUMN V}

\section{$\underline{\text { Introduction }}$}

It was noted in earlier discussions of experimentation with the water column that a vacuum had been forming in the reservoir. The vacuum was likely caused by a leak within the system on the discharge side of the pump, which unbalances the relationship between fluid supplied by the reservoir and fluid returned to the reservoir. The net loss results in vacuum formation and is cumulative over time. A potential solution to this problem is to leave the reservoir vented to the open air throughout the duration of the test. This will hopefully eliminate the issue of pressure fluctuation within the system. The experiment described in this chapter implemented this potential solution. Vented reservoirs have been used in other research facilities, but with the intent of allowing gas exchange (9). Accordingly, it is feasible to use the vented reservoir during regular laboratory experimentation without compromising the sterility of the system, as long as a filter is incorporated. In this experimental study the vent was meant to serve a different purpose other than facilitating $\mathrm{CO} 2$ exchange with the media - it was implemented to help regulate pressure fluctuations.

\section{Materials and Methods}

The same setup protocol used in the previous experiments (Water Column III and IV) was applied to set up this experiment, except the manual vent from the previous study was not included. The reservoir was placed at the same height (54 inches), the same connections and lengths of tubing were used, and all of the fittings were the same. However, this test differed in the type of reservoir modification used. Instead of using the 
normal reservoir with only an inlet and outlet mounted in the cap, this reservoir had a cap with three ports (Figure 11.1). Using this slightly modified setup, procedures to measure and record pressures over an 18 hour time span were carried out as described in the previous chapter. Data points were entered into the table after the experiment was concluded by scrolling through the 18 hours of data and manually recording the pressure measurements every hour.

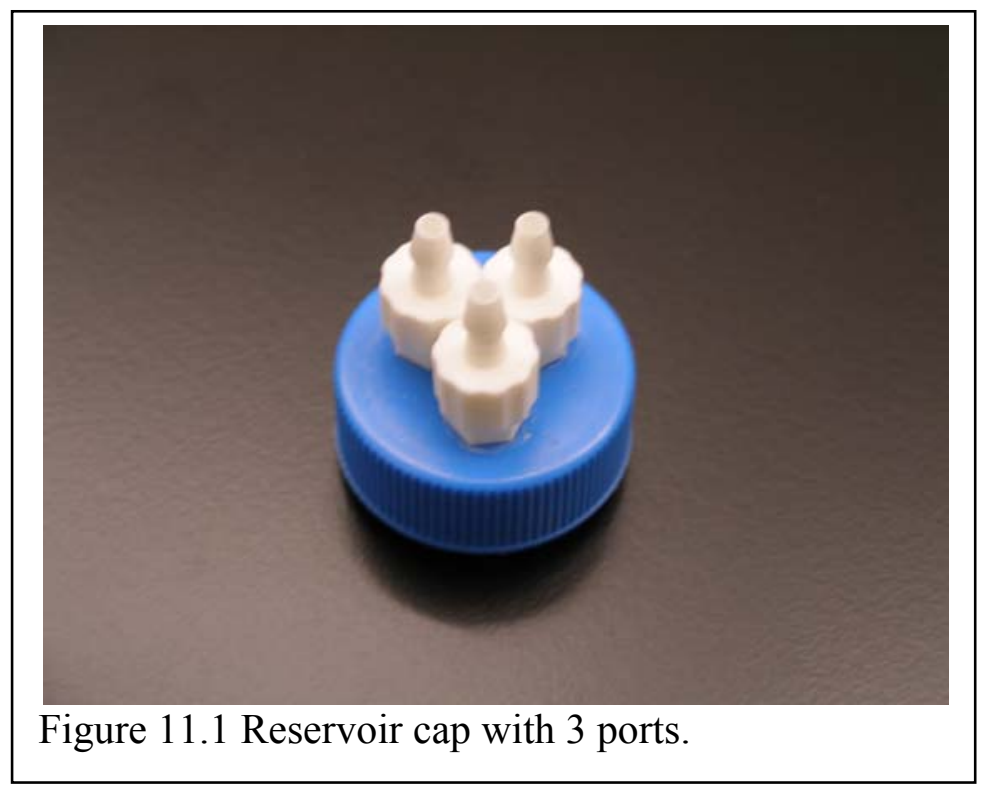

The reservoir cap had an inlet and outlet just like the previous system, but there was an additional panel mount that vented directly to the atmosphere. In this study, the newly designed bioreactor chamber was also implemented instead of the Lock N Lock. The Lock N Lock had proven inefficient at operating under dynamic conditions with elevated pressures. In addition, it was no longer necessary to characterize the formation of vacuums within the system. This study focused on eliminating pressure fluctuation 
rather than creating it. Measurements were taken continuously over an 18 hour period using the calibrated ADI pressure transducer and data acquisition equipment.

\section{$\underline{\text { Results }}$}

The beginning base-line pressure of the static system was $101 \mathrm{mmHg}$. Once the peristaltic pump had been started, the pressure wave oscillated between 119.22 and 80.64 mmHg. Table 11.1 below contains the data recorded over the following 18 hours. Once the pump had been stopped at the end of the experiment, the base-line pressure read between 101 and $102 \mathrm{mmHg}$. In addition, the new bioreactor did not show any signs of leakage. Figure 11.2 illustrates the pressure waveform generated by the peristaltic pump and is characteristic of all pressure waveforms the system generated under these applied conditions. 
Table 11.1 Systolic and diastolic pressures reported hourly for an 18 hour test.

\begin{tabular}{|r|r|l|}
\hline \multicolumn{1}{|r|}{ hour } & $\begin{array}{l}\text { systolic pressure } \\
(\mathrm{mmHg})\end{array}$ & $\begin{array}{l}\text { diastolic pressure } \\
(\mathrm{mmHg})\end{array}$ \\
\hline 1 & 120.14 & 80.23 \\
\hline 2 & 119.93 & 80.13 \\
\hline 3 & 120.39 & 79.72 \\
\hline 4 & 121.16 & 78.85 \\
\hline 5 & 121.11 & 78.75 \\
\hline 6 & 121.77 & 78.44 \\
\hline 7 & 121.57 & 78.14 \\
\hline 8 & 121.98 & 77.93 \\
\hline 9 & 122.54 & 78.44 \\
\hline 10 & 123.61 & 78.39 \\
\hline 11 & 123.51 & 78.29 \\
\hline 12 & 123.81 & 77.88 \\
\hline 13 & 123.2 & 77.42 \\
\hline 14 & 123.15 & 77.83 \\
\hline 15 & 123.87 & 77.12 \\
\hline 16 & 123.81 & 76.91 \\
\hline 17 & 124.17 & 76.96 \\
\hline 18 & 123.81 & 77.12 \\
\hline
\end{tabular}

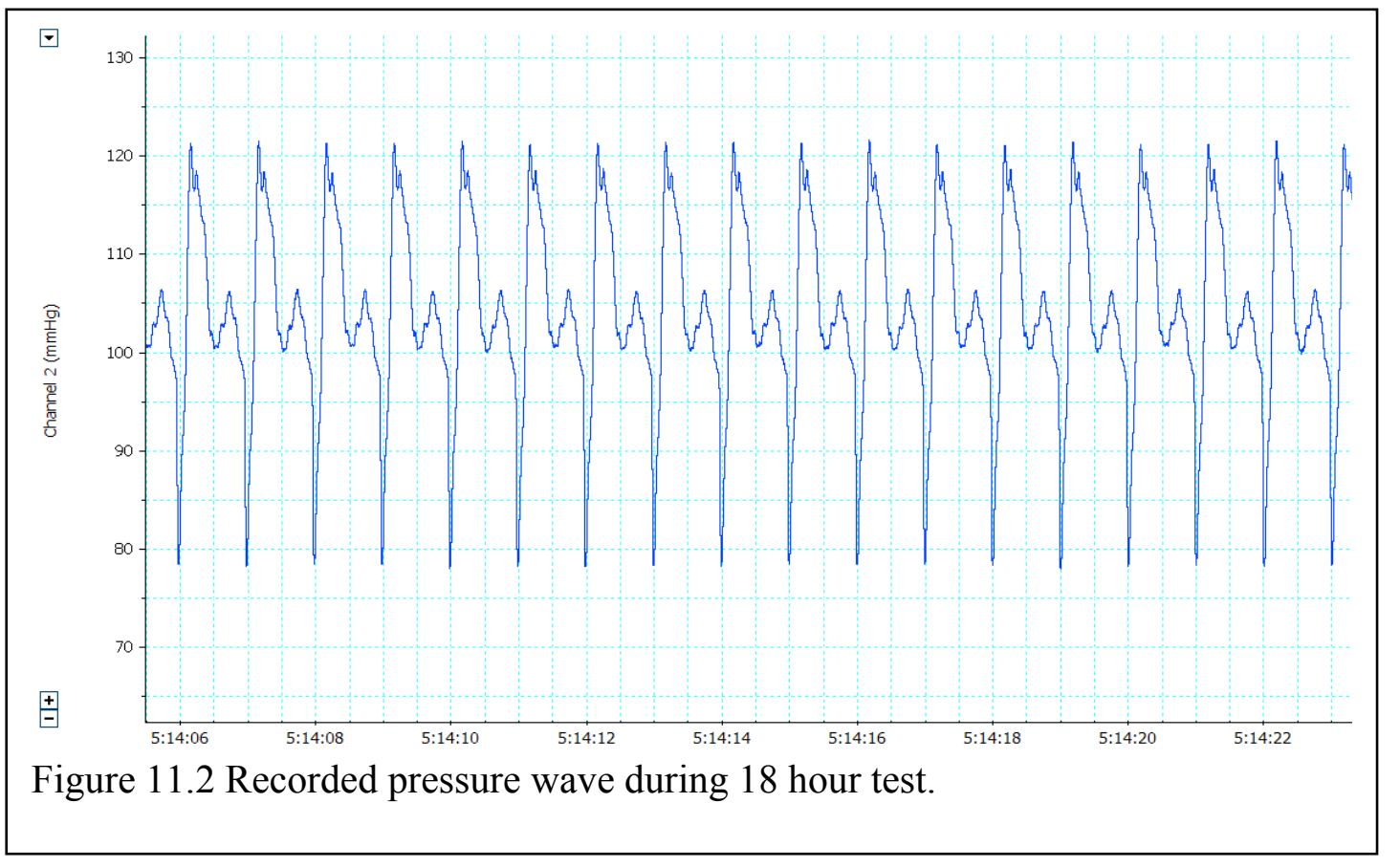




\section{Discussion and Conclusion}

Based on the data generated from this test, it appeared that the fluctuating pressures had been nearly eliminated. There was some small variation within the data, but not nearly as much as in previous studies. Although this was an encouraging result, the pressure wave at the end of the study was not the same as the beginning pressure wave. The float or variation in measurement was likely due to wear-related changes in the pump tubing over time. This assumption is based upon the changes in the pressure waveform over time. Instead of the entire pressure wave shifting up or down, as a result of pressurization or vacuum formation, the amplitude of the wave increased. The increase in pressure wave amplitude resulted in increased systolic pressures and decreased diastolic pressures, but maintained the same average pressure (approximately $100 \mathrm{mmHg}$ ). A simultaneous increase in systolic pressure and decrease in diastolic pressure cannot be accounted for by the previously explored pressure fluctuation mechanisms - especially considering the included vented reservoir. A shift in pressure wave magnitude is substantially different from an increase in wave amplitude. Just as a vacuum can be generated in the reservoir as fluid leaks out on the discharge side of the pump, pressure can be generated within the reservoir by leaks on the suction side of the pump. A leak on the suction side of the pump would result in air being pulled and forced into the system. System temperature may also play a role in pressure increase. Thermal expansion of the fluid within a closed system would result in an increased volume and pressure. However, the system was no longer closed. As a result, volume changes no longer resulted in pressure changes. Accordingly, thermal expansion or leaks on the suction side of the pump could not generate increased pressures in the system. Clearly, the increased 
pressure wave amplitude must have been due to some physical property of the system changing. Since the wave grew over time, the increase in amplitude was likely due to the degradation of the pump tubing over time. Nonetheless, a number of potential explanations were explored before dismantling the system.

The pump was stopped and restarted to see if any change in pressure would occur due to any potential operational or flow related factors. If a change was noted, attention could be focused on analyzing the potential factors and their relation to pressure. However, no changes occurred. After the pump had been stopped and restarted, a couple of bubbles were noticed within the tubing line in a few places throughout the system. Pockets of gas can alter pressure readings within the system since air is a different density and exhibits different fluid properties. For example, air is compressible while fluid is not. The presence of a less dense and more compressible fluid within the system should not result in elevated pressures. In fact, since air is less dense and compressible, an increased volume of air within the system lines would result in lower pressures and smaller pressure wave amplitudes. To remove the bubbles from the system, the system was primed by increasing the RPM of the pump. Priming the pump removed most of the bubbles and the system returned to $121 / 80 \mathrm{mmHg}$. After priming the pump a second time, a small leak was noticed. Therefore, moving the air bubbles out of the system was not the likely cause of the pressure wave amplitude reduction. In previous experiments, worn pump tubing was found to lose its shape and accumulate silicone debris as black or gray deposits on the outer tubing wall. Priming the pump may have cleared or shifted such an accumulation of debris to a position where it may not have impacted tubing occlusion any 
longer. This is plausible considering the tubing was so worn out that the second time the system was primed the tubing began to leak.

Removing the cartridge head from the pump revealed that the platinum cured tubing had been significantly degraded over the course of the experiment. The tubing had large debris deposits and a large crack in the longitudinal direction. New pump tubing had not been used in this experiment because the tubing is rated to endure many cycles and repeated use. It is easy to imagine the condition of the pump tubing affecting the pressure waves, especially since adjusting the occlusion of the cartridge played a role in determining the amplitude of the pressure wave when initially assembling the system. Cole Parmer had been contacted in regard to the condition of the pump tubing. The accumulation of worn debris on the outer surface of the tubing was considered normal with extended use. Future trials will be conducted with new pump tubing.

Another potential source of variation could be due to temperature. Fluid expands as it is heated. The cyclic nature of the peristaltic pump may elevate the temperature of the fluid slightly. An increase in temperature would cause the fluid to expand over time and result in higher pressures and larger amplitudes. Even if the pump itself did not change the temperature of the system significantly, ambient temperature of the room may fluctuate and have the same end effect. Even if this was the case, the vented reservoir should counteract these effects. The end goal is to run the bioreactor within an incubator. The incubator is maintained at $37^{\circ} \mathrm{C}$. Since this is a constant temperature, there should not be any volume fluctuations due to the environmental temperature. However, the $37^{\circ} \mathrm{C}$ temperature of the incubator is elevated compared to standard temperature and pressure (STP) conditions that designate $25^{\circ} \mathrm{C}$ as room temperature. If bioreactor materials 
assembled outside the incubator are near room temperature, thermal expansion will likely occur when the materials heat up to $37^{\circ} \mathrm{C}$ inside the incubator. The expansion may be minimal, but the vented reservoir would compensate for any volumetric changes.

Overall, the new bioreactor chamber proved to be durable and able to withstand the environmental conditions of the trial. Whether the increased stabilization of pressures was attributable to the addition of the new bioreactor or the vented reservoir, it was not specifically deduced. It was likely some combination of the two factors, but the vented reservoir was able to counteract volumetric fluctuations whereas the bioreactor chamber simply attempted to prevent them. At the end of the trial, the bioreactor chamber did not show any signs of leakage and there were no wet spots on the floor where the bioreactor had been sitting.

In the future, it will be important to use new pump tubing for experiments designed for prolonged periods of time in order to ensure similar testing conditions and comparable results. The short test demonstrated that the vented reservoir was effective in significantly reducing pressure fluctuation. A fluctuation of only a few $\mathrm{mmHg}$ is acceptable and attributable to nuisance factors. These results warranted an extended trial to assess the stability of the system under more realistic laboratory conditions. 


\section{Chapter 12: 72 HOUR TEST}

\section{Introduction}

The study conducted in Chapter 11 evaluated the efficacy of the permanently vented reservoir in preventing pressure fluctuations within the bioreactor system. The results from the study indicated that the system modification was successful in eliminating pressure oscillations within the bioreactor. However, the results also indicated that the pressure wave amplitude increased over time. Increasing pressure wave amplitude is undesirable, but it was attributed to the degradation of old pump tubing used in the setup. Accordingly, the undesirable results were independent of the system modification to the reservoir and could hypothetically be remedied with the inclusion of new pump tubing during system assembly. Therefore, the bioreactor system with the elevated and vented reservoir was ready for a long-term trial.

The purpose of the tests presented in this chapter was to run an extended trial to assess the stability and durability of the final backpressure system design. The longer the system can run without assistance or intervention, the more practical and successful the design is. As a minimum, 72 hours of operation without intervention is desirable. This would allow the system to run over a standard 3 day cell culture period without requiring a laboratory assistant to make additional adjustments. Intervention would be defined by a laboratory assistant needing to make any adjustment to the system to maintain the desired pressure levels. The experiment in this chapter was therefore conducted over three days and the system was periodically checked to confirm proper function. 


\section{Materials and Methods}

The system was set up using the same protocol described in Water Column Test V. The permanently vented reservoir and newly developed bioreactor were utilized in this system. The pressure transducer was calibrated using two point calibration and zeroed before use. Data points were entered into the table after the experiment was concluded by scrolling through the data and manually recording the pressure measurements every hour. The test was designed to run for 72 hours.

\section{$\underline{\text { Results: }}$}

Results for this study were only recorded for 13 hours before the test was aborted due to abnormal data collection. The base-line pressure of the system before testing was measured at $100 \mathrm{mmHg}$. The final base-line pressure of the system was $112 \mathrm{mmHg}$.. The measurements are listed below in Table 12.1. Once the pressure transducer was removed from the bioreactor system, the data acquisition equipment measured $12 \mathrm{mmHg}$ under atmospheric pressure.

Table 12.1 Systolic and diastolic pressures reported hourly
for a test terminated after 13 hours.
\begin{tabular}{|r|l|l|}
\hline \multicolumn{1}{|r|}{ hour } & $\begin{array}{l}\text { systolic pressure } \\
(\mathrm{mmHg})\end{array}$ & $\begin{array}{l}\text { diastolic pressure } \\
(\mathrm{mmHg})\end{array}$ \\
\hline 1 & 120 & 80 \\
\hline 3 & 122 & 82 \\
\hline 5 & 125 & 86 \\
\hline 7 & 127 & 88 \\
\hline 9 & 129 & 90 \\
\hline 11 & 131 & 91 \\
\hline 13 & 132 & 92 \\
\hline
\end{tabular}




\section{Discussion and Conclusion}

The test was terminated early, after only 13 hours, because of the inexplicable increasing trend in pressure. Data during this period was not consistent with previous studies and an explanation for the deviation could not be determined without inspection of the system. Before taking the system apart, potential sources of error were considered. These sources included increasing fluid or gas volume, a shrinking system, the development of flow restriction within the system, or pressure transducer drift. Increasing fluid or gas volume was dismissed based on environmental conditions. The trials were conducted within a laboratory maintained at a relatively constant temperature. Therefore, the gasses and fluids should not have been heating up and expanding within the system. In addition, the reservoir was vented and should have accommodated any volumetric changes of system constituents. One might propose that the extra-luminal space within the bioreactor chamber may have acted as a closed system, and that an increase in volume within this space may have exerted additional forces upon the system. However, previous tests showed that the permeability of the ePTFE scaffolding allows for trans-mural diffusion of pressure. Accordingly, the extra-luminal space would be at equilibrium with the luminal region of the bioreactor chamber and the vented reservoir would still account for such volumetric changes. The same reasoning can be applied to system shrinkage. If for any reason the tubing, reservoir, or bioreactor chamber constricted, the vented reservoir would have allowed fluid levels to freely adapt, thereby preventing system pressurization.

The most probable source of variation was concluded to be the pressure transducer itself. To inspect the transducer without altering the rest of the system and 
risking loss of potential evidence, a hemostat was used to clamp off the tubing downstream of the pressure transducer and the two-way valve upstream was closed. The transducer was then removed and inspected. The data acquisition equipment was left running to assess potential measurement drifts during inspection. Oddly enough, once the sensor was taken out of the system it read $12.32 \mathrm{mmHg}$. The transducer was zeroed before it had been inserted into the system. Logically, once the transducer was removed from the system it should have again read zero. During this trial the transducer did not maintain the zero calibration. Water was tapped out of the transducer and dried. The pressure transducer was then checked using the pressure cuff, and the pressure cuff was inflated until it read $100 \mathrm{mmHg}$. LabChart 7 was used to record data simultaneously. When the pressure cuff read $100 \mathrm{mmHg}$, LabChart measured $112 \mathrm{mmHg}$. The transducer was zeroed using the bridge pod amplifier and then connected to the pressure cuff and inflated until the gauge read $100 \mathrm{mmHg}$. The pressure cuff gauge and data acquisition software measurements were viewed simultaneously. The transducer measurement indicated by the data acquisition software reflected that of the gauge. The gauge measured $100 \mathrm{mmHg}$ while the software measured approximately $99.8 \mathrm{mmHg}$. It was therefore safe to conclude that the transducer was still calibrated correctly. Water was tapped out of the pressure transducer because the transducer was calibrated dry and liquid must not be introduced into the pressure cuff. The sensor was reattached to the system using the luer lock connections. The hemostat was removed, the two-way valve was opened, and the pressure was rechecked. The pressure then read $95 \mathrm{mmHg}$, which was to be expected given the drop in the 54 inch water column height and a couple of trapped air bubbles - artifacts from removing and reattaching the transducer. The system was then 
primed and the pressure returned to $100 \mathrm{mmHg}$. This effectively confirmed the drift in transducer readings. It had been previously calculated that the 54 inch water column height would generate approximately $100 \mathrm{mmHg}$. If the pressure transducer did not drift, dialing down the bridge pod amplifier would have resulted in a pressure reading of 88 $\mathrm{mmHg}$ from the water column.

The pressure transducer drifted over the course of the trial for some undetermined reason. It was odd that the measurements would shift. A new transducer was therefore used in subsequents tests and the 72 hour test was attempted once again. 


\section{Chapter 13: 72 HOUR TEST II}

\section{Introduction:}

Chapter 12 described a failed attempt at conducting a 72 hour test. Over the course of the 13 hours of operation, the data acquisition software recorded an increasing pressure over time. The pressure began at 120/80 $\mathrm{mmHg}$ and ended at $132 / 92 \mathrm{mmHg}-$ an average increase of $12 \mathrm{mmHg}$. Upon system inspection and disassembly, it was concluded that the pressure transducer readings drifted and were not indicative of the true bioreactor pressures. This was attributed to a potentially faulty pressure transducer.

Once again, an extended assessment of the back pressured bioreactor design was conducted. This study was a repeat of the first attempted 72 hour test and involved implementing a new pressure transducer. The goal of this study was to use the data collected from the test to determine whether further refinements of the system were necessary.

\section{Methods and Materials:}

The bioreactor design was set up the same way as in the previous study using the water column technique. This test was conducted for 72 hours. Measurements were continuously taken every minute (See Appendix A), and data was recorded into a table manually every hour. A different pressure transducer was included in this trial to see if the drifting measurements would be eliminated. The bioreactor system was periodically checked and monitored for drifting pressures throughout the duration of the trial. 


\section{$\underline{\text { Results: }}$}

The initial base-line pressure of the system was between 102 and $103 \mathrm{mmHg}$. Once the pump was started and the testing began, the pressure wave measured 121.34/82.12 mmHg. The final pressure recorded at the end of 72 hours of testing was 122/83 mmHg. The final base-line pressure was $102.52 \mathrm{mmHg}$. The data recorded over the duration of the test is listed below in Tables 13.1 and 13.2.

Figure 13.1 illustrates an example of the measured waveform recorded 15 hours into the study. In the previous experiment, the pressure drifted higher continually for 13 hours of operation. The zeroed calibration setting of the transducer was found to have shifted by $12 \mathrm{mmHg}$ - thus an inaccurate indication of the true pressure within the system. Even with the new transducer used in this study, a similar measurement drifting pattern occurred. Over the 72 hour period, the bioreactor was periodically checked. On 4 separate occasions a system intervention was conducted. The interventions occurred at 16, 20, 48, and 65 hours.

The initial base-line pressure measured was between 102 and $103 \mathrm{mmHg}$. This meant, with the normal protocol, the expected pressure wave would be around 122/182 mmHg. Once the pump had been started, the pressure wave measured 121.34/82.12 $\mathrm{mmHg}-$ close to the expected value. Over the following 16 hours, the pressures increased until reaching nearly $130 \mathrm{mmHg}$. At 16 hours the pump was shut down and a hemostat was used to clamp off the downstream tubing connecting to the pressure transducer. The two-way valve upstream of the transducer was also closed (Figure 13.2). As in previous experiments, this allowed the pressure transducer to be removed from the system without excessive fluid loss. 


\begin{tabular}{|c|c|c|c|c|c|c|c|c|}
\hline \multirow[b]{2}{*}{$\mathrm{Hr}$} & Systole & Diastole & \multirow[b]{2}{*}{$\mathrm{Hr}$} & \multirow{2}{*}{$\begin{array}{l}\text { Systole } \\
(\mathrm{mmHg})\end{array}$} & \multirow{2}{*}{$\frac{\text { Diastole }}{(\mathrm{mmHg})}$} & \multirow[b]{2}{*}{$\mathrm{Hr}$} & \multirow{2}{*}{$\frac{\text { Systole }}{(\mathrm{mmHg})}$} & \multirow{2}{*}{$\frac{\text { Diastole }}{(\mathrm{mmHg})}$} \\
\hline & $(\mathrm{mmHg})$ & $(\mathrm{mmHg})$ & & & & & & \\
\hline 1 & 122.93 & 83.17 & 25 & 122.47 & 81.92 & 49 & 123.05 & 82.95 \\
\hline 2 & 122.68 & 83.14 & 26 & 122.94 & 82.25 & 50 & 123.38 & 82.42 \\
\hline 3 & 123.41 & 86.83 & 27 & 122.56 & 82.61 & 51 & 122.96 & 83.73 \\
\hline 4 & 124.53 & 84.33 & 28 & 122.81 & 82.80 & 52 & 123.54 & 83.16 \\
\hline 5 & 125.03 & 87.15 & 29 & 121.85 & 83.98 & 53 & 123.87 & 82.67 \\
\hline 6 & 125.32 & 88.45 & 30 & 123.02 & 84.20 & 54 & 123.76 & 82.74 \\
\hline 7 & 126.09 & 87.62 & 31 & 123.57 & 85.54 & 55 & 123.78 & 82.65 \\
\hline 8 & 126.14 & 87.66 & 32 & 124.34 & 84.99 & 56 & 123.98 & 82.48 \\
\hline 9 & 126.37 & 89.06 & 33 & 124.98 & 84.35 & 57 & 123.02 & 83.69 \\
\hline 10 & 126.36 & 88.07 & 34 & 125.61 & 84.60 & 58 & 123.01 & 84.15 \\
\hline 11 & 126.51 & 87.86 & 35 & 126.07 & 84.05 & 59 & 122.98 & 84.12 \\
\hline 12 & 126.73 & 87.52 & 36 & 126.22 & 83.42 & 60 & 123.80 & 82.68 \\
\hline 13 & 127.00 & 87.55 & 37 & 126.19 & 84.63 & 61 & 123.92 & 82.54 \\
\hline 14 & 126.88 & 88.14 & 38 & 127.20 & 85.53 & 62 & 124.31 & 82.35 \\
\hline 15 & 127.03 & 86.87 & 39 & 128.85 & 85.86 & 63 & 124.32 & 83.12 \\
\hline$* 16$ & 128.29 & 83.28 & 40 & 129.36 & 85.70 & 64 & 124.25 & 83.10 \\
\hline 17 & 124.31 & 82.95 & 41 & 128.65 & 86.96 & $* 65$ & 124.11 & 83.41 \\
\hline 18 & 126.88 & 81.16 & 42 & 129.98 & 87.19 & 66 & 122.55 & 82.96 \\
\hline 19 & 127.45 & 81.66 & 43 & 129.67 & 88.52 & 67 & 122.21 & 82.76 \\
\hline$* 20$ & 127.99 & 82.22 & 44 & 130.20 & 88.73 & 68 & 122.18 & 82.62 \\
\hline 21 & 122.74 & 82.01 & 45 & 130.87 & 87.54 & 69 & 122.47 & 82.84 \\
\hline 22 & 121.71 & 81.76 & 46 & 130.27 & 89.42 & 70 & 122.39 & 82.91 \\
\hline 23 & 121.79 & 82.20 & 47 & 129.87 & 89.80 & 71 & 122.27 & 82.83 \\
\hline 24 & 121.79 & 82.28 & $* 48$ & 122.86 & 83.01 & 72 & 122.33 & 82.91 \\
\hline
\end{tabular}



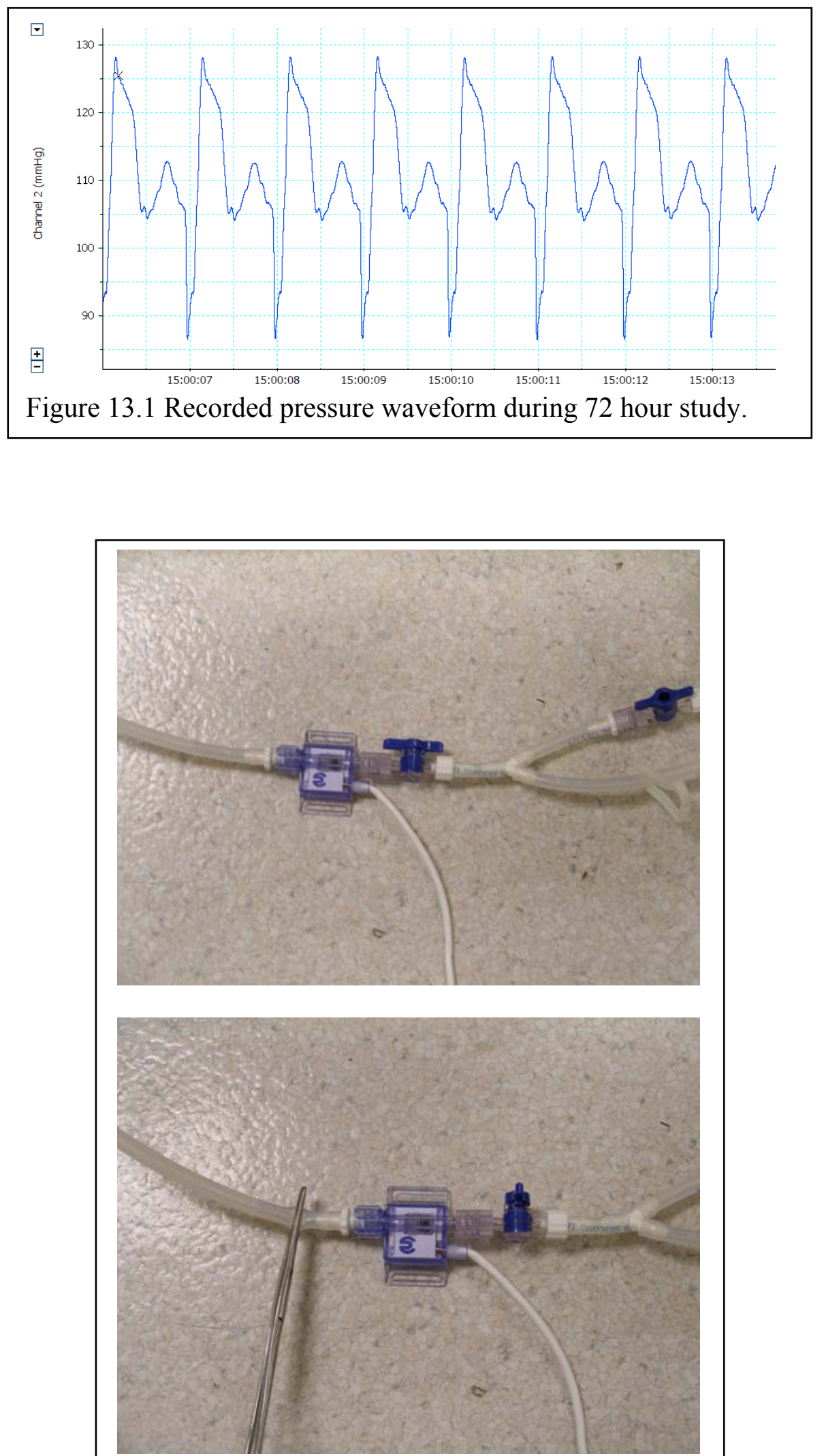

Figure 13.2 Illustration of the pressure transducer removal process during an intervention/evaluation. 
Once the pressure transducer was removed, the water within the sensor was tapped out and measurements were taken using the data acquisition software. The baseline pressure read $7 \mathrm{mmHg}$ instead of zero. The bridge-pod amplifier was used to zero the transducer. Calibration accuracy was confirmed using the pressure cuff by cross-checking gauge and LabChart pressure readings.

The pressure transducer was injected with fluid and reattached to the bioreactor. The hemostat was removed and the two-way valve was then opened. The pump was turned back on for a short period of time at a low RPM to minimize the introduction of air bubbles into the system and to facilitate the collection of any system bubbles in the reservoir air space. The pump was then turned off and base-line measurements were retaken. The base-line pressure had returned to $103 \mathrm{mmHg}$. The pump was turned on again and set back at the normal RPM. The resulting pressure wave measured 122/81 mmHg. The pressures concurred with the expected pressure waves based on initial and current base-line pressure readings and the test was continued.

At 20 hours, the bioreactor was checked again. Peak pressures had once again increased to $128 \mathrm{mmHg}$. Using the same method as before, the pressure transducer was removed from the system and pressure readings were checked. The transducer's atmospheric pressure read $4 \mathrm{mmHg}$. The bridge-pod amplifier was once again used to zero the transducer before reattaching it to the bioreactor. In-line base-line pressures read $103 \mathrm{mmHg}$ and 122/82 $\mathrm{mmHg}$ once the peristaltic pump had been turned back on.

The next check was taken 28 hours later at the 48 hour mark. For roughly 10 hours after restarting the system, the systolic pressure held relatively constant around 122 mmHg. After this point in time, the pattern drift repeated. A final peak pressure of 130 
$\mathrm{mmHg}$ was measured prior to stopping the system at hour 48 (Figure 13.3). The pressure transducer was removed and reassessed. The transducer's atmospheric pressure read 6 mmHg (Figure 13.3). The transducer was zeroed again and replaced into the system. Base-line pressure measured $103 \mathrm{mmHg}$. Once the pump was turned on, the pressure wave was roughly $122 / 83 \mathrm{mmHg}$. Once again, this was considered a reasonable pressure measurement for the system (based on the measured and previously calculated base-line pressures). The test was continued. 


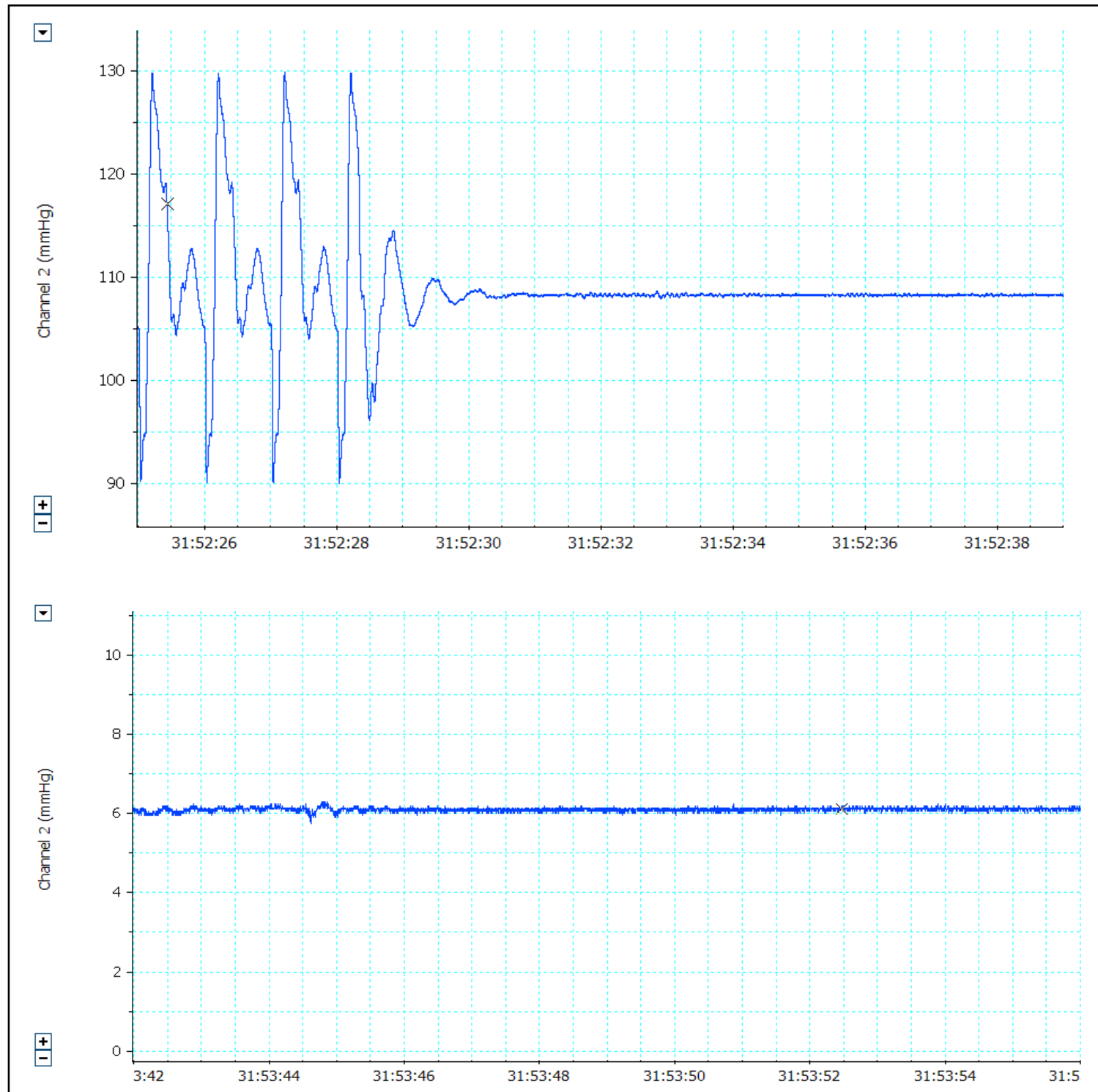

Figure 13.3 Example of drifting pressure transducer calibration. This data corresponds to the 48 hour system evaluation and intervention. Data acquisition had been temporarily shut off during the first 16 hour check, and time restarted.

The last system intervention occurred at 65 hours - a total of 17 hours later. Peak pressure readings were around $124 \mathrm{mmHg}$. This was a good sign that the pressure may have not drifted, but it was necessary to remove and evaluate the pressure transducer to ensure that this was actually true. The transducer's atmospheric pressure read 0.68 
mmHg. The transducer was placed back in-line with bioreactor without recalibration. Base-line peak pressure read $103 \mathrm{mmHg}$. When the pump was turned back on, the pressure wave oscillated between $123 \mathrm{mmHg}$ and $83 \mathrm{mmHg}$. There were no pressure drifts during this time period, and the pressure wave was consistent with expectations based on the resting base-line pressure of the system. The test continued until 72 hours at which point pressure had remained relatively constant and the system was shut down.

\section{Discussion and Conclusion}

Changing out transducers did not solve the problem with drifting measurements. The problem most likely was equipment related. Since changing the transducer did not solve the problem, the potential source would be the bridge pod amplifier. There are a number of reasons why faulty equipment could be the source of variation rather than some other phenomenon occurring with the bioreactor setup. First of all, in previous tests, the pressure fluctuation was intermittent but cyclic in nature. The pressure would increase and then drop. The pressure variation that occurred in this test demonstrated a positive bias and correlated with a calibration shift in the transducer. Furthermore, the atmospheric venting of the reservoir would compensate for any leaks or accumulations in volume. The configuration of the water column provided a constant backpressure as long as the reservoir elevation remained unchanged. The pressure variation in the tests conducted with sealed reservoirs also had erratic fluctuations in pressure, but this was in terms of cycle initiation - once the cycle began, it followed a consistent pattern. The pressure trends in this current study varied in frequency, rate of change, magnitude of change, and direction (an increase) of change without a pattern outside of the drift itself. 
In the closed system water column tests, a vacuum would be generated and the pressure would drop until a consistent minimum threshold was reached. Then the system would gain pressure within an 8-10 second period and return to close initial operating conditions, thus marking the end of the cycle. In this case, pressure just increased without reaching a threshold value.

The open system is not capable of increasing pressure over time under these conditions. Just like the vented reservoir prevented the system from generating a vacuum, it would also prevent a cumulative increase in pressure. The water column height remained unchanged; therefore the base-line pressure should have remained unchanged. Increase in pressure would require an increase in volume of fluid within a closed bioreactor system. There are two potential ways this may occur. The pump could pull in air from the outside environment, or the fluid within the system could undergo thermal expansion. If the pump had pulled in air, the gas bubbles in the line would have decreased the pressure since they are compressible. When the bubbles enter the vertical portion of the system, they displace fluid and reduce the pressure because they are less dense than the fluid that would have otherwise been there to make up the water column. As a result, measurements would initially decrease as air flowed through the lines and then increase as it accumulated in the reservoir - which the vented reservoir would not allow. Even if temperature changes were to cause fluid expansion in the system, the open system would have prevented pressure fluctuation stemming from this change as well. More importantly, a transducer that had been calibrated and zeroed before being placed in the system was removed multiple times only to find the transducer no longer read zero when it should. 
Barring the odd results provided by the data acquisition equipment, the system operated well over the extended trial. At three of the four system check-ups, equipment drift was corrected and normal pressure readings were restored. While the checks were not conducted at equally spaced intervals, they were performed throughout the duration of the test. The first check was conducted slightly after 13 hours of operation because in the previous study pressure had drifted within that time period. The next system evaluation was performed 4 hours later to assess whether or not the system correction had been maintained or if readings were continuing to drift. At each intervention, restored pressure would result in a characteristic pressure wave of approximately $122 / 82 \mathrm{mmHg}$. Regrettably, the skewed measurements prevented the analysis or detection of general variation trends within the data during the drifts. The test further lends to the credibility of the water column backpressure technique in that the system is durable, consistent, there are no detectable leaks or losses arising from the bioreactor chamber. Even if such variation was actually occurring, it would still be within physiologic ranges.

The water column vented reservoir technique for back-pressuring the system is an effective means of establishing desired pressures within the system for extended periods of time. While equipment problems may have skewed data during the study, the periodic system checks confirmed consistent pressure. The Chapter 11 study, which was conducted using the vented reservoir for 18 hours, illustrated how well the water column method works for back-pressuring the system. The longest duration at which a relatively constant pressure wave was maintained was 24 hours ( 48 through 72 hours). Even so, the pressure was consistently within the normal range for human blood pressure throughout all of the water column trials. It seems reasonable to presume that the stability of the 
pressure measurements observed in the 18 hour study and at the end of the 72 hour study would be characteristic of all the results in this study if the data was adjusted to compensate for the calibration drift. The restoration of expected pressures in the system was achieved several times during the 72 hour test by correcting the transducer zero calibration - no other changes were made.

Careful consideration was given the potential causes of the drifting pressure transducer measurements taken by the data acquisition equipment. As mentioned previously, the pressure transducer itself was ruled out because the drift had been observed in two trials with different transducers. The bridge pod amplifier became the next likely cause. The amplifier can be manually adjusted to amplify the recorded signal. Perhaps, manually increasing or decreasing signal amplification could impact the amplifier's sensitivity to drifting. This explanation would fit the trends in the data measurements because they increase in magnitude. The amplitude of the pressure wave remains relatively constant while the entire wave shifts upward. To test the hypothesis, trials can be conducted with the same setup from this experiment but with the bridge pod amplifier's intensity at different levels. The goal would be to observe a threshold amplifier intensity that denotes the point at which drift would no longer occur.

In summary, the inclusion of the new bioreactor chamber and water column in the bioreactor system effectively provided a means to accurately create a physiologic pressure wave and maintain it indefinitely. The drifting pressures may seem contrary to this conclusion, but the expected pressure measurements were reproduced each time the equipment had been recalibrated for the system. Now that an effective technique for back-pressuring the system has been developed, the modifications made to the system 
must be evaluated to ensure flow patterns have not been drastically altered. Changes in flow can potentially influence fluid velocity and wall shear stresses within the system. To estimate the generated flow conditions, computational modeling of the bioreactor system will be performed in the next chapter. 


\section{Chapter 14: INFLUENCE OF BIOREACTOR GEOMETRIES ON SCAFFOLD WALL SHEAR STRESS DISTRIBUTION}

\section{Introduction}

The previous chapters explored designing a new bioreactor chamber and developing a backpressure technique in order to accurately generate physiologic pressure waves accurately and consistently. However, this work did not consider the impact the system modifications may have had on flow conditions. Therefore, the purpose of this final study was to assess the potential effect bioreactor geometries may have upon fluid flow conditions within the bioreactor chamber. As mentioned previously, tissue engineering relies on controlling physiologic conditions. There are a variety of environmental factors that play a significant role in cell proliferation, differentiation, and phenotypic expression. Such factors include temperature, $\mathrm{CO} 2$ levels, $\mathrm{O} 2$ levels, $\mathrm{pH}$, pressure, growth factors, cell signaling, and mechanical stimulation (40). Cyclic strain is crucial to stimulate smooth muscle cells within the tunica media to grow, differentiate, and develop properties similar to native tissue $(46 ; 49)$. The tunica intima is composed of a monolayer of endothelial cells which align, directionally, with blood flow $(43,54)$. Endothelial cells require stimulation from wall shear stress in order for them to adhere to the scaffold, differentiate, and align properly $(37,43,54)$. Therefore, in addition to the focus on pressure described throughout this thesis, it was also necessary to consider implications of the new system for shear stress.

The newly designed bioreactor (Figure 14.1) has a couple geometries that may adversely affect the shear stress distribution within the scaffold region of the bioreactor chamber. The tubing connecting to each side of the bioreactor attaches at a swooping $90^{\circ}$ 
bend, and the luer lock barbs supporting the scaffold are undersized (Figure 14.2). Since shear stress is crucial to endothelial cell development, it was worth investigating the influence the new bioreactor geometries may have on flow conditions in addition to the scaffold/fitting interfaces. If problems were detected, they could be eliminated and lab research could progress. This would also be the first computational model created and applied directly to the bioreactor system. Previous characterizations of flow conditions were limited to hand calculations.

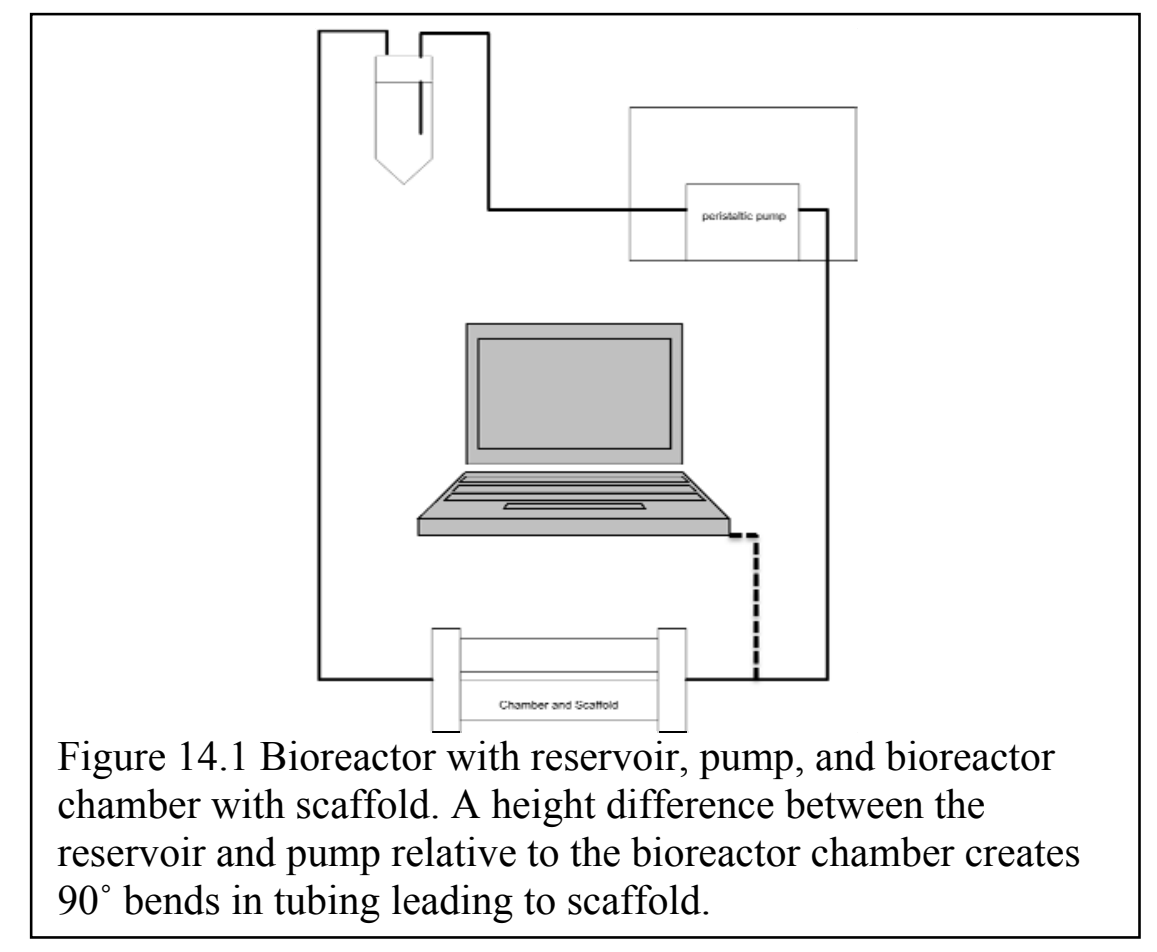




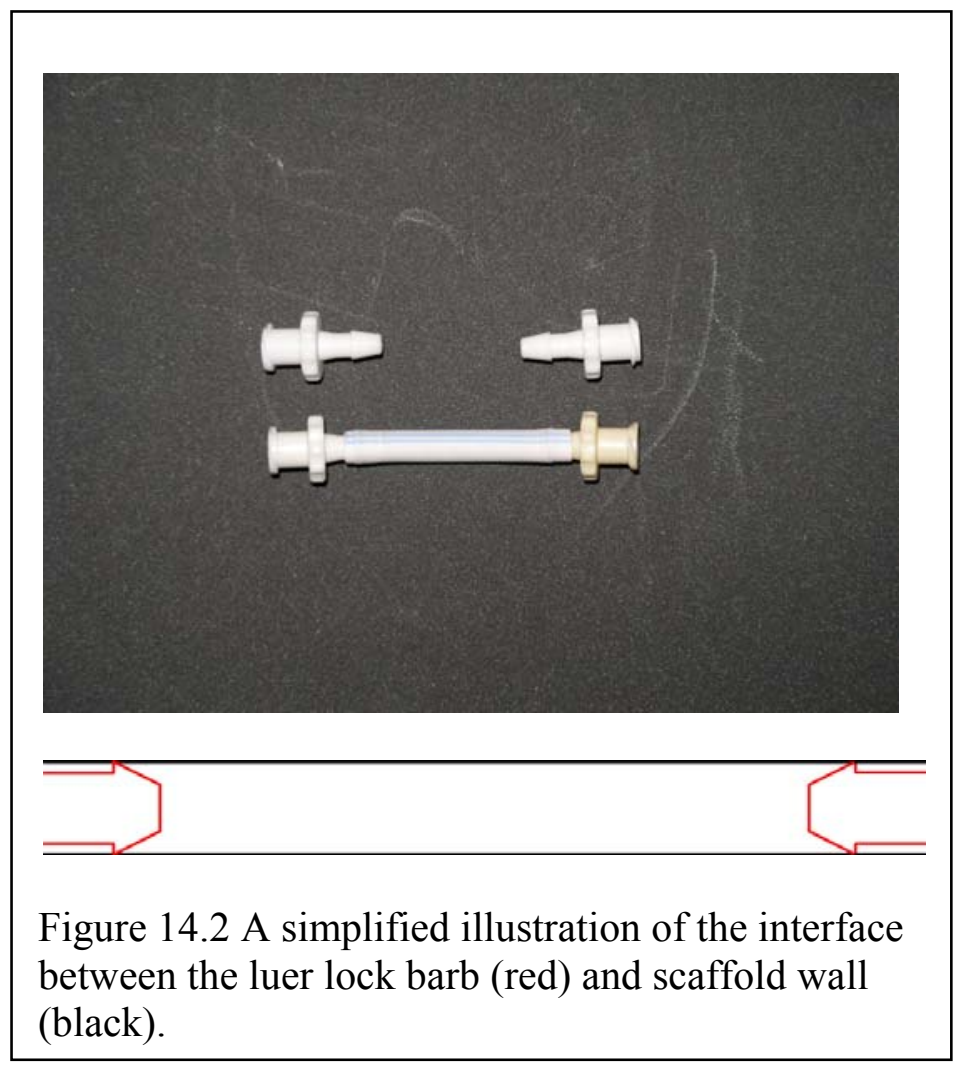

\section{Methods and Materials}

In order to properly model the current bioreactor system, part specification sheets were consulted and a series of measurements were taken with digital calipers for confirmation. Dimensions of interest included both the length and inner-diameter $(3 \times 0.4$ $\mathrm{cm})$ of the ePTFE scaffold, the length and inner-diameter $(2.54 \times 0.3 \mathrm{~cm})$ of the luer lock fittings supporting the scaffold, and the inner-diameter $(0.3 \mathrm{~cm})$ of the silicone tubing connecting to the luer lock fittings at $90^{\circ}$ angles. The scaffold length was based on the gap measurement between the tips of the inserted barbs. The surrounding bioreactor chamber was removed from the analysis because there was no trans-mural flow during normal operation. In addition, the primary focus was on the fluid dynamics within the scaffold. Using the recorded measurements, a three-dimensional model was generated 
using SolidWorks that accounted for the luer lock barb and scaffold diameter discrepancies as well as the angled tubing leading to the scaffold.

COMSOL software was used to perform the computational modeling necessary to characterize the velocity profiles and wall shear stresses within the system. The SolidWorks model of the abbreviated bioreactor was imported into the COMSOL program, and the physics model applied to the bioreactor design was the $3 \mathrm{D}$ transient incompressible Navier-Stokes model for laminar flow. Next, the density and dynamic viscosity of the fluid perfused through the system were set to $1000 \mathrm{~kg} / \mathrm{m}^{3}$ and $0.001 \mathrm{~Pa} \cdot \mathrm{s}$, respectively. After completing this step, the boundary conditions were defined for the system. The inlet and outlet corresponded with the angled silicone tubing ends at each side of the abbreviated bioreactor. All of the remaining walls were defined by the "noslip" condition. The outlet boundary condition was set so the pressure was zero. This was not indicative of the true base-line pressure within the system since it did not account for the 5333 Pa gauge pressure generated by the height differential $(\mathrm{P}=\rho \mathrm{gh})$ between the scaffold and the reservoir. However, this was acceptable because wall shear stress $\left(\tau_{\mathrm{w}}\right)$ is dependent on volumetric flowrate (Q) in Equation 14.1 below.

$$
\begin{aligned}
& \tau_{\mathrm{w}}=(4 \cdot \mu \cdot \mathrm{Q}) /\left(\pi \cdot \mathrm{R}^{3}\right) \\
& \mathrm{Q}=\left(\pi \cdot \mathrm{R}^{4} \cdot \Delta \mathrm{P}\right) /(8 \cdot \mu \cdot \mathrm{L})
\end{aligned}
$$

The volumetric flowrate is, in turn, dependent on change in pressure $(\Delta \mathrm{P})$ in Equation 14.2 (15). In effect, the base-line pressure would not affect velocity or wall shear stress, but the change in pressure would. The inlet boundary condition was more complex and accounted for the pulsatile nature of the bioreactor. Based on the specifications of the 
peristaltic pump, the volumetric flowrate for any given pump speed was known. It is typical to use a flowrate of $15 \mathrm{ml} / \mathrm{min}$ in the BVM Lab (12). Based on the Navier-Stokes equation for laminar flow in a pipe, the maximum velocity of fluid at the center of a pipe is twice the average velocity (16). Coupling this law with the flowrate equation, $Q=$ $\mathrm{V}_{\mathrm{ave}} \mathrm{A}_{\mathrm{c}}$, allowed the maximum fluid velocity within the scaffold to be determined. With this information, a sine wave was manipulated to model the cyclic pulses of flow (Equation 14.3).

$$
\mathrm{V}_{\mathrm{wave}}=\mathrm{V}_{\mathrm{ave}} * \sin (2 \pi * \mathrm{t}+(3 \pi / 2))+\text { Vave }
$$

The magnitude of the wave was set to $\mathrm{V}_{\text {ave }}(.0352 \mathrm{~m} / \mathrm{s})$ and shifted up by $\mathrm{V}_{\text {ave }}$ so that there was no negative velocity. In previous studies, fluid perfused through the system appeared to incrementally progress. To account for this, the sine wave was not shifted up any higher than $\mathrm{V}_{\text {ave }}$. This resulted in the lowest point of the wave being equal to zero and thus represented the very brief pauses that appeared to be generated by the pump. The wave velocity appropriately defines the velocities within the bioreactor since the peak velocity was equal to $V_{\max }$ (which is equal to twice $V_{\text {ave }}$ ), and mean velocity was equal to $V_{\text {ave. }}$. The frequency of the sine wave was set at $2 \pi$ to model a single pulse for every second which is equivalent to 60 pulses in a minute. The 60 pulses a minute model the healthy heart beat of a person and the estimated pulses of the peristaltic pump used at 20

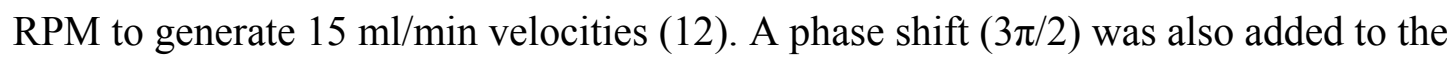
wave so that when time was equal to zero the velocity was also equal to zero and correlated to not having any fluid flow before the pump was turned on. 
The bioreactor model was meshed once and analyzed with COMSOL. The analysis provided transient data velocity profiles, and wall shear stresses. The velocity profiles depict how the bioreactor geometries influence the fluid flow within the system and where high or low wall shear stresses may occur. The wall shear stresses are used to assess the uniformity of the stress distribution along the surface of the scaffold based on flow parameters established in previous chapters.

\section{$\underline{\text { Results }}$}

The simulation was run for 60 seconds and data was gathered describing fluid velocity and wall shear stress within the simplified system model. While data was recorded for every 0.1 seconds, the velocity profile and wall shear stress measurements taken during peak velocity were the focus of analysis because they represented the upper limit of the bioreactor's capabilities under the preset conditions. If uniform wall shear stress was not achieved during peak velocity, it would never occur. Figure 14.3, below, illustrates the velocity profile of the fluid flow within the scaffold model at peak velocity. The figure shows a longitudinal, center-line cross section of the model. 


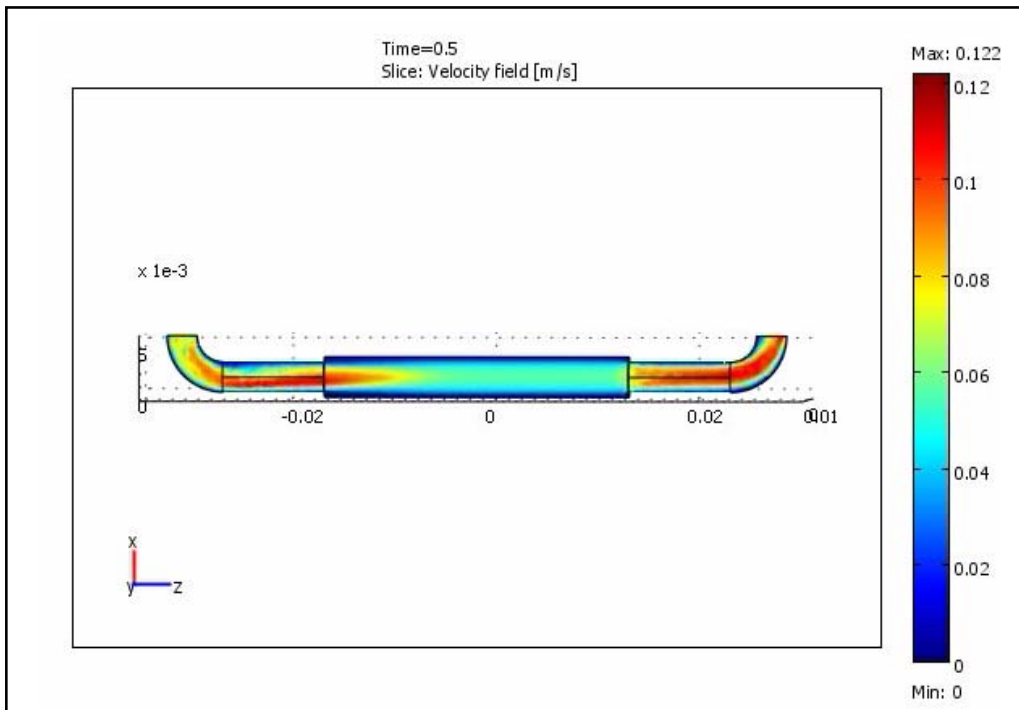

Figure 14.3 Fluid velocity profile at time 0.5 seconds.

The maximum velocity at this time point was $0.122 \mathrm{~m} / \mathrm{s}$, and the minimum velocity was zero as dictated by the no-slip boundary condition. The wall shear stress for the model was slightly more difficult to visualize as the shades of colors blend together. Figure 14.4 has two pictures illustrating the non-uniform distribution of shear stress along the wall of the scaffold. The maximum wall shear stress achieved within the scaffold region of the model was 2 dynes $/ \mathrm{cm}^{2}$ during peak velocity. There existed two transition regions where the colors changed from green to yellow and yellow to orange along the radial edge of the cross sections. This indicated that the wall shear stress increased along the length of the scaffold going from 0-2 dynes $/ \mathrm{cm}^{2}$. Approximately one third of the scaffold does not receive uniform shear stresses. 


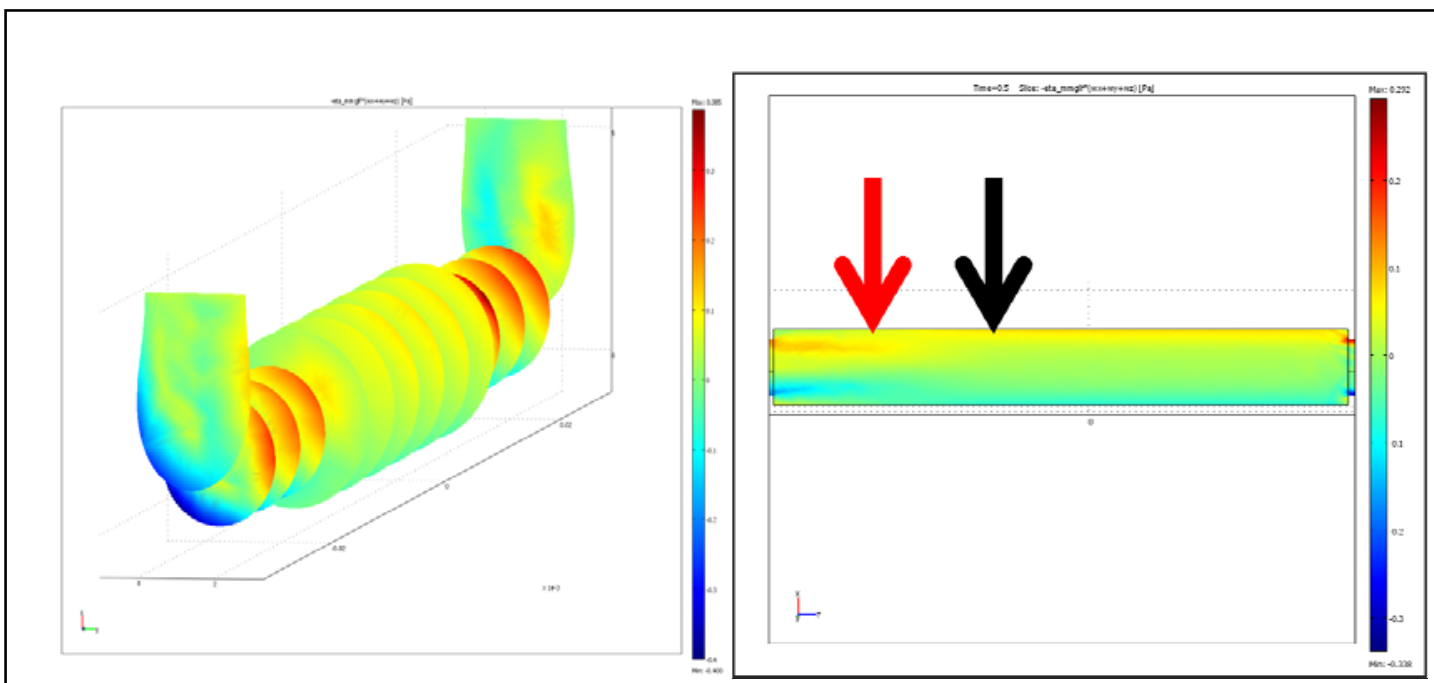

Figure 14.4 Radial cross sections of shear stress (left). Longitudinal cross section of shear stress (right). The red arrow signifies the transition from green to yellow. The black arrow signifies the transition from yellow to orange.

\section{Discussion and Conclusion}

The COMSOL computational model of the abbreviated bioreactor system appeared to be fairly accurate and a good estimate of the fluid dynamics and flow conditions within the bioreactor and corresponding small diameter arteries. The recorded maximum velocity was $12.2 \mathrm{~cm} / \mathrm{s}(0.122 \mathrm{~m} / \mathrm{s})$. This corresponds well with measured velocities of the brachial artery found in literature (31). A study performed on the effects of age on brachial artery myogenic responses in humans recorded mean blood velocities in the brachial artery of $5.83 \pm 0.32 \mathrm{~cm} / \mathrm{s}$ for young subjects and $7.44 \pm 0.74 \mathrm{~cm} / \mathrm{s}$ for older subjects (31). The mean blood velocities are equivalent to an average velocity. Applying the relationship between average velocity and max velocity to their results for young subjects equates to $11.66 \pm .64 \mathrm{~cm} / \mathrm{s}$ and the recorded maximum velocity from the simulation fell within that range. In addition, as was to be expected, the calculated average velocity determined from the volumetric flowrate of $15 \mathrm{ml} / \mathrm{min}$ also fell within 
the range of young and old subjects at $7.06 \mathrm{~cm} / \mathrm{s}$. Accordingly, the model reasonably characterized the flow conditions within the bioreactor as well as the brachial artery.

The wall shear stresses estimated by the computational simulation showed that the inlet transition from the luer lock barb to the scaffold generated a non uniform stress distribution. The maximum measured wall stress of 2 dynes $/ \mathrm{cm}^{2}$ was low, but this was expected. The media used within the bioreactor has a low viscosity that is less than blood, and viscosity plays a significant role in determining wall shear stress as can be seen in the above equation (Eqn. 14.2). Approximately 6-10 dynes $/ \mathrm{cm}^{2}$ are required for proper endothelial cell alignment (37). Figure 14.5 (below) shows the non uniform distribution of shear stress on the scaffold wall as it relates to the velocity profile within the system. 


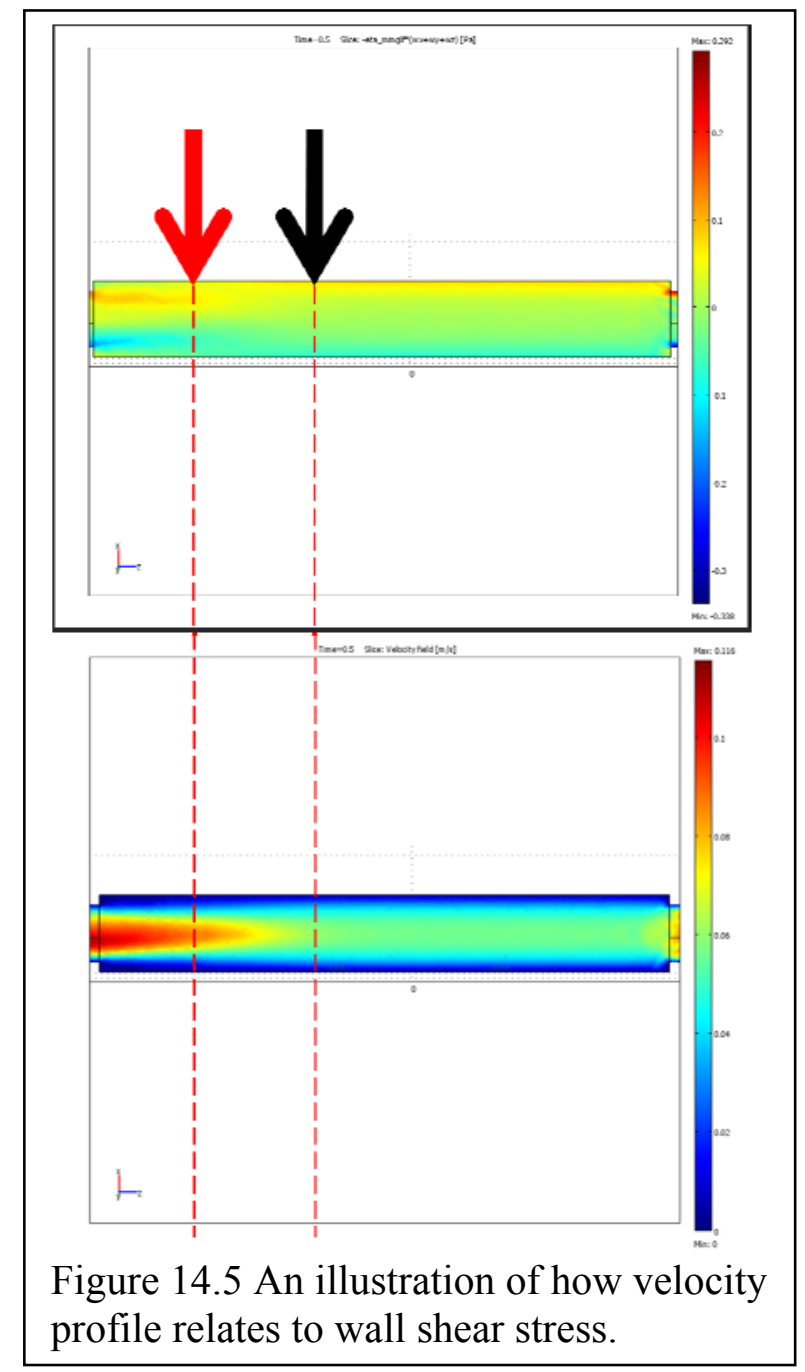

It can be seen that the smaller inlet diameter shields the proximal scaffold wall until the velocity profile fans out to the width of the scaffold. The scaffold is $3 \mathrm{~cm}$ long and approximately $1 \mathrm{~cm}$ of the length received a wall shear stress significantly less than the recorded max of 2 dynes $/ \mathrm{cm}^{2}$. The angled inlet and outlet have a negligible effect upon the shear stress as illustrated by the symmetrical and centered nature of the velocity profile. An example of a case where the angled inlet has an effect on velocity profile is shown in Figure 14.6. 


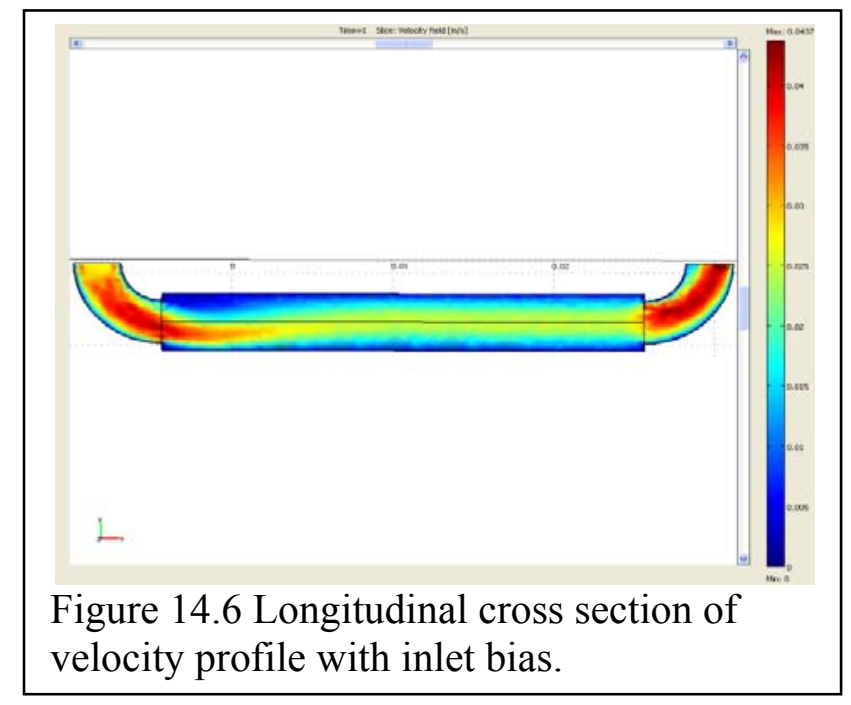

Since the geometries leading to the scaffold in the design did not have a noticeable effect upon the velocity profile or corresponding wall shear stress, only the barb and scaffold interface would need to be resolved to allow for a uniform shear stress distribution. Extending the length of the scaffold is not a reasonable solution because ePTFE is expensive and it would be a waste of resources. A simple solution would be to use a larger diameter barb that would stretch the scaffold slightly and provide a more even transition from the fitting to the scaffold.

Limitations of this model stem from the governing equation used to estimate the inlet boundary conditions. From the Navier-Stokes equation, the relationship between the maximum and minimum velocities can be derived for a parabolic velocity profile for fully developed laminar pipe flow. The fluid dynamics and model do not meet these requirements since flow within the system is pulsatile and the pipe has varying geometries and diameters. The model is also limited in that the mesh density is low because the operating computer did not have enough memory to complete the simulation with higher densities. Importing the bioreactor assembly from SolidWorks into 
COMSOL prevented further modifications to the geometry. As a result, the model could not be simplified and further meshed. Future work would include implementing a volumetric flowrate equation that more accurately accounted for the pulsatile nature of the flow conditions and recreating the bioreactor model within COMSOL. Creating the model within COMSOL would allow for geometry simplifications and greater mesh densities to be applied. It would also be useful to implement mixed boundary equations to further define environmental conditions.

The computational model can help predict the necessary viscosity to generate the ideal wall shear stresses. Dextran is used to alter the rheological properties of media within bioreactors to generate more physiologic conditions. The dextran increases fluid viscosity and as a result increases the wall shear stress within the system. Marc Dawson worked with increasing the viscosity of the bioreactor system to help generate ideal wall shear stresses (12). Dextran is made up of glucose and, accordingly, raised suspicions of its influence upon the endothelial cells in various concentrations. The tonicity of a solution can greatly affect the viability of a cell. The concern is that if the solute concentration becomes too high as a result of adding dextran to the media, a hyperosmotic solution would be created. This would result in the net movement of water out of the endothelial cells in contact with the media and kill the cells. Rouleau et al. studied the effect of dextran concentrations upon endothelial cells for this very reason. Dextran was found to increase endothelial cell viability, decrease their attachment to the surfaces of culture plates for concentrations up to $17.5 \%$ (45), and was able to generate viscosities near $20 \mathrm{cP}$. With the ability to produce such high viscosities and not negatively impact endothelial cell viability, increased dextran concentrations can be used 
within the bioreactor system. It is important to note that while viability was nearly unaffected by the $20 \%$ concentration of dextran, it did elicit a change in phenotypic expression.

The gathered data and the computational model are extremely useful for the Cal Poly BVM lab. With these results, the bioreactor can be modified so that wall shear stresses are uniformly distributed across the scaffold. Also, any design modifications or proposed changes in conditions can be assessed before they are actually implemented using the computational model. For example, geometries can be assessed for their impact on flow, or viscosity can be manipulated until the desired wall shear stress is achieved. Overall, the work in this chapter verified that the system modifications made to back-pressure the bioreactor did not have a significant impact upon the flow conditions within the bioreactor chamber. The computational model did, however, indicate a potential issue with the fit of the luer lock barbs and the ePTFE scaffolding. The discrepancy between the inner diameters of the two parts generated a region of nonuniform wall shear stress. As a result, modifications can be made to minimize the transition between the fittings and the scaffold to alleviate the problem. In addition, the computational model can be used to guide future work. 


\section{Chapter 15: DISCUSSION AND CONCLUSION}

In summary, the goal of this thesis was to improve physiologic pressure conditions within an in vitro blood vessel bioreactor system intended to assess intravascular devices. This goal was significant in that it could potentially contribute towards developing more complex tissue engineered blood vessels that include a tunica media. The newly designed bioreactor chamber can be rotated $360^{\circ}$. This will prevent cell sedimentation and justify the development of an automatic rotary system for the chambers.

There were 4 specific aims that comprised this project, and each will be summarized in turn. The first aim was to design a new bioreactor chamber that could withstand backpressures. This was accomplished by designing a more robust bioreactor chamber capable of administering elevated compressive forces to sealed edges, and by appropriate material selection of the silicone end plates that functioned as gaskets. The outcome was a bioreactor chamber that could withstand pressures $0-1000 \mathrm{mmHg}$.

The second aim was to establish a realistic base-line pressure to mimic systolic/diastolic conditions. This was accomplished by assessing various backpressure designs through a variety of trials. Results from this work led to the selection of the water column technique for back-pressuring the bioreactor system. Refinements in the design resulted in accurate and consistent pressure waves within the physiologic range.

The third aim was to characterize flow conditions through computational modeling. This was addressed in Chapter 14 and was accomplished by creating a simplified physical model of the bioreactor system in SolidWorks and importing the design into COMSOL. COMSOL was then used to set boundary conditions, flow 
parameters, and fluid properties in order to accurately simulate the operational flow conditions of the bioreactor. Results from this work indicated that the system modifications made to backpressure the bioreactor did not have a significant impact upon the flow conditions. However, the model did indicate that the wall shear stress within the bioreactor chamber may be non-uniform due to the interface between the luer lock fittings and ePTFE scaffolding.

The fourth aim was to propose a methodology to prevent cell sedimentation. This is addressed in Appendix D of this thesis with a suggested design of a rotary rack for the bioreactor chambers. The design includes a conceptual model generated in SolidWorks, specified parts necessary to accomplish task, and a proposed method of operation. The design is simple and scalable. The rack will allow the bioreactor chambers to be continually rotated automatically without laboratory assistant aid. This will free up laboratory assistants to perform other work and generate uniform cell dispersion across the bioreactor scaffold.

Overall, this work led to the development of a durable, reliable system that will provide the desired operating pressures and support further experimentation and optimization. This work is similar to some of the bioreactor systems researched for this project. For example, this system incorporated a vented reservoir. Chen et al. used vented reservoirs in order to allow media within the bioreactor system to exchange gases with the $\mathrm{CO} 2$ within the incubator (9). This allowed the system to maintain the correct media $\mathrm{pH}$ level. In the recently designed bioreactor system, the vented reservoirs allow the system to adapt to dynamic volumetric conditions. In another study, performed by Engbers-Buijtenhuijs et al., a bioreactor system using a peristaltic pump was back- 
pressured to $100 \mathrm{mmHg}$ (14). The newly designed bioreactor is also back-pressured to $100 \mathrm{mmHg}$. The systems are different in how the backpressure was established. In Engbers-Buijtenhuijs' trial, the system reservoir was pressurized - likely by compressed gas and a pressure regulator. In the new bioreactor system, a height differential was used to backpressure the bioreactor chamber to $100 \mathrm{mmHg}$.

Although significant progress was made through the accomplishments described in this thesis, there were several limitations and areas for improvement that were noted throughout the project. These areas for future work will be addressed below.

\section{Future Work}

There are several modifications that could readily be made to the recently designed bioreactor system to improve functionality while maintaining the desired pressure performance. First, there is an issue of potential contamination with a vented reservoir. Even in an aseptic incubator environment, the bioreactor cannot be open to the environment while maintaining sterility. To address this, the vented reservoir could be fitted with a purifying 0.22 micron filter which could easily be attached to the third panel mount in the cap using additional silicone tubing. The filter should be more than adequate to compensate for any pressure/volume fluctuation while ensuring sterile conditions.

There is also another potential solution to the problem of contamination. The filtered system is sterile, but still open to the incubator environment. The current reservoir is a hard-walled $50 \mathrm{ml}$ conical tube. The non-compliant container cannot adjust to changing pressures. If the reservoir was designed to be more compliant, it could change shape to adjust its volume and compensate for pressure fluctuations. IV style bags 
could be used to accomplish this, but would have to be configured so that the reservoir inlet returned to the airspace above the liquid level. If the inlet fed into the base of the bag, below the liquid level, fluid level heights within the bag would contribute to the base-line pressures and result in pressure variation. Another reservoir could also be designed to accomplish this task - if the IV bag or some variant is not practical. Another perk to using an IV bag would be the ability to suspend the reservoir from a rack and save space. These bags also have greater volume capacities than the current reservoirs and would not require the cap modifications or the expense of air purifying filters. The current reservoirs have sealant issues that do not withstand multiple sterilizations or uses. IV bags could be purchased already sterile.

The settings for the bioreactor reservoir height (54 inches) used in this work was based on water density at room temperature. Two main differences that must be considered during the use of the bioreactors to tissue engineer vessels are media density and environmental temperature. While a temperature/density chart for the DMEM media would be desirable for performing the necessary calculations to determine the appropriate reservoir heights, water is quite similar. Assuming that water and media have roughly the same density, a water temperature/density chart would be sufficient for providing necessary data to perform the needed calculations. A new height would be calculated based on $37^{\circ} \mathrm{C}$. Of course, if the mounting hardware for the reservoir was adjustable, the appropriate height for a given fluid and temperature can be arrived at empirically using feedback from the pressure transducer data acquisition equipment placed within the incubator. Additional detailed recommendations for future work regarding modifying the 
bioreactor chamber, back-pressuring the system, and designing a rotary bioreactor chamber rack can be found in Appendix D.

Another topic of interest for future research would be developing a more physiologic pressure waveform within the bioreactor. All of the experiments and designs conducted for this thesis were performed under the generalized definition of physiologic conditions for arterial pressure - the range of pressures and frequency of the wave. The coronary artery has a characteristic pressure wave that resembles a skewed parabola where the pressure steadily increases to a maximum and then, at a slightly slower rate, decreases back to a minimum (Figure 15.1). The pressure waves generated within the bioreactor system do not resemble the characteristic coronary artery waves very closely with regard to general shape, rates of increase and decrease, and the general smoothness of the curve. Now that constant backpressure has been achieved, efforts can be made to create a more accurate wave. Potential areas of investigation would include pumps, dampeners, and system geometry variations.

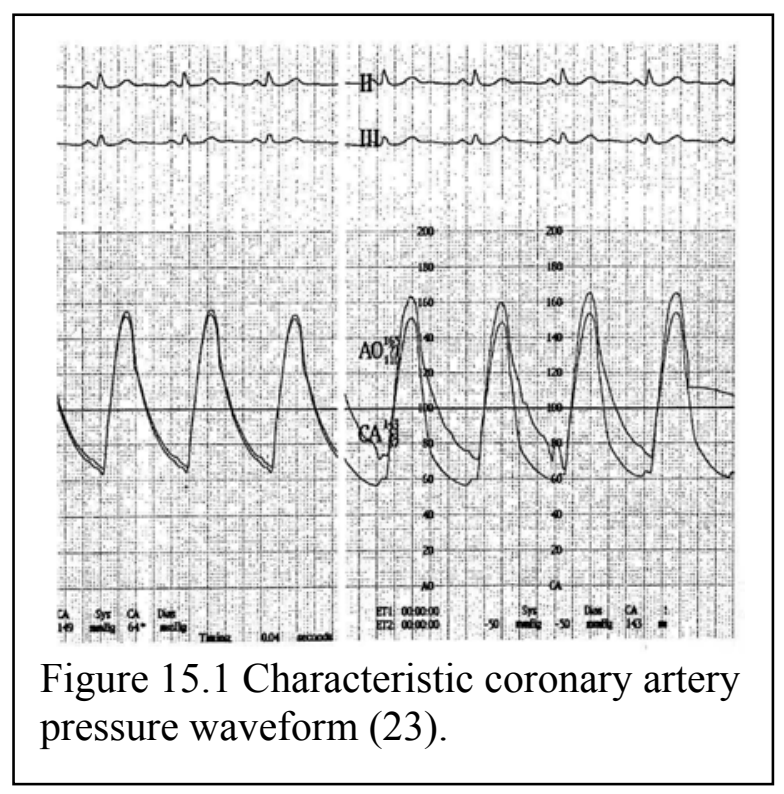




\section{Conclusion}

In summary, the ultimate goal of this thesis was to develop a method for providing accurate and sustainable pressure waves that are easily set and would replicate human physiologic pressure conditions. The study began with characterizing the current system used in the BVM laboratory. A number of system flaws made consistent pressurization of the system in the desired operating range difficult. Identifying specific concerns and resolving them led to designing a new bioreactor chamber. The chamber was redesigned and the bioreactor system modified to support physiologic pressure. Additionally, this robust and relatively user-friendly system provides a platform for future designs and optimization of the system.

After the manual pressure injection technique failed, pressure leak diagnostics confirmed the futility of patching up all of the micro leaks within the system. New methods intended to withstand system pressure-loss tendencies were employed. They ranged from simple regulators to more elaborate weighted syringe systems. Each technique was tried and assessed for potential viability. The numerous failed experiments helped increase overall knowledge and understanding of the system and allowed a practical solution to be devised.

The water column technique proved quite effective in producing consistently accurate pressure waveforms. The pressures are easily adjusted and can mimic virtually any potentially desired pressure range. It is also worth noting that the system can be applied to more than just the pulsatile bioreactor. The continuous flow perfusion system would react similarly to the back-pressuring, but there would not be discernable pulses. 
Some technical issues were encountered with the data acquisition equipment, specifically the transducer zero calibration. The experiments were well documented materials, set up, operation, observations and results were characterized in meticulous detail. The completeness of records allowed questionable or confusing results to be reviewed both during and after experimentation. Some of the results indicated system generated phenomena, such as vacuum formation, that needed to be dealt with. Other results indicated pressurization of an atmospherically vented open system - which is impossible. This led to trouble-shooting analysis and deconstruction of the system in search of evidence for or against the hypothesized explanation. As it turned out, the pressure transducer demonstrated random drift with no causal correlation. Further tests were required to confirm the impression that the system was still and had been operating at the appropriate pressures even though the transducer indicated contrary measurements.

Computational modeling performed using COMSOL provided valuable data characterizing the flow conditions within the newly designed bioreactor system. Using a height differential to establish the backpressure within the bioreactor chamber did not significantly influence the velocity profile of the fluid flowing through the scaffold. As a result, the wall shear stress distribution was not affected by that system modification. The COMSOL model also provided important information regarding the effect of the luer lock fittings and scaffold interface which resulted in a non-uniform wall shear stress distribution. The computational model will help future work when modifying system designs and flow conditions to improve the distribution and magnitude of wall shear stress. 
Ultimately and overall, the system proved to be a success. There is future work to be done assembling a bioreactor chamber rack and designing more scalable backpressuring systems, but based on the work performed in this project, the lab has a durable, reliable system that will provide the desired operating pressures and support further experimentation and optimization. The blood vessel mimic can now run under the human condition of $120 / 80 \mathrm{mmHg}$. In addition, significant progress was made in characterizing and documenting the intricacies of the pressure/flow environment. The research and progress made during this project will help direct future work and improvements to the bioreactor system necessary to create an effective assay for intravascular devices. 


\section{List of References}

1. Barbero AM, Frasch HF. Pig and guinea pig skin as surrogates for human in vitro penetration studies: A quantitative review. Toxicology in Vitro. 2009;23(1):1-13.

2. Black AF, Berthod F, L'heureux N, Germain L, Auger FA. In vitro reconstruction of a human capillary-like network in a tissue-engineered skin equivalent. FASEB J. 1998;12(13):1331-1340.

3. Blitterswijk CV, Thomsen P, Hubbell J, et al. Tissue Engineering. 1st ed. Academic Press; 2008.

4. Bode MK, Soini Y, Melkko J, et al. Increased amount of type III pN-collagen in human abdominal aortic aneurysms: evidence for impaired type III collagen fibrillogenesis. Journal of Vascular Surgery. 2000;32(6):1201-1207.

5. Browning CL, Culberson DE, Aragon IV, et al. The Developmentally Regulated Expression of Serum Response Factor Plays a Key Role in the Control of Smooth Muscle-Specific Genes. Developmental Biology. 1998;194(1):18-37.

6. Cevallos M, Riha GM, Wang X, et al. Cyclic strain induces expression of specific smooth muscle cell markers in human endothelial cells. Differentiation. 2006;74(9-10):552-561.

7. Charles S. Wallace, Sophie A. Strike, George A. Truskey. Smooth muscle cell rigidity and extracellular matrix organization influence endothelial cell spreading and adhesion formation in coculture. American Journal of Physiology - Heart and Circulatory Physiology. 2007;293(3):H1978-H1986.

8. Chen F, Zhou Y, Barnabas ST, Woodruff MA, Hutmacher DW. Engineering tubular bone constructs. J Biomech. 2007;40 Suppl 1:S73-79.

9. Chen J, Lin C. Dynamic seeding and perfusion culture of hepatocytes with galactosylated vegetable sponge in packed-bed bioreactor. J. Biosci. Bioeng. 2006;102(1):41-45.

10. Creager MA, Loscalzo J, Dzau VJ. Vascular medicine. Elsevier Health Sciences; 2006.

11. Dardik A, Liu A, Ballermann BJ. Chronic in vitro shear stress stimulates endothelial cell retention on prosthetic vascular grafts and reduces subsequent in vivo neointimal thickness. Journal of Vascular Surgery. 1999;29(1):157-167.

12. Dawson M. Implementation of Physiologic Flow Conditions in a Blood Vessel Mimic Bioreactor System for the Evaluation of Intravascular Devices. [master's thesis]. San Luis Obispo, CA: California Polytechnic State University; 2009. 
13. Ebara M, Yuki I, Murayama Y, et al. A rabbit model for efficacy evaluation of endovascular coil materials. Surgical Neurology. 2009;72(6):620-627.

14. Engbers-Buijtenhuijs P, Buttafoco L, Poot AA, et al. Biological characterisation of vascular grafts cultured in a bioreactor. Biomaterials. 2006;27(11):2390-2397.

15. Fournier RL. Basic Transport Phenomena in Biomedical Engineering. Philadelphia, Pa: Taylor \& Francis; 1999:312.

16. Fox RW, McDonald AT, Pritchard PJ. Introduction to Fluid Mechanics. Wiley; 2008 .

17. Grandas OH, Costanza MJ, Donnell RL, et al. Effect of retroviral transduction of canine microvascular endothelial cells on [beta]1 integrin subunit expression and cell retention to PTFE grafts. Cardiovascular Surgery. 2001;9(6):595-599.

18. Hahn MS, McHale MK, Wang E, Schmedlen RH, West JL. Physiologic pulsatile flow bioreactor conditioning of poly(ethylene glycol)-based tissue engineered vascular grafts. Ann Biomed Eng. 2007;35(2):190-200.

19. Hansson L, Lindholm LH, Ekbom T, et al. Randomised trial of old and new antihypertensive drugs in elderly patients: cardiovascular mortality and morbidity the Swedish Trial in Old Patients with Hypertension-2 study. The Lancet. 1999;354(9192):1751-1756

20. Hessle H, Engvall E. Type VI collagen. Studies on its localization, structure, and biosynthetic form with monoclonal antibodies. Journal of Biological Chemistry. 1984;259(6):3955.

21. Hirashima M, Kataoka H, Nishikawa S, Matsuyoshi N, Nishikawa S. Maturation of Embryonic Stem Cells Into Endothelial Cells in an In Vitro Model of Vasculogenesis. Blood. 1999;93(4):1253-1263.

22. Hoerstrup SP, Zünd G, Sodian R, et al. Tissue engineering of small caliber vascular grafts. European Journal of Cardio-Thoracic Surgery. 2001;20(1):164169.

23. Holmes D, Velappan P, Kern MJ. Coronary pressure notch: an early nonhyperemic visual indicator of the physiologic significance of a coronary artery stenosis. J Invasive Cardiol. 2004;16(11):617-620.

24. Iwasaki K, Kojima K, Kodama S, et al. Bioengineered three-layered robust and elastic artery using hemodynamically-equivalent pulsatile bioreactor. Circulation. 2008;118(14 Suppl):S52-57 
25. Jogi B, Brahmankar P, Nanda V, Prasad R. Some studies on fatigue crack growth rate of aluminum alloy 6061. Journal of Materials Processing Technology. 2008;201(1-3):380-384.

26. Kaplan AV, Baim DS, Smith JJ, et al. Medical device development: from prototype to regulatory approval. Circulation. 2004;109(25):3068-3072.

27. Laurencin CT, Nair LS. Nanotechnology and Tissue Engineering. CRC Press; 2008.

28. Lavender MD, Pang Z, Wallace CS, Niklason LE, Truskey GA. A system for the direct co-culture of endothelium on smooth muscle cells. Biomaterials. 2005;26(22):4642-4653.

29. Lehoux S, Tedgui A. Cellular mechanics and gene expression in blood vessels. $J$ Biomech. 2003;36(5):631-643.

30. Leifer S. Design and Optimization of a Blood Vessel Mimic Bioreactor System for the Evaluation of Intravascular Devices in Simple and Complex Vessel Geometries. [master's thesis]. San Luis Obispo, CA: California Polytechnic State University; 2008.

31. Lott MEJ, Herr MD, Sinoway LI. Effects of age on brachial artery myogenic responses in humans. Am. J. Physiol. Regul. Integr. Comp. Physiol. 2004;287(3):R586-591.

32. Narayanaswamy M, Wright KC, Kandarpa K. Animal Models for Atherosclerosis, Restenosis, and Endovascular Graft Research. Journal of Vascular and Interventional Radiology. 2000;11(1):5-17.

33. NetLibrary, Inc. Biology. New York: Macmillan Reference USA; 2002:4.

34. NetLibrary, Inc. The Cardiovascular System at a Glance. Oxford: Blackwell Science; 1999:125.

35. Nieponice A, Soletti L, Guan J, et al. Development of a tissue-engineered vascular graft combining a biodegradable scaffold, muscle-derived stem cells and a rotational vacuum seeding technique. Biomaterials. 2008;29(7):825-833.

36. Nilsson H, Aalkjær C. Vasomotion: Mechanisms and Physiological Importance. Molecular Interventions. 2003;3(2):79-89.

37. Ott MJ, Ballermann BJ. Shear stress-conditioned, endothelial cell-seeded vascular grafts: Improved cell adherence in response to in vitro shear stress. Surgery. 1995;117(3):334-339. 
38. Orlandi A, Ferlosio A, Gabbiani G, Spagnoli LG, Ehrlich PH. Phenotypic heterogeneity influences the behavior of rat aortic smooth muscle cells in collagen lattice. Experimental cell research. 2005;311(2):317-327.

39. Plenz GA, Deng MC, Robenek H, Vlölker W. Vascular collagens: spotlight on the role of type VIII collagen in atherogenesis. Atherosclerosis. 2003;166(1):1-11

40. Pörtner R, Nagel-Heyer S, Goepfert C, Adamietz P, Meenen NM. Bioreactor design for tissue engineering. Journal of Bioscience and Bioengineering. 2005;100(3):235-245.

41. Ranjan AK, Kumar U, Hardikar AA, et al. Human blood vessel-derived endothelial progenitors for endothelialization of small diameter vascular prosthesis. PLOS ONE. 2009;4(11):e7718.

42. Rashid ST, Salacinski HJ, Hamilton G, Seifalian AM. The use of animal models in developing the discipline of cardiovascular tissue engineering: a review.

Biomaterials. 2004;25(9):1627-1637.

43. Riha GM, Lin PH, Lumsden AB, Yao Q, Chen C. Roles of hemodynamic forces in vascular cell differentiation. Ann Biomed Eng. 2005;33(6):772-779.

44. Robert L. Mauck. Functional Tissue Engineering of Articular Cartilage Through Dynamic Loading of Chondrocyte-Seeded Agarose Gels. Journal of Biomechanical Engineering. 2000;122(3):252-260.

45. Rouleau L, Rossi J, Leask RL. Concentration and time effects of dextran exposure on endothelial cell viability, attachment, and inflammatory marker expression in vitro. Ann Biomed Eng. 2010;38(4):1451-1462.

46. Rzucidlo EM, Martin KA, Powell RJ. Regulation of vascular smooth muscle cell differentiation. Journal of Vascular Surgery. 2007;45(6, Supplement 1):A25-A32.

47. Sastri VR. Engineering Thermoplastics: Acrylics, Polycarbonates, Polyurethanes, Polyacetals, Polyesters, and Polyamides. In: Plastics in Medical Devices. Boston: William Andrew Publishing; 2010:121-173.

48. Shirota T, Yasui H, Shimokawa H, Matsuda T. Fabrication of endothelial progenitor cell (EPC)-seeded intravascular stent devices and in vitro endothelialization on hybrid vascular tissue. Biomaterials. 2003;24(13):22952302 .

49. Sterpetti AV, Cucina A, Santoro L, Cardillo B, Cavallaro A. Modulation of arterial smooth muscle cell growth by haemodynamic forces. European Journal of Vascular Surgery. 1992;6(1):16-20. 
50. Sugawara T, Kawase M, Lewén A, et al. Effect of hypotension severity on hippocampal CA1 neurons in a rat global ischemia model. Brain research. 2000;877(2):281-287.

51. Thomas AC, Campbell GR, Campbell JH. Advances in vascular tissue engineering. Cardiovascular Pathology. 12(5):271-276.

52. Thompson CA, Colon-Hernandez P, Pomerantseva I, et al. A novel pulsatile, laminar flow bioreactor for the development of tissue-engineered vascular structures. Tissue Eng. 2002;8(6):1083-1088.

53. Wang C, Cen L, Yin S, et al. A small diameter elastic blood vessel wall prepared under pulsatile conditions from polyglycolic acid mesh and smooth muscle cells differentiated from adipose-derived stem cells. Biomaterials. 2010;31(4):621-630.

54. Wang H, Yan S, Li M, et al. Shear stress induces endothelial differentiation from mouse embryo mesenchymal progenitor cells. Journal of Surgical Research. 2004;121(2):274.

55. Warnock JN, Konduri S, He Z, Yoganathan AP. Design of a sterile organ culture system for the ex vivo study of aortic heart valves. J Biomech Eng. 2005;127(5):857-861.

56. Williamson MR, Adams EF, Coombes AG. Gravity spun polycaprolactone fibres for soft tissue engineering: Interaction with fibroblasts and myoblasts in cell culture. Biomaterials. 2006;27(7):1019-1026.

57. Wise DL, NetLibrary, Inc. Biomaterials and Bioengineering Handbook. New York: Marcel Dekker; 2000:920.

58. Wu YF, Zhang J, Gu YQ, et al. Reendothelialization of tubular scaffolds by sedimentary and rotative forces: a first step toward tissue-engineered venous graft. Cardiovascular Revascularization Medicine. 9(4):238-247.

59. Yazdani SK, Tillman BW, Berry JL, Soker S, Geary RL. The fate of an endothelium layer after preconditioning. J. Vasc. Surg. 2010;51(1):174-183.

60. Zhang X, Wang X, Keshav V, et al. Dynamic culture conditions to generate silkbased tissue-engineered vascular grafts. Biomaterials. 2009;30(19):3213-3223. 
APPENDIX A: Raw Data

Contains:

1) Table A.1: 25 hour test

2) Table A.2: 31 hour test

3) Table A.3: 18 hour test

4) Table A.4: 72 hour test

Data is recorded at a minutely rate for all of the above trials

5) Table A.5: Weighted Syringe 


\begin{tabular}{|c|c|c|c|c|c|c|c|c|}
\hline $\begin{array}{l}\text { Table A.1: } 25 \text { Hou } \\
\text { Minimum }\end{array}$ & $\begin{array}{l}\text { Test } \\
\text { maximum }\end{array}$ & height & $\begin{array}{l}80.6255 \\
80.5623\end{array}$ & $\begin{array}{l}120.3077 \\
120.2748\end{array}$ & $\begin{array}{l}39.6822 \\
39.7125\end{array}$ & $\begin{array}{l}78.9256 \\
78.8936\end{array}$ & $\begin{array}{r}118.923 \\
118.9256\end{array}$ & $\begin{array}{l}39.9974 \\
40.0321\end{array}$ \\
\hline $\mathrm{mmHg}$ & $\mathrm{mmHg}$ & $\mathrm{mmHg}$ & 80.4921 & 120.267 & 39.7749 & 78.8702 & 118.904 & 40.0338 \\
\hline 79.7915 & 119.6055 & 39.814 & 80.4922 & 120.2287 & 39.7365 & 78.8589 & 118.8598 & 40.0009 \\
\hline 85.2281 & 123.9829 & 38.7548 & 80.3899 & 120.157 & 39.7671 & 78.8182 & 118.8434 & 40.0251 \\
\hline 84.4436 & 123.4373 & 38.9938 & 80.3605 & 120.1224 & 39.7619 & 78.7727 & 118.8175 & 40.0449 \\
\hline 83.8755 & 122.9706 & 39.0951 & 80.312 & 120.1146 & 39.8026 & 78.7507 & 118.7628 & 40.0122 \\
\hline 83.4919 & 122.6164 & 39.1245 & 80.2419 & 120.086 & 39.8442 & 78.7117 & 118.7671 & 40.0554 \\
\hline 83.2121 & 122.3973 & 39.1852 & 80.2107 & 120.08 & 39.8693 & 78.6996 & 118.7005 & 40.0009 \\
\hline 82.9851 & 122.1949 & 39.2098 & 80.1864 & 120.0445 & 39.858 & 78.6571 & 118.6849 & 40.0277 \\
\hline 82.7523 & 122.0734 & 39.3211 & 80.125 & 120.0038 & 39.8788 & 78.5974 & 118.6693 & 40.0719 \\
\hline 82.5982 & 121.9574 & 39.3592 & 80.0694 & 119.9583 & 39.8889 & 78.5454 & 118.6676 & 40.1221 \\
\hline 82.457 & 121.882 & 39.425 & 79.989 & 119.9137 & 39.9247 & 78.5385 & 118.6217 & 40.0832 \\
\hline 82.4458 & 121.7833 & 39.3376 & 79.9007 & 119.8808 & 39.9801 & 78.5058 & 118.6096 & 40.1039 \\
\hline 82.4579 & 121.6404 & 39.1826 & 79.8886 & 119.8384 & 39.9498 & 78.4874 & 118.5957 & 40.1083 \\
\hline 82.3315 & 121.5859 & 39.2544 & 79.8764 & 119.821 & 39.9446 & 78.4701 & 118.5515 & 40.0814 \\
\hline 82.2102 & 121.5218 & 39.3116 & 79.8357 & 119.8219 & 39.9862 & 78.4034 & 118.4918 & 40.0884 \\
\hline 82.0796 & 121.4453 & 39.3657 & 79.7959 & 119.7985 & 40.0026 & 78.4034 & 118.4684 & 40.065 \\
\hline 82.0171 & 121.4014 & 39.3843 & 79.7301 & 119.7803 & 40.0503 & 78.3523 & 118.4372 & 40.0849 \\
\hline 81.9582 & 121.3529 & 39.3947 & 79.6615 & 119.7434 & 40.0819 & 78.3004 & 118.4112 & 40.1109 \\
\hline 81.8742 & 121.3114 & 39.4371 & 79.5993 & 119.7292 & 40.1299 & 78.2233 & 118.4173 & 40.194 \\
\hline 81.8327 & 121.2464 & 39.4138 & 79.5863 & 119.6712 & 40.0849 & 78.149 & 118.3753 & 40.2263 \\
\hline 81.7521 & 121.1858 & 39.4337 & 79.5145 & 119.6366 & 40.1221 & 78.1124 & 118.3472 & 40.2347 \\
\hline 81.6716 & 121.1693 & 39.4978 & 79.4893 & 119.608 & 40.1187 & 78.1306 & 118.342 & 40.2113 \\
\hline 81.6274 & 121.1642 & 39.5367 & 79.5179 & 119.5708 & 40.0529 & 78.1696 & 118.29 & 40.1204 \\
\hline 81.6832 & 121.0832 & 39.4 & 79.6625 & 119.4902 & 39.8277 & 78.1306 & 118.2658 & 40.1351 \\
\hline 81.6889 & 121.0412 & 39.3523 & 79.6114 & 119.4565 & 39.845 & 78.0492 & 118.2476 & 40.1983 \\
\hline 81.6473 & 120.997 & 39.3497 & 79.5954 & 119.421 & 39.8255 & 77.9756 & 118.2536 & 40.278 \\
\hline 81.5893 & 120.9399 & 39.3505 & 79.5621 & 119.3984 & 39.8364 & 77.9289 & 118.2372 & 40.3083 \\
\hline 81.5426 & 120.9156 & 39.3731 & 79.5049 & 119.3811 & 39.8762 & 77.9482 & 118.1771 & 40.229 \\
\hline 81.4724 & 120.8619 & 39.3895 & 79.446 & 119.3378 & 39.8918 & 77.9938 & 118.1497 & 40.1559 \\
\hline 81.3815 & 120.8082 & 39.4268 & 79.44 & 119.3231 & 39.8831 & 77.986 & 118.1255 & 40.1394 \\
\hline 81.3183 & 120.8221 & 39.5038 & 79.4105 & 119.2668 & 39.8563 & 77.9583 & 118.1081 & 40.1498 \\
\hline 81.2715 & 120.7918 & 39.5203 & 79.33 & 119.2374 & 39.9074 & 77.9609 & 118.0285 & 40.0676 \\
\hline 81.1934 & 120.7916 & 39.5982 & 79.3006 & 119.2426 & 39.942 & 77.883 & 117.993 & 40.11 \\
\hline 81.1148 & 120.7485 & 39.6337 & 79.3023 & 119.2365 & 39.9342 & 77.7998 & 117.9912 & 40.1914 \\
\hline 81.0212 & 120.7156 & 39.6943 & 79.2801 & 119.2386 & 39.9585 & 77.8197 & 117.9549 & 40.1351 \\
\hline 80.9468 & 120.6619 & 39.7151 & 79.2538 & 119.2192 & 39.9654 & 77.7929 & 117.9159 & 40.123 \\
\hline 80.9165 & 120.6489 & 39.7324 & 79.2451 & 119.1932 & 39.9481 & 77.7376 & 117.9287 & 40.1911 \\
\hline 80.8896 & 120.616 & 39.7264 & 79.2928 & 119.1109 & 39.8182 & 77.7479 & 117.8657 & 40.1178 \\
\hline 80.8342 & 120.5684 & 39.7342 & 79.2053 & 119.0581 & 39.8528 & 77.6725 & 117.838 & 40.1654 \\
\hline 80.7563 & 120.5311 & 39.7749 & 79.136 & 119.0685 & 39.9325 & 77.6301 & 117.831 & 40.2009 \\
\hline 80.6657 & 120.5238 & 39.8581 & 79.1352 & 119.0062 & 39.871 & 77.6526 & 117.7332 & 40.0806 \\
\hline 80.6168 & 120.4826 & 39.8658 & 79.1308 & 118.9862 & 39.8554 & 77.6223 & 117.7401 & 40.1178 \\
\hline 80.5562 & 120.4714 & 39.9152 & 79.0687 & 118.9638 & 39.8951 & 77.631 & 117.7245 & 40.0936 \\
\hline 80.5614 & 120.4411 & 39.8797 & 78.9914 & 118.9499 & 39.9585 & 77.5686 & 117.7072 & 40.1386 \\
\hline 80.6342 & 120.3562 & 39.7221 & 78.9568 & 118.9455 & 39.9888 & 77.4769 & 117.7464 & 40.2695 \\
\hline
\end{tabular}




\begin{tabular}{|c|c|c|c|c|c|c|c|c|}
\hline 77.3798 & 117.7237 & 40.3438 & 76.2506 & 116.675 & 40.4244 & 75.6358 & 115.7657 & 40.1299 \\
\hline 77.3417 & 117.6856 & 40.3438 & 76.2181 & 116.6135 & 40.3955 & 75.7527 & 115.7588 & 40.0061 \\
\hline 77.3634 & 117.663 & 40.2997 & 76.1874 & 116.5832 & 40.3958 & 75.647 & 115.7371 & 40.0901 \\
\hline 77.3287 & 117.6518 & 40.323 & 76.1831 & 116.5832 & 40.4001 & 75.5741 & 115.7485 & 40.1744 \\
\hline 77.4318 & 117.5505 & 40.1187 & 76.0904 & 116.5953 & 40.5049 & 75.4877 & 115.7146 & 40.2269 \\
\hline 77.4309 & 117.5323 & 40.1013 & 76.1216 & 116.5477 & 40.4261 & 75.4565 & 115.725 & 40.2685 \\
\hline 77.3824 & 117.5262 & 40.1438 & 76.1424 & 116.5347 & 40.3923 & 75.4366 & 115.6964 & 40.2598 \\
\hline 77.3738 & 117.4557 & 40.0819 & 76.1588 & 116.5174 & 40.3585 & 75.2963 & 115.7025 & 40.4062 \\
\hline 77.3694 & 117.405 & 40.0355 & 76.1597 & 116.4377 & 40.278 & 75.3396 & 115.6549 & 40.3152 \\
\hline 77.3487 & 117.3851 & 40.0364 & 76.1749 & 116.4259 & 40.251 & 75.3465 & 115.6315 & 40.2849 \\
\hline 77.3504 & 117.3816 & 40.0312 & 76.1606 & 116.3788 & 40.2183 & 75.2989 & 115.6245 & 40.3256 \\
\hline 77.3885 & 117.3296 & 39.9411 & 76.1484 & 116.3537 & 40.2053 & 75.3469 & 115.597 & 40.2501 \\
\hline 77.4457 & 117.2985 & 39.8528 & 76.1865 & 116.3416 & 40.155 & 75.3682 & 115.5657 & 40.1975 \\
\hline 77.3565 & 117.2751 & 39.9186 & 76.2125 & 116.3121 & 40.0996 & 75.3985 & 115.5535 & 40.155 \\
\hline 77.3106 & 117.25 & 39.9394 & 76.2047 & 116.2844 & 40.0797 & 75.3786 & 115.5076 & 40.1291 \\
\hline 77.2619 & 117.2601 & 39.9982 & 76.1926 & 116.268 & 40.0754 & 75.3751 & 115.492 & 40.1169 \\
\hline 77.2222 & 117.2249 & 40.0026 & 76.145 & 116.2783 & 40.1334 & 75.4141 & 115.4808 & 40.0667 \\
\hline 77.1971 & 117.2205 & 40.0234 & 76.0806 & 116.2444 & 40.1638 & 75.4046 & 115.4418 & 40.0373 \\
\hline 77.1755 & 117.2205 & 40.0451 & 76.0263 & 116.2169 & 40.1905 & 75.3725 & 115.4695 & 40.097 \\
\hline 77.1192 & 117.2197 & 40.1005 & 75.9977 & 116.2402 & 40.2425 & 75.3389 & 115.4543 & 40.1153 \\
\hline 77.1114 & 117.1833 & 40.0719 & 76.009 & 116.2125 & 40.2035 & 75.3361 & 115.4834 & 40.1472 \\
\hline 77.0767 & 117.1781 & 40.1013 & 75.9397 & 116.164 & 40.2243 & 75.4124 & 115.4106 & 39.9983 \\
\hline 77.0153 & 117.2049 & 40.1897 & 75.8826 & 116.158 & 40.2754 & 75.4331 & 115.3899 & 39.9567 \\
\hline 76.9422 & 117.2002 & 40.258 & 75.8601 & 116.1606 & 40.3005 & 75.4297 & 115.3968 & 39.9671 \\
\hline 76.8386 & 117.1893 & 40.3507 & 75.8973 & 116.1822 & 40.2849 & 75.4184 & 115.3916 & 39.9732 \\
\hline 76.8031 & 117.1504 & 40.3473 & 75.8948 & 116.1484 & 40.2536 & 75.4011 & 115.3682 & 39.9671 \\
\hline 76.8048 & 117.1201 & 40.3152 & 75.8497 & 116.0982 & 40.2486 & 75.4323 & 115.3232 & 39.8909 \\
\hline 76.8222 & 117.088 & 40.2659 & 75.9709 & 116.0038 & 40.0329 & 75.4411 & 115.3301 & 39.8889 \\
\hline 76.8533 & 117.0343 & 40.181 & 75.9328 & 116.0004 & 40.0676 & 75.4998 & 115.2859 & 39.7861 \\
\hline 76.8525 & 117.0118 & 40.1594 & 75.8973 & 116.0333 & 40.136 & 75.4825 & 115.2435 & 39.761 \\
\hline 76.8603 & 116.9685 & 40.1083 & 75.8531 & 116.0324 & 40.1793 & 75.4245 & 115.2245 & 39.8 \\
\hline 76.8259 & 116.9174 & 40.0916 & 75.783 & 115.9865 & 40.2035 & 75.3708 & 115.1829 & 39.8121 \\
\hline 76.7962 & 116.8828 & 40.0866 & 75.7198 & 115.9839 & 40.2641 & 75.2747 & 115.1985 & 39.9238 \\
\hline 76.7096 & 116.8967 & 40.1871 & 75.745 & 115.959 & 40.214 & 75.227 & 115.2063 & 39.9792 \\
\hline 76.6749 & 116.9088 & 40.2338 & 75.6886 & 115.9597 & 40.2711 & 75.1898 & 115.2028 & 40.013 \\
\hline 76.6342 & 116.8958 & 40.2616 & 75.8176 & 115.9302 & 40.1126 & 75.1865 & 115.1962 & 40.0096 \\
\hline 76.6256 & 116.8715 & 40.246 & 75.8531 & 115.912 & 40.0589 & 75.1448 & 115.2019 & 40.0572 \\
\hline 76.5805 & 116.849 & 40.2685 & 75.8332 & 115.9224 & 40.0892 & 75.1967 & 115.2019 & 40.0052 \\
\hline 76.5572 & 116.83 & 40.2728 & 75.7795 & 115.9068 & 40.1273 & 75.2218 & 115.1881 & 39.9663 \\
\hline 76.4914 & 116.8023 & 40.3109 & 75.8133 & 115.9103 & 40.097 & 75.2348 & 115.1855 & 39.9507 \\
\hline 76.4489 & 116.7923 & 40.3435 & 75.8081 & 115.8887 & 40.0806 & 75.1967 & 115.1569 & 39.9602 \\
\hline 76.4281 & 116.7564 & 40.3282 & 75.8895 & 115.8692 & 39.9797 & 75.1387 & 115.169 & 40.0303 \\
\hline 76.3883 & 116.7538 & 40.3655 & 75.9163 & 115.8471 & 39.9307 & 75.2106 & 115.1128 & 39.9022 \\
\hline 76.2801 & 116.752 & 40.472 & 75.8938 & 115.828 & 39.9342 & 75.1883 & 115.1274 & 39.9392 \\
\hline 76.2307 & 116.746 & 40.5153 & 75.815 & 115.7934 & 39.9784 & 75.1119 & 115.0946 & 39.9827 \\
\hline 76.2082 & 116.7512 & 40.543 & 75.7336 & 115.7917 & 40.058 & 75.0755 & 115.098 & 40.0225 \\
\hline 76.1727 & 116.7261 & 40.5534 & 75.6548 & 115.7562 & 40.1013 & 75.0937 & 115.0703 & 39.9766 \\
\hline
\end{tabular}




\begin{tabular}{|c|c|c|c|c|c|c|c|c|}
\hline 75.085 & 115.0946 & 40.0096 & 74.2753 & 114.5048 & 40.2295 & 73.0539 & 113.3427 & 40.2889 \\
\hline 75.053 & 115.0617 & 40.0087 & 74.3437 & 114.4875 & 40.1438 & 73.0933 & 113.2985 & 40.2053 \\
\hline 75.0538 & 115.066 & 40.0121 & 74.3905 & 114.4061 & 40.0156 & 73.0197 & 113.2552 & 40.2356 \\
\hline 75.0443 & 115.027 & 39.9827 & 74.3602 & 114.3905 & 40.0303 & 72.9158 & 113.283 & 40.3672 \\
\hline 75.0634 & 115.0539 & 39.9905 & 74.329 & 114.4139 & 40.0849 & 72.8525 & 113.2847 & 40.4321 \\
\hline 75.0914 & 115.0446 & 39.9532 & 74.2277 & 114.2312 & 40.0035 & 72.8413 & 113.2942 & 40.4529 \\
\hline 75.1119 & 114.9837 & 39.8719 & 74.1437 & 114.1126 & 39.9689 & 72.9634 & 113.2656 & 40.3022 \\
\hline 75.1162 & 114.9898 & 39.8736 & 74.1259 & 114.0704 & 39.9444 & 73.0058 & 113.2353 & 40.2295 \\
\hline 75.1153 & 114.9681 & 39.8528 & 74.1134 & 114.058 & 39.9446 & 73.0257 & 113.2256 & 40.1999 \\
\hline 75.0651 & 114.95 & 39.8849 & 74.0484 & 114.026 & 39.9775 & 73.0993 & 113.179 & 40.0797 \\
\hline 75.0391 & 114.9387 & 39.8996 & 73.9541 & 114.0364 & 40.0823 & 73.0855 & 113.1669 & 40.0814 \\
\hline 75.0036 & 114.9162 & 39.9126 & 73.8926 & 113.9896 & 40.097 & 73.0301 & 113.1617 & 40.1317 \\
\hline 74.9101 & 114.911 & 40.0009 & 73.9497 & 113.9697 & 40.0199 & 73.0586 & 113.1098 & 40.0511 \\
\hline 74.9187 & 114.8834 & 39.9647 & 73.935 & 113.9515 & 40.0165 & 73.0699 & 113.0907 & 40.0208 \\
\hline 74.917 & 114.8893 & 39.9723 & 73.922 & 113.9437 & 40.0217 & 73.0283 & 113.0898 & 40.0615 \\
\hline 74.9317 & 114.8893 & 39.9576 & 73.8934 & 113.9585 & 40.0651 & 73.0093 & 113.089 & 40.0797 \\
\hline 74.9032 & 114.8919 & 39.9888 & 73.8908 & 113.922 & 40.0312 & 72.9703 & 113.0786 & 40.1083 \\
\hline 74.9153 & 114.8867 & 39.9714 & 73.8813 & 113.8666 & 39.9853 & 73.0063 & 113.0829 & 40.0766 \\
\hline 74.9439 & 114.8564 & 39.9126 & 73.8406 & 113.8528 & 40.0122 & 72.9686 & 113.05 & 40.0814 \\
\hline 74.9162 & 114.8278 & 39.9117 & 73.7592 & 113.8606 & 40.1013 & 72.9738 & 113.0084 & 40.0347 \\
\hline 74.8408 & 114.8287 & 39.9879 & 73.7488 & 113.8684 & 40.1195 & 72.9253 & 113.0119 & 40.0866 \\
\hline 74.8289 & 114.8165 & 39.9876 & 73.6847 & 113.8268 & 40.142 & 73.0171 & 112.9851 & 39.968 \\
\hline 74.7005 & 114.8149 & 40.1143 & 73.6631 & 113.8138 & 40.1507 & 73.0214 & 112.9851 & 39.9637 \\
\hline 74.7075 & 114.8382 & 40.1308 & 73.6987 & 113.8008 & 40.1021 & 72.9868 & 113.0171 & 40.0303 \\
\hline 74.6607 & 114.8045 & 40.1438 & 73.6614 & 113.7359 & 40.0745 & 72.9357 & 113.0275 & 40.0918 \\
\hline 74.6676 & 114.7776 & 40.11 & 73.6631 & 113.7133 & 40.0503 & 72.9191 & 113.0159 & 40.0968 \\
\hline 74.6875 & 114.7326 & 40.0451 & 73.6059 & 113.7367 & 40.1308 & 72.8803 & 112.9972 & 40.1169 \\
\hline 74.7187 & 114.6893 & 39.9706 & 73.5427 & 113.7402 & 40.1975 & 72.8898 & 112.9868 & 40.097 \\
\hline 74.73 & 114.678 & 39.9481 & 73.4613 & 113.7393 & 40.278 & 72.9573 & 112.9089 & 39.9515 \\
\hline 74.7232 & 114.6958 & 39.9726 & 73.4786 & 113.7038 & 40.2252 & 72.992 & 112.8491 & 39.8571 \\
\hline 74.6763 & 114.6841 & 40.0078 & 73.5332 & 113.6666 & 40.1334 & 72.9625 & 112.856 & 39.8935 \\
\hline 74.6278 & 114.7335 & 40.1057 & 73.4961 & 113.6537 & 40.1576 & 72.921 & 112.8612 & 39.9403 \\
\hline 74.5966 & 114.6633 & 40.0667 & 73.4639 & 113.6562 & 40.1923 & 72.8681 & 112.8136 & 39.9455 \\
\hline 74.5905 & 114.7196 & 40.1291 & 73.4665 & 113.619 & 40.1524 & 72.8275 & 112.7922 & 39.9647 \\
\hline 74.5074 & 114.7049 & 40.1975 & 73.4726 & 113.5402 & 40.0676 & 72.7737 & 112.7946 & 40.0208 \\
\hline 74.4676 & 114.7032 & 40.2356 & 73.4475 & 113.5644 & 40.1169 & 72.7616 & 112.7937 & 40.0321 \\
\hline 74.5325 & 114.6607 & 40.1282 & 73.3176 & 113.5583 & 40.2408 & 72.6906 & 112.8093 & 40.1187 \\
\hline 74.6201 & 114.5795 & 39.9594 & 73.2838 & 113.554 & 40.2702 & 72.6612 & 112.8058 & 40.1446 \\
\hline 74.5983 & 114.5629 & 39.9645 & 73.2128 & 113.5376 & 40.3248 & 72.6646 & 112.7859 & 40.1213 \\
\hline 74.5117 & 114.5862 & 40.0745 & 73.2353 & 113.5004 & 40.2651 & 72.6612 & 112.7816 & 40.1204 \\
\hline 74.4667 & 114.5724 & 40.1057 & 73.1634 & 113.4726 & 40.3092 & 72.7105 & 112.759 & 40.0485 \\
\hline 74.4009 & 114.5386 & 40.1377 & 73.192 & 113.425 & 40.233 & 72.6848 & 112.7437 & 40.059 \\
\hline 74.3792 & 114.5447 & 40.1654 & 73.1288 & 113.4224 & 40.2936 & 72.6672 & 112.7097 & 40.0425 \\
\hline 74.2866 & 114.5594 & 40.2728 & 73.1426 & 113.4155 & 40.2728 & 72.5884 & 112.714 & 40.1256 \\
\hline 74.2433 & 114.5248 & 40.2815 & 73.1357 & 113.3834 & 40.2477 & 72.6092 & 112.7054 & 40.0962 \\
\hline 74.2915 & 114.487 & 40.1955 & 73.1115 & 113.3722 & 40.2607 & 72.5953 & 112.6742 & 40.0788 \\
\hline 74.2736 & 114.5135 & 40.2399 & 73.0309 & 113.3964 & 40.3655 & 72.6187 & 112.6179 & 39.9992 \\
\hline
\end{tabular}




\begin{tabular}{|c|c|c|c|c|c|c|c|c|}
\hline 72.6083 & 112.6049 & 39.9966 & 77.5184 & 117.586 & 40.0676 & 76.2064 & 116.5788 & 40.3724 \\
\hline 72.5711 & 112.5936 & 40.0225 & 77.4465 & 117.5816 & 40.1351 & 76.1718 & 116.5234 & 40.3516 \\
\hline 72.5077 & 112.5922 & 40.0845 & 77.4353 & 117.5634 & 40.1282 & 76.1657 & 116.5174 & 40.3516 \\
\hline 72.4438 & 112.5478 & 40.1039 & 77.5201 & 117.4994 & 39.9792 & 76.138 & 116.4732 & 40.3352 \\
\hline 72.3364 & 112.5148 & 40.1784 & 77.4883 & 117.4601 & 39.9717 & 76.1536 & 116.4749 & 40.3213 \\
\hline 72.2992 & 112.501 & 40.2018 & 77.4379 & 117.4362 & 39.9983 & 76.2004 & 116.4368 & 40.2364 \\
\hline 72.3165 & 112.4672 & 40.1507 & 77.366 & 117.418 & 40.052 & 76.1485 & 116.4321 & 40.2836 \\
\hline 72.2334 & 112.456 & 40.2226 & 77.321 & 117.4041 & 40.0832 & 76.0627 & 116.4048 & 40.3421 \\
\hline 72.2282 & 112.4248 & 40.1966 & 77.2075 & 117.4162 & 40.2087 & 75.9882 & 116.4308 & 40.4425 \\
\hline 72.2576 & 112.4023 & 40.1446 & 77.1971 & 117.3972 & 40.2001 & 75.9596 & 116.4082 & 40.4486 \\
\hline 72.2329 & 112.4028 & 40.17 & 77.1504 & 117.327 & 40.1767 & 75.9034 & 116.4056 & 40.5023 \\
\hline 72.2178 & 112.3936 & 40.1758 & 77.1815 & 117.2526 & 40.071 & 75.8202 & 116.3979 & 40.5776 \\
\hline 72.2091 & 112.346 & 40.1369 & 77.1368 & 117.2222 & 40.0854 & 75.7908 & 116.3615 & 40.5707 \\
\hline 72.197 & 112.3434 & 40.1464 & 77.0932 & 117.1989 & 40.1057 & 75.8003 & 116.3286 & 40.5283 \\
\hline 72.2516 & 112.346 & 40.0944 & 77.0369 & 117.1443 & 40.1074 & 75.7459 & 116.2946 & 40.5487 \\
\hline 72.3399 & 112.3148 & 39.9749 & 77.0352 & 117.1374 & 40.1022 & 75.731 & 116.274 & 40.543 \\
\hline 72.3217 & 112.3053 & 39.9836 & 76.972 & 117.1045 & 40.1325 & 75.6877 & 116.2584 & 40.5707 \\
\hline 72.2992 & 112.2767 & 39.9775 & 76.9295 & 117.1054 & 40.1758 & 75.647 & 116.2316 & 40.5846 \\
\hline 72.2975 & 112.2499 & 39.9524 & 76.9148 & 117.0612 & 40.1464 & 75.6886 & 116.2203 & 40.5317 \\
\hline 72.2611 & 112.2399 & 39.9788 & 76.9191 & 117.0595 & 40.1403 & 75.6964 & 116.158 & 40.4616 \\
\hline 72.1667 & 112.2265 & 40.0598 & 76.8507 & 117.0447 & 40.194 & 75.8003 & 116.106 & 40.3057 \\
\hline 72.0636 & 112.2395 & 40.1758 & 76.8188 & 117.024 & 40.2052 & 75.8055 & 116.061 & 40.2555 \\
\hline 71.9918 & 112.2299 & 40.2382 & 76.8594 & 117.0006 & 40.1412 & 75.7509 & 116.0627 & 40.3118 \\
\hline 71.9961 & 112.1988 & 40.2027 & 76.8161 & 116.991 & 40.175 & 75.7177 & 116.0233 & 40.3056 \\
\hline 72.0048 & 112.1953 & 40.1905 & 76.7823 & 116.9304 & 40.1481 & 75.705 & 115.9744 & 40.2693 \\
\hline 71.9459 & 112.1832 & 40.2373 & 76.7468 & 116.9296 & 40.1827 & 75.6756 & 115.9683 & 40.2927 \\
\hline 71.8489 & 112.1685 & 40.3196 & 76.7927 & 116.8759 & 40.0832 & 75.641 & 115.9571 & 40.3161 \\
\hline 71.8303 & 112.1694 & 40.3391 & 76.739 & 116.8551 & 40.1161 & 75.6046 & 115.9181 & 40.3135 \\
\hline 71.8359 & 112.1407 & 40.3048 & 76.6897 & 116.817 & 40.1273 & 75.5648 & 115.8869 & 40.3222 \\
\hline 71.8671 & 112.1035 & 40.2364 & 76.6215 & 116.8153 & 40.1937 & 75.5847 & 115.8558 & 40.2711 \\
\hline 71.8801 & 112.0767 & 40.1966 & 76.5901 & 116.8083 & 40.2183 & 75.5526 & 115.8705 & 40.3178 \\
\hline 71.8688 & 112.0377 & 40.1689 & 76.6109 & 116.791 & 40.1802 & 75.6023 & 115.8137 & 40.2114 \\
\hline 71.8749 & 111.9944 & 40.1195 & 76.6299 & 116.7581 & 40.1282 & 75.5829 & 115.7787 & 40.1957 \\
\hline 71.816 & 112.0212 & 40.2053 & 76.5762 & 116.7512 & 40.175 & 75.5717 & 115.8159 & 40.2442 \\
\hline 71.8212 & 111.9892 & 40.168 & 76.5901 & 116.7252 & 40.1351 & 75.5336 & 115.7873 & 40.2538 \\
\hline 71.8153 & 111.9782 & 40.1629 & 76.377 & 116.7746 & 40.3975 & 75.4721 & 115.7666 & 40.2945 \\
\hline 71.7614 & 111.9996 & 40.2382 & 76.293 & 116.8066 & 40.5135 & 75.4479 & 115.7683 & 40.3204 \\
\hline 71.6844 & 112.0264 & 40.3421 & 76.3335 & 116.78 & 40.4466 & 75.4383 & 115.751 & 40.3126 \\
\hline 71.667 & 112.0204 & 40.3533 & 76.3 & 116.7451 & 40.4451 & 75.3665 & 115.7328 & 40.3663 \\
\hline 71.9138 & 112.2568 & 40.343 & 76.2948 & 116.7702 & 40.4754 & 75.3292 & 115.7291 & 40.3999 \\
\hline 77.0473 & 117.1486 & 40.1013 & 76.2835 & 116.7269 & 40.4434 & 75.3613 & 115.7007 & 40.3395 \\
\hline 77.6639 & 117.7392 & 40.0754 & 76.2757 & 116.7122 & 40.4365 & 75.3985 & 115.6652 & 40.2667 \\
\hline 77.6414 & 117.741 & 40.0996 & 76.2411 & 116.7174 & 40.4763 & 75.4124 & 115.6609 & 40.2486 \\
\hline 77.5817 & 117.7094 & 40.1277 & 76.2541 & 116.6897 & 40.4356 & 75.3682 & 115.6445 & 40.2763 \\
\hline 77.579 & 117.728 & 40.149 & 76.2766 & 116.6897 & 40.4131 & 75.4098 & 115.6531 & 40.2434 \\
\hline 77.5141 & 117.6786 & 40.1646 & 76.2947 & 116.6197 & 40.325 & 75.4894 & 115.5916 & 40.1022 \\
\hline 77.4881 & 117.6526 & 40.1646 & 76.248 & 116.5875 & 40.3395 & 75.55 & 115.5215 & 39.9714 \\
\hline
\end{tabular}




\begin{tabular}{|c|c|c|c|c|c|c|c|c|}
\hline 75.5362 & 115.5177 & 39.9814 & 74.9006 & 115.2236 & 40.323 & 74.6468 & 114.9707 & 40.3239 \\
\hline 75.5336 & 115.512 & 39.9784 & 74.9716 & 115.2045 & 40.233 & 74.6624 & 114.9811 & 40.3187 \\
\hline 75.5223 & 115.5016 & 39.9792 & 74.9628 & 115.212 & 40.2492 & 74.7049 & 114.982 & 40.2771 \\
\hline 75.5145 & 115.5024 & 39.9879 & 74.9369 & 115.1864 & 40.2494 & 74.7611 & 114.9863 & 40.2252 \\
\hline 75.4686 & 115.4678 & 39.9992 & 74.9213 & 115.1933 & 40.2719 & 74.7866 & 114.9821 & 40.1955 \\
\hline 75.3665 & 115.492 & 40.1256 & 74.9049 & 115.2054 & 40.3005 & 74.7031 & 115.0019 & 40.2988 \\
\hline 75.2851 & 115.4661 & 40.181 & 74.8971 & 115.1933 & 40.2962 & 74.6356 & 115.0218 & 40.3862 \\
\hline 75.2288 & 115.4765 & 40.2477 & 74.8997 & 115.1907 & 40.291 & 74.5559 & 115.0556 & 40.4997 \\
\hline 75.1698 & 115.486 & 40.3162 & 74.8512 & 115.1422 & 40.291 & 74.51 & 115.0547 & 40.5447 \\
\hline 75.1681 & 115.473 & 40.3048 & 74.8867 & 115.1483 & 40.2616 & 74.5005 & 115.053 & 40.5525 \\
\hline 75.2132 & 115.4349 & 40.2217 & 74.8932 & 115.1178 & 40.2246 & 74.5273 & 115.021 & 40.4936 \\
\hline 75.2825 & 115.4063 & 40.1239 & 74.8772 & 115.1015 & 40.2243 & 74.5905 & 115.001 & 40.4105 \\
\hline 75.2738 & 115.402 & 40.1282 & 74.8841 & 115.0937 & 40.2096 & 74.5849 & 114.9883 & 40.4034 \\
\hline 75.2262 & 115.3578 & 40.1317 & 74.8893 & 115.0842 & 40.1949 & 74.581 & 114.995 & 40.414 \\
\hline 75.1976 & 115.376 & 40.1784 & 74.8625 & 115.1006 & 40.2382 & 74.5879 & 115.0088 & 40.4209 \\
\hline 75.098 & 115.3803 & 40.2823 & 74.8694 & 115.0634 & 40.194 & 74.5914 & 114.9768 & 40.3854 \\
\hline 75.0791 & 115.3556 & 40.2765 & 74.8677 & 115.0669 & 40.1992 & 74.6183 & 114.9699 & 40.3516 \\
\hline 75.0599 & 115.3639 & 40.304 & 74.9101 & 115.066 & 40.1559 & 74.6841 & 114.9032 & 40.2191 \\
\hline 75.0712 & 115.3544 & 40.2832 & 74.9011 & 115.0332 & 40.1321 & 74.6832 & 114.9101 & 40.2269 \\
\hline 75.0755 & 115.3318 & 40.2564 & 74.8919 & 115.0374 & 40.1455 & 74.7005 & 114.9049 & 40.2044 \\
\hline 75.0443 & 115.3327 & 40.2884 & 74.8729 & 115.0322 & 40.1594 & 74.6624 & 114.8737 & 40.2114 \\
\hline 75.0053 & 115.3578 & 40.3525 & 74.8218 & 115.0097 & 40.1879 & 74.6286 & 114.8833 & 40.2546 \\
\hline 74.9664 & 115.3578 & 40.3914 & 74.7767 & 115.0288 & 40.252 & 74.6087 & 114.9101 & 40.3014 \\
\hline 75.0209 & 115.3925 & 40.3715 & 74.71 & 115.0288 & 40.3187 & 74.6027 & 114.9352 & 40.3326 \\
\hline 75.0331 & 115.3102 & 40.2771 & 74.6278 & 115.0513 & 40.4235 & 74.6027 & 114.943 & 40.3404 \\
\hline 75.1178 & 115.2939 & 40.1761 & 74.5481 & 115.0513 & 40.5032 & 74.5879 & 114.9361 & 40.3481 \\
\hline 75.1223 & 115.3544 & 40.2321 & 74.5377 & 115.0339 & 40.4962 & 74.581 & 114.9118 & 40.3308 \\
\hline 75.1084 & 115.2981 & 40.1897 & 74.5734 & 115.0341 & 40.4606 & 74.5628 & 114.9482 & 40.3854 \\
\hline 75.1352 & 115.3119 & 40.1767 & 74.5455 & 115.0573 & 40.5118 & 74.5602 & 114.9151 & 40.3549 \\
\hline 75.1465 & 115.279 & 40.1325 & 74.5109 & 115.0513 & 40.5404 & 74.5507 & 114.8945 & 40.3438 \\
\hline 75.0781 & 115.3145 & 40.2364 & 74.6174 & 114.9924 & 40.375 & 74.4918 & 114.9067 & 40.4148 \\
\hline 75.0296 & 115.2937 & 40.2641 & 74.5836 & 114.9933 & 40.4096 & 74.4581 & 114.937 & 40.4789 \\
\hline 75.0045 & 115.2712 & 40.2667 & 74.5498 & 114.9958 & 40.446 & 74.4598 & 114.8893 & 40.4295 \\
\hline 75.0799 & 115.2543 & 40.1744 & 74.4996 & 114.9967 & 40.4971 & 75.2374 & 114.497 & 39.2596 \\
\hline 75.1664 & 115.176 & 40.0096 & 74.4122 & 115.0383 & 40.6261 & 75.2842 & 114.3594 & 39.0752 \\
\hline 75.1526 & 115.195 & 40.0425 & 74.3417 & 115.0411 & 40.6994 & 75.3916 & 114.2961 & 38.9046 \\
\hline 75.1274 & 115.2071 & 40.0797 & 74.3879 & 115.0374 & 40.6495 & 75.3413 & 114.2658 & 38.9245 \\
\hline 75.0686 & 115.2019 & 40.1334 & 74.3715 & 115.0781 & 40.7067 & 75.2975 & 114.2765 & 38.979 \\
\hline 75.0504 & 115.2435 & 40.1931 & 74.4745 & 115.0513 & 40.5768 & 75.3128 & 114.2762 & 38.9635 \\
\hline 75.0729 & 115.2141 & 40.1412 & 74.5031 & 115.0348 & 40.5317 & 75.2521 & 114.2502 & 38.9981 \\
\hline 75.0616 & 115.1933 & 40.1317 & 74.497 & 115.0409 & 40.5439 & 75.2279 & 114.2901 & 39.0622 \\
\hline 75.0491 & 115.1838 & 40.1347 & 74.5429 & 115.0262 & 40.4832 & 75.2262 & 114.2987 & 39.0726 \\
\hline 74.9534 & 115.1976 & 40.2442 & 74.6001 & 114.9673 & 40.3672 & 75.2521 & 114.3126 & 39.0605 \\
\hline 74.885 & 115.2816 & 40.3966 & 74.5998 & 114.9821 & 40.3822 & 75.2106 & 114.323 & 39.1124 \\
\hline 74.8832 & 115.2989 & 40.4157 & 74.5706 & 114.9638 & 40.3932 & 75.1647 & 114.3013 & 39.1367 \\
\hline 74.8434 & 115.2712 & 40.4278 & 74.5983 & 114.9612 & 40.3629 & 75.1997 & 114.2624 & 39.0627 \\
\hline 74.8798 & 115.247 & 40.3672 & 74.6347 & 114.9491 & 40.3144 & 75.1647 & 114.2355 & 39.0708 \\
\hline
\end{tabular}




\begin{tabular}{|c|c|c|c|c|c|c|c|c|}
\hline 75.1647 & 114.2425 & 39.0778 & 75.6948 & 113.3991 & 37.7043 & 75.3266 & 113.2622 & 37.9356 \\
\hline 75.1933 & 114.1784 & 38.9851 & 75.673 & 113.3895 & 37.7165 & 75.3119 & 113.3167 & 38.0048 \\
\hline 75.1352 & 114.1966 & 39.0613 & 75.6886 & 113.3895 & 37.7009 & 75.2931 & 113.2714 & 37.9783 \\
\hline 75.0703 & 114.2303 & 39.16 & 75.6332 & 113.3704 & 37.7372 & 75.5431 & 113.1245 & 37.5814 \\
\hline 75.0686 & 114.2303 & 39.1618 & 75.634 & 113.3522 & 37.7182 & 75.5405 & 113.0855 & 37.545 \\
\hline 75.0954 & 114.2043 & 39.1089 & 75.6098 & 113.3678 & 37.758 & 75.5024 & 113.102 & 37.5996 \\
\hline 75.0817 & 114.1822 & 39.1005 & 75.5674 & 113.3141 & 37.7468 & 75.4219 & 113.1297 & 37.7078 \\
\hline 75.072 & 114.1559 & 39.0838 & 75.5613 & 113.3514 & 37.7901 & 75.4149 & 113.1513 & 37.7364 \\
\hline 75.0859 & 114.174 & 39.0882 & 75.5054 & 113.3101 & 37.8047 & 75.4141 & 113.1158 & 37.7017 \\
\hline 75.0677 & 114.1576 & 39.0899 & 75.5518 & 113.2778 & 37.726 & 75.4349 & 113.0656 & 37.6307 \\
\hline 75.1049 & 114.1628 & 39.0578 & 75.5059 & 113.257 & 37.7511 & 75.3469 & 113.0705 & 37.7237 \\
\hline 75.0746 & 114.1688 & 39.0942 & 75.5041 & 113.2223 & 37.7182 & 75.46 & 113.0613 & 37.6013 \\
\hline 75.0686 & 114.2 & 39.1315 & 75.5119 & 113.2042 & 37.6922 & 75.5232 & 113.0483 & 37.5251 \\
\hline 75.0937 & 114.1957 & 39.102 & 75.5596 & 113.1877 & 37.6281 & 75.3587 & 113.0717 & 37.713 \\
\hline 75.0808 & 114.1787 & 39.0979 & 75.5007 & 113.1617 & 37.661 & 75.4418 & 113.0102 & 37.5684 \\
\hline 75.0677 & 114.1948 & 39.1271 & 75.4392 & 113.1756 & 37.7364 & 75.4886 & 112.9825 & 37.4939 \\
\hline 75.0235 & 114.168 & 39.1444 & 75.4649 & 113.1463 & 37.6814 & 75.4825 & 113.0301 & 37.5476 \\
\hline 75.0426 & 114.1654 & 39.1228 & 75.4375 & 113.1557 & 37.7182 & 75.4808 & 113.0232 & 37.5424 \\
\hline 74.9984 & 114.1463 & 39.1479 & 75.4634 & 113.1557 & 37.6922 & 75.4966 & 113.0468 & 37.5502 \\
\hline 74.9698 & 114.168 & 39.1981 & 75.5249 & 113.1435 & 37.6186 & 75.1586 & 112.9807 & 37.8221 \\
\hline 74.9681 & 114.1524 & 39.1843 & 75.5215 & 113.1383 & 37.6169 & 74.5966 & 113.1886 & 38.592 \\
\hline 74.9239 & 114.1394 & 39.2155 & 75.4799 & 113.1236 & 37.6437 & 74.3108 & 113.2379 & 38.9271 \\
\hline 74.9046 & 114.1364 & 39.2318 & 75.4539 & 113.1712 & 37.7173 & 74.226 & 113.6813 & 39.4553 \\
\hline 74.9898 & 114.1169 & 39.1271 & 75.4539 & 113.1998 & 37.7459 & 74.1887 & 113.8173 & 39.6285 \\
\hline 74.9854 & 114.1255 & 39.1401 & 75.4843 & 113.178 & 37.6937 & 74.1853 & 113.7714 & 39.5861 \\
\hline 75.0919 & 114.142 & 39.0501 & 75.4981 & 113.1834 & 37.6853 & 74.239 & 113.7566 & 39.5177 \\
\hline 74.9958 & 114.1593 & 39.1635 & 75.4574 & 113.1929 & 37.7355 & 74.229 & 113.7717 & 39.5428 \\
\hline 74.9984 & 114.1403 & 39.1419 & 75.4747 & 113.1929 & 37.7182 & 74.219 & 113.7861 & 39.567 \\
\hline 75.8038 & 113.5921 & 37.7883 & 75.4877 & 113.1929 & 37.7052 & 74.2208 & 113.7844 & 39.5636 \\
\hline 75.8116 & 113.5774 & 37.7658 & 75.4591 & 113.2024 & 37.7433 & 74.2164 & 113.7896 & 39.5731 \\
\hline 75.7609 & 113.563 & 37.8021 & 75.473 & 113.2189 & 37.7459 & 74.1801 & 113.7956 & 39.6155 \\
\hline 75.7613 & 113.5523 & 37.7909 & 75.4764 & 113.2128 & 37.7364 & 74.0866 & 113.8277 & 39.7411 \\
\hline 75.7406 & 113.5566 & 37.8161 & 75.4455 & 113.1639 & 37.7184 & 74.0943 & 113.8103 & 39.716 \\
\hline 75.6773 & 113.5445 & 37.8671 & 75.4574 & 113.1409 & 37.6836 & 74.129 & 113.8077 & 39.6788 \\
\hline 75.7371 & 113.502 & 37.765 & 75.4046 & 113.179 & 37.7745 & 74.0916 & 113.7779 & 39.6863 \\
\hline 75.712 & 113.5124 & 37.8005 & 75.4409 & 113.1842 & 37.7433 & 74.1169 & 113.7419 & 39.6251 \\
\hline 75.7137 & 113.4665 & 37.7528 & 75.4271 & 113.1998 & 37.7728 & 74.2069 & 113.7445 & 39.5376 \\
\hline 75.6522 & 113.47 & 37.8178 & 75.4262 & 113.1678 & 37.7416 & 74.1775 & 113.7939 & 39.6164 \\
\hline 75.6271 & 113.4492 & 37.8221 & 75.4574 & 113.1712 & 37.7139 & 74.1801 & 113.8233 & 39.6432 \\
\hline 75.6058 & 113.4432 & 37.8373 & 75.4531 & 113.1574 & 37.7043 & 74.1662 & 113.8233 & 39.6571 \\
\hline 75.615 & 113.4267 & 37.8117 & 75.5708 & 113.1002 & 37.5294 & 74.1316 & 113.8242 & 39.6926 \\
\hline 75.5942 & 113.4085 & 37.8143 & 75.6023 & 113.1172 & 37.5149 & 74.161 & 113.7991 & 39.6381 \\
\hline 75.5881 & 113.399 & 37.8109 & 75.5518 & 113.1773 & 37.6255 & 74.1662 & 113.8077 & 39.6415 \\
\hline 75.6115 & 113.4042 & 37.7927 & 75.4938 & 113.2449 & 37.7511 & 74.1629 & 113.8131 & 39.6502 \\
\hline 75.6262 & 113.4328 & 37.8065 & 75.4331 & 113.2397 & 37.8065 & 74.129 & 113.8207 & 39.6917 \\
\hline 75.654 & 113.3999 & 37.7459 & 75.3829 & 113.2873 & 37.9044 & 74.1602 & 113.8328 & 39.6727 \\
\hline 75.6843 & 113.4016 & 37.7173 & 75.3708 & 113.2985 & 37.9278 & 74.2199 & 113.8181 & 39.5982 \\
\hline
\end{tabular}




\begin{tabular}{|c|c|c|c|c|c|c|c|c|}
\hline 74.2113 & 113.8484 & 39.6372 & 74.4044 & 114.0952 & 39.6909 & 74.4298 & 114.1479 & 39.718 \\
\hline 74.1809 & 113.864 & 39.6831 & 74.4693 & 114.1022 & 39.6329 & 74.4191 & 114.1351 & 39.716 \\
\hline 74.2104 & 113.8874 & 39.677 & 74.4771 & 114.103 & 39.6259 & 74.3767 & 114.1437 & 39.7671 \\
\hline 74.1636 & 113.8909 & 39.7272 & 74.4788 & 114.1307 & 39.6519 & 74.3593 & 114.1533 & 39.7939 \\
\hline 74.1497 & 113.9021 & 39.7524 & 74.4806 & 114.1255 & 39.645 & 74.3489 & 114.1498 & 39.8009 \\
\hline 74.1723 & 113.9186 & 39.7463 & 74.4321 & 114.1169 & 39.6848 & 74.381 & 114.1611 & 39.7801 \\
\hline 74.1809 & 113.9134 & 39.7324 & 74.3708 & 114.1153 & 39.7445 & 74.4329 & 114.1325 & 39.6995 \\
\hline 74.161 & 113.9272 & 39.7662 & 74.3507 & 114.1152 & 39.7645 & 74.5074 & 114.0874 & 39.58 \\
\hline 74.1047 & 113.9679 & 39.8632 & 74.3628 & 114.1316 & 39.7688 & 74.5452 & 114.1118 & 39.5665 \\
\hline 74.0874 & 113.9818 & 39.8944 & 74.413 & 114.0935 & 39.6805 & 74.5455 & 114.1074 & 39.5618 \\
\hline 74.0952 & 113.9428 & 39.8476 & 74.4122 & 114.071 & 39.6588 & 74.5498 & 114.1013 & 39.5515 \\
\hline 74.0891 & 113.9394 & 39.8502 & 74.491 & 114.0294 & 39.5385 & 74.536 & 114.1411 & 39.6051 \\
\hline 74.0942 & 113.9382 & 39.844 & 74.5308 & 114.0641 & 39.5333 & 74.5975 & 114.0944 & 39.4969 \\
\hline 74.0805 & 113.9601 & 39.8797 & 74.549 & 114.0701 & 39.5211 & 74.4771 & 114.1022 & 39.6251 \\
\hline 74.1827 & 113.9567 & 39.774 & 74.5082 & 114.0492 & 39.541 & 74.4122 & 114.0935 & 39.6813 \\
\hline 74.1498 & 113.9532 & 39.8035 & 74.497 & 114.026 & 39.5289 & 74.4338 & 114.1463 & 39.7125 \\
\hline 74.1273 & 113.9584 & 39.8312 & 74.497 & 114.0034 & 39.5064 & 74.4792 & 114.1311 & 39.652 \\
\hline 74.1195 & 113.9939 & 39.8745 & 74.4563 & 114.0138 & 39.5575 & 74.5204 & 114.1472 & 39.6268 \\
\hline 74.1628 & 113.9264 & 39.7636 & 74.4416 & 114.0312 & 39.5896 & 74.5299 & 114.1706 & 39.6406 \\
\hline 74.2113 & 113.9134 & 39.7021 & 74.3922 & 114.026 & 39.6337 & 74.5031 & 114.2069 & 39.7039 \\
\hline 74.2545 & 113.91 & 39.6555 & 74.3472 & 114.0563 & 39.7091 & 74.4936 & 114.2338 & 39.7402 \\
\hline 74.2632 & 113.9177 & 39.6545 & 74.3169 & 114.1082 & 39.7913 & 74.5481 & 114.1966 & 39.6484 \\
\hline 74.284 & 113.9238 & 39.6398 & 74.3259 & 114.0906 & 39.7647 & 74.5914 & 114.1706 & 39.5792 \\
\hline 74.2338 & 113.9498 & 39.716 & 74.5117 & 114.0597 & 39.548 & 74.594 & 114.181 & 39.587 \\
\hline 74.2831 & 113.9463 & 39.6632 & 74.4823 & 114.0554 & 39.5731 & 74.5849 & 114.2034 & 39.6185 \\
\hline 74.258 & 113.9402 & 39.6822 & 74.4624 & 114.0658 & 39.6034 & 74.5983 & 114.1966 & 39.5982 \\
\hline 74.2346 & 113.9974 & 39.7628 & 74.4511 & 114.084 & 39.6329 & 74.5888 & 114.2009 & 39.6121 \\
\hline 74.2771 & 114.032 & 39.755 & 74.4442 & 114.0935 & 39.6493 & 74.555 & 114.2087 & 39.6536 \\
\hline 74.281 & 114.0501 & 39.7691 & 74.4251 & 114.1273 & 39.7021 & 74.6113 & 114.1628 & 39.5515 \\
\hline 74.3074 & 114.0597 & 39.7524 & 74.426 & 114.116 & 39.69 & 74.6382 & 114.1775 & 39.5393 \\
\hline 74.2831 & 114.0623 & 39.7792 & 74.5232 & 114.1338 & 39.6106 & 74.6364 & 114.1749 & 39.5385 \\
\hline 74.2597 & 114.0736 & 39.8138 & 74.4624 & 114.1437 & 39.6813 & 74.6087 & 114.1645 & 39.5558 \\
\hline 74.2597 & 114.0597 & 39.8 & 74.4165 & 114.1559 & 39.7394 & 74.6915 & 114.1179 & 39.4265 \\
\hline 74.3533 & 114.0779 & 39.7246 & 74.2953 & 114.0996 & 39.8043 & 74.7421 & 114.0459 & 39.3038 \\
\hline 74.4096 & 114.0277 & 39.6181 & 74.2753 & 114.1082 & 39.8329 & 74.6962 & 114.0485 & 39.3523 \\
\hline 74.4364 & 113.9939 & 39.5575 & 74.2459 & 114.0987 & 39.8528 & 74.6737 & 114.0303 & 39.3566 \\
\hline 74.4492 & 114.0052 & 39.556 & 74.2684 & 114.116 & 39.8476 & 74.5637 & 114.0138 & 39.4501 \\
\hline 74.4736 & 114.0147 & 39.5411 & 74.3074 & 114.1325 & 39.8251 & 74.5065 & 114.0199 & 39.5134 \\
\hline 74.4529 & 114.013 & 39.5601 & 74.3779 & 114.1223 & 39.7445 & 74.4511 & 114.0519 & 39.6008 \\
\hline 74.4624 & 114.0069 & 39.5445 & 74.4589 & 114.103 & 39.6441 & 74.3689 & 114.0667 & 39.6978 \\
\hline 74.4953 & 114.0034 & 39.5082 & 74.3922 & 114.0926 & 39.7004 & 74.3197 & 114.0818 & 39.7621 \\
\hline 74.5005 & 114.0398 & 39.5393 & 74.3342 & 114.0926 & 39.7584 & 74.3299 & 114.1074 & 39.7775 \\
\hline 74.4381 & 114.0346 & 39.5965 & 74.3611 & 114.0961 & 39.735 & 74.3619 & 114.0693 & 39.7073 \\
\hline 74.426 & 114.0641 & 39.6381 & 74.2987 & 114.0874 & 39.7887 & 74.3992 & 114.0389 & 39.6398 \\
\hline 74.4321 & 114.1022 & 39.6701 & 74.2753 & 114.1143 & 39.839 & 74.5091 & 114.0502 & 39.5411 \\
\hline 74.392 & 114.0836 & 39.6916 & 74.2978 & 114.1186 & 39.8208 & 74.5039 & 114.0545 & 39.5506 \\
\hline 74.4139 & 114.0779 & 39.664 & 74.407 & 114.1524 & 39.7454 & 74.491 & 114.0597 & 39.5688 \\
\hline
\end{tabular}




\begin{tabular}{|c|c|c|c|c|c|c|c|c|}
\hline 74.4875 & 114.0745 & 39.587 & 74.6148 & 113.3964 & 38.7816 & 73.9653 & 113.302 & 39.3367 \\
\hline 74.5481 & 114.039 & 39.4908 & 74.5923 & 113.3938 & 38.8015 & 74.019 & 113.2933 & 39.2743 \\
\hline 74.5849 & 114.0378 & 39.4529 & 74.562 & 113.3687 & 38.8067 & 73.9567 & 113.2856 & 39.3289 \\
\hline 74.6096 & 114.0251 & 39.4155 & 74.5611 & 113.3938 & 38.8327 & 73.8649 & 113.302 & 39.4371 \\
\hline 74.6797 & 114.0173 & 39.3376 & 74.562 & 113.4273 & 38.8653 & 73.8467 & 113.2639 & 39.4172 \\
\hline 74.6988 & 114.0052 & 39.3064 & 74.5871 & 113.4354 & 38.8483 & 73.785 & 113.2687 & 39.4837 \\
\hline 74.6607 & 114 & 39.3393 & 74.5758 & 113.4189 & 38.8431 & 73.7791 & 113.263 & 39.4839 \\
\hline 74.5992 & 114.0182 & 39.419 & 74.5524 & 113.3782 & 38.8258 & 73.7254 & 113.263 & 39.5376 \\
\hline 74.5905 & 113.9931 & 39.4025 & 74.5161 & 113.3661 & 38.85 & 73.7532 & 113.2734 & 39.5203 \\
\hline 74.5438 & 113.9783 & 39.4345 & 74.4901 & 113.3929 & 38.9028 & 73.7384 & 113.2856 & 39.5471 \\
\hline 74.5056 & 114.0351 & 39.5295 & 74.4563 & 113.3808 & 38.9245 & 73.7298 & 113.2717 & 39.5419 \\
\hline 74.536 & 114.0173 & 39.4813 & 74.4407 & 113.3375 & 38.8968 & 73.7445 & 113.2249 & 39.4804 \\
\hline 74.478 & 114.0667 & 39.5887 & 74.4237 & 113.3885 & 38.9649 & 73.7125 & 113.2284 & 39.516 \\
\hline 74.4736 & 114.0537 & 39.58 & 74.381 & 113.3756 & 38.9946 & 73.7376 & 113.2284 & 39.4908 \\
\hline 74.4866 & 114.0623 & 39.5757 & 74.3576 & 113.3773 & 39.0197 & 73.7242 & 113.23 & 39.5058 \\
\hline 74.5065 & 114.0866 & 39.58 & 74.3437 & 113.3211 & 38.9773 & 73.7272 & 113.2293 & 39.5021 \\
\hline 74.5014 & 114.0606 & 39.5592 & 74.3429 & 113.3297 & 38.9868 & 73.7073 & 113.2691 & 39.5618 \\
\hline 75.0893 & 113.5427 & 38.4534 & 74.3039 & 113.3211 & 39.0171 & 73.9445 & 113.2552 & 39.3107 \\
\hline 75.0271 & 113.3339 & 38.3068 & 74.2649 & 113.3011 & 39.0362 & 73.9004 & 113.2535 & 39.3531 \\
\hline 75.0937 & 113.257 & 38.1633 & 74.2831 & 113.2795 & 38.9964 & 73.7696 & 113.2622 & 39.4926 \\
\hline 75.2556 & 113.58 & 38.3244 & 74.281 & 113.2705 & 38.9895 & 73.7973 & 113.2665 & 39.4692 \\
\hline 75.3197 & 113.6458 & 38.3261 & 74.2225 & 113.2856 & 39.063 & 73.8181 & 113.1816 & 39.3635 \\
\hline 75.2703 & 113.6164 & 38.346 & 74.2563 & 113.2604 & 39.0042 & 73.7727 & 113.1868 & 39.4141 \\
\hline 75.1985 & 113.6172 & 38.4188 & 74.2303 & 113.244 & 39.0137 & 73.7861 & 113.2189 & 39.4328 \\
\hline 75.137 & 113.6285 & 38.4915 & 74.161 & 113.2578 & 39.0968 & 73.8259 & 113.2215 & 39.3956 \\
\hline 75.0919 & 113.5878 & 38.4958 & 74.2831 & 113.2431 & 38.96 & 73.7601 & 113.263 & 39.503 \\
\hline 75.1231 & 113.5744 & 38.4513 & 74.3611 & 113.289 & 38.928 & 73.9212 & 113.2518 & 39.3306 \\
\hline 75.1049 & 113.6345 & 38.5296 & 74.2234 & 113.276 & 39.0527 & 73.7679 & 113.276 & 39.5082 \\
\hline 75.1326 & 113.5722 & 38.4395 & 74.2545 & 113.2784 & 39.0239 & 73.7047 & 113.2864 & 39.5818 \\
\hline 75.1292 & 113.5376 & 38.4084 & 74.1654 & 113.3011 & 39.1358 & 73.7644 & 113.2959 & 39.5315 \\
\hline 75.0807 & 113.5774 & 38.4967 & 74.1307 & 113.3037 & 39.173 & 73.8696 & 113.2855 & 39.4159 \\
\hline 75.0131 & 113.5921 & 38.579 & 74.0952 & 113.3011 & 39.2059 & 73.8423 & 113.3211 & 39.4787 \\
\hline 74.9759 & 113.5938 & 38.6179 & 74.0043 & 113.2985 & 39.2943 & 73.8666 & 113.2985 & 39.432 \\
\hline 74.9924 & 113.5878 & 38.5954 & 73.9662 & 113.3029 & 39.3367 & 73.7055 & 113.2778 & 39.5722 \\
\hline 74.9654 & 113.5515 & 38.5861 & 74.0528 & 113.2856 & 39.2328 & 73.7419 & 113.2622 & 39.5203 \\
\hline 74.9404 & 113.5731 & 38.6327 & 74.1506 & 113.3211 & 39.1704 & 73.722 & 113.2847 & 39.5627 \\
\hline 74.9213 & 113.5912 & 38.6699 & 74.1224 & 113.2855 & 39.1631 & 73.8207 & 113.2873 & 39.4666 \\
\hline 74.9118 & 113.5514 & 38.6396 & 73.9722 & 113.3098 & 39.3376 & 73.8294 & 113.2942 & 39.4649 \\
\hline 74.9058 & 113.5843 & 38.6786 & 74.0926 & 113.2856 & 39.1929 & 73.9154 & 113.3101 & 39.3948 \\
\hline 74.9101 & 113.606 & 38.6959 & 73.9359 & 113.3072 & 39.3713 & 73.9601 & 113.3176 & 39.3575 \\
\hline 74.8806 & 113.5488 & 38.6682 & 73.9489 & 113.2882 & 39.3393 & 73.9359 & 113.3159 & 39.38 \\
\hline 74.8434 & 113.5661 & 38.7227 & 73.9939 & 113.2925 & 39.2986 & 73.8952 & 113.315 & 39.4198 \\
\hline 74.8588 & 113.5286 & 38.6698 & 74.0147 & 113.2994 & 39.2847 & 73.9108 & 113.3124 & 39.4016 \\
\hline 74.7663 & 113.4536 & 38.6872 & 74.1039 & 113.2933 & 39.1895 & 73.7237 & 113.2925 & 39.5688 \\
\hline 74.6823 & 113.4665 & 38.7842 & 73.8925 & 113.2767 & 39.3842 & 73.8068 & 113.2968 & 39.49 \\
\hline 74.7075 & 113.4458 & 38.7383 & 73.8761 & 113.2994 & 39.4233 & 73.8675 & 113.2691 & 39.4016 \\
\hline 74.6659 & 113.4163 & 38.7504 & 73.8934 & 113.2821 & 39.3887 & 73.7524 & 113.2423 & 39.4899 \\
\hline
\end{tabular}




\begin{tabular}{|c|c|c|c|c|c|c|c|c|}
\hline 73.7913 & 113.2232 & 39.432 & 78.9152 & 117.6907 & 38.7755 & 77.5851 & 116.8395 & 39.2544 \\
\hline 73.799 & 113.2345 & 39.4354 & 78.6899 & 117.7261 & 39.0362 & 77.5842 & 116.8352 & 39.251 \\
\hline 73.7653 & 113.2353 & 39.4701 & 78.6805 & 117.6873 & 39.0068 & 77.6405 & 116.8317 & 39.1912 \\
\hline 73.709 & 113.2908 & 39.5818 & 78.6528 & 117.6379 & 38.9851 & 77.6002 & 116.7994 & 39.1992 \\
\hline 73.6769 & 113.2743 & 39.5974 & 78.7437 & 117.6345 & 38.8907 & 77.5773 & 116.7746 & 39.1973 \\
\hline 73.7133 & 113.27 & 39.5567 & 78.5645 & 117.6431 & 39.0786 & 77.5478 & 116.7416 & 39.1938 \\
\hline 73.6752 & 113.2604 & 39.5852 & 78.4277 & 117.6206 & 39.1929 & 77.5478 & 116.7425 & 39.1947 \\
\hline 73.674 & 113.2546 & 39.5806 & 78.4329 & 117.6474 & 39.2146 & 77.5019 & 116.7304 & 39.2284 \\
\hline 73.735 & 113.2388 & 39.5038 & 78.5446 & 117.5375 & 38.9929 & 77.4292 & 116.7425 & 39.3133 \\
\hline 73.78 & 113.108 & 39.328 & 78.6317 & 117.5138 & 38.8821 & 77.4006 & 116.6854 & 39.2847 \\
\hline 74.284 & 112.8959 & 38.6119 & 78.5498 & 117.4933 & 38.9435 & 77.3816 & 116.6845 & 39.3029 \\
\hline 74.2164 & 112.9998 & 38.7833 & 78.4103 & 117.5193 & 39.1089 & 77.4196 & 116.7677 & 39.3481 \\
\hline 74.2182 & 112.9885 & 38.7703 & 78.3151 & 117.521 & 39.2059 & 77.4049 & 116.7321 & 39.3272 \\
\hline 74.2918 & 112.9669 & 38.6751 & 78.2692 & 117.4734 & 39.2042 & 77.3539 & 116.7122 & 39.3583 \\
\hline 74.2234 & 112.9244 & 38.7011 & 78.2129 & 117.4621 & 39.2492 & 77.5773 & 116.4533 & 38.876 \\
\hline 74.1731 & 112.9548 & 38.7816 & 78.1964 & 117.4197 & 39.2233 & 77.7565 & 116.1095 & 38.353 \\
\hline 74.1294 & 112.9393 & 38.8098 & 78.2198 & 117.3799 & 39.16 & 77.5859 & 116.19 & 38.6041 \\
\hline 73.8666 & 112.8439 & 38.9773 & 78.1861 & 117.3651 & 39.1791 & 77.3539 & 116.2125 & 38.8587 \\
\hline 73.9298 & 112.8249 & 38.895 & 78.1199 & 117.3711 & 39.2512 & 77.5383 & 116.4316 & 38.8933 \\
\hline 73.8787 & 112.7937 & 38.915 & 78.0873 & 117.3504 & 39.2631 & 77.4552 & 116.403 & 38.9479 \\
\hline 73.7878 & 112.792 & 39.0042 & 78.0354 & 117.3452 & 39.3098 & 77.3659 & 116.4585 & 39.0926 \\
\hline 73.5713 & 112.7963 & 39.225 & 78.0077 & 117.3037 & 39.296 & 77.3539 & 116.4541 & 39.1003 \\
\hline 73.5843 & 112.7902 & 39.2059 & 78.0527 & 117.2993 & 39.2466 & 77.2967 & 116.429 & 39.1323 \\
\hline 73.6025 & 112.7608 & 39.1583 & 78.0752 & 117.2872 & 39.212 & 77.3253 & 116.3849 & 39.0596 \\
\hline 73.6071 & 112.7481 & 39.1411 & 78.0362 & 117.263 & 39.2267 & 77.2681 & 116.3857 & 39.1176 \\
\hline 73.6042 & 112.7045 & 39.1003 & 78.0691 & 117.2404 & 39.1713 & 77.2525 & 116.3632 & 39.1107 \\
\hline 73.6207 & 112.7209 & 39.1003 & 78.1252 & 117.2055 & 39.0803 & 77.2205 & 116.3355 & 39.115 \\
\hline 73.541 & 112.7114 & 39.1704 & 78.0276 & 117.1599 & 39.1323 & 77.3651 & 116.2472 & 38.8821 \\
\hline 73.5488 & 112.6846 & 39.1358 & 78.031 & 117.1703 & 39.1393 & 77.3157 & 116.2347 & 38.9191 \\
\hline 75.8272 & 114.8201 & 38.9929 & 78.0155 & 117.1054 & 39.0899 & 77.2344 & 116.2273 & 38.9929 \\
\hline 79.2018 & 118.1229 & 38.921 & 77.9384 & 117.1209 & 39.1826 & 77.159 & 116.2247 & 39.0656 \\
\hline 79.1378 & 118.1523 & 39.0146 & 77.928 & 117.1166 & 39.1886 & 77.0975 & 116.2082 & 39.1107 \\
\hline 79.1224 & 118.1436 & 39.0213 & 77.8795 & 117.1036 & 39.2241 & 77.0811 & 116.2013 & 39.1202 \\
\hline 79.1759 & 118.0744 & 38.8985 & 77.8267 & 117.1149 & 39.2882 & 77.1313 & 116.1666 & 39.0353 \\
\hline 79.0797 & 118.0744 & 38.9946 & 77.8442 & 117.1244 & 39.2802 & 77.217 & 116.1337 & 38.9167 \\
\hline 79.0278 & 118.0354 & 39.0076 & 77.8475 & 117.075 & 39.2276 & 77.2361 & 116.087 & 38.8509 \\
\hline 78.9983 & 118.0129 & 39.0146 & 77.8076 & 117.017 & 39.2094 & 77.1606 & 116.1088 & 38.9481 \\
\hline 78.9801 & 117.9817 & 39.0016 & 77.8215 & 116.9434 & 39.1219 & 77.0334 & 116.1381 & 39.1046 \\
\hline 78.9438 & 117.9479 & 39.0042 & 77.7271 & 116.9425 & 39.2155 & 77.0109 & 116.1398 & 39.1289 \\
\hline 78.9741 & 117.9228 & 38.9487 & 77.7184 & 116.9244 & 39.2059 & 77.0334 & 116.1363 & 39.1029 \\
\hline 78.9603 & 117.8864 & 38.9261 & 77.7002 & 116.9122 & 39.212 & 77.017 & 116.1476 & 39.1306 \\
\hline 78.9776 & 117.8432 & 38.8656 & 77.7522 & 116.901 & 39.1488 & 76.9876 & 116.1129 & 39.1254 \\
\hline 79.0364 & 117.7877 & 38.7513 & 77.735 & 116.9086 & 39.1737 & 76.881 & 116.1207 & 39.2397 \\
\hline 78.9758 & 117.7947 & 38.8188 & 77.7288 & 116.9062 & 39.1774 & 76.9278 & 116.0541 & 39.1263 \\
\hline 78.9039 & 117.7834 & 38.8795 & 77.6327 & 116.9036 & 39.2709 & 76.9052 & 116.0524 & 39.1472 \\
\hline 78.8814 & 117.7609 & 38.8795 & 77.6483 & 116.8759 & 39.2276 & 76.8265 & 116.0419 & 39.2155 \\
\hline 78.8693 & 117.7176 & 38.8483 & 77.5998 & 116.8837 & 39.2839 & 76.8005 & 116.035 & 39.2345 \\
\hline
\end{tabular}




\begin{tabular}{|c|c|c|c|c|c|c|c|c|}
\hline 76.8092 & 116.009 & 39.1999 & 75.5691 & 115.1439 & 39.5748 & 74.6183 & 114.4789 & 39.8606 \\
\hline 76.9572 & 115.9683 & 39.0111 & 75.6124 & 115.1647 & 39.5523 & 74.6324 & 114.4342 & 39.8017 \\
\hline 76.7537 & 115.9813 & 39.2276 & 75.5613 & 115.1483 & 39.587 & 74.6191 & 114.381 & 39.7619 \\
\hline 76.6871 & 115.9398 & 39.2527 & 75.5613 & 115.1569 & 39.5956 & 74.6745 & 114.3983 & 39.7238 \\
\hline 76.7269 & 115.9207 & 39.1938 & 75.5483 & 115.0876 & 39.5393 & 74.6815 & 114.4105 & 39.729 \\
\hline 76.7193 & 115.8903 & 39.171 & 75.7354 & 115.1015 & 39.3661 & 74.6875 & 114.3914 & 39.7039 \\
\hline 76.7347 & 115.8826 & 39.1479 & 75.6349 & 115.0876 & 39.4527 & 74.5412 & 114.3819 & 39.8407 \\
\hline 76.6394 & 115.8376 & 39.1981 & 75.627 & 115.0772 & 39.4503 & 74.4762 & 114.3905 & 39.9143 \\
\hline 76.5485 & 115.8618 & 39.3133 & 75.4227 & 115.0478 & 39.6251 & 74.4702 & 114.3775 & 39.9074 \\
\hline 76.5667 & 115.8384 & 39.2717 & 75.4357 & 115.0348 & 39.5991 & 74.5681 & 114.2879 & 39.7198 \\
\hline 76.6325 & 115.8185 & 39.186 & 75.4098 & 115.014 & 39.6043 & 74.5403 & 114.3169 & 39.7766 \\
\hline 76.5502 & 115.835 & 39.2847 & 75.3457 & 115.0114 & 39.6658 & 74.4503 & 114.278 & 39.8277 \\
\hline 76.3961 & 115.8081 & 39.412 & 75.3968 & 114.9829 & 39.5861 & 74.4814 & 114.2373 & 39.7558 \\
\hline 76.455 & 115.7617 & 39.3067 & 75.3223 & 114.95 & 39.6277 & 74.5048 & 114.2425 & 39.7376 \\
\hline 76.4801 & 115.7371 & 39.257 & 75.389 & 114.9274 & 39.5385 & 74.9594 & 113.8995 & 38.9401 \\
\hline 76.3675 & 115.7501 & 39.3826 & 75.3618 & 114.8887 & 39.5269 & 75.5015 & 113.4813 & 37.9797 \\
\hline 76.416 & 115.7146 & 39.2986 & 75.3552 & 114.9162 & 39.561 & 75.7613 & 113.3444 & 37.5831 \\
\hline 76.2939 & 115.6843 & 39.3904 & 75.3691 & 114.9144 & 39.5454 & 75.6684 & 113.3445 & 37.6761 \\
\hline 76.2636 & 115.6756 & 39.412 & 75.3639 & 114.8928 & 39.5289 & 75.654 & 113.3271 & 37.6732 \\
\hline 76.3727 & 115.66 & 39.2873 & 75.2773 & 114.898 & 39.6207 & 75.7406 & 113.3081 & 37.5675 \\
\hline 76.4299 & 115.6098 & 39.18 & 75.1197 & 114.8625 & 39.7428 & 75.7787 & 113.2769 & 37.4982 \\
\hline 76.4195 & 115.5544 & 39.1349 & 75.2877 & 114.8374 & 39.5497 & 75.7605 & 113.2708 & 37.5104 \\
\hline 76.2313 & 115.597 & 39.3657 & 75.2877 & 114.8287 & 39.5411 & 75.6314 & 113.2864 & 37.655 \\
\hline 76.1909 & 115.5501 & 39.3592 & 75.3041 & 114.8183 & 39.5142 & 75.5821 & 113.3263 & 37.7442 \\
\hline 76.048 & 115.5561 & 39.5082 & 75.25 & 114.7918 & 39.5419 & 75.5803 & 113.2795 & 37.6991 \\
\hline 76.0774 & 115.5215 & 39.4441 & 75.1439 & 114.749 & 39.6051 & 75.3187 & 113.3022 & 37.9836 \\
\hline 76.048 & 115.499 & 39.451 & 75.0807 & 114.756 & 39.6753 & 74.9231 & 113.302 & 38.3789 \\
\hline 75.9977 & 115.5146 & 39.5168 & 75.0495 & 114.7257 & 39.6762 & 74.7456 & 113.3401 & 38.5946 \\
\hline 75.9796 & 115.5042 & 39.5246 & 75.0105 & 114.7317 & 39.7212 & 74.5793 & 113.373 & 38.7937 \\
\hline 75.977 & 115.486 & 39.509 & 75.0582 & 114.6798 & 39.6216 & 74.5983 & 113.3193 & 38.721 \\
\hline 75.9106 & 115.4992 & 39.5886 & 75.0738 & 114.6884 & 39.6147 & 74.7464 & 113.2501 & 38.5036 \\
\hline 75.9267 & 115.4765 & 39.5497 & 74.9785 & 114.6417 & 39.6632 & 74.5395 & 113.2994 & 38.76 \\
\hline 76.0636 & 115.4869 & 39.4233 & 75.1055 & 114.657 & 39.5516 & 74.3914 & 113.3358 & 38.9444 \\
\hline 75.9345 & 115.447 & 39.5125 & 74.9187 & 114.6555 & 39.7368 & 74.4295 & 113.2795 & 38.85 \\
\hline 76.048 & 115.389 & 39.341 & 74.8356 & 114.6347 & 39.7991 & 74.784 & 113.0459 & 38.2619 \\
\hline 76.1502 & 115.4055 & 39.2553 & 74.8365 & 114.6321 & 39.7957 & 74.8157 & 113.0171 & 38.2014 \\
\hline 75.9267 & 115.4046 & 39.4778 & 74.7906 & 114.6252 & 39.8346 & 74.7897 & 113.0067 & 38.217 \\
\hline 75.7856 & 115.415 & 39.6294 & 74.8062 & 114.6174 & 39.8112 & 74.3368 & 113.1635 & 38.8266 \\
\hline 75.8287 & 115.3662 & 39.5375 & 74.8832 & 114.5836 & 39.7004 & 74.2502 & 113.1513 & 38.9011 \\
\hline 75.7856 & 115.3457 & 39.5601 & 74.7611 & 114.5914 & 39.8303 & 74.2156 & 113.1505 & 38.9349 \\
\hline 75.7882 & 115.3414 & 39.5532 & 74.85 & 114.5786 & 39.7286 & 74.3446 & 113.1098 & 38.7652 \\
\hline 75.757 & 115.3388 & 39.5818 & 74.6668 & 114.5984 & 39.9316 & 74.5282 & 113.0665 & 38.5383 \\
\hline 75.8471 & 115.3336 & 39.4865 & 74.775 & 114.5559 & 39.7809 & 74.3074 & 113.0573 & 38.7499 \\
\hline 75.7709 & 115.3232 & 39.5523 & 74.7568 & 114.5611 & 39.8043 & 74.1896 & 113.0717 & 38.8821 \\
\hline 75.615 & 115.2401 & 39.6251 & 74.6962 & 114.5222 & 39.826 & 74.1125 & 113.0465 & 38.934 \\
\hline 75.6323 & 115.2522 & 39.6199 & 74.678 & 114.5213 & 39.8433 & 74.1879 & 112.9885 & 38.8007 \\
\hline 75.7151 & 115.1856 & 39.4705 & 74.6486 & 114.4546 & 39.806 & 74.4 & 112.9504 & 38.5504 \\
\hline
\end{tabular}




\begin{tabular}{|c|c|c|c|c|c|}
\hline 74.3931 & 112.9859 & 38.5928 & 77.8691 & 117.4942 & 39.6251 \\
\hline 73.9731 & 113.0102 & 39.0371 & 77.7842 & 117.5124 & 39.7281 \\
\hline 73.78 & 113.0673 & 39.2873 & 77.8431 & 117.4872 & 39.6441 \\
\hline 74.0748 & 113.215 & 39.1402 & 77.8094 & 117.4717 & 39.6623 \\
\hline 79.1499 & 118.1142 & 38.9643 & 77.8483 & 117.4587 & 39.6103 \\
\hline 79.6461 & 118.6459 & 38.9998 & 77.9596 & 117.3685 & 39.4089 \\
\hline 79.6487 & 118.581 & 38.9323 & 77.9176 & 117.3565 & 39.4389 \\
\hline 79.685 & 118.4918 & 38.8067 & 77.8665 & 117.3807 & 39.5142 \\
\hline 79.6487 & 118.4563 & 38.8076 & 77.8388 & 117.3573 & 39.5185 \\
\hline 79.3006 & 118.5151 & 39.2146 & 77.7496 & 117.3842 & 39.6346 \\
\hline 79.298 & 118.4805 & 39.1826 & 77.799 & 117.3877 & 39.5887 \\
\hline 79.244 & 118.4335 & 39.1895 & 77.7375 & 117.3444 & 39.6069 \\
\hline 79.1698 & 118.4606 & 39.2908 & 77.7522 & 117.3288 & 39.5766 \\
\hline 79.136 & 118.4528 & 39.3168 & 77.7579 & 117.2918 & 39.5339 \\
\hline 79.0624 & 118.3549 & 39.2925 & 77.6682 & 117.3089 & 39.6406 \\
\hline 78.975 & 118.3013 & 39.3263 & 77.6699 & 117.2396 & 39.5696 \\
\hline 78.8667 & 118.361 & 39.4943 & 77.6067 & 117.1989 & 39.5922 \\
\hline 78.8451 & 118.2857 & 39.4406 & 77.7011 & 117.1634 & 39.4623 \\
\hline 78.8407 & 118.2415 & 39.4008 & 77.7505 & 117.1244 & 39.3739 \\
\hline 78.8246 & 118.2159 & 39.3912 & 77.6007 & 117.1071 & 39.5064 \\
\hline 78.7429 & 118.1766 & 39.4337 & 77.508 & 117.0915 & 39.5835 \\
\hline 78.7879 & 118.148 & 39.3601 & 77.5068 & 117.0645 & 39.5577 \\
\hline 78.7065 & 118.1393 & 39.4328 & 77.4673 & 117.0517 & 39.5844 \\
\hline 78.6467 & 118.1168 & 39.4701 & 77.5071 & 117.0274 & 39.5203 \\
\hline 78.6433 & 118.0475 & 39.4042 & 77.4803 & 117.0274 & 39.5471 \\
\hline 78.5827 & 118.064 & 39.4813 & 77.4179 & 117.0006 & 39.5826 \\
\hline 78.4813 & 118.0215 & 39.5402 & 77.411 & 116.9529 & 39.5419 \\
\hline 78.393 & 118.0415 & 39.6484 & 77.5487 & 116.927 & 39.3783 \\
\hline 78.3305 & 117.9639 & 39.6335 & 77.5348 & 116.8196 & 39.2847 \\
\hline 78.3896 & 117.9427 & 39.5532 & 77.5324 & 116.7853 & 39.2529 \\
\hline 78.4086 & 117.922 & 39.5134 & 77.4275 & 116.8083 & 39.3809 \\
\hline 78.3922 & 117.9116 & 39.5194 & 77.4465 & 116.8585 & 39.412 \\
\hline 78.4762 & 117.76 & 39.2839 & 77.3928 & 116.9096 & 39.5168 \\
\hline 78.5229 & 117.7107 & 39.1877 & 77.4379 & 116.9192 & 39.4813 \\
\hline 78.49 & 117.6778 & 39.1877 & 77.2846 & 116.8508 & 39.5662 \\
\hline 78.7256 & 117.5816 & 38.8561 & 77.4275 & 116.9183 & 39.4908 \\
\hline 78.541 & 117.6028 & 39.0618 & 77.3702 & 116.8891 & 39.5189 \\
\hline 78.5983 & 117.5349 & 38.9366 & & & \\
\hline 78.5385 & 117.5002 & 38.9617 & & & \\
\hline 78.3497 & 117.4691 & 39.1193 & & & \\
\hline 78.2839 & 117.4907 & 39.2068 & & & \\
\hline 78.1324 & 117.5565 & 39.4242 & & & \\
\hline 78.1228 & 117.5357 & 39.4129 & & & \\
\hline 78.0969 & 117.5054 & 39.4086 & & & \\
\hline 78.016 & 117.5182 & 39.5022 & & & \\
\hline 78.0501 & 117.4439 & 39.3938 & & & \\
\hline 78.0345 & 117.4829 & 39.4484 & & & \\
\hline
\end{tabular}




\begin{tabular}{|c|c|c|c|c|c|c|c|c|}
\hline \multicolumn{3}{|c|}{ Table A.2: 31 Hour Test } & 82.4639 & 122.2743 & 39.8104 & 82.6744 & 122.3782 & 39.7039 \\
\hline minimum & maximum & height & 82.4513 & 122.3138 & 39.8625 & 82.6086 & 122.4129 & 39.8043 \\
\hline $\mathrm{mmHg}$ & $\mathrm{mmHg}$ & $\mathrm{mmHg}$ & 82.4458 & 122.2769 & 39.8312 & 82.6285 & 122.483 & 39.8545 \\
\hline 82.6138 & 122.2189 & 39.6051 & 82.4458 & 122.283 & 39.8372 & 82.6548 & 122.3288 & 39.674 \\
\hline 82.6008 & 122.1999 & 39.5991 & 82.5289 & 122.2596 & 39.7307 & 82.7307 & 122.2501 & 39.5194 \\
\hline 82.5618 & 122.1964 & 39.6346 & 82.5791 & 122.2674 & 39.6883 & 82.7514 & 122.2423 & 39.4908 \\
\hline 82.5679 & 122.1938 & 39.6259 & 82.6345 & 122.257 & 39.6225 & 82.7177 & 122.2163 & 39.4986 \\
\hline 82.579 & 122.1799 & 39.6009 & 82.6371 & 122.2761 & 39.6389 & 82.7073 & 122.2189 & 39.5116 \\
\hline 82.528 & 122.1955 & 39.6675 & 82.6458 & 122.2709 & 39.6251 & 82.6726 & 122.2016 & 39.5289 \\
\hline 82.4683 & 122.2293 & 39.761 & 82.6046 & 122.2425 & 39.6379 & 82.6475 & 122.1418 & 39.4943 \\
\hline 82.4717 & 122.1999 & 39.7281 & 82.606 & 122.2778 & 39.6718 & 82.7263 & 122.0665 & 39.3402 \\
\hline 82.4977 & 122.2639 & 39.7662 & 82.6008 & 122.2709 & 39.6701 & 82.698 & 122.1068 & 39.4089 \\
\hline 82.5254 & 122.2518 & 39.7264 & 82.6112 & 122.2648 & 39.6536 & 82.7159 & 122.1366 & 39.4207 \\
\hline 82.586 & 122.2302 & 39.6441 & 82.6086 & 122.2657 & 39.6571 & 82.7549 & 122.1366 & 39.3817 \\
\hline 82.5852 & 122.2293 & 39.6441 & 82.7082 & 122.2206 & 39.5125 & 82.735 & 122.1236 & 39.3887 \\
\hline 82.8138 & 122.2042 & 39.3904 & 82.7592 & 122.2059 & 39.4467 & 82.7229 & 122.0994 & 39.3765 \\
\hline 82.6068 & 122.1574 & 39.5506 & 82.7662 & 122.2302 & 39.464 & 82.7705 & 122.0907 & 39.3202 \\
\hline 82.6432 & 122.1323 & 39.4891 & 82.7632 & 122.2337 & 39.4705 & 82.7921 & 122.0873 & 39.2951 \\
\hline 82.6146 & 122.1323 & 39.5177 & 82.7575 & 122.2224 & 39.4649 & 82.8692 & 122.037 & 39.1678 \\
\hline 82.6181 & 122.1661 & 39.548 & 82.7211 & 122.2371 & 39.516 & 82.8565 & 122.0311 & 39.1745 \\
\hline 82.5713 & 122.1782 & 39.6069 & 82.7419 & 122.2397 & 39.4978 & 82.8848 & 122.0284 & 39.1436 \\
\hline 82.5332 & 122.1929 & 39.6597 & 82.7696 & 122.2414 & 39.4718 & 82.8753 & 122.044 & 39.1687 \\
\hline 82.5254 & 122.1895 & 39.664 & 82.7783 & 122.244 & 39.4657 & 82.8398 & 122.076 & 39.2362 \\
\hline 82.5042 & 122.1747 & 39.6705 & 82.7402 & 122.2622 & 39.522 & 82.7991 & 122.1046 & 39.3055 \\
\hline 82.4527 & 122.1903 & 39.7376 & 82.7021 & 122.2423 & 39.5402 & 82.7774 & 122.1176 & 39.3402 \\
\hline 82.4466 & 122.186 & 39.7394 & 82.6751 & 122.2742 & 39.5991 & 82.7402 & 122.1678 & 39.4276 \\
\hline 82.4674 & 122.1756 & 39.7082 & 82.6856 & 122.2787 & 39.593 & 82.683 & 122.1635 & 39.4804 \\
\hline 82.483 & 122.1592 & 39.6762 & 82.6683 & 122.296 & 39.6277 & 82.6539 & 122.1544 & 39.5005 \\
\hline 82.6181 & 122.1635 & 39.5454 & 82.6103 & 122.3159 & 39.7056 & 82.6562 & 122.2007 & 39.5445 \\
\hline 82.6337 & 122.1938 & 39.5601 & 82.6077 & 122.3133 & 39.7056 & 82.6718 & 122.3133 & 39.6415 \\
\hline 82.6259 & 122.1609 & 39.535 & 82.6259 & 122.3739 & 39.748 & 82.7237 & 122.3375 & 39.6138 \\
\hline 82.6198 & 122.1747 & 39.5549 & 82.6666 & 122.3471 & 39.6805 & 82.6389 & 122.3592 & 39.7203 \\
\hline 82.5755 & 122.1702 & 39.5947 & 82.6406 & 122.3635 & 39.7229 & 82.6164 & 122.3722 & 39.7558 \\
\hline 82.4899 & 122.1895 & 39.6995 & 82.6319 & 122.3488 & 39.7169 & 82.638 & 122.3644 & 39.7264 \\
\hline 82.515 & 122.1869 & 39.6718 & 82.6301 & 122.3632 & 39.733 & 82.6787 & 122.4259 & 39.7472 \\
\hline 82.522 & 122.2128 & 39.6909 & 82.6389 & 122.3886 & 39.7498 & 82.7385 & 122.4241 & 39.6857 \\
\hline 82.4986 & 122.244 & 39.7454 & 82.6752 & 122.3436 & 39.6684 & 82.7579 & 122.3966 & 39.6388 \\
\hline 82.5497 & 122.2596 & 39.7099 & 82.6536 & 122.3332 & 39.6796 & 82.735 & 122.4146 & 39.6796 \\
\hline 82.5549 & 122.2501 & 39.6952 & 82.6155 & 122.3367 & 39.7212 & 82.7151 & 122.4034 & 39.6883 \\
\hline 82.5142 & 122.2544 & 39.7402 & 82.6354 & 122.3635 & 39.7281 & 82.7012 & 122.3904 & 39.6891 \\
\hline 82.4945 & 122.2548 & 39.7603 & 82.6389 & 122.3618 & 39.7229 & 82.7177 & 122.4034 & 39.6857 \\
\hline 82.4475 & 122.3124 & 39.8649 & 82.703 & 122.3367 & 39.6337 & 82.7402 & 122.393 & 39.6528 \\
\hline 82.4544 & 122.3055 & 39.8511 & 82.6953 & 122.3905 & 39.6951 & 82.7792 & 122.4354 & 39.6562 \\
\hline 82.4917 & 122.2761 & 39.7844 & 82.7012 & 122.4094 & 39.7082 & 82.8138 & 122.4319 & 39.6181 \\
\hline 82.5653 & 122.2613 & 39.6961 & 82.7185 & 122.3886 & 39.6701 & 82.809 & 122.4054 & 39.5965 \\
\hline 82.5176 & 122.2501 & 39.7324 & 82.6527 & 122.3947 & 39.742 & 82.7644 & 122.412 & 39.6476 \\
\hline 82.4657 & 122.2778 & 39.8121 & 82.6164 & 122.4137 & 39.7974 & 82.7489 & 122.4103 & 39.6614 \\
\hline
\end{tabular}




\begin{tabular}{|c|c|c|c|c|c|c|c|c|}
\hline 82.7558 & 122.4657 & 39.7099 & 82.851 & 122.4527 & 39.6017 & 82.7748 & 122.5791 & 39.8043 \\
\hline 82.7748 & 122.4596 & 39.6848 & 82.9255 & 122.4319 & 39.5064 & 82.7306 & 122.6521 & 39.9215 \\
\hline 82.7913 & 122.4709 & 39.6796 & 82.864 & 122.4501 & 39.5861 & 82.7419 & 122.6129 & 39.871 \\
\hline 82.7826 & 122.4544 & 39.6718 & 82.8259 & 122.4778 & 39.6519 & 82.7142 & 122.6276 & 39.9134 \\
\hline 82.8588 & 122.4302 & 39.5714 & 82.7896 & 122.4718 & 39.6822 & 82.7653 & 122.6224 & 39.8571 \\
\hline 82.8266 & 122.4372 & 39.6106 & 82.7991 & 122.4501 & 39.651 & 82.7913 & 122.632 & 39.8407 \\
\hline 82.9177 & 122.4008 & 39.483 & 82.8199 & 122.4588 & 39.6389 & 82.8406 & 122.6432 & 39.8026 \\
\hline 82.9298 & 122.4189 & 39.4891 & 82.8803 & 122.4336 & 39.5533 & 82.8242 & 122.6839 & 39.8597 \\
\hline 82.9125 & 122.4259 & 39.5134 & 82.935 & 122.4302 & 39.4952 & 82.8424 & 122.6476 & 39.8052 \\
\hline 82.864 & 122.4562 & 39.5922 & 83.0104 & 122.4163 & 39.406 & 82.9684 & 122.6045 & 39.6361 \\
\hline 82.8675 & 122.4163 & 39.5489 & 82.9783 & 122.4111 & 39.4328 & 83.0615 & 122.593 & 39.5315 \\
\hline 82.8337 & 122.4068 & 39.5731 & 82.9212 & 122.4172 & 39.496 & 83.1394 & 122.5705 & 39.4311 \\
\hline 82.858 & 122.406 & 39.548 & 82.9047 & 122.4363 & 39.5315 & 83.1992 & 122.5878 & 39.3887 \\
\hline 82.8689 & 122.3359 & 39.467 & 82.9194 & 122.4389 & 39.5194 & 83.1507 & 122.6025 & 39.4519 \\
\hline 82.8147 & 122.3791 & 39.5644 & 82.8935 & 122.4293 & 39.5359 & 83.1983 & 122.6285 & 39.4302 \\
\hline 82.8398 & 122.4181 & 39.5783 & 82.8601 & 122.4416 & 39.5815 & 83.1896 & 122.6432 & 39.4536 \\
\hline 82.7887 & 122.4441 & 39.6554 & 82.948 & 122.4189 & 39.4709 & 83.1446 & 122.6744 & 39.5298 \\
\hline 82.8181 & 122.4562 & 39.6381 & 82.9498 & 122.412 & 39.4623 & 83.1358 & 122.6768 & 39.541 \\
\hline 82.7904 & 122.4181 & 39.6277 & 83.0286 & 122.406 & 39.3774 & 83.1429 & 122.7038 & 39.561 \\
\hline 82.7939 & 122.3938 & 39.5999 & 82.974 & 122.4137 & 39.4397 & 83.1022 & 122.7168 & 39.6147 \\
\hline 82.7887 & 122.4086 & 39.6199 & 83.0952 & 122.3947 & 39.2995 & 83.0684 & 122.7298 & 39.6614 \\
\hline 82.7491 & 122.3861 & 39.637 & 83.0892 & 122.4285 & 39.3393 & 83.0805 & 122.7307 & 39.6502 \\
\hline 82.8277 & 122.4008 & 39.5731 & 83.1758 & 122.412 & 39.2362 & 83.0632 & 122.7064 & 39.6432 \\
\hline 82.7939 & 122.4068 & 39.6129 & 83.1648 & 122.3949 & 39.23 & 83.0104 & 122.7567 & 39.7463 \\
\hline 82.7973 & 122.4016 & 39.6043 & 83.1048 & 122.4146 & 39.3098 & 82.9705 & 122.7749 & 39.8043 \\
\hline 82.8822 & 122.3505 & 39.4683 & 83.09 & 122.457 & 39.367 & 83.0072 & 122.793 & 39.7859 \\
\hline 82.9151 & 122.341 & 39.4259 & 83.0424 & 122.4995 & 39.4571 & 83.0762 & 122.7896 & 39.7134 \\
\hline 82.9818 & 122.341 & 39.3592 & 82.9653 & 122.5003 & 39.535 & 83.142 & 122.7402 & 39.5982 \\
\hline 83.0199 & 122.3142 & 39.2943 & 82.8943 & 122.5471 & 39.6528 & 83.2303 & 122.6718 & 39.4415 \\
\hline 83.016 & 122.3147 & 39.2987 & 82.8632 & 122.5999 & 39.7368 & 83.3429 & 122.6467 & 39.3038 \\
\hline 83.0459 & 122.3566 & 39.3107 & 82.9593 & 122.5592 & 39.5999 & 83.3178 & 122.6597 & 39.3419 \\
\hline 83.0407 & 122.3254 & 39.2847 & 83.0028 & 122.5526 & 39.5498 & 83.3611 & 122.6675 & 39.3064 \\
\hline 82.9991 & 122.3704 & 39.3713 & 83.0822 & 122.5211 & 39.4389 & 83.3368 & 122.6779 & 39.341 \\
\hline 82.9844 & 122.3765 & 39.3921 & 83.0632 & 122.5038 & 39.4406 & 83.2776 & 122.6741 & 39.3965 \\
\hline 83.0017 & 122.4224 & 39.4207 & 82.9844 & 122.5073 & 39.5229 & 83.278 & 122.7194 & 39.4415 \\
\hline 82.9939 & 122.3982 & 39.4042 & 82.9411 & 122.5471 & 39.606 & 83.2442 & 122.7038 & 39.4597 \\
\hline 82.9922 & 122.4112 & 39.419 & 82.9368 & 122.5194 & 39.5826 & 83.1498 & 122.7021 & 39.5523 \\
\hline 82.9411 & 122.4163 & 39.4752 & 82.9515 & 122.5333 & 39.5818 & 83.0727 & 122.7298 & 39.6571 \\
\hline 82.8882 & 122.4336 & 39.5454 & 82.9896 & 122.5177 & 39.5281 & 82.9965 & 122.7567 & 39.7602 \\
\hline 82.8692 & 122.4467 & 39.5774 & 82.8671 & 122.5658 & 39.6987 & 83.0381 & 122.7636 & 39.7255 \\
\hline 82.8268 & 122.4259 & 39.5991 & 82.7168 & 122.6025 & 39.8857 & 83.0831 & 122.7445 & 39.6614 \\
\hline 82.819 & 122.464 & 39.645 & 82.7185 & 122.6216 & 39.903 & 83.0908 & 122.7323 & 39.6414 \\
\hline 82.7973 & 122.483 & 39.6857 & 82.7341 & 122.5843 & 39.8502 & 83.0805 & 122.7402 & 39.6597 \\
\hline 82.7489 & 122.4865 & 39.7376 & 82.7644 & 122.5939 & 39.8294 & 83.058 & 122.7333 & 39.6753 \\
\hline 82.754 & 122.496 & 39.742 & 82.8199 & 122.5532 & 39.7333 & 83.0485 & 122.7558 & 39.7073 \\
\hline 82.8129 & 122.4752 & 39.6623 & 82.8658 & 122.5696 & 39.7039 & 83.0857 & 122.7523 & 39.6666 \\
\hline 82.8222 & 122.4601 & 39.6379 & 82.8415 & 122.5688 & 39.7272 & 83.1437 & 122.761 & 39.6173 \\
\hline
\end{tabular}




\begin{tabular}{|c|c|c|c|c|c|c|c|c|}
\hline 83.2658 & 122.7298 & 39.464 & 83.9551 & 122.5272 & 38.572 & 83.6789 & 122.7203 & 39.0414 \\
\hline 83.3775 & 122.6839 & 39.3064 & 84.0054 & 122.49 & 38.4846 & 83.6434 & 122.7402 & 39.0968 \\
\hline 83.323 & 122.6467 & 39.3237 & 83.9829 & 122.5255 & 38.5426 & 83.6798 & 122.7671 & 39.0873 \\
\hline 83.3771 & 122.6371 & 39.26 & 83.9785 & 122.5358 & 38.5573 & 83.6486 & 122.8104 & 39.1618 \\
\hline 83.3672 & 122.6354 & 39.2683 & 84.0374 & 122.5497 & 38.5123 & 83.6876 & 122.793 & 39.1055 \\
\hline 83.3316 & 122.6554 & 39.3237 & 83.8801 & 122.5156 & 38.6354 & 83.8382 & 122.7766 & 38.9383 \\
\hline 83.2563 & 122.6874 & 39.4311 & 83.9863 & 122.4874 & 38.501 & 83.9057 & 122.8124 & 38.9067 \\
\hline 83.2173 & 122.7151 & 39.4978 & 84.0556 & 122.5229 & 38.4673 & 83.6754 & 122.8303 & 39.1548 \\
\hline 83.1792 & 122.7099 & 39.5307 & 84.0036 & 122.541 & 38.5374 & 83.7551 & 122.8277 & 39.0726 \\
\hline 83.1784 & 122.6935 & 39.5151 & 84.0391 & 122.5514 & 38.5123 & 83.5802 & 122.8355 & 39.2553 \\
\hline 83.1403 & 122.7151 & 39.5748 & 83.8495 & 122.5497 & 38.7002 & 83.6806 & 122.8052 & 39.1245 \\
\hline 83.164 & 122.7023 & 39.5383 & 83.7664 & 122.5436 & 38.7773 & 83.7413 & 122.7861 & 39.0449 \\
\hline 83.1792 & 122.7073 & 39.5281 & 83.9015 & 122.548 & 38.6465 & 83.5906 & 122.8571 & 39.2665 \\
\hline 83.2113 & 122.7047 & 39.4934 & 84.0079 & 122.5112 & 38.5033 & 83.6659 & 122.8285 & 39.1626 \\
\hline 83.1697 & 122.7281 & 39.5584 & 83.7646 & 122.561 & 38.7963 & 83.6893 & 122.8623 & 39.173 \\
\hline 83.1619 & 122.7532 & 39.5913 & 83.8209 & 122.5575 & 38.7366 & 83.6581 & 122.83 & 39.1719 \\
\hline 83.155 & 122.7679 & 39.6129 & 83.7309 & 122.5393 & 38.8085 & 83.7923 & 122.806 & 39.0137 \\
\hline 83.1671 & 122.7272 & 39.5601 & 83.7413 & 122.5766 & 38.8353 & 83.7075 & 122.8398 & 39.1323 \\
\hline 83.1377 & 122.7679 & 39.6303 & 83.7109 & 122.58 & 38.8691 & 83.5265 & 122.8537 & 39.3272 \\
\hline 83.1331 & 122.764 & 39.6308 & 83.6988 & 122.5982 & 38.8994 & 83.5689 & 122.8294 & 39.2605 \\
\hline 83.2321 & 122.7506 & 39.5185 & 83.6997 & 122.6051 & 38.9054 & 83.5689 & 122.8597 & 39.2908 \\
\hline 83.2295 & 122.7203 & 39.4908 & 83.8202 & 122.5534 & 38.7332 & 83.6962 & 122.7948 & 39.0986 \\
\hline 83.2373 & 122.7246 & 39.4874 & 83.6971 & 122.5566 & 38.8595 & 83.7248 & 122.8026 & 39.0778 \\
\hline 83.3498 & 122.6839 & 39.3341 & 83.6659 & 122.5783 & 38.9124 & 83.903 & 122.6997 & 38.7966 \\
\hline 83.4001 & 122.6718 & 39.2717 & 83.7655 & 122.5705 & 38.805 & 83.5438 & 122.7818 & 39.238 \\
\hline 83.3966 & 122.6484 & 39.2518 & 83.73 & 122.5696 & 38.8396 & 83.6027 & 122.7394 & 39.1367 \\
\hline 83.9525 & 122.3852 & 38.4326 & 83.7326 & 122.5791 & 38.8466 & 83.5819 & 122.7783 & 39.1964 \\
\hline 83.9339 & 122.4028 & 38.4689 & 83.8833 & 122.5722 & 38.6889 & 83.8452 & 122.7792 & 38.934 \\
\hline 83.9196 & 122.4103 & 38.4906 & 83.9196 & 122.5722 & 38.6526 & 83.7499 & 122.819 & 39.0691 \\
\hline 83.8703 & 122.451 & 38.5807 & 83.8995 & 122.5737 & 38.6742 & 83.7759 & 122.8242 & 39.0483 \\
\hline 83.7889 & 122.4683 & 38.6794 & 83.8989 & 122.6242 & 38.7253 & 83.8911 & 122.7593 & 38.8682 \\
\hline 83.7499 & 122.4882 & 38.7383 & 83.7257 & 122.6528 & 38.9271 & 83.8114 & 122.7481 & 38.9367 \\
\hline 83.8209 & 122.5159 & 38.695 & 83.704 & 122.6579 & 38.9539 & 84.1058 & 122.7038 & 38.598 \\
\hline 83.7508 & 122.5081 & 38.7574 & 83.7066 & 122.6952 & 38.9886 & 83.95 & 122.6952 & 38.7452 \\
\hline 83.9266 & 122.4415 & 38.5149 & 83.665 & 122.6857 & 39.0206 & 83.8244 & 122.7627 & 38.9383 \\
\hline 83.9488 & 122.438 & 38.4892 & 83.7975 & 122.6935 & 38.8959 & 83.8469 & 122.7766 & 38.9297 \\
\hline 83.8408 & 122.4484 & 38.6075 & 83.6451 & 122.7151 & 39.07 & 83.8867 & 122.7636 & 38.8769 \\
\hline 83.8157 & 122.49 & 38.6742 & 83.822 & 122.7102 & 38.8882 & 83.8772 & 122.7497 & 38.8725 \\
\hline 83.9144 & 122.5029 & 38.5885 & 83.6798 & 122.7671 & 39.0873 & 84.1673 & 122.6172 & 38.4499 \\
\hline 83.9205 & 122.5185 & 38.598 & 83.7205 & 122.7316 & 39.0111 & 84.347 & 122.5252 & 38.1782 \\
\hline 83.8053 & 122.5168 & 38.7115 & 83.7187 & 122.7679 & 39.0492 & 84.5215 & 122.4224 & 37.9009 \\
\hline 83.9361 & 122.47 & 38.5339 & 83.6728 & 122.7688 & 39.096 & 84.4479 & 122.4839 & 38.036 \\
\hline 83.8668 & 122.4692 & 38.6024 & 83.7447 & 122.7636 & 39.0189 & 84.5102 & 122.4441 & 37.9338 \\
\hline 83.9497 & 122.4671 & 38.5174 & 83.7404 & 122.7541 & 39.0137 & 84.4427 & 122.4319 & 37.9892 \\
\hline 83.8209 & 122.5281 & 38.7071 & 83.8841 & 122.748 & 38.8639 & 84.4436 & 122.4519 & 38.0083 \\
\hline 83.8088 & 122.5047 & 38.6959 & 83.829 & 122.7349 & 38.9059 & 84.4418 & 122.4562 & 38.0144 \\
\hline 83.9283 & 122.5029 & 38.5746 & 83.5854 & 122.7428 & 39.1574 & 84.3457 & 122.5315 & 38.1858 \\
\hline
\end{tabular}




\begin{tabular}{|c|c|c|c|c|c|c|c|c|}
\hline 84.1788 & 122.6089 & 38.4302 & 83.0467 & 123.1091 & 40.0624 & 82.8051 & 123.3741 & 40.569 \\
\hline 84.1179 & 122.6207 & 38.5028 & 82.9775 & 123.1091 & 40.1317 & 82.9601 & 123.3299 & 40.3698 \\
\hline 84.1725 & 122.5679 & 38.3954 & 82.9499 & 123.1472 & 40.1973 & 83.0052 & 123.3577 & 40.3525 \\
\hline 83.7785 & 122.7047 & 38.9262 & 82.9584 & 123.1862 & 40.2278 & 83.1082 & 123.284 & 40.1758 \\
\hline 83.9101 & 122.6987 & 38.7885 & 82.9437 & 123.181 & 40.2373 & 83.0627 & 123.2872 & 40.2246 \\
\hline 83.7698 & 122.7653 & 38.9955 & 82.9316 & 123.1706 & 40.239 & 83.045 & 123.3092 & 40.2641 \\
\hline 83.7984 & 122.7489 & 38.9505 & 83.1429 & 123.0693 & 39.9264 & 83.0251 & 123.3282 & 40.3031 \\
\hline 83.7776 & 122.7506 & 38.973 & 83.0476 & 123.0927 & 40.0451 & 83.0043 & 123.3334 & 40.3291 \\
\hline 83.7639 & 122.7472 & 38.9834 & 83.0623 & 123.0771 & 40.0147 & 82.9957 & 123.3629 & 40.3672 \\
\hline 83.9041 & 122.6935 & 38.7894 & 83.2095 & 122.9965 & 39.787 & 83.013 & 123.3741 & 40.3611 \\
\hline 83.943 & 122.722 & 38.779 & 83.3172 & 123.0053 & 39.6881 & 83.0086 & 123.3256 & 40.317 \\
\hline 83.833 & 122.7376 & 38.9046 & 83.2771 & 122.9836 & 39.7065 & 82.9506 & 123.3724 & 40.4218 \\
\hline 83.9378 & 122.6744 & 38.7366 & 83.2528 & 123.0061 & 39.7532 & 82.8759 & 123.3859 & 40.51 \\
\hline 83.9326 & 122.6805 & 38.7478 & 83.1602 & 123.0753 & 39.9152 & 82.7445 & 123.4434 & 40.6989 \\
\hline 83.7949 & 122.7108 & 38.9158 & 83.1307 & 123.0667 & 39.9359 & 82.8753 & 123.5456 & 40.6703 \\
\hline 83.8001 & 122.6822 & 38.8821 & 83.1178 & 123.1005 & 39.9827 & 83.0407 & 123.3949 & 40.3542 \\
\hline 83.8313 & 122.7437 & 38.9124 & 83.1065 & 123.1083 & 40.0018 & 83.0684 & 123.3031 & 40.2347 \\
\hline 83.6247 & 122.8089 & 39.1842 & 83.1559 & 123.0883 & 39.9325 & 83.0649 & 123.2078 & 40.1429 \\
\hline 83.5533 & 122.8822 & 39.3289 & 83.194 & 123.0667 & 39.8727 & 83.019 & 123.1975 & 40.1784 \\
\hline 83.549 & 122.8952 & 39.3462 & 83.2485 & 123.0793 & 39.8308 & 82.9627 & 123.1758 & 40.2131 \\
\hline 83.5481 & 122.9039 & 39.3557 & 83.1524 & 123.1446 & 39.9922 & 82.9186 & 123.1524 & 40.2338 \\
\hline 83.5767 & 122.8623 & 39.2856 & 83.1749 & 123.1342 & 39.9593 & 83.1939 & 122.9587 & 39.7647 \\
\hline 83.5334 & 122.8987 & 39.3653 & 83.1377 & 123.1585 & 40.0208 & 83.4563 & 122.7965 & 39.3402 \\
\hline 83.5793 & 122.8563 & 39.2769 & 83.0909 & 123.142 & 40.0511 & 83.6001 & 122.7073 & 39.1072 \\
\hline 83.446 & 122.9437 & 39.4978 & 83.0675 & 123.1412 & 40.0736 & 83.8893 & 122.606 & 38.7167 \\
\hline 83.4767 & 122.912 & 39.4353 & 83.0623 & 123.155 & 40.0927 & 84.1067 & 122.3696 & 38.2629 \\
\hline 83.5802 & 122.8389 & 39.2588 & 83.2745 & 123.0953 & 39.8208 & 84.2288 & 122.2795 & 38.0507 \\
\hline 83.5412 & 122.8857 & 39.3445 & 83.3886 & 123.0881 & 39.6996 & 84.3734 & 122.1687 & 37.7953 \\
\hline 83.452 & 122.8866 & 39.4345 & 83.4598 & 123.0554 & 39.5956 & 84.4695 & 122.0916 & 37.6221 \\
\hline 83.4321 & 122.8848 & 39.4527 & 83.3628 & 123.065 & 39.7021 & 84.2765 & 122.2028 & 37.9263 \\
\hline 83.3879 & 122.8952 & 39.5073 & 83.3083 & 123.0961 & 39.7879 & 84.4392 & 122.1089 & 37.6697 \\
\hline 83.4191 & 122.8918 & 39.4727 & 83.2676 & 123.1325 & 39.8649 & 84.4583 & 122.1323 & 37.674 \\
\hline 83.3637 & 122.9351 & 39.5714 & 83.2451 & 123.136 & 39.8909 & 84.5327 & 122.0942 & 37.5615 \\
\hline 83.3084 & 122.9322 & 39.6238 & 83.2494 & 123.1689 & 39.9195 & 84.3111 & 122.1947 & 37.8836 \\
\hline 83.2806 & 122.9472 & 39.6666 & 83.2451 & 123.1351 & 39.89 & 84.2885 & 122.2172 & 37.9286 \\
\hline 83.3065 & 122.9264 & 39.6199 & 83.2406 & 123.1613 & 39.9207 & 84.305 & 122.2241 & 37.9191 \\
\hline 83.2849 & 122.9359 & 39.651 & 83.2537 & 123.1749 & 39.9212 & 84.4254 & 122.173 & 37.7476 \\
\hline 83.2658 & 122.9541 & 39.6883 & 83.174 & 123.2018 & 40.0277 & 84.3083 & 122.1835 & 37.8752 \\
\hline 83.1836 & 122.9853 & 39.8017 & 83.129 & 123.2018 & 40.0728 & 84.3258 & 122.1635 & 37.8377 \\
\hline 83.1255 & 123.0234 & 39.8978 & 83.0415 & 123.2537 & 40.2122 & 84.4436 & 122.0933 & 37.6498 \\
\hline 83.1411 & 123.0572 & 39.916 & 83.0407 & 123.2485 & 40.2079 & 84.5172 & 122.0526 & 37.5355 \\
\hline 83.2062 & 123.0441 & 39.8379 & 83.0078 & 123.2633 & 40.2555 & 84.5648 & 122.031 & 37.4662 \\
\hline 83.2234 & 123.0554 & 39.832 & 82.9801 & 123.2797 & 40.2997 & 84.6228 & 121.9548 & 37.332 \\
\hline 83.3568 & 123.0061 & 39.6493 & 82.9067 & 123.3163 & 40.4096 & 84.7068 & 121.9548 & 37.248 \\
\hline 83.3663 & 122.9714 & 39.6051 & 82.7757 & 123.3923 & 40.6166 & 84.7804 & 121.8595 & 37.0791 \\
\hline 83.3013 & 123.0026 & 39.7013 & 82.8207 & 123.362 & 40.5413 & 84.7267 & 121.8637 & 37.137 \\
\hline 83.1948 & 123.0381 & 39.8433 & 82.8069 & 123.4027 & 40.5958 & 84.7709 & 121.8742 & 37.1034 \\
\hline
\end{tabular}




\begin{tabular}{|c|c|c|c|c|c|c|c|c|}
\hline 84.7553 & 121.8708 & 37.1155 & 84.7989 & 122.2962 & 37.4973 & 84.9467 & 122.5298 & 37.5831 \\
\hline 84.6869 & 121.9375 & 37.2506 & 84.492 & 122.4086 & 37.9165 & 84.9138 & 122.5177 & 37.6039 \\
\hline 84.7432 & 121.824 & 37.0808 & 84.744 & 122.3081 & 37.5641 & 84.9434 & 122.5235 & 37.5801 \\
\hline 84.7562 & 121.8379 & 37.0817 & 84.4557 & 122.4467 & 37.991 & 85.0116 & 122.5107 & 37.4991 \\
\hline 84.8289 & 121.8084 & 36.9795 & 84.7241 & 122.3523 & 37.6281 & 84.9623 & 122.5246 & 37.5623 \\
\hline 84.6774 & 121.8942 & 37.2168 & 84.6003 & 122.3635 & 37.7632 & 84.9614 & 122.5237 & 37.5623 \\
\hline 84.6333 & 121.8892 & 37.2559 & 84.5674 & 122.3886 & 37.8212 & 84.9129 & 122.5367 & 37.6238 \\
\hline 84.5908 & 121.9522 & 37.3614 & 84.4522 & 122.4501 & 37.9979 & 84.9112 & 122.5125 & 37.6013 \\
\hline 84.5388 & 122.0024 & 37.4636 & 84.1955 & 122.5667 & 38.3712 & 84.8523 & 122.5073 & 37.655 \\
\hline 84.4184 & 122.0994 & 37.681 & 84.1872 & 122.5462 & 38.359 & 84.9068 & 122.4709 & 37.5641 \\
\hline 84.4643 & 122.0535 & 37.5892 & 84.2426 & 122.4796 & 38.2369 & 85.0235 & 122.3887 & 37.3652 \\
\hline 84.2704 & 122.1531 & 37.8827 & 84.0651 & 122.5289 & 38.4638 & 85.0454 & 122.4224 & 37.377 \\
\hline 84.2756 & 122.1921 & 37.9165 & 84.2634 & 122.477 & 38.2135 & 84.9441 & 122.47 & 37.526 \\
\hline 84.1491 & 122.2934 & 38.1443 & 84.1006 & 122.5073 & 38.4066 & 84.7466 & 122.5809 & 37.8342 \\
\hline 84.2034 & 122.2205 & 38.017 & 84.1699 & 122.4596 & 38.2897 & 84.6228 & 122.6787 & 38.0559 \\
\hline 84.2401 & 122.173 & 37.933 & 84.4687 & 122.3289 & 37.8602 & 84.5128 & 122.7601 & 38.2473 \\
\hline 84.2357 & 122.1947 & 37.9589 & 84.4192 & 122.3552 & 37.936 & 84.5812 & 122.6727 & 38.0914 \\
\hline 84.3448 & 122.1141 & 37.7693 & 84.2963 & 122.3834 & 38.0871 & 84.6081 & 122.6753 & 38.0672 \\
\hline 84.1024 & 122.2284 & 38.1261 & 84.3613 & 122.3531 & 37.9918 & 84.7055 & 122.6512 & 37.9457 \\
\hline 83.9465 & 122.3185 & 38.372 & 84.434 & 122.3453 & 37.9113 & 84.7475 & 122.619 & 37.8715 \\
\hline 83.8088 & 122.4086 & 38.5998 & 84.1223 & 122.4596 & 38.3374 & 84.699 & 122.6458 & 37.9468 \\
\hline 83.7603 & 122.4215 & 38.6612 & 84.1179 & 122.5324 & 38.4144 & 84.6427 & 122.6969 & 38.0542 \\
\hline 83.7941 & 122.4562 & 38.6621 & 84.2236 & 122.4856 & 38.262 & 84.3024 & 122.8441 & 38.5417 \\
\hline 83.6388 & 122.5094 & 38.8706 & 84.1491 & 122.5627 & 38.4136 & 84.2712 & 122.8225 & 38.5513 \\
\hline 83.5516 & 122.5921 & 39.0405 & 84.176 & 122.5393 & 38.3633 & 84.2097 & 122.8649 & 38.6552 \\
\hline 83.5793 & 122.606 & 39.0267 & 84.1611 & 122.5358 & 38.3747 & 84.5431 & 122.6995 & 38.1564 \\
\hline 83.5629 & 122.6614 & 39.0986 & 84.3414 & 122.4804 & 38.1391 & 84.4115 & 122.748 & 38.3365 \\
\hline 83.7057 & 122.5861 & 38.8803 & 83.9474 & 122.6398 & 38.6924 & 84.2633 & 122.8019 & 38.5385 \\
\hline 83.6858 & 122.606 & 38.9202 & 84.0348 & 122.6121 & 38.5772 & 84.2262 & 122.7827 & 38.5565 \\
\hline 84.1145 & 122.3878 & 38.2733 & 84.0954 & 122.6467 & 38.5513 & 84.3007 & 122.7766 & 38.4759 \\
\hline 83.4693 & 122.6346 & 39.1652 & 84.1405 & 122.6034 & 38.4629 & 84.3734 & 122.7636 & 38.3902 \\
\hline 83.1913 & 122.7728 & 39.5815 & 84.3561 & 122.7411 & 38.385 & 84.4591 & 122.7038 & 38.2447 \\
\hline 83.5603 & 122.677 & 39.1167 & 84.3561 & 122.9177 & 38.5617 & 84.3483 & 122.7896 & 38.4413 \\
\hline 83.5759 & 122.5835 & 39.0076 & 84.4941 & 122.8908 & 38.3967 & 84.3189 & 122.7463 & 38.4274 \\
\hline 83.7161 & 122.651 & 38.9349 & 84.5743 & 122.7601 & 38.1858 & 84.363 & 122.7116 & 38.3486 \\
\hline 83.5282 & 122.6831 & 39.1548 & 84.8436 & 122.5497 & 37.7061 & 84.3699 & 122.7226 & 38.3527 \\
\hline 83.5499 & 122.7168 & 39.167 & 84.8904 & 122.5558 & 37.6654 & 84.2747 & 122.7939 & 38.5192 \\
\hline 83.8469 & 122.5774 & 38.7305 & 84.7787 & 122.6285 & 37.8498 & 84.3076 & 122.8242 & 38.5166 \\
\hline 84.3769 & 122.3843 & 38.0074 & 84.9727 & 122.4848 & 37.5121 & 84.3266 & 122.7974 & 38.4707 \\
\hline 84.4351 & 122.3376 & 37.9025 & 85.0411 & 122.4137 & 37.3727 & 84.2522 & 122.8337 & 38.5816 \\
\hline 84.253 & 122.477 & 38.2239 & 85.0212 & 122.4458 & 37.4246 & 84.0556 & 122.9506 & 38.895 \\
\hline 84.1102 & 122.4718 & 38.3616 & 85.0482 & 122.4433 & 37.3951 & 84.2054 & 122.8641 & 38.6586 \\
\hline 83.9803 & 122.5558 & 38.5755 & 85.0497 & 122.4596 & 37.4099 & 84.3708 & 122.8407 & 38.4699 \\
\hline 83.691 & 122.6761 & 38.9851 & 84.8852 & 122.5705 & 37.6853 & 84.0087 & 122.9904 & 38.9816 \\
\hline 83.3689 & 122.7653 & 39.3964 & 84.7917 & 122.5722 & 37.7805 & 83.8815 & 123.0372 & 39.1557 \\
\hline 84.4271 & 122.4302 & 38.0031 & 84.8826 & 122.5021 & 37.6195 & 83.7482 & 123.1619 & 39.4138 \\
\hline 84.6704 & 122.3107 & 37.6403 & 84.9441 & 122.5003 & 37.5563 & 83.9586 & 123.0346 & 39.076 \\
\hline
\end{tabular}




\begin{tabular}{|c|c|c|c|c|c|c|c|c|}
\hline 83.6893 & 123.1481 & 39.4588 & 85.4914 & 122.406 & 36.9146 & 85.723 & 122.5455 & 36.8225 \\
\hline 83.4988 & 123.2442 & 39.7454 & 85.3511 & 122.47 & 37.1189 & 85.72 & 122.5662 & 36.8462 \\
\hline 83.4901 & 123.2598 & 39.7697 & 85.3191 & 122.4631 & 37.1441 & 85.6784 & 122.5904 & 36.912 \\
\hline 83.5031 & 123.2728 & 39.7697 & 85.3095 & 122.4397 & 37.1302 & 85.6724 & 122.5852 & 36.9128 \\
\hline 83.6079 & 123.2291 & 39.6212 & 85.3294 & 122.4553 & 37.1259 & 85.7174 & 122.5679 & 36.8505 \\
\hline 83.6434 & 123.2537 & 39.6103 & 85.2515 & 122.5203 & 37.2688 & 85.6836 & 122.5817 & 36.8981 \\
\hline 83.7118 & 123.233 & 39.5211 & 85.198 & 122.5173 & 37.3194 & 85.6481 & 122.6276 & 36.9795 \\
\hline 83.756 & 123.2174 & 39.4614 & 85.1969 & 122.5601 & 37.3631 & 85.6923 & 122.5991 & 36.9068 \\
\hline 83.6702 & 123.2693 & 39.5991 & 85.0913 & 122.645 & 37.5537 & 85.693 & 122.5931 & 36.9 \\
\hline 83.7603 & 123.2208 & 39.4605 & 85.177 & 122.6198 & 37.4428 & 85.7226 & 122.5566 & 36.834 \\
\hline 83.9292 & 123.1446 & 39.2155 & 85.2368 & 122.6086 & 37.3718 & 85.7009 & 122.5714 & 36.8704 \\
\hline 83.9586 & 123.1299 & 39.1713 & 85.2957 & 122.5549 & 37.2592 & 85.7087 & 122.535 & 36.8262 \\
\hline 83.9048 & 123.1542 & 39.2494 & 85.3121 & 122.5636 & 37.2514 & 85.6862 & 122.5843 & 36.8981 \\
\hline 83.6018 & 123.3066 & 39.7047 & 85.4983 & 122.7212 & 37.2229 & 85.7538 & 122.5921 & 36.8384 \\
\hline 83.9153 & 123.1784 & 39.2631 & 85.3213 & 122.6988 & 37.3775 & 85.7815 & 122.5428 & 36.7613 \\
\hline 84.04 & 123.1325 & 39.0925 & 85.2351 & 122.6484 & 37.4134 & 85.7183 & 122.5965 & 36.8782 \\
\hline 83.9387 & 123.2338 & 39.2951 & 85.2931 & 122.5384 & 37.2454 & 85.6913 & 122.5975 & 36.9062 \\
\hline 84.2885 & 123.0346 & 38.7461 & 85.1944 & 122.5159 & 37.3216 & 85.6784 & 122.6354 & 36.957 \\
\hline 83.8815 & 123.1862 & 39.3047 & 85.171 & 122.4319 & 37.261 & 85.6836 & 122.6103 & 36.9267 \\
\hline 83.9075 & 123.2087 & 39.3012 & 85.158 & 122.4016 & 37.2436 & 85.7113 & 122.5947 & 36.8834 \\
\hline 83.9524 & 123.1384 & 39.186 & 85.1095 & 122.4267 & 37.3173 & 85.8291 & 122.5748 & 36.7457 \\
\hline 83.885 & 123.1645 & 39.2795 & 84.9623 & 122.4648 & 37.5026 & 85.8239 & 122.6086 & 36.7847 \\
\hline 83.833 & 123.2191 & 39.3861 & 85.0438 & 122.3746 & 37.3308 & 85.856 & 122.5791 & 36.7232 \\
\hline 83.8426 & 123.1983 & 39.3557 & 84.977 & 122.367 & 37.39 & 85.8802 & 122.5817 & 36.7015 \\
\hline 83.8157 & 123.2468 & 39.4311 & 84.8549 & 122.4172 & 37.5623 & 85.8305 & 122.5966 & 36.7662 \\
\hline 83.9188 & 123.1897 & 39.2709 & 84.7544 & 122.4458 & 37.6914 & 85.8282 & 122.5973 & 36.7691 \\
\hline 83.9889 & 123.1619 & 39.173 & 84.6826 & 122.4406 & 37.758 & 85.7927 & 122.6173 & 36.8245 \\
\hline 84.5232 & 122.8372 & 38.314 & 84.7103 & 122.4371 & 37.7269 & 85.8213 & 122.5965 & 36.7752 \\
\hline 85.1164 & 122.5402 & 37.4238 & 84.6878 & 122.4198 & 37.7321 & 85.733 & 122.6121 & 36.8791 \\
\hline 85.0482 & 122.5913 & 37.5431 & 84.6592 & 122.4588 & 37.7996 & 85.7694 & 122.6181 & 36.8488 \\
\hline 85.2238 & 122.5211 & 37.2973 & 84.828 & 122.3394 & 37.5114 & 85.8395 & 122.6008 & 36.7613 \\
\hline 85.261 & 122.5133 & 37.2523 & 84.893 & 122.3436 & 37.4506 & 85.8984 & 122.5705 & 36.6721 \\
\hline 85.352 & 122.4215 & 37.0696 & 84.9917 & 122.4865 & 37.4948 & 86.0947 & 122.5781 & 36.4834 \\
\hline 85.3546 & 122.4293 & 37.0748 & 85.2402 & 122.638 & 37.3978 & 86.1383 & 122.5246 & 36.3863 \\
\hline 85.2835 & 122.4735 & 37.19 & 85.5459 & 122.4744 & 36.9284 & 86.2084 & 122.5722 & 36.3638 \\
\hline 85.2203 & 122.509 & 37.2887 & 85.5381 & 122.4822 & 36.944 & 86.2785 & 122.5142 & 36.2357 \\
\hline 85.2273 & 122.5211 & 37.2939 & 85.4221 & 122.5566 & 37.1345 & 86.2422 & 122.5333 & 36.2911 \\
\hline 85.2473 & 122.5235 & 37.2762 & 85.4308 & 122.5428 & 37.112 & 86.1642 & 122.5774 & 36.4132 \\
\hline 85.1909 & 122.5575 & 37.3666 & 85.4178 & 122.58 & 37.1622 & 86.2283 & 122.5748 & 36.3465 \\
\hline 85.0575 & 122.651 & 37.5935 & 85.627 & 122.5138 & 36.8868 & 86.1997 & 122.6198 & 36.4201 \\
\hline 85.1026 & 122.5445 & 37.442 & 85.6533 & 122.457 & 36.8037 & 86.1651 & 122.6432 & 36.4781 \\
\hline 85.1614 & 122.5497 & 37.3883 & 85.5381 & 122.5324 & 36.9942 & 85.9952 & 122.7481 & 36.7529 \\
\hline 85.1831 & 122.5384 & 37.3554 & 85.6143 & 122.4432 & 36.8288 & 86.0136 & 122.7203 & 36.7067 \\
\hline 85.2177 & 122.4709 & 37.2532 & 85.5962 & 122.5211 & 36.925 & 85.9469 & 122.7593 & 36.8124 \\
\hline 85.2515 & 122.4397 & 37.1882 & 85.6473 & 122.5774 & 36.9302 & 85.901 & 122.7177 & 36.8167 \\
\hline 85.2605 & 122.4821 & 37.2216 & 85.7382 & 122.5272 & 36.789 & 85.8568 & 122.7333 & 36.8765 \\
\hline 85.2671 & 122.5099 & 37.2428 & 85.7486 & 122.5116 & 36.763 & 85.9322 & 122.7151 & 36.7829 \\
\hline
\end{tabular}




\begin{tabular}{|c|c|c|c|c|c|c|c|c|}
\hline 86.1183 & 122.6623 & 36.5439 & 86.5712 & 121.7877 & 35.2164 & 86.9852 & 121.3339 & 34.3487 \\
\hline 86.0681 & 122.6883 & 36.6201 & 86.5938 & 121.8024 & 35.2086 & 86.9705 & 121.3703 & 34.3998 \\
\hline 86.1714 & 122.6574 & 36.486 & 86.5686 & 121.7998 & 35.2311 & 86.9843 & 121.3754 & 34.3911 \\
\hline 86.1634 & 122.6458 & 36.4825 & 86.5314 & 121.7954 & 35.264 & 87.0181 & 121.359 & 34.3409 \\
\hline 86.1729 & 122.6242 & 36.4513 & 86.5105 & 121.8144 & 35.3039 & 87.1004 & 121.3114 & 34.211 \\
\hline 86.1859 & 122.5982 & 36.4123 & 86.4526 & 121.7643 & 35.3117 & 87.1004 & 121.3373 & 34.237 \\
\hline 86.101 & 122.6528 & 36.5517 & 86.4673 & 121.7348 & 35.2675 & 87.0672 & 121.3519 & 34.2847 \\
\hline 86.1071 & 122.658 & 36.5509 & 86.3799 & 121.7374 & 35.3576 & 87.0458 & 121.3997 & 34.3539 \\
\hline 86.1235 & 122.6441 & 36.5206 & 86.3435 & 121.7106 & 35.3671 & 86.993 & 121.3936 & 34.4007 \\
\hline 86.1945 & 122.6631 & 36.4686 & 86.3045 & 121.7088 & 35.4043 & 86.9765 & 121.398 & 34.4214 \\
\hline 86.1291 & 122.6495 & 36.5204 & 86.2751 & 121.6985 & 35.4234 & 87.0172 & 121.4672 & 34.45 \\
\hline 86.0698 & 122.7038 & 36.634 & 86.2552 & 121.6985 & 35.4433 & 87.0397 & 121.5166 & 34.4769 \\
\hline 86.0127 & 122.722 & 36.7093 & 86.1855 & 121.6981 & 35.5126 & 87.0311 & 121.5357 & 34.5046 \\
\hline 86.0188 & 122.7168 & 36.6981 & 86.1807 & 121.7504 & 35.5697 & 87.0406 & 121.5807 & 34.5401 \\
\hline 85.9642 & 122.7419 & 36.7778 & 86.2301 & 121.766 & 35.536 & 86.9554 & 121.5959 & 34.6405 \\
\hline 86.0664 & 122.6883 & 36.6219 & 86.2214 & 121.74 & 35.5186 & 86.9358 & 121.5712 & 34.6353 \\
\hline 86.0214 & 122.6961 & 36.6747 & 86.2387 & 121.74 & 35.5013 & 86.9358 & 121.5504 & 34.6146 \\
\hline 86.0006 & 122.7186 & 36.718 & 86.2448 & 121.7599 & 35.5152 & 86.9375 & 121.5244 & 34.5868 \\
\hline 86.0753 & 122.7032 & 36.6278 & 86.3166 & 121.7868 & 35.4701 & 86.928 & 121.5192 & 34.5912 \\
\hline 86.1893 & 122.6952 & 36.5058 & 86.3132 & 121.7911 & 35.4779 & 86.8873 & 121.5469 & 34.6596 \\
\hline 86.0387 & 122.7307 & 36.692 & 86.329 & 121.81 & 35.4809 & 86.8968 & 121.5504 & 34.6535 \\
\hline 85.8984 & 122.8268 & 36.9284 & 86.3184 & 121.7521 & 35.4338 & 86.8683 & 121.5867 & 34.7185 \\
\hline 85.843 & 122.8337 & 36.9908 & 86.256 & 121.7279 & 35.4719 & 86.8426 & 121.6364 & 34.7938 \\
\hline 85.8707 & 122.7939 & 36.9232 & 86.2335 & 121.7054 & 35.4719 & 86.9029 & 121.6231 & 34.7202 \\
\hline 86.8873 & 122.1366 & 35.2493 & 86.1547 & 121.7175 & 35.5628 & 86.8925 & 121.6352 & 34.7427 \\
\hline 86.967 & 122.0596 & 35.0926 & 86.1599 & 121.7175 & 35.5576 & 86.896 & 121.637 & 34.741 \\
\hline 86.9721 & 122.076 & 35.1039 & 86.2119 & 121.7244 & 35.5126 & 86.8925 & 121.6179 & 34.7254 \\
\hline 86.9886 & 122.0847 & 35.096 & 86.1677 & 121.7478 & 35.5801 & 86.9055 & 121.6248 & 34.7193 \\
\hline 86.941 & 122.0786 & 35.1376 & 86.1317 & 121.7439 & 35.6122 & 86.9185 & 121.6188 & 34.7003 \\
\hline 86.9254 & 122.0752 & 35.1497 & 86.1261 & 121.7392 & 35.613 & 86.9713 & 121.6223 & 34.6509 \\
\hline 86.9583 & 122.0977 & 35.1393 & 86.1149 & 121.7877 & 35.6728 & 86.9642 & 121.6197 & 34.6555 \\
\hline 86.8917 & 122.0985 & 35.2069 & 86.1634 & 121.7747 & 35.6113 & 86.9453 & 121.6197 & 34.6743 \\
\hline 86.7635 & 121.9652 & 35.2017 & 86.1945 & 121.8284 & 35.6338 & 86.9644 & 121.6335 & 34.6691 \\
\hline 86.7159 & 121.8578 & 35.1419 & 86.1989 & 121.8266 & 35.6277 & 86.9453 & 121.6508 & 34.7055 \\
\hline 86.6109 & 121.8249 & 35.214 & 86.1287 & 121.8812 & 35.7524 & 86.909 & 121.695 & 34.786 \\
\hline 86.534 & 121.7651 & 35.2311 & 86.1036 & 121.876 & 35.7724 & 86.9462 & 121.6604 & 34.7141 \\
\hline 86.5262 & 121.7556 & 35.2294 & 86.1449 & 121.8804 & 35.7355 & 86.9713 & 121.6751 & 34.7038 \\
\hline 86.5531 & 121.7175 & 35.1644 & 86.56 & 121.4811 & 34.9211 & 86.9298 & 121.6759 & 34.7462 \\
\hline 86.6258 & 121.6985 & 35.0727 & 86.8587 & 121.2845 & 34.4258 & 86.8682 & 121.6664 & 34.7982 \\
\hline 86.6223 & 121.7201 & 35.0978 & 86.8449 & 121.2958 & 34.4509 & 86.8319 & 121.6751 & 34.8432 \\
\hline 86.6111 & 121.7591 & 35.148 & 86.8994 & 121.288 & 34.3885 & 86.8319 & 121.6396 & 34.8077 \\
\hline 86.5634 & 121.753 & 35.1896 & 86.8951 & 121.2906 & 34.3955 & 86.838 & 121.6474 & 34.8094 \\
\hline 86.5453 & 121.7495 & 35.2043 & 86.9731 & 121.3166 & 34.3435 & 86.8492 & 121.6326 & 34.7834 \\
\hline 86.5272 & 121.7597 & 35.2325 & 87.0172 & 121.3122 & 34.295 & 86.8613 & 121.6361 & 34.7748 \\
\hline 86.5461 & 121.7357 & 35.1896 & 87.0155 & 121.3356 & 34.3201 & 86.8527 & 121.6638 & 34.8111 \\
\hline 86.5981 & 121.7357 & 35.1376 & 86.9668 & 121.3492 & 34.3824 & 86.8726 & 121.6924 & 34.8198 \\
\hline 86.5678 & 121.753 & 35.1852 & 86.9869 & 121.3347 & 34.3478 & 86.8631 & 121.6933 & 34.8302 \\
\hline
\end{tabular}




\begin{tabular}{|c|c|c|c|c|c|c|c|c|}
\hline 86.8206 & 121.7573 & 34.9367 & 80.3441 & 120.0817 & 39.7376 & 77.3642 & 117.2785 & 39.9143 \\
\hline 86.5834 & 121.8483 & 35.2649 & 80.2297 & 120.012 & 39.7824 & 77.4153 & 117.1616 & 39.7463 \\
\hline 86.579 & 121.8405 & 35.2614 & 79.9743 & 119.8453 & 39.871 & 77.5548 & 117.0387 & 39.4839 \\
\hline 86.5747 & 121.8335 & 35.2588 & 79.9881 & 119.8028 & 39.8147 & 77.6178 & 116.8593 & 39.2415 \\
\hline 86.6059 & 121.8483 & 35.2424 & 79.957 & 119.7812 & 39.8242 & 77.6595 & 116.7633 & 39.1037 \\
\hline 86.5972 & 121.9245 & 35.3273 & 79.9396 & 119.7595 & 39.8199 & 77.469 & 116.7096 & 39.2406 \\
\hline 86.5583 & 121.9262 & 35.368 & 79.9344 & 119.6747 & 39.7402 & 77.3469 & 116.6377 & 39.2908 \\
\hline 86.4275 & 121.8136 & 35.3861 & 80.0184 & 119.6037 & 39.5852 & 77.3097 & 116.5693 & 39.2596 \\
\hline 85.9458 & 121.6699 & 35.724 & 79.8894 & 119.5682 & 39.6788 & 77.0075 & 116.5156 & 39.5082 \\
\hline 85.4039 & 121.6578 & 36.2538 & 79.7387 & 119.5725 & 39.8338 & 77.0023 & 116.5165 & 39.5142 \\
\hline 85.1658 & 121.5019 & 36.3361 & 79.6492 & 119.5399 & 39.8907 & 76.8334 & 116.3944 & 39.561 \\
\hline 85.2134 & 121.8214 & 36.608 & 79.5534 & 119.4937 & 39.9403 & 76.6013 & 116.3684 & 39.7671 \\
\hline 85.1623 & 121.7764 & 36.6141 & 79.4348 & 119.414 & 39.9792 & 76.4057 & 116.2849 & 39.8793 \\
\hline 85.022 & 121.7184 & 36.6964 & 79.4331 & 119.3118 & 39.8788 & 76.4177 & 116.1848 & 39.7671 \\
\hline 84.9164 & 121.6716 & 36.7552 & 79.2694 & 119.3352 & 40.0658 & 76.4792 & 116.1052 & 39.6259 \\
\hline 84.8523 & 121.5902 & 36.7379 & 79.2079 & 119.2313 & 40.0234 & 76.4195 & 116.0099 & 39.5904 \\
\hline 84.7434 & 121.4893 & 36.7459 & 79.227 & 119.0893 & 39.8623 & 76.3848 & 115.9337 & 39.5489 \\
\hline 84.6938 & 121.3893 & 36.6955 & 79.0598 & 119.0806 & 40.0208 & 76.4879 & 115.8298 & 39.3419 \\
\hline 84.5327 & 121.3737 & 36.841 & 78.9119 & 118.9691 & 40.0572 & 76.3684 & 115.7778 & 39.4094 \\
\hline 84.5466 & 121.2663 & 36.7197 & 78.7983 & 118.8988 & 40.1005 & 76.1614 & 115.6938 & 39.5324 \\
\hline 84.4782 & 121.1971 & 36.7189 & 78.6935 & 118.794 & 40.1005 & 76.1291 & 115.715 & 39.5859 \\
\hline 84.4306 & 121.1451 & 36.7145 & 78.5792 & 118.7403 & 40.1611 & 75.9648 & 115.6904 & 39.7255 \\
\hline 84.3102 & 121.1027 & 36.7925 & 78.4762 & 118.6805 & 40.2044 & 75.8895 & 115.6557 & 39.7662 \\
\hline 84.253 & 120.9875 & 36.7345 & 78.4051 & 118.6468 & 40.2416 & 75.77 & 115.6081 & 39.8381 \\
\hline 84.118 & 120.9326 & 36.8146 & 78.3246 & 118.6078 & 40.2832 & 75.7076 & 115.6245 & 39.9169 \\
\hline 84.0504 & 120.8152 & 36.7648 & 78.3142 & 118.5732 & 40.259 & 75.5639 & 115.5449 & 39.981 \\
\hline 83.9378 & 120.7476 & 36.8098 & 78.2318 & 118.555 & 40.3232 & 75.4946 & 115.4756 & 39.981 \\
\hline 83.8131 & 120.6541 & 36.841 & 78.1964 & 118.4978 & 40.3014 & 75.3578 & 115.4202 & 40.0624 \\
\hline 83.7066 & 120.564 & 36.8574 & 78.1012 & 118.4649 & 40.3637 & 75.227 & 115.3697 & 40.1426 \\
\hline 83.6044 & 120.4705 & 36.8661 & 78.0293 & 118.4251 & 40.3958 & 75.3413 & 115.2833 & 39.942 \\
\hline 83.4919 & 120.3562 & 36.8643 & 77.9367 & 118.3697 & 40.433 & 75.3578 & 115.2634 & 39.9056 \\
\hline 83.3836 & 120.228 & 36.8444 & 77.8457 & 118.3186 & 40.4728 & 75.3794 & 115.2106 & 39.8312 \\
\hline 83.2617 & 120.123 & 36.8613 & 77.7574 & 118.2813 & 40.5239 & 75.3344 & 115.1838 & 39.8493 \\
\hline 83.1567 & 120.015 & 36.8583 & 77.7366 & 118.232 & 40.4954 & 75.4124 & 115.0902 & 39.6779 \\
\hline 83.0606 & 119.8617 & 36.8011 & 77.7147 & 118.1842 & 40.4695 & 75.0478 & 115.0599 & 40.0122 \\
\hline 82.9783 & 119.7864 & 36.8081 & 77.7461 & 118.1125 & 40.3663 & 75.1396 & 115.0798 & 39.9403 \\
\hline 82.8839 & 119.7673 & 36.8834 & 77.7946 & 118.0146 & 40.22 & 75.2896 & 114.9883 & 39.6987 \\
\hline 82.8181 & 119.7448 & 36.9267 & 77.7314 & 117.9635 & 40.2321 & 75.1621 & 114.9543 & 39.7922 \\
\hline 82.761 & 119.692 & 36.931 & 77.6292 & 117.9176 & 40.2884 & 75.2063 & 114.911 & 39.7047 \\
\hline 82.6978 & 119.5569 & 36.8592 & 77.6076 & 117.8735 & 40.2659 & 75.2374 & 114.8408 & 39.6034 \\
\hline 82.6892 & 119.4174 & 36.7283 & 77.5426 & 117.7704 & 40.2278 & 75.1699 & 114.8408 & 39.671 \\
\hline 82.599 & 119.3361 & 36.7371 & 77.5374 & 117.7505 & 40.2131 & 74.8434 & 114.8235 & 39.9801 \\
\hline 81.7764 & 119.5517 & 37.7753 & 77.5385 & 117.6812 & 40.1426 & 74.7845 & 114.7794 & 39.9948 \\
\hline 81.3434 & 119.7604 & 38.417 & 77.4612 & 117.6466 & 40.1853 & 74.7508 & 114.7534 & 40.0026 \\
\hline 80.9052 & 119.7232 & 38.818 & 77.3591 & 117.5609 & 40.2018 & 74.7029 & 114.7372 & 40.0343 \\
\hline 80.8186 & 120.0332 & 39.2146 & 77.2716 & 117.5764 & 40.3048 & 74.7759 & 114.6806 & 39.9048 \\
\hline 80.6723 & 119.9951 & 39.3228 & 77.1737 & 117.5375 & 40.3637 & 74.9075 & 114.6088 & 39.7013 \\
\hline
\end{tabular}




\begin{tabular}{|c|c|c|c|c|c|c|c|c|}
\hline 74.8096 & 114.5516 & 39.742 & 72.5373 & 112.7911 & 40.2538 & 75.9856 & 115.2201 & 39.2345 \\
\hline 74.7629 & 114.5048 & 39.742 & 72.5503 & 112.7305 & 40.1801 & 75.8551 & 115.2349 & 39.3798 \\
\hline 74.6832 & 114.4719 & 39.7887 & 72.9313 & 112.5824 & 39.651 & 75.873 & 115.0409 & 39.1678 \\
\hline 74.575 & 114.4719 & 39.897 & 72.4343 & 112.6603 & 40.226 & 75.8384 & 114.9863 & 39.1479 \\
\hline 74.6217 & 114.3827 & 39.761 & 72.5252 & 112.7045 & 40.1793 & 76.3294 & 114.5577 & 38.2283 \\
\hline 74.4483 & 114.3822 & 39.9339 & 72.7123 & 112.6517 & 39.9394 & 76.3069 & 114.5646 & 38.2577 \\
\hline 74.4321 & 114.3758 & 39.9437 & 74.4355 & 114.0329 & 39.5974 & 76.0965 & 114.5733 & 38.4768 \\
\hline 74.5057 & 114.3455 & 39.8398 & 78.1384 & 118.0749 & 39.9365 & 76.1025 & 114.4719 & 38.3694 \\
\hline 74.4849 & 114.3126 & 39.8277 & 77.9557 & 118.0371 & 40.0814 & 76.2324 & 114.2832 & 38.0507 \\
\hline 74.5221 & 114.2459 & 39.7238 & 77.87 & 117.9739 & 40.1039 & 76.0939 & 114.3276 & 38.2337 \\
\hline 74.2727 & 114.252 & 39.9792 & 77.7167 & 117.9315 & 40.2148 & 76.0809 & 114.2225 & 38.1417 \\
\hline 74.1134 & 114.1896 & 40.0762 & 77.6518 & 117.8553 & 40.2035 & 75.893 & 114.2087 & 38.3157 \\
\hline 74.032 & 114.1316 & 40.0996 & 77.6266 & 117.7635 & 40.1369 & 75.983 & 113.9766 & 37.9936 \\
\hline 74.1844 & 114.0407 & 39.8563 & 77.6916 & 117.6111 & 39.9195 & 75.9856 & 113.89 & 37.9044 \\
\hline 74.1656 & 113.9946 & 39.829 & 77.4803 & 117.5756 & 40.0953 & 76.2272 & 113.6562 & 37.429 \\
\hline 74.0199 & 113.9913 & 39.9714 & 77.5885 & 117.4699 & 39.8814 & 76.1597 & 113.5956 & 37.4359 \\
\hline 74.0043 & 113.9359 & 39.9316 & 77.3409 & 117.5132 & 40.1724 & 75.6652 & 113.8943 & 38.2291 \\
\hline 74.0251 & 113.8969 & 39.8719 & 77.1426 & 117.5184 & 40.3759 & 75.7424 & 113.6881 & 37.9457 \\
\hline 73.8224 & 113.8632 & 40.0407 & 77.4413 & 117.166 & 39.7246 & 75.5855 & 113.7263 & 38.1408 \\
\hline 73.7757 & 113.7965 & 40.0208 & 78.2415 & 116.623 & 38.3815 & 75.8886 & 113.8398 & 37.9511 \\
\hline 73.8458 & 113.7679 & 39.9221 & 78.225 & 116.5174 & 38.2923 & 76.3121 & 114.0121 & 37.7 \\
\hline 73.6839 & 113.7211 & 40.0373 & 78.2735 & 116.3987 & 38.1252 & 76.655 & 114.2728 & 37.6177 \\
\hline 73.5701 & 113.6326 & 40.0625 & 78.1341 & 116.3805 & 38.2464 & 76.9711 & 114.4468 & 37.4757 \\
\hline 73.515 & 113.6267 & 40.1117 & 77.9592 & 116.3719 & 38.4127 & 77.1668 & 114.7248 & 37.558 \\
\hline 73.5141 & 113.5462 & 40.0321 & 77.8574 & 116.3325 & 38.4751 & 77.3824 & 114.9474 & 37.5649 \\
\hline 73.3011 & 113.5358 & 40.2347 & 77.9375 & 116.1666 & 38.2291 & 77.6362 & 115.1552 & 37.519 \\
\hline 73.2977 & 113.4934 & 40.1957 & 77.7808 & 116.1848 & 38.404 & 77.9851 & 115.3089 & 37.3238 \\
\hline 73.2977 & 113.4094 & 40.1117 & 77.6388 & 116.1511 & 38.5123 & 78.1791 & 115.5094 & 37.3302 \\
\hline 73.2396 & 113.3877 & 40.1481 & 77.5435 & 116.1571 & 38.6136 & 78.2865 & 115.738 & 37.4515 \\
\hline 73.1331 & 113.3574 & 40.2243 & 77.4318 & 116.1086 & 38.6768 & 78.3809 & 116.0212 & 37.6403 \\
\hline 72.9993 & 113.3463 & 40.347 & 77.4275 & 116.0662 & 38.6387 & 78.6017 & 116.171 & 37.5692 \\
\hline 72.9617 & 113.2838 & 40.3222 & 77.3175 & 115.9986 & 38.6812 & 78.6935 & 116.416 & 37.7225 \\
\hline 73.0578 & 113.2206 & 40.1628 & 77.2249 & 115.9467 & 38.7218 & 78.9169 & 116.4992 & 37.5822 \\
\hline 72.9435 & 113.1808 & 40.2373 & 76.9979 & 115.9484 & 38.9505 & 79.2772 & 116.5329 & 37.2558 \\
\hline 72.9062 & 113.1357 & 40.2295 & 76.7763 & 116.016 & 39.2397 & 79.4589 & 116.6364 & 37.1775 \\
\hline 72.8638 & 113.1132 & 40.2494 & 76.6351 & 115.9857 & 39.3505 & 79.5751 & 116.7668 & 37.1917 \\
\hline 72.8603 & 113.0795 & 40.2191 & 76.2636 & 116.1181 & 39.8545 & 79.6348 & 116.9954 & 37.3606 \\
\hline 72.8837 & 113.0457 & 40.162 & 76.3389 & 116.0194 & 39.6805 & 79.8911 & 116.9564 & 37.0653 \\
\hline 72.9728 & 112.941 & 39.9682 & 76.3052 & 115.9735 & 39.6684 & 79.9362 & 117.1391 & 37.2029 \\
\hline 72.8075 & 112.9227 & 40.1152 & 76.0878 & 115.9389 & 39.8511 & 80.0895 & 117.2257 & 37.1363 \\
\hline 72.7538 & 112.9097 & 40.1559 & 76.056 & 115.9705 & 39.9145 & 80.086 & 117.431 & 37.345 \\
\hline 72.6871 & 112.8967 & 40.2096 & 76.3502 & 115.7181 & 39.3679 & 80.0687 & 117.6275 & 37.5589 \\
\hline 72.7131 & 112.8811 & 40.168 & 76.7789 & 115.3318 & 38.553 & 80.5627 & 117.4997 & 36.937 \\
\hline 73.0292 & 112.7556 & 39.7264 & 76.7156 & 115.2123 & 38.4967 & 80.9494 & 117.4136 & 36.4643 \\
\hline 72.7582 & 112.8292 & 40.071 & 76.2939 & 115.3751 & 39.0812 & 80.9113 & 117.7245 & 36.8133 \\
\hline 72.6075 & 112.8179 & 40.2105 & 76.2238 & 115.3604 & 39.1367 & 80.6731 & 118.0596 & 37.3865 \\
\hline 72.5791 & 112.7842 & 40.2052 & 76.2792 & 115.1768 & 38.8976 & 81.0931 & 117.9237 & 36.8306 \\
\hline
\end{tabular}




\begin{tabular}{|c|c|c|c|c|c|c|c|c|}
\hline 81.3079 & 117.954 & 36.6461 & 83.8763 & 120.2774 & 36.4011 & 84.3786 & 120.8368 & 36.4582 \\
\hline 81.4534 & 117.98 & 36.5266 & 83.8885 & 120.3068 & 36.4184 & 84.3873 & 120.8308 & 36.4435 \\
\hline 81.5625 & 118.0882 & 36.5258 & 83.937 & 120.2939 & 36.3569 & 84.4176 & 120.8463 & 36.4288 \\
\hline 81.7131 & 118.0882 & 36.375 & 83.9448 & 120.3242 & 36.3794 & 84.376 & 120.8377 & 36.4617 \\
\hline 81.766 & 118.1939 & 36.4279 & 84.0106 & 120.3207 & 36.3101 & 84.4106 & 120.8411 & 36.4305 \\
\hline 81.8699 & 118.2302 & 36.3604 & 84.014 & 120.3433 & 36.3292 & 84.3942 & 120.8637 & 36.4695 \\
\hline 81.9842 & 118.3125 & 36.3283 & 84.0348 & 120.3493 & 36.3145 & 84.3812 & 120.881 & 36.4998 \\
\hline 82.037 & 118.4294 & 36.3924 & 84.0643 & 120.3562 & 36.2919 & 84.3981 & 120.9035 & 36.5054 \\
\hline 81.9201 & 118.697 & 36.7769 & 84.0374 & 120.4333 & 36.3959 & 84.3864 & 120.9312 & 36.5448 \\
\hline 82.0232 & 118.8269 & 36.8037 & 84.0166 & 120.4333 & 36.4166 & 84.4124 & 120.997 & 36.5846 \\
\hline 82.0976 & 118.9204 & 36.8228 & 84.0625 & 120.4541 & 36.3915 & 84.3881 & 121.0533 & 36.6652 \\
\hline 82.3209 & 118.8704 & 36.5494 & 84.1032 & 120.4523 & 36.3491 & 84.473 & 121.0039 & 36.531 \\
\hline 82.2526 & 119.0304 & 36.7778 & 84.1344 & 120.5017 & 36.3673 & 84.4972 & 120.9788 & 36.4816 \\
\hline 82.4735 & 118.9793 & 36.5058 & 84.1902 & 120.5247 & 36.3345 & 84.4418 & 120.9771 & 36.5353 \\
\hline 82.638 & 118.988 & 36.35 & 84.2444 & 120.5545 & 36.3101 & 84.434 & 120.9918 & 36.5578 \\
\hline 82.6813 & 119.0581 & 36.3768 & 84.2643 & 120.5415 & 36.2772 & 84.421 & 120.944 & 36.523 \\
\hline 82.7705 & 119.1664 & 36.3959 & 84.2652 & 120.5571 & 36.2919 & 84.4193 & 121.0014 & 36.582 \\
\hline 82.8675 & 119.2201 & 36.3526 & 84.3154 & 120.5632 & 36.2478 & 84.4132 & 121.0109 & 36.5976 \\
\hline 82.9705 & 119.2287 & 36.2582 & 84.3223 & 120.5407 & 36.2183 & 84.421 & 120.9996 & 36.5786 \\
\hline 83.0318 & 119.2201 & 36.1883 & 84.3076 & 120.5606 & 36.253 & 84.4773 & 120.9563 & 36.479 \\
\hline 83.0294 & 119.2772 & 36.2478 & 84.2782 & 120.6134 & 36.3352 & 84.5206 & 120.9771 & 36.4565 \\
\hline 83.0615 & 119.3032 & 36.2417 & 84.2404 & 120.619 & 36.3786 & 84.4998 & 120.9771 & 36.4773 \\
\hline 83.1429 & 119.3551 & 36.2123 & 84.2582 & 120.6541 & 36.3959 & 84.7285 & 120.713 & 35.9845 \\
\hline 83.2035 & 119.3837 & 36.1802 & 84.273 & 120.6324 & 36.3595 & 85.1196 & 120.4146 & 35.295 \\
\hline 83.2416 & 119.4348 & 36.1932 & 84.3059 & 120.6558 & 36.35 & 85.0991 & 120.416 & 35.3169 \\
\hline 83.265 & 119.4842 & 36.2192 & 84.331 & 120.6203 & 36.2893 & 85.0411 & 120.4073 & 35.3662 \\
\hline 83.3109 & 119.5353 & 36.2244 & 84.3596 & 120.6671 & 36.3075 & 85.0315 & 120.4263 & 35.3948 \\
\hline 83.3438 & 119.6106 & 36.2668 & 84.4158 & 120.6697 & 36.2538 & 85.0281 & 120.4038 & 35.3757 \\
\hline 83.363 & 119.6799 & 36.3169 & 84.4548 & 120.7 & 36.2452 & 85.0497 & 120.3969 & 35.3472 \\
\hline 83.4615 & 119.7249 & 36.2634 & 84.4219 & 120.7009 & 36.279 & 85.0142 & 120.4099 & 35.3957 \\
\hline 83.4745 & 119.8072 & 36.3326 & 84.3875 & 120.7124 & 36.3248 & 84.9839 & 120.422 & 35.4381 \\
\hline 83.5828 & 119.8089 & 36.2261 & 84.3873 & 120.6913 & 36.3041 & 84.9172 & 120.4593 & 35.542 \\
\hline 83.6027 & 119.8531 & 36.2504 & 84.4132 & 120.7398 & 36.3266 & 84.9328 & 120.4657 & 35.5329 \\
\hline 83.601 & 119.8678 & 36.2668 & 84.4479 & 120.7424 & 36.2945 & 84.8835 & 120.4913 & 35.6078 \\
\hline 83.6261 & 119.8817 & 36.2556 & 84.4236 & 120.7485 & 36.3249 & 84.9138 & 120.519 & 35.6052 \\
\hline 83.6728 & 119.8834 & 36.2105 & 84.3829 & 120.7502 & 36.3673 & 85.0688 & 120.4558 & 35.387 \\
\hline 83.7198 & 119.9151 & 36.1953 & 84.3864 & 120.7589 & 36.3725 & 85.1155 & 120.4939 & 35.3783 \\
\hline 83.749 & 119.9371 & 36.188 & 84.4202 & 120.7641 & 36.3439 & 85.1684 & 120.5 & 35.3316 \\
\hline 83.8235 & 119.9665 & 36.143 & 84.4422 & 120.7397 & 36.2975 & 85.2047 & 120.5034 & 35.2987 \\
\hline 83.846 & 120.0297 & 36.1837 & 84.3977 & 120.7701 & 36.3725 & 85.1623 & 120.5441 & 35.3818 \\
\hline 83.8789 & 120.0652 & 36.1863 & 84.408 & 120.816 & 36.408 & 85.1169 & 120.5785 & 35.4615 \\
\hline 83.9032 & 120.1007 & 36.1976 & 84.4098 & 120.8082 & 36.3985 & 85.074 & 120.6273 & 35.5533 \\
\hline 83.9118 & 120.1466 & 36.2348 & 84.4418 & 120.8091 & 36.3673 & 85.0766 & 120.6134 & 35.5368 \\
\hline 83.8893 & 120.1908 & 36.3015 & 84.4661 & 120.8437 & 36.3777 & 85.0671 & 120.6281 & 35.5611 \\
\hline 83.8713 & 120.2164 & 36.3451 & 84.4366 & 120.8351 & 36.3985 & 85.1459 & 120.6047 & 35.4589 \\
\hline 83.8668 & 120.2402 & 36.3733 & 84.4029 & 120.8403 & 36.4374 & 85.2169 & 120.5753 & 35.3584 \\
\hline 83.9049 & 120.2185 & 36.3136 & 84.3585 & 120.8603 & 36.5019 & 85.1866 & 120.5684 & 35.3818 \\
\hline
\end{tabular}




\begin{tabular}{|c|c|c|c|c|c|c|c|c|}
\hline 85.1372 & 120.6073 & 35.4701 & 84.9805 & 120.2532 & 35.2727 & 85.1692 & 120.6316 & 35.4623 \\
\hline 85.1636 & 120.5564 & 35.3928 & 84.9692 & 120.2592 & 35.29 & 85.2801 & 120.5909 & 35.3108 \\
\hline 85.1554 & 120.5268 & 35.3714 & 84.9727 & 120.2748 & 35.3021 & 85.2558 & 120.5753 & 35.3195 \\
\hline 85.1779 & 120.5129 & 35.335 & 84.9663 & 120.2455 & 35.2792 & 85.2758 & 120.59 & 35.3143 \\
\hline 85.0766 & 120.5207 & 35.4442 & 84.9701 & 120.2687 & 35.2987 & 85.255 & 120.5917 & 35.3368 \\
\hline 85.0549 & 120.5225 & 35.4675 & 85.0376 & 120.3103 & 35.2727 & 85.2737 & 120.5441 & 35.2704 \\
\hline 85.0826 & 120.5216 & 35.439 & 85.0567 & 120.3086 & 35.2519 & 85.2645 & 120.5103 & 35.2459 \\
\hline 85.0645 & 120.5233 & 35.4589 & 85.1147 & 120.3112 & 35.1965 & 85.3043 & 120.4965 & 35.1922 \\
\hline 85.1086 & 120.5251 & 35.4164 & 85.1381 & 120.3406 & 35.2026 & 85.3485 & 120.5389 & 35.1904 \\
\hline 85.1398 & 120.5767 & 35.4369 & 85.1372 & 120.3545 & 35.2173 & 85.3537 & 120.5796 & 35.2259 \\
\hline 85.1571 & 120.5848 & 35.4277 & 85.1294 & 120.3346 & 35.2052 & 85.3658 & 120.5935 & 35.2277 \\
\hline 85.1666 & 120.5649 & 35.3983 & 85.0694 & 120.338 & 35.2686 & 85.3598 & 120.6645 & 35.3047 \\
\hline 85.1485 & 120.5554 & 35.4069 & 85.0125 & 120.3337 & 35.3212 & 85.3979 & 120.6957 & 35.2978 \\
\hline 85.1536 & 120.5251 & 35.3714 & 84.9675 & 120.293 & 35.3255 & 85.4358 & 120.7018 & 35.266 \\
\hline 85.1173 & 120.5294 & 35.4121 & 84.9874 & 120.3354 & 35.348 & 85.4247 & 120.7147 & 35.29 \\
\hline 85.1017 & 120.5389 & 35.4372 & 84.9753 & 120.3493 & 35.374 & 85.4134 & 120.7312 & 35.3177 \\
\hline 85.0852 & 120.4965 & 35.4113 & 84.9831 & 120.3848 & 35.4017 & 85.3701 & 120.7138 & 35.3437 \\
\hline 85.0852 & 120.4868 & 35.4016 & 85.0956 & 120.3718 & 35.2762 & 85.3381 & 120.7208 & 35.3827 \\
\hline 85.0887 & 120.4991 & 35.4104 & 85.0956 & 120.422 & 35.3264 & 85.3407 & 120.6844 & 35.3437 \\
\hline 85.0697 & 120.4861 & 35.4164 & 85.0746 & 120.4595 & 35.3849 & 85.3675 & 120.7615 & 35.3939 \\
\hline 85.0246 & 120.5095 & 35.4849 & 85.1017 & 120.4515 & 35.3498 & 85.4238 & 120.7641 & 35.3402 \\
\hline 85.0671 & 120.4861 & 35.419 & 85.0774 & 120.422 & 35.3446 & 85.4525 & 120.8225 & 35.3699 \\
\hline 85.1337 & 120.5121 & 35.3783 & 85.0255 & 120.4307 & 35.4052 & 85.5052 & 120.8697 & 35.3645 \\
\hline 85.0541 & 120.5476 & 35.4935 & 85.0272 & 120.4099 & 35.3827 & 85.5581 & 120.8983 & 35.3402 \\
\hline 85.0792 & 120.5484 & 35.4693 & 84.9527 & 120.4411 & 35.4883 & 85.5252 & 120.9529 & 35.4277 \\
\hline 85.0773 & 120.5327 & 35.4554 & 84.9857 & 120.4151 & 35.4294 & 85.487 & 120.984 & 35.497 \\
\hline 85.0012 & 120.5528 & 35.5515 & 84.9657 & 120.4567 & 35.4909 & 85.4862 & 121.0325 & 35.5463 \\
\hline 84.4152 & 120.282 & 35.8668 & 85.0059 & 120.4454 & 35.4395 & 85.5052 & 121.0585 & 35.5533 \\
\hline 85.1207 & 120.1622 & 35.0415 & 85.0532 & 120.4541 & 35.4009 & 85.5113 & 121.0836 & 35.5723 \\
\hline 85.0896 & 120.0548 & 34.9653 & 85.0887 & 120.4272 & 35.3385 & 85.5292 & 121.1255 & 35.5963 \\
\hline 85.0956 & 120.0964 & 35.0008 & 85.0948 & 120.4636 & 35.3688 & 85.5728 & 121.139 & 35.5663 \\
\hline 85.074 & 120.1241 & 35.0501 & 85.0852 & 120.4662 & 35.3809 & 85.6022 & 121.165 & 35.5628 \\
\hline 85.093 & 120.1328 & 35.0398 & 85.1277 & 120.461 & 35.3333 & 85.6429 & 121.1771 & 35.5342 \\
\hline 85.0913 & 120.1683 & 35.077 & 85.0818 & 120.4766 & 35.3948 & 85.6308 & 121.2005 & 35.5697 \\
\hline 85.0923 & 120.2032 & 35.1109 & 85.0359 & 120.5493 & 35.5134 & 85.6282 & 121.1953 & 35.5671 \\
\hline 85.0913 & 120.2116 & 35.1203 & 85.0385 & 120.5779 & 35.5394 & 85.5503 & 121.249 & 35.6988 \\
\hline 85.1147 & 120.2332 & 35.1186 & 85.0826 & 120.6366 & 35.554 & 85.5468 & 121.2499 & 35.7031 \\
\hline 85.1095 & 120.2722 & 35.1627 & 85.1918 & 120.642 & 35.4502 & 85.6226 & 121.2215 & 35.599 \\
\hline 85.1216 & 120.2817 & 35.1601 & 85.261 & 120.6602 & 35.3991 & 85.6403 & 121.2724 & 35.6321 \\
\hline 85.1389 & 120.2982 & 35.1593 & 85.216 & 120.6567 & 35.4407 & 85.649 & 121.3122 & 35.6632 \\
\hline 85.1225 & 120.2982 & 35.1757 & 85.216 & 120.6117 & 35.3957 & 85.6784 & 121.3287 & 35.6503 \\
\hline 85.0731 & 120.3268 & 35.2536 & 85.2151 & 120.6368 & 35.4216 & 85.7252 & 121.3434 & 35.6182 \\
\hline 85.0826 & 120.3397 & 35.2572 & 85.2541 & 120.5926 & 35.3385 & 85.7235 & 121.3642 & 35.6407 \\
\hline 85.0506 & 120.306 & 35.2554 & 85.2333 & 120.6099 & 35.3766 & 85.6854 & 121.3625 & 35.6771 \\
\hline 85.0229 & 120.3354 & 35.3125 & 85.2861 & 120.5688 & 35.2827 & 85.6637 & 121.3417 & 35.678 \\
\hline 85.0021 & 120.3216 & 35.3195 & 85.2342 & 120.6082 & 35.374 & 85.655 & 121.3607 & 35.7057 \\
\hline 85.0177 & 120.2887 & 35.271 & 85.1926 & 120.5926 & 35.4 & 85.6772 & 121.3836 & 35.7064 \\
\hline
\end{tabular}




\begin{tabular}{|c|c|c|c|c|c|c|c|c|}
\hline 85.7027 & 121.4343 & 35.7317 & 85.7166 & 124.5887 & 38.8721 & 85.1528 & 124.512 & 39.3592 \\
\hline 85.7252 & 121.4733 & 35.7481 & 86.2254 & 124.5629 & 38.3375 & 85.2065 & 124.5319 & 39.3254 \\
\hline 85.7659 & 121.4967 & 35.7308 & 85.6168 & 124.5088 & 38.8919 & 85.2065 & 124.5466 & 39.3402 \\
\hline 85.8274 & 121.553 & 35.7256 & 86.1434 & 124.5105 & 38.3671 & 85.2006 & 124.5892 & 39.3886 \\
\hline 85.8698 & 121.5538 & 35.684 & 85.9507 & 124.4623 & 38.5116 & 85.1285 & 124.5215 & 39.393 \\
\hline 85.9027 & 121.6075 & 35.7048 & 85.7747 & 124.4061 & 38.6314 & 85.3058 & 124.5089 & 39.2031 \\
\hline 85.9503 & 121.663 & 35.7126 & 86.1253 & 124.3989 & 38.2736 & 85.2752 & 124.4723 & 39.1971 \\
\hline 85.9353 & 121.6752 & 35.7399 & 86.0923 & 124.3816 & 38.2893 & 85.0056 & 124.4609 & 39.4553 \\
\hline 85.9642 & 121.6915 & 35.7273 & 85.884 & 124.3628 & 38.4787 & 84.9796 & 124.46 & 39.4804 \\
\hline 86.0231 & 121.7643 & 35.7412 & 85.8973 & 124.3394 & 38.4421 & 85.1159 & 124.4357 & 39.3197 \\
\hline 86.0595 & 121.7868 & 35.7273 & 85.8909 & 124.3314 & 38.4405 & 84.8497 & 124.4505 & 39.6008 \\
\hline 86.0785 & 121.8595 & 35.781 & 85.1994 & 124.3462 & 39.1469 & 85.0298 & 124.4306 & 39.4008 \\
\hline 86.0872 & 121.9098 & 35.8226 & 85.7844 & 124.3777 & 38.5933 & 85.0427 & 124.428 & 39.3853 \\
\hline 86.1296 & 121.9236 & 35.794 & 86.1528 & 124.4264 & 38.2736 & 85.0606 & 124.4101 & 39.3495 \\
\hline 86.1807 & 122.0033 & 35.8226 & 85.6317 & 124.4766 & 38.8449 & 85.035 & 124.422 & 39.387 \\
\hline 86.2084 & 122.0284 & 35.8201 & 85.0697 & 124.5077 & 39.438 & 84.8462 & 124.4687 & 39.6225 \\
\hline 86.1937 & 122.1185 & 35.9248 & 85.8607 & 124.4856 & 38.6249 & 85.0929 & 124.4944 & 39.4015 \\
\hline 86.2742 & 122.0483 & 35.7741 & 85.2879 & 124.4652 & 39.1774 & 85.3391 & 124.5319 & 39.1928 \\
\hline 86.2751 & 122.0474 & 35.7724 & 85.0116 & 124.4237 & 39.412 & 85.3835 & 124.5963 & 39.2129 \\
\hline 86.2742 & 122.0855 & 35.8113 & 85.2138 & 124.3914 & 39.1775 & 85.408 & 124.6537 & 39.2457 \\
\hline 86.2638 & 122.07 & 35.8061 & 85.9819 & 124.4288 & 38.4469 & 85.2198 & 124.6971 & 39.4773 \\
\hline 86.2326 & 122.0881 & 35.8555 & 85.4153 & 124.4741 & 39.0588 & 85.0315 & 124.68 & 39.6484 \\
\hline 86.237 & 122.0942 & 35.8572 & 85.2701 & 124.4927 & 39.2227 & 85.207 & 124.6528 & 39.4458 \\
\hline 86.2471 & 122.135 & 35.8879 & 85.1277 & 124.531 & 39.4034 & 84.9813 & 124.6402 & 39.6588 \\
\hline 86.3409 & 122.2631 & 35.9222 & 85.1216 & 124.5345 & 39.4129 & 85.1926 & 124.5855 & 39.393 \\
\hline 86.4924 & 122.3756 & 35.8832 & 85.0614 & 124.4782 & 39.4168 & 85.3818 & 124.5587 & 39.1769 \\
\hline 86.534 & 122.4891 & 35.9551 & 85.4111 & 124.4037 & 38.9926 & 85.1679 & 124.5055 & 39.3376 \\
\hline 86.6145 & 122.5644 & 35.9499 & 85.3558 & 124.3669 & 39.011 & 84.9407 & 124.4553 & 39.5146 \\
\hline 86.7107 & 122.6069 & 35.8962 & 84.8852 & 124.3743 & 39.4891 & 84.932 & 124.4245 & 39.4926 \\
\hline 86.7626 & 122.651 & 35.8884 & 84.8722 & 124.4037 & 39.5315 & 84.9372 & 124.4774 & 39.5402 \\
\hline 86.8033 & 122.7316 & 35.9282 & 84.8601 & 124.4427 & 39.5826 & 84.9735 & 124.4851 & 39.5116 \\
\hline 86.8276 & 122.7587 & 35.9311 & 85.2553 & 124.464 & 39.2087 & 85.259 & 124.5821 & 39.3231 \\
\hline 86.844 & 122.8138 & 35.9698 & 85.314 & 124.5126 & 39.1986 & 85.1164 & 124.641 & 39.5246 \\
\hline 86.8536 & 122.8216 & 35.9681 & 85.3245 & 124.4936 & 39.169 & 85.1597 & 124.6618 & 39.5021 \\
\hline 86.8839 & 122.8485 & 35.9646 & 85.2897 & 124.5143 & 39.2246 & 85.3808 & 124.663 & 39.2823 \\
\hline 86.8943 & 122.8675 & 35.9733 & 85.3173 & 124.4933 & 39.176 & 85.3598 & 124.6376 & 39.2778 \\
\hline 86.9202 & 122.8944 & 35.9741 & 85.9172 & 124.4951 & 38.5778 & 85.1337 & 124.6419 & 39.5082 \\
\hline 85.8889 & 123.323 & 37.4342 & 85.1892 & 124.5208 & 39.3317 & 85.3143 & 124.5992 & 39.2848 \\
\hline 85.1433 & 123.7473 & 38.6041 & 85.6424 & 124.4989 & 38.8565 & 85.2947 & 124.5923 & 39.2976 \\
\hline 84.9152 & 123.8114 & 38.8962 & 85.4538 & 124.4875 & 39.0336 & 85.2845 & 124.5906 & 39.3061 \\
\hline 86.255 & 124.3164 & 38.0614 & 84.9831 & 124.4566 & 39.4735 & 85.4957 & 124.6324 & 39.1367 \\
\hline 86.1016 & 124.4655 & 38.3639 & 85.2177 & 124.4514 & 39.2336 & 86.1866 & 123.9163 & 37.7297 \\
\hline 86.3077 & 124.5137 & 38.206 & 85.0255 & 124.4193 & 39.3938 & 82.6276 & 120.8818 & 38.2542 \\
\hline 86.1583 & 124.5477 & 38.3894 & 85.4086 & 124.4179 & 39.0093 & 83.0303 & 121.1399 & 38.1096 \\
\hline 86.3572 & 124.5813 & 38.2241 & 85.0497 & 124.3596 & 39.3098 & 83.1359 & 121.4543 & 38.3183 \\
\hline 86.1639 & 124.6036 & 38.4397 & 85.2999 & 124.3931 & 39.0932 & 83.4633 & 121.5192 & 38.0559 \\
\hline 86.299 & 124.6064 & 38.3074 & 85.3646 & 124.4305 & 39.066 & 83.6503 & 121.5841 & 37.9338 \\
\hline
\end{tabular}




\begin{tabular}{|c|c|c|c|c|c|c|c|c|}
\hline 83.9465 & 121.6084 & 37.6619 & 81.2602 & 120.215 & 38.9548 & 78.4684 & 118.2164 & 39.748 \\
\hline 83.9785 & 121.7218 & 37.7433 & 81.2005 & 120.1129 & 38.9124 & 78.4528 & 118.167 & 39.7143 \\
\hline 84.2501 & 121.6488 & 37.3986 & 81.1537 & 120.0938 & 38.9401 & 78.5039 & 118.1549 & 39.651 \\
\hline 84.1621 & 121.895 & 37.7329 & 81.1338 & 120.0427 & 38.9089 & 78.451 & 118.1359 & 39.6848 \\
\hline 83.9222 & 122.1574 & 38.2352 & 81.0745 & 119.9909 & 38.9164 & 78.3445 & 118.0796 & 39.735 \\
\hline 84.079 & 122.1609 & 38.0819 & 80.9944 & 119.976 & 38.9816 & 78.3402 & 118.0371 & 39.6969 \\
\hline 84.4029 & 122.0509 & 37.6481 & 81.0134 & 119.9163 & 38.9028 & 78.2071 & 118.0027 & 39.7956 \\
\hline 84.3275 & 122.1566 & 37.829 & 80.8896 & 119.8938 & 39.0042 & 78.1445 & 117.9687 & 39.8242 \\
\hline 84.3344 & 122.1661 & 37.8316 & 80.7666 & 119.8609 & 39.0942 & 78.1124 & 117.9211 & 39.8086 \\
\hline 84.2756 & 122.2206 & 37.9451 & 80.6948 & 119.8392 & 39.1444 & 78.1012 & 117.8856 & 39.7844 \\
\hline 84.236 & 122.1588 & 37.9228 & 80.6255 & 119.7925 & 39.167 & 78.1575 & 117.8077 & 39.6502 \\
\hline 84.0469 & 122.2717 & 38.2248 & 80.4904 & 119.7483 & 39.2579 & 78.0691 & 117.7505 & 39.6814 \\
\hline 84.0426 & 122.3419 & 38.2993 & 80.3592 & 119.6702 & 39.3111 & 78.0181 & 117.7011 & 39.6831 \\
\hline 83.8538 & 122.354 & 38.5002 & 80.2194 & 119.6652 & 39.4458 & 77.9531 & 117.6085 & 39.6554 \\
\hline 83.7785 & 122.257 & 38.4785 & 80.2029 & 119.608 & 39.4051 & 77.9517 & 117.5349 & 39.5833 \\
\hline 83.8244 & 122.1306 & 38.3062 & 80.0678 & 119.5673 & 39.4995 & 77.8648 & 117.515 & 39.6502 \\
\hline 83.5897 & 122.1202 & 38.5305 & 80.0834 & 119.4885 & 39.4051 & 77.6994 & 117.5002 & 39.8009 \\
\hline 83.5733 & 122.0431 & 38.4699 & 79.8808 & 119.4868 & 39.606 & 77.6492 & 117.4422 & 39.7931 \\
\hline 83.5181 & 121.9897 & 38.4716 & 79.8089 & 119.4339 & 39.6251 & 77.5799 & 117.3816 & 39.8017 \\
\hline 83.4875 & 121.8673 & 38.3798 & 79.8894 & 119.3898 & 39.5004 & 77.6145 & 117.327 & 39.7125 \\
\hline 83.4901 & 121.8379 & 38.3478 & 79.7461 & 119.3619 & 39.6159 & 77.5227 & 117.3011 & 39.7783 \\
\hline 83.407 & 121.8128 & 38.4058 & 79.6114 & 119.3673 & 39.7558 & 77.5193 & 117.2283 & 39.7091 \\
\hline 83.1186 & 121.7452 & 38.6266 & 79.6106 & 119.3482 & 39.7376 & 77.4372 & 117.1843 & 39.7471 \\
\hline 83.0234 & 121.6777 & 38.6543 & 79.5907 & 119.285 & 39.6943 & 77.4976 & 117.1227 & 39.6251 \\
\hline 82.9922 & 121.6015 & 38.6093 & 79.4989 & 119.2296 & 39.7307 & 77.4543 & 117.1157 & 39.6614 \\
\hline 82.8277 & 121.5183 & 38.6907 & 79.4634 & 119.1932 & 39.7298 & 77.2413 & 117.1374 & 39.8961 \\
\hline 82.772 & 121.5078 & 38.7358 & 79.4296 & 119.1499 & 39.7203 & 77.2577 & 117.127 & 39.8693 \\
\hline 82.9108 & 121.5192 & 38.6084 & 79.3716 & 119.0789 & 39.7073 & 77.1088 & 117.0672 & 39.9585 \\
\hline 82.8043 & 121.4283 & 38.624 & 79.288 & 118.9646 & 39.6766 & 77.1633 & 117.0291 & 39.8658 \\
\hline 82.625 & 121.378 & 38.753 & 79.3846 & 118.8494 & 39.4649 & 77.1374 & 116.9486 & 39.8112 \\
\hline 82.5783 & 121.2889 & 38.7106 & 79.4166 & 118.7394 & 39.3228 & 76.9369 & 116.9668 & 40.0299 \\
\hline 82.4025 & 121.2204 & 38.818 & 79.3291 & 118.6615 & 39.3324 & 76.9486 & 116.8863 & 39.9377 \\
\hline 82.3323 & 121.139 & 38.8067 & 79.2027 & 118.626 & 39.4233 & 76.9183 & 116.8508 & 39.9325 \\
\hline 82.2561 & 121.0377 & 38.7816 & 79.1161 & 118.6563 & 39.5402 & 76.875 & 116.8023 & 39.9273 \\
\hline 82.0526 & 121.0031 & 38.9505 & 79.0416 & 118.6442 & 39.6025 & 76.8299 & 116.7538 & 39.9238 \\
\hline 81.9245 & 120.959 & 39.0345 & 79.0529 & 118.5983 & 39.5454 & 76.7182 & 116.7087 & 39.9905 \\
\hline 81.966 & 120.8862 & 38.9202 & 79.0157 & 118.5351 & 39.5194 & 76.6992 & 116.6429 & 39.9437 \\
\hline 81.8768 & 120.8706 & 38.9938 & 78.7075 & 118.4757 & 39.7683 & 76.642 & 116.604 & 39.9619 \\
\hline 81.8933 & 120.8039 & 38.9106 & 78.8892 & 118.3991 & 39.5099 & 76.6827 & 116.5416 & 39.8589 \\
\hline 81.9262 & 120.7234 & 38.7972 & 78.6849 & 118.3974 & 39.7125 & 76.6347 & 116.4858 & 39.8511 \\
\hline 81.8084 & 120.6385 & 38.8301 & 78.8814 & 118.3082 & 39.4268 & 76.545 & 116.4671 & 39.9221 \\
\hline 81.6517 & 120.5969 & 38.9453 & 78.8537 & 118.3082 & 39.4545 & 76.5554 & 116.3849 & 39.8294 \\
\hline 81.6439 & 120.5242 & 38.8803 & 78.8286 & 118.3047 & 39.4761 & 76.5987 & 116.3225 & 39.7238 \\
\hline 81.4568 & 120.4798 & 39.023 & 78.684 & 118.2917 & 39.6077 & 76.481 & 116.3009 & 39.8199 \\
\hline 81.4057 & 120.416 & 39.0102 & 78.4164 & 118.335 & 39.9186 & 76.4273 & 116.2437 & 39.8164 \\
\hline 81.3694 & 120.3744 & 39.005 & 78.5234 & 118.2494 & 39.726 & 76.3199 & 116.2446 & 39.9247 \\
\hline 81.2793 & 120.2904 & 39.0111 & 78.458 & 118.2233 & 39.7653 & 76.242 & 116.2264 & 39.9844 \\
\hline
\end{tabular}




\begin{tabular}{|c|c|c|c|c|c|}
\hline 76.1784 & 116.1449 & 39.9665 & 74.3896 & 114.51 & 40.1204 \\
\hline 76.1129 & 116.0645 & 39.9515 & 74.3836 & 114.4789 & 40.0953 \\
\hline 76.1207 & 116.0367 & 39.916 & & & \\
\hline 76.0861 & 116.0168 & 39.9307 & & & \\
\hline 76.1744 & 115.9415 & 39.7671 & & & \\
\hline 76.1476 & 115.8861 & 39.7385 & & & \\
\hline 76.0393 & 115.8445 & 39.8052 & & & \\
\hline 75.9943 & 115.8003 & 39.8061 & & & \\
\hline 75.8807 & 115.7502 & 39.8696 & & & \\
\hline 75.8237 & 115.7111 & 39.8875 & & & \\
\hline 75.8116 & 115.6791 & 39.8675 & & & \\
\hline 75.8436 & 115.628 & 39.7844 & & & \\
\hline 75.7345 & 115.6072 & 39.8727 & & & \\
\hline 75.7163 & 115.5622 & 39.8459 & & & \\
\hline 75.5362 & 115.5622 & 40.026 & & & \\
\hline 75.5578 & 115.4981 & 39.9403 & & & \\
\hline 75.5248 & 115.4613 & 39.9365 & & & \\
\hline 75.5345 & 115.3639 & 39.8294 & & & \\
\hline 75.4487 & 115.3639 & 39.9152 & & & \\
\hline 75.3881 & 115.324 & 39.9359 & & & \\
\hline 75.3128 & 115.2297 & 39.9169 & & & \\
\hline 75.2054 & 115.0054 & 39.8 & & & \\
\hline 75.1448 & 114.9898 & 39.845 & & & \\
\hline 75.027 & 114.9881 & 39.9611 & & & \\
\hline 74.9804 & 114.9645 & 39.9841 & & & \\
\hline 74.872 & 114.9984 & 40.1265 & & & \\
\hline 74.8581 & 114.9681 & 40.11 & & & \\
\hline 74.9049 & 114.9647 & 40.0598 & & & \\
\hline 74.898 & 114.9551 & 40.0572 & & & \\
\hline 74.8486 & 114.9283 & 40.0797 & & & \\
\hline 74.8408 & 114.9153 & 40.0745 & & & \\
\hline 74.8711 & 114.8634 & 39.9922 & & & \\
\hline 74.7461 & 114.8544 & 40.1083 & & & \\
\hline 74.7585 & 114.749 & 39.9905 & & & \\
\hline 74.6823 & 114.7846 & 40.1022 & & & \\
\hline 74.6261 & 114.7742 & 40.1481 & & & \\
\hline 74.7256 & 114.7031 & 39.9775 & & & \\
\hline 74.646 & 114.7006 & 40.0546 & & & \\
\hline 74.6105 & 114.7031 & 40.0927 & & & \\
\hline 74.4988 & 114.698 & 40.1992 & & & \\
\hline 74.5187 & 114.6746 & 40.1559 & & & \\
\hline 74.4836 & 114.6077 & 40.1241 & & & \\
\hline 74.4667 & 114.5828 & 40.1161 & & & \\
\hline 74.4936 & 114.5629 & 40.0693 & & & \\
\hline 74.4122 & 114.5819 & 40.1698 & & & \\
\hline 74.4702 & 114.5239 & 40.0537 & & & \\
\hline 74.4234 & 114.5274 & 40.1039 & & & \\
\hline
\end{tabular}




\begin{tabular}{|c|c|c|c|c|c|c|c|c|}
\hline \multicolumn{3}{|c|}{ Table A.3: 18 Hour Test } & 80.138 & 119.9068 & 39.7688 & 79.8522 & 120.0566 & 40.2044 \\
\hline minimum & maximum & height & 80.0548 & 119.9492 & 39.8944 & 79.8456 & 120.0393 & 40.1937 \\
\hline $\mathrm{mmHg}$ & $\mathrm{mmHg}$ & $\mathrm{mmHg}$ & 80.0314 & 119.9241 & 39.8926 & 79.8955 & 120.0419 & 40.1464 \\
\hline 80.519 & 119.6513 & 39.1323 & 80.015 & 119.9838 & 39.9689 & 79.9275 & 120.0626 & 40.1351 \\
\hline 80.5935 & 119.6981 & 39.1046 & 80.1172 & 119.9968 & 39.8797 & 79.9396 & 120.054 & 40.1143 \\
\hline 80.5882 & 119.6799 & 39.0917 & 80.1198 & 119.9812 & 39.8615 & 79.9085 & 120.1146 & 40.2061 \\
\hline 80.6705 & 119.6608 & 38.9903 & 80.1717 & 119.9821 & 39.8104 & 79.9422 & 120.1311 & 40.1888 \\
\hline 80.6627 & 119.6652 & 39.0024 & 80.0923 & 119.9935 & 39.9013 & 79.8998 & 120.157 & 40.2572 \\
\hline 80.5926 & 119.66 & 39.0674 & 80.0574 & 120.0159 & 39.9585 & 79.8669 & 120.1873 & 40.3204 \\
\hline 80.5129 & 119.653 & 39.1401 & 80.0825 & 120.0237 & 39.9411 & 79.8121 & 120.2191 & 40.4069 \\
\hline 80.506 & 119.6236 & 39.1176 & 80.0652 & 120.0219 & 39.9567 & 79.7907 & 120.2765 & 40.4858 \\
\hline 80.4921 & 119.679 & 39.1869 & 80.1007 & 120.0219 & 39.9212 & 79.7465 & 120.2722 & 40.5257 \\
\hline 80.551 & 119.6626 & 39.1115 & 80.0851 & 120.0271 & 39.942 & 79.6885 & 120.3016 & 40.6131 \\
\hline 80.4711 & 119.702 & 39.2309 & 80.0462 & 120.0678 & 40.0217 & 79.7665 & 120.325 & 40.5586 \\
\hline 80.4255 & 119.7214 & 39.296 & 80.0747 & 120.0903 & 40.0156 & 79.718 & 120.3562 & 40.6382 \\
\hline 80.3822 & 119.7353 & 39.3531 & 80.1081 & 120.0446 & 39.9365 & 79.6487 & 120.3501 & 40.7015 \\
\hline 80.3943 & 119.75 & 39.3557 & 80.1362 & 120.0765 & 39.9403 & 79.6825 & 120.3614 & 40.6789 \\
\hline 80.4159 & 119.7214 & 39.3055 & 80.1336 & 120.0124 & 39.8788 & 79.6501 & 120.3353 & 40.6853 \\
\hline 80.4194 & 119.7301 & 39.3107 & 80.0877 & 120.0609 & 39.9732 & 79.7855 & 120.2956 & 40.5101 \\
\hline 80.3588 & 119.7916 & 39.4328 & 80.0574 & 120.0237 & 39.9663 & 79.8305 & 120.2861 & 40.4555 \\
\hline 80.383 & 119.7647 & 39.3817 & 80.0747 & 120.0592 & 39.9844 & 79.8617 & 120.2713 & 40.4096 \\
\hline 80.4948 & 119.7302 & 39.2353 & 80.1683 & 120.0297 & 39.8615 & 79.8678 & 120.3103 & 40.4425 \\
\hline 80.4332 & 119.7647 & 39.3315 & 80.1665 & 120.0297 & 39.8632 & 79.9327 & 120.2904 & 40.3577 \\
\hline 80.4324 & 119.7864 & 39.354 & 80.19 & 120.0103 & 39.8202 & 79.8643 & 120.3597 & 40.4954 \\
\hline 80.3986 & 119.7925 & 39.3938 & 80.2557 & 119.9397 & 39.6839 & 79.8357 & 120.3856 & 40.5499 \\
\hline 80.3951 & 119.7977 & 39.4025 & 80.1916 & 119.9682 & 39.7766 & 79.8104 & 120.3618 & 40.5514 \\
\hline 80.2566 & 119.7916 & 39.535 & 80.1267 & 120.0107 & 39.884 & 79.8937 & 120.3389 & 40.4451 \\
\hline 80.2436 & 119.8297 & 39.5861 & 80.0704 & 120.0583 & 39.9879 & 79.8418 & 120.3475 & 40.5058 \\
\hline 80.3735 & 119.7873 & 39.4138 & 80.0366 & 120.0566 & 40.0199 & 79.9353 & 120.3129 & 40.3776 \\
\hline 80.3441 & 119.7595 & 39.4155 & 80.0843 & 120.0756 & 39.9914 & 79.9613 & 120.2878 & 40.3265 \\
\hline 80.3865 & 119.8401 & 39.4536 & 80.0488 & 120.1007 & 40.052 & 79.9422 & 120.3164 & 40.3741 \\
\hline 80.4064 & 119.7492 & 39.3428 & 79.9812 & 120.0644 & 40.0832 & 80.0063 & 120.3016 & 40.2953 \\
\hline 80.2912 & 119.8721 & 39.5809 & 80.0059 & 120.0261 & 40.0202 & 80.0167 & 120.2376 & 40.2208 \\
\hline 80.2237 & 119.8964 & 39.6727 & 80.0609 & 119.9717 & 39.9108 & 79.9838 & 120.2402 & 40.2564 \\
\hline 80.1864 & 119.8765 & 39.69 & 80.0366 & 119.9899 & 39.9533 & 79.9795 & 120.2728 & 40.2933 \\
\hline 80.1466 & 119.8721 & 39.7255 & 80.0903 & 119.9968 & 39.9065 & 80.0107 & 120.2661 & 40.2555 \\
\hline 80.1683 & 119.8903 & 39.7221 & 80.06 & 120.0089 & 39.9489 & 79.9353 & 120.254 & 40.3187 \\
\hline 80.157 & 119.899 & 39.742 & 80.0418 & 120.0107 & 39.9689 & 79.9422 & 120.2462 & 40.304 \\
\hline 80.1451 & 119.8482 & 39.7031 & 80.1405 & 120.0219 & 39.8814 & 79.9717 & 120.2454 & 40.2737 \\
\hline 80.1466 & 119.8756 & 39.729 & 80.1769 & 119.9778 & 39.8009 & 79.9691 & 120.2376 & 40.2685 \\
\hline 80.1362 & 119.8955 & 39.7593 & 80.102 & 119.9645 & 39.8625 & 79.9786 & 120.2722 & 40.2936 \\
\hline 80.0765 & 119.8877 & 39.8112 & 80.1258 & 120.0003 & 39.8745 & 79.9466 & 120.2739 & 40.3274 \\
\hline 80.0522 & 119.9258 & 39.8736 & 80.0617 & 120.0038 & 39.942 & 79.9126 & 120.308 & 40.3955 \\
\hline 80.0981 & 119.9657 & 39.8675 & 79.9907 & 120.0609 & 40.0702 & 79.8937 & 120.3094 & 40.4157 \\
\hline 80.0808 & 119.9769 & 39.8961 & 79.9163 & 119.9934 & 40.0771 & 79.9024 & 120.3129 & 40.4105 \\
\hline 80.0219 & 119.996 & 39.974 & 79.9604 & 119.9795 & 40.0191 & 79.9215 & 120.2748 & 40.3533 \\
\hline 80.079 & 119.9997 & 39.9207 & 79.8513 & 120.0323 & 40.181 & 79.957 & 120.2635 & 40.3066 \\
\hline
\end{tabular}




\begin{tabular}{|c|c|c|c|c|c|c|c|c|}
\hline 79.8929 & 120.2748 & 40.3819 & 79.5508 & 120.894 & 41.3431 & 78.9845 & 121.3564 & 42.3719 \\
\hline 79.9379 & 120.3077 & 40.3698 & 79.485 & 120.9096 & 41.4245 & 79.0183 & 121.3157 & 42.2974 \\
\hline 79.9786 & 120.2549 & 40.2763 & 79.3941 & 120.9407 & 41.5467 & 78.9914 & 121.2819 & 42.2905 \\
\hline 79.9777 & 120.3239 & 40.3461 & 79.4365 & 120.9087 & 41.4722 & 78.9914 & 121.2646 & 42.2732 \\
\hline 79.9396 & 120.3423 & 40.4027 & 79.4824 & 120.9096 & 41.4271 & 78.9758 & 121.2187 & 42.2429 \\
\hline 79.9145 & 120.383 & 40.4685 & 79.3717 & 120.9837 & 41.612 & 78.9594 & 121.2222 & 42.2628 \\
\hline 79.8548 & 120.3744 & 40.5196 & 79.2677 & 121.0724 & 41.8047 & 78.9057 & 121.2334 & 42.3278 \\
\hline 79.8738 & 120.3856 & 40.5118 & 79.3248 & 121.0784 & 41.7536 & 78.8528 & 121.2418 & 42.3889 \\
\hline 79.879 & 120.3744 & 40.4954 & 79.3776 & 121.0412 & 41.6636 & 78.891 & 121.2326 & 42.3416 \\
\hline 79.8721 & 120.3683 & 40.4962 & 79.3958 & 121.0498 & 41.654 & 78.9091 & 121.2239 & 42.3148 \\
\hline 79.8392 & 120.3839 & 40.5447 & 79.4486 & 121.0325 & 41.5839 & 78.9057 & 121.2161 & 42.3104 \\
\hline 79.8139 & 120.3609 & 40.547 & 79.3811 & 121.1364 & 41.7553 & 78.9351 & 121.1979 & 42.2628 \\
\hline 79.7985 & 120.3545 & 40.556 & 79.3525 & 121.1754 & 41.8229 & 78.9836 & 121.1546 & 42.171 \\
\hline 79.7119 & 120.3744 & 40.6625 & 79.3602 & 121.1739 & 41.8137 & 78.9784 & 121.2014 & 42.223 \\
\hline 79.7214 & 120.3536 & 40.6322 & 79.4027 & 121.1702 & 41.7675 & 78.9091 & 121.1832 & 42.2741 \\
\hline 79.6747 & 120.4168 & 40.7422 & 79.3517 & 121.1823 & 41.8307 & 78.9665 & 121.1114 & 42.1449 \\
\hline 79.6253 & 120.3839 & 40.7586 & 79.4573 & 121.1382 & 41.6809 & 78.9031 & 121.1425 & 42.2394 \\
\hline 79.6599 & 120.4281 & 40.7681 & 79.459 & 121.146 & 41.6869 & 78.8676 & 121.1719 & 42.3044 \\
\hline 79.377 & 124.2254 & 44.8484 & 79.3672 & 121.1607 & 41.7935 & 78.8979 & 121.1763 & 42.2784 \\
\hline 79.9258 & 120.3732 & 40.4474 & 79.3127 & 121.1719 & 41.8593 & 78.8944 & 121.217 & 42.3226 \\
\hline 79.8548 & 120.4835 & 40.6287 & 79.3742 & 121.1728 & 41.7986 & 78.8243 & 121.2265 & 42.4022 \\
\hline 79.7699 & 120.5484 & 40.7785 & 79.4043 & 121.1801 & 41.7758 & 78.8364 & 121.2759 & 42.4395 \\
\hline 79.7916 & 120.5441 & 40.7526 & 79.4867 & 121.1771 & 41.6904 & 78.9143 & 121.2508 & 42.3364 \\
\hline 79.7742 & 120.577 & 40.8028 & 79.4495 & 121.1875 & 41.738 & 78.8828 & 121.255 & 42.3722 \\
\hline 79.7829 & 120.6151 & 40.8322 & 79.3491 & 121.2456 & 41.8965 & 78.8433 & 121.2871 & 42.4438 \\
\hline 79.7786 & 120.6489 & 40.8703 & 79.3439 & 121.23 & 41.8861 & 78.8615 & 121.3399 & 42.4784 \\
\hline 79.7292 & 120.6775 & 40.9483 & 79.3265 & 121.2118 & 41.8852 & 78.7585 & 121.2837 & 42.5252 \\
\hline 79.7531 & 120.7388 & 40.9857 & 79.2616 & 121.1642 & 41.9026 & 78.7827 & 121.2871 & 42.5044 \\
\hline 79.7335 & 120.7927 & 41.0591 & 79.2295 & 121.1685 & 41.9389 & 78.8009 & 121.2992 & 42.4984 \\
\hline 79.6712 & 120.8178 & 41.1466 & 79.2492 & 121.1519 & 41.9027 & 78.8251 & 121.2845 & 42.4594 \\
\hline 79.7387 & 120.7918 & 41.053 & 79.2157 & 121.1728 & 41.9571 & 78.8243 & 121.2715 & 42.4473 \\
\hline 79.7119 & 120.8022 & 41.0903 & 79.1447 & 121.146 & 42.0013 & 78.8431 & 121.2559 & 42.4127 \\
\hline 79.7067 & 120.8455 & 41.1388 & 79.2218 & 121.1901 & 41.9684 & 78.8355 & 121.314 & 42.4784 \\
\hline 79.7223 & 120.8671 & 41.1448 & 79.194 & 121.1702 & 41.9762 & 78.8122 & 121.2698 & 42.4577 \\
\hline 79.7292 & 120.8126 & 41.0834 & 79.0962 & 121.2334 & 42.1372 & 78.7195 & 121.3192 & 42.5997 \\
\hline 79.798 & 120.789 & 40.991 & 79.0901 & 121.2828 & 42.1927 & 78.729 & 121.3763 & 42.6473 \\
\hline 79.7595 & 120.8385 & 41.079 & 79.0035 & 121.301 & 42.2974 & 78.684 & 121.3884 & 42.7045 \\
\hline 79.6998 & 120.8394 & 41.1396 & 79.0132 & 121.2682 & 42.255 & 78.755 & 121.411 & 42.656 \\
\hline 79.7725 & 120.823 & 41.0504 & 78.9784 & 121.2811 & 42.3026 & 78.6961 & 121.4378 & 42.7417 \\
\hline 79.7283 & 120.8082 & 41.0799 & 78.9576 & 121.2871 & 42.3295 & 78.6606 & 121.4491 & 42.7885 \\
\hline 79.7309 & 120.8619 & 41.131 & 78.9628 & 121.3088 & 42.3459 & 78.6564 & 121.4752 & 42.8188 \\
\hline 79.718 & 120.881 & 41.163 & 78.9663 & 121.333 & 42.3667 & 78.5983 & 121.4283 & 42.83 \\
\hline 79.6071 & 120.8611 & 41.254 & 78.9931 & 121.2906 & 42.2974 & 78.6095 & 121.4664 & 42.8569 \\
\hline 79.5907 & 120.8663 & 41.2756 & 78.9888 & 121.3088 & 42.32 & 78.5446 & 121.4594 & 42.9149 \\
\hline 79.5461 & 120.8269 & 41.2808 & 78.9559 & 121.3469 & 42.391 & 78.6112 & 121.4352 & 42.824 \\
\hline 79.5318 & 120.8498 & 41.318 & 79.0148 & 121.359 & 42.3442 & 78.6545 & 121.4499 & 42.7954 \\
\hline 79.5552 & 120.8671 & 41.312 & 78.9726 & 121.3607 & 42.3881 & 78.6035 & 121.4569 & 42.8534 \\
\hline
\end{tabular}




\begin{tabular}{|c|c|c|c|c|c|c|c|c|}
\hline 78.5991 & 121.4941 & 42.895 & 78.3644 & 121.4421 & 43.0777 & 78.0769 & 121.7539 & 43.6769 \\
\hline 78.6493 & 121.462 & 42.8127 & 78.3731 & 121.4084 & 43.0353 & 78.0631 & 121.7348 & 43.6717 \\
\hline 78.7403 & 121.4491 & 42.7088 & 78.4554 & 121.398 & 42.9426 & 78.1298 & 121.7244 & 43.5947 \\
\hline 78.6996 & 121.482 & 42.7824 & 78.5119 & 121.3607 & 42.8488 & 78.2337 & 121.6171 & 43.3834 \\
\hline 78.6606 & 121.4612 & 42.8006 & 78.4891 & 121.3798 & 42.8906 & 78.2475 & 121.5582 & 43.3106 \\
\hline 78.5844 & 121.514 & 42.9296 & 78.3774 & 121.4439 & 43.0664 & 78.2151 & 121.632 & 43.417 \\
\hline 78.6242 & 121.5036 & 42.8794 & 78.4917 & 121.3928 & 42.901 & 78.2545 & 121.6647 & 43.4102 \\
\hline 78.5697 & 121.5001 & 42.9305 & 78.4268 & 121.4551 & 43.0283 & 78.2371 & 121.6638 & 43.4267 \\
\hline 78.5879 & 121.5391 & 42.9513 & 78.4606 & 121.4196 & 42.959 & 78.1237 & 121.7114 & 43.5877 \\
\hline 78.5983 & 121.4964 & 42.8981 & 78.4649 & 121.391 & 42.9261 & 78.0691 & 121.7807 & 43.7116 \\
\hline 78.5783 & 121.5409 & 42.9625 & 78.3974 & 121.443 & 43.0456 & 78.0553 & 121.8197 & 43.7644 \\
\hline 78.6147 & 121.5677 & 42.953 & 78.3261 & 121.5184 & 43.1923 & 77.9808 & 121.8249 & 43.8441 \\
\hline 78.5983 & 121.4958 & 42.8976 & 78.3939 & 121.4854 & 43.0915 & 78.0181 & 121.8128 & 43.7947 \\
\hline 78.5948 & 121.4768 & 42.882 & 78.3809 & 121.5183 & 43.1374 & 78.0635 & 121.7668 & 43.7032 \\
\hline 78.5558 & 121.4681 & 42.9123 & 78.3999 & 121.5279 & 43.1279 & 78.0605 & 121.7565 & 43.696 \\
\hline 78.5931 & 121.4032 & 42.8101 & 78.4432 & 121.5642 & 43.121 & 78.0562 & 121.8067 & 43.7505 \\
\hline 78.6312 & 121.3928 & 42.7616 & 78.387 & 121.5859 & 43.1989 & 78.0778 & 121.7773 & 43.6995 \\
\hline 78.5128 & 121.4532 & 42.9404 & 78.4095 & 121.5521 & 43.1426 & 78.135 & 121.7357 & 43.6007 \\
\hline 78.5792 & 121.4248 & 42.8456 & 78.3168 & 121.5997 & 43.2829 & 78.1393 & 121.7028 & 43.5635 \\
\hline 78.5636 & 121.4343 & 42.8707 & 78.2767 & 121.632 & 43.3553 & 78.141 & 121.6404 & 43.4994 \\
\hline 78.5723 & 121.4569 & 42.8846 & 78.2155 & 121.6751 & 43.4596 & 78.1029 & 121.7262 & 43.6232 \\
\hline 78.5454 & 121.4941 & 42.9487 & 78.1609 & 121.6803 & 43.5193 & 78.0521 & 121.7175 & 43.6654 \\
\hline 78.4943 & 121.5244 & 43.0301 & 78.2415 & 121.6543 & 43.4128 & 78.0276 & 121.747 & 43.7194 \\
\hline 78.5333 & 121.5469 & 43.0136 & 78.2233 & 121.7045 & 43.4812 & 78.0657 & 121.7002 & 43.6345 \\
\hline 78.5307 & 121.5036 & 42.9729 & 78.2068 & 121.7097 & 43.5029 & 78.1142 & 121.6898 & 43.5756 \\
\hline 78.4873 & 121.4655 & 42.9783 & 78.2259 & 121.6855 & 43.4596 & 78.1124 & 121.682 & 43.5696 \\
\hline 78.4926 & 121.4447 & 42.9521 & 78.219 & 121.6811 & 43.4622 & 78.096 & 121.6586 & 43.5626 \\
\hline 78.49 & 121.4594 & 42.9694 & 78.2318 & 121.6857 & 43.4539 & 78.0969 & 121.6266 & 43.5297 \\
\hline 78.3939 & 121.5088 & 43.1149 & 78.2086 & 121.7028 & 43.4942 & 78.0674 & 121.669 & 43.6016 \\
\hline 78.406 & 121.5123 & 43.1063 & 78.1774 & 121.7019 & 43.5245 & 78.0213 & 121.6664 & 43.6451 \\
\hline 78.4536 & 121.4854 & 43.0318 & 78.1462 & 121.6855 & 43.5392 & 78.0821 & 121.6612 & 43.5791 \\
\hline 78.535 & 121.4049 & 42.8699 & 78.1817 & 121.6794 & 43.4977 & 78.0726 & 121.6924 & 43.6198 \\
\hline 78.5775 & 121.4187 & 42.8413 & 78.2172 & 121.6508 & 43.4336 & 78.0804 & 121.6915 & 43.6111 \\
\hline 78.4608 & 121.4453 & 42.9844 & 78.2207 & 121.6751 & 43.4544 & 78.083 & 121.6673 & 43.5843 \\
\hline 78.4181 & 121.4664 & 43.0482 & 78.2744 & 121.6889 & 43.4145 & 78.1055 & 121.6482 & 43.5427 \\
\hline 78.3393 & 121.4465 & 43.1071 & 78.1851 & 121.7457 & 43.5605 & 78.0856 & 121.6725 & 43.5869 \\
\hline 78.3307 & 121.462 & 43.1314 & 78.1601 & 121.8015 & 43.6414 & 78.1289 & 121.6604 & 43.5315 \\
\hline 78.3324 & 121.4283 & 43.0959 & 78.1765 & 121.8136 & 43.6371 & 78.0997 & 121.6549 & 43.5553 \\
\hline 78.3108 & 121.4084 & 43.0976 & 78.135 & 121.818 & 43.683 & 78.0951 & 121.6439 & 43.5488 \\
\hline 78.3064 & 121.4291 & 43.1227 & 78.0891 & 121.8509 & 43.7618 & 78.0821 & 121.6396 & 43.5574 \\
\hline 78.3575 & 121.3858 & 43.0283 & 78.0077 & 121.8864 & 43.8787 & 78.0172 & 121.6933 & 43.6761 \\
\hline 78.334 & 121.3977 & 43.0637 & 78.0562 & 121.876 & 43.8198 & 78.0224 & 121.7599 & 43.7376 \\
\hline 78.3653 & 121.3685 & 43.0032 & 78.1661 & 121.85 & 43.6839 & 78.0198 & 121.7478 & 43.728 \\
\hline 78.4415 & 121.3417 & 42.9002 & 78.075 & 121.8434 & 43.7684 & 78.0588 & 121.7279 & 43.6691 \\
\hline 78.4147 & 121.3555 & 42.9409 & 78.1047 & 121.8318 & 43.7272 & 78.0319 & 121.7513 & 43.7194 \\
\hline 78.3982 & 121.3893 & 42.9911 & 78.0813 & 121.8483 & 43.767 & 78.0276 & 121.8284 & 43.8008 \\
\hline 78.3757 & 121.4006 & 43.0249 & 78.0657 & 121.8439 & 43.7783 & 78.0468 & 121.8566 & 43.8098 \\
\hline
\end{tabular}




\begin{tabular}{|c|c|c|c|c|c|c|c|c|}
\hline 78.1185 & 121.8387 & 43.7202 & 77.9955 & 121.7521 & 43.7566 & 78.2181 & 123.2719 & 45.0538 \\
\hline 78.1471 & 121.8309 & 43.6839 & 77.964 & 121.7967 & 43.8327 & 78.1912 & 123.2806 & 45.0893 \\
\hline 78.1194 & 121.8292 & 43.7098 & 77.9903 & 121.8361 & 43.8458 & 78.1722 & 123.2936 & 45.1214 \\
\hline 78.044 & 121.8699 & 43.8259 & 78.0856 & 121.792 & 43.7064 & 78.2027 & 123.2943 & 45.0915 \\
\hline 78.0847 & 121.8448 & 43.7601 & 78.1523 & 121.7695 & 43.6172 & 78.1471 & 123.297 & 45.15 \\
\hline 78.1332 & 121.8284 & 43.6951 & 78.2094 & 121.7314 & 43.5219 & 78.1436 & 123.2988 & 45.1551 \\
\hline 78.1384 & 121.8015 & 43.6631 & 78.1583 & 121.747 & 43.5886 & 78.1869 & 123.22 & 45.033 \\
\hline 78.075 & 121.8346 & 43.7596 & 78.1531 & 121.7625 & 43.6094 & 78.2571 & 123.1602 & 44.9031 \\
\hline 78.0562 & 121.8708 & 43.8146 & 78.0865 & 121.8327 & 43.7462 & 78.3463 & 123.1221 & 44.7759 \\
\hline 78.0328 & 121.8881 & 43.8553 & 78.0398 & 121.7871 & 43.7473 & 78.3194 & 123.1221 & 44.8027 \\
\hline 77.9358 & 121.9808 & 44.045 & 78.0033 & 121.8162 & 43.8129 & 78.2761 & 123.1628 & 44.8867 \\
\hline 77.986 & 121.9929 & 44.0069 & 78.012 & 121.8543 & 43.8423 & 78.2644 & 123.1234 & 44.859 \\
\hline 78.0657 & 122.0024 & 43.9367 & 77.9531 & 121.9011 & 43.948 & 78.3255 & 123.0745 & 44.749 \\
\hline 78.1358 & 121.9349 & 43.799 & 77.9488 & 121.9141 & 43.9653 & 78.3896 & 123.0442 & 44.6546 \\
\hline 78.083 & 121.9989 & 43.9159 & 77.9401 & 121.9305 & 43.9904 & 78.3922 & 123.0728 & 44.6806 \\
\hline 78.0653 & 121.9483 & 43.8829 & 77.9652 & 121.882 & 43.9168 & 78.3878 & 123.0771 & 44.6893 \\
\hline 78.0943 & 121.9046 & 43.8103 & 78.0059 & 121.9201 & 43.9142 & 78.3774 & 123.0381 & 44.6607 \\
\hline 78.0743 & 121.889 & 43.8146 & 78.0477 & 121.8707 & 43.823 & 78.3688 & 123.0217 & 44.6529 \\
\hline 78.0388 & 121.8794 & 43.8406 & 78.0657 & 121.8206 & 43.7549 & 78.3523 & 123.0632 & 44.7109 \\
\hline 78.0778 & 121.8881 & 43.8103 & 78.0553 & 121.8509 & 43.7956 & 78.2767 & 123.0423 & 44.7656 \\
\hline 78.0631 & 121.8838 & 43.8207 & 78.0111 & 121.8994 & 43.8882 & 78.232 & 123.0217 & 44.7897 \\
\hline 78.038 & 121.8898 & 43.8519 & 77.9834 & 121.9184 & 43.935 & 78.3315 & 122.9965 & 44.665 \\
\hline 78.0129 & 121.9098 & 43.8969 & 77.9791 & 121.9262 & 43.9471 & 78.3913 & 122.9463 & 44.555 \\
\hline 78.0424 & 121.854 & 43.8116 & 77.9349 & 121.9531 & 44.0181 & 78.4069 & 122.9506 & 44.5438 \\
\hline 78.0025 & 121.8898 & 43.8874 & 77.8882 & 122.0258 & 44.1376 & 78.3168 & 122.9749 & 44.6581 \\
\hline 77.999 & 121.8803 & 43.8813 & 77.9041 & 121.9879 & 44.0838 & 78.2536 & 122.974 & 44.7204 \\
\hline 78.0016 & 121.9002 & 43.8986 & 77.9661 & 121.9695 & 44.0034 & 78.2804 & 123.0043 & 44.7239 \\
\hline 78.0562 & 121.8569 & 43.8008 & 78.0475 & 121.9427 & 43.8952 & 78.2415 & 123.0177 & 44.7762 \\
\hline 78.0284 & 121.8136 & 43.7852 & 77.9869 & 121.9712 & 43.9844 & 78.232 & 123.052 & 44.82 \\
\hline 78.0544 & 121.8327 & 43.7783 & 77.941 & 122.0215 & 44.0805 & 78.2155 & 123.0442 & 44.8287 \\
\hline 78.1012 & 121.8422 & 43.741 & 77.9384 & 122.0232 & 44.0848 & 78.1722 & 123.0494 & 44.8772 \\
\hline 78.1675 & 121.7871 & 43.6196 & 77.9098 & 121.9773 & 44.0675 & 78.1774 & 123.0407 & 44.8633 \\
\hline 78.1263 & 121.8032 & 43.6769 & 77.9237 & 121.9756 & 44.0519 & 78.1895 & 123.0875 & 44.898 \\
\hline 78.096 & 121.8335 & 43.7376 & 77.8856 & 122.0346 & 44.149 & 78.2155 & 123.0676 & 44.8521 \\
\hline 78.0336 & 121.8309 & 43.7973 & 77.8362 & 122.0821 & 44.2459 & 78.2986 & 123.0719 & 44.7733 \\
\hline 78.0466 & 121.7946 & 43.7479 & 77.8674 & 122.102 & 44.2346 & 78.334 & 123.0749 & 44.7409 \\
\hline 78.044 & 121.8145 & 43.7705 & 77.8916 & 122.1262 & 44.2346 & 78.2995 & 123.1186 & 44.8192 \\
\hline 78.1176 & 121.7703 & 43.6527 & 77.8501 & 122.1297 & 44.2797 & 78.2657 & 123.1697 & 44.904 \\
\hline 78.1038 & 121.7781 & 43.6743 & 77.8431 & 122.0812 & 44.2381 & 78.2172 & 123.1775 & 44.9603 \\
\hline 78.0953 & 121.7571 & 43.6618 & 77.8518 & 122.0786 & 44.2268 & 78.2233 & 123.1862 & 44.9629 \\
\hline 78.1246 & 121.7599 & 43.6354 & 77.8553 & 122.057 & 44.2017 & 78.2086 & 123.2243 & 45.0157 \\
\hline 78.115 & 121.7556 & 43.6406 & 77.838 & 122.0478 & 44.2098 & 78.1817 & 123.2278 & 45.046 \\
\hline 78.1627 & 121.74 & 43.5773 & 77.9644 & 122.5367 & 44.5723 & 78.1592 & 123.239 & 45.0798 \\
\hline 78.1523 & 121.7132 & 43.5609 & 78.2285 & 123.2763 & 45.0478 & 78.1437 & 123.2379 & 45.0942 \\
\hline 78.1722 & 121.714 & 43.5418 & 78.232 & 123.2823 & 45.0504 & 78.0536 & 123.2737 & 45.2201 \\
\hline 78.1298 & 121.7045 & 43.5748 & 78.2519 & 123.323 & 45.0711 & 78.0449 & 123.2875 & 45.2426 \\
\hline 78.1263 & 121.7028 & 43.5765 & 78.2094 & 123.317 & 45.1075 & 78.1549 & 123.2139 & 45.059 \\
\hline
\end{tabular}




\begin{tabular}{|c|c|c|c|c|c|c|c|c|}
\hline 78.3038 & 123.1464 & 44.8425 & 78.2519 & 123.4096 & 45.1577 & 78.0345 & 123.7083 & 45.6738 \\
\hline 78.322 & 123.1282 & 44.8062 & 78.1809 & 123.3975 & 45.2166 & 78.0588 & 123.7534 & 45.6946 \\
\hline 78.2285 & 123.1914 & 44.9629 & 78.193 & 123.3481 & 45.1551 & 77.9981 & 123.7543 & 45.7561 \\
\hline 78.2086 & 123.1741 & 44.9655 & 78.2155 & 123.3299 & 45.1144 & 77.9843 & 123.7742 & 45.7899 \\
\hline 78.2068 & 123.1836 & 44.9768 & 78.2068 & 123.3949 & 45.1881 & 78.0085 & 123.7785 & 45.77 \\
\hline 78.1737 & 123.1613 & 44.9876 & 78.2415 & 123.3706 & 45.1292 & 77.9826 & 123.7248 & 45.7423 \\
\hline 78.2034 & 123.1827 & 44.9794 & 78.2242 & 123.349 & 45.1248 & 77.9877 & 123.6937 & 45.7059 \\
\hline 78.1679 & 123.1464 & 44.9785 & 78.2195 & 123.4035 & 45.184 & 78.0051 & 123.7413 & 45.7362 \\
\hline 78.1713 & 123.1498 & 44.9785 & 78.2155 & 123.3603 & 45.1448 & 78.0068 & 123.7014 & 45.6946 \\
\hline 78.1038 & 123.1819 & 45.0781 & 78.2268 & 123.3646 & 45.1378 & 78.0662 & 123.6863 & 45.6201 \\
\hline 78.1124 & 123.2018 & 45.0893 & 78.1774 & 123.4036 & 45.2262 & 78.0536 & 123.7136 & 45.66 \\
\hline 78.1462 & 123.1767 & 45.0304 & 78.1402 & 123.3888 & 45.2487 & 78.0293 & 123.7309 & 45.7016 \\
\hline 78.1488 & 123.181 & 45.0322 & 78.1073 & 123.3984 & 45.2911 & 77.9843 & 123.711 & 45.7267 \\
\hline 78.1543 & 123.2176 & 45.0634 & 78.0622 & 123.452 & 45.3898 & 77.9609 & 123.7162 & 45.7553 \\
\hline 78.1376 & 123.1923 & 45.0547 & 78.0423 & 123.4746 & 45.4323 & 78.025 & 123.717 & 45.692 \\
\hline 78.1869 & 123.1931 & 45.0062 & 78.1041 & 123.4396 & 45.3356 & 77.9999 & 123.7023 & 45.7024 \\
\hline 78.1635 & 123.1975 & 45.0339 & 78.1116 & 123.4261 & 45.3145 & 77.9756 & 123.7101 & 45.7345 \\
\hline 78.1653 & 123.233 & 45.0677 & 78.1289 & 123.4417 & 45.3128 & 78.0169 & 123.6819 & 45.665 \\
\hline 78.1913 & 123.2399 & 45.0486 & 78.1047 & 123.4694 & 45.3647 & 78.0068 & 123.6391 & 45.6323 \\
\hline 78.1506 & 123.3031 & 45.1525 & 78.1168 & 123.4754 & 45.3587 & 77.9479 & 123.6521 & 45.7042 \\
\hline 78.1402 & 123.3048 & 45.1647 & 78.0969 & 123.4832 & 45.3864 & 77.98 & 123.6123 & 45.6323 \\
\hline 78.1904 & 123.3154 & 45.125 & 78.0795 & 123.5092 & 45.4297 & 78.031 & 123.5932 & 45.5622 \\
\hline 78.1921 & 123.3144 & 45.1222 & 78.0761 & 123.465 & 45.389 & 78.0267 & 123.5733 & 45.5466 \\
\hline 78.1869 & 123.2797 & 45.0928 & 78.1719 & 123.4951 & 45.3232 & 77.999 & 123.5664 & 45.5673 \\
\hline 78.2337 & 123.2979 & 45.0642 & 78.1272 & 123.4737 & 45.3465 & 77.9419 & 123.6486 & 45.7068 \\
\hline 78.2242 & 123.2944 & 45.0703 & 78.1073 & 123.5239 & 45.4167 & 77.9587 & 123.5955 & 45.6368 \\
\hline 78.2666 & 123.2988 & 45.0322 & 78.1757 & 123.5213 & 45.3457 & 78.0068 & 123.5724 & 45.5656 \\
\hline 78.1436 & 123.4079 & 45.2643 & 78.2077 & 123.5109 & 45.3032 & 78.0856 & 123.5499 & 45.4643 \\
\hline 78.0674 & 123.4443 & 45.3768 & 78.1627 & 123.5031 & 45.3405 & 78.0492 & 123.5733 & 45.5241 \\
\hline 78.1182 & 123.4238 & 45.3056 & 78.1081 & 123.5222 & 45.4141 & 78.0085 & 123.6287 & 45.6202 \\
\hline 78.0432 & 123.4564 & 45.4132 & 78.025 & 123.5871 & 45.5622 & 77.9981 & 123.6607 & 45.6626 \\
\hline 78.0865 & 123.4295 & 45.3431 & 78.0336 & 123.6264 & 45.5928 & 77.96 & 123.6512 & 45.6912 \\
\hline 77.9851 & 123.5014 & 45.5163 & 78.0198 & 123.6045 & 45.5847 & 77.8665 & 123.7162 & 45.8497 \\
\hline 78.0051 & 123.4945 & 45.4894 & 78.0388 & 123.5958 & 45.557 & 77.8812 & 123.6907 & 45.8095 \\
\hline 78.0172 & 123.5127 & 45.4955 & 78.0423 & 123.6166 & 45.5743 & 77.8726 & 123.7499 & 45.8774 \\
\hline 78.0666 & 123.4884 & 45.4219 & 78.0129 & 123.659 & 45.6462 & 77.8951 & 123.7283 & 45.8332 \\
\hline 78.1471 & 123.4572 & 45.3102 & 77.967 & 123.6564 & 45.6895 & 77.8916 & 123.7352 & 45.8436 \\
\hline 78.1516 & 123.4819 & 45.3303 & 77.9834 & 123.6599 & 45.6765 & 77.9289 & 123.7023 & 45.7735 \\
\hline 78.1956 & 123.5213 & 45.3257 & 77.9938 & 123.6486 & 45.6548 & 77.883 & 123.7032 & 45.8202 \\
\hline 78.1549 & 123.517 & 45.3621 & 77.9385 & 123.6678 & 45.7293 & 77.8232 & 123.7785 & 45.9553 \\
\hline 78.1791 & 123.4884 & 45.3093 & 77.9202 & 123.7014 & 45.7812 & 77.8449 & 123.7785 & 45.9337 \\
\hline 78.1835 & 123.4893 & 45.3058 & 77.9193 & 123.7118 & 45.7925 & 77.7755 & 123.829 & 46.0535 \\
\hline 78.1047 & 123.504 & 45.3994 & 77.9713 & 123.6746 & 45.7033 & 77.8284 & 123.8296 & 46.0012 \\
\hline 78.0336 & 123.4867 & 45.453 & 77.999 & 123.6911 & 45.692 & 77.8535 & 123.8261 & 45.9726 \\
\hline 78.1488 & 123.485 & 45.3361 & 78.0362 & 123.6677 & 45.6314 & 77.8752 & 123.7863 & 45.9111 \\
\hline 78.2388 & 123.3991 & 45.1603 & 78.0475 & 123.685 & 45.6375 & 77.8986 & 123.8088 & 45.9103 \\
\hline 78.2822 & 123.3629 & 45.0807 & 78.0276 & 123.7118 & 45.6843 & 77.883 & 123.8218 & 45.9389 \\
\hline
\end{tabular}




\begin{tabular}{|c|c|c|c|c|c|c|c|c|}
\hline 77.9029 & 123.8028 & 45.8999 & 77.6344 & 123.6988 & 46.0644 & 77.2395 & 123.8357 & 46.5961 \\
\hline 77.8977 & 123.853 & 45.9553 & 77.6284 & 123.7006 & 46.0722 & 77.3409 & 123.8513 & 46.5104 \\
\hline 77.8636 & 123.8431 & 45.9795 & 77.6673 & 123.6486 & 45.9813 & 77.2872 & 123.866 & 46.5788 \\
\hline 77.7721 & 123.8616 & 46.0895 & 77.65 & 123.7283 & 46.0783 & 77.2751 & 123.8521 & 46.5771 \\
\hline 77.8258 & 123.8417 & 46.0159 & 77.5976 & 123.6537 & 46.0561 & 77.295 & 123.8729 & 46.5779 \\
\hline 77.8665 & 123.8911 & 46.0246 & 77.4933 & 123.6772 & 46.1839 & 77.2803 & 123.8452 & 46.5649 \\
\hline 77.805 & 123.8842 & 46.0791 & 77.5755 & 123.6668 & 46.0913 & 77.2425 & 123.8721 & 46.6296 \\
\hline 77.8223 & 123.8859 & 46.0635 & 77.5348 & 123.6365 & 46.1017 & 77.2066 & 123.9041 & 46.6974 \\
\hline 77.779 & 123.9335 & 46.1545 & 77.5097 & 123.5577 & 46.048 & 77.1971 & 123.8686 & 46.6715 \\
\hline 77.8535 & 123.9396 & 46.0861 & 77.521 & 123.614 & 46.093 & 77.2569 & 123.8599 & 46.603 \\
\hline 77.8116 & 123.9814 & 46.1698 & 77.4595 & 123.6711 & 46.2116 & 77.2309 & 123.8573 & 46.6264 \\
\hline 77.8596 & 123.9656 & 46.106 & 77.4223 & 123.6391 & 46.2168 & 77.2603 & 123.8192 & 46.5589 \\
\hline 77.844 & 123.9682 & 46.1242 & 77.4474 & 123.6564 & 46.209 & 77.2127 & 123.9058 & 46.6931 \\
\hline 77.8752 & 123.9569 & 46.0817 & 77.4319 & 123.6739 & 46.242 & 77.1642 & 123.9067 & 46.7425 \\
\hline 77.8223 & 124.0071 & 46.1848 & 77.3729 & 123.6815 & 46.3086 & 77.1245 & 123.9541 & 46.8296 \\
\hline 77.7176 & 124.0426 & 46.3251 & 77.4136 & 123.6521 & 46.2385 & 77.1027 & 123.9647 & 46.862 \\
\hline 77.7236 & 124.0383 & 46.3147 & 77.3634 & 123.6677 & 46.3043 & 77.1599 & 123.9223 & 46.7624 \\
\hline 77.7245 & 124.0219 & 46.2974 & 77.34 & 123.6729 & 46.3329 & 77.056 & 124.0565 & 47.0005 \\
\hline 77.7262 & 124.0097 & 46.2835 & 77.4231 & 123.6304 & 46.2073 & 77.0741 & 124.0859 & 47.0118 \\
\hline 77.6856 & 124.0149 & 46.3292 & 77.3045 & 123.6694 & 46.3649 & 76.9953 & 124.0617 & 47.0663 \\
\hline 77.6639 & 123.9976 & 46.3337 & 77.3002 & 123.6694 & 46.3692 & 76.9936 & 124.1033 & 47.1096 \\
\hline 77.6734 & 123.9941 & 46.3207 & 77.3588 & 123.6616 & 46.3028 & 76.9191 & 124.1197 & 47.2006 \\
\hline 77.7028 & 123.9855 & 46.2826 & 77.3443 & 123.6928 & 46.3485 & 76.9685 & 124.1041 & 47.1356 \\
\hline 77.6925 & 123.9863 & 46.2939 & 77.3062 & 123.7421 & 46.4359 & 76.9783 & 124.1329 & 47.1546 \\
\hline 77.7531 & 123.9543 & 46.2012 & 77.3305 & 123.7924 & 46.4619 & 77.0499 & 124.0807 & 47.0308 \\
\hline 77.7617 & 123.9578 & 46.196 & 77.3989 & 123.7344 & 46.3355 & 77.0542 & 124.0747 & 47.0204 \\
\hline 77.8223 & 123.9292 & 46.1068 & 77.4024 & 123.7196 & 46.3173 & 77.017 & 124.0816 & 47.0646 \\
\hline 77.7799 & 123.9285 & 46.1486 & 77.4015 & 123.7188 & 46.3173 & 76.9512 & 124.1232 & 47.172 \\
\hline 77.721 & 123.9205 & 46.1995 & 77.4257 & 123.6902 & 46.2645 & 76.9512 & 124.1232 & 47.172 \\
\hline 77.7002 & 123.9205 & 46.2203 & 77.439 & 123.703 & 46.264 & 77.0525 & 124.0643 & 47.0118 \\
\hline 77.7513 & 123.8738 & 46.1224 & 77.4335 & 123.6443 & 46.2108 & 77.043 & 124.0392 & 46.9962 \\
\hline 77.8189 & 123.8296 & 46.0107 & 77.4465 & 123.6746 & 46.2281 & 76.9836 & 124.0827 & 47.0991 \\
\hline 77.7167 & 123.8599 & 46.1432 & 77.4751 & 123.7387 & 46.2636 & 76.972 & 124.0426 & 47.0707 \\
\hline 77.7401 & 123.8374 & 46.0973 & 77.495 & 123.717 & 46.222 & 76.985 & 124.0565 & 47.0715 \\
\hline 77.6803 & 123.8547 & 46.1744 & 77.5054 & 123.6997 & 46.1943 & 76.9806 & 124.0322 & 47.0516 \\
\hline 77.6909 & 123.755 & 46.0641 & 77.4829 & 123.6945 & 46.2116 & 76.9503 & 124.1085 & 47.1581 \\
\hline 77.6968 & 123.8019 & 46.1051 & 77.5071 & 123.6841 & 46.177 & 76.952 & 124.0418 & 47.0897 \\
\hline 77.6942 & 123.8106 & 46.1164 & 77.5306 & 123.7127 & 46.1821 & 76.9036 & 124.0859 & 47.1824 \\
\hline 77.6318 & 123.724 & 46.0921 & 77.4647 & 123.7395 & 46.2748 & 76.9235 & 124.0582 & 47.1348 \\
\hline 77.6734 & 123.6729 & 45.9995 & 77.4864 & 123.7482 & 46.2619 & 77.0443 & 124.0113 & 46.967 \\
\hline 77.6466 & 123.6703 & 46.0237 & 77.411 & 123.808 & 46.3969 & 77.1452 & 123.9266 & 46.7814 \\
\hline 77.6673 & 123.6581 & 45.9908 & 77.4682 & 123.808 & 46.3398 & 77.2465 & 123.9145 & 46.668 \\
\hline 77.6743 & 123.704 & 46.0298 & 77.4292 & 123.8166 & 46.3874 & 77.2811 & 123.885 & 46.6039 \\
\hline 77.5843 & 123.6458 & 46.0614 & 77.3573 & 123.8192 & 46.4619 & 77.4101 & 123.8868 & 46.4766 \\
\hline 77.5998 & 123.737 & 46.1372 & 77.321 & 123.7075 & 46.3866 & 77.54 & 123.6841 & 46.1441 \\
\hline 77.5721 & 123.659 & 46.0869 & 77.2293 & 123.8413 & 46.612 & 77.4171 & 123.7621 & 46.345 \\
\hline 77.5877 & 123.7318 & 46.1441 & 77.1876 & 123.8426 & 46.655 & 77.1729 & 123.7231 & 46.5502 \\
\hline
\end{tabular}




\begin{tabular}{|c|c|c|c|c|c|c|c|c|}
\hline 77.2699 & 123.8545 & 46.5847 & 77.0412 & 124.0643 & 47.023 & 77.2664 & 123.9101 & 46.6437 \\
\hline 77.3452 & 123.8755 & 46.5303 & 77.0664 & 124.1067 & 47.0404 & 77.301 & 123.9006 & 46.5996 \\
\hline 77.3738 & 123.8634 & 46.4896 & 77.0972 & 124.0245 & 46.9273 & 77.2465 & 123.9543 & 46.7078 \\
\hline 77.3417 & 123.84 & 46.4983 & 77.1408 & 124.0201 & 46.8793 & 77.1504 & 124.0089 & 46.8585 \\
\hline 77.3677 & 123.8989 & 46.5312 & 77.133 & 124.0227 & 46.8897 & 77.1439 & 123.9708 & 46.8269 \\
\hline 77.3132 & 123.8166 & 46.5035 & 77.1322 & 123.9967 & 46.8646 & 77.2309 & 123.9682 & 46.7373 \\
\hline 77.3088 & 123.9041 & 46.5953 & 77.1599 & 123.9898 & 46.8299 & 77.3591 & 123.9517 & 46.5927 \\
\hline 77.3036 & 123.8383 & 46.5346 & 77.0975 & 123.9898 & 46.8923 & 77.3668 & 123.9205 & 46.5537 \\
\hline 77.2884 & 123.8845 & 46.5961 & 77.0949 & 124.0279 & 46.933 & 77.3954 & 123.9517 & 46.5563 \\
\hline 77.2439 & 123.8868 & 46.6429 & 77.0811 & 124.0877 & 47.0066 & 77.3729 & 123.9257 & 46.5528 \\
\hline 77.269 & 123.9041 & 46.6351 & 77.0813 & 124.0686 & 46.9872 & 77.2136 & 124.0366 & 46.823 \\
\hline 77.3071 & 123.8703 & 46.5632 & 77.1123 & 124.1587 & 47.0464 & 77.1893 & 124.0167 & 46.8273 \\
\hline 77.3097 & 123.8885 & 46.5788 & 77.0837 & 124.1033 & 47.0196 & 77.1606 & 124.0237 & 46.863 \\
\hline 77.3642 & 123.8625 & 46.4983 & 77.1123 & 124.131 & 47.0187 & 77.1079 & 124.0634 & 46.9555 \\
\hline 77.2924 & 123.7863 & 46.4939 & 77.0741 & 124.0712 & 46.9971 & 77.0603 & 124.0729 & 47.0127 \\
\hline 77.3227 & 123.8469 & 46.5242 & 77.0915 & 124.1067 & 47.0153 & 77.0274 & 124.0548 & 47.0274 \\
\hline 77.3826 & 123.8607 & 46.4781 & 77.0828 & 124.1517 & 47.0689 & 76.9772 & 124.0591 & 47.0819 \\
\hline 77.3391 & 123.7993 & 46.4602 & 77.1209 & 124.1344 & 47.0135 & 76.9261 & 124.1033 & 47.1772 \\
\hline 77.2811 & 123.795 & 46.5139 & 77.1474 & 124.0686 & 46.9212 & 76.9313 & 124.0859 & 47.1547 \\
\hline 77.3409 & 123.8071 & 46.4662 & 77.1564 & 124.0123 & 46.8559 & 76.8654 & 124.0219 & 47.1564 \\
\hline 77.3885 & 123.7404 & 46.3519 & 77.0741 & 123.9301 & 46.8559 & 76.8699 & 124.0809 & 47.211 \\
\hline 77.4275 & 123.633 & 46.2056 & 77.1348 & 123.924 & 46.7892 & 76.8291 & 124.1041 & 47.275 \\
\hline 77.2967 & 123.7292 & 46.4325 & 77.1573 & 123.8781 & 46.7208 & 76.8066 & 124.1552 & 47.3486 \\
\hline 77.2569 & 123.7292 & 46.4723 & 77.1278 & 123.8816 & 46.7537 & 76.7866 & 124.2028 & 47.4162 \\
\hline 77.2906 & 123.653 & 46.3623 & 77.1443 & 123.8547 & 46.7104 & 76.778 & 124.1812 & 47.4032 \\
\hline 77.3227 & 123.6361 & 46.3134 & 77.2023 & 123.8946 & 46.6922 & 76.7485 & 124.1396 & 47.3911 \\
\hline 77.2681 & 123.6356 & 46.3675 & 77.1547 & 123.9231 & 46.7684 & 76.8057 & 124.1067 & 47.301 \\
\hline 77.2655 & 123.7153 & 46.4498 & 77.1967 & 123.9497 & 46.7529 & 76.8949 & 124.124 & 47.2291 \\
\hline 77.3062 & 123.7413 & 46.4351 & 77.146 & 123.9344 & 46.7884 & 76.8862 & 124.0937 & 47.2075 \\
\hline 77.3201 & 123.8062 & 46.4861 & 77.2352 & 123.9673 & 46.7321 & 76.9968 & 124.0633 & 47.0665 \\
\hline 77.2733 & 123.8296 & 46.5563 & 77.2828 & 123.9482 & 46.6654 & 77.0014 & 124.0903 & 47.0889 \\
\hline 77.2984 & 123.8426 & 46.5442 & 77.2915 & 123.872 & 46.5805 & 77.0109 & 124.0097 & 46.9988 \\
\hline 77.321 & 123.7802 & 46.4593 & 77.2395 & 123.8859 & 46.6463 & 76.9417 & 124.0608 & 47.1192 \\
\hline 77.3069 & 123.8607 & 46.5538 & 77.2742 & 123.8842 & 46.61 & 76.9858 & 124.1214 & 47.1356 \\
\hline 77.2629 & 123.7984 & 46.5355 & 77.3149 & 123.8521 & 46.5372 & 77.0395 & 124.0643 & 47.0248 \\
\hline 77.2889 & 123.8019 & 46.513 & 77.3853 & 123.8184 & 46.4332 & 76.9988 & 124.0288 & 47.03 \\
\hline 77.2404 & 123.8469 & 46.6065 & 77.3642 & 123.7976 & 46.4333 & 77.0473 & 124.0634 & 47.0161 \\
\hline 77.2967 & 123.8504 & 46.5537 & 77.3591 & 123.8296 & 46.4706 & 77.0214 & 124.0827 & 47.0612 \\
\hline 77.3642 & 123.7932 & 46.429 & 77.3781 & 123.7932 & 46.4151 & 76.959 & 124.0885 & 47.1296 \\
\hline 77.3729 & 123.9335 & 46.5606 & 77.4136 & 123.7958 & 46.3822 & 76.92 & 124.1266 & 47.2066 \\
\hline 77.3339 & 124.0011 & 46.6671 & 77.4145 & 123.7872 & 46.3727 & 76.8836 & 124.1509 & 47.2672 \\
\hline 77.3157 & 123.9479 & 46.6322 & 77.4093 & 123.7794 & 46.3701 & 76.8377 & 124.2124 & 47.3746 \\
\hline 77.2777 & 124.0011 & 46.7234 & 77.4249 & 123.7629 & 46.3381 & 76.8906 & 124.1162 & 47.2257 \\
\hline 77.185 & 124.0461 & 46.8611 & 77.4487 & 123.799 & 46.3503 & 76.8646 & 124.1492 & 47.2846 \\
\hline 77.1174 & 124.1266 & 47.0092 & 77.4214 & 123.827 & 46.4056 & 76.8932 & 124.1128 & 47.2196 \\
\hline 77.1382 & 124.1024 & 46.9642 & 77.3816 & 123.8192 & 46.4376 & 76.9589 & 124.1681 & 47.2092 \\
\hline 77.1876 & 124.1509 & 46.9633 & 77.3201 & 123.8608 & 46.5407 & 76.8802 & 124.1881 & 47.308 \\
\hline
\end{tabular}




\begin{tabular}{|c|c|c|c|c|c|c|c|c|}
\hline 76.8732 & 124.2609 & 47.3876 & 76.847 & 124.2888 & 47.4418 & 76.5927 & 124.2357 & 47.6431 \\
\hline 76.8325 & 124.2098 & 47.3772 & 76.8282 & 124.2609 & 47.4327 & 76.6931 & 124.2357 & 47.5426 \\
\hline 76.8966 & 124.1821 & 47.2854 & 76.8144 & 124.3024 & 47.4881 & 76.7519 & 124.295 & 47.5431 \\
\hline 76.8888 & 124.2028 & 47.314 & 76.9122 & 124.247 & 47.3348 & 76.7078 & 124.2479 & 47.54 \\
\hline 76.8525 & 124.1743 & 47.3218 & 76.7607 & 124.305 & 47.5444 & 76.7685 & 124.234 & 47.4656 \\
\hline 76.8308 & 124.1708 & 47.34 & 76.6126 & 124.2739 & 47.6613 & 76.7858 & 124.2262 & 47.4404 \\
\hline 76.8197 & 124.1576 & 47.3378 & 76.7676 & 124.2332 & 47.4656 & 76.7979 & 124.2297 & 47.4318 \\
\hline 76.8317 & 124.1474 & 47.3157 & 76.7278 & 124.2444 & 47.5166 & 76.8395 & 124.2479 & 47.4084 \\
\hline 76.9027 & 124.1569 & 47.2543 & 76.7977 & 124.2562 & 47.4585 & 76.8299 & 124.2002 & 47.3703 \\
\hline 76.907 & 124.176 & 47.269 & 76.8611 & 124.2453 & 47.3842 & 76.7728 & 124.215 & 47.4422 \\
\hline 76.9486 & 124.1422 & 47.1936 & 76.8689 & 124.1907 & 47.3218 & 76.7677 & 124.2826 & 47.5149 \\
\hline 76.9417 & 124.1604 & 47.2188 & 76.8178 & 124.1933 & 47.3755 & 76.7399 & 124.2695 & 47.5296 \\
\hline 76.9927 & 124.1855 & 47.1928 & 76.8083 & 124.2132 & 47.4049 & 76.7382 & 124.3223 & 47.5842 \\
\hline 76.9598 & 124.1708 & 47.211 & 76.7321 & 124.1994 & 47.4673 & 76.7598 & 124.3509 & 47.5911 \\
\hline 77.0012 & 124.1391 & 47.1379 & 76.7641 & 124.1907 & 47.4266 & 76.7459 & 124.3994 & 47.6535 \\
\hline 77.0101 & 124.1595 & 47.1495 & 76.7918 & 124.176 & 47.3842 & 76.7589 & 124.3717 & 47.6128 \\
\hline 76.9728 & 124.1266 & 47.1538 & 76.8197 & 124.1734 & 47.3537 & 76.7936 & 124.3648 & 47.5712 \\
\hline 76.9624 & 124.1838 & 47.2214 & 76.8187 & 124.1907 & 47.372 & 76.7754 & 124.4479 & 47.6725 \\
\hline 76.933 & 124.1214 & 47.1884 & 76.7866 & 124.1275 & 47.3409 & 76.7951 & 124.4306 & 47.6356 \\
\hline 76.9425 & 124.1258 & 47.1832 & 76.8923 & 124.079 & 47.1867 & 76.7892 & 124.4981 & 47.7089 \\
\hline 76.9417 & 124.1379 & 47.1962 & 76.9044 & 124.0851 & 47.1807 & 76.7373 & 124.48 & 47.7427 \\
\hline 77.0057 & 124.1085 & 47.1027 & 76.8758 & 124.124 & 47.2482 & 76.7295 & 124.5631 & 47.8336 \\
\hline 76.9809 & 124.0545 & 47.0736 & 76.9183 & 124.1492 & 47.2309 & 76.6602 & 124.5241 & 47.8639 \\
\hline 77.062 & 124.0435 & 46.9815 & 76.9702 & 124.0981 & 47.1278 & 76.5693 & 124.6003 & 48.031 \\
\hline 77.03 & 124.0721 & 47.0421 & 76.9395 & 124.0668 & 47.1273 & 76.4593 & 124.6332 & 48.1739 \\
\hline 77.153 & 124.0331 & 46.8802 & 76.9174 & 124.1145 & 47.1971 & 76.6117 & 124.6367 & 48.025 \\
\hline 77.1642 & 124.079 & 46.9148 & 76.804 & 124.1258 & 47.3218 & 76.5651 & 124.6473 & 48.0822 \\
\hline 77.1252 & 124.0426 & 46.9174 & 76.9391 & 124.111 & 47.172 & 76.4706 & 124.7242 & 48.2536 \\
\hline 77.1105 & 124.0461 & 46.9356 & 77.0326 & 124.1535 & 47.1209 & 76.8569 & 124.4408 & 47.5839 \\
\hline 77.0958 & 124.0963 & 47.0005 & 77.0638 & 124.092 & 47.0282 & & & \\
\hline 77.1443 & 124.1024 & 46.9581 & 77.0716 & 124.1301 & 47.0585 & & & \\
\hline 77.1351 & 124.0985 & 46.9635 & 77.0283 & 124.1717 & 47.1434 & & & \\
\hline 77.1157 & 124.1059 & 46.9901 & 76.9339 & 124.2202 & 47.2863 & & & \\
\hline 77.0231 & 124.1353 & 47.1122 & 76.7906 & 124.2536 & 47.4629 & & & \\
\hline 76.9997 & 124.1457 & 47.146 & 76.8577 & 124.2565 & 47.3989 & & & \\
\hline 77.0118 & 124.1569 & 47.1451 & 76.8663 & 124.1803 & 47.314 & & & \\
\hline 76.9919 & 124.1621 & 47.1703 & 76.8265 & 124.1093 & 47.2828 & & & \\
\hline 76.978 & 124.1916 & 47.2136 & 76.8438 & 124.0825 & 47.2387 & & & \\
\hline 76.9798 & 124.1673 & 47.1876 & 76.8551 & 124.0937 & 47.2387 & & & \\
\hline 76.9712 & 124.1893 & 47.218 & 76.8464 & 124.1007 & 47.2543 & & & \\
\hline 76.9633 & 124.1751 & 47.2118 & 76.8758 & 124.1336 & 47.2577 & & & \\
\hline 77.0404 & 124.1535 & 47.1131 & 76.9272 & 124.1646 & 47.2374 & & & \\
\hline 77.0343 & 124.0989 & 47.0646 & 76.9746 & 124.1076 & 47.133 & & & \\
\hline 77.0031 & 124.2409 & 47.2378 & 77.0101 & 124.1734 & 47.1633 & & & \\
\hline 76.9624 & 124.2583 & 47.2958 & 76.9494 & 124.1604 & 47.211 & & & \\
\hline 76.9165 & 124.2669 & 47.3504 & 76.9936 & 124.1777 & 47.1841 & & & \\
\hline 76.9096 & 124.2756 & 47.366 & 76.8074 & 124.2392 & 47.4318 & & & \\
\hline
\end{tabular}




\begin{tabular}{|c|c|c|c|c|c|c|c|c|}
\hline \multicolumn{3}{|c|}{ Table A.4: 72 Hour Test } & 82.5764 & 122.8344 & 40.258 & 83.31 & 122.5714 & 39.2614 \\
\hline minimum & maximum & height & 82.5886 & 122.8467 & 40.2581 & 83.2632 & 122.554 & 39.2908 \\
\hline $\mathrm{mmHg}$ & $\mathrm{mmHg}$ & $\mathrm{mmHg}$ & 82.625 & 122.89 & 40.265 & 83.2767 & 122.5552 & 39.2785 \\
\hline 82.1219 & 121.3403 & 39.2184 & 82.6588 & 122.9151 & 40.2564 & 83.2451 & 122.5298 & 39.2847 \\
\hline 82.0284 & 120.9009 & 38.8725 & 82.7004 & 122.9775 & 40.2771 & 83.2165 & 122.5081 & 39.2917 \\
\hline 81.9392 & 120.8974 & 38.9583 & 82.7082 & 123.0061 & 40.2979 & 83.1619 & 122.4674 & 39.3055 \\
\hline 81.8329 & 120.9238 & 39.0908 & 82.6856 & 122.9775 & 40.2919 & 83.1325 & 122.4458 & 39.3133 \\
\hline 81.7937 & 120.9875 & 39.1938 & 83.1351 & 122.8242 & 39.6891 & 83.1108 & 122.4233 & 39.3124 \\
\hline 81.7192 & 121.0195 & 39.3003 & 83.1833 & 122.8803 & 39.6969 & 83.1152 & 122.4423 & 39.3272 \\
\hline 81.6776 & 121.0732 & 39.3956 & 83.2173 & 122.9351 & 39.7177 & 83.1108 & 122.4302 & 39.3194 \\
\hline 81.6742 & 121.0828 & 39.4086 & 83.2451 & 122.9593 & 39.7143 & 83.1375 & 122.4618 & 39.3243 \\
\hline 81.3103 & 121.7687 & 40.4585 & 83.3135 & 122.9879 & 39.6744 & 83.1048 & 122.4735 & 39.3687 \\
\hline 81.5529 & 120.9044 & 39.3514 & 83.2251 & 122.9567 & 39.7316 & 83.0719 & 122.4648 & 39.393 \\
\hline 81.5651 & 120.9477 & 39.3826 & 83.181 & 122.9498 & 39.7688 & 83.0719 & 122.4622 & 39.3904 \\
\hline 81.5008 & 120.9564 & 39.4555 & 83.1792 & 122.9203 & 39.7411 & 83.0874 & 122.4692 & 39.3817 \\
\hline 81.4508 & 120.9381 & 39.4874 & 83.1758 & 122.9342 & 39.7584 & 83.0952 & 122.4848 & 39.3895 \\
\hline 81.4369 & 120.9468 & 39.5099 & 83.1675 & 122.9454 & 39.778 & 83.0918 & 122.4969 & 39.4051 \\
\hline 81.5036 & 121.0724 & 39.5688 & 83.1723 & 122.9437 & 39.7714 & 83.0814 & 122.5081 & 39.4268 \\
\hline 81.6447 & 121.2707 & 39.6259 & 83.1273 & 122.9351 & 39.8078 & 83.0785 & 122.49 & 39.4115 \\
\hline 81.7694 & 121.4032 & 39.6337 & 83.103 & 122.9177 & 39.8147 & 83.084 & 122.5263 & 39.4423 \\
\hline 81.8656 & 121.5478 & 39.6822 & 83.1342 & 122.9567 & 39.8225 & 83.0424 & 122.5367 & 39.4943 \\
\hline 81.9608 & 121.6699 & 39.7091 & 83.1351 & 122.9576 & 39.8225 & 83.0727 & 122.5506 & 39.4778 \\
\hline 81.98 & 121.7316 & 39.7515 & 83.0883 & 122.9273 & 39.839 & 83.0684 & 122.5618 & 39.4934 \\
\hline 82.0491 & 121.8413 & 39.7922 & 83.0294 & 122.8935 & 39.8641 & 83.0545 & 122.5653 & 39.5108 \\
\hline 82.0777 & 121.8872 & 39.8095 & 82.9605 & 122.8899 & 39.9295 & 83.0364 & 122.574 & 39.5376 \\
\hline 82.0448 & 121.9418 & 39.897 & 82.9662 & 122.8866 & 39.9204 & 83.0684 & 122.5731 & 39.5047 \\
\hline 82.0691 & 121.9678 & 39.8987 & 82.9636 & 122.8684 & 39.9048 & 83.0653 & 122.6116 & 39.5463 \\
\hline 82.0318 & 121.979 & 39.9472 & 82.8943 & 122.8372 & 39.9429 & 83.1195 & 122.6597 & 39.5402 \\
\hline 82.063 & 122.0206 & 39.9576 & 82.8831 & 122.8 & 39.9169 & 83.1481 & 122.6839 & 39.5359 \\
\hline 82.095 & 122.0916 & 39.9966 & 82.8614 & 122.7783 & 39.9169 & 83.1385 & 122.6822 & 39.5437 \\
\hline 82.1016 & 122.1156 & 40.014 & 82.864 & 122.7991 & 39.9351 & 83.1472 & 122.6857 & 39.5385 \\
\hline 82.0994 & 122.154 & 40.0546 & 82.845 & 122.7801 & 39.9351 & 83.1143 & 122.6675 & 39.5532 \\
\hline 82.1461 & 122.2094 & 40.0632 & 82.7922 & 122.7525 & 39.9603 & 83.1273 & 122.7125 & 39.5852 \\
\hline 82.1886 & 122.2509 & 40.0624 & 82.8095 & 122.7671 & 39.9576 & 83.1186 & 122.7082 & 39.5896 \\
\hline 82.1669 & 122.2847 & 40.1178 & 82.7965 & 122.7489 & 39.9524 & 83.1314 & 122.7349 & 39.6035 \\
\hline 82.1323 & 122.2475 & 40.1152 & 82.832 & 122.8606 & 40.0286 & 83.1299 & 122.7394 & 39.6095 \\
\hline 82.1479 & 122.283 & 40.1351 & 82.8268 & 122.8736 & 40.0468 & 83.1481 & 122.761 & 39.6129 \\
\hline 82.1981 & 122.3297 & 40.1317 & 82.8684 & 122.8918 & 40.0234 & 83.1472 & 122.787 & 39.6398 \\
\hline 82.2379 & 122.4129 & 40.175 & 82.8415 & 122.8935 & 40.052 & 83.1524 & 122.7861 & 39.6337 \\
\hline 82.2813 & 122.4574 & 40.1761 & 82.8164 & 122.8589 & 40.0425 & 83.181 & 122.8242 & 39.6432 \\
\hline 82.3046 & 122.4787 & 40.1741 & 82.7679 & 122.819 & 40.0511 & 83.2381 & 122.8121 & 39.574 \\
\hline 82.3652 & 122.5636 & 40.1983 & 82.801 & 122.8424 & 40.0413 & 84.6973 & 122.4631 & 37.7658 \\
\hline 82.3921 & 122.5991 & 40.207 & 82.7887 & 122.8285 & 40.0399 & 84.4113 & 122.6028 & 38.1914 \\
\hline 82.3851 & 122.6753 & 40.2901 & 82.7532 & 122.8043 & 40.0511 & 84.5215 & 122.5783 & 38.0568 \\
\hline 82.5064 & 122.735 & 40.2286 & 82.7298 & 122.7749 & 40.0451 & 84.5743 & 122.6198 & 38.0455 \\
\hline 82.528 & 122.7714 & 40.2434 & 83.4079 & 122.6129 & 39.2051 & 84.1552 & 122.6822 & 38.527 \\
\hline 82.5618 & 122.8026 & 40.2408 & 83.3646 & 122.593 & 39.2284 & 84.1119 & 122.6779 & 38.566 \\
\hline
\end{tabular}




\begin{tabular}{|c|c|c|c|c|c|c|c|c|}
\hline 84.1102 & 122.6657 & 38.5556 & 86.8613 & 123.4512 & 36.5898 & 85.5295 & 124.2929 & 38.7634 \\
\hline 84.1136 & 122.6857 & 38.572 & 86.838 & 123.4642 & 36.6262 & 84.9926 & 124.2756 & 39.283 \\
\hline 84.1162 & 122.7108 & 38.5946 & 85.83 & 123.4971 & 37.6671 & 84.602 & 124.215 & 39.6129 \\
\hline 84.1448 & 122.7471 & 38.6024 & 85.6637 & 123.5066 & 37.8429 & 84.4981 & 124.3873 & 39.8892 \\
\hline 84.1497 & 122.7684 & 38.6187 & 85.7243 & 123.5516 & 37.8273 & 84.2054 & 124.2583 & 40.0529 \\
\hline 84.1595 & 122.7757 & 38.6162 & 85.7347 & 123.5854 & 37.8507 & 84.3925 & 124.4505 & 40.058 \\
\hline 84.2037 & 122.8459 & 38.6422 & 85.7327 & 123.6273 & 37.8946 & 84.3561 & 124.4704 & 40.1143 \\
\hline 84.2808 & 122.8744 & 38.5937 & 85.707 & 123.6478 & 37.9408 & 84.3864 & 124.4635 & 40.0771 \\
\hline 84.2998 & 122.9134 & 38.6136 & 85.6464 & 123.6642 & 38.0178 & 84.4466 & 124.4923 & 40.0457 \\
\hline 84.2929 & 122.9654 & 38.6725 & 85.5996 & 123.6893 & 38.0897 & 84.4436 & 124.4851 & 40.0416 \\
\hline 84.3137 & 122.981 & 38.6673 & 85.5468 & 123.6581 & 38.1113 & 84.4072 & 124.5042 & 40.097 \\
\hline 85.707 & 122.7965 & 37.0895 & 85.5598 & 123.6885 & 38.1287 & 84.4011 & 124.5103 & 40.1091 \\
\hline 85.9538 & 122.7957 & 36.8419 & 85.5962 & 123.7179 & 38.1217 & 84.3734 & 124.4981 & 40.1247 \\
\hline 86.0127 & 122.7965 & 36.7838 & 85.5814 & 123.7447 & 38.1633 & 84.3336 & 124.5397 & 40.2061 \\
\hline 86.0473 & 122.8407 & 36.7933 & 85.6067 & 123.7752 & 38.1685 & 84.3422 & 124.5544 & 40.2122 \\
\hline 86.0084 & 122.8511 & 36.8427 & 85.5936 & 123.8088 & 38.2153 & 84.3414 & 124.5648 & 40.2234 \\
\hline 86.0421 & 122.8848 & 36.8427 & 85.6291 & 123.8088 & 38.1798 & 84.2915 & 124.5725 & 40.2809 \\
\hline 86.1192 & 122.9342 & 36.815 & 85.6438 & 123.8158 & 38.172 & 84.3111 & 124.5579 & 40.2468 \\
\hline 86.1331 & 122.9749 & 36.8418 & 85.6602 & 123.8218 & 38.1616 & 84.3241 & 124.5917 & 40.2676 \\
\hline 86.1556 & 122.9792 & 36.8236 & 85.6481 & 123.8383 & 38.1901 & 84.3613 & 124.6194 & 40.2581 \\
\hline 86.115 & 123.0009 & 36.886 & 85.6429 & 123.8694 & 38.2265 & 84.441 & 124.6505 & 40.2096 \\
\hline 86.1694 & 123.0026 & 36.8332 & 85.6464 & 123.8894 & 38.243 & 84.389 & 124.6705 & 40.2815 \\
\hline 86.1894 & 122.9991 & 36.8098 & 85.656 & 123.8924 & 38.2364 & 84.4427 & 124.7042 & 40.2616 \\
\hline 86.1062 & 123.0104 & 36.9042 & 85.694 & 123.9292 & 38.2352 & 84.4029 & 124.7276 & 40.3248 \\
\hline 86.1227 & 123.0078 & 36.8851 & 85.7087 & 123.9465 & 38.2378 & 84.6571 & 124.6614 & 40.0043 \\
\hline 86.0854 & 123.0208 & 36.9354 & 85.7018 & 123.9881 & 38.2863 & 85.5598 & 124.5293 & 38.9695 \\
\hline 86.0984 & 123.0234 & 36.925 & 85.7157 & 124.0002 & 38.2845 & 85.8551 & 124.538 & 38.6829 \\
\hline 86.127 & 123.0502 & 36.9232 & 85.7425 & 123.9907 & 38.2482 & 85.8352 & 124.5293 & 38.6941 \\
\hline 86.0789 & 123.0353 & 36.9564 & 85.7295 & 124.0011 & 38.2715 & 85.8906 & 124.5406 & 38.65 \\
\hline 86.088 & 123.0381 & 36.9501 & 85.688 & 124.0019 & 38.314 & 85.8906 & 124.5501 & 38.6595 \\
\hline 86.1911 & 123.0459 & 36.8548 & 85.6437 & 124.0087 & 38.365 & 85.9555 & 124.5995 & 38.6439 \\
\hline 86.2595 & 123.071 & 36.8115 & 85.6438 & 124.0288 & 38.385 & 86.0032 & 124.628 & 38.6249 \\
\hline 86.3643 & 123.1074 & 36.7431 & 85.7313 & 124.053 & 38.3218 & 85.9749 & 124.6139 & 38.6389 \\
\hline 86.3799 & 123.1386 & 36.7587 & 85.7287 & 124.1041 & 38.3755 & 85.9893 & 124.6497 & 38.6604 \\
\hline 86.4258 & 123.1446 & 36.7189 & 85.7538 & 124.1613 & 38.4075 & 85.9382 & 124.6826 & 38.7444 \\
\hline 86.5279 & 123.1784 & 36.6505 & 85.7685 & 124.1708 & 38.4023 & 85.9209 & 124.6878 & 38.7669 \\
\hline 86.544 & 123.2071 & 36.6631 & 85.8274 & 124.1786 & 38.3512 & 86.0231 & 124.7276 & 38.7045 \\
\hline 86.5461 & 123.2148 & 36.6686 & 85.8386 & 124.1976 & 38.359 & 86.0032 & 124.7683 & 38.7652 \\
\hline 86.5167 & 123.2234 & 36.7067 & 85.8551 & 124.1981 & 38.343 & 86.0621 & 124.738 & 38.676 \\
\hline 86.6085 & 123.2078 & 36.5994 & 85.8993 & 124.215 & 38.3157 & 85.8456 & 124.7848 & 38.9392 \\
\hline 86.6959 & 123.2737 & 36.5777 & 86.3548 & 124.0885 & 37.7338 & 85.9168 & 124.8244 & 38.9076 \\
\hline 86.6899 & 123.2892 & 36.5994 & 86.398 & 124.0946 & 37.6965 & 86.0638 & 124.8393 & 38.7755 \\
\hline 86.7141 & 123.3239 & 36.6098 & 86.4483 & 124.131 & 37.6827 & 86.0603 & 124.8636 & 38.8033 \\
\hline 86.6985 & 123.3265 & 36.6279 & 86.4665 & 124.1976 & 37.7312 & 86.1175 & 124.874 & 38.7565 \\
\hline 86.773 & 123.3894 & 36.6164 & 86.4387 & 124.2626 & 37.8238 & 86.3409 & 124.9103 & 38.5694 \\
\hline 86.8388 & 123.4165 & 36.5777 & 86.4976 & 124.273 & 37.7754 & 86.3799 & 124.9259 & 38.5461 \\
\hline 86.8709 & 123.4235 & 36.5526 & 85.8481 & 124.1558 & 38.3077 & 86.3115 & 124.9164 & 38.605 \\
\hline
\end{tabular}




\begin{tabular}{|c|c|c|c|c|c|c|c|c|}
\hline 86.2898 & 124.9112 & 38.6214 & 87.0674 & 125.1364 & 38.0689 & 88.453 & 125.3745 & 36.9215 \\
\hline 86.3801 & 124.9442 & 38.5641 & 87.0692 & 125.1554 & 38.0862 & 88.5578 & 125.3745 & 36.8167 \\
\hline 86.34 & 124.9545 & 38.6145 & 86.9679 & 125.1467 & 38.1789 & 88.563 & 125.3892 & 36.8262 \\
\hline 86.3392 & 124.9381 & 38.5989 & 87.0047 & 125.1248 & 38.1201 & 88.6253 & 125.4126 & 36.7873 \\
\hline 86.3192 & 124.9458 & 38.6266 & 86.928 & 125.1719 & 38.2438 & 88.7102 & 125.436 & 36.7258 \\
\hline 86.3773 & 124.9528 & 38.5755 & 86.9497 & 125.1762 & 38.2265 & 88.6062 & 125.4305 & 36.8243 \\
\hline 86.4058 & 124.9701 & 38.5642 & 86.9211 & 125.1658 & 38.2447 & 88.6643 & 125.4412 & 36.7769 \\
\hline 86.4405 & 124.9675 & 38.527 & 86.9445 & 125.1944 & 38.2499 & 88.7673 & 125.4438 & 36.6764 \\
\hline 86.5046 & 124.9658 & 38.4612 & 86.9194 & 125.2359 & 38.3166 & 88.815 & 125.4689 & 36.6539 \\
\hline 86.5002 & 124.9857 & 38.4854 & 86.9133 & 125.2455 & 38.3322 & 88.7977 & 125.4585 & 36.6608 \\
\hline 86.5343 & 125.0085 & 38.4742 & 86.9142 & 125.2507 & 38.3365 & 88.7769 & 125.4715 & 36.6946 \\
\hline 86.5089 & 124.9796 & 38.4707 & 86.8977 & 125.2385 & 38.3408 & 88.7639 & 125.4905 & 36.7267 \\
\hline 86.5539 & 124.9917 & 38.4378 & 86.9488 & 125.2368 & 38.288 & 88.757 & 125.4897 & 36.7327 \\
\hline 86.5262 & 125.0013 & 38.4751 & 87.0259 & 125.2645 & 38.2386 & 88.8405 & 125.5282 & 36.6878 \\
\hline 86.5219 & 125.0169 & 38.495 & 86.7124 & 125.3364 & 38.624 & 88.9466 & 125.5581 & 36.6115 \\
\hline 86.5557 & 125.0454 & 38.4898 & 86.6388 & 125.358 & 38.7193 & 88.9501 & 125.5667 & 36.6167 \\
\hline 86.5288 & 125.0446 & 38.5158 & 86.6145 & 125.3442 & 38.7297 & 88.9908 & 125.5685 & 36.5777 \\
\hline 86.4846 & 125.0298 & 38.5452 & 86.5738 & 125.3797 & 38.8059 & 89.1094 & 125.5763 & 36.4669 \\
\hline 86.2031 & 125.0807 & 38.8777 & 86.4691 & 125.3927 & 38.9236 & 89.1155 & 125.6378 & 36.5223 \\
\hline 86.1054 & 125.0922 & 38.9868 & 86.3677 & 125.4126 & 39.0449 & 88.9726 & 125.6204 & 36.6479 \\
\hline 86.0248 & 125.1225 & 39.0977 & 86.2885 & 125.3891 & 39.1005 & 88.9215 & 125.6473 & 36.7258 \\
\hline 85.9451 & 125.1667 & 39.2215 & 86.2933 & 125.3563 & 39.063 & 88.8427 & 125.6637 & 36.821 \\
\hline 85.8811 & 125.2065 & 39.3254 & 86.2465 & 125.3632 & 39.1167 & 88.7709 & 125.7009 & 36.93 \\
\hline 85.875 & 125.2281 & 39.3531 & 86.1599 & 125.3979 & 39.238 & 88.6825 & 125.7044 & 37.022 \\
\hline 86.5418 & 125.0939 & 38.5521 & 86.1235 & 125.3771 & 39.2536 & 88.5794 & 125.7287 & 37.1493 \\
\hline 87.1073 & 125.0524 & 37.9451 & 86.0387 & 125.4308 & 39.3921 & 88.5188 & 125.746 & 37.2272 \\
\hline 87.0417 & 125.042 & 38.0003 & 86.0664 & 125.4256 & 39.3592 & 88.5084 & 125.7711 & 37.2627 \\
\hline 87.1679 & 125.0463 & 37.8784 & 86.1192 & 125.436 & 39.3168 & 88.4946 & 125.8127 & 37.3181 \\
\hline 87.1575 & 125.0359 & 37.8784 & 86.0868 & 125.4534 & 39.3666 & 88.5093 & 125.8413 & 37.332 \\
\hline 87.1012 & 125.0246 & 37.9234 & 86.0924 & 125.4403 & 39.348 & 88.5751 & 125.8664 & 37.2913 \\
\hline 87.0172 & 125.0679 & 38.0507 & 86.1028 & 125.462 & 39.3592 & 88.4494 & 125.9105 & 37.4612 \\
\hline 86.0153 & 125.223 & 39.2077 & 86.0785 & 125.4472 & 39.3687 & 88.3257 & 125.933 & 37.6074 \\
\hline 86.0421 & 125.216 & 39.1739 & 86.0742 & 125.4386 & 39.3644 & 88.3179 & 125.9391 & 37.6212 \\
\hline 86.0621 & 125.2524 & 39.1903 & 86.1668 & 125.4273 & 39.2605 & 88.4036 & 125.9478 & 37.5441 \\
\hline 86.056 & 125.2375 & 39.1816 & 86.2326 & 125.4239 & 39.1912 & 88.2919 & 125.9876 & 37.6957 \\
\hline 86.0387 & 125.2307 & 39.1921 & 86.2326 & 125.4265 & 39.1938 & 88.4539 & 125.9019 & 37.448 \\
\hline 86.0369 & 125.2411 & 39.2042 & 86.2286 & 125.4578 & 39.2291 & 88.7422 & 125.843 & 37.1008 \\
\hline 86.0032 & 125.2663 & 39.2631 & 86.2422 & 125.4481 & 39.2059 & 88.705 & 125.8612 & 37.1562 \\
\hline 85.9192 & 125.3087 & 39.3895 & 86.2794 & 125.4897 & 39.2103 & 88.7357 & 125.8771 & 37.1414 \\
\hline 86.101 & 125.2541 & 39.1531 & 86.2223 & 125.4983 & 39.2761 & 88.6279 & 125.8681 & 37.2402 \\
\hline 86.8397 & 125.1069 & 38.2672 & 86.4734 & 125.4776 & 39.0042 & 88.4019 & 125.862 & 37.4601 \\
\hline 86.9514 & 125.113 & 38.1616 & 88.188 & 125.3139 & 37.1259 & 88.4746 & 125.8664 & 37.3917 \\
\hline 86.8655 & 125.1186 & 38.2531 & 88.3794 & 125.2983 & 36.9189 & 88.5119 & 125.882 & 37.3701 \\
\hline 86.9679 & 125.1138 & 38.146 & 88.453 & 125.3243 & 36.8713 & 88.472 & 125.901 & 37.429 \\
\hline 86.9705 & 125.0905 & 38.12 & 88.3983 & 125.3248 & 36.9265 & 88.3664 & 125.9512 & 37.5848 \\
\hline 87.006 & 125.1009 & 38.0949 & 88.537 & 125.3563 & 36.8193 & 88.3725 & 125.9616 & 37.5892 \\
\hline 87.0718 & 125.1286 & 38.0568 & 88.5292 & 125.3503 & 36.8211 & 88.3745 & 125.966 & 37.5916 \\
\hline
\end{tabular}




\begin{tabular}{|c|c|c|c|c|c|c|c|c|}
\hline 88.2685 & 125.9755 & 37.7069 & 86.3158 & 126.3349 & 40.0191 & 88.2928 & 126.1972 & 37.9044 \\
\hline 88.2391 & 126.037 & 37.7979 & 86.3546 & 126.2911 & 39.9365 & 88.2036 & 126.1859 & 37.9823 \\
\hline 88.1404 & 126.0404 & 37.9 & 86.2733 & 126.3236 & 40.0503 & 88.1187 & 126.2076 & 38.0888 \\
\hline 88.1733 & 126.0344 & 37.8611 & 86.2153 & 126.3401 & 40.1247 & 88.2547 & 126.2101 & 37.9554 \\
\hline 88.0659 & 126.0326 & 37.9667 & 86.7618 & 126.263 & 39.5012 & 88.3127 & 126.2171 & 37.9044 \\
\hline 88.0243 & 126.0759 & 38.0516 & 88.2062 & 125.9686 & 37.7624 & 88.5517 & 126.205 & 37.6532 \\
\hline 87.9118 & 126.0508 & 38.1391 & 88.214 & 125.9928 & 37.7788 & 88.2989 & 126.2162 & 37.9174 \\
\hline 88.0107 & 126.0453 & 38.0346 & 88.3447 & 125.9703 & 37.6255 & 88.2382 & 126.192 & 37.9537 \\
\hline 87.9325 & 126.0716 & 38.1391 & 88.2322 & 126.0145 & 37.7823 & 88.2027 & 126.2015 & 37.9988 \\
\hline 87.8581 & 126.0647 & 38.2066 & 88.3005 & 125.9757 & 37.6752 & 88.1421 & 126.2032 & 38.0611 \\
\hline 87.7481 & 126.0638 & 38.3157 & 88.1066 & 126.0214 & 37.9148 & 88.2642 & 126.2015 & 37.9373 \\
\hline 87.6251 & 126.0967 & 38.4716 & 88.0936 & 126.0283 & 37.9347 & 88.201 & 126.181 & 37.98 \\
\hline 87.5983 & 126.0907 & 38.4924 & 88.3318 & 126.0439 & 37.7121 & 88.4201 & 126.1998 & 37.7797 \\
\hline 87.4857 & 126.0863 & 38.6006 & 88.046 & 126.0881 & 38.0421 & 88.5509 & 126.1954 & 37.6446 \\
\hline 87.4459 & 126.0586 & 38.6127 & 87.9568 & 126.1002 & 38.1434 & 88.2417 & 126.2327 & 37.991 \\
\hline 87.3818 & 126.0794 & 38.6976 & 87.9274 & 126.0811 & 38.1538 & 88.1239 & 126.2396 & 38.1157 \\
\hline 87.5843 & 126.0577 & 38.4733 & 87.8771 & 126.0933 & 38.2161 & 88.2642 & 126.2431 & 37.9789 \\
\hline 87.5524 & 126.0552 & 38.5028 & 88.1252 & 126.1052 & 37.98 & 88.0503 & 126.2673 & 38.217 \\
\hline 87.2216 & 126.0681 & 38.8466 & 88.1664 & 126.1392 & 37.9728 & 88.104 & 126.2803 & 38.1763 \\
\hline 87.193 & 126.0907 & 38.8976 & 87.962 & 126.1166 & 38.1546 & 88.378 & 126.255 & 37.877 \\
\hline 87.1887 & 126.0837 & 38.895 & 88.0815 & 126.1236 & 38.0421 & 88.1716 & 126.2587 & 38.0871 \\
\hline 87.2389 & 126.1114 & 38.8725 & 88.0555 & 126.1244 & 38.0689 & 88.4365 & 126.2396 & 37.8031 \\
\hline 87.2069 & 126.1028 & 38.8959 & 88.1222 & 126.0993 & 37.9771 & 88.6383 & 126.2535 & 37.6151 \\
\hline 87.3324 & 126.1114 & 38.779 & 88.2313 & 126.114 & 37.8827 & 88.802 & 126.2171 & 37.4151 \\
\hline 87.3606 & 126.1123 & 38.7517 & 88.1309 & 126.1123 & 37.9814 & 88.737 & 126.2283 & 37.4913 \\
\hline 87.2761 & 126.1149 & 38.8388 & 88.149 & 126.1227 & 37.9737 & 88.6002 & 126.2405 & 37.6403 \\
\hline 87.2787 & 126.0915 & 38.8128 & 87.6618 & 126.1405 & 38.4786 & 88.4625 & 126.2439 & 37.7814 \\
\hline 87.1843 & 126.1201 & 38.9357 & 87.6892 & 126.1435 & 38.4543 & 88.3629 & 126.2543 & 37.8914 \\
\hline 87.1289 & 126.153 & 39.0241 & 87.8702 & 126.1392 & 38.269 & 88.5005 & 126.2523 & 37.7519 \\
\hline 87.1289 & 126.1998 & 39.0708 & 87.7879 & 126.127 & 38.3391 & 88.3456 & 126.2621 & 37.9165 \\
\hline 87.1731 & 126.2015 & 39.0284 & 87.6433 & 126.1366 & 38.4932 & 88.7266 & 126.2604 & 37.5337 \\
\hline 87.0865 & 126.179 & 39.0925 & 87.7923 & 126.1097 & 38.3174 & 88.6149 & 126.2509 & 37.6359 \\
\hline 87.1219 & 126.2004 & 39.0785 & 87.7187 & 126.14 & 38.4214 & 88.8375 & 126.2638 & 37.4264 \\
\hline 87.0501 & 126.2128 & 39.1626 & 87.5766 & 126.1712 & 38.5946 & 89.0531 & 126.3115 & 37.2584 \\
\hline 86.8536 & 126.2638 & 39.4103 & 87.6671 & 126.1757 & 38.5086 & 89.3233 & 126.302 & 36.9787 \\
\hline 86.9228 & 126.2587 & 39.3358 & 87.3376 & 126.2864 & 38.9487 & 88.9994 & 126.3331 & 37.3337 \\
\hline 86.9124 & 126.2517 & 39.3393 & 86.8518 & 126.4249 & 39.5731 & 89.2563 & 126.3193 & 37.063 \\
\hline 86.8717 & 126.2751 & 39.4034 & 86.9029 & 126.3972 & 39.4943 & 89.1977 & 126.3392 & 37.1415 \\
\hline 86.7799 & 126.2907 & 39.5108 & 87.9906 & 126.2431 & 38.2525 & 89.1666 & 126.3305 & 37.164 \\
\hline 86.7817 & 126.2994 & 39.5177 & 88.5041 & 126.1729 & 37.6688 & 89.1648 & 126.3626 & 37.1977 \\
\hline 86.7836 & 126.2937 & 39.5102 & 88.4469 & 126.1547 & 37.7078 & 88.9509 & 126.3868 & 37.4359 \\
\hline 86.754 & 126.3184 & 39.5644 & 88.5058 & 126.1582 & 37.6524 & 89.0687 & 126.3782 & 37.3095 \\
\hline 86.7055 & 126.2968 & 39.5913 & 88.3481 & 126.1801 & 37.832 & 88.7266 & 126.3513 & 37.6247 \\
\hline 86.663 & 126.2942 & 39.6311 & 88.3508 & 126.1928 & 37.842 & 88.4305 & 126.25 & 37.8195 \\
\hline 86.5323 & 126.3106 & 39.7783 & 88.3777 & 126.1902 & 37.8126 & 88.0926 & 126.2788 & 38.1862 \\
\hline 86.5089 & 126.308 & 39.7991 & 88.3491 & 126.1824 & 37.8334 & 88.0486 & 126.2881 & 38.2395 \\
\hline 86.3833 & 126.3219 & 39.9385 & 88.3664 & 126.1911 & 37.8247 & 87.8953 & 126.2768 & 38.3815 \\
\hline
\end{tabular}




\begin{tabular}{|c|c|c|c|c|c|c|c|c|}
\hline 88.4591 & 126.2249 & 37.7658 & 88.2859 & 126.3167 & 38.0308 & 87.7568 & 126.4041 & 38.6474 \\
\hline 88.3993 & 126.2439 & 37.8446 & 88.1335 & 126.3288 & 38.1953 & 87.727 & 126.4127 & 38.6856 \\
\hline 88.3508 & 126.2656 & 37.9148 & 87.51 & 126.3756 & 38.8656 & 87.7992 & 126.3972 & 38.598 \\
\hline 88.3638 & 126.2708 & 37.907 & 87.5351 & 126.3418 & 38.8067 & 87.8537 & 126.4396 & 38.5859 \\
\hline 88.4539 & 126.269 & 37.8152 & 87.7117 & 126.3002 & 38.5885 & 87.9828 & 126.4492 & 38.4664 \\
\hline 88.5701 & 126.2567 & 37.6867 & 87.5792 & 126.308 & 38.7288 & 87.9403 & 126.4691 & 38.5287 \\
\hline 88.3231 & 126.2587 & 37.9356 & 88.7474 & 126.3106 & 37.5632 & 88.0157 & 126.4656 & 38.4499 \\
\hline 88.2651 & 126.2734 & 38.0083 & 87.7182 & 126.3651 & 38.6469 & 88.0295 & 126.457 & 38.4274 \\
\hline 88.2201 & 126.2959 & 38.0758 & 87.9481 & 126.3695 & 38.4214 & 88.0139 & 126.47 & 38.456 \\
\hline 88.2625 & 126.3054 & 38.0429 & 88.0772 & 126.3652 & 38.288 & 87.9869 & 126.4558 & 38.4689 \\
\hline 88.02 & 126.3167 & 38.2967 & 88.162 & 126.347 & 38.185 & 87.91 & 126.4812 & 38.5712 \\
\hline 88.1187 & 126.3375 & 38.2187 & 88.2989 & 126.353 & 38.0542 & 87.9187 & 126.4916 & 38.5729 \\
\hline 88.0252 & 126.3331 & 38.3079 & 88.2911 & 126.4223 & 38.1313 & 87.93 & 126.5063 & 38.5764 \\
\hline 87.9226 & 126.3563 & 38.4337 & 88.5231 & 126.4405 & 37.9174 & 87.8485 & 126.4855 & 38.637 \\
\hline 88.0971 & 126.3409 & 38.2438 & 88.4928 & 126.431 & 37.9382 & 87.6719 & 126.4994 & 38.8275 \\
\hline 88.1473 & 126.3383 & 38.191 & 88.504 & 126.4479 & 37.9439 & 87.8815 & 126.4795 & 38.598 \\
\hline 88.2798 & 126.3375 & 38.0577 & 88.653 & 126.3877 & 37.7347 & 87.9724 & 126.4734 & 38.501 \\
\hline 88.3231 & 126.3331 & 38.01 & 88.4738 & 126.3963 & 37.9226 & 87.9129 & 126.4743 & 38.5614 \\
\hline 88.3725 & 126.3279 & 37.9555 & 88.5993 & 126.3712 & 37.7719 & 87.8373 & 126.4803 & 38.6431 \\
\hline 88.3603 & 126.3435 & 37.9832 & 88.4305 & 126.4154 & 37.9849 & 87.8607 & 126.5003 & 38.6396 \\
\hline 88.3872 & 126.3626 & 37.9754 & 88.9414 & 126.3989 & 37.4575 & 87.9256 & 126.521 & 38.5954 \\
\hline 88.2908 & 126.3536 & 38.0628 & 88.9319 & 126.4258 & 37.4939 & 87.7628 & 126.5375 & 38.7747 \\
\hline 88.1984 & 126.3678 & 38.1694 & 88.9613 & 126.4665 & 37.5052 & 87.781 & 126.5228 & 38.7418 \\
\hline 88.2711 & 126.347 & 38.0758 & 88.9515 & 126.469 & 37.5176 & 87.8304 & 126.5158 & 38.6855 \\
\hline 88.4296 & 126.3375 & 37.9078 & 87.8312 & 126.4318 & 38.6006 & 87.8667 & 126.5158 & 38.6491 \\
\hline 88.4019 & 126.366 & 37.9641 & 87.5368 & 126.4448 & 38.908 & 87.6698 & 126.5034 & 38.8336 \\
\hline 88.2365 & 126.3608 & 38.1243 & 87.7628 & 126.3998 & 38.637 & 87.7819 & 126.5158 & 38.734 \\
\hline 88.1949 & 126.3652 & 38.1702 & 87.5533 & 126.4622 & 38.9089 & 87.7204 & 126.528 & 38.8076 \\
\hline 88.621 & 126.3427 & 37.7217 & 87.4866 & 126.4769 & 38.9903 & 87.7949 & 126.5236 & 38.7288 \\
\hline 88.3648 & 126.3536 & 37.9888 & 87.4658 & 126.4336 & 38.9678 & 87.7853 & 126.5271 & 38.7418 \\
\hline 88.479 & 126.3236 & 37.8446 & 87.5481 & 126.4362 & 38.8881 & 87.6788 & 126.528 & 38.8492 \\
\hline 88.408 & 126.3349 & 37.9269 & 87.6654 & 126.4541 & 38.7887 & 88.1283 & 126.5366 & 38.4084 \\
\hline 88.3491 & 126.3444 & 37.9953 & 87.5472 & 126.4881 & 38.9409 & 87.7238 & 126.5418 & 38.818 \\
\hline 88.2737 & 126.3496 & 38.0758 & 87.6693 & 126.4474 & 38.7781 & 87.7085 & 126.5466 & 38.838 \\
\hline 88.188 & 126.3253 & 38.1373 & 87.4381 & 126.4691 & 39.031 & 87.8702 & 126.5098 & 38.6396 \\
\hline 88.1378 & 126.3496 & 38.2118 & 87.7481 & 126.4154 & 38.6673 & 87.8737 & 126.5081 & 38.6344 \\
\hline 88.4669 & 126.3427 & 37.8758 & 87.9559 & 126.4154 & 38.4595 & 87.9213 & 126.5158 & 38.5946 \\
\hline 88.3292 & 126.3227 & 37.9936 & 87.923 & 126.4033 & 38.4803 & 88.0044 & 126.5184 & 38.514 \\
\hline 88.0618 & 126.3501 & 38.2883 & 87.7464 & 126.4379 & 38.6915 & 87.8529 & 126.5522 & 38.6993 \\
\hline 87.9776 & 126.3297 & 38.3521 & 87.6996 & 126.4457 & 38.7461 & 87.9291 & 126.528 & 38.5989 \\
\hline 87.917 & 126.3158 & 38.3988 & 87.7667 & 126.4188 & 38.6522 & 87.9516 & 126.5349 & 38.5833 \\
\hline 88.2426 & 126.2994 & 38.0568 & 88.0139 & 126.4206 & 38.4066 & 87.5351 & 126.5825 & 39.0475 \\
\hline 88.6063 & 126.289 & 37.6827 & 88.0278 & 126.405 & 38.3772 & 87.6856 & 126.5527 & 38.8671 \\
\hline 88.6556 & 126.2846 & 37.629 & 87.9724 & 126.4379 & 38.4655 & 87.5498 & 126.5635 & 39.0137 \\
\hline 88.4608 & 126.3002 & 37.8394 & 87.9577 & 126.418 & 38.4603 & 87.6416 & 126.5912 & 38.9496 \\
\hline 88.4461 & 126.3245 & 37.8784 & 87.852 & 126.4275 & 38.5755 & 87.9092 & 126.5869 & 38.6777 \\
\hline 88.2723 & 126.3193 & 38.047 & 87.8425 & 126.3963 & 38.5539 & 88.1941 & 126.586 & 38.3919 \\
\hline
\end{tabular}




\begin{tabular}{|c|c|c|c|c|c|c|c|c|}
\hline 88.2382 & 126.5912 & 38.353 & 87.7524 & 126.7739 & 39.0215 & 87.4545 & 126.9488 & 39.4943 \\
\hline 88.3309 & 126.6042 & 38.2733 & 87.6026 & 126.7704 & 39.1678 & 87.5048 & 126.9497 & 39.4449 \\
\hline 88.3274 & 126.605 & 38.2776 & 87.846 & 126.7505 & 38.9046 & 87.4563 & 126.9887 & 39.5324 \\
\hline 88.2609 & 126.6294 & 38.3685 & 87.613 & 126.7635 & 39.1505 & 87.5784 & 126.9826 & 39.4042 \\
\hline 88.3569 & 126.6284 & 38.2716 & 87.6087 & 126.7652 & 39.1566 & 87.4104 & 126.9835 & 39.5731 \\
\hline 88.2963 & 126.657 & 38.3607 & 87.868 & 126.7404 & 38.8724 & 87.3662 & 127.0146 & 39.6484 \\
\hline 88.0902 & 126.6206 & 38.5305 & 87.7931 & 126.7445 & 38.9513 & 87.6061 & 127.0051 & 39.399 \\
\hline 88.3248 & 126.6354 & 38.3105 & 87.5992 & 126.7782 & 39.1791 & 87.5517 & 127.0037 & 39.452 \\
\hline 88.3465 & 126.638 & 38.2915 & 88.0521 & 126.7401 & 38.6881 & 87.6823 & 127.0528 & 39.3705 \\
\hline 88.472 & 126.6622 & 38.1902 & 87.8867 & 126.767 & 38.8803 & 87.7342 & 127.0389 & 39.3047 \\
\hline 88.2642 & 126.6709 & 38.4066 & 87.658 & 126.7756 & 39.1176 & 87.729 & 127.0302 & 39.3012 \\
\hline 88.5516 & 126.6637 & 38.1122 & 87.6494 & 126.7895 & 39.1401 & 87.7645 & 127.0398 & 39.2752 \\
\hline 88.4954 & 126.6665 & 38.1711 & 87.4424 & 126.7921 & 39.3497 & 87.4814 & 127.0493 & 39.5679 \\
\hline 88.6201 & 126.6804 & 38.0603 & 87.4989 & 126.7906 & 39.2917 & 87.3238 & 127.1082 & 39.7844 \\
\hline 88.3257 & 126.6674 & 38.3417 & 87.4251 & 126.8103 & 39.3852 & 87.4511 & 127.0649 & 39.6138 \\
\hline 88.7587 & 126.6882 & 37.9295 & 87.4935 & 126.8111 & 39.3176 & 87.2073 & 127.076 & 39.8687 \\
\hline 88.666 & 126.715 & 38.049 & 87.4234 & 126.8207 & 39.3973 & 88.4625 & 127.045 & 38.5824 \\
\hline 88.7214 & 126.6735 & 37.952 & 87.3523 & 126.8674 & 39.5151 & 87.9966 & 127.0354 & 39.0388 \\
\hline 89.1761 & 126.7254 & 37.5493 & 87.5004 & 126.851 & 39.3506 & 88.007 & 127.0372 & 39.0301 \\
\hline 88.5057 & 126.7412 & 38.2355 & 87.7022 & 126.8224 & 39.1202 & 87.7914 & 127.0354 & 39.244 \\
\hline 87.729 & 126.5635 & 38.8344 & 87.7264 & 126.8345 & 39.1081 & 87.8849 & 127.0683 & 39.1834 \\
\hline 87.7005 & 126.5643 & 38.8639 & 87.5931 & 126.8249 & 39.2318 & 87.9568 & 127.0458 & 39.089 \\
\hline 87.7585 & 126.5721 & 38.8136 & 87.7117 & 126.7878 & 39.076 & 88.0685 & 127.038 & 38.9695 \\
\hline 87.6788 & 126.6128 & 38.934 & 87.7524 & 126.7826 & 39.0301 & 87.8125 & 126.9016 & 39.0891 \\
\hline 87.7801 & 126.6319 & 38.8518 & 87.7628 & 126.7722 & 39.0094 & 88.0624 & 126.8631 & 38.8007 \\
\hline 87.684 & 126.6224 & 38.9383 & 87.7594 & 126.7618 & 39.0024 & 88.1239 & 126.9029 & 38.779 \\
\hline 87.6 & 126.6371 & 39.0371 & 87.6953 & 126.7869 & 39.0916 & 88.1672 & 126.896 & 38.7288 \\
\hline 87.6539 & 126.6294 & 38.9755 & 87.7827 & 126.7687 & 38.986 & 88.1265 & 126.9038 & 38.7773 \\
\hline 87.8078 & 126.6466 & 38.8388 & 87.6875 & 126.7895 & 39.102 & 88.1257 & 126.8925 & 38.7669 \\
\hline 87.7706 & 126.6457 & 38.8751 & 87.7852 & 126.7941 & 39.0089 & 88.3292 & 126.9185 & 38.5894 \\
\hline 87.5957 & 126.6648 & 39.0691 & 87.8572 & 126.7904 & 38.9332 & 88.369 & 126.9246 & 38.5556 \\
\hline 87.6199 & 126.6787 & 39.0587 & 87.9109 & 126.7956 & 38.8847 & 88.4194 & 126.9412 & 38.5218 \\
\hline 87.497 & 126.7202 & 39.2233 & 87.6468 & 126.7964 & 39.1496 & 88.2538 & 126.9341 & 38.6803 \\
\hline 87.3801 & 126.7029 & 39.3228 & 87.6234 & 126.8163 & 39.1929 & 88.143 & 126.9428 & 38.7998 \\
\hline 87.5879 & 126.7098 & 39.1219 & 87.6624 & 126.8345 & 39.1722 & 88.1464 & 126.9159 & 38.7695 \\
\hline 87.4865 & 126.7069 & 39.2203 & 87.7282 & 126.8337 & 39.1055 & 88.1638 & 126.9445 & 38.7807 \\
\hline 87.393 & 126.7194 & 39.3263 & 87.7845 & 126.8354 & 39.0509 & 87.7715 & 126.9402 & 39.1687 \\
\hline 87.5446 & 126.7202 & 39.1756 & 87.7667 & 126.8602 & 39.0935 & 87.8122 & 126.9246 & 39.1124 \\
\hline 87.5844 & 126.7185 & 39.1341 & 87.6693 & 126.8804 & 39.2111 & 87.8009 & 126.9332 & 39.1323 \\
\hline 87.5221 & 126.7358 & 39.2137 & 87.7031 & 126.8735 & 39.1704 & 87.8565 & 126.9236 & 39.0671 \\
\hline 87.5879 & 126.7453 & 39.1574 & 87.5654 & 126.8718 & 39.3064 & 88.0789 & 126.9255 & 38.8466 \\
\hline 87.742 & 126.741 & 38.999 & 87.5576 & 126.864 & 39.3064 & 88.0356 & 126.9203 & 38.8847 \\
\hline 87.7109 & 126.7297 & 39.0189 & 87.4519 & 126.8458 & 39.3939 & 88.0079 & 126.9306 & 38.9228 \\
\hline 87.5412 & 126.7562 & 39.2151 & 87.374 & 126.8622 & 39.4882 & 88.2192 & 126.928 & 38.7089 \\
\hline 87.4511 & 126.7704 & 39.3194 & 87.4935 & 126.8874 & 39.3938 & 88.2434 & 126.9289 & 38.6855 \\
\hline 87.6165 & 126.728 & 39.1115 & 87.4989 & 126.8972 & 39.3983 & 88.1508 & 126.9177 & 38.7669 \\
\hline 87.8018 & 126.7497 & 38.9479 & 87.4355 & 126.9255 & 39.49 & 88.1005 & 126.9272 & 38.8266 \\
\hline
\end{tabular}




\begin{tabular}{|c|c|c|c|c|c|c|c|c|}
\hline 87.8512 & 126.8945 & 39.0433 & 88.2954 & 127.0328 & 38.7374 & 86.3903 & 127.0813 & 40.6911 \\
\hline 88.0624 & 126.8995 & 38.837 & 88.2287 & 127.025 & 38.7963 & 86.2976 & 127.1004 & 40.8028 \\
\hline 87.9022 & 126.8778 & 38.9756 & 88.2371 & 127.0293 & 38.7922 & 86.2811 & 127.1238 & 40.8426 \\
\hline 87.813 & 126.8995 & 39.0864 & 88.1698 & 127.0268 & 38.8569 & 86.1764 & 127.18 & 41.0037 \\
\hline 87.9516 & 126.8891 & 38.9375 & 88.0728 & 127.0172 & 38.9444 & 86.0188 & 127.1922 & 41.1734 \\
\hline 87.6754 & 126.8882 & 39.2129 & 87.9464 & 127.032 & 39.0856 & 85.974 & 127.2266 & 41.2526 \\
\hline 87.5515 & 126.883 & 39.3315 & 87.839 & 127.077 & 39.238 & 85.9218 & 127.2502 & 41.3284 \\
\hline 87.3229 & 126.9029 & 39.58 & 87.9109 & 127.0553 & 39.1444 & 85.8447 & 127.2718 & 41.4271 \\
\hline 87.5446 & 126.8934 & 39.3488 & 87.8702 & 127.0398 & 39.1696 & 85.6931 & 127.3541 & 41.661 \\
\hline 87.8363 & 126.891 & 39.0547 & 87.9057 & 127.0415 & 39.1358 & 85.6152 & 127.361 & 41.7458 \\
\hline 87.7135 & 126.8744 & 39.1609 & 87.9118 & 127.0458 & 39.1341 & 85.6802 & 127.2528 & 41.5726 \\
\hline 87.8234 & 126.8544 & 39.031 & 87.9569 & 127.0531 & 39.0961 & 85.7139 & 127.2112 & 41.4973 \\
\hline 87.6693 & 126.864 & 39.1947 & 87.7853 & 127.0796 & 39.2943 & 85.7815 & 127.2303 & 41.4488 \\
\hline 87.6788 & 126.9194 & 39.2406 & 87.9776 & 127.0034 & 39.0258 & 85.819 & 127.2619 & 41.4429 \\
\hline 87.6407 & 126.9151 & 39.2743 & 88.1135 & 126.9566 & 38.8431 & 85.8135 & 127.2614 & 41.4479 \\
\hline 87.7576 & 126.8891 & 39.1315 & 88.2088 & 126.9705 & 38.7617 & 85.8049 & 127.2485 & 41.4436 \\
\hline 87.7368 & 126.8822 & 39.1453 & 88.1802 & 126.9705 & 38.7903 & 85.7893 & 127.2424 & 41.4531 \\
\hline 88.356 & 126.8883 & 38.5324 & 87.9577 & 126.9722 & 39.0146 & 85.8473 & 127.2744 & 41.4271 \\
\hline 88.0356 & 126.8596 & 38.824 & 87.8633 & 126.9662 & 39.1029 & 85.649 & 127.2285 & 41.5796 \\
\hline 87.9871 & 126.8466 & 38.8595 & 88.0257 & 126.9958 & 38.9702 & 85.6369 & 127.2259 & 41.5891 \\
\hline 87.9196 & 126.8596 & 38.9401 & 87.8191 & 126.9921 & 39.173 & 85.6724 & 127.2407 & 41.5683 \\
\hline 88.0139 & 126.851 & 38.837 & 87.7316 & 126.9939 & 39.2622 & 85.6833 & 127.2592 & 41.5759 \\
\hline 88.1421 & 126.8804 & 38.7383 & 87.6442 & 126.993 & 39.3488 & 85.6983 & 127.2831 & 41.5848 \\
\hline 88.1378 & 126.8726 & 38.7348 & 87.574 & 126.9921 & 39.4181 & 85.7148 & 127.3004 & 41.5856 \\
\hline 88.1153 & 126.864 & 38.7487 & 87.5515 & 127.0198 & 39.4683 & 85.7468 & 127.3368 & 41.5899 \\
\hline 88.1833 & 126.8813 & 38.698 & 87.5307 & 127.012 & 39.4813 & 85.7494 & 127.348 & 41.5986 \\
\hline 88.1811 & 126.8874 & 38.7063 & 87.529 & 127.0311 & 39.5021 & 85.7269 & 127.3584 & 41.6315 \\
\hline 88.2937 & 126.9246 & 38.6309 & 87.52 & 127.054 & 39.5339 & 85.7209 & 127.3359 & 41.6151 \\
\hline 88.2469 & 126.9315 & 38.6846 & 87.5515 & 127.0571 & 39.5056 & 85.6265 & 127.3411 & 41.7146 \\
\hline 88.2278 & 126.9549 & 38.727 & 87.4078 & 127.038 & 39.6303 & 85.6129 & 127.3905 & 41.7776 \\
\hline 88.3292 & 126.9618 & 38.6327 & 87.3039 & 127.0354 & 39.7316 & 85.6594 & 127.4026 & 41.7432 \\
\hline 88.3465 & 126.9826 & 38.6361 & 87.2597 & 127.0216 & 39.7619 & 84.8332 & 127.3853 & 42.552 \\
\hline 88.4443 & 126.9783 & 38.5339 & 87.1428 & 127.0198 & 39.8771 & 84.2375 & 127.7576 & 43.5202 \\
\hline 88.5375 & 126.9914 & 38.454 & 87.0839 & 127.012 & 39.9282 & 82.9498 & 127.5524 & 44.6027 \\
\hline 88.6175 & 127.0034 & 38.3859 & 87.0146 & 127.0224 & 40.0078 & 83.1229 & 128.2712 & 45.1482 \\
\hline 88.5612 & 126.9211 & 38.3599 & 86.9915 & 127.0337 & 40.0422 & 83.2416 & 128.3171 & 45.0755 \\
\hline 88.6262 & 126.9237 & 38.2975 & 87.0051 & 127.0484 & 40.0433 & 83.1654 & 128.3136 & 45.1482 \\
\hline 88.7258 & 126.9358 & 38.2101 & 86.8709 & 127.0354 & 40.1646 & 83.1939 & 128.3277 & 45.1338 \\
\hline 88.7613 & 126.9566 & 38.1953 & 86.7462 & 127.0095 & 40.2633 & 83.194 & 128.3552 & 45.1612 \\
\hline 88.7301 & 126.9783 & 38.2482 & 86.6726 & 127.0233 & 40.3507 & 83.213 & 128.3734 & 45.1603 \\
\hline 88.7353 & 126.9757 & 38.2404 & 86.6985 & 127.0224 & 40.3239 & 83.194 & 128.3976 & 45.2036 \\
\hline 88.7815 & 127.0046 & 38.2232 & 86.6414 & 127.0337 & 40.3923 & 83.194 & 128.4054 & 45.2114 \\
\hline 88.7587 & 127.006 & 38.2473 & 86.5695 & 127.0398 & 40.4702 & 83.1688 & 128.3586 & 45.1898 \\
\hline 88.6695 & 127.0164 & 38.3469 & 86.4964 & 127.0249 & 40.5285 & 83.2329 & 128.3708 & 45.1378 \\
\hline 88.5465 & 127.006 & 38.4595 & 86.4032 & 127.0623 & 40.659 & 83.2234 & 128.3448 & 45.1214 \\
\hline 88.4729 & 127.0294 & 38.5565 & 86.3227 & 127.0605 & 40.7378 & 83.2918 & 128.2781 & 44.9863 \\
\hline 88.4426 & 127.0432 & 38.6006 & 86.5063 & 127.0753 & 40.569 & 83.2814 & 128.2634 & 44.982 \\
\hline
\end{tabular}




\begin{tabular}{|c|c|c|c|c|c|c|c|c|}
\hline 83.1818 & 128.2357 & 45.0538 & 82.3574 & 123.5949 & 41.2375 & 82.024 & 125.5927 & 43.5687 \\
\hline 83.1983 & 128.2539 & 45.0556 & 82.5402 & 123.5793 & 41.0392 & 81.9799 & 125.6802 & 43.7003 \\
\hline 83.284 & 128.2677 & 44.9837 & 82.5332 & 123.5837 & 41.0504 & 82.0621 & 125.6733 & 43.6111 \\
\hline 83.2797 & 128.2738 & 44.9941 & 82.5139 & 123.6361 & 41.1222 & 82.0084 & 125.7451 & 43.7367 \\
\hline 83.278 & 128.2937 & 45.0157 & 82.5488 & 123.7491 & 41.2003 & 82.0933 & 125.7841 & 43.6908 \\
\hline 83.2849 & 128.2963 & 45.0114 & 82.5194 & 123.7577 & 41.2384 & 82.0664 & 125.7308 & 43.6645 \\
\hline 83.2676 & 128.3145 & 45.0469 & 82.4865 & 123.7828 & 41.2964 & 82.0076 & 125.7218 & 43.7142 \\
\hline 83.2987 & 128.3621 & 45.0634 & 82.6683 & 123.8668 & 41.1985 & 81.8794 & 125.7867 & 43.9073 \\
\hline 83.2511 & 128.3318 & 45.0807 & 82.6042 & 123.9231 & 41.3189 & 81.9322 & 125.8542 & 43.922 \\
\hline 83.2234 & 128.3656 & 45.1422 & 82.6796 & 123.9422 & 41.2626 & 81.8915 & 125.9417 & 44.0502 \\
\hline 83.2676 & 128.3508 & 45.0833 & 82.7913 & 123.9604 & 41.1691 & 81.7539 & 126.0413 & 44.2874 \\
\hline 83.1888 & 128.3474 & 45.1586 & 83.11 & 123.9967 & 40.8868 & 81.7608 & 126.0976 & 44.3368 \\
\hline 83.2312 & 128.3976 & 45.1664 & 83.0001 & 123.9664 & 40.9663 & 81.7106 & 126.1469 & 44.4364 \\
\hline 83.2745 & 128.4123 & 45.1378 & 82.8424 & 124.0357 & 41.1933 & 81.6266 & 126.1773 & 44.5507 \\
\hline 83.2624 & 128.4193 & 45.1569 & 82.929 & 124.0903 & 41.1613 & 81.5711 & 126.2128 & 44.6416 \\
\hline 83.2397 & 128.3956 & 45.1559 & 82.7766 & 124.1414 & 41.3648 & 81.624 & 126.2587 & 44.6347 \\
\hline 83.2554 & 128.3993 & 45.1439 & 82.8744 & 124.1535 & 41.2791 & 81.5088 & 126.3634 & 44.8547 \\
\hline 83.2684 & 128.4253 & 45.1569 & 82.7497 & 124.1968 & 41.4471 & 81.4655 & 126.4457 & 44.9802 \\
\hline 83.2832 & 128.4158 & 45.1326 & 82.8303 & 124.2332 & 41.4029 & 81.3979 & 126.4396 & 45.0417 \\
\hline 83.2797 & 128.4054 & 45.1257 & 82.9567 & 124.3102 & 41.3535 & 81.3616 & 126.5037 & 45.1422 \\
\hline 83.3039 & 128.4106 & 45.1067 & 82.8997 & 124.3425 & 41.4429 & 81.3165 & 126.5003 & 45.1837 \\
\hline 83.2953 & 128.4071 & 45.1118 & 82.819 & 124.3249 & 41.506 & 81.3165 & 126.5202 & 45.2036 \\
\hline 83.2589 & 128.4444 & 45.1855 & 82.6172 & 124.363 & 41.7458 & 81.3026 & 126.5378 & 45.2351 \\
\hline 83.2424 & 128.4334 & 45.1911 & 82.5081 & 124.3596 & 41.8515 & 81.1892 & 126.5929 & 45.4037 \\
\hline 83.2321 & 128.4314 & 45.1993 & 82.6042 & 124.3838 & 41.7796 & 81.2074 & 126.6743 & 45.4669 \\
\hline 83.2321 & 128.4426 & 45.2106 & 82.6112 & 124.3994 & 41.7883 & 81.1944 & 126.7168 & 45.5223 \\
\hline 83.2069 & 128.4677 & 45.2608 & 82.6354 & 124.396 & 41.7605 & 81.2074 & 126.6622 & 45.4548 \\
\hline 83.2095 & 128.4677 & 45.2582 & 82.4631 & 124.4462 & 41.9831 & 81.1468 & 126.7064 & 45.5596 \\
\hline 83.3031 & 128.4132 & 45.1101 & 82.5156 & 124.5143 & 41.9987 & 81.1529 & 126.7289 & 45.576 \\
\hline 83.2806 & 128.3837 & 45.1032 & 82.5765 & 124.5527 & 41.9762 & 81.191 & 126.7652 & 45.5743 \\
\hline 83.2987 & 128.3517 & 45.053 & 82.5072 & 124.6644 & 42.1572 & 81.196 & 126.8188 & 45.6227 \\
\hline 83.378 & 128.4361 & 45.0581 & 82.3808 & 124.7649 & 42.384 & 81.1849 & 126.8328 & 45.6479 \\
\hline 83.3827 & 128.4045 & 45.0218 & 82.4206 & 124.8922 & 42.4715 & 81.1659 & 126.883 & 45.7172 \\
\hline 83.4053 & 128.4019 & 44.9967 & 82.5679 & 124.8445 & 42.2767 & 81.3373 & 126.8415 & 45.5041 \\
\hline 81.1618 & 142.1615 & 60.9997 & 82.5012 & 124.9528 & 42.4516 & 81.4109 & 126.8172 & 45.4063 \\
\hline 83.8097 & 127.8927 & 44.0831 & 82.0838 & 125.0922 & 43.0084 & 81.3815 & 126.8051 & 45.4236 \\
\hline 82.2107 & 129.8027 & 47.592 & 82.1536 & 125.0834 & 42.9298 & 81.3893 & 126.8328 & 45.4435 \\
\hline 83.7698 & 127.9698 & 44.2 & 82.1366 & 125.139 & 43.0023 & 81.3226 & 126.87 & 45.5474 \\
\hline 84.1136 & 127.9776 & 43.864 & 82.1661 & 125.1805 & 43.0145 & 81.3246 & 126.8452 & 45.5205 \\
\hline 84.7875 & 127.8159 & 43.0285 & 82.2951 & 125.2152 & 42.9201 & 81.2975 & 126.8761 & 45.5786 \\
\hline 84.3405 & 127.7672 & 43.4267 & 82.2821 & 125.2922 & 43.0101 & 81.3728 & 126.9064 & 45.5336 \\
\hline 84.0365 & 127.7732 & 43.7367 & 82.1738 & 125.3849 & 43.211 & 81.475 & 126.8908 & 45.4158 \\
\hline 82.254 & 123.4379 & 41.1839 & 82.1548 & 125.4732 & 43.3184 & 81.4646 & 126.8778 & 45.4132 \\
\hline 82.289 & 123.4304 & 41.1414 & 82.2085 & 125.4758 & 43.2673 & 81.4542 & 126.941 & 45.4868 \\
\hline 82.2873 & 123.478 & 41.1907 & 82.0681 & 125.6049 & 43.5368 & 81.4005 & 126.9177 & 45.5171 \\
\hline 82.3505 & 123.5231 & 41.1726 & 82.0007 & 125.6256 & 43.625 & 81.4031 & 126.9462 & 45.5431 \\
\hline 82.315 & 123.5889 & 41.2739 & 82.147 & 125.6282 & 43.4812 & 81.3942 & 126.9535 & 45.5593 \\
\hline
\end{tabular}




\begin{tabular}{|c|c|c|c|c|c|c|c|c|}
\hline 81.4811 & 126.9419 & 45.4608 & 81.669 & 127.4953 & 45.8263 & 81.9504 & 127.8061 & 45.8557 \\
\hline 81.4949 & 126.928 & 45.4331 & 81.6656 & 127.5094 & 45.8438 & 81.947 & 127.7914 & 45.8445 \\
\hline 81.4612 & 126.9471 & 45.4859 & 81.7478 & 127.5022 & 45.7544 & 82.0292 & 127.7585 & 45.7293 \\
\hline 81.4127 & 126.9939 & 45.5812 & 81.8153 & 127.529 & 45.7137 & 82.0514 & 127.7481 & 45.6967 \\
\hline 81.3988 & 127.0172 & 45.6184 & 81.8699 & 127.5351 & 45.6652 & 82.0206 & 127.7559 & 45.7353 \\
\hline 81.4586 & 126.9913 & 45.5327 & 81.9236 & 127.4996 & 45.576 & 82.0258 & 127.7602 & 45.7345 \\
\hline 81.4897 & 126.9739 & 45.4842 & 81.9141 & 127.4632 & 45.5492 & 81.9686 & 127.8191 & 45.8505 \\
\hline 81.5202 & 126.9844 & 45.4642 & 81.8959 & 127.5048 & 45.6089 & 81.9721 & 127.8901 & 45.9181 \\
\hline 81.52 & 126.9688 & 45.4487 & 81.8863 & 127.5074 & 45.621 & 81.927 & 127.9586 & 46.0315 \\
\hline 81.5408 & 126.9679 & 45.4271 & 81.8426 & 127.4865 & 45.6439 & 82.0076 & 127.9785 & 45.9709 \\
\hline 81.5079 & 126.9506 & 45.4426 & 81.8794 & 127.5334 & 45.6539 & 82.1687 & 127.9958 & 45.8271 \\
\hline 81.4456 & 126.9532 & 45.5076 & 81.9158 & 127.5221 & 45.6063 & 82.224 & 127.9974 & 45.7734 \\
\hline 81.4179 & 126.9532 & 45.5353 & 81.9426 & 127.5715 & 45.6288 & 82.2708 & 127.9447 & 45.6739 \\
\hline 81.4412 & 126.9653 & 45.524 & 81.9011 & 127.5524 & 45.6513 & 82.2596 & 127.9187 & 45.6591 \\
\hline 81.3616 & 126.9913 & 45.6297 & 81.9253 & 127.4979 & 45.5725 & 82.2778 & 127.9057 & 45.628 \\
\hline 81.3211 & 127.046 & 45.7249 & 81.8863 & 127.5238 & 45.6375 & 82.2353 & 127.9343 & 45.699 \\
\hline 81.3702 & 127.0614 & 45.6912 & 81.8855 & 127.5767 & 45.6912 & 82.192 & 128.0079 & 45.8159 \\
\hline 81.3849 & 127.0727 & 45.6877 & 81.803 & 127.6063 & 45.8033 & 82.2371 & 128.0045 & 45.7674 \\
\hline 81.3711 & 127.1246 & 45.7535 & 81.7937 & 127.6174 & 45.8237 & 82.2397 & 128.0079 & 45.7683 \\
\hline 81.3772 & 127.1567 & 45.7795 & 81.6906 & 127.6122 & 45.9215 & 82.3086 & 127.9921 & 45.6835 \\
\hline 81.3564 & 127.1809 & 45.8245 & 81.6733 & 127.6477 & 45.9744 & 82.3947 & 127.9802 & 45.5855 \\
\hline 81.333 & 127.1766 & 45.8436 & 81.927 & 127.5498 & 45.6228 & 82.3739 & 127.9629 & 45.589 \\
\hline 81.4386 & 127.2086 & 45.77 & 82.044 & 127.5126 & 45.4686 & 82.4544 & 127.9646 & 45.5102 \\
\hline 81.4672 & 127.1913 & 45.7241 & 82.0353 & 127.4866 & 45.4513 & 82.5124 & 127.8927 & 45.3803 \\
\hline 81.4762 & 127.1905 & 45.7143 & 82.0587 & 127.4979 & 45.4392 & 82.3765 & 127.9248 & 45.5483 \\
\hline 81.4837 & 127.2078 & 45.7241 & 81.9461 & 127.5654 & 45.6193 & 82.3063 & 128.0183 & 45.712 \\
\hline 81.4386 & 127.187 & 45.7483 & 81.9439 & 127.616 & 45.6721 & 82.2197 & 128.0512 & 45.8315 \\
\hline 81.4014 & 127.2537 & 45.8523 & 81.953 & 127.633 & 45.6799 & 82.186 & 128.1075 & 45.9215 \\
\hline 81.4265 & 127.258 & 45.8315 & 81.9478 & 127.6243 & 45.6765 & 82.2381 & 128.1982 & 45.9601 \\
\hline 81.5235 & 127.2156 & 45.692 & 81.9747 & 127.594 & 45.6193 & 82.1868 & 128.2019 & 46.0151 \\
\hline 81.5313 & 127.2104 & 45.6791 & 81.9089 & 127.6113 & 45.7024 & 82.1946 & 128.2487 & 46.054 \\
\hline 81.5573 & 127.2207 & 45.6635 & 81.9322 & 127.6407 & 45.7085 & 82.2275 & 128.2824 & 46.0549 \\
\hline 81.5581 & 127.2266 & 45.6685 & 81.9193 & 127.6537 & 45.7345 & 82.2466 & 128.3197 & 46.0731 \\
\hline 81.5443 & 127.2597 & 45.7154 & 81.9037 & 127.6754 & 45.7717 & 82.3531 & 128.2348 & 45.8817 \\
\hline 81.6179 & 127.2303 & 45.6124 & 81.9113 & 127.7129 & 45.8015 & 82.5246 & 128.1664 & 45.6418 \\
\hline 81.6569 & 127.1948 & 45.5379 & 81.869 & 127.7551 & 45.886 & 82.5956 & 128.1517 & 45.5561 \\
\hline 81.6898 & 127.1948 & 45.505 & 81.8145 & 127.7888 & 45.9744 & 82.6116 & 128.1639 & 45.5523 \\
\hline 81.6222 & 127.2389 & 45.6167 & 81.7824 & 127.8157 & 46.0332 & 82.6034 & 128.2132 & 45.6098 \\
\hline 81.5824 & 127.2814 & 45.699 & 81.8353 & 127.8035 & 45.9683 & 82.6068 & 128.2807 & 45.6739 \\
\hline 81.5374 & 127.3091 & 45.7717 & 81.8474 & 127.8061 & 45.9588 & 82.6328 & 128.2313 & 45.5985 \\
\hline 81.5026 & 127.3235 & 45.8209 & 81.8526 & 127.7923 & 45.9397 & 82.1167 & 129.235 & 47.1183 \\
\hline 81.4707 & 127.374 & 45.9033 & 81.8552 & 127.7602 & 45.9051 & 82.7662 & 128.0564 & 45.2902 \\
\hline 81.4741 & 127.4113 & 45.9371 & 81.7906 & 127.8107 & 46.02 & 81.6369 & 122.3064 & 40.6694 \\
\hline 81.4915 & 127.4286 & 45.9371 & 81.7227 & 127.8113 & 46.0887 & 81.9218 & 122.3306 & 40.4088 \\
\hline 81.5486 & 127.4139 & 45.8652 & 81.7755 & 127.8425 & 46.067 & 81.8578 & 122.4224 & 40.5646 \\
\hline 81.6638 & 127.4502 & 45.7864 & 81.8586 & 127.7984 & 45.9397 & 81.8482 & 122.4111 & 40.5629 \\
\hline 81.7123 & 127.4268 & 45.7146 & 81.9097 & 127.7732 & 45.8635 & 81.7448 & 122.4997 & 40.7549 \\
\hline
\end{tabular}




\begin{tabular}{|c|c|c|c|c|c|c|c|c|}
\hline 81.6162 & 122.6796 & 41.0634 & 82.1089 & 122.7887 & 40.6798 & 81.7963 & 121.6266 & 39.8303 \\
\hline 81.5824 & 122.5757 & 40.9933 & 82.0752 & 122.9366 & 40.8615 & 81.7616 & 121.8335 & 40.0719 \\
\hline 81.598 & 122.5921 & 40.9942 & 82.0699 & 122.8848 & 40.8149 & 81.7218 & 121.8465 & 40.1247 \\
\hline 81.5928 & 122.6614 & 41.0686 & 81.9496 & 122.8788 & 40.9292 & 81.8047 & 121.7254 & 39.9207 \\
\hline 81.5478 & 122.8372 & 41.2895 & 81.9729 & 122.8727 & 40.8998 & 81.8301 & 121.7513 & 39.9212 \\
\hline 81.63 & 122.8641 & 41.234 & 81.9998 & 122.8519 & 40.8521 & 81.8673 & 121.5911 & 39.7238 \\
\hline 81.6647 & 122.8363 & 41.1717 & 82.0136 & 122.8026 & 40.7889 & 81.8959 & 121.7348 & 39.839 \\
\hline 81.7008 & 122.83 & 41.1293 & 82.095 & 122.7861 & 40.6911 & 81.8742 & 121.6543 & 39.7801 \\
\hline 81.7227 & 122.7359 & 41.0132 & 82.044 & 122.8173 & 40.7733 & 81.8283 & 121.8119 & 39.9836 \\
\hline 81.7677 & 122.554 & 40.7863 & 81.9518 & 122.8371 & 40.8852 & 81.8058 & 121.7279 & 39.9221 \\
\hline 81.7452 & 122.5402 & 40.795 & 81.4975 & 121.4343 & 39.9368 & 81.8647 & 121.8067 & 39.942 \\
\hline 81.7495 & 122.4839 & 40.7344 & 81.5997 & 121.5703 & 39.9706 & 81.9457 & 121.6452 & 39.6996 \\
\hline 81.7287 & 122.5047 & 40.7759 & 81.6395 & 121.5279 & 39.8883 & 81.9911 & 121.6655 & 39.6744 \\
\hline 81.759 & 122.5653 & 40.8062 & 81.669 & 121.5634 & 39.8944 & 82.0223 & 121.6283 & 39.606 \\
\hline 81.7192 & 122.5869 & 40.8677 & 81.7062 & 121.43 & 39.7238 & 82.0292 & 121.8379 & 39.8086 \\
\hline 81.7202 & 122.7825 & 41.0623 & 81.7573 & 121.4863 & 39.729 & 81.9556 & 121.7461 & 39.7905 \\
\hline 81.7738 & 122.6943 & 40.9206 & 81.7547 & 121.6197 & 39.8649 & 81.8742 & 121.766 & 39.8918 \\
\hline 81.8491 & 122.7064 & 40.8573 & 81.7369 & 121.5607 & 39.8238 & 81.9158 & 121.8327 & 39.9169 \\
\hline 81.8967 & 122.69 & 40.7933 & 81.6274 & 121.6006 & 39.9732 & 81.8846 & 121.7314 & 39.8468 \\
\hline 81.9331 & 122.6761 & 40.743 & 81.5876 & 121.5902 & 40.0026 & 81.8417 & 121.9113 & 40.0695 \\
\hline 81.94 & 122.7186 & 40.7785 & 81.5503 & 121.7374 & 40.1871 & 81.8656 & 121.792 & 39.9264 \\
\hline 81.9288 & 122.7861 & 40.8573 & 81.5417 & 121.8015 & 40.2598 & 81.882 & 121.7617 & 39.8797 \\
\hline 81.9314 & 122.574 & 40.6426 & 81.5382 & 121.8465 & 40.3083 & 81.8335 & 121.7556 & 39.9221 \\
\hline 81.934 & 122.8216 & 40.8876 & 81.6776 & 121.8188 & 40.1412 & 81.8638 & 121.8803 & 40.0165 \\
\hline 82.0144 & 122.7428 & 40.7284 & 81.7694 & 121.6378 & 39.8684 & 81.9115 & 121.7201 & 39.8086 \\
\hline 81.9781 & 122.7168 & 40.7387 & 81.7443 & 121.7478 & 40.0035 & 81.9868 & 122.0829 & 40.0962 \\
\hline 81.9167 & 122.8597 & 40.9431 & 81.8708 & 121.7078 & 39.837 & 81.8786 & 121.7859 & 39.9074 \\
\hline 81.8811 & 122.774 & 40.8928 & 81.7997 & 121.7409 & 39.9411 & 82.0604 & 121.8379 & 39.7775 \\
\hline 81.9218 & 122.6683 & 40.7465 & 81.7997 & 121.7946 & 39.9948 & 82.2038 & 121.8531 & 39.6493 \\
\hline 81.9374 & 122.7783 & 40.8409 & 81.8205 & 121.5443 & 39.7238 & 82.27 & 122.6147 & 40.3447 \\
\hline 81.9946 & 122.7445 & 40.75 & 81.9963 & 121.6352 & 39.6389 & 82.2414 & 121.7582 & 39.5168 \\
\hline 81.9929 & 122.7428 & 40.75 & 82.0916 & 121.6517 & 39.5601 & 82.2284 & 121.9539 & 39.7255 \\
\hline 82.0241 & 122.6301 & 40.606 & 81.9496 & 121.7123 & 39.7628 & 82.1609 & 121.6586 & 39.4978 \\
\hline 82.0067 & 122.6562 & 40.6495 & 81.8881 & 121.6647 & 39.7766 & 82.1453 & 121.8457 & 39.7004 \\
\hline 82.0015 & 122.7238 & 40.7222 & 81.8611 & 121.7597 & 39.8986 & 82.1279 & 121.7807 & 39.6528 \\
\hline 81.8075 & 122.147 & 40.3395 & 81.759 & 121.6811 & 39.9221 & 82.1409 & 122.0189 & 39.8779 \\
\hline 82.0232 & 122.8225 & 40.7993 & 81.7911 & 121.7123 & 39.9212 & 82.0479 & 121.9941 & 39.9462 \\
\hline 81.979 & 122.7004 & 40.7214 & 81.7677 & 121.7158 & 39.9481 & 81.9972 & 121.9946 & 39.9974 \\
\hline 81.9911 & 122.8156 & 40.8244 & 81.7764 & 121.6664 & 39.89 & 82.0414 & 121.986 & 39.9446 \\
\hline 82.1228 & 122.6874 & 40.5646 & 81.7755 & 121.8907 & 40.1152 & 82.0474 & 121.9738 & 39.9264 \\
\hline 82.1509 & 122.7076 & 40.5567 & 81.8266 & 121.7461 & 39.9195 & 82.1461 & 121.8309 & 39.6848 \\
\hline 82.1591 & 122.742 & 40.5828 & 81.837 & 121.7348 & 39.8978 & 82.2102 & 121.8032 & 39.593 \\
\hline 82.1513 & 122.7827 & 40.6313 & 81.869 & 121.7104 & 39.8414 & 82.1851 & 121.9063 & 39.7212 \\
\hline 82.0933 & 122.8502 & 40.7569 & 81.8344 & 121.6404 & 39.8061 & 82.2414 & 121.8794 & 39.6381 \\
\hline 82.0682 & 122.8493 & 40.7811 & 81.8508 & 121.7123 & 39.8615 & 82.1677 & 121.7844 & 39.6167 \\
\hline 82.0067 & 122.7982 & 40.7915 & 81.8673 & 121.6448 & 39.7775 & 82.2319 & 122.0604 & 39.8286 \\
\hline 82.0448 & 122.7342 & 40.6893 & 81.8526 & 121.6915 & 39.839 & 82.2457 & 121.7807 & 39.535 \\
\hline
\end{tabular}




\begin{tabular}{|c|c|c|c|c|c|c|c|c|}
\hline 82.315 & 121.9158 & 39.6008 & 82.4674 & 121.7816 & 39.3142 & 82.2284 & 122.0985 & 39.8701 \\
\hline 82.3245 & 121.8561 & 39.5315 & 82.4553 & 121.6179 & 39.1626 & 82.2082 & 122.0557 & 39.8475 \\
\hline 82.3063 & 121.8292 & 39.5229 & 82.3375 & 121.7582 & 39.4207 & 82.3237 & 122.1124 & 39.7887 \\
\hline 82.205 & 121.7937 & 39.5887 & 82.2682 & 121.6768 & 39.4086 & 82.3124 & 122.1046 & 39.7922 \\
\hline 82.1557 & 121.7591 & 39.6034 & 82.3323 & 121.9695 & 39.6372 & 82.3219 & 122.0795 & 39.7576 \\
\hline 82.1007 & 121.6364 & 39.5357 & 82.2613 & 121.8509 & 39.5896 & 82.2674 & 122.1288 & 39.8615 \\
\hline 82.1851 & 121.8223 & 39.6372 & 82.263 & 121.8439 & 39.5809 & 82.2596 & 122.0648 & 39.8052 \\
\hline 82.1357 & 121.9072 & 39.7714 & 82.2469 & 121.7897 & 39.5428 & 82.328 & 122.063 & 39.735 \\
\hline 82.0976 & 121.8422 & 39.7446 & 82.2059 & 121.8509 & 39.645 & 82.2812 & 122.1384 & 39.8571 \\
\hline 82.1444 & 121.9617 & 39.8173 & 82.2128 & 122.0041 & 39.7913 & 82.2928 & 122.098 & 39.8053 \\
\hline 82.1202 & 121.8768 & 39.7567 & 82.1141 & 121.8699 & 39.7558 & 82.2163 & 122.115 & 39.8987 \\
\hline 82.1687 & 121.8994 & 39.7307 & 82.0249 & 121.9626 & 39.9377 & 82.018 & 122.0206 & 40.0026 \\
\hline 82.1652 & 121.7149 & 39.5497 & 82.0587 & 121.8734 & 39.8147 & 81.9946 & 122.1903 & 40.1957 \\
\hline 82.2549 & 121.7536 & 39.4987 & 82.199 & 121.9124 & 39.7134 & 82.0214 & 122.2648 & 40.2434 \\
\hline 82.3522 & 121.811 & 39.4588 & 82.3055 & 121.8093 & 39.5038 & 81.9851 & 122.2544 & 40.2693 \\
\hline 82.2985 & 121.643 & 39.3445 & 82.3553 & 121.8593 & 39.504 & 82.0249 & 122.2483 & 40.2234 \\
\hline 82.3401 & 121.8309 & 39.4908 & 82.2873 & 121.792 & 39.5047 & 82.0821 & 122.1514 & 40.0693 \\
\hline 82.431 & 121.876 & 39.4449 & 82.244 & 121.7747 & 39.5307 & 82.1791 & 122.1685 & 39.9894 \\
\hline 82.4354 & 121.6422 & 39.2068 & 82.1964 & 121.8665 & 39.6701 & 82.2128 & 122.1643 & 39.9515 \\
\hline 82.4146 & 121.6439 & 39.2293 & 82.2985 & 121.8673 & 39.5688 & 82.2552 & 122.1392 & 39.884 \\
\hline 82.3903 & 121.6404 & 39.2501 & 82.2587 & 121.7894 & 39.5307 & 82.2561 & 122.1037 & 39.8476 \\
\hline 82.3368 & 121.7553 & 39.4185 & 82.2882 & 122.0526 & 39.7645 & 82.1877 & 122.147 & 39.9593 \\
\hline 82.3107 & 121.579 & 39.2683 & 82.1773 & 121.9453 & 39.7679 & 82.2622 & 122.1756 & 39.9134 \\
\hline 82.2717 & 121.7773 & 39.5056 & 82.1034 & 121.8522 & 39.7489 & 82.1704 & 122.2328 & 40.0624 \\
\hline 82.1972 & 121.7608 & 39.5636 & 82.0968 & 121.9383 & 39.8416 & 82.0847 & 122.2432 & 40.1585 \\
\hline 82.3323 & 121.8284 & 39.496 & 82.0916 & 122.0241 & 39.9325 & 82.0373 & 122.246 & 40.2087 \\
\hline 82.2552 & 121.7184 & 39.4631 & 82.1141 & 122.0163 & 39.9022 & 82.0076 & 122.2483 & 40.2408 \\
\hline 82.2509 & 121.7989 & 39.548 & 82.0994 & 121.9712 & 39.8719 & 81.9998 & 122.3029 & 40.3031 \\
\hline 82.2951 & 121.8994 & 39.6043 & 82.1721 & 121.8768 & 39.7047 & 81.9513 & 122.3306 & 40.3793 \\
\hline 82.2587 & 121.8621 & 39.6034 & 82.2613 & 121.7738 & 39.5125 & 81.9296 & 122.4752 & 40.5456 \\
\hline 82.1932 & 121.6919 & 39.4987 & 82.2076 & 121.8803 & 39.6727 & 82.0414 & 122.4215 & 40.3802 \\
\hline 82.27 & 121.669 & 39.399 & 82.2812 & 121.9782 & 39.6969 & 82.0561 & 122.4189 & 40.3629 \\
\hline 82.3219 & 121.9964 & 39.6744 & 82.3051 & 121.9527 & 39.6476 & 82.0344 & 122.4371 & 40.4027 \\
\hline 82.2137 & 121.7547 & 39.5411 & 82.2925 & 122.0128 & 39.7203 & 82.1695 & 122.4172 & 40.2477 \\
\hline 82.2838 & 121.8907 & 39.6069 & 82.2102 & 121.8924 & 39.6822 & 82.2839 & 122.4689 & 40.1849 \\
\hline 82.2345 & 121.9548 & 39.7203 & 82.2544 & 121.9357 & 39.6813 & 82.2102 & 122.4207 & 40.2105 \\
\hline 82.121 & 121.9028 & 39.7818 & 82.2345 & 121.9037 & 39.6692 & 82.1635 & 122.4501 & 40.2867 \\
\hline 82.1487 & 121.8976 & 39.7489 & 82.179 & 121.9834 & 39.8043 & 82.1505 & 122.4778 & 40.3274 \\
\hline 82.1862 & 121.9042 & 39.718 & 82.0881 & 122.0345 & 39.9463 & 82.05 & 122.5861 & 40.5361 \\
\hline 82.1427 & 121.8794 & 39.7368 & 82.0249 & 121.9608 & 39.9359 & 82.0621 & 122.6398 & 40.5776 \\
\hline 82.1981 & 121.798 & 39.5999 & 81.9466 & 121.9976 & 40.051 & 82.0474 & 122.7004 & 40.653 \\
\hline 82.3263 & 121.9098 & 39.5835 & 81.8534 & 122.0639 & 40.2105 & 82.0292 & 122.522 & 40.4928 \\
\hline 82.4544 & 121.7712 & 39.3168 & 81.9686 & 122.044 & 40.0754 & 82.0065 & 122.6204 & 40.6139 \\
\hline 82.4648 & 121.805 & 39.3402 & 82.1028 & 122.0015 & 39.8987 & 81.8803 & 122.5783 & 40.698 \\
\hline 82.5237 & 121.8933 & 39.3696 & 82.1323 & 121.9842 & 39.8519 & 81.9279 & 122.7047 & 40.7768 \\
\hline 82.4579 & 121.7452 & 39.2873 & 82.2033 & 122.063 & 39.8597 & 81.9738 & 122.729 & 40.7552 \\
\hline 82.424 & 121.7835 & 39.3595 & 82.1531 & 122.0128 & 39.8597 & 82.0769 & 122.6657 & 40.5889 \\
\hline
\end{tabular}




\begin{tabular}{|c|c|c|c|c|c|c|c|c|}
\hline 82.1409 & 122.567 & 40.4261 & 82.2223 & 122.9177 & 40.6954 & 82.5384 & 122.6285 & 40.0901 \\
\hline 82.2544 & 122.5575 & 40.3031 & 82.2319 & 122.8952 & 40.6634 & 82.5055 & 122.6346 & 40.1291 \\
\hline 82.3366 & 122.5358 & 40.1992 & 82.2449 & 122.9463 & 40.7015 & 82.599 & 122.6285 & 40.0295 \\
\hline 82.2469 & 122.5341 & 40.2871 & 82.2959 & 122.9403 & 40.6443 & 82.6562 & 122.593 & 39.9368 \\
\hline 82.2293 & 122.6458 & 40.4166 & 82.3254 & 122.9247 & 40.5993 & 82.6285 & 122.5644 & 39.9359 \\
\hline 82.1825 & 122.664 & 40.4815 & 82.3421 & 122.7534 & 40.4113 & 82.606 & 122.5307 & 39.9247 \\
\hline 82.199 & 122.5289 & 40.33 & 82.1877 & 122.8164 & 40.6287 & 82.5964 & 122.6173 & 40.0208 \\
\hline 82.199 & 122.5999 & 40.401 & 82.2648 & 122.8667 & 40.6019 & 82.5958 & 122.5217 & 39.9259 \\
\hline 82.1202 & 122.6623 & 40.5421 & 82.2128 & 122.9342 & 40.7214 & 82.6181 & 122.5696 & 39.9515 \\
\hline 82.0907 & 122.7246 & 40.6339 & 82.1357 & 122.7697 & 40.6339 & 82.5687 & 122.5012 & 39.9325 \\
\hline 81.9903 & 122.6571 & 40.6668 & 82.1626 & 122.8095 & 40.6469 & 82.5505 & 122.5376 & 39.987 \\
\hline 82.0998 & 122.6829 & 40.5831 & 82.2908 & 122.8285 & 40.5378 & 82.5947 & 122.5298 & 39.9351 \\
\hline 82.2197 & 122.6164 & 40.3966 & 82.3011 & 122.8649 & 40.5638 & 82.6164 & 122.4891 & 39.8727 \\
\hline 82.2752 & 122.7038 & 40.4287 & 82.239 & 122.7728 & 40.5338 & 82.5757 & 122.541 & 39.9654 \\
\hline 82.2743 & 122.6649 & 40.3906 & 82.2197 & 122.8589 & 40.6391 & 82.4899 & 122.522 & 40.0321 \\
\hline 82.3418 & 122.6831 & 40.3412 & 82.2353 & 122.7428 & 40.5075 & 82.4134 & 122.5235 & 40.1101 \\
\hline 82.3947 & 122.6372 & 40.2425 & 82.2526 & 122.774 & 40.5213 & 82.4224 & 122.5618 & 40.1394 \\
\hline 82.4466 & 122.7194 & 40.2728 & 82.2215 & 122.7896 & 40.5681 & 82.4267 & 122.5532 & 40.1265 \\
\hline 82.5228 & 122.6311 & 40.1083 & 82.1617 & 122.729 & 40.5672 & 82.3618 & 122.5748 & 40.2131 \\
\hline 82.565 & 122.7789 & 40.214 & 82.1279 & 122.6597 & 40.5317 & 82.3626 & 122.5246 & 40.162 \\
\hline 82.5367 & 122.8554 & 40.3187 & 82.121 & 122.7852 & 40.6642 & 82.4068 & 122.5956 & 40.1888 \\
\hline 82.3055 & 122.8311 & 40.5257 & 82.0373 & 122.7684 & 40.7311 & 82.3817 & 122.6129 & 40.2312 \\
\hline 82.0058 & 122.9359 & 40.9301 & 82.0275 & 122.7775 & 40.75 & 82.4189 & 122.6337 & 40.2148 \\
\hline 82.0465 & 122.8727 & 40.8262 & 81.9461 & 122.7835 & 40.8374 & 82.4196 & 122.5358 & 40.1162 \\
\hline 82.1002 & 122.9455 & 40.8452 & 82.0795 & 122.7021 & 40.6227 & 82.4865 & 122.6216 & 40.1351 \\
\hline 82.0336 & 122.9247 & 40.8911 & 82.0388 & 122.7168 & 40.6781 & 82.4908 & 122.5783 & 40.0875 \\
\hline 82.179 & 122.9714 & 40.7924 & 81.9651 & 122.664 & 40.6989 & 82.5904 & 122.5445 & 39.9541 \\
\hline 82.1967 & 122.9675 & 40.7707 & 82.0249 & 122.6415 & 40.6166 & 82.6848 & 122.6077 & 39.923 \\
\hline 82.2085 & 122.9125 & 40.7041 & 82.1011 & 122.7212 & 40.6201 & 82.7237 & 122.5003 & 39.7766 \\
\hline 82.2708 & 123.0199 & 40.7491 & 82.0232 & 122.7331 & 40.7099 & 82.7766 & 122.4493 & 39.6727 \\
\hline 82.2665 & 122.9732 & 40.7067 & 81.9877 & 122.8822 & 40.8946 & 82.6848 & 122.4804 & 39.7957 \\
\hline 82.2977 & 122.9732 & 40.6755 & 82.0249 & 122.8944 & 40.8695 & 82.7772 & 122.468 & 39.6907 \\
\hline 82.2171 & 123.0156 & 40.7985 & 82.1894 & 122.8251 & 40.6356 & 82.7722 & 122.4605 & 39.6883 \\
\hline 82.1314 & 123.0095 & 40.8781 & 82.4163 & 122.6493 & 40.233 & 82.7047 & 122.5021 & 39.7974 \\
\hline 82.0933 & 122.9697 & 40.8764 & 82.4267 & 122.5991 & 40.1724 & 82.832 & 122.4536 & 39.6216 \\
\hline 82.1765 & 123.0159 & 40.8394 & 82.4345 & 122.6883 & 40.2538 & 82.8354 & 122.509 & 39.6736 \\
\hline 82.1046 & 123.0017 & 40.8972 & 82.3773 & 122.5705 & 40.1931 & 82.7549 & 122.5636 & 39.8086 \\
\hline 82.2353 & 122.9965 & 40.7612 & 82.3579 & 122.6697 & 40.3118 & 82.6397 & 122.5965 & 39.9567 \\
\hline 82.2206 & 122.9939 & 40.7733 & 82.3739 & 122.6276 & 40.2538 & 82.6146 & 122.6796 & 40.065 \\
\hline 82.2102 & 122.9584 & 40.7482 & 82.4917 & 122.6675 & 40.1758 & 82.5403 & 122.6988 & 40.1585 \\
\hline 82.1661 & 122.8623 & 40.6963 & 82.4908 & 122.6502 & 40.1594 & 82.5479 & 122.6987 & 40.1507 \\
\hline 82.1453 & 122.9004 & 40.7552 & 82.5505 & 122.7697 & 40.2191 & 82.593 & 122.6372 & 40.0442 \\
\hline 82.2544 & 122.9481 & 40.6937 & 82.5393 & 122.7229 & 40.1836 & 82.7463 & 122.6458 & 39.8996 \\
\hline 82.2483 & 122.884 & 40.6356 & 82.5618 & 122.6328 & 40.071 & 82.8259 & 122.5592 & 39.7333 \\
\hline 82.2839 & 122.8644 & 40.5805 & 82.4969 & 122.6476 & 40.1507 & 82.851 & 122.6198 & 39.7688 \\
\hline 82.3046 & 122.9125 & 40.6079 & 82.5324 & 122.6727 & 40.1403 & 82.7099 & 122.6519 & 39.942 \\
\hline 82.2942 & 122.8736 & 40.5794 & 82.5394 & 122.6803 & 40.1409 & 82.4882 & 122.8069 & 40.3187 \\
\hline
\end{tabular}




\begin{tabular}{|c|c|c|c|c|c|c|c|c|}
\hline 82.5592 & 122.7705 & 40.2113 & 82.5133 & 122.9091 & 40.3958 & 84.5007 & 121.7539 & 37.2532 \\
\hline 82.5588 & 122.7957 & 40.2369 & 82.425 & 122.9732 & 40.5482 & 84.447 & 121.7599 & 37.3129 \\
\hline 82.5505 & 122.7653 & 40.2148 & 82.4674 & 123.0269 & 40.5594 & 84.4245 & 121.8578 & 37.4333 \\
\hline 82.6042 & 122.6935 & 40.0892 & 82.5112 & 123.1207 & 40.6095 & 84.3353 & 121.908 & 37.5727 \\
\hline 82.5722 & 122.5497 & 39.9775 & 82.6571 & 123.0589 & 40.4018 & 84.447 & 121.7998 & 37.3528 \\
\hline 82.6034 & 122.6147 & 40.0113 & 82.6796 & 122.9801 & 40.3005 & 84.4703 & 121.8232 & 37.3528 \\
\hline 82.5549 & 122.5731 & 40.0182 & 82.6155 & 123.0217 & 40.4062 & 84.5596 & 121.8569 & 37.2973 \\
\hline 82.5938 & 122.7038 & 40.11 & 82.4995 & 122.9567 & 40.4573 & 84.492 & 121.8673 & 37.3753 \\
\hline 82.696 & 122.651 & 39.955 & 82.6285 & 122.9203 & 40.2919 & 84.6297 & 121.8223 & 37.1926 \\
\hline 82.6046 & 122.7305 & 40.1259 & 82.6761 & 122.9342 & 40.2581 & 84.5709 & 121.8916 & 37.3207 \\
\hline 82.6856 & 122.729 & 40.0433 & 82.664 & 122.8718 & 40.2079 & 84.4669 & 121.8933 & 37.4264 \\
\hline 82.7159 & 122.7723 & 40.0563 & 82.6636 & 122.9217 & 40.258 & 84.3829 & 122.057 & 37.674 \\
\hline 82.7991 & 122.748 & 39.9489 & 82.6259 & 122.9411 & 40.3152 & 84.6479 & 121.8846 & 37.2367 \\
\hline 82.7696 & 122.7671 & 39.9974 & 82.6553 & 122.8831 & 40.2278 & 84.4915 & 122.0046 & 37.5132 \\
\hline 82.7956 & 122.7238 & 39.9282 & 82.6112 & 122.929 & 40.3178 & 84.3119 & 122.07 & 37.758 \\
\hline 82.7947 & 122.8649 & 40.0702 & 82.5653 & 122.9446 & 40.3793 & 84.1907 & 122.1635 & 37.9728 \\
\hline 82.8502 & 122.8346 & 39.9844 & 82.5956 & 122.9489 & 40.3533 & 84.15 & 122.3635 & 38.2135 \\
\hline 82.7041 & 122.8679 & 40.1638 & 82.638 & 122.9922 & 40.3542 & 84.1136 & 122.3904 & 38.2767 \\
\hline 82.787 & 122.832 & 40.0451 & 82.6146 & 122.8692 & 40.2546 & 84.2045 & 122.4094 & 38.2049 \\
\hline 82.6519 & 122.8216 & 40.1698 & 82.5852 & 122.838 & 40.2528 & 84.1474 & 122.4458 & 38.2984 \\
\hline 82.7324 & 122.8355 & 40.1031 & 82.5618 & 122.8259 & 40.2641 & 84.1786 & 122.4432 & 38.2646 \\
\hline 82.806 & 122.8173 & 40.0113 & 82.5012 & 122.8511 & 40.3499 & 84.1849 & 122.4389 & 38.254 \\
\hline 82.8441 & 122.8537 & 40.0096 & 82.5012 & 122.942 & 40.4408 & 83.9179 & 122.49 & 38.572 \\
\hline 82.7844 & 122.7904 & 40.0061 & 82.4094 & 122.9169 & 40.5075 & 83.924 & 122.5307 & 38.6067 \\
\hline 82.8528 & 122.7342 & 39.8814 & 82.4536 & 122.8303 & 40.3767 & 84.0028 & 122.5428 & 38.54 \\
\hline 82.8477 & 122.8151 & 39.9673 & 82.4839 & 122.8459 & 40.362 & 84.1846 & 122.4285 & 38.2438 \\
\hline 82.7956 & 122.8182 & 40.0225 & 82.593 & 122.6848 & 40.0918 & 84.3119 & 122.4553 & 38.1434 \\
\hline 82.8372 & 122.8441 & 40.007 & 82.6011 & 122.7895 & 40.1884 & 84.1231 & 122.5237 & 38.4006 \\
\hline 82.8692 & 122.7974 & 39.9282 & 82.5514 & 122.7835 & 40.2321 & 84.0634 & 122.3895 & 38.3261 \\
\hline 82.9246 & 122.7064 & 39.7818 & 82.4657 & 122.6069 & 40.1412 & 84.0554 & 122.4548 & 38.3993 \\
\hline 82.8822 & 122.7506 & 39.8684 & 82.5895 & 122.5627 & 39.9732 & 84.0417 & 122.4536 & 38.4118 \\
\hline 82.9454 & 122.8121 & 39.8667 & 83.5265 & 122.0977 & 38.5712 & 83.9231 & 122.4891 & 38.566 \\
\hline 82.9688 & 122.7307 & 39.7619 & 83.7525 & 121.9842 & 38.2317 & 83.8278 & 122.386 & 38.5582 \\
\hline 82.8909 & 122.7851 & 39.8942 & 84.1275 & 121.8335 & 37.7061 & 84.0253 & 122.4544 & 38.4292 \\
\hline 82.8562 & 122.8043 & 39.9481 & 83.9829 & 121.8517 & 37.8689 & 84.2678 & 122.3358 & 38.068 \\
\hline 82.8943 & 122.8485 & 39.9541 & 83.9779 & 121.8188 & 37.8408 & 84.2323 & 122.4891 & 38.2568 \\
\hline 82.987 & 122.7125 & 39.7255 & 83.9976 & 121.889 & 37.8914 & 84.3197 & 122.4977 & 38.178 \\
\hline 83.0693 & 122.7272 & 39.658 & 84.0288 & 121.7721 & 37.7433 & 84.303 & 122.5596 & 38.2566 \\
\hline 83.0961 & 122.7523 & 39.6562 & 84.0002 & 121.7877 & 37.7875 & 84.4513 & 122.5817 & 38.1304 \\
\hline 83.0424 & 122.7359 & 39.6935 & 84.0132 & 121.7392 & 37.726 & 84.5786 & 122.5895 & 38.0109 \\
\hline 82.9541 & 122.7203 & 39.7662 & 84.0521 & 121.7054 & 37.6532 & 84.5778 & 122.5445 & 37.9667 \\
\hline 82.8495 & 122.8212 & 39.9717 & 84.3292 & 121.6266 & 37.2973 & 84.6193 & 122.593 & 37.9737 \\
\hline 82.774 & 122.8779 & 40.1039 & 84.4219 & 121.4958 & 37.0739 & 84.544 & 122.7108 & 38.1668 \\
\hline 82.6865 & 122.8667 & 40.1801 & 84.4677 & 121.5096 & 37.0419 & 84.2175 & 122.9212 & 38.7037 \\
\hline 82.6345 & 122.897 & 40.2624 & 84.5258 & 121.6863 & 37.1605 & 84.2756 & 122.9385 & 38.663 \\
\hline 82.6207 & 122.9913 & 40.3707 & 84.4531 & 121.6378 & 37.1848 & 84.1259 & 123.0106 & 38.8847 \\
\hline 82.4665 & 122.8926 & 40.4261 & 84.5908 & 121.7755 & 37.1848 & 84.2184 & 123.0277 & 38.8093 \\
\hline
\end{tabular}




\begin{tabular}{|c|c|c|c|c|c|c|c|c|}
\hline 84.2097 & 123.0217 & 38.8119 & 85.4208 & 123.1771 & 37.7563 & 85.0272 & 124.0201 & 38.9929 \\
\hline 84.2219 & 123.0468 & 38.8249 & 85.4775 & 123.1888 & 37.7113 & 85.0463 & 124.0678 & 39.0215 \\
\hline 84.1638 & 122.9099 & 38.7461 & 85.3935 & 123.2364 & 37.8429 & 85.0658 & 124.0184 & 38.9525 \\
\hline 84.337 & 122.9377 & 38.6006 & 85.5174 & 123.3031 & 37.7857 & 85.106 & 123.9838 & 38.8777 \\
\hline 84.176 & 123.045 & 38.8691 & 85.5061 & 123.3377 & 37.8316 & 85.1233 & 123.9716 & 38.8483 \\
\hline 84.1959 & 123.0017 & 38.8059 & 85.5607 & 123.4607 & 37.9 & 85.0194 & 123.9872 & 38.9678 \\
\hline 84.1699 & 123.0857 & 38.9158 & 85.481 & 123.4408 & 37.9598 & 85.0593 & 123.9595 & 38.9002 \\
\hline 84.2008 & 123.0582 & 38.8574 & 85.6083 & 123.4053 & 37.797 & 84.9882 & 124.0669 & 39.0786 \\
\hline 84.266 & 123.0459 & 38.7799 & 85.6776 & 123.4858 & 37.8083 & 84.9882 & 124.1613 & 39.173 \\
\hline 84.1474 & 123.2087 & 39.0613 & 85.5373 & 123.5023 & 37.965 & 84.9761 & 124.1448 & 39.1687 \\
\hline 84.1275 & 123.297 & 39.1696 & 85.5156 & 123.562 & 38.0464 & 84.9654 & 124.0633 & 39.0979 \\
\hline 84.3258 & 123.2174 & 38.8916 & 85.5875 & 123.5239 & 37.9364 & 84.9242 & 124.137 & 39.2129 \\
\hline 84.4773 & 123.11 & 38.6327 & 85.6022 & 123.6322 & 38.0299 & 84.9484 & 124.0712 & 39.1228 \\
\hline 84.3137 & 123.239 & 38.9254 & 85.5477 & 123.5724 & 38.0247 & 84.9276 & 124.195 & 39.2674 \\
\hline 84.2426 & 123.3403 & 39.0977 & 85.6057 & 123.5923 & 37.9866 & 85.0757 & 124.1483 & 39.0726 \\
\hline 84.0951 & 123.3568 & 39.2617 & 85.6507 & 123.6798 & 38.0291 & 84.9735 & 124.2106 & 39.2371 \\
\hline 84.1006 & 123.3594 & 39.2588 & 85.3026 & 123.7257 & 38.4231 & 85.0298 & 124.1803 & 39.1505 \\
\hline 84.2028 & 122.9481 & 38.7452 & 84.9804 & 123.6968 & 38.7165 & 85.0792 & 124.1907 & 39.1115 \\
\hline 84.1716 & 122.9827 & 38.811 & 85.016 & 123.6157 & 38.5998 & 85.0809 & 124.1821 & 39.1012 \\
\hline 84.0824 & 123.0346 & 38.9522 & 85.029 & 123.6348 & 38.6058 & 85.0271 & 124.2853 & 39.2582 \\
\hline 84.0565 & 123.1429 & 39.0864 & 85.2376 & 123.6114 & 38.3737 & 85.0506 & 124.2591 & 39.2085 \\
\hline 84.0426 & 123.0918 & 39.0492 & 85.3883 & 123.6348 & 38.2464 & 85.093 & 124.2505 & 39.1574 \\
\hline 83.9058 & 123.2087 & 39.3029 & 85.3009 & 123.6226 & 38.3218 & 85.0593 & 124.3189 & 39.2596 \\
\hline 83.8238 & 123.1604 & 39.3366 & 85.2169 & 123.7958 & 38.579 & 85.0082 & 124.2721 & 39.264 \\
\hline 84.0807 & 123.1897 & 39.1089 & 85.184 & 123.7153 & 38.5313 & 85.0887 & 124.2487 & 39.16 \\
\hline 83.9872 & 123.2148 & 39.2276 & 85.2931 & 123.7365 & 38.4434 & 85.0038 & 124.3379 & 39.3341 \\
\hline 84.2816 & 123.1143 & 38.8327 & 85.2853 & 123.7196 & 38.4344 & 84.996 & 124.3457 & 39.3497 \\
\hline 83.7516 & 123.3048 & 39.5532 & 85.3424 & 123.6859 & 38.3434 & 84.9689 & 124.3188 & 39.3498 \\
\hline 83.9811 & 123.297 & 39.3159 & 85.332 & 123.7569 & 38.4248 & 84.9675 & 124.3189 & 39.3514 \\
\hline 84.654 & 123.0442 & 38.3902 & 85.3658 & 123.7101 & 38.3443 & 84.9787 & 124.383 & 39.4042 \\
\hline 84.945 & 122.9567 & 38.0118 & 85.3424 & 123.7118 & 38.3694 & 85.0298 & 124.3379 & 39.3081 \\
\hline 84.9266 & 122.9833 & 38.0567 & 85.3572 & 123.8166 & 38.4595 & 85.0298 & 124.389 & 39.3592 \\
\hline 84.7146 & 123.0632 & 38.3486 & 85.3061 & 123.8738 & 38.5677 & 85.0593 & 124.3492 & 39.2899 \\
\hline 84.8064 & 123.0121 & 38.2057 & 85.2552 & 123.8589 & 38.6037 & 84.99 & 124.389 & 39.399 \\
\hline 84.8081 & 123.0805 & 38.2724 & 85.2402 & 123.9405 & 38.7002 & 84.8549 & 124.4756 & 39.6207 \\
\hline 84.9129 & 123.0953 & 38.1824 & 85.1762 & 123.9266 & 38.7504 & 84.8667 & 124.4967 & 39.63 \\
\hline 84.9008 & 123.084 & 38.1832 & 85.1614 & 123.8859 & 38.7245 & 84.9302 & 124.4739 & 39.5437 \\
\hline 84.7969 & 123.0676 & 38.2707 & 85.1095 & 123.9439 & 38.8344 & 84.9302 & 124.5007 & 39.5705 \\
\hline 85.132 & 123.0788 & 37.9468 & 85.0272 & 123.9491 & 38.9219 & 84.9579 & 124.5648 & 39.6069 \\
\hline 84.9804 & 123.1005 & 38.1201 & 84.9536 & 123.9855 & 39.0319 & 84.8731 & 124.5925 & 39.7195 \\
\hline 85.0116 & 123.1559 & 38.1443 & 84.9605 & 123.9387 & 38.9782 & 84.7761 & 124.6731 & 39.897 \\
\hline 85.268 & 123.0511 & 37.7831 & 85.0103 & 123.955 & 38.9446 & 84.8263 & 124.725 & 39.8987 \\
\hline 85.1493 & 123.1212 & 37.9719 & 84.9857 & 123.9638 & 38.9782 & 84.8159 & 124.7978 & 39.9818 \\
\hline 85.2299 & 123.1005 & 37.8706 & 84.9779 & 123.9621 & 38.9842 & 84.7619 & 124.5161 & 39.7542 \\
\hline 85.1995 & 123.2052 & 38.0057 & 84.9883 & 123.9491 & 38.9609 & 84.7579 & 124.6012 & 39.8433 \\
\hline 85.2325 & 123.1819 & 37.9494 & 85.0948 & 123.9543 & 38.8595 & 84.822 & 124.5302 & 39.7082 \\
\hline 85.2879 & 123.1628 & 37.8749 & 85.0333 & 123.9318 & 38.8985 & 84.8774 & 124.4323 & 39.5549 \\
\hline
\end{tabular}




\begin{tabular}{|c|c|c|c|c|c|c|c|c|}
\hline 84.9103 & 124.4947 & 39.5844 & 84.2756 & 125.0835 & 40.808 & 84.377 & 125.6577 & 41.2808 \\
\hline 84.9701 & 124.5155 & 39.5454 & 83.9006 & 125.1745 & 41.2739 & 84.4505 & 125.6447 & 41.1942 \\
\hline 84.9276 & 124.4124 & 39.4848 & 83.8235 & 125.2117 & 41.3882 & 84.7224 & 125.5867 & 40.8643 \\
\hline 84.9432 & 124.3786 & 39.4354 & 83.7352 & 125.3312 & 41.596 & 84.822 & 125.5598 & 40.7378 \\
\hline 85.0368 & 124.3778 & 39.341 & 83.756 & 125.3892 & 41.6332 & 84.6912 & 125.5667 & 40.8755 \\
\hline 84.8757 & 124.4315 & 39.5558 & 83.7525 & 125.4013 & 41.6488 & 84.602 & 125.6196 & 41.0175 \\
\hline 84.9969 & 124.4834 & 39.4865 & 83.7462 & 125.4181 & 41.6719 & 84.1275 & 125.8949 & 41.7675 \\
\hline 84.6219 & 124.6549 & 40.0329 & 83.7984 & 125.4187 & 41.6203 & 83.6166 & 125.9651 & 42.3485 \\
\hline 84.7847 & 124.5345 & 39.7498 & 83.7742 & 125.4343 & 41.6601 & 84.4087 & 125.6542 & 41.2455 \\
\hline 84.9501 & 124.4748 & 39.5246 & 83.7534 & 125.3823 & 41.6289 & 84.0513 & 125.6551 & 41.6038 \\
\hline 84.9242 & 124.4505 & 39.5263 & 83.9352 & 125.3373 & 41.402 & 84.2297 & 125.5728 & 41.3431 \\
\hline 84.8739 & 124.5605 & 39.6865 & 83.665 & 125.4334 & 41.7683 & 84.1898 & 125.552 & 41.3622 \\
\hline 84.8738 & 124.5064 & 39.6326 & 83.6902 & 125.4559 & 41.7657 & 84.3266 & 125.4715 & 41.1448 \\
\hline 84.8705 & 124.5562 & 39.6857 & 83.8512 & 125.4031 & 41.5518 & 84.5327 & 125.3693 & 40.8366 \\
\hline 84.9675 & 124.5492 & 39.5818 & 83.8678 & 125.3186 & 41.4508 & 84.5059 & 125.365 & 40.8591 \\
\hline 84.9138 & 124.5336 & 39.6199 & 83.8737 & 125.3182 & 41.4445 & 84.4903 & 125.3104 & 40.8201 \\
\hline 84.744 & 124.6021 & 39.858 & 83.9889 & 125.2758 & 41.2869 & 84.3171 & 125.3732 & 41.0561 \\
\hline 84.8203 & 124.6124 & 39.7922 & 83.9387 & 125.2411 & 41.3024 & 84.4479 & 125.2974 & 40.8495 \\
\hline 84.8991 & 124.557 & 39.658 & 83.9067 & 125.255 & 41.3483 & 84.279 & 125.3329 & 41.0539 \\
\hline 84.8393 & 124.6298 & 39.7905 & 83.9188 & 125.2654 & 41.3466 & 84.4158 & 125.2957 & 40.8799 \\
\hline 84.8384 & 124.6047 & 39.7662 & 84.014 & 125.2498 & 41.2358 & 84.2859 & 125.3312 & 41.0453 \\
\hline 84.6641 & 124.7539 & 40.0898 & 84.0833 & 125.2186 & 41.1353 & 84.2842 & 125.4135 & 41.1293 \\
\hline 84.5838 & 124.7787 & 40.1949 & 83.9136 & 125.2498 & 41.3362 & 84.2574 & 125.4187 & 41.1613 \\
\hline 84.7172 & 124.6843 & 39.9671 & 83.9154 & 125.2587 & 41.3433 & 84.3076 & 125.4117 & 41.1041 \\
\hline 84.6349 & 124.7493 & 40.1143 & 84.1024 & 125.2455 & 41.1431 & 84.488 & 125.3547 & 40.8667 \\
\hline 84.3396 & 124.9025 & 40.5629 & 84.0885 & 125.2463 & 41.1578 & 84.3007 & 125.4438 & 41.1431 \\
\hline 84.1231 & 125.029 & 40.9058 & 84.1413 & 125.2299 & 41.0886 & 84.2903 & 125.4213 & 41.131 \\
\hline 84.2089 & 124.9701 & 40.7612 & 84.2487 & 125.2723 & 41.0236 & 84.1266 & 125.475 & 41.3483 \\
\hline 84.1257 & 125.048 & 40.9223 & 84.2219 & 125.2671 & 41.0453 & 84.0184 & 125.5408 & 41.5224 \\
\hline 84.3453 & 124.9794 & 40.6342 & 84.1604 & 125.3009 & 41.1405 & 84.1231 & 125.4966 & 41.3735 \\
\hline 84.3007 & 124.8904 & 40.5898 & 84.2764 & 125.3165 & 41.0401 & 84.1249 & 125.4698 & 41.3449 \\
\hline 84.1266 & 124.9857 & 40.8591 & 84.2765 & 125.3001 & 41.0235 & 83.9837 & 125.5468 & 41.5631 \\
\hline 84.0236 & 125.0134 & 40.9898 & 84.344 & 125.3139 & 40.9699 & 84.1102 & 125.5382 & 41.428 \\
\hline 84.1335 & 125.0272 & 40.8937 & 84.0374 & 125.3987 & 41.3613 & 84.0449 & 125.5723 & 41.5274 \\
\hline 84.111 & 125.0359 & 40.9249 & 84.1734 & 125.4213 & 41.2479 & 84.0305 & 125.5659 & 41.5354 \\
\hline 84.0166 & 125.0905 & 41.0738 & 84.1353 & 125.4646 & 41.3293 & 83.9777 & 125.5936 & 41.6159 \\
\hline 84.04 & 125.0913 & 41.0513 & 84.2981 & 125.5027 & 41.2046 & 83.8798 & 125.6378 & 41.7579 \\
\hline 84.0361 & 125.0437 & 41.0077 & 84.1812 & 125.5971 & 41.4159 & 83.8616 & 125.6715 & 41.8099 \\
\hline 84.3916 & 124.9519 & 40.5603 & 84.2167 & 125.6888 & 41.4722 & 83.8296 & 125.694 & 41.8645 \\
\hline 84.35 & 124.9813 & 40.6313 & 84.2246 & 125.7079 & 41.4834 & 83.8088 & 125.7044 & 41.8956 \\
\hline 83.9413 & 125.139 & 41.1977 & 84.2686 & 125.6862 & 41.4176 & 83.911 & 125.7079 & 41.7969 \\
\hline 84.1396 & 125.1329 & 40.9933 & 84.1188 & 125.72 & 41.6012 & 83.9806 & 125.6912 & 41.7106 \\
\hline 84.4938 & 124.9406 & 40.4469 & 84.1197 & 125.7356 & 41.6159 & 83.9162 & 125.7001 & 41.7839 \\
\hline 84.0383 & 125.0974 & 41.0591 & 83.9993 & 125.7668 & 41.7675 & 83.8469 & 125.7555 & 41.9086 \\
\hline 83.859 & 125.1597 & 41.3007 & 84.0495 & 125.7832 & 41.7337 & 83.8876 & 125.746 & 41.8584 \\
\hline 83.6907 & 125.2992 & 41.6085 & 84.0504 & 125.7971 & 41.7467 & 83.7205 & 125.8066 & 42.0861 \\
\hline 83.9855 & 125.2074 & 41.2219 & 84.8506 & 125.5338 & 40.6833 & 83.7794 & 125.8439 & 42.0645 \\
\hline
\end{tabular}




\begin{tabular}{|c|c|c|c|c|c|c|c|c|}
\hline 83.8486 & 125.8629 & 42.0143 & 83.3775 & 126.3782 & 43.0006 & 83.6711 & 125.9062 & 42.2351 \\
\hline 83.9344 & 125.862 & 41.9277 & 83.3923 & 126.334 & 42.9417 & 83.7101 & 125.8568 & 42.1468 \\
\hline 83.9955 & 125.8938 & 41.8983 & 83.381 & 126.295 & 42.914 & 83.6538 & 125.8456 & 42.1918 \\
\hline 84.0227 & 125.8846 & 41.8619 & 83.517 & 126.2188 & 42.7019 & 83.6425 & 125.8378 & 42.1953 \\
\hline 83.9681 & 125.9209 & 41.9528 & 83.2979 & 126.2479 & 42.9501 & 83.5516 & 125.8222 & 42.2706 \\
\hline 83.9196 & 125.9504 & 42.0307 & 83.2528 & 126.2838 & 43.0309 & 83.5421 & 125.8257 & 42.2836 \\
\hline 83.9456 & 125.9582 & 42.0125 & 83.3256 & 126.2457 & 42.9201 & 83.6863 & 125.8163 & 42.13 \\
\hline 83.9335 & 125.9538 & 42.0203 & 83.3005 & 126.1824 & 42.882 & 83.7222 & 125.7555 & 42.0333 \\
\hline 83.8937 & 125.9668 & 42.0732 & 83.226 & 126.1651 & 42.9391 & 83.7231 & 125.7685 & 42.0454 \\
\hline 83.833 & 126.043 & 42.21 & 83.1983 & 126.1366 & 42.9383 & 83.7023 & 125.7148 & 42.0125 \\
\hline 83.8255 & 126.0647 & 42.2392 & 83.0736 & 126.1305 & 43.0569 & 83.7222 & 125.688 & 41.9658 \\
\hline 83.8997 & 126.0318 & 42.132 & 83.0467 & 126.1478 & 43.1011 & 83.7231 & 125.7079 & 41.9848 \\
\hline 83.8486 & 126.0915 & 42.2429 & 83.0195 & 126.1484 & 43.1289 & 83.7014 & 125.7356 & 42.0342 \\
\hline 83.8192 & 126.1392 & 42.32 & 82.9827 & 126.1443 & 43.1617 & 83.6858 & 125.7763 & 42.0905 \\
\hline 83.8885 & 126.1158 & 42.2273 & 82.9913 & 126.1236 & 43.1322 & 83.6802 & 125.7397 & 42.0595 \\
\hline 83.9707 & 126.0794 & 42.1087 & 83.1732 & 126.0811 & 42.908 & 83.633 & 125.7529 & 42.1199 \\
\hline 84.0028 & 126.1132 & 42.1104 & 83.058 & 126.0638 & 43.0058 & 83.6642 & 125.7581 & 42.0939 \\
\hline 84.0695 & 126.0716 & 42.0022 & 83.1255 & 126.1625 & 43.037 & 83.7309 & 125.7122 & 41.9814 \\
\hline 84.0537 & 126.0762 & 42.0225 & 83.0407 & 126.2465 & 43.2058 & 83.8304 & 125.6871 & 41.8567 \\
\hline 84.0504 & 125.9001 & 41.8497 & 82.9905 & 126.2491 & 43.2587 & 83.8088 & 125.6837 & 41.8749 \\
\hline 83.9474 & 125.8447 & 41.8974 & 83.0671 & 126.2321 & 43.165 & 83.8746 & 125.7001 & 41.8255 \\
\hline 83.9292 & 125.8785 & 41.9493 & 82.9342 & 126.2587 & 43.3245 & 83.9153 & 125.7356 & 41.8203 \\
\hline 83.8235 & 125.9296 & 42.1061 & 83.0338 & 126.2916 & 43.2578 & 83.8079 & 125.7229 & 41.915 \\
\hline 83.8149 & 126.0196 & 42.2048 & 83.0615 & 126.3253 & 43.2639 & 83.8296 & 125.7607 & 41.9311 \\
\hline 83.7594 & 126.0361 & 42.2767 & 83.084 & 126.4691 & 43.3851 & 83.7949 & 125.7936 & 41.9987 \\
\hline 83.6841 & 126.1296 & 42.4455 & 83.0459 & 126.5228 & 43.4769 & 83.8114 & 125.8361 & 42.0247 \\
\hline 83.7181 & 126.2012 & 42.4832 & 83.1004 & 126.5843 & 43.4838 & 84.0175 & 125.7824 & 41.7649 \\
\hline 83.6988 & 126.211 & 42.5122 & 83.3905 & 126.463 & 43.0725 & 84.0798 & 125.7746 & 41.6947 \\
\hline 83.6599 & 126.2154 & 42.5555 & 83.4274 & 126.3387 & 42.9113 & 84.1595 & 125.772 & 41.6125 \\
\hline 83.6408 & 126.2682 & 42.6274 & 83.4105 & 126.3063 & 42.8958 & 84.0513 & 125.8248 & 41.7735 \\
\hline 83.6599 & 126.2812 & 42.6213 & 83.4208 & 126.2742 & 42.8534 & 84.3021 & 125.7802 & 41.4781 \\
\hline 83.6451 & 126.2552 & 42.6101 & 83.4226 & 126.2283 & 42.8058 & 84.3015 & 125.8023 & 41.5008 \\
\hline 83.6529 & 126.2465 & 42.5936 & 83.336 & 126.1972 & 42.8612 & 84.3847 & 125.7962 & 41.4116 \\
\hline 83.5914 & 126.2846 & 42.6932 & 83.3195 & 126.2128 & 42.8932 & 84.5102 & 125.7945 & 41.2843 \\
\hline 83.5672 & 126.3271 & 42.7599 & 83.4373 & 126.1513 & 42.714 & 84.5856 & 125.8153 & 41.2297 \\
\hline 83.8819 & 126.2497 & 42.3678 & 83.5256 & 126.1807 & 42.6551 & 84.6869 & 125.7677 & 41.0808 \\
\hline 84.0729 & 126.1409 & 42.068 & 83.588 & 126.1435 & 42.5555 & 84.6315 & 125.7763 & 41.1448 \\
\hline 84.0963 & 126.1504 & 42.0541 & 83.5348 & 126.1634 & 42.6285 & 84.6384 & 125.8846 & 41.2462 \\
\hline 83.9976 & 126.1452 & 42.1476 & 83.5516 & 126.1859 & 42.6343 & 84.7311 & 125.9686 & 41.2375 \\
\hline 83.9707 & 126.1236 & 42.1528 & 83.4719 & 126.2136 & 42.7417 & 84.7117 & 125.9995 & 41.2878 \\
\hline 83.8686 & 126.1686 & 42.3 & 83.4399 & 126.2024 & 42.7625 & 84.6999 & 126.0534 & 41.3535 \\
\hline 83.8737 & 126.1833 & 42.3096 & 83.5646 & 126.1608 & 42.5962 & 84.7085 & 126.1045 & 41.396 \\
\hline 83.769 & 126.2431 & 42.4741 & 83.6547 & 126.1565 & 42.5018 & 84.6523 & 126.1322 & 41.48 \\
\hline 83.6502 & 126.3334 & 42.6832 & 83.73 & 126.0448 & 42.3148 & 84.66 & 126.1591 & 41.499 \\
\hline 83.5005 & 126.431 & 42.9305 & 83.6971 & 126.0049 & 42.3078 & 84.6306 & 126.1937 & 41.5631 \\
\hline 83.2961 & 126.4327 & 43.1366 & 83.704 & 125.952 & 42.248 & 84.7302 & 126.2716 & 41.5415 \\
\hline 83.4122 & 126.4041 & 42.992 & 83.7118 & 125.901 & 42.1892 & 84.6704 & 126.2751 & 41.6047 \\
\hline
\end{tabular}




\begin{tabular}{|c|c|c|c|c|c|c|c|c|}
\hline 84.8421 & 126.2215 & 41.3794 & 85.1805 & 127.1523 & 41.9718 & 85.655 & 128.6548 & 42.9997 \\
\hline 84.9415 & 126.2595 & 41.318 & 85.3883 & 127.1264 & 41.738 & 85.6828 & 128.6981 & 43.0153 \\
\hline 84.9198 & 126.3071 & 41.3873 & 85.3345 & 127.1394 & 41.8049 & 85.6429 & 128.7648 & 43.1218 \\
\hline 84.9025 & 126.3383 & 41.4358 & 85.3199 & 127.1194 & 41.7995 & 85.5771 & 128.8141 & 43.237 \\
\hline 84.8306 & 126.3877 & 41.557 & 85.3745 & 127.1549 & 41.7805 & 85.4992 & 128.8589 & 43.3597 \\
\hline 84.835 & 126.4137 & 41.5787 & 85.487 & 127.148 & 41.661 & 85.3684 & 128.8609 & 43.4925 \\
\hline 84.7259 & 126.4422 & 41.7164 & 85.3753 & 127.2259 & 41.8506 & 85.2809 & 128.9492 & 43.6683 \\
\hline 84.7181 & 126.5297 & 41.8116 & 85.3286 & 127.2528 & 41.9242 & 85.2827 & 128.9241 & 43.6414 \\
\hline 84.7214 & 126.5545 & 41.8331 & 85.4515 & 127.2181 & 41.7666 & 85.2498 & 128.9085 & 43.6588 \\
\hline 84.751 & 126.6726 & 41.9216 & 85.5321 & 127.245 & 41.7129 & 85.2957 & 128.9042 & 43.6085 \\
\hline 84.9527 & 126.6388 & 41.6861 & 85.5327 & 127.2072 & 41.6745 & 85.3857 & 128.8903 & 43.5046 \\
\hline 84.8566 & 126.696 & 41.8393 & 85.4845 & 127.3004 & 41.816 & 85.5113 & 128.8661 & 43.3548 \\
\hline 84.8107 & 126.7687 & 41.958 & 85.4446 & 127.3671 & 41.9225 & 85.3979 & 128.9338 & 43.5359 \\
\hline 84.9917 & 126.7609 & 41.7692 & 85.203 & 127.5905 & 42.3875 & 85.5702 & 128.8349 & 43.2647 \\
\hline 84.9605 & 126.793 & 41.8324 & 85.1978 & 127.6459 & 42.4481 & 85.7685 & 128.8271 & 43.0586 \\
\hline 84.9631 & 126.8466 & 41.8835 & 85.2446 & 127.6563 & 42.4118 & 85.7616 & 128.828 & 43.0664 \\
\hline 84.887 & 126.8381 & 41.9511 & 85.2013 & 127.7022 & 42.5009 & 85.688 & 128.8626 & 43.1747 \\
\hline 84.822 & 126.7774 & 41.9554 & 85.061 & 127.7559 & 42.6949 & 85.5338 & 128.9051 & 43.3713 \\
\hline 84.7466 & 126.7938 & 42.0472 & 84.917 & 127.8406 & 42.9236 & 85.4403 & 128.9458 & 43.5055 \\
\hline 84.7778 & 126.8406 & 42.0628 & 84.8393 & 127.8893 & 43.05 & 85.6828 & 128.8981 & 43.2154 \\
\hline 84.8471 & 126.9047 & 42.0576 & 84.9908 & 127.8434 & 42.8525 & 85.6287 & 128.9435 & 43.3148 \\
\hline 84.6479 & 126.7722 & 42.1243 & 85.0411 & 127.8018 & 42.7607 & 85.8161 & 128.8748 & 43.0586 \\
\hline 84.7614 & 126.8302 & 42.0688 & 85.1138 & 127.7862 & 42.6724 & 85.8023 & 128.8895 & 43.0872 \\
\hline 84.7804 & 126.8501 & 42.0697 & 85.1155 & 127.8217 & 42.7062 & 85.8672 & 128.8592 & 42.992 \\
\hline 84.8315 & 126.8945 & 42.063 & 85.0359 & 127.846 & 42.8101 & 85.9365 & 128.8462 & 42.9097 \\
\hline 84.8558 & 126.9739 & 42.1182 & 85.0593 & 127.8841 & 42.8248 & 86.0309 & 128.802 & 42.7711 \\
\hline 84.8341 & 127.0034 & 42.1693 & 85.1231 & 127.8468 & 42.7237 & 86.0759 & 128.7405 & 42.6646 \\
\hline 84.9969 & 126.9384 & 41.9415 & 85.1441 & 127.8313 & 42.6871 & 86.1244 & 128.7656 & 42.6412 \\
\hline 84.9848 & 126.9246 & 41.9398 & 85.1346 & 127.8529 & 42.7183 & 86.13 & 128.814 & 42.684 \\
\hline 84.9501 & 126.9904 & 42.0403 & 85.2307 & 127.8243 & 42.5936 & 86.1435 & 128.8193 & 42.6759 \\
\hline 84.9363 & 127.0372 & 42.1009 & 85.19 & 127.8183 & 42.6282 & 86.0724 & 128.8427 & 42.7703 \\
\hline 84.8696 & 127.1593 & 42.2897 & 85.2273 & 127.7888 & 42.5616 & 86.0854 & 128.8661 & 42.7807 \\
\hline 84.9725 & 127.1923 & 42.2198 & 85.2472 & 127.8018 & 42.5546 & 86.0534 & 128.8869 & 42.8335 \\
\hline 85.0532 & 127.0155 & 41.9623 & 85.1104 & 127.8607 & 42.7503 & 86.0378 & 128.8661 & 42.8283 \\
\hline 84.9415 & 127.0389 & 42.0974 & 85.131 & 127.9164 & 42.7853 & 86.1339 & 128.8531 & 42.7192 \\
\hline 84.8436 & 127.0839 & 42.2403 & 85.132 & 127.9196 & 42.7876 & 86.1123 & 128.8003 & 42.688 \\
\hline 84.7995 & 127.1506 & 42.3511 & 85.1173 & 127.9404 & 42.8231 & 86.085 & 128.792 & 42.7069 \\
\hline 84.7544 & 127.1991 & 42.4447 & 85.1502 & 127.9075 & 42.7573 & 86.0785 & 128.8037 & 42.7252 \\
\hline 84.6271 & 127.2181 & 42.591 & 85.1805 & 127.8789 & 42.6984 & 86.1937 & 128.7942 & 42.6005 \\
\hline 84.5734 & 127.2233 & 42.6499 & 85.2411 & 127.8451 & 42.604 & 86.14 & 128.8063 & 42.6663 \\
\hline 84.5549 & 127.2345 & 42.6796 & 85.184 & 127.8598 & 42.6759 & 86.0586 & 128.8029 & 42.7443 \\
\hline 84.7042 & 127.2156 & 42.5113 & 85.2558 & 127.8676 & 42.6118 & 85.9945 & 128.8254 & 42.8309 \\
\hline 84.8168 & 127.2138 & 42.397 & 85.3371 & 127.8406 & 42.5035 & 86.0075 & 128.8453 & 42.8378 \\
\hline 85.016 & 127.1774 & 42.1615 & 85.3831 & 127.8685 & 42.4854 & 86.0153 & 128.8557 & 42.8404 \\
\hline 84.8159 & 127.1645 & 42.3485 & 85.3216 & 127.9031 & 42.5815 & 86.0621 & 128.8184 & 42.7563 \\
\hline 84.8791 & 127.1965 & 42.3174 & 85.4879 & 128.6591 & 43.1712 & 86.0785 & 128.8392 & 42.7607 \\
\hline 85.048 & 127.1852 & 42.1372 & 85.578 & 128.6669 & 43.0889 & 86.011 & 128.8306 & 42.8196 \\
\hline
\end{tabular}




\begin{tabular}{|c|c|c|c|c|c|c|c|c|}
\hline 85.9815 & 128.8418 & 42.8603 & 86.4362 & 129.2627 & 42.8266 & 86.9298 & 128.6383 & 41.7086 \\
\hline 86.0785 & 128.7856 & 42.707 & 86.4474 & 129.2411 & 42.7936 & 86.9518 & 128.6308 & 41.6789 \\
\hline 86.0647 & 128.8081 & 42.7434 & 86.4682 & 129.254 & 42.7859 & 86.9635 & 128.6591 & 41.6956 \\
\hline 86.0802 & 128.8176 & 42.7374 & 86.4491 & 129.2255 & 42.7763 & 86.8977 & 128.7007 & 41.803 \\
\hline 86.1755 & 128.8271 & 42.6516 & 86.482 & 129.2644 & 42.7824 & 86.8449 & 128.7206 & 41.8757 \\
\hline 86.3392 & 128.815 & 42.4758 & 86.489 & 129.2696 & 42.7807 & 86.7297 & 128.8193 & 42.0896 \\
\hline 86.3581 & 128.8122 & 42.4541 & 86.5617 & 129.2506 & 42.6889 & 86.747 & 128.8375 & 42.0905 \\
\hline 86.3002 & 128.8332 & 42.533 & 86.5854 & 129.2633 & 42.6779 & 86.7375 & 128.8488 & 42.1113 \\
\hline 86.2881 & 128.8505 & 42.5624 & 86.65 & 129.2393 & 42.5893 & 86.7072 & 128.9051 & 42.1979 \\
\hline 86.185 & 128.912 & 42.727 & 86.6301 & 129.254 & 42.6239 & 86.6726 & 128.9162 & 42.2436 \\
\hline 86.1045 & 128.9284 & 42.824 & 86.5522 & 129.3458 & 42.7936 & 86.7444 & 128.8869 & 42.1424 \\
\hline 86.0075 & 128.9951 & 42.9876 & 86.5851 & 129.3536 & 42.7685 & 86.734 & 128.9362 & 42.2022 \\
\hline 86.0439 & 128.9761 & 42.9322 & 86.5427 & 129.364 & 42.8214 & 86.6145 & 128.9882 & 42.3737 \\
\hline 86.0265 & 128.9761 & 42.9495 & 86.5072 & 129.4047 & 42.8976 & 86.6388 & 128.9778 & 42.339 \\
\hline 85.9758 & 128.9734 & 42.9976 & 86.4907 & 129.3995 & 42.9088 & 86.6016 & 128.9908 & 42.3892 \\
\hline 85.9529 & 128.9778 & 43.0249 & 86.4214 & 129.4463 & 43.0249 & 86.5938 & 129.0878 & 42.494 \\
\hline 85.972 & 128.9891 & 43.0171 & 86.5889 & 129.4218 & 42.8329 & 86.6448 & 129.1389 & 42.494 \\
\hline 86.0508 & 128.9605 & 42.9097 & 86.6907 & 129.3874 & 42.6967 & 86.592 & 129.1597 & 42.5676 \\
\hline 86.1322 & 128.9388 & 42.8066 & 86.786 & 129.3727 & 42.5867 & 86.6039 & 129.1866 & 42.5827 \\
\hline 86.2898 & 128.8955 & 42.6057 & 86.7349 & 129.3311 & 42.5962 & 86.6474 & 129.2411 & 42.5936 \\
\hline 86.3409 & 128.8739 & 42.533 & 86.7652 & 129.3606 & 42.5953 & 86.6319 & 129.3545 & 42.7226 \\
\hline 86.3565 & 128.854 & 42.4975 & 86.7739 & 129.3441 & 42.5702 & 86.6431 & 129.4879 & 42.8447 \\
\hline 86.2594 & 128.8959 & 42.6365 & 86.7626 & 129.3372 & 42.5746 & 86.5487 & 129.5762 & 43.0275 \\
\hline 86.1911 & 128.9267 & 42.7356 & 86.7193 & 129.4143 & 42.6949 & 86.6293 & 129.6429 & 43.0136 \\
\hline 86.1885 & 128.9336 & 42.7452 & 86.6638 & 129.4368 & 42.773 & 86.6024 & 129.7442 & 43.1418 \\
\hline 86.1538 & 128.9639 & 42.8101 & 86.5219 & 129.4688 & 42.9469 & 86.5505 & 129.9312 & 43.3808 \\
\hline 86.0802 & 129.0228 & 42.9426 & 86.3478 & 129.5381 & 43.1903 & 86.5634 & 129.709 & 43.1456 \\
\hline 85.9893 & 129.086 & 43.0967 & 86.2439 & 129.5996 & 43.3557 & 86.5955 & 129.9208 & 43.3254 \\
\hline 85.8967 & 129.1337 & 43.237 & 86.0075 & 129.7702 & 43.7627 & 86.76 & 129.9347 & 43.1747 \\
\hline 85.8906 & 129.1597 & 43.2691 & 85.9755 & 129.8083 & 43.8328 & 86.7765 & 129.9191 & 43.1426 \\
\hline 85.9732 & 129.1549 & 43.1817 & 86.0603 & 129.7485 & 43.6882 & 87.0813 & 129.8126 & 42.7313 \\
\hline 85.9841 & 129.1458 & 43.1617 & 86.2465 & 129.7009 & 43.4544 & 87.1558 & 129.7771 & 42.6213 \\
\hline 85.9997 & 129.164 & 43.1643 & 87.2214 & 128.8052 & 41.5838 & 87.2043 & 129.7381 & 42.5339 \\
\hline 85.959 & 129.2194 & 43.2604 & 88.3967 & 128.4972 & 40.1005 & 87.1783 & 129.7442 & 42.5659 \\
\hline 86.0179 & 129.2341 & 43.2162 & 87.665 & 128.3803 & 40.7153 & 87.217 & 129.7169 & 42.4999 \\
\hline 85.9477 & 129.2904 & 43.3427 & 87.2251 & 129.1917 & 41.9666 & 87.1973 & 129.7416 & 42.5442 \\
\hline 85.7096 & 129.3692 & 43.6596 & 87.0778 & 128.7847 & 41.7069 & 87.2588 & 129.6723 & 42.4135 \\
\hline 85.6429 & 129.4229 & 43.78 & 87.0726 & 128.7102 & 41.6376 & 87.3489 & 129.6992 & 42.3503 \\
\hline 85.797 & 129.3839 & 43.587 & 87.154 & 128.6357 & 41.4817 & 87.2935 & 129.7225 & 42.4291 \\
\hline 85.8733 & 129.3779 & 43.5046 & 87.1281 & 128.6661 & 41.538 & 87.2952 & 129.7295 & 42.4343 \\
\hline 85.8447 & 129.4194 & 43.5748 & 87.1245 & 128.5858 & 41.4614 & 87.3775 & 129.6879 & 42.3104 \\
\hline 85.9651 & 129.3744 & 43.4094 & 86.954 & 128.6894 & 41.7354 & 87.3541 & 129.7433 & 42.3892 \\
\hline 86.1114 & 129.3484 & 43.237 & 86.8241 & 128.6565 & 41.8324 & 87.3077 & 129.8015 & 42.4938 \\
\hline 86.263 & 129.3008 & 43.0379 & 86.7964 & 128.6539 & 41.8575 & 87.2199 & 129.8273 & 42.6075 \\
\hline 86.4604 & 129.2047 & 42.7443 & 86.9029 & 128.6808 & 41.7779 & 87.2268 & 129.8412 & 42.6144 \\
\hline 86.4405 & 129.222 & 42.7815 & 86.9341 & 128.6609 & 41.7268 & 87.1662 & 129.8741 & 42.7079 \\
\hline 86.448 & 129.2562 & 42.8082 & 86.9427 & 128.6738 & 41.7311 & 87.1887 & 129.8576 & 42.6689 \\
\hline
\end{tabular}




\begin{tabular}{|c|c|c|c|c|c|c|c|c|}
\hline 87.2839 & 129.8827 & 42.5988 & 87.1133 & 130.469 & 43.3557 & 88.511 & 129.8334 & 41.3224 \\
\hline 87.2138 & 129.8931 & 42.6793 & 87.1662 & 130.4638 & 43.2976 & 88.2919 & 129.946 & 41.654 \\
\hline 87.1411 & 129.8923 & 42.7512 & 87.0926 & 130.5045 & 43.4119 & 88.2382 & 129.9633 & 41.725 \\
\hline 87.0857 & 129.8922 & 42.8065 & 87.1177 & 130.4915 & 43.3738 & 88.4262 & 129.9356 & 41.5094 \\
\hline 87.0475 & 129.8983 & 42.8508 & 87.0683 & 130.4855 & 43.4171 & 88.3898 & 129.9321 & 41.5423 \\
\hline 87.0224 & 129.9243 & 42.9019 & 87.1289 & 130.4807 & 43.3518 & 88.6989 & 129.7494 & 41.0504 \\
\hline 87.0709 & 129.9027 & 42.8317 & 87.1887 & 130.4829 & 43.2942 & 88.7717 & 129.7199 & 40.9483 \\
\hline 87.019 & 129.9139 & 42.895 & 87.1921 & 130.4733 & 43.2812 & 88.6581 & 129.7698 & 41.1116 \\
\hline 86.9791 & 129.9027 & 42.9235 & 87.1679 & 130.4474 & 43.2795 & 88.3387 & 129.9979 & 41.6592 \\
\hline 86.9999 & 129.9243 & 42.9244 & 87.1973 & 130.4889 & 43.2916 & 88.6452 & 129.8949 & 41.2496 \\
\hline 87.0172 & 129.9295 & 42.9123 & 87.1947 & 130.5071 & 43.3124 & 88.4634 & 130.01 & 41.5467 \\
\hline 87.0708 & 129.9407 & 42.8699 & 87.1705 & 130.514 & 43.3435 & 87.5619 & 130.3833 & 42.8214 \\
\hline 87.0804 & 129.9598 & 42.8794 & 87.1333 & 130.4993 & 43.3661 & 87.8901 & 130.269 & 42.3788 \\
\hline 87.0822 & 129.9823 & 42.9002 & 87.5394 & 130.2543 & 42.7149 & 88.085 & 130.2222 & 42.1372 \\
\hline 87.1506 & 130.0083 & 42.8577 & 87.762 & 130.2023 & 42.4403 & 88.6548 & 130.0533 & 41.3986 \\
\hline 87.1939 & 129.9841 & 42.7902 & 88.4868 & 129.8091 & 41.3224 & 88.4652 & 130.2032 & 41.738 \\
\hline 87.2077 & 130.0326 & 42.8248 & 88.5301 & 129.6368 & 41.1067 & 88.4114 & 130.2949 & 41.8835 \\
\hline 87.2493 & 130.049 & 42.7997 & 88.4565 & 129.6714 & 41.215 & 88.9241 & 130.0975 & 41.1734 \\
\hline 87.2103 & 130.0836 & 42.8733 & 88.4045 & 129.7 & 41.2955 & 89.3692 & 129.8879 & 40.5187 \\
\hline 87.0674 & 130.1685 & 43.1011 & 88.6158 & 129.5736 & 40.9578 & 89.3077 & 129.92 & 40.6123 \\
\hline 87.0822 & 130.1794 & 43.0972 & 88.7206 & 129.5164 & 40.7959 & 88.9873 & 130.036 & 41.0487 \\
\hline 87.0285 & 130.2335 & 43.205 & 88.6426 & 129.5753 & 40.9327 & 88.8877 & 130.1157 & 41.228 \\
\hline 87.0397 & 130.2387 & 43.1989 & 88.5375 & 129.6235 & 41.0861 & 88.8661 & 130.1183 & 41.2522 \\
\hline 87.0882 & 130.2222 & 43.134 & 88.5422 & 129.6662 & 41.1241 & 88.8946 & 130.1798 & 41.2851 \\
\hline 86.9834 & 130.2724 & 43.289 & 88.582 & 129.6039 & 41.0219 & 88.4582 & 130.3794 & 41.9212 \\
\hline 86.9168 & 130.3374 & 43.4206 & 88.5881 & 129.6758 & 41.0877 & 88.7284 & 130.185 & 41.4566 \\
\hline 86.9375 & 130.3183 & 43.3808 & 88.4998 & 129.713 & 41.2133 & 88.9795 & 130.0845 & 41.105 \\
\hline 86.8432 & 130.3668 & 43.5237 & 88.5327 & 129.6983 & 41.1656 & 88.64 & 130.2776 & 41.6376 \\
\hline 86.8505 & 130.4031 & 43.5526 & 88.5214 & 129.6749 & 41.1535 & 88.6851 & 130.2352 & 41.5501 \\
\hline 86.8899 & 130.3677 & 43.4778 & 88.5846 & 129.7043 & 41.1197 & 89.1224 & 130.0646 & 40.9422 \\
\hline 86.9514 & 130.3885 & 43.4371 & 88.6493 & 129.6359 & 40.9865 & 89.041 & 130.0481 & 41.0071 \\
\hline 86.941 & 130.3893 & 43.4483 & 88.5586 & 129.7234 & 41.1648 & 88.9856 & 130.075 & 41.0894 \\
\hline 86.9826 & 130.3486 & 43.3661 & 88.5292 & 129.8117 & 41.2825 & 88.8713 & 130.0904 & 41.2191 \\
\hline 87.2008 & 130.2534 & 43.0526 & 88.5119 & 129.8663 & 41.3544 & 88.8678 & 130.0741 & 41.2063 \\
\hline 87.1402 & 130.3296 & 43.1894 & 88.5249 & 129.8576 & 41.3328 & 88.4192 & 130.3027 & 41.8835 \\
\hline 87.0726 & 130.3694 & 43.2968 & 88.5067 & 129.8871 & 41.3804 & 88.4019 & 130.366 & 41.964 \\
\hline 87.0285 & 130.4084 & 43.38 & 88.55 & 129.8992 & 41.3492 & 88.7292 & 130.2205 & 41.4912 \\
\hline 87.051 & 130.4326 & 43.3816 & 88.3967 & 129.9044 & 41.5077 & 88.6045 & 130.2646 & 41.6601 \\
\hline 87.0796 & 130.4179 & 43.3383 & 88.3287 & 129.8816 & 41.553 & 88.3759 & 130.3582 & 41.9822 \\
\hline 87.2718 & 130.3504 & 43.0786 & 88.4201 & 129.8169 & 41.3968 & 88.6903 & 130.2075 & 41.5172 \\
\hline 87.2493 & 130.3019 & 43.0526 & 88.5405 & 129.7589 & 41.2184 & 88.5912 & 130.2657 & 41.6745 \\
\hline 87.1653 & 130.359 & 43.1937 & 88.5552 & 129.7494 & 41.1942 & 88.7318 & 130.2092 & 41.4774 \\
\hline 87.1627 & 130.3937 & 43.231 & 88.5543 & 129.7797 & 41.2254 & 88.5032 & 130.3201 & 41.8168 \\
\hline 87.1947 & 130.3668 & 43.1721 & 88.6063 & 129.7433 & 41.137 & 88.44 & 130.3911 & 41.9511 \\
\hline 87.1263 & 130.4155 & 43.2892 & 88.5751 & 129.7684 & 41.1933 & 88.9423 & 130.1945 & 41.2522 \\
\hline 87.0822 & 130.4534 & 43.3712 & 88.2833 & 129.9226 & 41.6393 & 88.9111 & 130.1304 & 41.2193 \\
\hline 87.1185 & 130.4586 & 43.3401 & 88.4309 & 129.8464 & 41.4155 & 88.6807 & 130.2213 & 41.5406 \\
\hline
\end{tabular}




\begin{tabular}{|c|c|c|c|c|c|c|c|c|}
\hline 88.4504 & 130.3434 & 41.893 & 87.0302 & 131.1098 & 44.0796 & 87.3879 & 131.0345 & 43.6466 \\
\hline 88.3296 & 130.3776 & 42.048 & 87.1835 & 131.0362 & 43.8527 & 87.3749 & 131.0405 & 43.6657 \\
\hline 88.5257 & 130.3434 & 41.8177 & 87.3956 & 130.9678 & 43.5722 & 87.3697 & 131.0769 & 43.7072 \\
\hline 88.8617 & 130.1867 & 41.325 & 87.5042 & 130.8718 & 43.3676 & 87.4433 & 131.0492 & 43.6059 \\
\hline 88.9934 & 130.0793 & 41.086 & 87.561 & 130.876 & 43.315 & 87.4727 & 131.076 & 43.6033 \\
\hline 88.6989 & 130.2785 & 41.5796 & 87.5472 & 130.8864 & 43.3392 & 87.409 & 131.1237 & 43.7147 \\
\hline 88.4876 & 130.3417 & 41.8541 & 87.5134 & 130.8821 & 43.3687 & 87.5316 & 131.089 & 43.5574 \\
\hline 88.3621 & 130.4023 & 42.0403 & 87.5446 & 130.8725 & 43.328 & 87.6909 & 131.0596 & 43.3687 \\
\hline 87.8841 & 130.6006 & 42.7166 & 87.6476 & 130.8881 & 43.2405 & 87.6269 & 131.0717 & 43.4449 \\
\hline 88.1798 & 130.5053 & 42.3255 & 87.555 & 130.8994 & 43.3444 & 87.7031 & 131.0449 & 43.3418 \\
\hline 88.072 & 130.5634 & 42.4914 & 87.5645 & 130.9003 & 43.3357 & 87.7585 & 131.0518 & 43.2933 \\
\hline 88.0287 & 130.5842 & 42.5555 & 87.4716 & 130.929 & 43.4575 & 87.8009 & 131.0674 & 43.2665 \\
\hline 88.1473 & 130.566 & 42.4187 & 87.4415 & 130.9462 & 43.5046 & 88.1906 & 130.8907 & 42.7001 \\
\hline 87.9655 & 130.6032 & 42.6378 & 87.4623 & 130.9678 & 43.5055 & 89.2387 & 130.3415 & 41.1028 \\
\hline 88.1031 & 130.5305 & 42.4273 & 87.3679 & 130.9747 & 43.6068 & 89.4333 & 130.2897 & 40.8565 \\
\hline 87.7723 & 130.6993 & 42.927 & 87.3601 & 130.9756 & 43.6155 & 89.4125 & 130.3001 & 40.8876 \\
\hline 87.9317 & 130.6483 & 42.7166 & 87.3775 & 130.9643 & 43.5869 & 89.448 & 130.3071 & 40.8591 \\
\hline 87.8856 & 130.6815 & 42.7959 & 87.3757 & 130.9539 & 43.5782 & 89.6091 & 130.2387 & 40.6296 \\
\hline 87.7264 & 130.7262 & 42.9997 & 87.3342 & 130.9496 & 43.6155 & 89.532 & 130.2473 & 40.7153 \\
\hline 87.4996 & 130.7764 & 43.2769 & 87.3209 & 130.9423 & 43.6213 & 89.4965 & 130.2733 & 40.7768 \\
\hline 87.6953 & 130.7323 & 43.037 & 87.29 & 130.9825 & 43.6925 & 89.4212 & 130.2707 & 40.8495 \\
\hline 87.7221 & 130.6993 & 42.9772 & 87.2701 & 130.9513 & 43.6813 & 89.4527 & 130.3045 & 40.8518 \\
\hline 87.6762 & 130.721 & 43.0448 & 87.245 & 130.986 & 43.741 & 89.4402 & 130.3278 & 40.8876 \\
\hline 87.6104 & 130.7903 & 43.1799 & 87.2632 & 130.9297 & 43.6665 & 89.4142 & 130.3313 & 40.9171 \\
\hline 87.4857 & 130.8734 & 43.3877 & 87.251 & 130.9314 & 43.6804 & 89.4696 & 130.2897 & 40.8201 \\
\hline 87.3509 & 130.9238 & 43.5729 & 87.2701 & 131.0276 & 43.7575 & 89.5294 & 130.2534 & 40.724 \\
\hline 87.2467 & 130.9652 & 43.7185 & 87.2891 & 130.9643 & 43.6752 & 89.5692 & 130.2101 & 40.6408 \\
\hline 87.3584 & 130.9375 & 43.5791 & 87.3456 & 130.929 & 43.5834 & 89.4809 & 130.2811 & 40.8002 \\
\hline 87.4312 & 130.9513 & 43.5202 & 87.4095 & 130.9817 & 43.5722 & 89.5251 & 130.2681 & 40.743 \\
\hline 87.4935 & 130.9054 & 43.4119 & 87.2978 & 131.0232 & 43.7254 & 89.4712 & 130.2772 & 40.806 \\
\hline 87.5229 & 130.8821 & 43.3591 & 87.2103 & 131.0475 & 43.8371 & 89.4307 & 130.2837 & 40.853 \\
\hline 87.6017 & 130.8751 & 43.2734 & 87.1064 & 131.0856 & 43.9792 & 89.3969 & 130.3183 & 40.9214 \\
\hline 87.5195 & 130.9011 & 43.3816 & 87.2008 & 131.0631 & 43.8623 & 89.4766 & 130.2915 & 40.8149 \\
\hline 87.5732 & 130.863 & 43.2898 & 87.303 & 131.0128 & 43.7098 & 89.5459 & 130.2707 & 40.7248 \\
\hline 87.3967 & 130.9282 & 43.5315 & 87.3705 & 130.9825 & 43.612 & 89.558 & 130.2629 & 40.7049 \\
\hline 87.4762 & 130.9063 & 43.4301 & 87.3749 & 130.9877 & 43.6129 & 89.5545 & 130.2785 & 40.724 \\
\hline 87.5013 & 130.9003 & 43.399 & 87.3456 & 131.0171 & 43.6715 & 89.5424 & 130.2742 & 40.7318 \\
\hline 87.5377 & 130.8942 & 43.3565 & 87.3151 & 130.9877 & 43.6726 & 89.4835 & 130.2923 & 40.8088 \\
\hline 87.4372 & 130.8673 & 43.4301 & 87.2865 & 131.0327 & 43.7462 & 89.5531 & 130.2587 & 40.7055 \\
\hline 87.645 & 130.85 & 43.205 & 87.2216 & 131.083 & 43.8614 & 89.5251 & 130.3027 & 40.7777 \\
\hline 87.6953 & 130.8232 & 43.1279 & 87.193 & 131.0353 & 43.8423 & 89.4818 & 130.3521 & 40.8703 \\
\hline 87.6199 & 130.8249 & 43.205 & 87.1783 & 131.0769 & 43.8986 & 89.4766 & 130.3296 & 40.853 \\
\hline 87.5931 & 130.8462 & 43.2531 & 87.2718 & 131.0942 & 43.8224 & 89.5164 & 130.2828 & 40.7664 \\
\hline 87.4156 & 130.9479 & 43.5323 & 87.3965 & 131.0483 & 43.6518 & 89.5606 & 130.2196 & 40.659 \\
\hline 87.3515 & 130.9912 & 43.6397 & 87.5799 & 130.944 & 43.3641 & 89.6749 & 130.1945 & 40.5196 \\
\hline 87.1263 & 131.089 & 43.9627 & 87.5463 & 130.9522 & 43.4059 & 89.6099 & 130.2438 & 40.6339 \\
\hline 86.9627 & 131.1445 & 44.1818 & 87.3714 & 131.0362 & 43.6648 & 89.7346 & 130.1891 & 40.4545 \\
\hline
\end{tabular}




\begin{tabular}{|c|c|c|c|c|c|c|c|c|}
\hline 89.7164 & 130.1235 & 40.407 & 89.9736 & 129.7303 & 39.7567 & 82.7203 & 123.0156 & 40.2953 \\
\hline 89.6593 & 130.1547 & 40.4954 & 90.0218 & 129.7389 & 39.7172 & 82.7185 & 122.9723 & 40.2538 \\
\hline 89.5883 & 130.2283 & 40.64 & 90.0239 & 129.7511 & 39.7272 & 82.6882 & 122.9957 & 40.3074 \\
\hline 89.5917 & 130.2352 & 40.6434 & 89.9788 & 129.7935 & 39.8147 & 82.6787 & 123.0295 & 40.3507 \\
\hline 89.674 & 130.178 & 40.504 & 90.0048 & 129.7658 & 39.761 & 82.7099 & 122.9801 & 40.2702 \\
\hline 89.655 & 130.1642 & 40.5092 & 89.9962 & 129.7546 & 39.7584 & 82.9056 & 122.9688 & 40.0632 \\
\hline 89.668 & 130.1798 & 40.5118 & 89.9736 & 129.7814 & 39.8078 & 82.8926 & 122.9515 & 40.0589 \\
\hline 89.6677 & 130.1759 & 40.5082 & 90.0135 & 129.7546 & 39.7411 & 82.8539 & 122.978 & 40.1241 \\
\hline 89.6376 & 130.1486 & 40.511 & 90.0195 & 129.7702 & 39.7506 & 82.8259 & 122.955 & 40.1291 \\
\hline 89.6082 & 130.185 & 40.5768 & 89.894 & 129.9231 & 40.029 & 82.8173 & 122.9584 & 40.1412 \\
\hline 89.6342 & 130.1702 & 40.5361 & 89.8697 & 129.9607 & 40.091 & 82.7514 & 122.9541 & 40.2027 \\
\hline 89.6013 & 130.1997 & 40.5984 & 89.9355 & 129.8325 & 39.897 & 82.78 & 122.9368 & 40.1568 \\
\hline 89.5649 & 130.2482 & 40.6833 & 89.9555 & 129.8291 & 39.8736 & 82.7514 & 122.9238 & 40.1724 \\
\hline 89.6143 & 130.2646 & 40.6504 & 89.9373 & 129.8888 & 39.9515 & 82.6865 & 123.0182 & 40.3317 \\
\hline 89.6125 & 130.2343 & 40.6218 & 89.9295 & 129.8905 & 39.9611 & 82.6709 & 122.9939 & 40.323 \\
\hline 89.6879 & 130.1477 & 40.4598 & 89.9632 & 129.9624 & 39.9992 & 82.6934 & 122.9888 & 40.2953 \\
\hline 89.713 & 130.1226 & 40.4096 & 89.997 & 129.9945 & 39.9974 & 82.7385 & 122.9181 & 40.1796 \\
\hline 89.7052 & 130.1988 & 40.4936 & 89.9909 & 129.9653 & 39.9744 & 82.7896 & 122.884 & 40.0944 \\
\hline 89.7416 & 130.2404 & 40.4988 & 90.0031 & 129.9823 & 39.9792 & 82.8467 & 122.8831 & 40.0364 \\
\hline 89.6723 & 130.2603 & 40.588 & 89.9355 & 130.0447 & 40.1091 & 82.8051 & 122.8718 & 40.0667 \\
\hline 89.6394 & 130.249 & 40.6097 & 89.9425 & 130.0542 & 40.1117 & 82.7887 & 122.8623 & 40.0736 \\
\hline 89.5935 & 130.288 & 40.6945 & 89.8966 & 130.0784 & 40.1819 & 82.8095 & 122.8632 & 40.0537 \\
\hline 89.5961 & 130.2672 & 40.6712 & 89.9078 & 130.03 & 40.1221 & 82.7982 & 122.8727 & 40.0745 \\
\hline 89.5461 & 130.2728 & 40.7267 & 89.7312 & 130.0871 & 40.3559 & 82.7255 & 122.9437 & 40.2183 \\
\hline 89.5917 & 130.2828 & 40.6911 & 89.8576 & 130.0369 & 40.1793 & 82.6434 & 122.9895 & 40.3461 \\
\hline 89.6498 & 130.2854 & 40.6356 & 90.0103 & 129.9477 & 39.9374 & 82.5583 & 123.0381 & 40.4798 \\
\hline 89.5848 & 129.6844 & 40.0996 & 90.0057 & 129.9832 & 39.9775 & 82.5202 & 123.0277 & 40.5075 \\
\hline 89.474 & 128.3058 & 38.8318 & 82.7393 & 122.8138 & 40.0745 & 82.5384 & 123.0346 & 40.4962 \\
\hline 89.5051 & 128.6903 & 39.1851 & 82.838 & 122.658 & 39.8199 & 82.5168 & 123.0866 & 40.5698 \\
\hline 89.4956 & 129.8169 & 40.3213 & 82.832 & 122.7956 & 39.9637 & 82.6103 & 123.0485 & 40.4382 \\
\hline 89.4021 & 129.9901 & 40.588 & 82.8865 & 122.52 & 39.6335 & 82.7584 & 123.0043 & 40.246 \\
\hline 89.7337 & 129.8957 & 40.162 & 82.8822 & 122.6528 & 39.7705 & 82.7437 & 122.9939 & 40.2503 \\
\hline 89.8784 & 129.8048 & 39.9264 & 82.8614 & 122.6502 & 39.7887 & 82.7279 & 123.0318 & 40.3038 \\
\hline 89.8065 & 129.8723 & 40.0658 & 82.9896 & 122.6813 & 39.6917 & 82.7203 & 123.0771 & 40.3568 \\
\hline 89.9174 & 129.8204 & 39.903 & 83.0719 & 122.6987 & 39.6268 & 82.6796 & 123.1186 & 40.4391 \\
\hline 89.8844 & 129.8481 & 39.9637 & 83.2399 & 122.677 & 39.4371 & 82.6675 & 123.0831 & 40.4157 \\
\hline 89.8221 & 129.8602 & 40.0381 & 83.3429 & 122.6805 & 39.3376 & 82.6302 & 123.1299 & 40.4997 \\
\hline 89.913 & 129.8221 & 39.9091 & 83.2468 & 122.7775 & 39.5307 & 82.6605 & 123.0814 & 40.4209 \\
\hline 89.9217 & 129.7944 & 39.8727 & 83.1754 & 122.7966 & 39.6212 & 82.6649 & 123.0693 & 40.4044 \\
\hline 89.9026 & 129.7883 & 39.8857 & 83.0104 & 122.8606 & 39.8502 & 82.7653 & 123.0269 & 40.2615 \\
\hline 90.0244 & 129.7275 & 39.7031 & 82.9168 & 122.9082 & 39.9914 & 82.594 & 123.0908 & 40.4968 \\
\hline 89.9598 & 129.7806 & 39.8208 & 82.7852 & 122.8926 & 40.1074 & 82.7272 & 123.0606 & 40.3334 \\
\hline 89.9503 & 129.7606 & 39.8104 & 82.7125 & 122.9333 & 40.2208 & 82.722 & 123.0502 & 40.3282 \\
\hline 89.9927 & 129.7641 & 39.7714 & 82.7159 & 122.9411 & 40.2252 & 82.7099 & 123.0554 & 40.3455 \\
\hline 89.9364 & 129.778 & 39.8416 & 82.6943 & 122.9671 & 40.2728 & 82.7341 & 123.0676 & 40.3334 \\
\hline 89.9754 & 129.7589 & 39.7835 & 82.6943 & 122.9515 & 40.2572 & 82.7082 & 123.0727 & 40.3646 \\
\hline 89.9892 & 129.7165 & 39.7272 & 82.6777 & 122.986 & 40.3083 & 82.6527 & 123.1273 & 40.4746 \\
\hline
\end{tabular}




\begin{tabular}{|c|c|c|c|c|c|c|c|c|}
\hline 82.664 & 123.0814 & 40.4174 & 82.6865 & 123.2425 & 40.556 & 82.664 & 123.3966 & 40.7326 \\
\hline 82.6883 & 123.1348 & 40.4466 & 82.6675 & 123.2555 & 40.588 & 82.6649 & 123.394 & 40.7292 \\
\hline 82.7991 & 123.1126 & 40.3135 & 82.6943 & 123.2433 & 40.5491 & 82.6345 & 123.401 & 40.7664 \\
\hline 82.8961 & 123.0667 & 40.1706 & 82.7323 & 123.2441 & 40.5117 & 82.6034 & 123.4113 & 40.808 \\
\hline 82.8787 & 123.1256 & 40.2468 & 82.7454 & 123.2356 & 40.4902 & 82.6432 & 123.4217 & 40.7785 \\
\hline 82.9558 & 123.0546 & 40.0987 & 82.7549 & 123.2459 & 40.491 & 82.565 & 123.4273 & 40.8623 \\
\hline 82.9359 & 123.0494 & 40.1135 & 82.5869 & 123.2659 & 40.6789 & 82.4847 & 123.4512 & 40.9664 \\
\hline 82.8935 & 123.0866 & 40.1931 & 82.5367 & 123.3161 & 40.7794 & 82.6293 & 123.4469 & 40.8175 \\
\hline 82.8303 & 123.0831 & 40.2529 & 82.5142 & 123.3499 & 40.8357 & 82.6605 & 123.4408 & 40.7803 \\
\hline 82.8231 & 123.0652 & 40.2422 & 82.4839 & 123.3473 & 40.8634 & 83.0095 & 123.3525 & 40.343 \\
\hline 82.8112 & 123.084 & 40.2728 & 82.5479 & 123.349 & 40.801 & 83.1654 & 123.336 & 40.1706 \\
\hline 82.6735 & 123.1325 & 40.459 & 82.4954 & 123.3436 & 40.8482 & 83.3845 & 123.2546 & 39.8701 \\
\hline 82.6094 & 123.1533 & 40.5439 & 82.4388 & 123.3325 & 40.8937 & 83.5265 & 123.0416 & 39.5151 \\
\hline 82.4795 & 123.1975 & 40.7179 & 82.4605 & 123.3412 & 40.8807 & 83.8493 & 122.882 & 39.0327 \\
\hline 82.4873 & 123.1983 & 40.711 & 82.4414 & 123.3802 & 40.9387 & 83.8547 & 122.832 & 38.9773 \\
\hline 82.4267 & 123.2269 & 40.8002 & 82.4181 & 123.3568 & 40.9387 & 83.9422 & 122.8069 & 38.8647 \\
\hline 82.4691 & 123.226 & 40.7569 & 82.3791 & 123.3819 & 41.0028 & 83.7594 & 122.8918 & 39.1323 \\
\hline 82.4709 & 123.2373 & 40.7664 & 82.4267 & 123.3758 & 40.9491 & 83.9058 & 122.8563 & 38.9505 \\
\hline 82.5685 & 123.1974 & 40.6289 & 82.4258 & 123.381 & 40.9552 & 84.0322 & 122.8104 & 38.7781 \\
\hline 82.5748 & 123.1983 & 40.6235 & 82.4328 & 123.3966 & 40.9639 & 83.937 & 122.8545 & 38.9176 \\
\hline 82.69 & 123.1697 & 40.4798 & 82.4742 & 123.4106 & 40.9363 & 83.8521 & 122.8831 & 39.031 \\
\hline 82.4821 & 123.233 & 40.7508 & 82.4605 & 123.3984 & 40.9379 & 83.827 & 122.8805 & 39.0535 \\
\hline 82.5228 & 123.1949 & 40.672 & 82.554 & 123.4105 & 40.8565 & 83.8431 & 122.8362 & 38.9931 \\
\hline 82.5999 & 123.168 & 40.5681 & 82.5731 & 123.3984 & 40.8253 & 83.9785 & 122.8658 & 38.8873 \\
\hline 82.6649 & 123.1152 & 40.4503 & 82.5765 & 123.3966 & 40.8201 & 83.8634 & 122.9203 & 39.057 \\
\hline 82.5904 & 123.1923 & 40.6019 & 82.5748 & 123.3984 & 40.8236 & 83.885 & 122.9039 & 39.0189 \\
\hline 82.6002 & 123.1947 & 40.5945 & 82.6432 & 123.368 & 40.7248 & 83.6728 & 123.0017 & 39.3289 \\
\hline 82.6415 & 123.168 & 40.5265 & 82.5947 & 123.3507 & 40.756 & 83.6555 & 123.0113 & 39.3557 \\
\hline 82.4077 & 123.6071 & 41.1994 & 82.6927 & 123.3172 & 40.6245 & 83.5871 & 123.0502 & 39.4631 \\
\hline 82.6198 & 123.2052 & 40.5854 & 82.6553 & 123.3308 & 40.6755 & 83.743 & 122.9922 & 39.2492 \\
\hline 82.7047 & 123.1853 & 40.4806 & 82.6709 & 123.3343 & 40.6634 & 83.7753 & 123.0001 & 39.2247 \\
\hline 82.8337 & 123.1628 & 40.3291 & 82.5618 & 123.3473 & 40.7855 & 83.7369 & 122.9654 & 39.2284 \\
\hline 82.8684 & 123.1715 & 40.3031 & 82.5324 & 123.3888 & 40.8565 & 83.6971 & 123.013 & 39.3159 \\
\hline 82.8588 & 123.1533 & 40.2945 & 82.4362 & 123.4252 & 40.989 & 83.7118 & 123.0398 & 39.328 \\
\hline 82.8556 & 123.1604 & 40.3047 & 82.4388 & 123.4477 & 41.0089 & 83.5862 & 123.0753 & 39.4891 \\
\hline 82.7809 & 123.181 & 40.4001 & 82.4094 & 123.4798 & 41.0704 & 83.6876 & 123.0277 & 39.3402 \\
\hline 82.6874 & 123.213 & 40.5257 & 82.5244 & 123.3947 & 40.8703 & 83.53 & 123.0927 & 39.5627 \\
\hline 82.6752 & 123.2572 & 40.582 & 82.5453 & 123.3784 & 40.8331 & 83.5248 & 123.1394 & 39.6147 \\
\hline 82.5973 & 123.2494 & 40.6521 & 82.5479 & 123.3992 & 40.8513 & 83.5207 & 123.1331 & 39.6123 \\
\hline 82.5835 & 123.2858 & 40.7023 & 82.6761 & 123.3932 & 40.717 & 83.7421 & 123.1083 & 39.3661 \\
\hline 82.6605 & 123.2589 & 40.5984 & 82.6934 & 123.3854 & 40.6919 & 84.3838 & 122.968 & 38.5842 \\
\hline 82.6164 & 123.2607 & 40.6443 & 82.6709 & 123.3906 & 40.7196 & 83.6936 & 123.1126 & 39.419 \\
\hline 82.5852 & 123.2652 & 40.68 & 82.6068 & 123.4018 & 40.795 & 83.7794 & 123.1212 & 39.3419 \\
\hline 82.5653 & 123.2745 & 40.7093 & 82.58 & 123.3854 & 40.8054 & 83.801 & 123.1083 & 39.3073 \\
\hline 82.5679 & 123.2685 & 40.7006 & 82.6011 & 123.3912 & 40.7901 & 84.3067 & 123.0087 & 38.7019 \\
\hline 82.6138 & 123.2338 & 40.6201 & 82.6657 & 123.368 & 40.7023 & 84.1959 & 123.0615 & 38.8656 \\
\hline 82.7133 & 123.2026 & 40.4893 & 82.7125 & 123.3845 & 40.672 & 84.4184 & 123.0441 & 38.6257 \\
\hline
\end{tabular}




\begin{tabular}{|c|c|c|c|c|c|c|c|c|}
\hline 84.4895 & 123.045 & 38.5556 & 83.0467 & 123.6114 & 40.5646 & 82.7809 & 123.8478 & 41.0669 \\
\hline 84.4825 & 122.9567 & 38.4742 & 83.0054 & 123.6334 & 40.628 & 82.7584 & 123.8495 & 41.0911 \\
\hline 84.8514 & 122.8944 & 38.0429 & 83.039 & 123.6798 & 40.6408 & 82.7341 & 123.8556 & 41.1215 \\
\hline 84.5215 & 122.974 & 38.4525 & 82.9013 & 123.7655 & 40.8643 & 82.7737 & 123.8237 & 41.05 \\
\hline 84.5302 & 122.9368 & 38.4066 & 83.0433 & 123.6755 & 40.6322 & 82.7748 & 123.8218 & 41.047 \\
\hline 84.544 & 122.9229 & 38.3789 & 82.9853 & 123.7144 & 40.7292 & 82.7012 & 123.8365 & 41.1353 \\
\hline 84.4695 & 123.0078 & 38.5383 & 83.097 & 123.6564 & 40.5594 & 82.6692 & 123.8608 & 41.1916 \\
\hline 84.3911 & 123.0009 & 38.6099 & 83.0883 & 123.653 & 40.5646 & 82.6328 & 123.8928 & 41.26 \\
\hline 84.1976 & 123.1169 & 38.9193 & 83.0545 & 123.6564 & 40.6019 & 82.6034 & 123.8729 & 41.2695 \\
\hline 84.0244 & 123.142 & 39.1176 & 83.0124 & 123.659 & 40.6465 & 82.6103 & 123.892 & 41.2817 \\
\hline 83.8945 & 123.1862 & 39.2917 & 82.89 & 123.704 & 40.814 & 82.6787 & 123.8798 & 41.2011 \\
\hline 84.0643 & 123.1663 & 39.102 & 82.7471 & 123.7335 & 40.9864 & 82.6504 & 123.8457 & 41.1953 \\
\hline 84.0695 & 123.1438 & 39.0743 & 82.5895 & 123.756 & 41.1665 & 82.6155 & 123.8764 & 41.2609 \\
\hline 84.4392 & 123.0745 & 38.6353 & 82.5436 & 123.7707 & 41.2271 & 82.5938 & 123.8504 & 41.2565 \\
\hline 84.7951 & 122.9697 & 38.1746 & 82.6423 & 123.7655 & 41.1232 & 82.5921 & 123.8902 & 41.2981 \\
\hline 84.4836 & 123.0344 & 38.5509 & 82.6484 & 123.7508 & 41.1024 & 82.5627 & 123.8902 & 41.3276 \\
\hline 83.9456 & 123.1827 & 39.2371 & 82.6778 & 123.756 & 41.0782 & 82.5514 & 123.9058 & 41.3544 \\
\hline 83.9101 & 123.2018 & 39.2917 & 82.6169 & 123.7858 & 41.1689 & 82.5557 & 123.9179 & 41.3622 \\
\hline 84.0132 & 123.1801 & 39.167 & 82.6614 & 123.7768 & 41.1154 & 82.5609 & 123.9708 & 41.4098 \\
\hline 84.3266 & 123.116 & 38.7894 & 82.6449 & 123.8019 & 41.157 & 82.5447 & 123.9206 & 41.3759 \\
\hline 84.195 & 123.1074 & 38.9124 & 82.567 & 123.8209 & 41.254 & 82.4787 & 123.9318 & 41.4531 \\
\hline 83.9872 & 123.1793 & 39.1921 & 82.638 & 123.8253 & 41.1873 & 82.5055 & 123.9604 & 41.4549 \\
\hline 84.2063 & 123.1195 & 38.9132 & 82.7289 & 123.8071 & 41.0782 & 82.5029 & 123.9318 & 41.4289 \\
\hline 83.9196 & 123.1723 & 39.2527 & 82.6761 & 123.8209 & 41.1448 & 82.5566 & 123.9335 & 41.3769 \\
\hline 83.8881 & 123.2097 & 39.3216 & 82.6943 & 123.7984 & 41.1041 & 82.5194 & 123.9015 & 41.3821 \\
\hline 83.9344 & 123.2087 & 39.2743 & 82.735 & 123.8026 & 41.0676 & 82.5341 & 123.8868 & 41.3527 \\
\hline 84.1266 & 123.155 & 39.0284 & 82.8043 & 123.795 & 40.9907 & 82.5098 & 123.9101 & 41.4003 \\
\hline 84.1361 & 123.1767 & 39.0405 & 82.7783 & 123.7456 & 40.9673 & 82.5051 & 123.9365 & 41.4314 \\
\hline 84.1656 & 123.1619 & 38.9964 & 82.7229 & 123.7785 & 41.0556 & 82.522 & 123.9153 & 41.3934 \\
\hline 84.2366 & 123.1316 & 38.895 & 82.6908 & 123.769 & 41.0782 & 82.5272 & 123.9223 & 41.3951 \\
\hline 84.0582 & 123.1905 & 39.1323 & 82.6475 & 123.7863 & 41.1388 & 82.5964 & 123.8703 & 41.2739 \\
\hline 84.0547 & 123.1853 & 39.1306 & 82.7982 & 123.7499 & 40.9517 & 82.5843 & 123.8573 & 41.273 \\
\hline 83.9814 & 123.2423 & 39.2609 & 82.8424 & 123.7837 & 40.9413 & 82.5912 & 123.8513 & 41.26 \\
\hline 83.9924 & 123.2555 & 39.2631 & 82.7497 & 123.769 & 41.0193 & 82.5852 & 123.9205 & 41.3354 \\
\hline 83.9655 & 123.2884 & 39.3228 & 82.7341 & 123.7893 & 41.0553 & 82.5272 & 123.9353 & 41.4081 \\
\hline 83.9144 & 123.2858 & 39.3713 & 82.7151 & 123.7924 & 41.0773 & 82.5393 & 123.9231 & 41.3838 \\
\hline 83.9681 & 123.2624 & 39.2943 & 82.6545 & 123.8106 & 41.1561 & 82.4945 & 123.9294 & 41.4349 \\
\hline 83.8408 & 123.3499 & 39.509 & 82.6146 & 123.8565 & 41.2418 & 82.5055 & 123.9101 & 41.4046 \\
\hline 83.7958 & 123.3247 & 39.5289 & 82.6597 & 123.8625 & 41.2029 & 82.5254 & 123.9145 & 41.389 \\
\hline 83.7768 & 123.3438 & 39.567 & 82.7021 & 123.8461 & 41.144 & 82.4847 & 123.9361 & 41.4514 \\
\hline 83.7727 & 123.3445 & 39.5718 & 82.6345 & 123.8452 & 41.2107 & 82.3514 & 123.9543 & 41.6029 \\
\hline 83.3342 & 123.5014 & 40.1672 & 82.5921 & 123.8573 & 41.2652 & 82.3964 & 123.9249 & 41.5285 \\
\hline 83.0017 & 123.6729 & 40.6712 & 82.5746 & 123.8307 & 41.2561 & 82.3618 & 123.9205 & 41.5588 \\
\hline 82.9203 & 123.6538 & 40.7335 & 82.8865 & 123.7846 & 40.898 & 82.3462 & 123.9656 & 41.6194 \\
\hline 83.1619 & 123.543 & 40.3811 & 82.696 & 123.8668 & 41.1708 & 82.2496 & 123.9734 & 41.7239 \\
\hline 83.1576 & 123.5308 & 40.3733 & 82.6553 & 123.8963 & 41.241 & 82.2033 & 123.9777 & 41.7744 \\
\hline 83.142 & 123.5577 & 40.4157 & 82.7298 & 123.8599 & 41.1301 & 82.3297 & 123.9119 & 41.5822 \\
\hline
\end{tabular}




\begin{tabular}{|c|c|c|c|c|c|c|c|c|}
\hline 82.3661 & 123.9032 & 41.5371 & 82.4605 & 123.8279 & 41.3674 & 82.554 & 123.8279 & 41.2739 \\
\hline 82.367 & 123.9257 & 41.5588 & 82.4458 & 123.8703 & 41.4245 & 82.4998 & 123.8484 & 41.3486 \\
\hline 82.4094 & 123.9353 & 41.5259 & 82.4639 & 123.8764 & 41.4124 & 82.4215 & 123.8452 & 41.4237 \\
\hline 82.4345 & 123.9153 & 41.4808 & 82.4579 & 123.8911 & 41.4332 & 82.3739 & 123.9136 & 41.5397 \\
\hline 82.3903 & 123.8998 & 41.5094 & 82.4501 & 123.8807 & 41.4306 & 82.3185 & 123.9153 & 41.5969 \\
\hline 82.3192 & 123.9417 & 41.6226 & 82.457 & 123.8859 & 41.4289 & 82.2622 & 123.9621 & 41.6999 \\
\hline 82.3037 & 123.924 & 41.6203 & 82.4328 & 123.9162 & 41.4834 & 82.2968 & 123.9699 & 41.6731 \\
\hline 82.3652 & 123.9179 & 41.5527 & 82.4152 & 123.918 & 41.5028 & 82.3185 & 123.9517 & 41.6332 \\
\hline 82.4155 & 123.8521 & 41.4367 & 82.3618 & 123.9075 & 41.5458 & 82.3722 & 123.9699 & 41.5977 \\
\hline 82.4605 & 123.8487 & 41.3882 & 82.4042 & 123.879 & 41.4748 & 82.3967 & 123.918 & 41.5213 \\
\hline 82.4804 & 123.8443 & 41.3639 & 82.5713 & 123.8097 & 41.2384 & 82.3375 & 123.9335 & 41.596 \\
\hline 82.5644 & 123.8339 & 41.2695 & 82.5627 & 123.8357 & 41.273 & 82.3955 & 123.95 & 41.5544 \\
\hline 82.6207 & 123.8235 & 41.2029 & 82.5514 & 123.8149 & 41.2635 & 82.4276 & 123.9301 & 41.5025 \\
\hline 82.6495 & 123.8325 & 41.183 & 82.5523 & 123.7958 & 41.2436 & 82.3462 & 123.9534 & 41.6073 \\
\hline 82.5869 & 123.8175 & 41.2306 & 82.6406 & 123.7491 & 41.1085 & 82.3366 & 123.9413 & 41.6047 \\
\hline 82.5549 & 123.8253 & 41.2704 & 82.6257 & 123.7338 & 41.1081 & 82.2977 & 123.9543 & 41.6566 \\
\hline 82.5791 & 123.8547 & 41.2756 & 82.7099 & 123.717 & 41.0071 & 82.3297 & 123.9777 & 41.648 \\
\hline 82.638 & 123.8729 & 41.2349 & 82.6475 & 123.7274 & 41.0799 & 82.3174 & 124.0008 & 41.6833 \\
\hline 82.6579 & 123.8461 & 41.1881 & 82.6112 & 123.7543 & 41.1431 & 82.2604 & 124.0331 & 41.7727 \\
\hline 82.6744 & 123.8539 & 41.1795 & 82.6345 & 123.7499 & 41.1154 & 82.2405 & 124.0461 & 41.8056 \\
\hline 82.7315 & 123.801 & 41.0695 & 82.6172 & 123.7482 & 41.131 & 82.2336 & 124.0149 & 41.7813 \\
\hline 82.7182 & 123.8351 & 41.1169 & 82.5757 & 123.7473 & 41.1717 & 82.4215 & 123.9846 & 41.5631 \\
\hline 82.7142 & 123.7924 & 41.0782 & 82.5107 & 123.7759 & 41.2652 & 82.5237 & 123.9093 & 41.3856 \\
\hline 82.7463 & 123.7699 & 41.0236 & 82.52 & 123.8017 & 41.2816 & 82.5211 & 123.9223 & 41.4012 \\
\hline 82.7523 & 123.7846 & 41.0323 & 82.5003 & 123.8054 & 41.305 & 82.5272 & 123.937 & 41.4098 \\
\hline 82.683 & 123.8218 & 41.1388 & 82.4847 & 123.8209 & 41.3362 & 82.5015 & 123.9241 & 41.4226 \\
\hline 82.6475 & 123.8409 & 41.1933 & 82.4596 & 123.8504 & 41.3908 & 82.5488 & 123.937 & 41.3882 \\
\hline 82.6146 & 123.8642 & 41.2496 & 82.47 & 123.8088 & 41.3388 & 82.6371 & 123.9058 & 41.2687 \\
\hline 82.5514 & 123.9041 & 41.3527 & 82.4596 & 123.8002 & 41.3405 & 82.722 & 123.9275 & 41.2055 \\
\hline 82.6011 & 123.8889 & 41.2878 & 82.5358 & 123.8513 & 41.3154 & 82.7307 & 123.9266 & 41.1959 \\
\hline 82.6397 & 123.9093 & 41.2695 & 82.5462 & 123.892 & 41.3457 & 82.6735 & 123.8937 & 41.2202 \\
\hline 82.5462 & 123.8764 & 41.3302 & 82.6786 & 123.8607 & 41.1821 & 82.6718 & 123.885 & 41.2132 \\
\hline 82.5358 & 123.898 & 41.3622 & 82.7133 & 123.8279 & 41.1145 & 82.6744 & 123.8764 & 41.202 \\
\hline 82.5912 & 123.8798 & 41.2886 & 82.7099 & 123.8279 & 41.118 & 82.6539 & 123.8924 & 41.2385 \\
\hline 82.5757 & 123.8989 & 41.3232 & 82.6813 & 123.8348 & 41.1535 & 82.7437 & 123.8876 & 41.144 \\
\hline 82.5731 & 123.8677 & 41.2947 & 82.6752 & 123.8184 & 41.1431 & 82.6778 & 123.8972 & 41.2193 \\
\hline 82.5367 & 123.8132 & 41.2765 & 82.6545 & 123.7802 & 41.1258 & 82.6484 & 123.9119 & 41.2635 \\
\hline 82.5523 & 123.7976 & 41.2453 & 82.6034 & 123.7802 & 41.1769 & 82.6207 & 123.898 & 41.2773 \\
\hline 82.594 & 123.8105 & 41.2165 & 82.5272 & 123.8253 & 41.2981 & 82.5982 & 123.9171 & 41.3189 \\
\hline 82.6016 & 123.8114 & 41.2098 & 82.47 & 123.8599 & 41.3899 & 82.6051 & 123.9318 & 41.3267 \\
\hline 82.5765 & 123.8461 & 41.2695 & 82.4372 & 123.8959 & 41.4587 & 82.5687 & 123.9119 & 41.3431 \\
\hline 82.6285 & 123.8244 & 41.1959 & 82.4527 & 123.8972 & 41.4445 & 82.528 & 123.8894 & 41.3613 \\
\hline 82.5783 & 123.8478 & 41.2695 & 82.4674 & 123.866 & 41.3986 & 82.4918 & 123.9347 & 41.4429 \\
\hline 82.5289 & 123.8547 & 41.3258 & 82.4137 & 123.9049 & 41.4912 & 82.4995 & 123.911 & 41.4116 \\
\hline 82.528 & 123.8668 & 41.3388 & 82.3609 & 123.9396 & 41.5787 & 82.5064 & 123.9145 & 41.4081 \\
\hline 82.4899 & 123.8547 & 41.3648 & 82.4475 & 123.8963 & 41.4488 & 82.4787 & 123.8972 & 41.4185 \\
\hline 82.4813 & 123.8704 & 41.3891 & 82.5237 & 123.8729 & 41.3492 & 82.4544 & 123.9318 & 41.4774 \\
\hline
\end{tabular}




\begin{tabular}{|c|c|c|c|c|c|c|c|c|}
\hline 82.4752 & 123.9699 & 41.4947 & 84.0244 & 123.1325 & 39.1081 & 83.4901 & 123.0286 & 39.5385 \\
\hline 82.4813 & 123.9915 & 41.5103 & 83.9525 & 123.136 & 39.1834 & 83.6945 & 122.961 & 39.2666 \\
\hline 82.4821 & 123.9829 & 41.5008 & 84.0236 & 123.1169 & 39.0934 & 83.6867 & 122.9541 & 39.2674 \\
\hline 82.4962 & 123.9673 & 41.471 & 84.0755 & 123.0883 & 39.0128 & 83.7265 & 122.9732 & 39.2466 \\
\hline 82.5003 & 123.9456 & 41.4453 & 84.0175 & 123.1585 & 39.141 & 83.5707 & 123.0346 & 39.464 \\
\hline 82.4925 & 123.9569 & 41.4644 & 84.0871 & 123.1172 & 39.0301 & 83.5897 & 123.0234 & 39.4337 \\
\hline 82.5609 & 123.9231 & 41.3622 & 84.0149 & 123.1377 & 39.1228 & 83.6157 & 123.0243 & 39.4086 \\
\hline 82.4639 & 123.9422 & 41.4782 & 83.9915 & 123.1827 & 39.1912 & 83.6714 & 123.0415 & 39.3701 \\
\hline 82.5064 & 123.9327 & 41.4263 & 83.8668 & 123.0286 & 39.1618 & 83.7776 & 122.9766 & 39.199 \\
\hline 82.4847 & 123.8764 & 41.3916 & 83.7629 & 123.0554 & 39.2925 & 83.6685 & 123.0364 & 39.3679 \\
\hline 82.5254 & 123.9006 & 41.3752 & 83.7629 & 123.0381 & 39.2752 & 83.6702 & 123.0139 & 39.3436 \\
\hline 82.6116 & 123.8378 & 41.2262 & 83.6824 & 123.0381 & 39.3557 & 83.6538 & 123.0641 & 39.4103 \\
\hline 82.5315 & 123.8738 & 41.3423 & 83.749 & 123.0087 & 39.2596 & 83.5897 & 123.0095 & 39.4198 \\
\hline 82.4943 & 123.8781 & 41.3838 & 83.6793 & 123.0247 & 39.3454 & 83.2702 & 123.155 & 39.8849 \\
\hline 82.4484 & 123.924 & 41.4756 & 83.7508 & 122.9879 & 39.2371 & 83.1377 & 123.1801 & 40.0425 \\
\hline 82.3817 & 123.9136 & 41.5319 & 83.698 & 123.026 & 39.328 & 83.2441 & 123.2203 & 39.9762 \\
\hline 82.3531 & 123.9361 & 41.583 & 83.704 & 123.0217 & 39.3176 & 83.2624 & 123.2269 & 39.9645 \\
\hline 82.3427 & 123.9682 & 41.6255 & 83.5689 & 123.0693 & 39.5004 & 83.4945 & 123.116 & 39.6216 \\
\hline 82.4518 & 123.9179 & 41.4661 & 83.5152 & 123.1135 & 39.5982 & 84.0149 & 122.9784 & 38.9635 \\
\hline 82.4319 & 123.9039 & 41.4719 & 83.1905 & 123.2589 & 40.0684 & 83.9344 & 123.0494 & 39.115 \\
\hline 82.4778 & 123.9119 & 41.4341 & 82.9939 & 123.3577 & 40.3637 & 83.7655 & 123.0546 & 39.2891 \\
\hline 82.451 & 123.943 & 41.4921 & 83.2846 & 123.2176 & 39.933 & 83.5135 & 123.1221 & 39.6086 \\
\hline 83.2113 & 123.6737 & 40.4625 & 83.2676 & 123.1992 & 39.9316 & 83.6893 & 123.0667 & 39.3774 \\
\hline 83.6711 & 123.4209 & 39.7498 & 83.4165 & 123.1412 & 39.7246 & 83.6811 & 123.0608 & 39.3798 \\
\hline 84.0348 & 123.2295 & 39.1947 & 83.2355 & 123.2104 & 39.9749 & 83.9058 & 123.0485 & 39.1427 \\
\hline 83.8919 & 123.2728 & 39.3809 & 83.2095 & 123.2122 & 40.0026 & 84.0132 & 123.0061 & 38.9929 \\
\hline 83.8478 & 123.2763 & 39.4285 & 83.2399 & 123.1983 & 39.9585 & 84.0365 & 123.032 & 38.9955 \\
\hline 83.7815 & 123.3189 & 39.5375 & 83.0848 & 123.226 & 40.1412 & 84.2115 & 123.0069 & 38.7955 \\
\hline 83.788 & 123.3118 & 39.5237 & 83.0554 & 123.2485 & 40.1931 & 84.1578 & 123.0191 & 38.8613 \\
\hline 83.8841 & 123.2537 & 39.3696 & 83.112 & 123.2106 & 40.0986 & 84.3076 & 122.961 & 38.6534 \\
\hline 83.7941 & 123.2771 & 39.483 & 83.1022 & 123.2364 & 40.1343 & 84.2314 & 123.0061 & 38.7747 \\
\hline 83.7629 & 123.3048 & 39.5419 & 83.4901 & 123.0927 & 39.6025 & 84.2404 & 122.9956 & 38.7552 \\
\hline 83.7768 & 123.2901 & 39.5134 & 83.4382 & 123.11 & 39.6718 & 84.0582 & 123.052 & 38.9938 \\
\hline 83.8573 & 123.2321 & 39.3748 & 83.5109 & 123.0788 & 39.5679 & 84.0495 & 123.123 & 39.0734 \\
\hline 83.7681 & 123.2927 & 39.5246 & 83.6001 & 123.045 & 39.4449 & 83.7361 & 123.2096 & 39.4735 \\
\hline 83.689 & 123.3322 & 39.6432 & 83.4156 & 123.1057 & 39.69 & 83.8071 & 123.1827 & 39.3757 \\
\hline 83.6425 & 123.3325 & 39.69 & 83.3351 & 123.1507 & 39.8156 & 83.7161 & 123.1949 & 39.4787 \\
\hline 83.6902 & 123.2936 & 39.6034 & 83.6705 & 123.0106 & 39.3401 & 83.6702 & 123.2061 & 39.5359 \\
\hline 83.8686 & 123.2494 & 39.3809 & 83.4503 & 123.0866 & 39.6363 & 83.7395 & 123.1663 & 39.4268 \\
\hline 83.8763 & 123.2511 & 39.3748 & 83.3446 & 123.0797 & 39.735 & 84.5258 & 122.9023 & 38.3764 \\
\hline 83.7846 & 123.2503 & 39.4657 & 83.4633 & 123.0433 & 39.58 & 84.3968 & 123.0043 & 38.6075 \\
\hline 83.8971 & 123.207 & 39.3098 & 83.5629 & 122.9758 & 39.4129 & 84.2504 & 123.0416 & 38.7911 \\
\hline 83.9422 & 123.149 & 39.2068 & 83.6373 & 122.9377 & 39.3003 & 84.0244 & 123.1169 & 39.0925 \\
\hline 83.9361 & 123.1663 & 39.2302 & 83.5655 & 123.0052 & 39.4397 & 83.9049 & 123.1585 & 39.2536 \\
\hline 83.8669 & 123.1577 & 39.2908 & 83.7802 & 122.9463 & 39.1661 & 84.0097 & 123.1065 & 39.0968 \\
\hline 83.9881 & 123.1412 & 39.1531 & 83.4702 & 122.9974 & 39.5272 & 83.9093 & 123.155 & 39.2458 \\
\hline 83.9647 & 123.1498 & 39.1851 & 83.3851 & 123.067 & 39.6819 & 83.8686 & 123.1481 & 39.2795 \\
\hline
\end{tabular}




\begin{tabular}{|c|c|c|c|c|c|c|c|c|}
\hline 83.8652 & 123.1912 & 39.326 & 84.2721 & 122.9619 & 38.6898 & 82.7454 & 123.84 & 41.0946 \\
\hline 83.8019 & 123.2252 & 39.4233 & 84.4522 & 122.9022 & 38.4499 & 82.7402 & 123.7958 & 41.0556 \\
\hline 83.8556 & 123.2087 & 39.3531 & 84.4078 & 122.9313 & 38.5235 & 82.7298 & 123.8036 & 41.0738 \\
\hline 84.0227 & 123.1983 & 39.1756 & 84.4626 & 122.9073 & 38.4447 & 82.6778 & 123.8209 & 41.1431 \\
\hline 84.26 & 123.1316 & 38.8717 & 84.2193 & 123.0572 & 38.8379 & 82.6645 & 123.844 & 41.1795 \\
\hline 83.8157 & 123.2373 & 39.4216 & 84.3474 & 123.0494 & 38.7019 & 82.7185 & 123.7785 & 41.06 \\
\hline 83.6157 & 123.3022 & 39.6865 & 84.2582 & 123.058 & 38.7998 & 82.7012 & 123.7828 & 41.0816 \\
\hline 83.6547 & 123.3092 & 39.6545 & 84.26 & 123.0277 & 38.7678 & 82.6882 & 123.801 & 41.1128 \\
\hline 83.7923 & 123.2918 & 39.4995 & 84.2946 & 123.0398 & 38.7452 & 82.735 & 123.827 & 41.092 \\
\hline 83.881 & 123.267 & 39.386 & 84.3197 & 123.0217 & 38.7019 & 82.6848 & 123.7872 & 41.1024 \\
\hline 83.7984 & 123.2641 & 39.4657 & 84.3353 & 123.0407 & 38.7054 & 82.6787 & 123.808 & 41.1293 \\
\hline 83.8495 & 123.2581 & 39.4086 & 84.3083 & 122.9956 & 38.6874 & 82.6597 & 123.8175 & 41.1578 \\
\hline 83.6642 & 123.304 & 39.6398 & 84.3258 & 122.9983 & 38.6725 & 82.6285 & 123.827 & 41.1985 \\
\hline 83.6503 & 123.3455 & 39.6952 & 84.2877 & 123.0381 & 38.7504 & 82.7147 & 123.7946 & 41.0799 \\
\hline 83.691 & 123.3092 & 39.6181 & 84.3388 & 123.0234 & 38.6846 & 82.8432 & 123.7837 & 40.9405 \\
\hline 83.7698 & 123.2633 & 39.4934 & 84.3665 & 122.9974 & 38.6309 & 82.8987 & 123.7491 & 40.8504 \\
\hline 83.6694 & 123.3031 & 39.6337 & 84.4306 & 122.9602 & 38.5296 & 82.8822 & 123.7603 & 40.8781 \\
\hline 83.6115 & 123.363 & 39.7515 & 84.4332 & 122.9325 & 38.4993 & 82.832 & 123.7898 & 40.9578 \\
\hline 83.7854 & 123.1671 & 39.3817 & 84.4375 & 122.9316 & 38.4941 & 82.8554 & 123.8158 & 40.9604 \\
\hline 83.7984 & 123.0684 & 39.27 & 84.4703 & 122.9093 & 38.439 & 82.903 & 123.7586 & 40.8556 \\
\hline 83.9829 & 122.9688 & 38.986 & 84.3015 & 123.0043 & 38.7028 & 82.9307 & 123.7872 & 40.8565 \\
\hline 84.1569 & 122.9203 & 38.7634 & 84.2504 & 123.0381 & 38.7877 & 82.8169 & 123.7541 & 40.9372 \\
\hline 84.247 & 122.8848 & 38.6379 & 84.2297 & 123.0424 & 38.8128 & 82.7757 & 123.7915 & 41.0158 \\
\hline 84.3076 & 122.8632 & 38.5556 & 84.318 & 123.0416 & 38.7236 & 82.748 & 123.814 & 41.066 \\
\hline 84.1673 & 122.9342 & 38.7669 & 84.3907 & 122.9948 & 38.6041 & 82.7489 & 123.7984 & 41.0496 \\
\hline 84.0281 & 122.9736 & 38.9455 & 84.4219 & 122.9654 & 38.5435 & 82.7116 & 123.7915 & 41.0799 \\
\hline 84.0314 & 122.9749 & 38.9435 & 84.4323 & 122.9879 & 38.5556 & 82.7099 & 123.7612 & 41.0513 \\
\hline 84.0374 & 122.968 & 38.9306 & 84.51 & 122.9613 & 38.4513 & 82.612 & 123.8149 & 41.2029 \\
\hline 84.0019 & 122.9749 & 38.973 & 84.5189 & 122.9671 & 38.4482 & 82.6475 & 123.8469 & 41.1994 \\
\hline 83.95 & 123 & 39.0501 & 84.486 & 122.9974 & 38.5114 & 82.683 & 123.8801 & 41.1971 \\
\hline 83.9136 & 122.9784 & 39.0648 & 84.4583 & 123.0312 & 38.5729 & 82.7272 & 123.8703 & 41.1431 \\
\hline 83.8668 & 123.0468 & 39.18 & 83.9326 & 123.2511 & 39.3185 & 82.8467 & 123.879 & 41.0323 \\
\hline 83.9093 & 123.0684 & 39.1592 & 83.0978 & 123.5456 & 40.4477 & 82.7618 & 123.9023 & 41.1405 \\
\hline 83.8775 & 123.0476 & 39.1701 & 82.9593 & 123.5326 & 40.5733 & 82.7047 & 123.9283 & 41.2236 \\
\hline 83.9759 & 123.0069 & 39.031 & 82.8865 & 123.7205 & 40.834 & 82.6934 & 123.8746 & 41.1812 \\
\hline 84.0045 & 123.0087 & 39.0042 & 82.8803 & 123.7567 & 40.8764 & 82.6345 & 123.827 & 41.1925 \\
\hline 84.0842 & 122.9818 & 38.8976 & 82.7982 & 123.7716 & 40.9734 & 82.6623 & 123.801 & 41.1388 \\
\hline 83.8902 & 123.0814 & 39.1912 & 82.7229 & 123.7231 & 41.0002 & 82.661 & 123.755 & 41.094 \\
\hline 84.0132 & 123.0208 & 39.0076 & 82.7748 & 123.7603 & 40.9855 & 82.6778 & 123.7612 & 41.0834 \\
\hline 84.0201 & 123.0312 & 39.0111 & 82.8467 & 123.7993 & 40.9526 & 82.677 & 123.7854 & 41.1085 \\
\hline 84.0365 & 123.0502 & 39.0137 & 82.7644 & 123.8071 & 41.0427 & 82.5159 & 123.8062 & 41.2903 \\
\hline 84.1277 & 122.9877 & 38.8601 & 82.6874 & 123.8313 & 41.144 & 82.4639 & 123.8244 & 41.3605 \\
\hline 84.3509 & 122.9359 & 38.585 & 82.6319 & 123.827 & 41.1951 & 82.4406 & 123.8539 & 41.4133 \\
\hline 84.163 & 122.9939 & 38.831 & 82.6293 & 123.8334 & 41.2041 & 82.4336 & 123.8582 & 41.4245 \\
\hline 84.4375 & 122.8909 & 38.4534 & 82.6415 & 123.8322 & 41.1907 & 82.4709 & 123.8573 & 41.3864 \\
\hline 84.3821 & 122.8961 & 38.514 & 82.6804 & 123.8417 & 41.1613 & 82.5429 & 123.8378 & 41.2949 \\
\hline 84.4037 & 122.8814 & 38.4777 & 82.7047 & 123.8963 & 41.1916 & 82.5419 & 123.8833 & 41.3414 \\
\hline
\end{tabular}




\begin{tabular}{|c|c|c|c|c|c|c|c|c|}
\hline 82.5791 & 123.9162 & 41.3371 & 82.5579 & 123.9215 & 41.3636 & 82.7437 & 124.4851 & 41.7415 \\
\hline 82.5367 & 123.9379 & 41.4012 & 82.5358 & 123.9197 & 41.3838 & 82.7644 & 124.4315 & 41.667 \\
\hline 82.5445 & 123.9075 & 41.3631 & 82.5185 & 123.911 & 41.3925 & 82.6936 & 124.4976 & 41.804 \\
\hline 82.5176 & 123.8798 & 41.3622 & 82.5826 & 123.9309 & 41.3483 & 82.7696 & 124.3656 & 41.596 \\
\hline 82.599 & 123.8625 & 41.2635 & 82.4562 & 123.9396 & 41.4834 & 82.8467 & 124.3362 & 41.4895 \\
\hline 82.6475 & 123.8651 & 41.2176 & 82.4276 & 123.9335 & 41.5059 & 82.8813 & 124.3163 & 41.4349 \\
\hline 82.6504 & 123.8739 & 41.2235 & 82.4769 & 123.9205 & 41.4436 & 82.8796 & 124.3544 & 41.4748 \\
\hline 82.5973 & 123.9015 & 41.3042 & 82.4328 & 123.9231 & 41.4904 & 82.8891 & 124.3379 & 41.4488 \\
\hline 82.5644 & 123.8807 & 41.3163 & 82.4628 & 123.9074 & 41.4446 & 82.8692 & 124.3492 & 41.48 \\
\hline 82.5324 & 123.8989 & 41.3665 & 82.5341 & 123.937 & 41.4029 & 82.8363 & 124.3362 & 41.4999 \\
\hline 82.6406 & 123.8781 & 41.2375 & 82.5324 & 123.8963 & 41.3639 & 82.8521 & 124.3804 & 41.5283 \\
\hline 82.6986 & 123.8383 & 41.1396 & 82.528 & 123.898 & 41.37 & 82.9143 & 124.415 & 41.5008 \\
\hline 82.7636 & 123.8513 & 41.0877 & 82.5358 & 123.9734 & 41.4375 & 82.9013 & 124.376 & 41.4748 \\
\hline 82.7012 & 123.879 & 41.1777 & 82.5116 & 124.1266 & 41.6151 & 82.8406 & 124.389 & 41.5484 \\
\hline 82.6848 & 123.8556 & 41.1708 & 82.8138 & 123.956 & 41.1422 & 82.8363 & 124.4133 & 41.577 \\
\hline 82.625 & 123.8634 & 41.2384 & 83.2944 & 124.2288 & 40.9344 & 82.8562 & 124.4167 & 41.5605 \\
\hline 82.5523 & 123.9396 & 41.3873 & 82.5438 & 124.3029 & 41.7591 & 82.9073 & 124.3812 & 41.4739 \\
\hline 82.6406 & 123.8894 & 41.2488 & 82.4717 & 124.2816 & 41.8099 & 82.8943 & 124.4003 & 41.506 \\
\hline 82.5246 & 123.8928 & 41.3683 & 82.4674 & 124.2938 & 41.8264 & 82.8821 & 124.413 & 41.5309 \\
\hline 82.5202 & 123.892 & 41.3717 & 82.522 & 124.2418 & 41.7198 & 83.0511 & 124.3353 & 41.2843 \\
\hline 82.541 & 123.9266 & 41.3856 & 82.528 & 124.2141 & 41.6861 & 83.0346 & 124.3111 & 41.2765 \\
\hline 82.5419 & 123.9344 & 41.3925 & 82.4856 & 124.2505 & 41.7649 & 83.0433 & 124.3284 & 41.2851 \\
\hline 82.5003 & 123.9223 & 41.4219 & 82.4743 & 124.2782 & 41.8038 & 83.0623 & 124.2202 & 41.1578 \\
\hline 82.6143 & 123.8625 & 41.2482 & 82.4553 & 124.2773 & 41.822 & 83.0415 & 124.1942 & 41.1526 \\
\hline 82.5376 & 123.8573 & 41.3198 & 82.4423 & 124.2998 & 41.8575 & 83.0779 & 124.1873 & 41.1093 \\
\hline 82.47 & 123.8712 & 41.4012 & 82.3817 & 124.3126 & 41.9309 & 83.0814 & 124.1777 & 41.0963 \\
\hline 82.5428 & 123.8296 & 41.2869 & 82.373 & 124.3068 & 41.9337 & 83.0277 & 124.1691 & 41.1414 \\
\hline 82.5046 & 123.8972 & 41.3925 & 82.3072 & 124.3544 & 42.0472 & 83.0345 & 124.2104 & 41.1759 \\
\hline 82.4769 & 123.9041 & 41.4271 & 82.3289 & 124.3553 & 42.0264 & 83.0069 & 124.2028 & 41.1959 \\
\hline 82.4423 & 123.9292 & 41.4869 & 82.3263 & 124.344 & 42.0177 & 83.0242 & 124.2202 & 41.1959 \\
\hline 82.4241 & 123.9231 & 41.499 & 82.3254 & 124.3708 & 42.0454 & 82.9983 & 124.2496 & 41.2514 \\
\hline 82.38 & 123.8995 & 41.5195 & 82.2934 & 124.3682 & 42.0749 & 83.0138 & 124.2453 & 41.2314 \\
\hline 82.3765 & 123.9335 & 41.557 & 82.3756 & 124.3466 & 41.971 & 83.0822 & 124.279 & 41.1968 \\
\hline 82.4224 & 123.9353 & 41.5129 & 82.3579 & 124.317 & 41.9591 & 83.0225 & 124.3284 & 41.3059 \\
\hline 82.5523 & 123.9067 & 41.3544 & 82.3635 & 124.3215 & 41.958 & 82.9688 & 124.3674 & 41.3986 \\
\hline 82.5739 & 123.8937 & 41.3198 & 82.4224 & 124.3241 & 41.9017 & 82.9411 & 124.3575 & 41.4164 \\
\hline 82.6484 & 123.8513 & 41.2029 & 82.5306 & 124.2868 & 41.7562 & 82.8761 & 124.428 & 41.5518 \\
\hline 82.6025 & 123.8539 & 41.2514 & 82.5739 & 124.2903 & 41.7164 & 82.942 & 124.383 & 41.441 \\
\hline 82.5549 & 123.8755 & 41.3206 & 82.6371 & 124.3042 & 41.667 & 82.9108 & 124.4046 & 41.4938 \\
\hline 82.5879 & 123.8739 & 41.2861 & 82.7107 & 124.273 & 41.5622 & 83.097 & 124.3449 & 41.2479 \\
\hline 82.5462 & 123.8634 & 41.3172 & 82.7004 & 124.273 & 41.5726 & 83.0467 & 124.3172 & 41.2704 \\
\hline 82.4995 & 123.8946 & 41.3951 & 82.6988 & 124.3135 & 41.6146 & 83.0338 & 124.3197 & 41.286 \\
\hline 82.502 & 123.8876 & 41.3856 & 82.6986 & 124.3232 & 41.6246 & 83.0355 & 124.3535 & 41.318 \\
\hline 82.4882 & 123.911 & 41.4228 & 82.6623 & 124.3466 & 41.6843 & 83.0873 & 124.3417 & 41.2543 \\
\hline 82.4527 & 123.9361 & 41.4834 & 82.6926 & 124.3232 & 41.6306 & 83.0883 & 124.305 & 41.2167 \\
\hline 82.4232 & 123.9422 & 41.5189 & 82.6865 & 124.3449 & 41.6584 & 83.1307 & 124.3284 & 41.1977 \\
\hline 82.5211 & 123.9517 & 41.4306 & 82.7636 & 124.3743 & 41.6107 & 83.1238 & 124.3275 & 41.2037 \\
\hline
\end{tabular}




\begin{tabular}{|c|c|c|c|c|c|c|c|c|}
\hline 83.1714 & 124.3197 & 41.1483 & 83.1896 & 124.2037 & 41.0141 & 82.8777 & 124.1743 & 41.2966 \\
\hline 83.2 & 124.3163 & 41.1163 & 83.1723 & 124.1743 & 41.002 & 82.8259 & 124.2228 & 41.3968 \\
\hline 83.1333 & 124.3249 & 41.1916 & 83.1455 & 124.1864 & 41.0409 & 82.7792 & 124.2626 & 41.4834 \\
\hline 83.1619 & 124.2842 & 41.1223 & 83.1264 & 124.1968 & 41.0704 & 82.7644 & 124.3362 & 41.5718 \\
\hline 83.1613 & 124.2677 & 41.1063 & 83.1602 & 124.2002 & 41.0401 & 82.8112 & 124.3414 & 41.5302 \\
\hline 83.1437 & 124.2894 & 41.1457 & 83.1013 & 124.2531 & 41.1518 & 82.8112 & 124.3146 & 41.5034 \\
\hline 83.1697 & 124.2955 & 41.1258 & 83.1067 & 124.2263 & 41.1196 & 82.9324 & 124.2938 & 41.3613 \\
\hline 83.1654 & 124.2383 & 41.073 & 83.0355 & 124.2479 & 41.2124 & 83.0381 & 124.2487 & 41.2107 \\
\hline 83.1325 & 124.2427 & 41.1102 & 83.0147 & 124.2617 & 41.247 & 83.0838 & 124.2236 & 41.1398 \\
\hline 83.1714 & 124.247 & 41.0756 & 83.058 & 124.2756 & 41.2176 & 83.2347 & 124.1466 & 40.9119 \\
\hline 83.1688 & 124.2461 & 41.0773 & 83.0078 & 124.299 & 41.2912 & 83.1957 & 124.1561 & 40.9604 \\
\hline 83.1992 & 124.2764 & 41.0773 & 83.0762 & 124.2912 & 41.215 & 83.1636 & 124.1466 & 40.9829 \\
\hline 83.1745 & 124.2501 & 41.0755 & 83.1082 & 124.2513 & 41.1431 & 83.1671 & 124.1223 & 40.9552 \\
\hline 83.2052 & 124.2548 & 41.0496 & 83.1585 & 124.2063 & 41.0479 & 83.2459 & 124.1691 & 40.9232 \\
\hline 83.2485 & 124.2383 & 40.9898 & 83.1305 & 124.1857 & 41.0553 & 83.2511 & 124.1301 & 40.879 \\
\hline 83.2338 & 124.2617 & 41.0279 & 83.0883 & 124.1795 & 41.0911 & 83.271 & 124.1171 & 40.8461 \\
\hline 83.194 & 124.2401 & 41.0461 & 83.0667 & 124.1665 & 41.0998 & 83.2882 & 124.1126 & 40.8245 \\
\hline 83.1533 & 124.2635 & 41.1102 & 83.0329 & 124.1864 & 41.1535 & 83.3741 & 124.0781 & 40.7041 \\
\hline 83.1446 & 124.2591 & 41.1145 & 83.0303 & 124.1907 & 41.1604 & 83.4009 & 124.0755 & 40.6746 \\
\hline 83.1385 & 124.2661 & 41.1275 & 83.0242 & 124.1873 & 41.163 & 83.3715 & 124.0764 & 40.7049 \\
\hline 83.1622 & 124.2553 & 41.0931 & 83.0034 & 124.1682 & 41.1648 & 83.2901 & 124.0911 & 40.801 \\
\hline 83.2199 & 124.208 & 40.9881 & 83.0848 & 124.1474 & 41.0626 & 83.3291 & 124.0652 & 40.7361 \\
\hline 83.2719 & 124.2158 & 40.9439 & 83.1816 & 124.095 & 40.9134 & 83.3672 & 124.092 & 40.7248 \\
\hline 83.2476 & 124.2236 & 40.976 & 83.226 & 124.0582 & 40.8322 & 83.3723 & 124.0712 & 40.6989 \\
\hline 83.2754 & 124.2046 & 40.9292 & 83.2199 & 124.0652 & 40.8452 & 83.2838 & 124.1232 & 40.8394 \\
\hline 83.2953 & 124.2228 & 40.9275 & 83.1992 & 124.0859 & 40.8868 & 83.4174 & 124.1171 & 40.6997 \\
\hline 83.3161 & 124.2392 & 40.9232 & 83.1472 & 124.118 & 40.9708 & 83.4849 & 124.079 & 40.5941 \\
\hline 83.2702 & 124.2505 & 40.9803 & 83.0667 & 124.1275 & 41.0608 & 83.5144 & 124.0721 & 40.5577 \\
\hline 83.1895 & 124.2271 & 41.0376 & 83.0545 & 124.1431 & 41.0886 & 83.517 & 124.0894 & 40.5724 \\
\hline 83.1992 & 124.2487 & 41.0496 & 82.9749 & 124.1639 & 41.189 & 83.4615 & 124.0816 & 40.6201 \\
\hline 83.1152 & 124.2115 & 41.0963 & 83.0112 & 124.1604 & 41.1492 & 83.4875 & 124.0617 & 40.5742 \\
\hline 83.0978 & 124.1976 & 41.0998 & 83.0177 & 124.1576 & 41.1398 & 83.5222 & 124.0461 & 40.5239 \\
\hline 82.9783 & 124.2669 & 41.2886 & 83.0069 & 124.1691 & 41.1622 & 83.4599 & 124.0545 & 40.5945 \\
\hline 82.9879 & 124.2739 & 41.286 & 83.0467 & 124.1543 & 41.1076 & 82.9091 & 122.0041 & 39.0951 \\
\hline 83.0476 & 124.2669 & 41.2193 & 83.1585 & 124.0972 & 40.9387 & 82.9164 & 122.0302 & 39.1138 \\
\hline 83.1567 & 124.2366 & 41.0799 & 83.1714 & 124.0331 & 40.8617 & 82.9428 & 122.0362 & 39.0934 \\
\hline 83.1914 & 124.2297 & 41.0383 & 83.1645 & 124.0703 & 40.9058 & 82.929 & 122.0855 & 39.1566 \\
\hline 83.2459 & 124.2069 & 40.961 & 83.174 & 124.053 & 40.879 & 82.9255 & 122.1479 & 39.2224 \\
\hline 83.3005 & 124.1959 & 40.8954 & 83.1706 & 124.0548 & 40.8842 & 82.9593 & 122.1643 & 39.2051 \\
\hline 83.3169 & 124.1777 & 40.8608 & 83.1648 & 124.0853 & 40.9205 & 83.0034 & 122.1453 & 39.1419 \\
\hline 83.3109 & 124.1881 & 40.8773 & 83.1247 & 124.1145 & 40.9898 & 82.9705 & 122.1141 & 39.1436 \\
\hline 83.3022 & 124.1821 & 40.8799 & 83.0736 & 124.1396 & 41.066 & 83.0234 & 122.2068 & 39.1834 \\
\hline 83.2953 & 124.202 & 40.9067 & 83.0199 & 124.1457 & 41.1258 & 83.0043 & 122.2042 & 39.1999 \\
\hline 83.2442 & 124.1795 & 40.9353 & 82.9255 & 124.2141 & 41.2886 & 83.0265 & 122.2971 & 39.2706 \\
\hline 83.2139 & 124.1847 & 40.9708 & 82.8926 & 124.1639 & 41.2713 & 83.0156 & 122.309 & 39.2934 \\
\hline 83.164 & 124.2078 & 41.0438 & 82.922 & 124.1976 & 41.2756 & 82.9671 & 122.3012 & 39.3341 \\
\hline 83.1662 & 124.2046 & 41.0383 & 82.9151 & 124.1708 & 41.2557 & 82.9662 & 122.3531 & 39.3869 \\
\hline
\end{tabular}




\begin{tabular}{|c|c|c|c|c|c|c|c|c|}
\hline 82.9411 & 122.3653 & 39.4242 & 82.8077 & 122.0821 & 39.2743 & 82.8142 & 122.2337 & 39.4194 \\
\hline 82.9272 & 122.3176 & 39.3904 & 82.7514 & 122.1453 & 39.3938 & 82.7688 & 122.2172 & 39.4484 \\
\hline 82.9143 & 122.4068 & 39.4926 & 82.7722 & 122.128 & 39.3557 & 82.7844 & 122.3124 & 39.5281 \\
\hline 82.9065 & 122.4596 & 39.5532 & 82.8121 & 122.1358 & 39.3237 & 82.7965 & 122.2942 & 39.4978 \\
\hline 82.8865 & 122.4962 & 39.6097 & 82.7956 & 122.2016 & 39.406 & 82.7532 & 122.425 & 39.6718 \\
\hline 82.9515 & 122.4354 & 39.4839 & 82.7757 & 122.2068 & 39.4311 & 82.7982 & 122.3427 & 39.5445 \\
\hline 82.942 & 122.4285 & 39.4865 & 82.7922 & 122.2715 & 39.4793 & 82.8207 & 122.2648 & 39.4441 \\
\hline 82.9333 & 122.4501 & 39.5168 & 82.7644 & 122.1929 & 39.4285 & 82.806 & 122.2258 & 39.4198 \\
\hline 82.9082 & 122.4874 & 39.5792 & 82.767 & 122.2925 & 39.5255 & 82.831 & 122.2557 & 39.4247 \\
\hline 82.8969 & 122.4917 & 39.5948 & 82.7921 & 122.3038 & 39.5116 & 82.8207 & 122.3081 & 39.4874 \\
\hline 82.9567 & 122.5107 & 39.5541 & 82.8181 & 122.3523 & 39.5341 & 82.8103 & 122.373 & 39.5627 \\
\hline 82.9636 & 122.4865 & 39.5229 & 82.7965 & 122.3419 & 39.5454 & 82.7852 & 122.2951 & 39.5099 \\
\hline 83.008 & 122.5015 & 39.4934 & 82.8424 & 122.3384 & 39.496 & 82.7618 & 122.309 & 39.5471 \\
\hline 82.974 & 122.5298 & 39.5558 & 82.787 & 122.3046 & 39.5177 & 82.8207 & 122.2622 & 39.4415 \\
\hline 82.9454 & 122.5012 & 39.5558 & 82.8142 & 122.2724 & 39.4582 & 82.7956 & 122.212 & 39.4164 \\
\hline 82.961 & 122.4917 & 39.5307 & 82.845 & 122.0977 & 39.2527 & 82.7844 & 122.2345 & 39.4501 \\
\hline 82.9428 & 122.5021 & 39.5592 & 82.8606 & 122.147 & 39.2865 & 82.7341 & 122.2742 & 39.5401 \\
\hline 82.8796 & 122.5436 & 39.664 & 82.8536 & 122.1159 & 39.2622 & 82.7237 & 122.3038 & 39.58 \\
\hline 82.8761 & 122.5003 & 39.6242 & 82.9316 & 122.1358 & 39.2042 & 82.7151 & 122.2951 & 39.58 \\
\hline 82.8354 & 122.4475 & 39.6121 & 82.9307 & 122.0726 & 39.1419 & 82.7116 & 122.2258 & 39.5142 \\
\hline 82.9076 & 122.4997 & 39.5921 & 82.8831 & 122.1618 & 39.2787 & 82.6969 & 122.2354 & 39.5385 \\
\hline 82.9506 & 122.4588 & 39.5082 & 82.845 & 122.2042 & 39.3592 & 82.7376 & 122.2457 & 39.5082 \\
\hline 83 & 122.4544 & 39.4545 & 82.8257 & 122.1544 & 39.3287 & 82.7757 & 122.3107 & 39.535 \\
\hline 82.9619 & 122.4553 & 39.4934 & 82.7818 & 122.1288 & 39.3471 & 82.8034 & 122.2839 & 39.4804 \\
\hline 82.9801 & 122.464 & 39.4839 & 82.8017 & 122.1392 & 39.3376 & 82.8283 & 122.1764 & 39.3481 \\
\hline 82.9627 & 122.5168 & 39.5541 & 82.7999 & 122.2302 & 39.4302 & 82.7913 & 122.2198 & 39.4285 \\
\hline 82.9705 & 122.4813 & 39.5108 & 82.8043 & 122.1436 & 39.3393 & 82.7558 & 122.1938 & 39.438 \\
\hline 82.9558 & 122.4181 & 39.4623 & 82.8337 & 122.1947 & 39.3609 & 82.7489 & 122.1999 & 39.451 \\
\hline 82.9261 & 122.4662 & 39.5401 & 82.8112 & 122.1643 & 39.3531 & 82.7887 & 122.1124 & 39.3237 \\
\hline 82.9428 & 122.535 & 39.5922 & 82.8346 & 122.1791 & 39.3445 & 82.8329 & 122.154 & 39.3211 \\
\hline 82.9766 & 122.4475 & 39.4709 & 82.8327 & 122.1729 & 39.3401 & 82.8528 & 122.0959 & 39.2432 \\
\hline 82.9896 & 122.4874 & 39.4978 & 82.8138 & 122.225 & 39.4112 & 82.8363 & 122.147 & 39.3107 \\
\hline 82.9688 & 122.5402 & 39.5714 & 82.864 & 122.186 & 39.322 & 82.809 & 122.083 & 39.2741 \\
\hline 83.0164 & 122.5263 & 39.5099 & 82.8173 & 122.2553 & 39.438 & 82.7947 & 122.1427 & 39.348 \\
\hline 82.9454 & 122.4441 & 39.4986 & 82.7887 & 122.2648 & 39.4761 & 82.8043 & 122.1306 & 39.3263 \\
\hline 82.9653 & 122.5592 & 39.5939 & 82.8121 & 122.1782 & 39.3661 & 82.8008 & 122.0985 & 39.2977 \\
\hline 82.9578 & 122.6142 & 39.6564 & 82.8398 & 122.2102 & 39.3705 & 82.8225 & 122.1098 & 39.2873 \\
\hline 82.9168 & 122.4596 & 39.5428 & 82.8259 & 122.2293 & 39.4034 & 82.8121 & 122.0829 & 39.2709 \\
\hline 82.9264 & 122.4778 & 39.5515 & 82.8609 & 122.2178 & 39.3569 & 82.7861 & 122.1167 & 39.3306 \\
\hline 82.8831 & 122.3341 & 39.451 & 82.9091 & 122.1635 & 39.2544 & 82.7982 & 122.1115 & 39.3133 \\
\hline 82.9394 & 122.412 & 39.4727 & 82.9091 & 122.1444 & 39.2354 & 82.7755 & 122.0822 & 39.3067 \\
\hline 82.9281 & 122.2475 & 39.3194 & 82.8701 & 122.1228 & 39.2527 & 82.7298 & 122.0708 & 39.341 \\
\hline 82.8813 & 122.1314 & 39.2501 & 82.8718 & 122.2206 & 39.3488 & 82.7159 & 122.1297 & 39.4138 \\
\hline 82.8346 & 122.1756 & 39.341 & 82.9021 & 122.218 & 39.3159 & 82.6614 & 122.1202 & 39.4588 \\
\hline 82.7956 & 122.1115 & 39.3159 & 82.8701 & 122.2518 & 39.3817 & 82.6943 & 122.1306 & 39.4363 \\
\hline 82.7975 & 122.046 & 39.2485 & 82.8519 & 122.218 & 39.3661 & 82.6978 & 122.1695 & 39.4718 \\
\hline 82.793 & 122.0223 & 39.2293 & 82.8251 & 122.3081 & 39.483 & 82.6839 & 122.1029 & 39.419 \\
\hline
\end{tabular}




\begin{tabular}{|c|c|c|c|c|c|c|c|c|}
\hline 82.6449 & 122.1046 & 39.4597 & 82.7523 & 122.315 & 39.5627 & 82.8173 & 122.4233 & 39.606 \\
\hline 82.6484 & 122.1185 & 39.4701 & 82.7185 & 122.2977 & 39.5792 & 82.8337 & 122.4683 & 39.6346 \\
\hline 82.6125 & 122.1306 & 39.5181 & 82.7116 & 122.2865 & 39.5748 & 82.8562 & 122.4631 & 39.6069 \\
\hline 82.6025 & 122.1185 & 39.516 & 82.7584 & 122.3202 & 39.5618 & 82.8614 & 122.4631 & 39.6017 \\
\hline 82.6683 & 122.1436 & 39.4752 & 82.7878 & 122.2953 & 39.5075 & 82.8787 & 122.4709 & 39.5922 \\
\hline 82.7237 & 122.1522 & 39.4285 & 82.8155 & 122.2908 & 39.4752 & 82.8493 & 122.4822 & 39.6329 \\
\hline 82.6683 & 122.1505 & 39.4822 & 82.7748 & 122.3289 & 39.5541 & 82.838 & 122.49 & 39.652 \\
\hline 82.6562 & 122.0821 & 39.4259 & 82.7965 & 122.3393 & 39.5428 & 82.8632 & 122.4553 & 39.5922 \\
\hline 82.69 & 122.1773 & 39.4874 & 82.7748 & 122.373 & 39.5982 & 82.871 & 122.5064 & 39.6355 \\
\hline 82.6813 & 122.0916 & 39.4103 & 82.7982 & 122.3471 & 39.5489 & 82.8484 & 122.5203 & 39.6718 \\
\hline 82.6663 & 122.1949 & 39.5287 & 82.7618 & 122.4397 & 39.6779 & 82.8701 & 122.47 & 39.5999 \\
\hline 82.6181 & 122.1124 & 39.4943 & 82.774 & 122.4103 & 39.6363 & 82.8684 & 122.4951 & 39.6268 \\
\hline 82.6094 & 122.2224 & 39.6129 & 82.7843 & 122.4063 & 39.622 & 82.8987 & 122.412 & 39.5134 \\
\hline 82.6293 & 122.1834 & 39.5541 & 82.8034 & 122.4293 & 39.6259 & 82.8987 & 122.3627 & 39.464 \\
\hline 82.6285 & 122.1877 & 39.5592 & 82.7445 & 122.3834 & 39.6389 & 82.8653 & 122.4178 & 39.5524 \\
\hline 82.6614 & 122.1375 & 39.4761 & 82.7523 & 122.4544 & 39.7021 & 82.8051 & 122.4553 & 39.6502 \\
\hline 82.6371 & 122.1626 & 39.5255 & 82.7636 & 122.4484 & 39.6848 & 82.8476 & 122.4345 & 39.587 \\
\hline 82.6484 & 122.1618 & 39.5134 & 82.7445 & 122.4389 & 39.6943 & 82.8458 & 122.4458 & 39.5999 \\
\hline 82.6381 & 122.1747 & 39.5366 & 82.7826 & 122.4207 & 39.6381 & 82.871 & 122.4311 & 39.5601 \\
\hline 82.6501 & 122.199 & 39.5489 & 82.7601 & 122.4345 & 39.6744 & 82.8268 & 122.4778 & 39.651 \\
\hline 82.6415 & 122.1773 & 39.5359 & 82.7385 & 122.4794 & 39.741 & 82.8545 & 122.4596 & 39.6051 \\
\hline 82.5835 & 122.2535 & 39.6701 & 82.7445 & 122.4042 & 39.6597 & 82.8225 & 122.4354 & 39.6129 \\
\hline 82.5523 & 122.2085 & 39.6562 & 82.7246 & 122.4034 & 39.6788 & 82.8636 & 122.4072 & 39.5436 \\
\hline 82.5817 & 122.1721 & 39.5904 & 82.7592 & 122.4726 & 39.7134 & 82.8103 & 122.4215 & 39.6112 \\
\hline 82.5705 & 122.2224 & 39.6519 & 82.7748 & 122.3696 & 39.5948 & 82.8502 & 122.5627 & 39.7125 \\
\hline 82.5549 & 122.1999 & 39.645 & 82.7558 & 122.4354 & 39.6796 & 82.8251 & 122.4943 & 39.6692 \\
\hline 82.6284 & 122.3033 & 39.6749 & 82.8086 & 122.4172 & 39.6086 & 82.8095 & 122.4276 & 39.6181 \\
\hline 82.6259 & 122.2726 & 39.6467 & 82.8406 & 122.4709 & 39.6303 & 82.8173 & 122.4648 & 39.6476 \\
\hline 82.6995 & 122.2163 & 39.5168 & 82.8521 & 122.409 & 39.5568 & 82.8675 & 122.4311 & 39.5636 \\
\hline 82.7315 & 122.2042 & 39.4727 & 82.8597 & 122.4441 & 39.5844 & 82.8441 & 122.4189 & 39.5748 \\
\hline 82.7203 & 122.2094 & 39.4891 & 82.8935 & 122.3912 & 39.4978 & 82.8424 & 122.4539 & 39.6115 \\
\hline 82.7246 & 122.2709 & 39.5463 & 82.9047 & 122.4103 & 39.5056 & 82.8519 & 122.4363 & 39.5844 \\
\hline 82.7454 & 122.2916 & 39.5463 & 82.8787 & 122.393 & 39.5142 & 82.8822 & 122.3471 & 39.4649 \\
\hline 82.7965 & 122.2518 & 39.4553 & 82.9073 & 122.4094 & 39.5021 & 82.8857 & 122.3765 & 39.4908 \\
\hline 82.7913 & 122.2654 & 39.474 & 82.9134 & 122.3575 & 39.4441 & 82.877 & 122.4267 & 39.5497 \\
\hline 82.7558 & 122.27 & 39.5142 & 82.89 & 122.4544 & 39.5644 & 82.9255 & 122.4337 & 39.5082 \\
\hline 82.7627 & 122.2925 & 39.5298 & 82.8645 & 122.4557 & 39.5912 & 82.948 & 122.3713 & 39.4233 \\
\hline 82.7237 & 122.3124 & 39.5887 & 82.8926 & 122.4622 & 39.5696 & 82.9584 & 122.3609 & 39.4025 \\
\hline 82.7272 & 122.2414 & 39.5142 & 82.838 & 122.4735 & 39.6355 & 82.9349 & 122.4354 & 39.5005 \\
\hline 82.7315 & 122.2761 & 39.5445 & 82.8008 & 122.5012 & 39.7004 & 82.9307 & 122.3661 & 39.4354 \\
\hline 82.7168 & 122.2267 & 39.5099 & 82.8562 & 122.4423 & 39.5861 & 82.9134 & 122.3904 & 39.477 \\
\hline 82.7099 & 122.3462 & 39.6363 & 82.877 & 122.5073 & 39.6303 & 82.9125 & 122.3713 & 39.4588 \\
\hline 82.7218 & 122.2953 & 39.5736 & 82.832 & 122.5549 & 39.7229 & 82.8701 & 122.3488 & 39.4787 \\
\hline 82.7133 & 122.2683 & 39.5549 & 82.8311 & 122.4709 & 39.6398 & 82.89 & 122.3895 & 39.4995 \\
\hline 82.7125 & 122.2908 & 39.5783 & 82.8134 & 122.4883 & 39.6749 & 82.8883 & 122.4086 & 39.5203 \\
\hline 82.7471 & 122.2916 & 39.5445 & 82.7965 & 122.412 & 39.6155 & 82.8813 & 122.4467 & 39.5653 \\
\hline 82.7627 & 122.302 & 39.5393 & 82.8129 & 122.47 & 39.6571 & 82.8653 & 122.4266 & 39.5612 \\
\hline
\end{tabular}




\begin{tabular}{|c|c|c|c|c|c|c|c|c|}
\hline 82.8225 & 122.4241 & 39.6017 & 82.774 & 122.4865 & 39.7125 & 82.6848 & 122.3479 & 39.6632 \\
\hline 82.7852 & 122.4345 & 39.6493 & 82.8803 & 122.4618 & 39.5815 & 82.6978 & 122.3375 & 39.6398 \\
\hline 82.7636 & 122.4761 & 39.7125 & 82.7289 & 122.3107 & 39.5818 & 82.7731 & 122.3453 & 39.5722 \\
\hline 82.7991 & 122.4302 & 39.6311 & 82.793 & 122.3098 & 39.5168 & 82.8098 & 122.3429 & 39.5331 \\
\hline 82.8216 & 122.406 & 39.5844 & 82.8484 & 122.2743 & 39.4259 & 82.8138 & 122.3462 & 39.5324 \\
\hline 82.8684 & 122.3895 & 39.5211 & 82.8502 & 122.289 & 39.4389 & 82.8441 & 122.3211 & 39.477 \\
\hline 82.8796 & 122.4068 & 39.5272 & 82.8354 & 122.2778 & 39.4423 & 82.7965 & 122.3064 & 39.5099 \\
\hline 82.9229 & 122.4008 & 39.4778 & 82.832 & 122.2951 & 39.4631 & 82.8684 & 122.2908 & 39.4224 \\
\hline 82.9517 & 122.3658 & 39.4141 & 82.8233 & 122.2934 & 39.4701 & 82.8424 & 122.3159 & 39.4735 \\
\hline 82.9896 & 122.3644 & 39.3748 & 82.793 & 122.2916 & 39.4986 & 82.8545 & 122.3237 & 39.4692 \\
\hline 82.9705 & 122.3644 & 39.3938 & 82.8081 & 122.3182 & 39.5102 & 82.8484 & 122.3427 & 39.4943 \\
\hline 82.9472 & 122.3609 & 39.4138 & 82.806 & 122.2683 & 39.4623 & 82.871 & 122.3168 & 39.4458 \\
\hline 82.9757 & 122.3107 & 39.335 & 82.8207 & 122.2864 & 39.4657 & 82.8195 & 122.3244 & 39.5049 \\
\hline 83.0684 & 122.3315 & 39.2631 & 82.7913 & 122.283 & 39.4917 & 82.8242 & 122.354 & 39.5298 \\
\hline 83.058 & 122.3098 & 39.2518 & 82.7549 & 122.3072 & 39.5523 & 82.8242 & 122.3401 & 39.516 \\
\hline 83.0545 & 122.3159 & 39.2614 & 82.7575 & 122.3046 & 39.5471 & 82.8329 & 122.3367 & 39.5038 \\
\hline 83.0618 & 122.3332 & 39.2714 & 82.8259 & 122.2986 & 39.4727 & 82.8536 & 122.3601 & 39.5064 \\
\hline 83.0459 & 122.3644 & 39.3185 & 82.7965 & 122.2977 & 39.5012 & 82.8813 & 122.3297 & 39.4484 \\
\hline 83.0424 & 122.3479 & 39.3055 & 82.7878 & 122.3226 & 39.5348 & 82.8909 & 122.3124 & 39.4216 \\
\hline 83.0978 & 122.3583 & 39.2605 & 82.793 & 122.315 & 39.522 & 82.9117 & 122.3323 & 39.4207 \\
\hline 83.0736 & 122.3618 & 39.2882 & 82.7514 & 122.322 & 39.5705 & & & \\
\hline 83.0502 & 122.3349 & 39.2847 & 82.7445 & 122.3228 & 39.5783 & & & \\
\hline 83.1074 & 122.3116 & 39.2042 & 82.7064 & 122.3064 & 39.5999 & & & \\
\hline 83.0727 & 122.3306 & 39.2579 & 82.7159 & 122.3471 & 39.6311 & & & \\
\hline 83.053 & 122.3024 & 39.2494 & 82.7809 & 122.3419 & 39.561 & & & \\
\hline 83.0996 & 122.322 & 39.2224 & 82.7575 & 122.3254 & 39.5679 & & & \\
\hline 83.0381 & 122.3211 & 39.283 & 82.7658 & 122.357 & 39.5912 & & & \\
\hline 83.0632 & 122.3185 & 39.2553 & 82.7411 & 122.3393 & 39.5982 & & & \\
\hline 83.084 & 122.3323 & 39.2484 & 82.7575 & 122.3419 & 39.5844 & & & \\
\hline 83.0545 & 122.386 & 39.3315 & 82.7714 & 122.3583 & 39.587 & & & \\
\hline 83.0649 & 122.3488 & 39.2839 & 82.7575 & 122.3323 & 39.5748 & & & \\
\hline 82.9601 & 122.3808 & 39.4207 & 82.722 & 122.3575 & 39.6355 & & & \\
\hline 82.9596 & 122.3852 & 39.4256 & 82.6943 & 122.3696 & 39.6753 & & & \\
\hline 82.9498 & 122.3687 & 39.419 & 82.6752 & 122.3514 & 39.6762 & & & \\
\hline 82.9298 & 122.4302 & 39.5004 & 82.698 & 122.3614 & 39.6634 & & & \\
\hline 82.9428 & 122.4189 & 39.4761 & 82.6675 & 122.3514 & 39.6839 & & & \\
\hline 82.9342 & 122.4276 & 39.4934 & 82.7021 & 122.38 & 39.6779 & & & \\
\hline 82.8917 & 122.4008 & 39.509 & 82.69 & 122.373 & 39.6831 & & & \\
\hline 82.858 & 122.3912 & 39.5333 & 82.6848 & 122.3817 & 39.6969 & & & \\
\hline 82.8441 & 122.38 & 39.5359 & 82.7463 & 122.3523 & 39.606 & & & \\
\hline 82.8204 & 122.4248 & 39.6044 & 82.7558 & 122.3332 & 39.5774 & & & \\
\hline 82.7913 & 122.4337 & 39.6424 & 82.735 & 122.3436 & 39.6086 & & & \\
\hline 82.754 & 122.4293 & 39.6753 & 82.7367 & 122.3174 & 39.5806 & & & \\
\hline 82.7237 & 122.4562 & 39.7324 & 82.722 & 122.3315 & 39.6095 & & & \\
\hline 82.7601 & 122.4527 & 39.6926 & 82.7125 & 122.3202 & 39.6077 & & & \\
\hline 82.7385 & 122.47 & 39.7316 & 82.6952 & 122.3315 & 39.6363 & & & \\
\hline 82.767 & 122.4891 & 39.7221 & 82.6926 & 122.3185 & 39.6259 & & & \\
\hline
\end{tabular}




\begin{tabular}{|c|c|c|c|c|c|c|c|}
\hline \multicolumn{2}{|c|}{$\begin{array}{l}\text { Table A.5: } \\
\text { weighted syringe }\end{array}$} & \multirow{2}{*}{\begin{tabular}{|l|}
45 \\
46 \\
\end{tabular}} & \multirow{2}{*}{$\begin{array}{l}181.0812 \\
180.3838 \\
\end{array}$} & \multirow{2}{*}{$\frac{90}{91}$} & \multirow{2}{*}{$\begin{array}{r}165.3492 \\
165.036 \\
\end{array}$} & \multirow{2}{*}{$\begin{array}{l}135 \\
136 \\
\end{array}$} & \multirow{2}{*}{$\begin{array}{r}151.087 \\
150.7423\end{array}$} \\
\hline Time(min) & $\mathrm{mmHg}$ & & & & & & \\
\hline 1 & 218.95 & 47 & 179.9273 & 92 & 164.7752 & 137 & 150.6299 \\
\hline 2 & 214.68 & 48 & 179.6504 & 93 & 164.5115 & 138 & 150.1093 \\
\hline 3 & 212.04 & 49 & 179.259 & 94 & 164.2071 & 139 & 150.0012 \\
\hline 5 & 209.83 & 50 & 179.0298 & 95 & 164.0137 & 140 & 149.6571 \\
\hline 6 & 208.3 & 51 & 178.484 & 96 & 163.6458 & 141 & 149.1915 \\
\hline 7 & 206.86 & 52 & 177.9055 & 97 & 163.2462 & 142 & 149.0317 \\
\hline 8 & 205.65 & 53 & 177.6589 & 98 & 162.8455 & 143 & 148.7439 \\
\hline 9 & 204.46 & 54 & 177.233 & 99 & 162.5805 & 144 & 148.3753 \\
\hline 10 & 203.21 & 55 & 176.811 & 100 & 162.0779 & 145 & 148.0959 \\
\hline 11 & 202.3 & 56 & 176.3679 & 101 & 161.8876 & 146 & 147.5288 \\
\hline 12 & 201.27 & 57 & 175.811 & 102 & 161.8684 & 147 & 147.2822 \\
\hline 13 & 200.35 & 58 & 175.6312 & 103 & 161.49 & 148 & 147.1424 \\
\hline 14 & 199.73 & 59 & 175.302 & 104 & 161.2459 & 149 & 146.7285 \\
\hline 15 & 198.69 & 60 & 174.8506 & 105 & 160.5396 & 150 & 146.6057 \\
\hline 16 & 197.78 & 61 & 174.3716 & 106 & 160.3155 & 151 & 146.3033 \\
\hline 17 & 196.99 & 62 & 173.99 & 107 & 159.8565 & 152 & 146.0971 \\
\hline 18 & 196 & 63 & 173.4758 & 108 & 159.6382 & 153 & 145.8319 \\
\hline 19 & 195.49 & 64 & 173.3824 & 109 & 159.3272 & 154 & 145.4129 \\
\hline 20 & 194.82 & 65 & 172.784 & 110 & 158.9871 & 155 & 145.1309 \\
\hline 21 & 194.08 & 66 & 172.4068 & 111 & 158.9047 & 156 & 144.7524 \\
\hline 22 & 193.19 & 67 & 172.1561 & 112 & 158.4544 & 157 & 144.5713 \\
\hline 23 & 192.54 & 68 & 171.5845 & 113 & 158.1571 & 158 & 144.2425 \\
\hline 24 & 192 & 69 & 171.3841 & 114 & 157.7984 & 159 & 144.0555 \\
\hline 25 & 191.34 & 70 & 170.8575 & 115 & 157.5629 & 160 & 143.6622 \\
\hline 26 & 190.77 & 71 & 170.6497 & 116 & 157.2732 & 161 & 143.4298 \\
\hline 27 & 190.18 & 72 & 170.3527 & 117 & 156.8806 & 162 & 143.2827 \\
\hline 28 & 189.62 & 73 & 170.2541 & 118 & 156.4867 & 163 & 143.0633 \\
\hline 29 & 189.15 & 74 & 169.8262 & 119 & 156.0214 & 164 & 142.7087 \\
\hline 30 & 188.45 & 75 & 169.5719 & 120 & 155.8841 & 165 & 142.5227 \\
\hline 31 & 187.94 & 76 & 169.4818 & 121 & 155.6152 & 166 & 142.1136 \\
\hline 32 & 187.32 & 77 & 169.0145 & 122 & 155.4636 & 167 & 141.9763 \\
\hline 33 & 186.66 & 78 & 168.7429 & 123 & 155.1561 & 168 & 141.4958 \\
\hline 34 & 186.25 & 79 & 168.5544 & 124 & 154.8401 & 169 & 141.341 \\
\hline 35 & 185.55 & 80 & 168.2443 & 125 & 154.4671 & 170 & 141.0671 \\
\hline 36 & 185.03 & 81 & 167.9755 & 126 & 154.0703 & 171 & 140.7205 \\
\hline 37 & 184.43 & 82 & 167.6244 & 127 & 153.9207 & 172 & 140.4361 \\
\hline 38 & 183.99 & 83 & 167.3106 & 128 & 153.4801 & 173 & 140.319 \\
\hline 39 & 183.48 & 84 & 166.9933 & 129 & 153.1682 & 174 & 140.0205 \\
\hline 40 & 182.88 & 85 & 166.3691 & 130 & 152.7995 & 175 & 139.7181 \\
\hline 41 & 182.53 & 86 & 166.5751 & 131 & 152.4962 & 176 & 139.4866 \\
\hline 42 & 182 & 87 & 166.0463 & 132 & 152.0202 & 177 & 139.1166 \\
\hline 43 & 181.58 & 88 & 165.5293 & 133 & 151.8173 & 178 & 138.9547 \\
\hline 44 & 181.2 & 89 & 165.4641 & 134 & 151.5451 & 179 & 138.5903 \\
\hline
\end{tabular}




\begin{tabular}{|c|c|c|c|c|c|c|c|}
\hline 180 & 138.34 & 225 & 128.5714 & 270 & 120.278 & 315 & 113.5115 \\
\hline 181 & 138.23 & 226 & 127.9874 & 271 & 120.1263 & 316 & 113.3827 \\
\hline 182 & 138.11 & 227 & 127.6422 & 272 & 119.9236 & 317 & 113.3072 \\
\hline 183 & 137.84 & 228 & 127.7147 & 273 & 119.7374 & 318 & 113.2598 \\
\hline 184 & 137.56 & 229 & 127.6121 & 274 & 119.5527 & 319 & 113.0321 \\
\hline 185 & 137.31 & 230 & 127.5189 & 275 & 119.3846 & 320 & 112.9743 \\
\hline 186 & 136.93 & 231 & 127.2948 & 276 & 119.2982 & 321 & 112.8605 \\
\hline 187 & 136.76 & 232 & 127.1388 & 277 & 119.0955 & 322 & 112.7438 \\
\hline 188 & 136.53 & 233 & 127.0079 & 278 & 118.9441 & 323 & 112.6541 \\
\hline 189 & 136.42 & 234 & 126.7966 & 279 & 118.801 & 324 & 112.4494 \\
\hline 190 & 136.03 & 235 & 126.5642 & 280 & 118.7362 & 325 & 112.2606 \\
\hline 191 & 135.91 & 236 & 126.398 & 281 & 118.4883 & 326 & 112.1861 \\
\hline 192 & 135.64 & 237 & 126.2672 & 282 & 118.31 & 327 & 111.9834 \\
\hline 193 & 135.32 & 238 & 126.1539 & 283 & 118.2208 & 328 & 111.8708 \\
\hline 194 & 135.05 & 239 & 125.9021 & 284 & 118.048 & 329 & 111.7332 \\
\hline 195 & 134.9 & 240 & 125.6857 & 285 & 117.8734 & 330 & 111.6338 \\
\hline 196 & 134.69 & 241 & 125.4903 & 286 & 117.7677 & 331 & 111.4912 \\
\hline 197 & 134.47 & 242 & 125.2833 & 287 & 117.6374 & 332 & 111.3663 \\
\hline 198 & 134.16 & 243 & 125.1227 & 288 & 117.4454 & 333 & 111.2777 \\
\hline 199 & 134.05 & 244 & 125.051 & 289 & 117.372 & 334 & 111.1208 \\
\hline 200 & 133.76 & 245 & 124.7147 & 290 & 117.0854 & 335 & 110.9357 \\
\hline 201 & 133.56 & 246 & 124.5651 & 291 & 116.9589 & 336 & 110.9205 \\
\hline 202 & 133.3 & 247 & 124.4846 & 292 & 116.8226 & 337 & 110.7264 \\
\hline 203 & 133.15 & 248 & 124.2522 & 293 & 116.7216 & & \\
\hline 204 & 132.9 & 249 & 124.0663 & 294 & 116.4858 & & \\
\hline 205 & 132.65 & 250 & 123.8364 & 295 & 116.3649 & & \\
\hline 206 & 132.39 & 251 & 123.6216 & 296 & 116.1799 & & \\
\hline 207 & 132.31 & 252 & 123.5399 & 297 & 116.1001 & & \\
\hline 208 & 132.03 & 253 & 123.3954 & 298 & 116.0087 & & \\
\hline 209 & 131.9 & 254 & 123.0747 & 299 & 115.8289 & & \\
\hline 210 & 131.69 & 255 & 122.8197 & 300 & 115.6243 & & \\
\hline 211 & 131.44 & 256 & 122.8122 & 301 & 115.5045 & & \\
\hline 212 & 131.19 & 257 & 122.6105 & 302 & 115.3534 & & \\
\hline 213 & 130.98 & 258 & 122.4087 & 303 & 115.2734 & & \\
\hline 214 & 130.87 & 259 & 122.2153 & 304 & 115.0175 & & \\
\hline 215 & 130.62 & 260 & 122.0698 & 305 & 114.8814 & & \\
\hline 216 & 130.49 & 261 & 121.9446 & 306 & 114.7801 & & \\
\hline 217 & 130.17 & 262 & 121.6759 & 307 & 114.5727 & & \\
\hline 218 & 130.05 & 263 & 121.5427 & 308 & 114.4062 & & \\
\hline 219 & 129.75 & 264 & 121.3939 & 309 & 114.3247 & & \\
\hline 220 & 129.56 & 265 & 121.1901 & 310 & 114.1373 & & \\
\hline 221 & 129.23 & 266 & 120.9779 & 311 & 114.0071 & & \\
\hline 222 & 129.06 & 267 & 120.7944 & 312 & 113.9003 & & \\
\hline 223 & 128.95 & 268 & 120.6003 & 313 & 113.7152 & & \\
\hline 224 & 128.51 & 269 & 120.4542 & 314 & 113.5735 & & \\
\hline
\end{tabular}


APPENDIX B: Qualitative Leak Diagnostic Testing

$\underline{\text { Introduction }}$

The pressure test of the bioreactor chamber indicated the presence of a small leak within the system. The location of the leak has yet to be determined. In an attempt to isolate the source, smaller and simpler systems will be assembled, monitored for drops in pressure, and inspected for leaks. The tests will be conducted under semi-static conditions. Meaning, the systems will not have fluid flow and will undergo an initial pressurization. This simplification is justified in terms of reducing variables for the source of the leakage. If the system cannot maintain constant pressures in a static state, then it will not be able to with pulsatile flow.

\section{Materials and Methods Setup 1}

The first setup will include a 12" length of silicone tubing, 2 barbed male luer lock fittings, a pressure transducer and related data acquisition software, 2 female luer lock plugs, 1 two-way stop valve, and a luer lock style syringe. The two barbed luer lock fittings are each inserted into the 12" length of silicone tubing. On one end the pressure transducer was attached, and the two-way valve was attached to the opposing end of the tubing. Once the transducer is attached to the open system, it is zeroed using the bridge pod. The syringe is filled with water and connected to the stop valve. If the stop valve is closed, it is opened to allow water to flow through the length of tubing. Once water shoots out from the transducer side of the system, the transducer is capped with a female luer lock plug. Before removing the syringe, the stop valve is closed to prevent water from exiting the system.

The syringe is filled with water once again and attached to the stop valve. Water is then forced into the system once the valve is opened. The amount of water introduced is determined by the pressure reading on the data acquisition software. Once the pressure reaches $120 \mathrm{mmHg}$, the stop valve is closed to maintain a constant pressure. The pressure will be monitored until it drops $10 \mathrm{mmHg}$. The $10 \mathrm{mmHg}$ limit 
is based on the presumption that if the system was to drop $10 \mathrm{mmHg}$ within 24 hours it would not be an effective system capable of maintaining an acceptably constant pressure.

\section{$\underline{\text { Results and Discussion }}$}

The pressure dropped at roughly $1 \mathrm{mmHg}$ every two minutes. Since the pressure declined without the bioreactor chamber in place, it is safe to conclude that the system is subject to significant pressure loss independent of the bioreactor chamber. In fact the chamber might not be the source of the leak. It seems incongruous that the leak should be more pronounced without the bioreactor chamber. This could be attributed to simple connection variability either between the silicone tubing and the barbs or the luer lock fittings themselves. Accordingly, modifications would be made to Setup 1 to eliminate one of the variables.

\section{Methods and Materials Setup 2}

Setup 2 includes all of the parts described in Setup 1 except the addition of spring clamps. The spring clamps are brass loops used to secure micro-tubing over barbed fitting and prevent slippage. The hope here is to have the clamps squeeze the tubing and prevent the possibility of fluid pushing around the barb over time. Previous leak testing trials indicated a tendency for the silicone to balloon around the barb and then expel fluids.

In order to apply the spring clamps, the two barbed male luer lock fittings must be removed from the 12 " length of silicone tubing. Using vice clamps, pinch the "ears" of the spring clamp and slide it onto the tubing. Repeat this process for both spring clamps. Once this is completed, re-attach the barbed fittings that had been previously removed. Use the vice clamp again to pinch the ears of the spring clamp and slide it over the barb. It should squeeze the tubing just behind the edge of the barb. Repeat the process for both barbs. Once this portion of the setup is complete, zero the pressure transducer using the bridge pod. It is ok if the transducer is not exactly zero, it is rather sensitive. 
Take the luer lock syringe full of water and inject it into the system as described before. Be sure to cap off the end of the transducer and to turn off the two-way valve. Refill the syringe with fluid. Start recording live data with the data acquisition software and after attaching the syringe to the two-way valve inject the fluid into the system. Inject enough fluid to raise the pressure up to $120 \mathrm{mmHg}$. Accurately injecting enough fluid is possible using the display on the software. Once the appropriate pressure is reached, close the two-way valve. The pressure will be monitored once again until $10 \mathrm{mmHg}$ of pressure is lost from the system.

\section{$\underline{\text { Results and Discussion }}$}

The pressure dropped at roughly $1 \mathrm{mmHg}$ per 3 minutes. The rate of decrease was not substantially different from the rate recorded for setup 1. Upon disassembly, it was found that the spring clamps were slightly deformed. The deformations were likely due to using the vice clamps to apply the spring clamps. Since they are deformed, their effectiveness for sealing the tube to barb connection was compromised. Given the slight improvement that was realized, it would be worthwhile to pursue enhancing the integrity of the seal between the tubing and barb connection.

\section{Methods and Materials Setup 3}

Setup 3 is the same as setup 1 except with the addition of twist ties. String or twine was a potential option. Using masonry string, it was found that the quality of the string made it slick and difficult to tie a tight knot that would not slip and loosen on the tube over the barb. Twist ties do not fall victim to slippage. Toying around with grocery store style paper covered twist ties, proof of concept was achieved. A tight seal can be achieved using the twist ties. The only issue is that the paper on these twist ties rips and tears. It's not too bad, but eventually the wire gets worn out and breaks or doesn't twist so well without the paper. As a remedy, heavy duty twist ties were used for securing electrical cords. 
The parts are put together the same as they were for the first setup. This time the barbed fittings do not have to be removed like they were to accommodate the spring clamps. Once everything is in place, the pressure transducer is zeroed. The twist ties are then placed at each end of the silicone tubing. The tie is tightened just behind the edge of the barb. The tie is twisted until its ability to squeeze the tubing has been maxed out.

The luer lock syringe is filled with fluid, and the fluid is injected into the system. Once water shoots out the end of the transducer, the transducer is capped and the two-way valve is closed. Based on the pressure readings displayed on the data acquisition software, inject additional fluid into the closed system until $120 \mathrm{mmHg}$ is shown. Then close off the two-way valve for a final time and monitor the system until it drops $10 \mathrm{mmHg}$.

\section{$\underline{\text { Results and Discussion }}$}

The system lost $1 \mathrm{mmHg}$ per every 10 minutes. The rate of pressure loss from the system has dramatically improved with the enhanced seal. Accordingly, it appears that some pressure continues to be lost through the connection between the tubing and barbed fittings. Nonetheless, there is still an unacceptable loss in pressure. Further tightening of the twist ties is impractical and unlikely to yield much more improvement in the rate of leakage. The next option is exploring the luer lock connections. They are the last remaining variable. The rate of leakage has been somewhat erratic over the test series and the rate here is half that of the original setup with the bioreactor chamber. This suggests that either the bioreactor chamber is contributing to the leakage or the additional connections to the chamber are leaking. Given the empirical evidence from previous trials conducted, the leaks are independent of the new bioreactor chamber. The next setup to be tested will become even simpler in order to isolate the luer lock fitting variable. 
This is the final setup to test whether or not the luer lock connections leak. There are only a few parts required for the test since it has been reduced greatly in complexity. The simplification is done in order to help reduce the number of variables affecting the drop in pressure. It is appropriate to note that the vendor supplying the luer lock fittings claims an air-tight connection to 20 psi.

This setup requires a single ADI pressure transducer, two stop valves, and one luer lock style syringe. The ADI pressure transducer is calibrated as usual. Using the bridge pod, zero the transducer. Once this is complete, attach both of the stop valves to each end of the ADI instrument. Make sure they are in the open position. Attaching the valves while they are closed will pressurize the interior of the system. Check that the transducer still reads zero once the valves have been added. Fill the syringe full of water and screw it on to one of the stop valves. Inject fluid through the system. Once the water shoots out the opposite stop valve, close both valves (starting with the farthest valve from the syringe). Refilling the syringe will not be necessary since the volume of the system is much smaller than that of the syringe. Press the "start" button on the LabChart software so it begins collecting data. Once the software begins collecting data, open the valve the syringe is attached to and inject water into the system until the desired $100 \mathrm{mmHg}$ pressure is achieved. This will be a small amount. Hold it steady by applying pressure to the syringe plunger while closing the valve.

Since this is a qualitative test, watch the pressure drop until it loses $10 \mathrm{mmHg}$. At this point, the system will be outside the acceptable range for fluctuation.

\section{$\underline{\text { Results and Discussion }}$}

The system leaks quickly - roughly $1 \mathrm{mmHg}$ per second. The results indicate that the luer lock fittings are at least causing some of the issues with the dropping pressure measurements. Some future testing should be conducted to see if different fittings leak at different rates. There are a variety of valves and fittings used in the bioreactor system and potentially substitutes. For example, there are multiple 3 
way valves to pick from. The potential source of leakage could be the ADI pressure transducer itself. Multiple ADI transducers should be tested as well to test whether or not the pressure drop depends on the transducer being used.

It is problematic that the luer lock fittings and/or pressure transducers are leaking. The ADI transducer is the only means of taking live pressure measurements within the system. If the leak can be accurately measured from the smallest system tested, the system can be rebuilt and compared to the initial leak. If there is a discrepancy at any stage where the larger system leaks more than the smaller system did, then additional leaks exist beyond the luer lock fittings. In which case, the system can be slowly rebuilt with the intention of locating and addressing the potential additional leaks.

Logically, the pressures drop faster with the smaller systems because with larger volumes, a micro leak at a specific rate represents a smaller percentage of the overall volume than is the case with the same micro leak and a much smaller volume.

\section{Methods and Materials Setup 5}

This test seeks to determine whether or not different luer lock valves leak at different rates. To perform the test, three different luer lock valves were used: two different three-way valves, and one twoway valve (Figure A.1). The ADI pressure transducer was filled with water and then capped at one end using a luer lock plug. Next, the valve in question would be filled with water and attached to the pressure transducer. Depending on the style of the valve, a luer lock plug would be added to the extra port of the three-way valve in order to provide a seal during the test. The plug would be added while the valve was open in order to prevent any excess pressure buildup beyond that applied intentionally by the syringe. The two-way valve would not require any modifications. Once the data acquisition software was enabled, a syringe full of water would be attached to the valve. Fluid would then be injected into the confined space to elevate the internal pressure. Once the pressure was elevated to $100 \mathrm{mmHg}$, the valve would be closed. Pressure would be monitored on the LabChart software. The pressure would be allowed to drop 10 
$\mathrm{mmHg}$, and the length of time that this would require was recorded. The average time for each valve would then be compared to the others, and the shortest duration would indicate the leakiest valve. The fact that the pressure is dropping would also indicate an issue with the luer lock interface and seal.

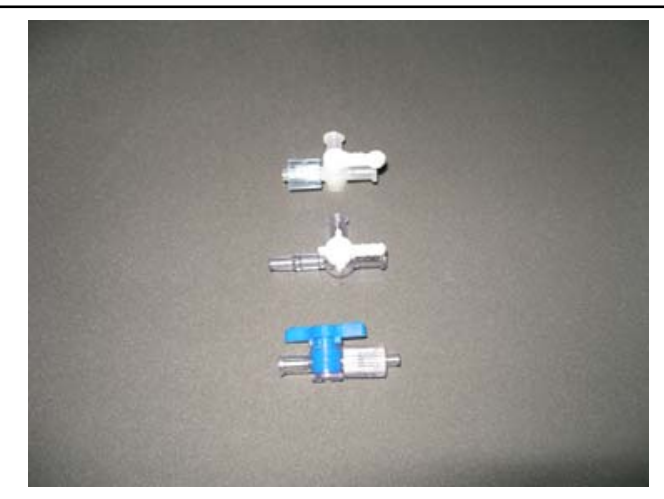

Figure A.1 The three different valves used to assess potential pressure.

\section{$\underline{\text { Results and Discussion: }}$}

Out of the three valves tested, the two-way valve had the slowest rate of leakage. The three-way valves both leaked quite fast. The two-way valve leak was on the order of minutes, while the others were on the order of seconds. This is a somewhat surprising find because luer lock valves and fittings are marketed and represented as being pressure and leak tight up to 20 psi. 20 psi would be roughly equivalent to $1,000 \mathrm{mmHg}$, and well above the intended operational pressures of the bioreactor. Perhaps there is a minimum pressure required for the fittings to seal properly. Regardless, the fittings are an integral component of the current bioreactor system. Replacing them would not make sense - they are top of the line products used for this very purpose. There might be a way to ensure an adequate seal using Teflon tape and o-rings, but a quantitative look at the system is required to assess the practicality of repairing leaks. 
Table A.6 General liner model ANOVA table.

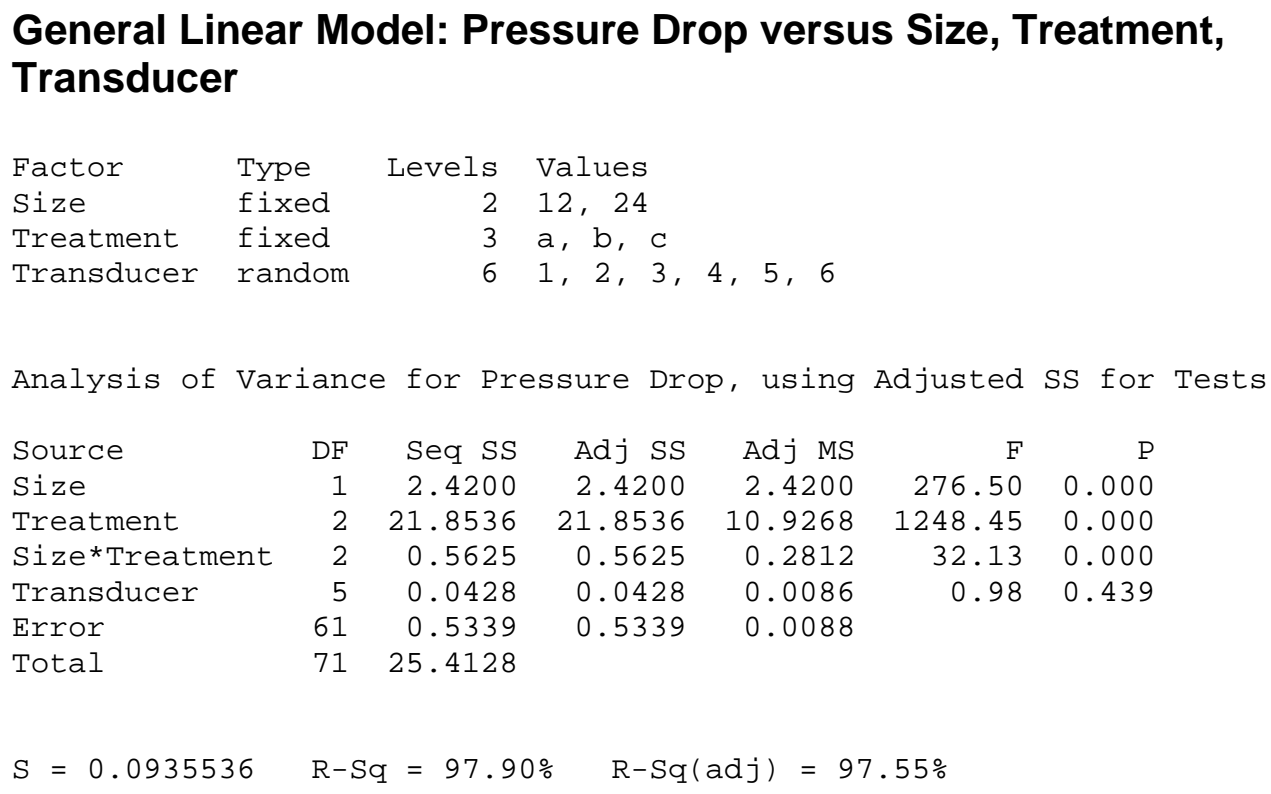

Table A.7 Least squares means for pressure drop.

\begin{tabular}{|c|c|}
\hline \multicolumn{2}{|c|}{$\begin{array}{l}\text { Least Squares } \\
\text { Pressure Drop }\end{array}$} \\
\hline Treatment & Mean \\
\hline $\mathrm{a}$ & 0.6708 \\
\hline b & 0.2500 \\
\hline C & 1.5708 \\
\hline \multicolumn{2}{|l|}{ Size } \\
\hline 12 & 1.0139 \\
\hline 24 & $\odot .6472$ \\
\hline \multicolumn{2}{|c|}{ Size*Treatment } \\
\hline 12 & 0.9167 \\
\hline 12 & 0.3083 \\
\hline 12 & 1.8167 \\
\hline 24 & 0.4250 \\
\hline 24 & 0.1917 \\
\hline 24 & 1.3250 \\
\hline
\end{tabular}


Table A.8 Tukey pairwise comparison of treatment factors.

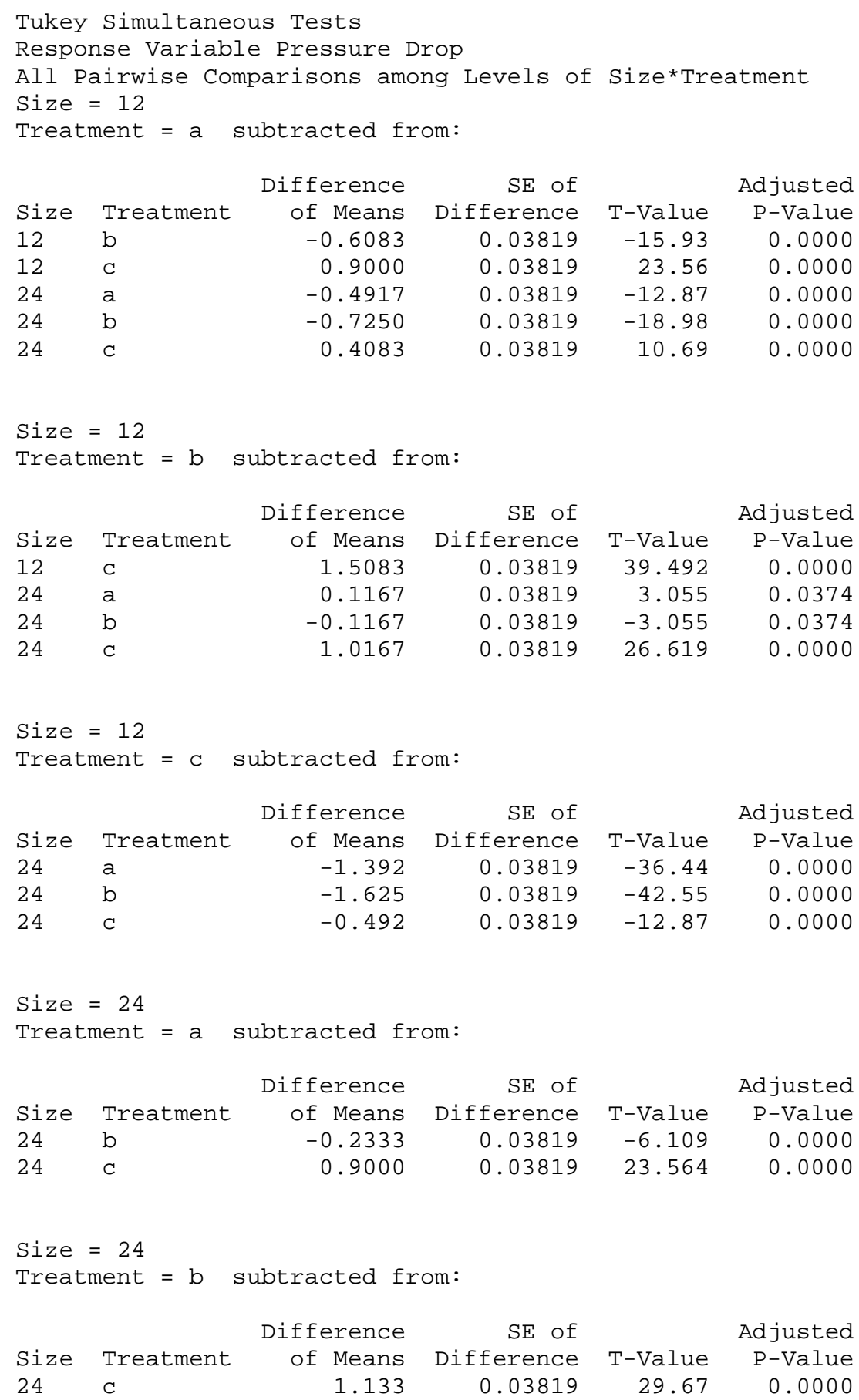


Table A.9 Data measurements for pressure leakage.

\begin{tabular}{|c|c|c|c|c|c|c|c|}
\hline Transducer & Size & Treatment & $\begin{array}{l}\text { Pressure } \\
\text { Drop } \\
(\mathrm{mmHg})\end{array}$ & Transducer & Size & Treatment & $\begin{array}{l}\text { Pressure } \\
\text { Drop } \\
(\mathrm{mmHg})\end{array}$ \\
\hline 1 & 12 & A & 1 & 1 & 24 & $a$ & 0.5 \\
\hline 2 & 12 & A & 0.8 & 2 & 24 & $a$ & 0.4 \\
\hline 3 & 12 & $a$ & 0.9 & 3 & 24 & $a$ & 0.5 \\
\hline 4 & 12 & $a$ & 0.8 & 4 & 24 & a & 0.4 \\
\hline 5 & 12 & $a$ & 0.9 & 5 & 24 & $a$ & 0.4 \\
\hline 6 & 12 & $a$ & 0.9 & 6 & 24 & $a$ & 0.5 \\
\hline 1 & 12 & $b$ & 0.2 & 1 & 24 & $\mathrm{~b}$ & 0.2 \\
\hline 2 & 12 & $\mathrm{~b}$ & 0.2 & 2 & 24 & $b$ & 0.2 \\
\hline 3 & 12 & $b$ & 0.3 & 3 & 24 & $b$ & 0.2 \\
\hline 4 & 12 & $\mathrm{~b}$ & 0.3 & 4 & 24 & $\mathrm{~b}$ & 0.3 \\
\hline 5 & 12 & $b$ & 0.3 & 5 & 24 & $b$ & 0.3 \\
\hline 6 & 12 & $b$ & 0.3 & 6 & 24 & $b$ & 0.2 \\
\hline 1 & 12 & $a$ & 0.9 & 1 & 12 & $\mathrm{C}$ & 1.8 \\
\hline 2 & 12 & $a$ & 0.9 & 2 & 12 & $\mathrm{C}$ & 1.8 \\
\hline 3 & 12 & $a$ & 1 & 3 & 12 & $\mathrm{C}$ & 1.7 \\
\hline 4 & 12 & $a$ & 1.2 & 4 & 12 & $\mathrm{C}$ & 1.6 \\
\hline 5 & 12 & $a$ & 0.8 & 5 & 12 & C & 1.9 \\
\hline 6 & 12 & $a$ & 0.9 & 6 & 12 & $\mathrm{C}$ & 2 \\
\hline 1 & 12 & $b$ & 0.2 & 1 & 24 & $\mathrm{C}$ & 1.4 \\
\hline 2 & 12 & $\mathrm{~b}$ & 0.4 & 2 & 24 & $\mathrm{C}$ & 1.1 \\
\hline 3 & 12 & $b$ & 0.4 & 3 & 24 & C & 1.4 \\
\hline 4 & 12 & $b$ & 0.4 & 4 & 24 & $\mathrm{C}$ & 1.3 \\
\hline 5 & 12 & $b$ & 0.4 & 5 & 24 & C & 1.5 \\
\hline 6 & 12 & $b$ & 0.3 & 6 & 24 & C & 1.3 \\
\hline 1 & 24 & $a$ & 0.4 & 1 & 12 & $\mathrm{C}$ & 1.8 \\
\hline 2 & 24 & $a$ & 0.4 & 2 & 12 & C & 1.9 \\
\hline 3 & 24 & $a$ & 0.4 & 3 & 12 & C & 1.9 \\
\hline 4 & 24 & $a$ & 0.4 & 4 & 12 & C & 1.8 \\
\hline 5 & 24 & $a$ & 0.3 & 5 & 12 & $\mathrm{C}$ & 1.8 \\
\hline 6 & 24 & $a$ & 0.5 & 6 & 12 & C & 1.8 \\
\hline 1 & 24 & $b$ & 0.2 & 1 & 24 & $\mathrm{C}$ & 1.3 \\
\hline 2 & 24 & $b$ & 0.2 & 2 & 24 & $\mathrm{C}$ & 1.1 \\
\hline 3 & 24 & $b$ & 0.1 & 3 & 24 & $\mathrm{C}$ & 1.4 \\
\hline 4 & 24 & $b$ & 0.1 & 4 & 24 & C & 1.3 \\
\hline 5 & 24 & $b$ & 0.1 & 5 & 24 & C & 1.4 \\
\hline 6 & 24 & $b$ & 0.2 & 6 & 24 & C & 1.4 \\
\hline
\end{tabular}




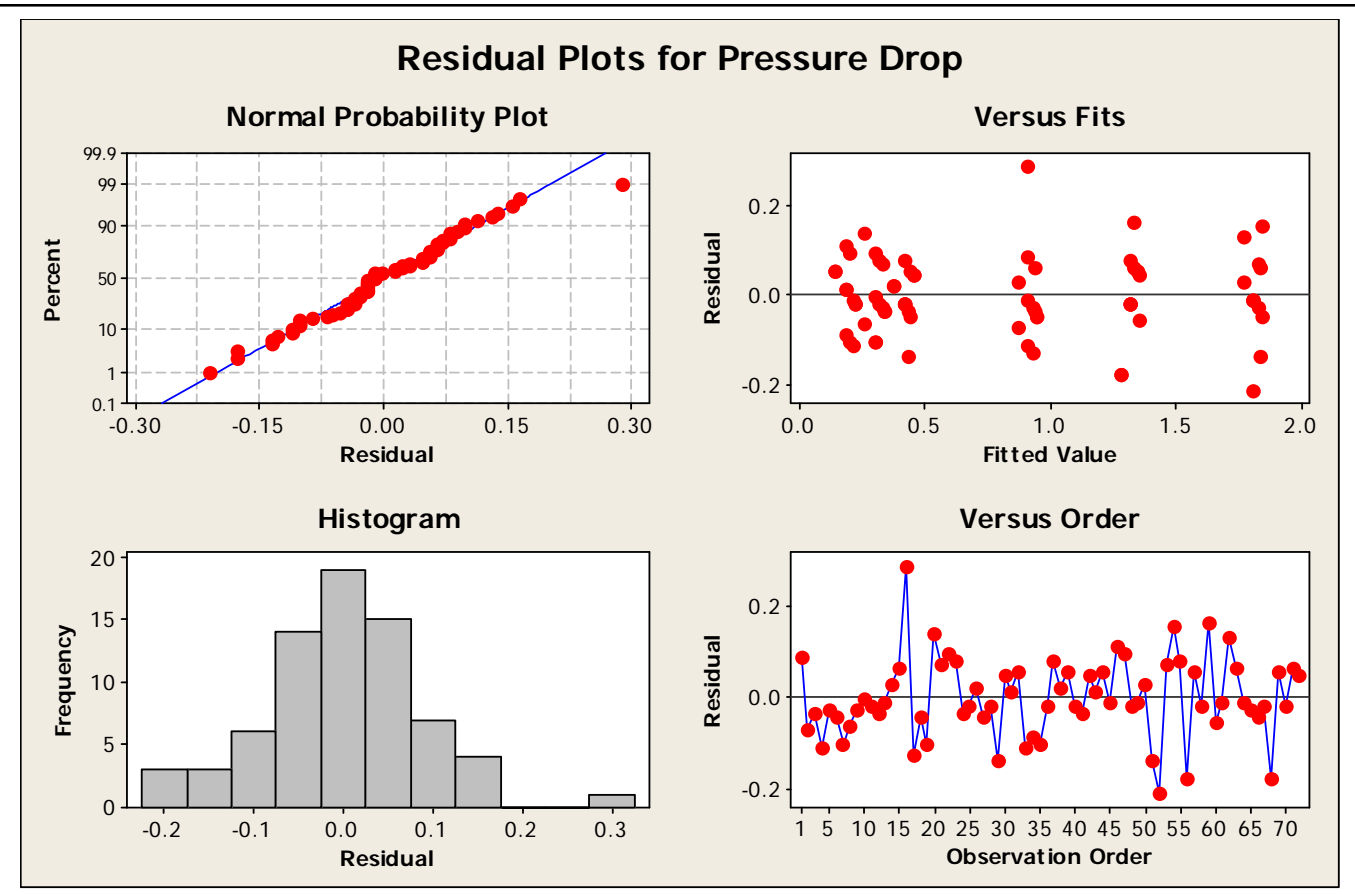

Figure A.2 Statistical charts used for assessing quality of data. 


\section{APPENDIX D: Design Considerations}

\section{Considerations for Future Prototype}

Before completely committing to a design, it is important to first test the theoretical basis on a simplified version of the system. While making and evaluating prototypes can be time consuming and expensive in the short-term, this approach can be very cost effective in the long term. Working with medical grade, sterilized equipment is expensive. Purchasing such equipment without testing equivalent models can be inefficient and wasteful. Accordingly, a prototype has been designed to assess a specific system configuration that should provide the same consistent backpressure as the water column technique while reducing the amount of space required.

This prototype is intended to backpressure only one bioreactor at this time. A successful test would warrant increasing the number of bioreactors pressurized until system capacity limitations are realized. From any hardware, garden supply, or home improvement store a garden sprayer should be readily available. A garden sprayer consists of a canister typically capable of holding one to two gallons of fluid, a lid with built in pump mechanism, a hose with attached spray trigger and nozzle, and a pressure relief vent. Depending on the design, some of these sprayers have triggers on the pump handle that is attached to the lid - this sprayer design should not be used as it would require taping down the trigger for use without a laboratory assistant. This equipment is typically used for spraying insecticides or fertilizer around the garden or landscape. The pressure vent is a useful feature, but not entirely necessary. Most pressure vents will "pop" or relieve when 45 psi is exceeded within the canister. Some models have this feature and some do not. Regardless, some " $\mathrm{x}$ " number of pumps to pressurize the 
canister can be correlated with a target range for internal pressure using the data acquisition software if need be.

There are a couple of options when it comes to rigging the pressurized canister to the bioreactor. The spray hose can be entirely removed from the sprayer by unscrewing the connecting adaptor. Over the last few years, the hoses used in the system have become much more robust compared to the previous hoses which were not much different than the polymer tubing currently used in the tissue engineering laboratory. The more robust tubing is a little more difficult to work with, but it holds pressure well. If it is too difficult, it can be removed and a new adapter can be fitted to the system. They are typically $3 / 8$ inch to $1 / 4$ inch adaptors. Using tubing with dimensions similar to the silicone tubing of the bioreactor, the adaptor would need to be $3 / 8$ inch to $1 / 8$ inch unless the silicone can stretch to accommodate a slightly larger barb. The other option would require removing the spray wand from the tubing and adapting the original tubing to the next portion of the system.

Either way, the spray canister is then connected to a pressure regulator capable of operating within a 1-5 psi range. A 1-10 psi range would work too if the more precise regulator is not available. Since the goal is to backpressure the system to $100 \mathrm{mmHg}$, the regulator only needs to pressurize the system to 2 psi. Specific connectors and/or adaptors may need to be used to connect the equipment and tubing. There are specific flanged brass fittings used for gas lines and micro tubing that would need to be used upstream of regulator. This portion of the system would be subject to elevated pressures that the silicone tubing would not withstand (potentially $45 \mathrm{psi}$ ). The silicone tubing would 
balloon and either leak at the fittings or just be blown off altogether. Downstream of the regulator, typical bioreactor materials can be used.

The pressurized canister is then connected to a 3 port reservoir, not unlike the vented reservoir used in previous tests. Once the system is connected, the regulator can be adjusted to provide $2 \mathrm{psi}$ in the system. The regulator would be attached directly to the reservoir because the reservoir acts as the high point of the system and contains a pocket of gas. Once the regulator is opened, there may be an initial redistribution of gases within the system. Running the pump, or priming the pump, should reestablish standard conditions.

Using this technique, the bioreactor setup would not utilize the height of a water column to provide base-line, back pressure levels. The bioreactors and reservoirs would all be maintained on a level surface with backpressure now provided by virtue of pressurized air space in the reservoir.

The volume of air provided by the canister should be more than adequate to pressurize the system for an extended period of time. Tests can be run to quantify the duration of available supply, but even a one gallon canister should maintain pressure while making up losses due to leaks for several days. Also, while the canister can be pressurized to roughly $45 \mathrm{psi}, 10 \mathrm{psi}$ should easily support a trial. This pressure would be sufficient to easily maintain a 2 psi backpressure. Gases would likely bleed out of the system faster than fluid would (based on previous studies), but tests would have to be run in order to properly characterize the limitations of the setup. It would not be difficult to upgrade the reservoir to improve the seal. Other sources of compressed air are readily 
available and capable of sustaining the system for longer durations of time. This model is just good for assessing the theory. 


\section{Considerations for Future Bioreactor Pressurization}

Assuming that the pressurized canister works in pressuring the system, there are a variety of ways to produce a final design. There is a lot of room for customization. There is room to work with system configuration, sources of compressed air, air filtration, interface between air and fluid, and scalability.

The prototype design can easily be scaled up to pressurize multiple systems at once by simply branching the tubing downstream from the regulator off to the other systems. This can be space effective and well organized with a properly designed manifold. A silent lab bench air compressor could be used to provide a continuous source of compressed air and, if desired, can be connected to an air receiver in order to reduce cycling. Pneumatic syringes can be pressurized to deliver media at a rate to maintain 2 psi within the system. If multiple IV bags are used for reservoirs, they can all be placed inside a pressurized canister that would maintain the 2 psi for the system. Filters can be placed in-line downstream of the pressure regulator to provide sterile gas. There are many ways to go about optimizing this system, but using an air compressor, which only takes up 1 cubic foot of space, to pressurize the bioreactors indefinitely would be well worth it. 


\section{Backpressure Regulators}

The backpressure regulator is a potential solution for establishing a desired baseline pressure and minimizing consumed space. Marc Dawson used a ratchet clamp to restrict flow and back pressure the bioreactor system. The ratchet clamp was able to provide flow resistance and thereby elevate pressures within the systems, but the pressures were hard to accurately set or control. This led to further research of system back-pressuring options - which, in turn, led to research on controlling back pressure through the application of pressure regulating devices. A backpressure regulator is a valve that installs in line on a system subject to pressurization for the express purpose of maintaining a preset pressure upstream (Figure A.3). The back pressure regulator is placed downstream of components that require a nominal pressure higher than the normal unregulated line pressure. The regulator is set to a desired operating level and will then relieve as necessary to maintain desired inlet or upstream pressure. The back pressure regulator is also used to provide high pressure dissipation in order to protect sensitive equipment. If, for whatever reason, target pressure is exceeded, the backpressure regulator will open and release fluid from the system in order to restore the desired maximum pressure level. 


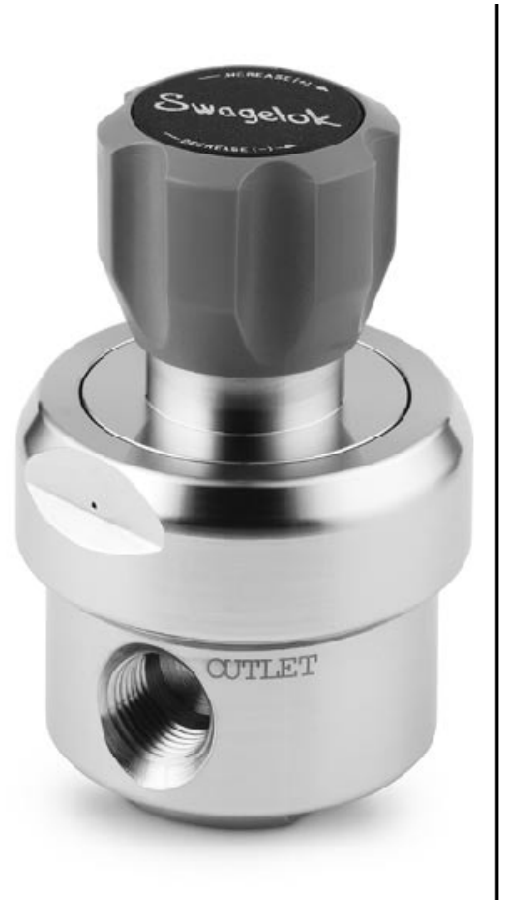

Materials of Construction

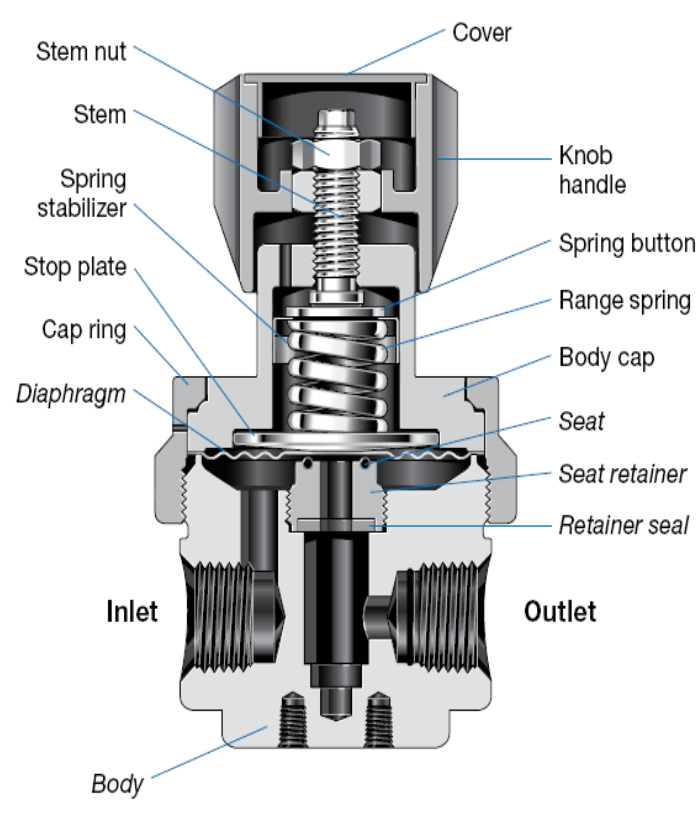

Figure A.3 Back-pressure regulator schematic.

For use in the Cal Poly system, the backpressure regulator would be connected inline downstream of the bioreactor chamber. The backpressure regulator is set by tightening or loosening its cap. Tightening the cap compresses a spring that seals off flow with a diaphragm. Higher pressures will raise the diaphragm and allow fluid flow. The regulators of interest are able to control pressures through a range of 0 to 10 psi. Roughly $50 \mathrm{mmHg}$ equates to $1 \mathrm{psi}$. The operational pressure would be at roughly $2 \mathrm{psi}$ in order to maintain a constant pressure of $100 \mathrm{mmHg}$.

In conversation with technical support from Swagelok, a company who carries a wide variety of backpressure regulators, the practicality of using such devices was reviewed. An estimate for a single regulator was $\$ 200$ to $\$ 300$ depending on material of construction. The company was unwilling to provide a sample regulator for testing 
purposes, but was open to the possibility of returning the regulator if it was not what the lab needed (a re-stocking fee would be assessed). The device is autoclavable with a maximum operating temperature of $200{ }^{\circ} \mathrm{C}$. Technicians from Swagelok were consulted specifically about the system configuration that was intended to be used. They did not feel that the regulator was sensitive enough to function properly at the range of physiological pressures that the system would be operated at. 2 psi is on the low end of the 0 to 10 psi regulatory ability of the device. In addition, the threading of the regulators determines the degree of control available when setting the pressure limit. Fine tuning on the scale of mmHg may be difficult. Therefore there are a number of potential factors that should be taken into consideration if backpressure regulators are considered for the bioreactor system.

While the backpressure regulator in principal may provide an effective solution, the devices readily available at a reasonable price are not sensitive enough to meet the requirements of lab's application. It would, however, be very interesting to see how well they work when there is more discretionary funding available. The backpressure regulator is still a potential solution for establishing a desired base-line pressure and minimizing consumed space, but the right specifications must be met. 


\section{Considerations for Bioreactor Chamber Modifications}

The redesigned bioreactor chamber also has room for improvement. The current Lock and Lock bioreactor system has potential to suffer from cell sedimentation during the early stages of vessel cultivation. The circular design of the newly designed bioreactor chamber would allow it to be rotated $360^{\circ}$. To facilitate this capability, a rack could be designed to suspend the chamber in an individual cradle and allow either manual or automatic rotation. Rotation of $360^{\circ}$ or any subset thereof could easily be managed. After $360^{\circ}$ of rotation was achieved, direction of rotation could be reversed and repeated - this way rotary unions are not required and the tubing will not twist excessively. If continual $360^{\circ}$ rotation is desired, rotary unions would have to be placed at both end of the bioreactor system. There are a number of such fittings on the market. Colder Products Company has a medical grade barbed swivel connector that would be compatible with the bioreactor system. The fitting is referred to as the SRC series (Figure A.4)

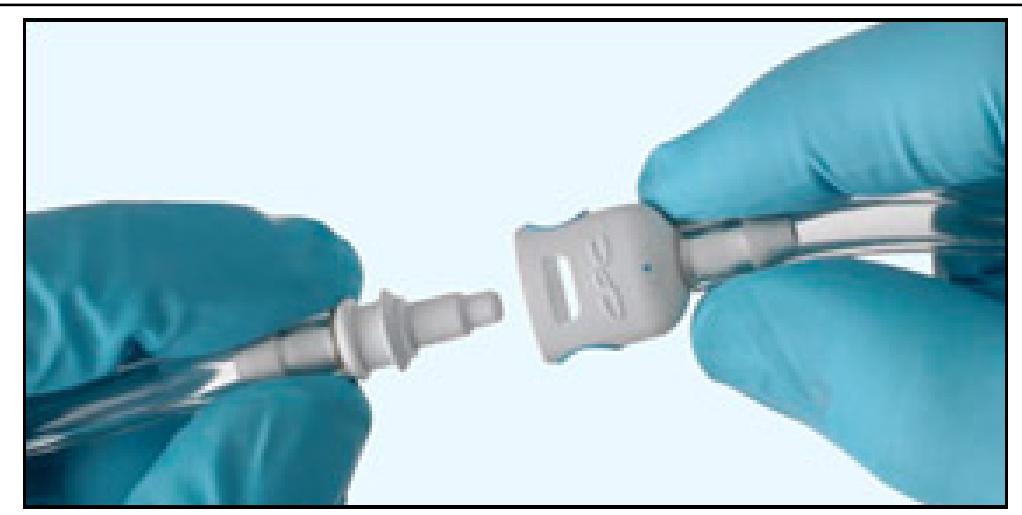

Figure A.4 SRC fitting from Colder Products Company. 
In addition, the panel mount luer lock fittings could be replaced. They are not intended to bridge dissimilar environments or maintain any sort of seal. They have worked well enough for laboratory purposes, but bulk head fittings would be better options. They are slightly more expensive, but they are intended to operate under conditions in which two different environments are bridged. The chamber end plates of the newly designed bioreactor chamber have a large enough diameter to accommodate a range of fitting dimensions. The flexible end plates of the bioreactor chamber could be replaced with more rigid materials to accommodate more substantial fittings if necessary. The other upside to the design is that the silicone discs could be made of a thinner, more compliant, and less stiff material. While pulsing pressure waves may cause slight mechanical stimulation, more mechanical stimulation could be applied to the system at later stages of vessel development. The flexible walls would accommodate the attachment of a mechanical apparatus intended to apply cyclic tensile loads to the vessel/scaffold.

Recent findings within the lab have indicated that the endothelial cells have been migrating into the scaffold. Whether this is due to pressure sodding or some other factor, it is unclear. It has been hypothesized that the stretching of the ePTFE scaffold during the loading process may be accountable. The flexible walls of the new bioreactor chamber may potentially alleviate the issue. The walls are flexible, and as such, they do not overly stretch the scaffold. 


\section{Considerations for Future Bioreactor Chamber Rack}

In an effort to propose future work for this project, initial research and design was performed for the development of a bioreactor chamber rack that would organize the bioreactors in a space saving arrangement, and rotate the chambers to prevent cell sedimentation. These are important parameters as the lab looks to scale up the number of potential tests that could be conducted at a single time. Dr. Kristen Cardinal used to periodically rotate the chambers by hand at set intervals when she was performing her research. This can be time consuming and space limiting depending on stack configuration and the number of chambers. The following design would eliminate the need for manual performance of such operations.

While the most recently designed bioreactor presented in this thesis has square end caps, the end caps could be modified to a circular geometry without much difficulty. The following bioreactor chamber rack design is based on the use of a bioreactor with circular end caps or, at least, circular projections from the end caps. The bioreactor would rest between two slotted walls made of delrin. Delrin, with the proper surface finish, is very slick. It is often used on production line conveyers to facilitate product movement down the line. Once the bioreactor chamber is placed with one end cap in both slots of the opposing walls, a guard is placed over the top of the bioreactor chamber. The guard is slotted just like the supporting walls, but it ensures that the bioreactor does not walk out of the rack as it rotates.

On one side of the rack, a compact DC gearbox motor is attached. These motors are relatively inexpensive, require 12 VDC to operate, and are capable of delivering 20$50 \mathrm{lbs}$ of torque at variable RPM (P/N 6409K13, McMaster-Carr). A carbon-zinc high 
voltage battery can power the system (P/N 7143K37, McMaster-Carr) - capable of delivering $45 \mathrm{~V}$. These powerful batteries take up minimal space (3.6" x 1.05 " x 0.63 "). The rotary motor will be linked with all of the bioreactor chambers using a gear and belt system - not unlike a bicycle chain. The bioreactors would have to be equipped with gears on one end, which is easily accomplished. The other option would be to use polyethylene belts. These industrial belts are basically large rubber-bands that are tubular. They coil around the driving motor and then loop over the various components of a system that are desired to turn. The bioreactors would then require a groove or slot to run around the entire circumference of the chamber to act as a guide for the belt. The belt then rotates the bioreactors based on the frictional interface between the rubber and the bioreactor material.

These materials and options are readily available and generally inexpensive. The last issue concerning the rotating bioreactors is the rotation itself. There are two different avenues to take: $180^{\circ}$ rotation, or $360^{\circ}$ rotation. Rotating the chamber only halfway around and then turning it back to its original position accomplishes all the motion necessary to prevent sedimentation. The problem here, however, is finding and equipping a rotary motor that oscillates between $180^{\circ}$ turns and the original set point. The advantage in having a bioreactor that only turns part of the way over and then reverses is that the tubes connecting to it will never tangle and expensive rotary unions are not required. This would justify using such a motor or system, but there is yet a simpler solution out there swivel connectors. There are a variety of plastic luer lock style fittings that are medical grade and designed to rotate $360^{\circ}$, as mentioned previously. They are generally used for medical equipment to prevent tangling or kinking tubing, but they would work just as 
well within the bioreactor system. Below is a diagram illustrating the proposed assembly and use of the bioreactor chamber rack (Figure A.5).

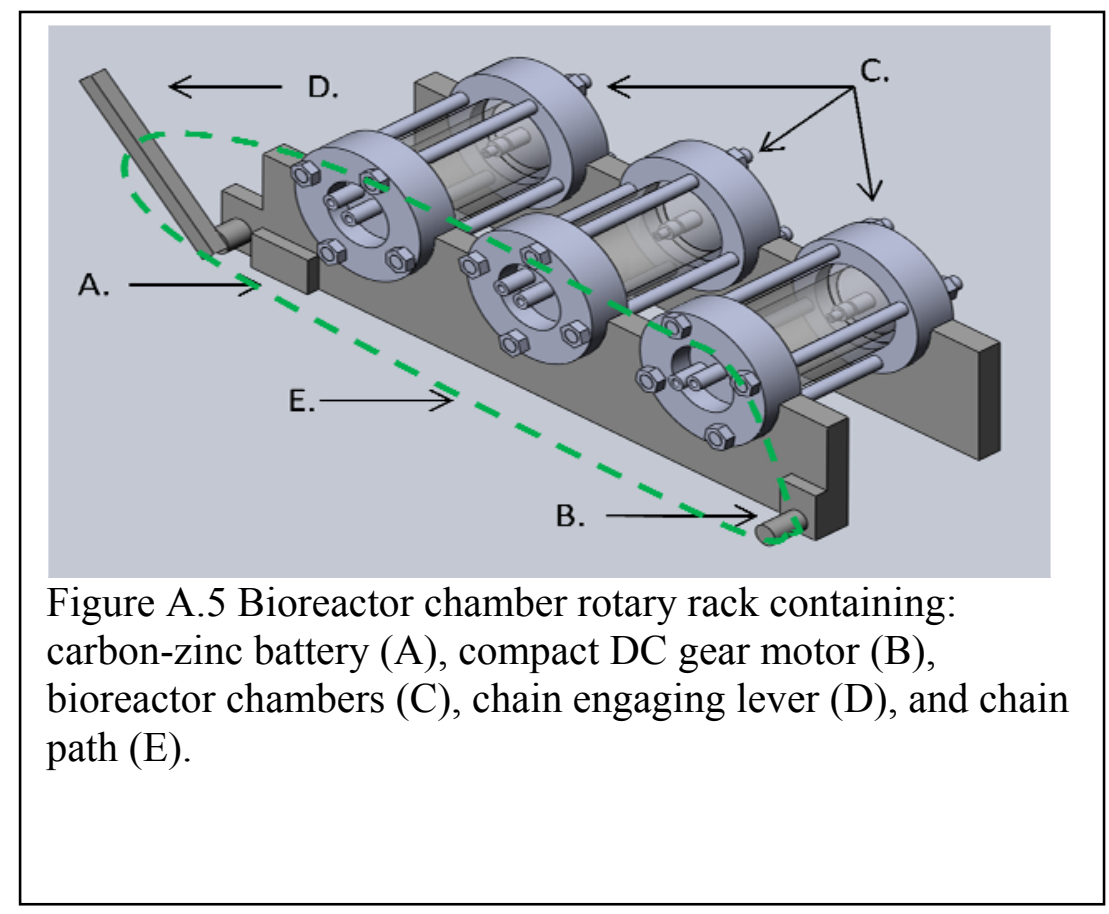

An additional modification that may be of interest may be to customize the bioreactor chamber rack to allow the bioreactor chambers to be tilted (Figure A.6). Currently, all of the bioreactors lay horizontal and parallel to the flat surface they are placed on. Accordingly, they have orientation in an $\mathrm{x}$ and $\mathrm{y}$ planes. The addition of a third dimension (z coordinate) would provide unique flow conditions that may better mimic the random geometries found within the human body. This can be done using the rotating bioreactor rack as well. The wall upon which the bioreactors rest would need to be affixed with a binder-style hinge (Figure A.6). Then, one end could be elevated by increasing the supporting wall's height. The height could be increased by either including adjustable legs or providing a stair-step graduation. As one end elevates, the hinges allow 
the bioreactor to tilt in accommodation. The slotted grooves and lock-bar would prevent the bioreactor chamber from falling. A basic illustration of this concept is included below.

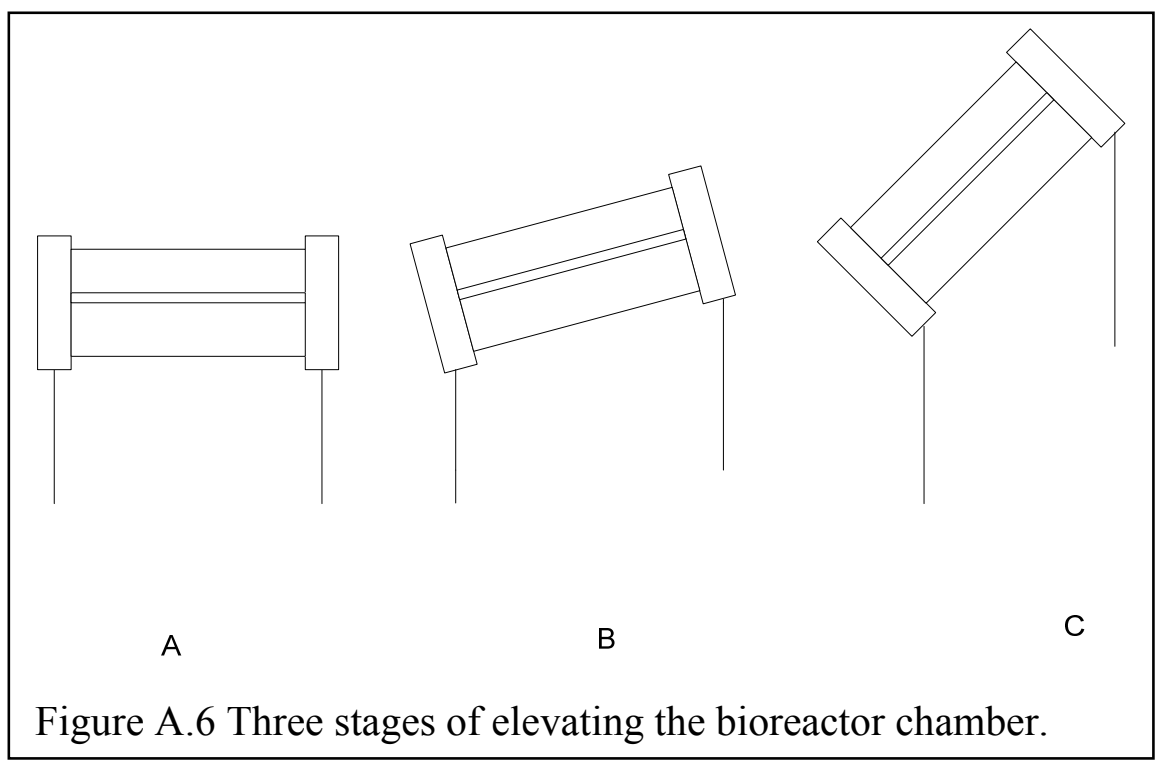




\section{Cautionary Note Regarding Bioreactor Stacking}

Using the incubator to house a large number of bioreactors is a long-term goal in the BVM laboratory. To accomplish this goal, the setup would have to be highly scalable and consume a minimal amount of space. In the past, this was accomplished this by stacking bioreactor chambers on top of one another. This exposes each chamber to varying pressures because of the transfer of loading forces in the stack. The chamber on bottom of the stack is compressed by the 2 fluid filled chambers on top of it. The solid wall reservoir would not compensate for the compression and the vessel would be exposed to elevated pressures. The height of the stack of bioreactor chambers also has a potentially significant drawback. The top chamber may be in a vacuum if it is above its corresponding reservoir. It would be interesting to see if stack order has influenced results at all. 